\title{
The Asiago Database on Photometric Systems (ADPS)^
}

\section{Census parameters for 167 photometric systems}

\author{
D. Moro ${ }^{1,2}$ and U. Munari $\mathbf{i}^{1,3}$ \\ 1 Osservatorio Astronomico di Padova, Sede di Asiago, I-36012 Asiago (VI), Italy \\ 2 Osservatorio Astrofisico di Asiago, Dipartimento di Astronomia, Università di Padova, I-36012 Asiago (VI), Italy \\ ${ }^{3}$ CISAS, Centro Interdipartimentale di Studi ed Attività Spaziali, Padova, Italy
}

Received May 9; accepted August 14, 2000

\begin{abstract}
The Asiago Database on Photometric Systems (ADPS) is a compilation of basic information and reference data on 167 optical, ultraviolet and infrared photometric systems. Thirty-four additional systems are briefly described. In compiling this census we have relied on published information only. In Paper II the photometric systems will be inter-compared, calibrated and parameterised by means of synthetic photometry using uniform criteria and the same set of input spectra and extinction laws.
\end{abstract}

Key words: photometry — astronomical data bases surveys

\section{Introduction}

When we became involved in the design of the photometric system for GAIA, which is under evaluation by ESA as the next Cornertone 5 mission, we realized how useful it would have been to have a nearly complete census of the existing ground-based and space-born photometric systems. The completion of the Asiago proposal for the GAIA photometric system (Munari 1998) was originally conceived to follow the realization of the Asiago Database on Photometric Systems (ADPS), but actually the reverse happened because the preparation of ADPS has been a tougher task than anticipated. This paper intends to summarise this extensive census in an interesting way for the general reader.

Some compilations of photometric systems have already been presented, among others by Lamla (1982),

\footnotetext{
Send offprint requests to: U. Munari;

e-mail: munari@pd.astro.it

* The ADPS is reachable at

http://ulisse.pd.astro.it/Astro/ADPS/ or via

http://www.pd.astro.it/
}

Bessell (1993), in the books by Golay (1974) and Straižys (1995) and on the world wide web by Mermilliod et al. (1997). The latter focuses mainly on the data collected in various photometric systems (with entries for more than $210^{5}$ stars). At the same time, the Mermilliod et al. www version available at the time of submission of the ADPS provides a census of photometric systems by listing and describing 82 of them. Even if our compilation was independent, we undoubtedly benefited by checking against Mermilliod et al.'s work.

In compiling the ADPS we have tried to be as complete as possible for the optical region (from 0.3 to $1.0 \mu \mathrm{m}$, where GAIA is currently planned to collect data), but effort has also been made to include ultraviolet as well as infrared photometric systems (even if at a lower degree of completeness). The ADPS www site will be regularly updated as new information becomes available.

In this paper we rely only on data from literature (instrumental set-ups, transmission curves, calibration and transformation functions, zero points, etc.), with preference for those provided by the authors of the photometric systems in the original papers. Unfortunately, accurate and complete information is available only for a small minority of the surveyed systems. We have been able to locate in literature (or derive ourselves from published graphs) the tabular band transmission profiles for 105 systems. Calibration and transformation functions with any kind of accuracy have been published only for a fraction of the 167 surveyed systems. Even when calibration and transformation functions are available, it is not always easy to inter-compare them because of the heterogeneous methods adopted by the different authors (see Sect. 2). As a contribution toward a clarification of the whole issue, in a forthcoming Paper II we will inter-compare, calibrate and parameterise the systems by means of synthetic photometry using uniform criteria and the same set of synthetic and observed input spectra and extinction laws. 
GAIA is a candidate ESA Cornerstone 5 mission designed to obtain extremely precise astrometry (in the micro-arcsec regime), multi-band photometry and medium/high resolution spectroscopy for a large sample of stars. The goals as depicted in the mission Red Book call for astrometry and broad band photometry to be collected for all stars down to $V \sim 20$ mag over the entire sky ( $\sim 10^{9}$ stars), with brighter magnitude limits for spectroscopy and intermediate band photometry. Each target star should be measured over a hundred times during the five year mission life-time, in a fashion similar to the highly successful Hipparcos operational mode. The astrophysical guidelines of the GAIA mission are discussed by Gilmore et al. (1998, and references therein) and Perryman (1999). An overview of the GAIA payload and spacecraft is presented by Mérat et al. (1998).

\section{The Database}

The 167 systems included in the ADPS are listed in Table 1, in order of publication year. The systems are documented by individual figures that conform to the common layout.

The reference sources are given in square brackets for all the information provided with a given photometric system and are also listed in Table 2. No information is entered if not present in literature. The information provided with the systems surveyed in the ADPS are grouped into five main blocks:

System Code Name. This is formed from the system name generally used in the literature, the author(s) name(s) and the year of publication of the original paper introducing the system. A short sentence describing the system main usage or aim follows (normally as given by the authors themselves).

General Information. The authors of the system (not listed for space missions), the telescope and detector originally adopted, and the main reference article are given here. The main reference article is generally the one introducing the photometric system; however, some of these historical articles contain little information and in such cases a more recent, informative paper is taken instead.

System Description. Basic information like the filters used, the wavelength and width of the bands, the zero points and the flux calibrations are reported here. The literature is heterogeneous regarding these parameters, with values sometimes referring only to the filters, or to the filters+detector combination or also including the contribution of the telescope optics and atmosphere. So different quantities as $\lambda_{\text {peak }}$ (peak transmission), $\lambda_{\mathrm{c}}$ (unspecified band "centre"), $\lambda_{\text {eff }}$ (generally without specification of the energy distribution of the input source weighting the transmission profile) or $\lambda_{0}$ (again unspecified) are provided by the authors of the photometric systems as the wavelength of the bands. The situation is somewhat more confused when it comes to the width of the bands: the authors have used values ranging from the FWHM of a Gaussian approximation to the band, to something resembling the full width at zero transmission, or half of the total width at $50 \%$ transmission, or even unspecified concepts as "band-width", "band-pass" or "half width". There are cases where the values given for the wavelength and width of the bands are missing or they disagree with the plotted and/or tabulated transmission profiles. In such cases we have computed on the transmission profiles two quantities and reported them in the ADPS with the aim of clarifying the situation: the WHM (width at half maximum: the full wavelength span between the points where the transmission reaches half of the maximum value) and $\lambda_{\mathrm{c}}$ (central lambda: the wavelength halfway between these half-maximum transmission points). In Paper II we plan to homogenise and standardise the situation regarding the wavelength and width of the bands for all systems with published transmission curves by means of synthetic photometry against the same set of observed and synthetic spectra. Finally, for some systems references are also provided to papers specifically devoted to the porting of the system to a different detector (like photographic plates or CCDs for systems originally designed for photo-multipliers).

System Analysis. This section deals with assorted information like the most frequently used colour indices and their aim (preserving as much as possible the terminology used by the original authors), reddening-free parameters, reddening ratios and transformation to other systems. Transformations are only to chronologically earlier systems in order to avoid duplication.

Transmission Curves. The last section is devoted to the presentation of the band transmission profiles both in graphical and tabular form. The transmission curves are not plotted and tabulated if they refer to square bands (essentially flat 1.0 transmission within the band and 0.0 outside), such as those provided by diaphragms placed on the focal plane of a spectrograph in front of a photo-multiplier tube or the square bands synthesised from fluxed digital spectra. When the transmission curves have been plotted but not tabulated by the authors, we have reconstructed them by measuring the plotted profile. In such a case, the reader is warned by a sentence like "As derived from Fig. Y of $[\mathrm{XXX}]$ ".

\section{Additional systems}

In this section 34 additional systems are briefly described. These systems do not appear in the main article body because - to our own judgment - they (a) lack some or most of the basic information, and/or (b) have been poorly documented, and/or $(c)$ had little or no follow-ups in the literature, and/or $(d)$ have been applied to very few objects, if not to just a single one. Finally, $(e)$ some 
Table 1. List of the photometric systems included in the Asiago Database on Photometric Systems (ADPS). The fourth column gives the number of bands, the fifth the figure which describes the system and the sixth lists if the band transmission profiles are given in the ADPS (the systems with square bands are labelled "sqr")

\begin{tabular}{|c|c|c|c|c|c|}
\hline system & year & authors & 1. of bands & fig. & res. \\
\hline$C_{1}$ & 1940 & Stebbins et al. & 2 & 1 & \\
\hline UVBGRI & 1943 & Stebbins and Withford & 6 & $2 a, b$ & yes \\
\hline$R G U$ & 1946 & Becker & 3 & $3 a, b$ & yes \\
\hline$R I$ & 1951 & Kron and Smith & 2 & $4 a, b$ & yes \\
\hline $\mathrm{BCD}$ & 1952 & Chalonge and Divan & 3 & 5 & \\
\hline$U B V$ & 1953 & Johnson and Morgan & 3 & $6 \mathrm{a}, \mathrm{b}, \mathrm{c}$ & yes \\
\hline POSS I & 1955 & & 2 & 7 & yes \\
\hline$P V$ & 1955 & Eggen & 2 & $8 \mathrm{a}, \mathrm{b}$ & yes \\
\hline Aerobee UV-55 & 1955 & & 2 & 9 & yes \\
\hline uvby $H \beta$ & 1956 & Strömgren and Crawford & 6 & $10 \mathrm{a}, \mathrm{b}, \mathrm{c}$ & yes \\
\hline Aerobee UV-57 & 1957 & & 1 & 11 & \\
\hline$U_{c} B V$ & 1958 & Arp & 3 & $12 \mathrm{a}, \mathrm{b}$ & yes \\
\hline ubgyri & 1958 & Bahng & 6 & $13 \mathrm{a}, \mathrm{b}$ & yes \\
\hline$U V B G R$ & 1958 & Tifft & 5 & $14 \mathrm{a}, \mathrm{b}$ & yes \\
\hline 5 colors & 1959 & Borgman & 5 & $15 \mathrm{a}, \mathrm{b}$ & yes \\
\hline \multirow{2}{*}{$K L M N P Q R$} & 1960 & Borgman & 7 & $16 \mathrm{a}, \mathrm{b}$ & yes \\
\hline & 1960 & Deeming & 3 & 17 & sqr \\
\hline \multirow[t]{2}{*}{$U B V$} & 1960 & Eggen and Sandage & 3 & 18 & \\
\hline & 1960 & Griffin and Redman & 6 & 19 & sqr \\
\hline USNO & 1960 & Kron and Mayall & 3 & 20 & \\
\hline \multirow[t]{2}{*}{$V B L U W$} & 1960 & Walraven and Walraven & 5 & $21 a, b$ & yes \\
\hline & 1961 & Griffin & 3 & 22 & sqr \\
\hline 8 colors & 1961 & Tifft & 8 & $23 a, b$ & yes \\
\hline$H \gamma$ & 1962 & Bappu et al. & 3 & 24 & \\
\hline Geneva & 1962 & Golay & 7 & $25 \mathrm{a}, \mathrm{b}$ & yes \\
\hline \multirow[t]{2}{*}{$u b v r_{20}$} & 1963 & Sandage and Smith & 4 & $26 a, b$ & yes \\
\hline & 1964 & Barbier and Morguleff & 8 & 27 & sqr \\
\hline$H \gamma$ & 1964 & Beer & 3 & 28 & sqr \\
\hline$H \alpha$ & 1964 & Peat & 1 & 29 & sqr \\
\hline Aerobee UV-64 & 1964 & & 4 & 30 & \\
\hline$H \alpha, \beta, \gamma, \delta$ & 1965 & Abt and Golson & 8 & 31 & \\
\hline$R I$ & 1965 & Eggen & 2 & 32 & \\
\hline \multirow[t]{2}{*}{$U B V R I(J H K L M N)$} & 1965 & Johnson & 11 & $33 a, b, c$ & yes \\
\hline & 1965 & Miner & 7 & 34 & \\
\hline Vilnius & 1965 & Straižys et al. & 8 & $35 \mathrm{a}, \mathrm{b}$ & yes \\
\hline$U V B Y$ & 1966 & Kruszewski & 4 & $36 a, b$ & yes \\
\hline \multirow[t]{2}{*}{4 colors } & 1966 & Neff and Travis & 4 & 37 & yes \\
\hline & 1966 & Scarfe & 22 & 38 & sqr \\
\hline$u b V t$ & 1966 & Smak & 6 & 39 & yes \\
\hline$u b v r$ & 1966 & Westerlund & 4 & 40 & \\
\hline 12 colors & 1966 & Wood & 12 & $41 \mathrm{a}, \mathrm{b}, \mathrm{c}$ & yes \\
\hline \multirow[t]{2}{*}{ ri } & 1967 & Argue & 2 & $42 \mathrm{a}, \mathrm{b}$ & yes \\
\hline & 1967 & Boyce & 6 & 43 & sqr \\
\hline 6265102 & 1967 & Eggen & 3 & $44 \mathrm{a}, \mathrm{b}$ & yes \\
\hline LPL & 1967 & Johnson et al. & 8 & $45 \mathrm{a}, \mathrm{b}$ & yes \\
\hline 27 colors & 1967 & Wing & 27 & 46 & sqr \\
\hline $\mathrm{H} \alpha$ & 1968 & Andrews & 3 & 47 & sqr \\
\hline DDO & 1968 & McClure and Van den Bergh & 7 & $48 \mathrm{a}, \mathrm{b}, \mathrm{c}$ & yes \\
\hline$H \beta, \gamma$ & 1968 & Sinnerstad et al. & 4 & $49 \mathrm{a}, \mathrm{b}$ & yes \\
\hline u'ubvv' & 1968 & Smith & 5 & 50 & \\
\hline jhk & 1969 & Bahng & 3 & 51 & \\
\hline \multirow[t]{2}{*}{ Ca II K } & 1969 & Henry & 2 & 52 & sqr \\
\hline & 1969 & Newell et al. & 4 & $53 \mathrm{a}, \mathrm{b}$ & yes \\
\hline
\end{tabular}


Table 1. continued

\begin{tabular}{|c|c|c|c|c|c|}
\hline system & year & authors & nds & fig. & res. \\
\hline & 1969 & Spinrad and Taylor & 33 & 54 & sqr \\
\hline$H \alpha, \beta$ & 1969 & Tebbe & 4 & 55 & \\
\hline gnkmfu & 1970 & Dickow et al. & 6 & 56 & \\
\hline Uppsala & 1970 & Häggkvist and Oja & 3 & 57 & \\
\hline$R I$ & 1970 & Jacobsen & 2 & $58 \mathrm{a}, \mathrm{b}$ & yes \\
\hline nh & 1970 & Landolt & 2 & 59 & \\
\hline & 1970 & Morguleff et al. & 12 & 60 & sqr \\
\hline OAO2 WEP & 1970 & & 16 & 61 & \\
\hline $\mathrm{H} \gamma$ & 1971 & Häggkvist & 3 & 62 & \\
\hline DAO & 1971 & Hill et al. & 6 & 63 & sqr \\
\hline 5 colors & 1971 & Lockwood and Wing & 5 & 64 & \\
\hline & 1971 & Mendoza & 3 & 65 & yes \\
\hline & 1971 & Williams et al. & 9 & 66 & sqr \\
\hline 8 colors & 1971 & Wing & 8 & 67 & \\
\hline Aerobee IR-71 & 1971 & & 4 & 68 & \\
\hline & 1972 & Jones and Dixon & 4 & 69 & \\
\hline$H \alpha, \beta$ & 1972 & Peton et al. & 5 & $70 \mathrm{a}, \mathrm{b}$ & yes \\
\hline$u_{A} b_{A} \beta_{A} y_{A}$ & 1972 & Wickramasinghe and Strittmatter & 4 & 71 & \\
\hline TD1 & 1972 & & 25 & 72 & sqr \\
\hline 24 colors & 1973 & Chapman et al. & 24 & 73 & \\
\hline 10 colors & 1973 & Faber & 10 & 74 & \\
\hline$J H K L$ SAAO & 1973 & Glass & 7 & $75 \mathrm{a}, \mathrm{b}$ & yes \\
\hline$W B V R$ & 1973 & Straižys & 4 & $76 a, b$ & yes \\
\hline MSSO & 1973 & Thomas et al. & 11 & 77 & \\
\hline & 1974 & Alexander and Branch & 3 & 78 & \\
\hline$U B V R$ & 1974 & Cathey & 4 & 79 & \\
\hline$H \alpha, \beta, \gamma$ & 1974 & Feinstein & 6 & 80 & \\
\hline UBVRI & 1974 & Fernie & 5 & 81 & \\
\hline$H \alpha, \beta$ & 1974 & Guinan and McCook & 4 & 82 & \\
\hline$N Q$ & 1974 & Low and Rieke & 2 & 83 & yes \\
\hline $\mathrm{H} \alpha$ & 1974 & Vidal & 2 & 84 & yes \\
\hline ANS & 1974 & & 6 & $85 a, b$ & yes \\
\hline $\mathrm{H} \alpha$ & 1975 & Dachs and Schmidt-Kaler & 2 & 86 & \\
\hline & 1975 & Helt and Gyldenkerne & 9 & 87 & \\
\hline UBViyz & 1975 & Jennens and Helfer & 6 & 88 & \\
\hline 8 colors & 1975 & Morguleff et al. & 8 & 89 & sqr \\
\hline ri & 1975 & Weistrop & 2 & 90 & \\
\hline D2B AURA & 1975 & & 4 & 91 & sqr \\
\hline Washington & 1976 & Canterna & 4 & $92 \mathrm{a}, \mathrm{b}, \mathrm{c}$ & yes \\
\hline$R I$ & 1976 & Cousins & 2 & 93 a,b,c & yes \\
\hline uvby & 1976 & Eggen & 4 & 94 & \\
\hline 13 colors & 1976 & Johnson and Mitchell & 13 & $95 \mathrm{a}, \mathrm{b}, \mathrm{c}$ & yes \\
\hline $\operatorname{vbyg}_{1} g_{2}$ & 1976 & Maitzen & 5 & $96 a, b$ & yes \\
\hline uvgr & 1976 & Thuan and Gunn & 4 & $97 \mathrm{a}, \mathrm{b}$ & yes \\
\hline Apollo-Soyuz & 1976 & & 5 & 98 & \\
\hline Trieste & 1977 & Cester et al. & 8 & $99 a, b$ & yes \\
\hline $\mathrm{JHK} \mathrm{H}_{2} \mathrm{O} \mathrm{CO}$ & 1977 & Persson et al. & 6 & $100 \mathrm{a}, \mathrm{b}$ & yes \\
\hline$u b V r$ & 1978 & Sandage and Visvanathan & 4 & 101 & \\
\hline 8 colors & 1978 & White and Wing & 8 & 102 & \\
\hline$U^{\prime} J F$ & 1979 & van der Kruit & 3 & $103 \mathrm{a}, \mathrm{b}$ & yes \\
\hline$B V R I$ & 1979 & Kunkel and Rydgren & 4 & 104 & \\
\hline ri & 1979 & Wade et al. & 2 & 105 & \\
\hline$B_{J} R_{F}$ & 1980 & Couch and Newell & 2 & $106 \mathrm{a}, \mathrm{b}$ & yes \\
\hline UBVRI & 1980 & Neckel and Chini & 5 & 107 & \\
\hline uvgr $39_{B} 39_{N}$ & 1980 & Zinn & 6 & $108 \mathrm{a}, \mathrm{b}$ & \\
\hline VJHKLM ESO & 1981 & Engels et al. & 6 & 109 & \\
\hline$\Delta a$ & 1981 & Joncas and Borra & 3 & 110 & yes \\
\hline
\end{tabular}


Table 1. continued

\begin{tabular}{|c|c|c|c|c|c|}
\hline system & year & authors & nds & fig. & res. \\
\hline & 1981 & Jones et al. & 4 & $111 \mathrm{a}, \mathrm{b}$ & yes \\
\hline$H \alpha$ & 1981 & Strauss and Ducati & 2 & 112 & \\
\hline JHKL CTIO & 1982 & Elias et al. & 4 & 113 & \\
\hline JHKL MSO & 1982 & Jones and Hyland & 3 & 114 & yes \\
\hline \multirow[t]{2}{*}{ VilGen } & 1982 & North et al. & 7 & $115 \mathrm{a}, \mathrm{b}$ & yes \\
\hline & 1982 & Solheim et al. & 10 & $116 \mathrm{a}, \mathrm{b}$ & yes \\
\hline 8 colors & 1982 & Tedesco et al. & 8 & 117 & \\
\hline$J H K L '$ AAO & 1983 & Allen and Cragg & 4 & 118 & \\
\hline$U B V R I$ & 1983 & Landolt & 5 & $119 a, b$ & yes \\
\hline griz & 1983 & Schneider et al. & 4 & 120 & yes \\
\hline$R G U$ & 1983 & Trefzger $e t a l$. & 3 & 121 & yes \\
\hline IRAS & 1983 & & 5 & $122 \mathrm{a}, \mathrm{b}$ & yes \\
\hline VWFSC Spacelab 1 & 1983 & & 3 & 123 & yes \\
\hline JHKLMNQ OAN & 1984 & Roth et al. & 13 & 124 & \\
\hline$W B V R$ & 1985 & Khalliullin et al. & 4 & $125 \mathrm{a}, \mathrm{b}$ & yes \\
\hline \multirow[t]{3}{*}{$J_{n} K_{n} L_{n} M_{n}$} & 1986 & Leggett et al. & 4 & $126 \mathrm{a}, \mathrm{b}$ & yes \\
\hline & 1986 & Park and Lee & 5 & $127 \mathrm{a}, \mathrm{b}$ & yes \\
\hline & 1987 & Kenyon and Fernandez-Castro & 8 & 128 & sqr \\
\hline$\psi(25)$ & 1987 & Mendoza & 3 & 129 & yes \\
\hline VJHKLL'M & 1988 & Bessell and Brett & 8 & $130 \mathrm{a}, \mathrm{b}$ & yes \\
\hline 7781 & 1989 & Cook and Aaronson & 2 & 131 & \\
\hline$g_{4} r_{4} i_{4} z_{4}$ & 1989 & Schneider et al. & 4 & 132 & yes \\
\hline FOC HST & 1989 & & 44 & $133 \mathrm{a}, \mathrm{b}$ & yes \\
\hline WFPC1 HST & 1989 & & 21 & $134 \mathrm{a}, \mathrm{b}$ & yes \\
\hline Hipparcos & 1989 & & 3 & $135 \mathrm{a}, \mathrm{b}$ & yes \\
\hline UBVRI & 1990 & Bessell & 5 & $136 \mathrm{a}, \mathrm{b}$ & yes \\
\hline$J H K L$ SAAO & 1990 & Carter & 4 & 137 & \\
\hline Guide Star Catalogue & 1990 & Lasker et al. & 4 & 138 & \\
\hline UIT & 1990 & & 12 & $139 a, b$ & yes \\
\hline$J H K L ' M$ ESO & 1991 & Bouchet et al. & 5 & $140 a, b$ & \\
\hline POSS II & 1991 & Reid et al. & 3 & 141 & yes \\
\hline CaII & 1991 & Twarog et al. & 2 & 142 & yes \\
\hline 20 colors & 1992 & Bastiaansen & 20 & $143 \mathrm{a}, \mathrm{b}$ & yes \\
\hline MACHO & 1992 & & 2 & 144 & yes \\
\hline SCAS & 1993 & Clark et al. & 7 & $145 a, b$ & yes \\
\hline WFPC2 HST & 1993 & & 41 & $146 \mathrm{a}, \mathrm{b}, \mathrm{c}$ & yes \\
\hline$J H K L '$ CST & 1994 & Alonso et al. & 4 & $147 \mathrm{a}, \mathrm{b}, \mathrm{c}$ & yes \\
\hline DENIS & 1994 & Epchtein et al. & 3 & $148 \mathrm{a}, \mathrm{b}$ & yes \\
\hline JHKL MSSSO & 1994 & McGregor & 4 & $149 a, b$ & yes \\
\hline IRTF NSFCAM & 1994 & Shure et al. & 18 & $150 a, b$ & yes \\
\hline FPBS & 1995 & Brewer et al. & 4 & 151 & \\
\hline ISOCAM ISO & 1995 & & 21 & $152 \mathrm{a}, \mathrm{b}, \mathrm{c}$ & yes \\
\hline BATC & 1996 & Fan et al. & 15 & $153 \mathrm{a}, \mathrm{b}$ & yes \\
\hline Sloan DSS & 1996 & Fukugita el al. & 5 & $154 \mathrm{a}, \mathrm{b}$ & yes \\
\hline StrömVil & 1996 & Straižys et al. & 7 & $155 \mathrm{a}, \mathrm{b}$ & yes \\
\hline ESO MIR & 1996 & Van der Bliek et al. & 5 & 156 & \\
\hline ESO NIR & 1996 & Van der Bliek et al. & 5 & 157 & \\
\hline \multirow[t]{2}{*}{ MANIAC } & 1997 & Böker et al. & 14 & 158 & \\
\hline & 1997 & Damineli et al. & 3 & 159 & yes \\
\hline TNG & 1997 & Marchetti et al. & 8 & 160 & \\
\hline $\mathrm{UBV}(\mathrm{RI})_{M W}$ & 1997 & Sandage & 5 & 161 & \\
\hline UWTAT & 1997 & Strassmeier et al. & 13 & 162 & \\
\hline NICMOS HST & 1997 & & 32 & $163 \mathrm{a}, \mathrm{b}, \mathrm{c}$ & yes \\
\hline \multirow[t]{2}{*}{ STIS HST } & 1997 & & 16 & $164 a, b$ & yes \\
\hline & 1998 & Royer et al. & 5 & 165 & \\
\hline Asiago GAIA & 1998 & Munari & 10 & $166 \mathrm{a}, \mathrm{b}, \mathrm{c}$ & yes \\
\hline Geneva GAIA & 1999 & Grenon et al. & 14 & $167 \mathrm{a}, \mathrm{b}$ & yes \\
\hline
\end{tabular}


systems based on spectral scanner data seem to resemble the classification of very-low dispersion spectra more than the conventional photometric techniques.

After the author name, year of publication and the reference paper (for the reference code numbers see Table 2), for each of the 34 additional systems three types of basic information are given. "Instr:" reports if the system has been obtained with a photometer (+filters) or if it has been designed on spectral scanner data. "Features:" lists the system's goals and the lines/bands/continuum the system is aimed to. Finally, "Bands:" gives the wavelength and width of the system bands.

Kraft - 1960 [170] Instr: photometer. Features: $G$-band in $F-G$ stars. Bands: at $4305(\Delta \lambda=10 \AA)$ and $4290 \AA$ $(\Delta \lambda=200 \AA)$.

Gutierrez-Moreno et al. - 1967 [129] Instr: spectral scanner. Features: $\mathrm{H} \beta, \mathrm{H} \gamma, \mathrm{H} \delta$ in B-A stars. Bands: set by the $50 \div 80 \AA$ slit width.

McNamara et al. - 1970 [205] Instr: photometer. Features: CaII K, $\mathrm{H} \delta$ and $G$-band in RR Lyr stars. Bands: at 3933, 4100 and $4305 \AA(\Delta \lambda=30 \AA$ for all of them).

Wawrukiewicz - 1971 [268][315] Instr: photometer. Features: $\mathrm{TiO}$ and $\mathrm{CN}$ in $\mathrm{M}$ stars. Bands: at 7125 $(\Delta \lambda=55 \AA), 7170(\Delta \lambda=302 \AA)$ and two at $7935 \AA$ $(\Delta \lambda=135$ and $500 \AA)$.

Lutz and Lutz - 1972 [194] Instr: photometer. Features: $\mathrm{H} \gamma$ in O-F stars. Bands: two at $\mathrm{H} \gamma(\Delta \lambda=30$ and $150 \AA$ ).

Seeds - 1972 [265] Instr: photometer. Features: $\mathrm{C}_{2}$ in Carbon stars. Bands: at 5165, 5635, 5300 and $5780 \AA$ $(\Delta \lambda=100 \AA$ for all of them).

Caplan - 1973 [59] Instr: spectral scanner. Features: $\mathrm{H} \alpha$ in G-M stars. Bands: at $6548(\Delta \lambda=24 \AA), 6563$ $(\Delta \lambda=4.9 \AA)$ and $6577 \AA(\Delta \lambda=24 \AA)$.

Cherepashchuk and Khaliullin - 1973 [67] Instr: photometer. Features: continuum in Wolf Rayet stars. Bands: at $4244(\Delta \lambda=49.5 \AA), 4789(\Delta \lambda=61 \AA), 5806(\Delta \lambda=$ $95 \AA), 6320(\Delta \lambda=91 \AA)$ and $7512 \AA(\Delta \lambda=109 \AA)$.

Jones and Carrick - 1973 [159] Instr: spectral scanner. Features: $\mathrm{H} \alpha$ and $\mathrm{H} \beta$ in early type stars. Bands: at 4761 , $4861,4961,6487,6563$ and $6642 \AA(\Delta \lambda=30.6 \AA$ for all of them).

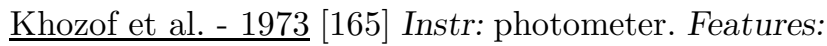
$\mathrm{H}^{-}$opacity in A-M stars. Bands: $h^{\prime}(1.58 \mu \mathrm{m}), h^{\prime \prime}$ $(1.70 \mu \mathrm{m})$ and $k(2.15 \mu \mathrm{m})$.

Faÿ et al. - 1974 [104] Instr: spectral scanner. Features: $\mathrm{Na} \mathrm{I}, \mathrm{C}_{2}$ and $\mathrm{CN}$ in carbon stars. Bands: $20 \AA$-width bandpasses between 5000 and $7000 \AA$, with $5610,5710,5730,5820$ and 6780 as the most relevant wavelengths.

Gustafsson et al. - 1974 [127][128][231] Instr: echelle spectrophotometer. Features: Metallicity and microturbolence in F-G-K stars. Bands: at 4794.5, 4799.5, 4897.0, 4911.0, 5801.5 and $5805.5 \AA(\Delta \lambda=3 \AA$ for all of them).
Nissen - 1974 [232][233] Instr: echelle spectrophotometer. Features: HeI (4026 $\AA$ ) in B dwarfs. Bands: at 4016 $(\Delta \lambda=6 \AA), 4026(\Delta \lambda=14 \AA)$ and $4036(\Delta \lambda=6 \AA)$.

Sorvari - 1974 [274] Instr: photometer. Features: OI $7772 \AA$ in A-F stars. Bands: two at $7772 \AA(\Delta \lambda=10$ and $60 \AA$ ).

Greenstein - 1976 [121] Instr: multichannel spectrophotometer. Features: classification of WDs. Bands: at $3571,4255,4717,5405,6579,6944,8000$ and $10000 \AA$. [168] adopted somewhat different wavelengths.

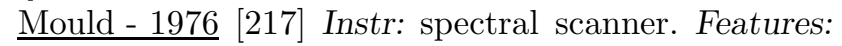
$\mathrm{CaH}$ and $\mathrm{TiO}$ in $\mathrm{M}$ dwarfs. Bands: at 6540, 6830, 7035, 7100, 7500, 7812 and $8130 \AA$. [218] has slightly modified the bands to investigate T Tau stars of the M type.

Dzervitis - 1977 [89] Instr: photometer. Features: classification of carbon stars via $\mathrm{CN}$ and $\mathrm{C}_{2}$ absorption bands. Bands: at $5135(\Delta \lambda=70 \AA), 5300(\Delta \lambda=200 \AA), 6775$ $(\Delta \lambda=250 \AA), 7785(\Delta \lambda=170 \AA), 8025(\Delta \lambda=250 \AA)$ and $8850 \AA(\Delta \lambda=300 \AA)$.

Yamashita et al. - 1977 [333] Instr: multichannel spectrophotometer. Features: Classification of Carbon stars via BaII, LiI, NaI lines and $\mathrm{CN}, \mathrm{C}_{2}$ bands. Bands: at 4160 $(\Delta \lambda=80 \AA), 4227(\Delta \lambda=10 \AA), 4234(\Delta \lambda=30 \AA), 4237$ $(\Delta \lambda=30 \AA), 4554(\Delta \lambda=10 \AA), 4575(\Delta \lambda=10 \AA), 5150$ $(\Delta \lambda=10 \AA), 5193(\Delta \lambda=10 \AA), 5893(\Delta \lambda=15 \AA), 5893$ $(\Delta \lambda=150 \AA), 6708(\Delta \lambda=8 \AA)$ and $6708 \AA(\Delta \lambda=30 \AA)$.

Mould and McElroy - 1978 [219] Instr: spectral scanner. Features: $\mathrm{CaH}$ and $\mathrm{TiO}$ bands in $\mathrm{M}$ dwarfs. Bands: at $7540(\Delta \lambda=50 \AA), 6880(\Delta \lambda=80 \AA), 7120(\Delta \lambda=60 \AA)$ and $10175 \AA(\Delta \lambda=280 \AA)$.

Pedersen and Rudkjøbing 1978 [241] Instr: echelle spectrophotometer. Features: Ca II K in B-F stars. Bands: at $3929.4(\Delta \lambda=4.3 \AA), 3933.7(\Delta \lambda=4.3 \AA)$, $3938.0(\Delta \lambda=4.3 \AA)$ and at $3925.9(\Delta \lambda=7.8 \AA), 3933.7$ $(\Delta \lambda=7.8 \AA), 3941.5 \AA(\Delta \lambda=7.8 \AA)$.

Pilachowski - 1978 [246] Instr: photometer. Features: $\mathrm{CO}$ in cool giants. Bands: at $2.17(\Delta \lambda=0.098 \mu \mathrm{m}), 2.2$ $(\Delta \lambda=0.40 \mu \mathrm{m})$ and $2.41 \mu \mathrm{m}(\Delta \lambda=0.088 \mu \mathrm{m})$.

Cohen - 1979 [72] Instr: spectral scanner. Features: $\mathrm{NaI}$ and $\mathrm{C}_{2}$ in Carbon stars. Bands: at 5165, 5185, 5220, $5635,5660,5720,5893,6070,6168,6192,6195,6206,6260$, 6615,6880 and $7830 \AA(\Delta \lambda=7 \AA$ for all of them).

Mould and Aaronson - 1980 [1][220] Instr: digital spectra. Features: $\mathrm{C}_{2}$ and $\mathrm{TiO}$ in $\mathrm{M}$ giants and Carbon stars. Bands: at 4930, 5615, 5690, 6150, 6250 and $6540 \AA$ ( $\Delta \lambda=15 \AA$ for all of them).

Avetisyan et al. - 1981 [13] Instr: photometer. Features: Continuum away from absorption bands in Carbon stars. Bands: at 3600, 3700, 4800, 5200, 5670, 6160 and $6690 \AA(120 \leq \Delta \lambda \leq 200 \AA)$.

Wing and Rinsland - 1981 [330] Instr: infrared spectral scanner $(\lambda / \Delta \lambda=450)$. Features: energy distribution of the continuum and the $\mathrm{CO}, \mathrm{CN}, \mathrm{C}_{2}, \mathrm{OH}, \mathrm{H}_{2}, \mathrm{SiO}$ absorption bands in late type stars. Bands: 13 bandpasses in the $1-4 \mu \mathrm{m}$ range including bands at 1.04, 1.29, 2.10, 2.28 and $4.00 \mu \mathrm{m}$. 
Avetisyan and Melik-Alaverdyan - 1982 [14] Instr: photometer. Features: $\mathrm{H}_{2} \mathrm{O}$ and $\mathrm{CO}$ bands in $\mathrm{M}$ giants. Bands: at $0.98(\Delta \lambda=0.03 \mu \mathrm{m}), 1.47(\Delta \lambda=0.03 \mu \mathrm{m})$, $1.54(\Delta \lambda=0.03 \mu \mathrm{m}), 1.72(\Delta \lambda=0.04 \mu \mathrm{m}), 1.75$ $(\Delta \lambda=0.03 \mu \mathrm{m}), 1.98(\Delta \lambda=0.05 \mu \mathrm{m}), 2.02(\Delta \lambda=$ $0.05 \mu \mathrm{m}), 2.17(\Delta \lambda=0.05 \mu \mathrm{m}), 2.29(\Delta \lambda=0.06 \mu \mathrm{m})$ and $2.39 \mu \mathrm{m}(\Delta \lambda=0.04 \mu \mathrm{m})$.

Yorka - 1983 [334] Instr: spectral scanner. Features: $\mathrm{NH}, \mathrm{CN}, \mathrm{CH}$ and $\mathrm{C}_{2}$ in Carbon stars. Bands: at 3366, $3410,3875,3908,4305,4325,4702,4766,5630$ and $5655 \AA$ $(\Delta \lambda=20 \AA$ for all of them).

Hartwick et al. - 1984 [134] Instr: spectral scanner. Features: $\mathrm{CaH}$ and $\mathrm{TiO}$ absorption bands in $\mathrm{M}$ dwarfs. Bands: at $6540(\Delta \lambda=32 \AA), 6830(\Delta \lambda=32 \AA), 7099$ $(\Delta \lambda=30 \AA)$ and $7500 \AA(\Delta \lambda=32 \AA)$.

McWilliam and Lambert - 1984 [206] Instr: photometer. Features: $\mathrm{CO}$ in M giants. Bands: at 2.17 and $2.40 \mu \mathrm{m}$.

Nersisian - 1984 [226] Instr: photometer. Features: continuum in Carbon and $\mathrm{S}$ stars. Bands: at 4020 $(\Delta \lambda=120 \AA), 4200(\Delta \lambda=120 \AA), 4660(\Delta \lambda=110 \AA)$, $4910(\Delta \lambda=130 \AA), 5167(\Delta \lambda=120 \AA), 5670$ $(\Delta \lambda=120 \AA), 5830(\Delta \lambda=100 \AA), 6160(\Delta \lambda=110 \AA)$ and $6360 \AA(\Delta \lambda=120 \AA)$.

Herbst et al. - 1987 [138] Instr: photometer. Features: $\mathrm{H} \alpha$ in $\mathrm{K}$ and $\mathrm{M}$ stars. Bands: a wide $(\Delta \lambda=150 \AA)$ and a narrow $(\Delta \lambda=30 \AA)$ band centered on $\mathrm{H} \alpha$. A third, narrower filter $(\Delta \lambda=8 \AA)$ has been added by [139].

Faulkner et al. - 1988 [103] Instr: photometer. Features: molecular opacity in the violet continuum on Carbon stars. Bands: at $3410(\Delta \lambda=90 \AA), 3500$ $(\Delta \lambda=314 \AA), 3676(\Delta \lambda=130 \AA), 3792(\Delta \lambda=116 \AA)$, $4090(\Delta \lambda=168 \AA)$ and $4520 \AA(\Delta \lambda=111 \AA)$.

Elsner et al. - 1999 [98] Instr: binning of $\lambda$-dispersed fringes by a Fizeau interferometer. Features: photometry by the DIVA astrometric satellite. Bands: 32 or
66 Gaussians (monochromatic instrumental PSF), generously overlapping and covering the whole $3500-11000 \AA$ range.

Vansevičius et al. - 1999 [308] Instr: CCD. Features: $\mathrm{Mg}$ in galaxies. Bands: two at $5190 \AA(\Delta \lambda=80$ and $450 \AA$ ).

Acknowledgements. This work has been partially supported by grants from the Osservatorio Astronomico di Padova and CISAS/Agenzia Spaziale Italiana. We would like to thank J.-C. Mermilliod, J. Lequeux and A. Henden for their comments on a preliminary version of the ADPS. Dedicated to Francesco.

\section{References}

Bessell M.S., 1993, in Stellar photometry: current tecniques and future developments, IAU Coll. 136, Butler C.J., Elliott I. (eds.). Cambridge Univ. Press, p. 22

Gilmore G., Perryman M., Lindegren L., Favata F., Hoeg E., Lattanzi M.G., Luri X., Mignard F., Roeser S., de Zeeuw P.T., 1998, Proc. SPIE Conf. 3350, p. 541

Golay M., 1974, Introduction to Astronomical Photometry, Ap. Sp. Sci. Library. Reidel, Dordrecht

Lamla E., 1982, in Landolt-Börnstein Series 2b, Schaifers K., Voight H.H. (eds.). Springer, Berlin

Merat P., Safa F., Camus J.P., Pace O., Perryman M.A.C., 1998, in Proceedings of the ESA Leiden Workshop on GAIA, 23-27 Nov. 1998, Baltic Astron. 8, 1

Mermilliod J.-C., Mermilliod M., Hauck B., 1997, A\&AS 124, 349, http://obswww.unige.ch/gcpd/gcpd.html

Munari U., 1998, in Proceedings of the ESA Leiden Workshop on GAIA, 23-27 Nov. 1998, Baltic Astron. 8, 73

Perryman M.A.C., 1999, S\&T 97, 48

Straižys V., 1995, Multicolor Stellar Photometry. Pachart Publishing House 


\section{C1 - Stebbins et al. - 1940}

Colors of stars. Interstellar reddening.

\section{GENERAL INFORMATION}

AUTHORS J. Stebbins, C. M., Huffer, A. E. Whitford

TELESCOPE $\quad 0.38 \mathrm{~m}$ (refractor), Madison Obs.; $1.52 \mathrm{~m}$ and 2.54m (reflectors), Mount Wilson Obs.

DETECTOR potassium cells

MAIN ARTICLE Stebbins, J., Huffer, C. M., Whitford, A. E. 1940, ApJ 91, 20

\section{SYSTEM DESCRIPTION}

\begin{tabular}{|c|cc|}
\hline \multicolumn{3}{|c|}{ BANDS DESCRIPTION [285], pg. 189} \\
\hline band & $\lambda_{0}(\AA)$ & bandpass $(\AA)$ \\
\hline violet & 4260 & $3600-4800$ \\
\hline blue & 4770 & $4000-5600$ \\
\hline
\end{tabular}

ZERO POINT: The zero point is set by standard stars of the Polar Sequence. [276]

\section{SYSTEM ANALYSIS}

RELATIONS WITH OTHER SYSTEMS [150]

$U B V$ - Johnson and Morgan - 1953

\begin{tabular}{|c|c|c|c|}
\hline$(B-V)$ & $=$ & $+0.30+2.06 C_{1}$ & for reddened and unreddened $\mathrm{O}$ and B0 Ia - B2 Ia stars \\
\hline$(B-V)$ & $=$ & $+0.18+1.76 C_{1}$ & for unreddened B1-A7 main \\
\hline$(B-V)$ & $=$ & $+0.27+1.37 C_{1}$ & for yellow giant stars \\
\hline
\end{tabular}

Fig. 1. The photometric system $C_{1}$ - Stebbins et al. - 1940 


\section{UVBGRI - Stebbins and Whitford - 1943}

General purpose system. Reddening law from O and B stars. Later revised by Stebbin and Kron [279].

\section{GENERAL INFORMATION}

$\begin{array}{ll}\text { AUTHORS } & \text { J. Stebbins and A. E. Whitford } \\ \text { TELESCOPE } & 1.52 \mathrm{~m} \text { (reflector), Mount Wilson; } \\ & 0.91 \mathrm{~m} \text { Crossley (reflector), Lick Observatory } \\ \text { DETECTOR } & \text { Western Electric D97087 cesium-oxide cell } \\ \text { MAIN ARTICLE } & \text { Stebbins, J., Whitford, A. E. 1943, ApJ 98, 20 }\end{array}$

SYSTEM DESCRIPTION

\begin{tabular}{|c|c|c|c|c|c|}
\hline \multicolumn{4}{|c|}{ BANDS DESCRIPTION } & \multirow[b]{2}{*}{$W H M(\AA)$} & \multirow[b]{2}{*}{$\lambda_{c}(\AA)$} \\
\hline band & filter [277] & $\lambda_{\text {peak }}(\AA)[230]$ & half-width $(\AA)$ [117], pg. 113 & & \\
\hline$U$ & $2 \mathrm{~mm}$ UG1 & 3530 & $\sim 800$ & 415 & 3465 \\
\hline$X(\#)$ & $2 \mathrm{~mm} \mathrm{UG1}+2 \mathrm{~mm} \mathrm{RG1}$ & & & & \\
\hline$V$ & $3 \mathrm{~mm} \mathrm{BG} 12+2 \mathrm{~mm} \mathrm{GG} 13$ & 4220 & $\sim 800$ & 805 & 4185 \\
\hline$B$ & $\begin{array}{l}2 \mathrm{~mm} \text { Corning } 038+ \\
5 \mathrm{~mm} \text { Corning } 430\end{array}$ & 4880 & $\sim 800$ & 1120 & 4800 \\
\hline$G$ & $\begin{array}{l}\text { 2mm Corning } 338+ \\
2 \mathrm{~mm} \mathrm{BG18}\end{array}$ & 5700 & $\sim 800$ & 1090 & 5550 \\
\hline$R$ & $\begin{array}{l}2 \mathrm{~mm} \mathrm{RG} 1+ \\
3 \mathrm{~mm} \text { Corning } 396\end{array}$ & 7190 & $\sim 1500$ & 1680 & 6960 \\
\hline$I$ & $2 \mathrm{~mm}$ Corning 254 & 10300 & $\sim 1500$ & 1805 & 10115 \\
\hline
\end{tabular}

(\#) It measures the red leak of the $U$ band.

Starting with [278] the $X$ band was unnecessary because the $U$ band included a red-leak suppressor.

\section{SYSTEM ANALYSIS}

RELATIONS WITH OTHER SYSTEMS [86]

UBVRI(JHKLMN) - Johnson - 1965

$(R-I)_{\text {Johnson }}=0.842( \pm 0.010)(R-I)_{\text {St-Whit }}+0.38( \pm 0.003)$

Fig. 2. The photometric system UVBGRI - Stebbin and Withford - 1943 


\section{TRANSMISSION CURVES [211]}

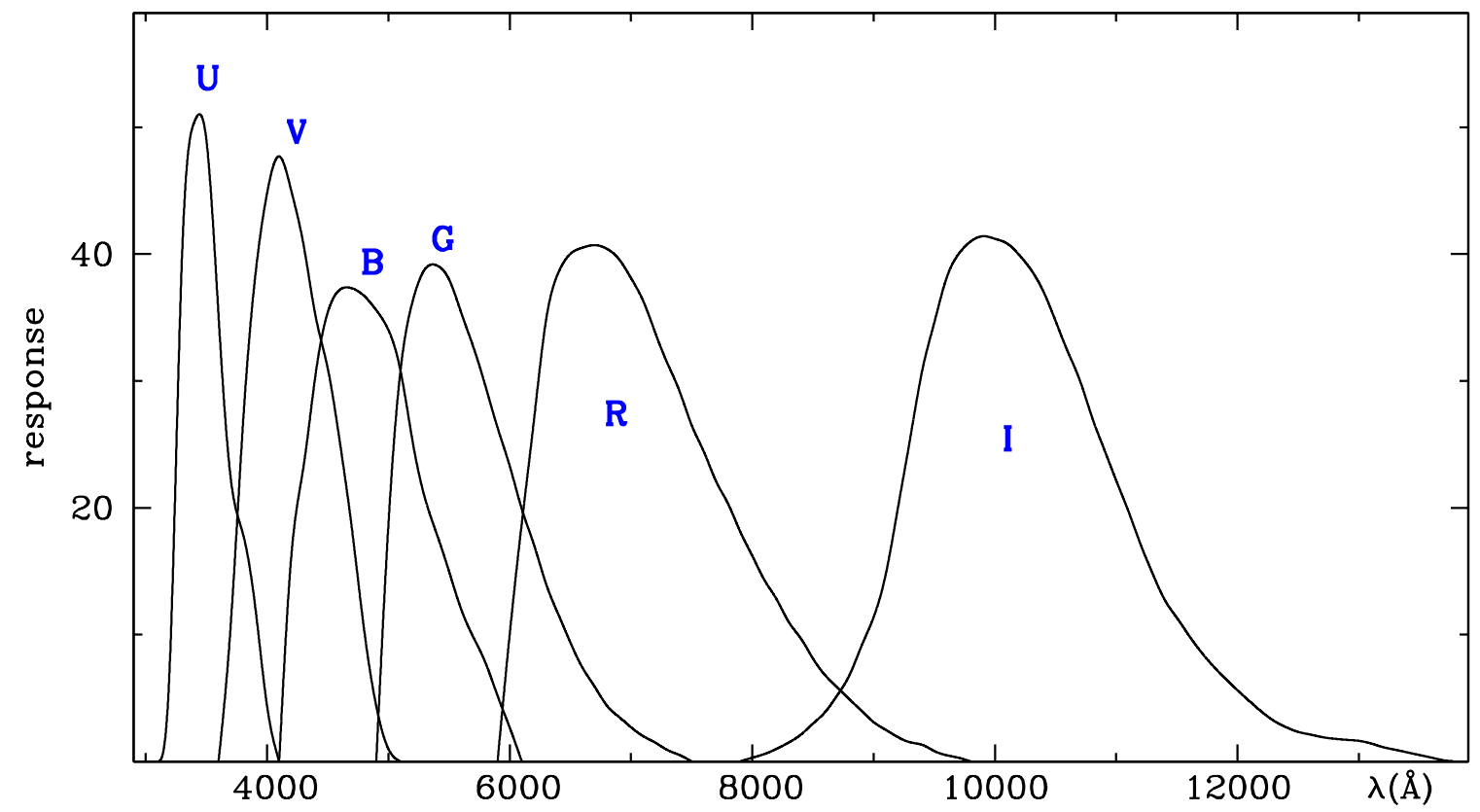

\begin{tabular}{|c|c|c|c|c|c|c|c|c|c|c|c|c|c|c|c|}
\hline \multicolumn{2}{|c|}{$U$} & \multicolumn{2}{|c|}{$V$} & \multicolumn{2}{|c|}{$B$} & \multicolumn{2}{|c|}{$G$} & \multicolumn{4}{|c|}{$R$} & \multicolumn{4}{|c|}{$I$} \\
\hline$\lambda(\mathrm{A})$ & $\Upsilon(\%)$ & $\lambda(\mathrm{A})$ & $\Upsilon(\%)$ & $\lambda(\mathrm{A})$ & $\Upsilon(\%)$ & $\lambda(\mathrm{A})$ & $\Upsilon(\%)$ & $\lambda(\mathrm{A})$ & $\Upsilon(\%)$ & $\lambda(\mathrm{A})$ & $\Upsilon(\%)$ & $\lambda(\mathrm{A})$ & $\Upsilon(\%)$ & $\lambda(\mathrm{A})$ & $\Upsilon(\%)$ \\
\hline 3100 & 0.0 & 3600 & 0.0 & 4100 & 0.0 & 4900 & 0.0 & 5900 & 0.0 & 8900 & 4.0 & 7900 & 0.0 & 10900 & 24.6 \\
\hline 3200 & 8.0 & 3700 & 10.7 & 4200 & 16.2 & 5000 & 18.6 & 6000 & 10.0 & 9000 & 3.1 & 8000 & 0.3 & 11000 & 22.1 \\
\hline 3300 & 40.0 & 3800 & 26.4 & 4300 & 23.2 & 5100 & 30.8 & 6100 & 18.8 & 9100 & 2.5 & 8100 & 0.6 & 11100 & 19.7 \\
\hline 3400 & 50.4 & 3900 & 38.0 & 4400 & 30.5 & 5200 & 36.3 & 6200 & 26.9 & 9200 & 1.9 & 8200 & 1.0 & 11200 & 17.1 \\
\hline 3500 & 49.0 & 4000 & 44.8 & 4500 & 35.4 & 5300 & 38.8 & 6300 & 34.8 & 9300 & 1.5 & 8300 & 1.5 & 11300 & 14.8 \\
\hline 3600 & 35.2 & 4100 & 47.7 & 4600 & 37.2 & 5400 & 39.1 & 6400 & 38.5 & 9400 & 1.3 & 8400 & 2.1 & 11400 & 12.7 \\
\hline 3700 & 22.4 & 4200 & 45.0 & 4700 & 37.3 & 5500 & 38.0 & 6500 & 40.0 & 9500 & 0.8 & 8500 & 3.0 & 11500 & 11.3 \\
\hline 3800 & 18.0 & 4300 & 40.8 & 4800 & 36.7 & 5600 & 35.4 & 6600 & 40.5 & 9600 & 0.5 & 8600 & 3.9 & 11600 & 9.9 \\
\hline 3900 & 12.4 & 4400 & 35.1 & 4900 & 35.6 & 5700 & 32.7 & 6700 & 40.7 & 9700 & 0.3 & 8700 & 5.2 & 11700 & 8.6 \\
\hline 4000 & 4.4 & 4500 & 31.0 & 5000 & 34.0 & 5800 & 29.6 & 6800 & 40.4 & 9800 & 0.0 & 8800 & 6.8 & 11800 & 7.5 \\
\hline 4100 & 0.0 & 4600 & 25.2 & 5100 & 30.8 & 5900 & 26.3 & 6900 & 39.6 & & & 8900 & 9.1 & 11900 & 6.5 \\
\hline & & 4700 & 18.3 & 5200 & 25.2 & 6000 & 23.3 & 7000 & 38.1 & & & 9000 & 11.4 & 12000 & 5.6 \\
\hline & & 4800 & 10.4 & 5300 & 20.8 & 6100 & 19.9 & 7100 & 36.3 & & & 9100 & 14.8 & 12100 & 4.7 \\
\hline & & 4900 & 4.4 & 5400 & 17.9 & 6200 & 17.1 & 7200 & 33.8 & & & 9200 & 20.0 & 12200 & 3.9 \\
\hline & & 5000 & 1.0 & 5500 & 15.0 & 6300 & 14.0 & 7300 & 31.4 & & & 9300 & 25.4 & 12300 & 3.2 \\
\hline & & 5100 & 0.0 & 5600 & 12.0 & 6400 & 11.6 & 7400 & 29.2 & & & 9400 & 30.8 & 12400 & 2.7 \\
\hline & & & & 5700 & 9.8 & 6500 & 9.4 & 7500 & 26.5 & & & 9500 & 34.6 & 12500 & 2.3 \\
\hline & & & & 5800 & 7.8 & 6600 & 7.4 & 7600 & 24.4 & & & 9600 & 38.1 & 12600 & 2.1 \\
\hline & & & & 5900 & 5.2 & 6700 & 5.9 & 7700 & 22.1 & & & 9700 & 40.0 & 12700 & 1.9 \\
\hline & & & & 6000 & 2.7 & 6800 & 4.4 & 7800 & 20.3 & & & 9800 & 41.0 & 12800 & 1.8 \\
\hline & & & & 6100 & 0.0 & 6900 & 3.5 & 7900 & 18.1 & & & 9900 & 41.4 & 12900 & 1.7 \\
\hline & & & & & & 7000 & 2.7 & 8000 & 16.2 & & & 10000 & 41.2 & 13000 & 1.6 \\
\hline & & & & & & 7100 & 2.0 & 8100 & 14.3 & & & 10100 & 40.8 & 13100 & 1.4 \\
\hline & & & & & & 7200 & 1.5 & 8200 & 12.8 & & & 10200 & 39.9 & 13200 & 1.1 \\
\hline & & & & & & 7300 & 0.9 & 8300 & 11.0 & & & 10300 & 38.7 & 13300 & 0.9 \\
\hline & & & & & & 7400 & 0.5 & 8400 & 9.7 & & & 10400 & 37.0 & 13400 & 0.7 \\
\hline & & & & & & 7500 & 0.0 & 8500 & 8.1 & & & 10500 & 34.7 & 13500 & 0.5 \\
\hline & & & & & & & & 8600 & 6.8 & & & 10600 & 32.3 & 13600 & 0.3 \\
\hline & & & & & & & & 8700 & 5.8 & & & 10700 & 30.0 & 13700 & 0.1 \\
\hline & & & & & & & & 8800 & 4.9 & & & 10800 & 27.1 & 13800 & 0.0 \\
\hline
\end{tabular}

Fig. 2. continued 


\title{
$R G U$ - Becker - 1946
}

Broad-band photographic system. Revised in 1967 and 1973.

\section{GENERAL INFORMATION}

\author{
AUTHORS W. Becker \\ DETECTOR photographic plates \\ MAIN ARTICLE Becker, W. 1946, Veröff. Univ. Strernwarte Göttingen 79
}

\section{SYSTEM DESCRIPTION}

\begin{tabular}{|c|c|c|c|c|c|c|c|}
\hline \multicolumn{6}{|c|}{ BANDS DESCRIPTION [54] } & \multirow[b]{2}{*}{ WHM $(\AA)$} & \multirow[b]{2}{*}{$\lambda_{c}(\AA)$} \\
\hline band & emulsion [178] & filter [178] & $\lambda_{0}(\AA)$ & $\lambda_{\text {eff }}(\AA)(\#)$ & half-width $(\AA)$ & & \\
\hline \multicolumn{8}{|c|}{1967} \\
\hline$U$ & $103 \mathrm{aO}$ & $2 \mathrm{~mm}$ UG2 & 3599 & 3634 & 520 & & \\
\hline$G$ & $103 \mathrm{aO}$ & $2 \mathrm{~mm}$ GG 5 & 4685 & 4657 & 475 & & \\
\hline$R$ & $103 \mathrm{aE}$ & 2mm RG 610 & 6435 & 6419 & 425 & & \\
\hline \multicolumn{8}{|c|}{1973} \\
\hline$U$ & $103 \mathrm{aO}$ & 2mm UG2 & 3593 & 3628 & 530 & 535 & 3600 \\
\hline$G$ & $103 \mathrm{aO}$ & $2 \mathrm{~mm}$ GG 5 & 4658 & 4630 & 495 & 500 & 4670 \\
\hline$R$ & $103 \mathrm{aE}$ & 2mm RG 610 & 6407 & 6393 & 430 & 430 & 6418 \\
\hline
\end{tabular}

(\#) For a A0V star.

ZERO POINT: $(U-G)=(G-R)=0.00$ for B0V stars. [285], pg. 195

\section{SYSTEM ANALYSIS}

REDDENING RATIOS [117], pg 110

$E(U-G) / E(G-R)=0.70 \quad A_{G}=2.69 E(G-R)$

RELATIONS WITH OTHER SYSTEMS [55]

UBV - Johnson and Morgan - 1953

For O-K, population I stars. Values of $\Delta_{i}$ are tabulated in [55]

\begin{tabular}{|ll|}
\hline$(U-G)$ & $=1.17(U-B)+0.15(B-V)+1.31+\Delta_{1}$ \\
\hline$(G-R)$ & $=1.27(B-V)-0.04(U-B)+0.34+\Delta_{2}$ \\
\hline$G$ & $=V+0.63(B-V)-0.01(U-B)+\Delta_{3}$ \\
\hline$U_{R G U}$ & $=U_{U B V}+0.16(U-B)-0.22(B-V)+1.31+\Delta_{4}$ \\
\hline$R$ & $=V-0.64(B-V)+0.03(U-B)-0.34+\Delta_{5}$ \\
\hline
\end{tabular}

Fig. 3. The photometric system RGU - Becker - 1946 


\section{TRANSMISSION CURVES [54]}

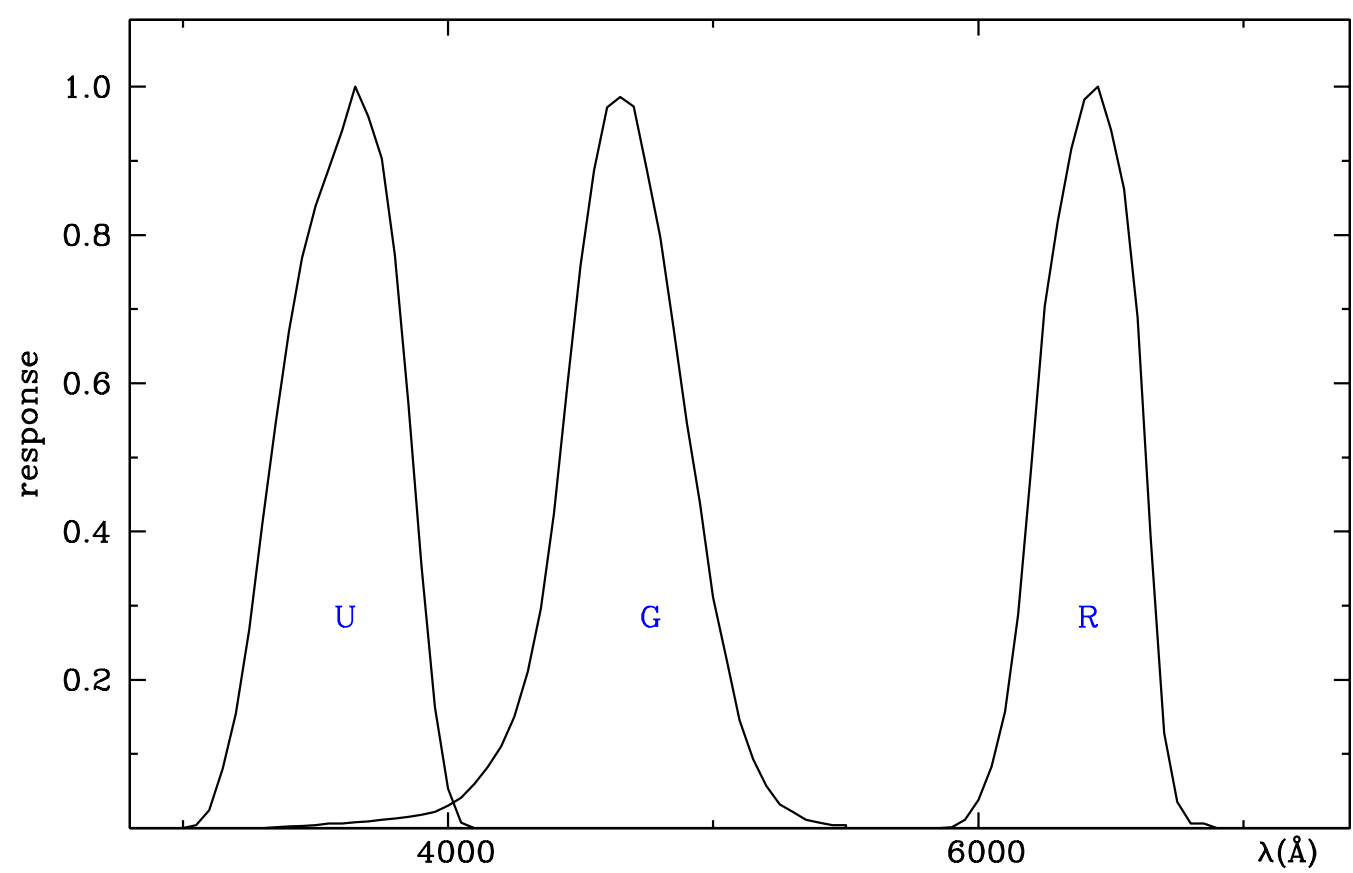

\begin{tabular}{|cc|cc|cc|cc|}
\hline \multicolumn{3}{|c|}{$U$} & \multicolumn{3}{|c|}{$G$} & \multicolumn{2}{c|}{$R$} \\
\hline$\lambda(\mathrm{A})$ & $\Upsilon$ & $\lambda(\mathrm{A})$ & $\Upsilon$ & $\lambda(\mathrm{A})$ & $\Upsilon$ & $\lambda(\mathrm{A})$ & $\Upsilon$ \\
\hline 3000 & 0.000 & 3300 & 0.000 & 4450 & 0.598 & 5850 & 0.000 \\
3050 & 0.004 & 3350 & 0.001 & 4500 & 0.759 & 5900 & 0.001 \\
3100 & 0.024 & 3400 & 0.002 & 4550 & 0.887 & 5950 & 0.011 \\
3150 & 0.080 & 3450 & 0.003 & 4600 & 0.972 & 6000 & 0.038 \\
3200 & 0.154 & 3500 & 0.004 & 4650 & 0.986 & 6050 & 0.083 \\
3250 & 0.268 & 3550 & 0.006 & 4700 & 0.973 & 6100 & 0.157 \\
3300 & 0.411 & 3600 & 0.006 & 4750 & 0.888 & 6150 & 0.288 \\
3350 & 0.548 & 3650 & 0.008 & 4800 & 0.797 & 6200 & 0.487 \\
3400 & 0.670 & 3700 & 0.009 & 4850 & 0.676 & 6250 & 0.704 \\
3450 & 0.769 & 3750 & 0.011 & 4900 & 0.546 & 6300 & 0.819 \\
3500 & 0.839 & 3800 & 0.013 & 4950 & 0.438 & 6350 & 0.916 \\
3550 & 0.889 & 3850 & 0.015 & 5000 & 0.311 & 6400 & 0.983 \\
3600 & 0.941 & 3900 & 0.018 & 5050 & 0.228 & 6450 & 1.000 \\
3650 & 1.000 & 3950 & 0.022 & 5100 & 0.145 & 6500 & 0.942 \\
3700 & 0.960 & 4000 & 0.030 & 5150 & 0.093 & 6550 & 0.862 \\
3750 & 0.903 & 4050 & 0.041 & 5200 & 0.057 & 6600 & 0.689 \\
3800 & 0.773 & 4100 & 0.060 & 5250 & 0.032 & 6650 & 0.392 \\
3850 & 0.573 & 4150 & 0.082 & 5300 & 0.022 & 6700 & 0.128 \\
3900 & 0.354 & 4200 & 0.110 & 5350 & 0.011 & 6750 & 0.035 \\
3950 & 0.163 & 4250 & 0.150 & 5400 & 0.007 & 6800 & 0.006 \\
4000 & 0.053 & 4300 & 0.211 & 5450 & 0.004 & 6850 & 0.006 \\
4050 & 0.007 & 4350 & 0.296 & 5500 & 0.004 & 6900 & 0.000 \\
4100 & 0.000 & 4400 & 0.424 & 5550 & 0.000 & & \\
\hline
\end{tabular}

Fig. 3. continued 


\section{RI - Kron and Smith - 1951}

General purpose red and near-infrared photometry.

\section{GENERAL INFORMATION}

$\begin{array}{ll}\text { AUTHORS } & \text { G. E. Kron and J. L. Smith } \\ \text { TELESCOPE } & \text { 0.60m (refractor) and 0.91m Crossley (reflector), Lick Obs.; } \\ & 1.52 \mathrm{~m} \text { and 2.54m (reflectors), Mount Wilson Obs. } \\ \text { DETECTOR } & \text { Continental Electric type CE25A/B j and e photocells } \\ \text { MAIN ARTICLE } & \text { Kron, G. E., Smith, J. L. 1951, ApJ 113, 324 }\end{array}$

\section{SYSTEM DESCRIPTION}

\begin{tabular}{|c|c|c|c|c|c|}
\hline \multicolumn{4}{|c|}{ BANDS DESCRIPTION (\#) } & \multirow{2}{*}{\multicolumn{2}{|c|}{$W H M\left(\AA \quad \lambda_{c}(\AA)\right.$}} \\
\hline band & filter [172] & $\lambda_{e f f}(\AA)[31]$ & bandpass $(\AA)$ [31] & & \\
\hline$R$ & $\begin{array}{l}\text { 2mm Schott OG1 + 2mm Schott BG21 + } \\
1 \mathrm{~mm} \text { Schott BG17 }\end{array}$ & 6500 & $5600-7400$ & 2340 & 6780 \\
\hline$R_{\text {diff }}$ & 2mm Schott OG2 - I band & & & 1610 & 6690 \\
\hline$I$ & cemented gelatin Wratten $88 \mathrm{~A}$ & 8250 & $7500-9000$ & 1540 & 8275 \\
\hline
\end{tabular}

(\#) Up to Oct 16, 1949 the $R$ band has been obtained as the difference between the readings through the $2 \mathrm{~mm}$ Schott OG2 filter and the Wratten 88A (the latter defining the $I$ band). In this way the infrared transmission of the OG2 filter was accounted for and eliminated. After that date the $R$ band has been obtained via the combination of 2mm Schott OG1 + 2mm Schott BG21 + 1mm Schott BG17 which provided infrared leak suppression.

ZERO POINT: The magnitude scale is linked to the Polar Sequence, and the $(R-I)$ color is zero for unreddened A0V stars. [172]

Fig. 4. The photometric system $R I-$ Kron and Smith - 1951 


\section{TRANSMISSION CURVES}

As derived from Fig. 2 of [172].

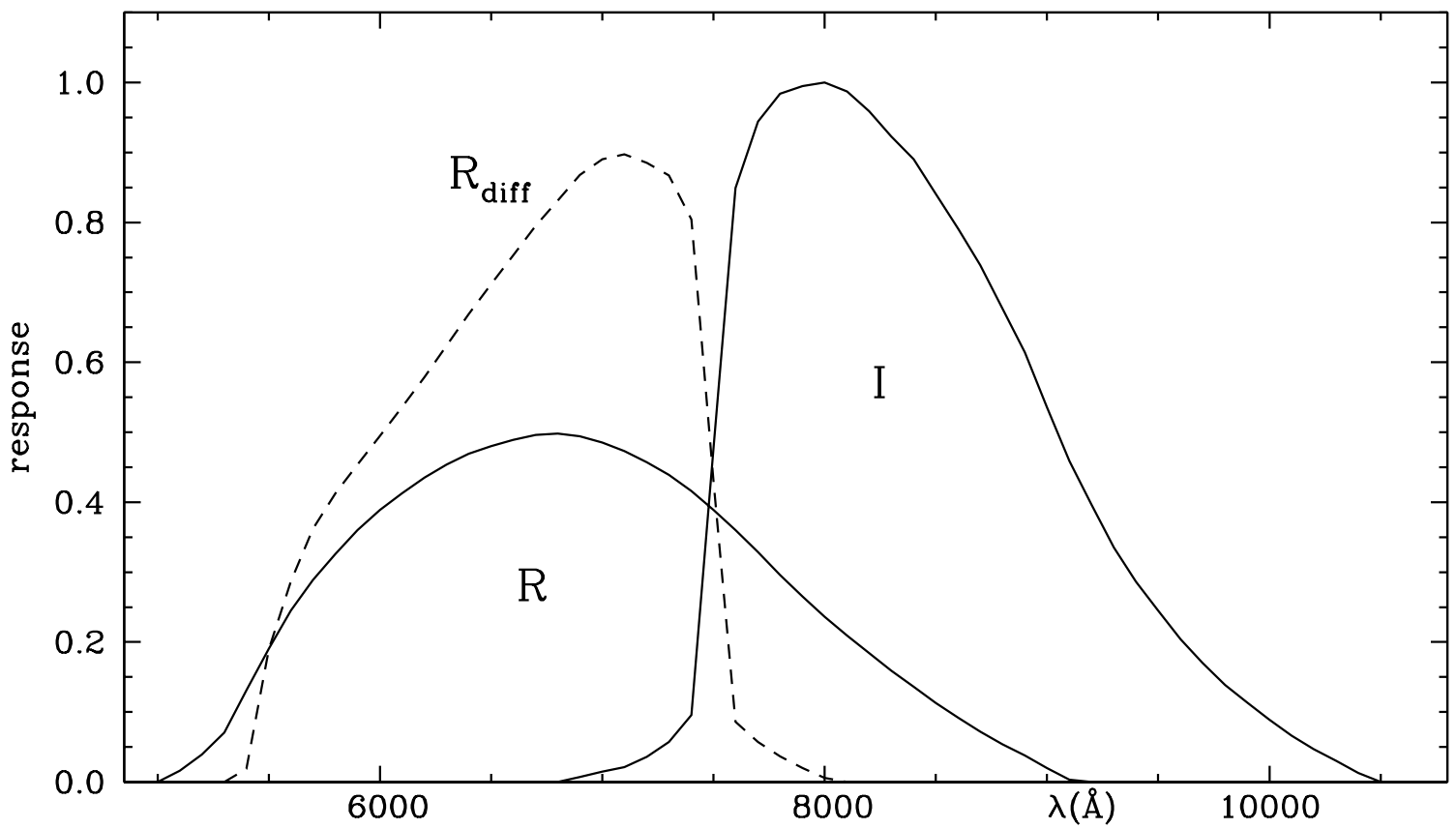

\begin{tabular}{|cc|cc|cc|ccc|cc|cc|}
\hline \multicolumn{7}{|c|}{$R$} & \multicolumn{6}{c|}{$R_{\text {diff }}$} \\
\hline$\lambda(\mathrm{A})$ & $\Upsilon$ & $\lambda(\mathrm{A})$ & $\Upsilon$ & $\lambda(\mathrm{A})$ & $\Upsilon$ & $\lambda(\mathrm{A})$ & $\Upsilon$ & $\lambda(\mathrm{A})$ & $\Upsilon$ & $\lambda(\mathrm{A})$ & $\Upsilon$ \\
\hline 5000 & 0.000 & 7200 & 0.457 & 5300 & 0.000 & 7500 & 0.431 & 6800 & 0.000 & 9000 & 0.536 \\
5100 & 0.016 & 7300 & 0.439 & 5400 & 0.019 & 7600 & 0.086 & 6900 & 0.007 & 9100 & 0.459 \\
5200 & 0.039 & 7400 & 0.416 & 5500 & 0.190 & 7700 & 0.057 & 7000 & 0.015 & 9200 & 0.397 \\
5300 & 0.071 & 7500 & 0.389 & 5600 & 0.287 & 7800 & 0.037 & 7100 & 0.021 & 9300 & 0.335 \\
5400 & 0.131 & 7600 & 0.360 & 5700 & 0.362 & 7900 & 0.020 & 7200 & 0.036 & 9400 & 0.286 \\
5500 & 0.191 & 7700 & 0.329 & 5800 & 0.413 & 8000 & 0.006 & 7300 & 0.057 & 9500 & 0.245 \\
5600 & 0.245 & 7800 & 0.296 & 5900 & 0.454 & 8100 & 0.000 & 7400 & 0.096 & 9600 & 0.204 \\
5700 & 0.289 & 7900 & 0.265 & 6000 & 0.495 & & & 7500 & 0.476 & 9700 & 0.170 \\
5800 & 0.326 & 8000 & 0.236 & 6100 & 0.536 & & & 7600 & 0.849 & 9800 & 0.139 \\
5900 & 0.360 & 8100 & 0.209 & 6200 & 0.579 & & & 7700 & 0.944 & 9900 & 0.114 \\
6000 & 0.389 & 8200 & 0.184 & 6300 & 0.624 & & & 7800 & 0.984 & 10000 & 0.089 \\
6100 & 0.413 & 8300 & 0.159 & 6400 & 0.669 & & & 7900 & 0.995 & 10100 & 0.066 \\
6200 & 0.435 & 8400 & 0.136 & 6500 & 0.712 & & & 8000 & 1.000 & 10200 & 0.047 \\
6300 & 0.454 & 8500 & 0.113 & 6600 & 0.753 & & & 8100 & 0.987 & 10300 & 0.030 \\
6400 & 0.469 & 8600 & 0.092 & 6700 & 0.795 & & & 8200 & 0.959 & 10400 & 0.013 \\
6500 & 0.480 & 8700 & 0.072 & 6800 & 0.831 & & & 8300 & 0.923 & 10500 & 0.000 \\
6600 & 0.489 & 8800 & 0.054 & 6900 & 0.868 & & & 8400 & 0.890 & & \\
6700 & 0.496 & 8900 & 0.038 & 7000 & 0.890 & & & 8500 & 0.841 & & \\
6800 & 0.498 & 9000 & 0.020 & 7100 & 0.897 & & & 8600 & 0.791 & & \\
6900 & 0.494 & 9100 & 0.003 & 7200 & 0.885 & & & 8700 & 0.739 & & \\
7000 & 0.485 & 9200 & 0.000 & 7300 & 0.867 & & & 8800 & 0.677 & & \\
7100 & 0.473 & & & 7400 & 0.804 & & & 8900 & 0.614 & & \\
\hline
\end{tabular}

Fig. 4. continued 


\section{BCD - Chalonge and Divan - 1952}

Classification of early type stars from the region around the Balmer jump as recorded on prismatic spectra.

\section{GENERAL INFORMATION}

AUTHORS D. Chalonge and L. Divan

TELESCOPE Cassegrain coudè focus, Jungfraujoch Observatory

DETECTOR Ilford Zènith and Kodak 103a-O plates

MAIN ARTICLE Chalonge D., Divan L. 1952, Ann. Astron. 15, 201

\section{SYSTEM DESCRIPTION}

Three quantities $\left(D, \lambda_{1}\right.$ and $\left.\Phi_{b}\right)$ are defined over the 3300-4600 $\AA$ portion of photographic prism spectra.

$D$ measures the magintude of the Balmer discontinuity, $\lambda_{1}$ indicates the effective location of the Balmer discontinuity in the spectrum and $\Phi_{b}$ is related to the slope of the continuum in the blue region of the spectrum longward of the Balmer discontinuity ( $3800-4800 \AA$ ) [65].

SYSTEM ANALYSIS

RELATIONS WITH OTHER SYSTEMS [26]

UBV - Johnson and Morgan - 1953

$D=0.525(Q+1) \quad$ for stars $\mathrm{O} 9-\mathrm{A} 2$, lum. class $\mathrm{V}$

Fig. 5. The photometric system BCD - Chalonge and Divan - 1952 


\section{UBV - Johnson and Morgan - 1953}

General purpose system. Due to problems in the reconstruction of the original system, two versions have become popular: the USA [198] and the Vilnius [15].

\section{GENERAL INFORMATION}

\section{AUTHORS \\ H. L. Johnson and W. W. Morgan \\ TELESCOPE $0.33 \mathrm{~m}$ and $2.08 \mathrm{~m}$ (reflectors) Mc Donald Observatory \\ DETECTOR RCA 1P21 (about refrigeration see [149]) \\ MAIN ARTICLE Johnson H.L., Morgan W.W. 1953, ApJ 117, 313}

\begin{tabular}{|c|c|c|c|c|c|c|}
\hline \multicolumn{7}{|c|}{ SYSTEM DESCRIPTION } \\
\hline \multicolumn{4}{|c|}{ BANDS DESCRIPTION [178], pg. 50} & & & FLUX CALIB. (\#) \\
\hline band & filter & alternative & $\lambda_{0}(\AA) \quad \lambda_{e f f}(\AA)(\#)$ & $W H M(\AA)$ & $\lambda_{c}(\AA)$ & $\left(\mathrm{erg} \mathrm{cm}{ }^{-2} \sec ^{-1} \AA^{-1}\right)$ \\
\hline \multicolumn{7}{|c|}{ photoelectric USA } \\
\hline$U$ & Corning 9863 & $2 \mathrm{~mm}$ UG2 & 3600 & 700 & 3500 & $3.9810^{-9}$ \\
\hline$B$ & $\begin{array}{l}\text { Corning } 5030+ \\
2 \mathrm{~mm} \text { GG13 }\end{array}$ & $\begin{array}{l}1 \mathrm{~mm} \mathrm{BG} 12+ \\
2 \mathrm{~mm} \mathrm{GG13}\end{array}$ & 4400 & 985 & 4380 & $6.9510^{-9}$ \\
\hline$V$ & Corning 3384 & $2 \mathrm{~mm}$ GG11 & 5500 & 870 & 5465 & $3.6310^{-9}$ \\
\hline \multicolumn{7}{|c|}{ photoelectric Vilnius } \\
\hline$U$ & & & & 400 & 3660 & $4.2210^{-9}$ \\
\hline$B$ & & & & 1000 & 4405 & $6.4010^{-9}$ \\
\hline$V$ & & & & 825 & 5425 & $3.7510^{-9}$ \\
\hline \multicolumn{7}{|c|}{ photographic } \\
\hline & plate & filter & & & & \\
\hline$U$ & $103 \mathrm{a}-\mathrm{O}$ & 2mm UG2 & & 630 & 3475 & \\
\hline$B$ & $103 \mathrm{a}-\mathrm{O}$ & $2 \mathrm{~mm}$ GG13 & & 1115 & 4385 & \\
\hline$V_{G}$ & $103 a-G$ & 2mm GG11 & & 580 & 5440 & \\
\hline$V_{D}$ & 103a-D & 2mm GG11 & & 1400 & 5725 & \\
\hline
\end{tabular}

(\#) For an unreddened A0V star with V =0.00. [178], pg. 50-54

ZERO POINT: The mean colors of the six unreddened A0 V stars $\alpha$ Lyr, $\gamma$ UMa, 109 Vir, $\alpha$ CrB, $\gamma$ Oph and HR 3314 are $U-B=B-V=0.00$. [156]

PHOTOGRAPHIC VERSION: Bell, R. A. 1972, MNRAS 159, 357

\section{SYSTEM ANALYSIS}

REDDENING-FREE PARAMETERS [285], pg. 241

$Q_{U B V}=(U-B)-[E(U-B) / E(B-V)](B-V)$

REDDENING RATIOS

$$
\begin{aligned}
& E(U-B) / E(B-V)=0.72+0.05 E(B-V) \quad[285], \text { pg. } 227 \\
& A_{U}=4.40 E(B-V) \quad A_{B}=4.10 E(B-V) \quad A_{V}=3.10 E(B-V) \quad[258]
\end{aligned}
$$

Fig. 6. The photometric system $U B V-$ Johnson and Morgan - 1953 
The photographic bands are derived from Fig. 1 of [29].

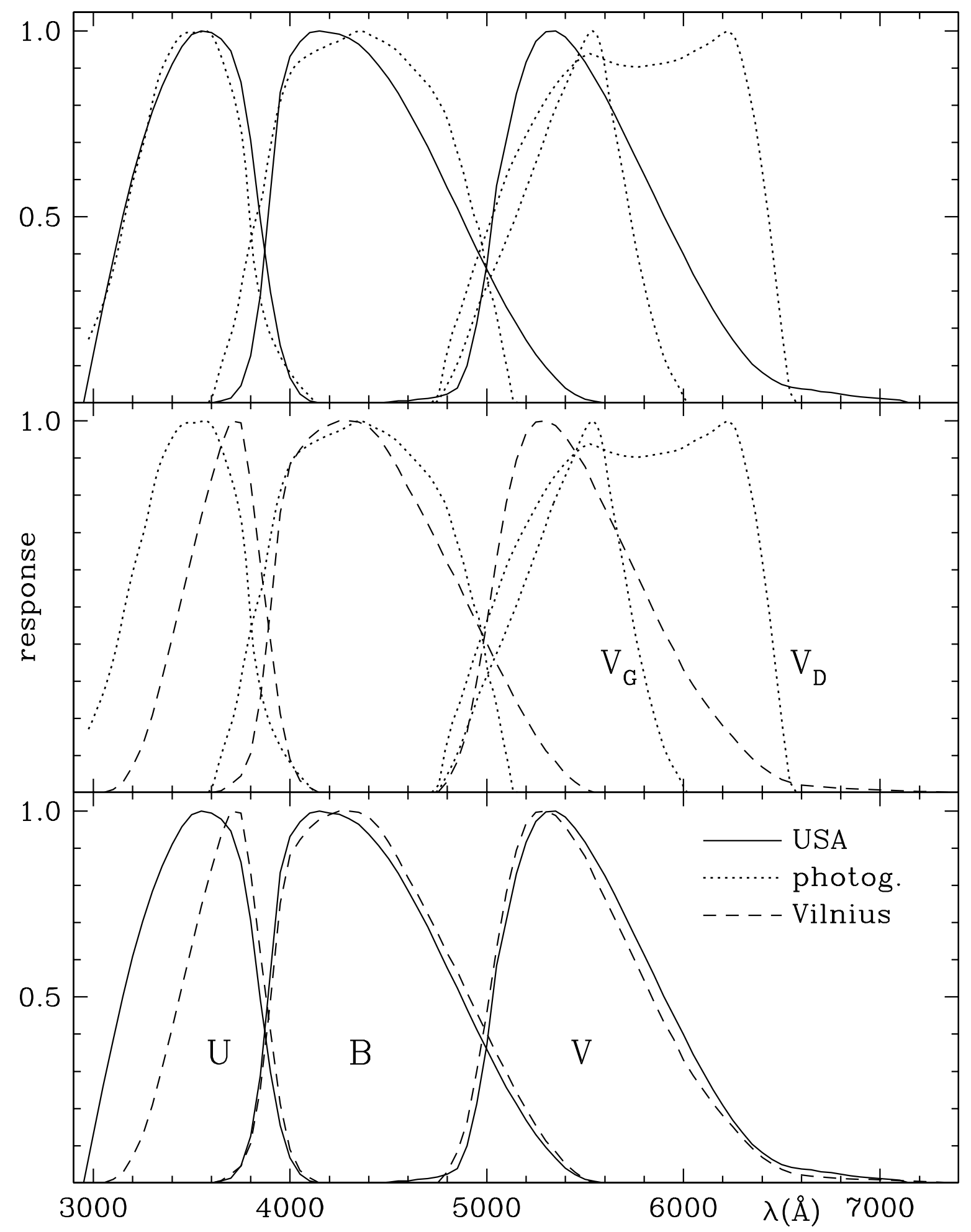

Fig. 6. continued 


\begin{tabular}{|c|c|c|c|c|c|c|c|c|c|c|c|c|c|c|c|c|c|c|c|}
\hline \multicolumn{6}{|c|}{ USA } & \multicolumn{6}{|c|}{ Vilnius } & \multicolumn{8}{|c|}{ Photographic } \\
\hline \multicolumn{2}{|c|}{$U$} & \multicolumn{2}{|c|}{$B$} & & & & & & & & & & & & & & & & $D$ \\
\hline$\lambda(\AA)$ & $\Upsilon$ & $\lambda(\AA)$ & $\Upsilon$ & $\lambda(\AA)$ & $\Upsilon$ & $\lambda(\AA)$ & $\Upsilon$ & $\lambda(\AA)$ & $\Upsilon$ & $\lambda(\AA)$ & $\Upsilon$ & $\lambda(\AA)$ & $\Upsilon$ & $\lambda(\AA)$ & $\Upsilon$ & $\lambda(\AA)$ & $\Upsilon$ & $\lambda(\AA)$ & $\Upsilon$ \\
\hline 2950 & 0.000 & 3600 & 0.000 & 4450 & 0.000 & 3050 & 0.000 & 3600 & 0.000 & 4750 & 0.000 & 2975 & 0.171 & 3585 & 0.000 & 4740 & 0.000 & 4720 & 0.000 \\
\hline 3000 & 0.131 & 3650 & 0.005 & 4500 & 0.002 & 3100 & 0.009 & 3650 & 0.006 & 4800 & 0.030 & 2995 & 0.195 & 3610 & 0.024 & 4760 & 0.011 & 4755 & 0.022 \\
\hline 3050 & 0.260 & 3700 & 0.013 & 4550 & 0.005 & 3150 & 0.028 & 3700 & 0.023 & 4850 & 0.084 & 3015 & 0.220 & 3635 & 0.074 & 4780 & 0.030 & 4790 & 0.115 \\
\hline 3100 & 0.384 & 3750 & 0.046 & 4600 & 0.005 & 3200 & 0.071 & 3750 & 0.045 & 4900 & 0.163 & 3035 & 0.246 & 3660 & 0.119 & 4800 & 0.049 & 4825 & 0.190 \\
\hline 3150 & 0.502 & 3800 & 0.126 & 4650 & 0.009 & 3250 & 0.128 & 3800 & 0.106 & 4950 & 0.301 & 3055 & 0.275 & 3685 & 0.159 & 4820 & 0.070 & 4860 & 0.239 \\
\hline 3200 & 0.609 & 3850 & 0.290 & 4700 & 0.012 & 3300 & 0.209 & 3850 & 0.254 & 5000 & 0.458 & 3075 & 0.307 & 3710 & 0.198 & 4840 & 0.093 & 4895 & 0.297 \\
\hline 3250 & 0.703 & 3900 & 0.568 & 4750 & 0.016 & 3350 & 0.309 & 3900 & 0.492 & 5050 & 0.630 & 3095 & 0.344 & 3735 & 0.255 & 4860 & 0.118 & 4930 & 0.356 \\
\hline 3300 & 0.784 & 3950 & 0.834 & 4800 & 0.023 & 3400 & 0.417 & 3950 & 0.752 & 5100 & 0.780 & 3115 & 0.387 & 3760 & 0.338 & 4880 & 0.144 & 4965 & 0.410 \\
\hline 3350 & 0.852 & 4000 & 0.931 & 4850 & 0.039 & 3450 & 0.527 & 4000 & 0.881 & 5150 & 0.895 & 3135 & 0.436 & 3785 & 0.402 & 4900 & 0.174 & 5000 & 0.461 \\
\hline 3400 & 0.911 & 4050 & 0.971 & 4900 & 0.100 & 3500 & 0.635 & 4050 & 0.923 & 5200 & 0.967 & 3155 & 0.486 & 3810 & 0.462 & 4920 & 0.205 & 5035 & 0.511 \\
\hline 3450 & 0.958 & 4100 & 0.995 & 4950 & 0.213 & 3550 & 0.744 & 4100 & 0.955 & 5250 & 0.997 & 3175 & 0.536 & 3835 & 0.512 & 4940 & 0.236 & 5070 & 0.566 \\
\hline 3500 & 0.990 & 4150 & 1.000 & 5000 & 0.371 & 3600 & 0.844 & 4150 & 0.977 & 5300 & 1.000 & 3195 & 0.583 & 3860 & 0.555 & 4960 & 0.264 & 5105 & 0.617 \\
\hline 3550 & 1.000 & 4200 & 0.995 & 5050 & 0.548 & 3650 & 0.934 & 4200 & 0.990 & 5350 & 0.988 & 3215 & 0.625 & 3885 & 0.640 & 4980 & 0.290 & 5140 & 0.658 \\
\hline 3600 & 0.995 & 4250 & 0.991 & 5100 & 0.705 & 3700 & 1.000 & 4250 & 1.000 & 5400 & 0.958 & 3235 & 0.661 & 3910 & 0.718 & 5000 & 0.314 & 5175 & 0.695 \\
\hline 3650 & 0.978 & 4300 & 0.980 & 5150 & 0.831 & 3750 & 0.995 & 4300 & 1.000 & 5450 & 0.919 & 3255 & 0.698 & 3935 & 0.782 & 5020 & 0.338 & 5210 & 0.730 \\
\hline 3700 & 0.946 & 4350 & 0.964 & 5200 & 0.916 & 3800 & 0.834 & 4350 & 0.997 & 5500 & 0.877 & 3275 & 0.743 & 3960 & 0.828 & 5040 & 0.362 & 5245 & 0.764 \\
\hline 3750 & 0.862 & 4400 & 0.938 & 5250 & 0.972 & 3850 & 0.616 & 4400 & 0.984 & 5550 & 0.819 & 3295 & 0.793 & 3985 & 0.865 & 5060 & 0.387 & 5280 & 0.797 \\
\hline 3800 & 0.705 & 4450 & 0.907 & 5300 & 0.998 & 3900 & 0.408 & 4450 & 0.958 & 5600 & 0.765 & 3315 & 0.840 & 4010 & 0.894 & 5080 & 0.412 & 5315 & 0.828 \\
\hline 3850 & 0.492 & 4500 & 0.872 & 5350 & 1.000 & 3950 & 0.213 & 4500 & 0.916 & 5650 & 0.711 & 3335 & 0.879 & 4035 & 0.912 & 5100 & 0.438 & 5350 & 0.855 \\
\hline 3900 & 0.297 & 4550 & 0.832 & 5400 & 0.984 & 4000 & 0.090 & 4550 & 0.871 & 5700 & 0.657 & 3355 & 0.907 & 4060 & 0.923 & 5120 & 0.464 & 5385 & 0.878 \\
\hline 3950 & 0.154 & 4600 & 0.786 & 5450 & 0.954 & 4050 & 0.033 & 4600 & 0.820 & 5750 & 0.602 & 3375 & 0.928 & 4085 & 0.932 & 5140 & 0.490 & 5420 & 0.898 \\
\hline 4000 & 0.067 & 4650 & 0.739 & 5500 & 0.916 & 4100 & 0.014 & 4650 & 0.775 & 5800 & 0.545 & 3395 & 0.949 & 4110 & 0.940 & 5160 & 0.518 & 5455 & 0.917 \\
\hline 4050 & 0.023 & 4700 & 0.688 & 5550 & 0.872 & 4150 & 0.000 & 4700 & 0.723 & 5850 & 0.488 & 3415 & 0.968 & 4135 & 0.947 & 5180 & 0.546 & 5490 & 0.931 \\
\hline 4100 & 0.005 & 4750 & 0.633 & 5600 & 0.826 & & & 4750 & 0.672 & 5900 & 0.434 & 3435 & 0.983 & 4160 & 0.953 & 5200 & 0.575 & 5525 & 0.938 \\
\hline 4150 & 0.000 & 4800 & 0.578 & 5650 & 0.775 & & & 4800 & 0.617 & 5950 & 0.386 & 3455 & 0.991 & 4185 & 0.959 & 5220 & 0.603 & 5560 & 0.932 \\
\hline & & 4850 & 0.524 & 5700 & 0.722 & & & 4850 & 0.569 & 6000 & 0.331 & 3475 & 0.995 & 4210 & 0.965 & 5240 & 0.631 & 5595 & 0.923 \\
\hline & & 4900 & 0.467 & 5750 & 0.668 & & & 4900 & 0.511 & 6050 & 0.289 & 3495 & 0.995 & 4235 & 0.970 & 5260 & 0.659 & 5630 & 0.916 \\
\hline & & 4950 & 0.412 & 5800 & 0.613 & & & 4950 & 0.457 & 6100 & 0.250 & 3515 & 0.995 & 4260 & 0.976 & 5280 & 0.689 & 5665 & 0.910 \\
\hline & & 5000 & 0.359 & 5850 & 0.559 & & & 5000 & 0.402 & 6150 & 0.214 & 3535 & 0.997 & 4285 & 0.983 & 5300 & 0.720 & 5700 & 0.906 \\
\hline & & 5050 & 0.307 & 5900 & 0.503 & & & 5050 & 0.347 & 6200 & 0.181 & 3555 & 0.999 & 4310 & 0.991 & 5320 & 0.751 & 5735 & 0.904 \\
\hline & & 5100 & 0.257 & 5950 & 0.450 & & & 5100 & 0.299 & 6250 & 0.151 & 3575 & 1.000 & 4335 & 0.998 & 5340 & 0.779 & 5770 & 0.903 \\
\hline & & 5150 & 0.212 & 6000 & 0.399 & & & 5150 & 0.244 & 6300 & 0.120 & 3595 & 0.994 & 4360 & 1.000 & 5360 & 0.805 & 5805 & 0.905 \\
\hline & & 5200 & 0.168 & 6050 & 0.346 & & & 5200 & 0.199 & 6350 & 0.093 & 3615 & 0.978 & 4385 & 0.996 & 5380 & 0.829 & 5840 & 0.908 \\
\hline & & 5250 & 0.130 & 6100 & 0.297 & & & 5250 & 0.154 & 6400 & 0.069 & 3635 & 0.952 & 4410 & 0.988 & 5400 & 0.852 & 5875 & 0.911 \\
\hline & & 5300 & 0.095 & 6150 & 0.251 & & & 5300 & 0.113 & 6450 & 0.051 & 3655 & 0.922 & 4435 & 0.981 & 5420 & 0.876 & 5910 & 0.914 \\
\hline & & 5350 & 0.066 & 6200 & 0.209 & & & 5350 & 0.084 & 6500 & 0.036 & 3675 & 0.891 & 4460 & 0.975 & 5440 & 0.901 & 5945 & 0.918 \\
\hline & & 5400 & 0.040 & 6250 & 0.169 & & & 5400 & 0.051 & 6550 & 0.027 & 3695 & 0.858 & 4485 & 0.969 & 5460 & 0.926 & 5980 & 0.923 \\
\hline & & 5450 & 0.022 & 6300 & 0.135 & & & 5450 & 0.029 & 6600 & 0.021 & 3715 & 0.821 & 4510 & 0.960 & 5480 & 0.953 & 6015 & 0.933 \\
\hline & & 5500 & 0.009 & 6350 & 0.104 & & & 5500 & 0.010 & 6650 & 0.018 & 3735 & 0.779 & 4535 & 0.950 & 5500 & 0.977 & 6050 & 0.944 \\
\hline & & 5550 & 0.004 & 6400 & 0.081 & & & 5550 & 0.000 & 6700 & 0.016 & 3755 & 0.723 & 4560 & 0.937 & 5520 & 0.994 & 6085 & 0.954 \\
\hline & & 5600 & 0.000 & 6450 & 0.063 & & & & & 6750 & 0.014 & 3775 & 0.630 & 4585 & 0.923 & 5540 & 1.000 & 6120 & 0.963 \\
\hline & & & & 6500 & 0.049 & & & & & 6800 & 0.012 & 3795 & 0.494 & 4610 & 0.909 & 5560 & 0.990 & 6155 & 0.975 \\
\hline & & & & 6550 & 0.042 & & & & & 6850 & 0.011 & 3815 & 0.384 & 4635 & 0.895 & 5580 & 0.958 & 6190 & 0.990 \\
\hline & & & & 6600 & 0.037 & & & & & 6900 & 0.010 & 3835 & 0.319 & 4660 & 0.881 & 5600 & 0.902 & 6225 & 1.000 \\
\hline & & & & 6650 & 0.035 & & & & & 6950 & 0.009 & 3855 & 0.262 & 4685 & 0.866 & 5620 & 0.833 & 6260 & 0.987 \\
\hline & & & & 6700 & 0.030 & & & & & 7000 & 0.008 & 3875 & 0.220 & 4710 & 0.850 & 5640 & 0.767 & 6295 & 0.930 \\
\hline & & & & 6750 & 0.028 & & & & & 7050 & 0.007 & 3895 & 0.190 & 4735 & 0.829 & 5660 & 0.709 & 6330 & 0.849 \\
\hline & & & & 6800 & 0.023 & & & & & 7100 & 0.006 & 3915 & 0.164 & 4760 & 0.806 & 5680 & 0.655 & 6365 & 0.751 \\
\hline & & & & 6850 & 0.019 & & & & & 7150 & 0.005 & 3935 & 0.141 & 4785 & 0.783 & 5700 & 0.597 & 6400 & 0.623 \\
\hline & & & & 6900 & 0.016 & & & & & 7200 & 0.004 & 3955 & 0.121 & 4810 & 0.748 & 5720 & 0.531 & 6435 & 0.487 \\
\hline & & & & 6950 & 0.014 & & & & & 7250 & 0.003 & 3975 & 0.102 & 4835 & 0.701 & 5740 & 0.469 & 6470 & 0.326 \\
\hline & & & & 7000 & 0.012 & & & & & 7300 & 0.002 & 3995 & 0.085 & 4860 & 0.656 & 5760 & 0.415 & 6505 & 0.169 \\
\hline & & & & 7050 & 0.009 & & & & & 7350 & 0.001 & 4015 & 0.070 & 4885 & 0.612 & 5780 & 0.365 & 6540 & 0.029 \\
\hline & & & & 7100 & 0.007 & & & & & 7400 & 0.000 & 4035 & 0.056 & 4910 & 0.556 & 5800 & 0.318 & 6575 & 0.000 \\
\hline & & & & 7150 & 0.000 & & & & & & & 4055 & 0.043 & 4935 & 0.505 & 5820 & 0.274 & & \\
\hline & & & & & & & & & & & & 4075 & 0.031 & 4960 & 0.471 & 5840 & 0.232 & & \\
\hline & & & & & & & & & & & & 4095 & 0.020 & 4985 & 0.400 & 5860 & 0.192 & & \\
\hline & & & & & & & & & & & & 4115 & 0.009 & 5010 & 0.312 & 5880 & 0.156 & & \\
\hline & & & & & & & & & & & & 4135 & 0.000 & 5035 & 0.270 & 5900 & 0.124 & & \\
\hline & & & & & & & & & & & & & & 5060 & 0.208 & 5920 & 0.098 & & \\
\hline & & & & & & & & & & & & & & 5085 & 0.138 & 5940 & 0.076 & & \\
\hline & & & & & & & & & & & & & & 5110 & 0.077 & 5960 & 0.058 & & \\
\hline & & & & & & & & & & & & & & 5135 & 0.005 & 5980 & 0.040 & & \\
\hline & & & & & & & & & & & & & & 5160 & 0.000 & 6000 & 0.017 & & \\
\hline & & & & & & & & & & & & & & & & 6020 & 0.000 & & \\
\hline
\end{tabular}

Fig. 6. continued 


\section{POSS I - 1955}

First photographic Palomar Observatory Sky Survey.

\section{GENERAL INFORMATION}

TELESCOPE $\quad 1.2 \mathrm{~m}$ Schmidt, Palomar Obs.

MAIN ARTICLE Lund, J. M., Dixon, R. S. 1973, PASP 85, 230

\section{SYSTEM DESCRIPTION}

\begin{tabular}{|l|cc|}
\hline \multicolumn{3}{|c|}{ BANDS DESCRIPTION [192] } \\
\hline \multicolumn{1}{|c|}{ band } & emulsion & lter \\
\hline blue & Eastman 103a-O & none \\
\hline amber (\#) & Eastman 103a-E & amber Plexiglas \\
\hline red & Eastman 103a-E & red Plexiglas 2444 \\
\hline
\end{tabular}

(\#) Used in the so called "Whiteoak Estension" to declination zones -36 and $-42^{\circ}$.

\section{TRANSMISSION CURVES}

As derived by combining the sensitivity/trasmission of photographic plates, lters, atmosphere and BK-7 glass [5], [167], [263]

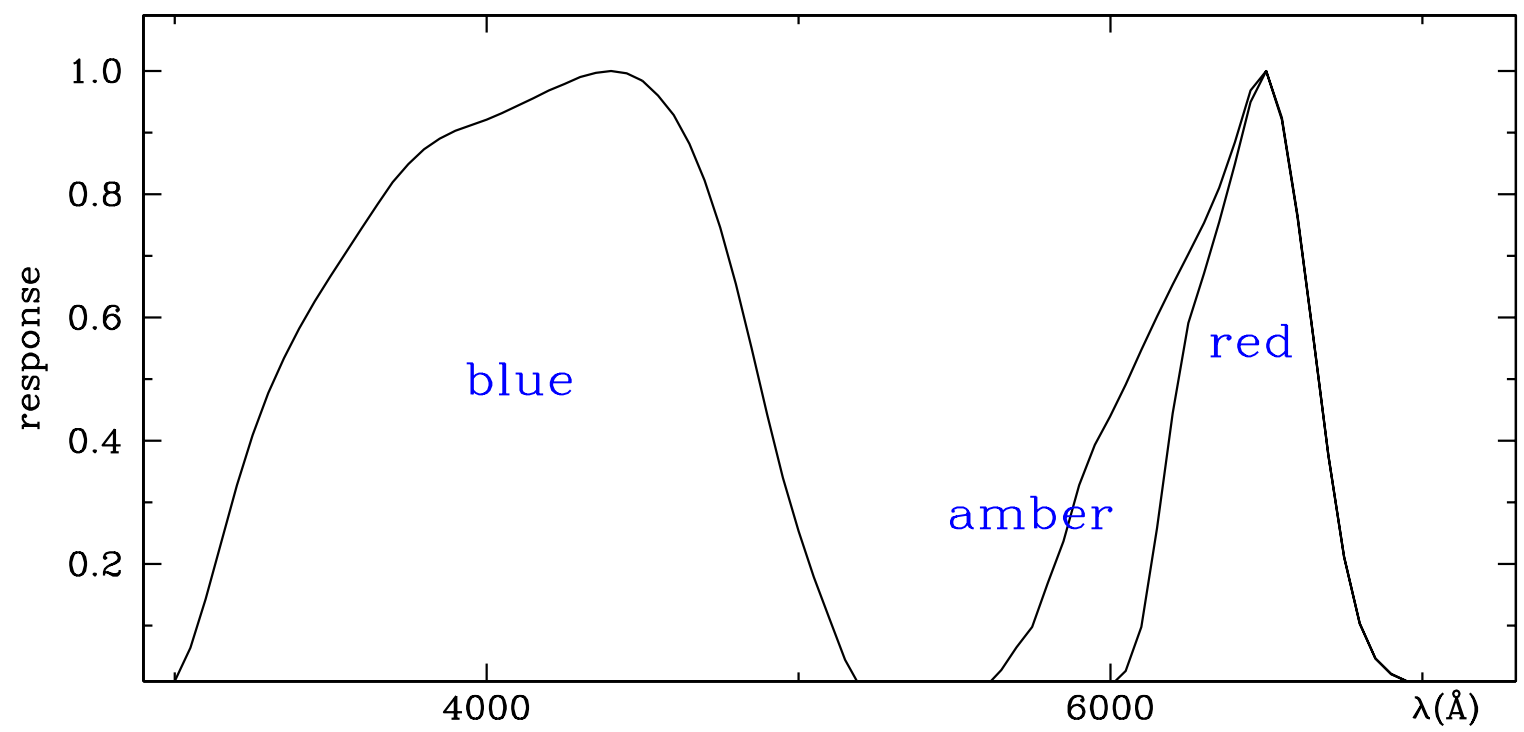

\begin{tabular}{|c|c|c|c|c|c|c|c|c|c|c|c|c|c|c|c|c|c|}
\hline \multicolumn{8}{|c|}{ blue } & \multicolumn{6}{|c|}{ amber } & \multicolumn{4}{|c|}{ red } \\
\hline$\lambda(\mathrm{A})$ & $\Upsilon$ & $\lambda(\mathrm{A})$ & $\Upsilon$ & $\lambda(\mathrm{A})$ & $\Upsilon$ & $\lambda(\mathrm{A})$ & $\Upsilon$ & $\lambda(\mathrm{A})$ & $\Upsilon$ & $\lambda(\mathrm{A})$ & $\Upsilon$ & $\lambda(\mathrm{A})$ & $\Upsilon$ & $\lambda(\mathrm{A})$ & $\Upsilon$ & $\lambda(\mathrm{A})$ & $\Upsilon$ \\
\hline 2950 & 0.000 & 3550 & 0.706 & 4100 & 0.943 & 4700 & 0.822 & 5600 & 0.000 & 6200 & 0.653 & 6800 & 0.104 & 5950 & 0.000 & 6550 & 0.924 \\
\hline 3000 & 0.010 & 3600 & 0.745 & 4150 & 0.955 & 4750 & 0.746 & 5650 & 0.028 & 6250 & 0.703 & 6850 & 0.047 & 6000 & 0.002 & 6600 & 0.765 \\
\hline 3050 & 0.064 & 3650 & 0.783 & 4200 & 0.968 & 4800 & 0.654 & 5700 & 0.065 & 6300 & 0.753 & 6900 & 0.021 & 6050 & 0.026 & 6650 & 0.570 \\
\hline 3100 & 0.143 & 3700 & 0.819 & 4250 & 0.979 & 4850 & 0.550 & 5750 & 0.098 & 6350 & 0.810 & 6950 & 0.010 & 6100 & 0.098 & 6700 & 0.376 \\
\hline 3150 & 0.235 & 3750 & 0.849 & 4300 & 0.990 & 4900 & 0.442 & 5800 & 0.169 & 6400 & 0.884 & 7000 & 0.004 & 6150 & 0.258 & 6750 & 0.211 \\
\hline 3200 & 0.328 & 3800 & 0.873 & 4350 & 0.997 & 4950 & 0.340 & 5850 & 0.237 & 6450 & 0.968 & 7050 & 0.002 & 6200 & 0.444 & 6800 & 0.103 \\
\hline 3250 & 0.409 & 3850 & 0.890 & 4400 & 1.000 & 5000 & 0.253 & 5900 & 0.328 & 6500 & 1.000 & 7100 & 0.000 & 6250 & 0.591 & 6850 & 0.047 \\
\hline 3300 & 0.477 & 3900 & 0.903 & 4450 & 0.996 & 5050 & 0.179 & 5950 & 0.393 & 6550 & 0.921 & & & 6300 & 0.671 & 6900 & 0.022 \\
\hline 3350 & 0.534 & 3950 & 0.912 & 4500 & 0.984 & 5100 & 0.111 & 6000 & 0.439 & 6600 & 0.762 & & & 6350 & 0.755 & 6950 & 0.010 \\
\hline 3400 & 0.582 & 4000 & 0.921 & 4550 & 0.960 & 5150 & 0.044 & 6050 & 0.491 & 6650 & 0.569 & & & 6400 & 0.849 & 7000 & 0.004 \\
\hline 3450 & 0.626 & 4050 & 0.931 & 4600 & 0.928 & 5200 & 0.000 & 6100 & 0.547 & 6700 & 0.376 & & & 6450 & 0.949 & 7050 & 0.000 \\
\hline 3500 & 0.667 & & & 4650 & 0.882 & & & 6150 & 0.601 & 6750 & 0.212 & & & 6500 & 1.000 & & \\
\hline
\end{tabular}

Fig. 7. The photometric system POSS I - 1955 


\section{$P V$ - Eggen - 1955}

General purpose system.

\section{GENERAL INFORMATION}

$\begin{array}{ll}\text { AUTHORS } & \text { O. J. Eggen } \\ \text { TELESCOPE } & 0.30 \mathrm{~m} \text { (refractor), Mt. Hamilton; 0.23m (refractor), Mt. Stromlo } \\ \text { DETECTOR } & 1 \mathrm{P} 21 \\ \text { MAIN ARTICLE } & \text { Eggen, O.J. 1955, AJ 60, 65 }\end{array}$

SYSTEM DESCRIPTION

\begin{tabular}{|c|c|c|c|c|}
\hline \multicolumn{3}{|c|}{ BANDS DESCRIPTION [91] } & \multirow[b]{2}{*}{ WHM $(\AA)$} & \multirow[b]{2}{*}{$\lambda_{c}(\AA)$} \\
\hline band & filter & $\lambda_{\text {peak }}(\AA)$ & & \\
\hline$P_{5330}$ & Corning 5330 & 4000 & 1025 & 4175 \\
\hline$P_{5562}$ & Corning 5562 & 4200 & 1015 & 4280 \\
\hline V & Corning 3385 & 5300 & 670 & 5290 \\
\hline
\end{tabular}

$P_{5330}$ and $P_{5562}$ are two versions of the same $P$ band, the first realized in late 40's with a Corning 5330 filter, the second in early 50's with a Corning 5562 filter.

ZERO POINT: The PV system is defined by the magnitudes of six Polar Sequence stars listed in [91]

\section{SYSTEM ANALYSIS}

RELATIONS WITH OTHER SYSTEMS [91]

UBV - Johnson and Morgan - 1953

\begin{tabular}{|c|c|c|}
\hline$V_{J}$ & $=V_{E}$ & \\
\hline$(B-V)_{J}$ & $=0.964(P-V)_{E}+0.120$ & for $(B-V)_{J}<+1.0$ \\
\hline$(B-V)_{J}$ & $1.19(P-V)_{E}-0.10$ & for $+1.0<(B-V)_{J}<+1.5$ dwarfs \\
\hline
\end{tabular}

Fig. 8. The photometric system $P V-$ Eggen - 1955 
D. Moro and U. Munari: The Asiago Database on Photometric Systems (ADPS). I.

TRANSMISSION CURVES [91]

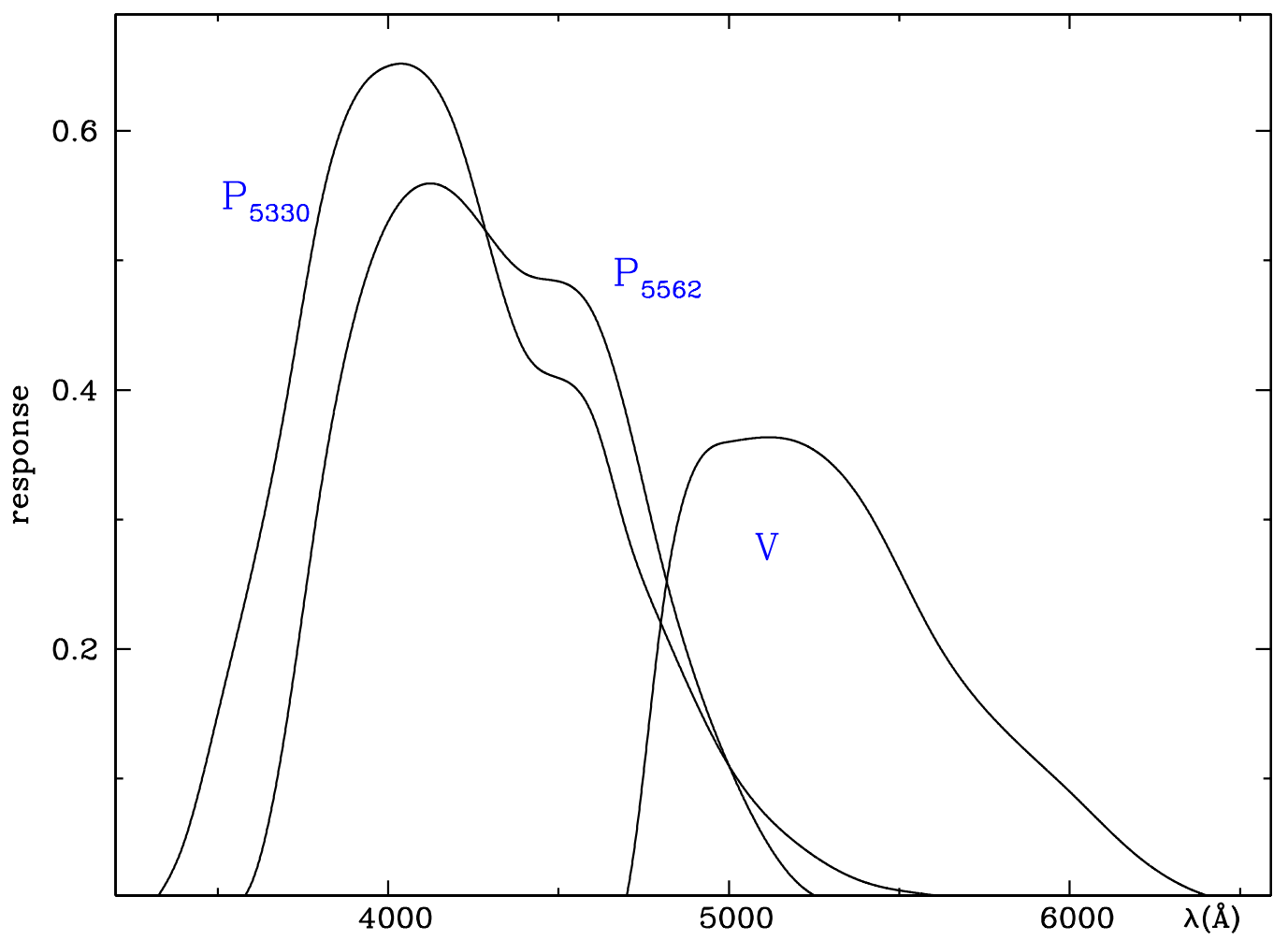

\begin{tabular}{|cc|cc|cc|}
\hline \multicolumn{5}{|c|}{$P$} & \multicolumn{2}{c|}{$V$} \\
\cline { 1 - 4 } \multicolumn{2}{|c|}{$P_{5330}$} & \multicolumn{2}{c|}{$P_{5562}$} & & \\
\cline { 1 - 4 }$\lambda(\mathrm{A})$ & $\Upsilon$ & $\lambda(\mathrm{A})$ & $\Upsilon$ & $\lambda(\mathrm{A})$ & $\Upsilon$ \\
\hline 3300 & 0.00 & 3500 & 0.00 & 4600 & 0.00 \\
3400 & 0.05 & 3600 & 0.02 & 4700 & 0.01 \\
3500 & 0.15 & 3700 & 0.14 & 4800 & 0.22 \\
3600 & 0.26 & 3800 & 0.32 & 5000 & 0.36 \\
3700 & 0.39 & 4000 & 0.53 & 5200 & 0.36 \\
3800 & 0.54 & 4200 & 0.55 & 5400 & 0.31 \\
4000 & 0.65 & 4400 & 0.49 & 5600 & 0.21 \\
4200 & 0.60 & 4600 & 0.46 & 5800 & 0.14 \\
4400 & 0.43 & 4700 & 0.38 & 6000 & 0.09 \\
4600 & 0.38 & 4800 & 0.27 & 6200 & 0.04 \\
4700 & 0.29 & 5000 & 0.11 & 6400 & 0.01 \\
4800 & 0.22 & 5200 & 0.02 & 6600 & 0.00 \\
5000 & 0.11 & 5400 & 0.00 & & \\
5200 & 0.05 & & & & \\
5400 & 0.02 & & & & \\
5600 & 0.01 & & & & \\
5800 & 0.00 & & & & \\
\hline
\end{tabular}

Fig. 8. continued 


\section{Aerobee UV-55 -1955}

Ultraviolet observations by an Aerobee rocket o wn on November 17, 1955.

\section{GENERAL INFORMATION}

DETECTOR UV photon counter

MAIN ARTICLE Kupperian, J. E. Boggess, A., Milligan, J. E. 1958, ApJ 128, 45

\section{SYSTEM DESCRIPTION}

\begin{tabular}{|c|c|}
\hline \multicolumn{2}{|c|}{ BANDS DESCRIPTION [37] } \\
\hline band & bandwidth (A) \\
\hline 120 & $1100-1340$ \\
\hline 130 & $1225-1350$ \\
\hline
\end{tabular}

The 120 band was found to be useless because of the strong geocoronal Ly $\alpha$ (1216A) contamination.

The wavelength limits of the 130 band are set by the calcium oride window of the tube (transparent to $\lambda>1225 \mathrm{~A}$ ) and the photosensitive gas in the tube (nitric oxide ionized by photons of $\lambda<1350 \mathrm{~A}$ ).

TRANSMISSION CURVES

As derived from Fig 1 of [177]

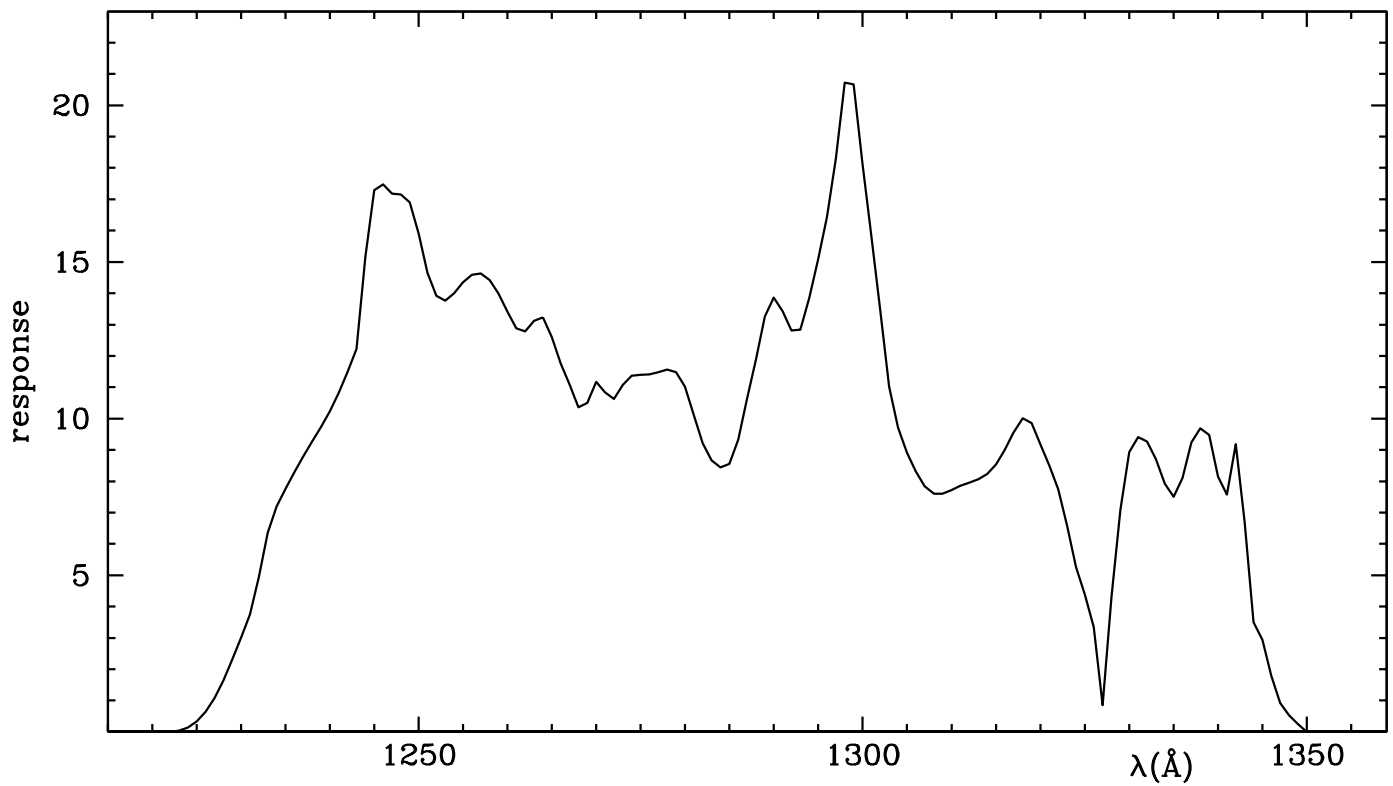

\begin{tabular}{|cc|cc|cc|cc|cc|cc|cc|cc|}
\hline \multicolumn{10}{|c|}{130} \\
\hline$\lambda(\mathrm{A})$ & $\Upsilon$ & $\lambda(\mathrm{A})$ & $\Upsilon$ & $\lambda(\mathrm{A})$ & $\Upsilon$ & $\lambda(\mathrm{A})$ & $\Upsilon$ & $\lambda(\mathrm{A})$ & $\Upsilon$ & $\lambda(\mathrm{A})$ & $\Upsilon$ & $\lambda(\mathrm{A})$ & $\Upsilon$ & $\lambda(\mathrm{A})$ & $\Upsilon$ \\
\hline 1220 & 0.0 & 1244 & 13.6 & 1262 & 12.7 & 1280 & 10.9 & 1297 & 18.7 & 1314 & 8.3 & 1329 & 6.3 & 1344 & 4.5 \\
1226 & 0.6 & 1244 & 15.1 & 1264 & 13.2 & 1281 & 9.6 & 1298 & 21.0 & 1317 & 9.4 & 1330 & 8.3 & 1345 & 3.0 \\
1228 & 1.5 & 1244 & 16.6 & 1266 & 12.1 & 1284 & 8.4 & 1300 & 19.0 & 1318 & 10.0 & 1331 & 9.4 & 1346 & 1.5 \\
1230 & 3.0 & 1247 & 17.2 & 1267 & 10.8 & 1286 & 9.0 & 1301 & 16.6 & 1320 & 9.0 & 1333 & 8.7 & 1348 & 0.6 \\
1232 & 4.5 & 1249 & 16.6 & 1269 & 10.2 & 1287 & 10.5 & 1301 & 15.1 & 1322 & 7.5 & 1336 & 7.9 & 1350 & 0.0 \\
1233 & 6.0 & 1251 & 15.1 & 1270 & 11.2 & 1288 & 12.1 & 1302 & 12.7 & 1323 & 6.0 & 1336 & 8.6 & & \\
1235 & 7.5 & 1252 & 13.8 & 1272 & 10.6 & 1289 & 13.7 & 1304 & 9.7 & 1325 & 4.5 & 1338 & 9.7 & & \\
1238 & 9.0 & 1255 & 14.2 & 1273 & 11.2 & 1293 & 12.8 & 1306 & 8.5 & 1326 & 3.0 & 1339 & 9.0 & & \\
1241 & 10.5 & 1257 & 14.6 & 1275 & 11.4 & 1294 & 13.6 & 1307 & 7.7 & 1327 & 1.2 & 1341 & 7.5 & & \\
1243 & 12.1 & 1260 & 13.6 & 1277 & 11.5 & 1295 & 15.7 & 1311 & 7.8 & 1328 & 3.6 & 1342 & 9.0 & & \\
\hline
\end{tabular}

Fig. 9. The photometric system Aerobee UV-55 - 1955 


\section{uvby $H \beta$ - Strömgren and Crawford - 1956}

Classification of F stars, later extended to other spectral types.

\section{GENERAL INFORMATION}

AUTHORS

TELESCOPE

DETECTOR

MAIN ARTICLE
B. Strömgren and D. L. Crawford

$0.80 \mathrm{~m}$ (reflector), Haute-Provence Obs.

RCA 1P21 (refrigerated)

Strömgren, B. 1956, Vistas in Astronomy 2, 1337

\section{SYSTEM DESCRIPTION}

\begin{tabular}{|c|lccc|c|}
\hline \multicolumn{5}{|c|}{ BANDS DESCRIPTION [178] pg 58 } & FLUX CALIBRATION [178] pg 58 \\
\hline band & \multicolumn{1}{|c|}{ filter } & $\lambda_{\text {peak }}(\AA)$ & half-width $(\AA)$ & $\lambda_{\text {eff }}(\AA)$ & $\left(\mathrm{erg} \mathrm{cm}^{-2} \mathrm{sec}^{-1} \AA^{-1}\right)(\#)$ \\
\hline$u$ & 8 mm SchottUG11+ & & & & \\
& 1mm SchottWG3 & 3500 & 300 & 3450 & $3.2510^{-9}$ \\
\hline$V$ & interference & 4110 & 190 & 4110 & $7.1810^{-9}$ \\
\hline$b$ & interference & 4670 & 180 & 4670 & $5.8110^{-9}$ \\
\hline$y$ & interference & 5470 & 230 & 5480 & $3.7010^{-9}$ \\
\hline$[79]$ & KPNO 212 & 4859 & 30 & & \\
$H \beta_{\text {narrow }}$ & KPNO 9 & 4858 & 29 & & \\
& KPNO 216 & 4868 & 26 & & \\
\hline$[79]$ & KPNO 214 & 4890 & 145 & & \\
$H \beta_{\text {wide }}$ & KPNO 10 & 4850 & 136 & & \\
& KPNO 225 & 4870 & 210 & & \\
\hline
\end{tabular}

(\#) Fluxes for an A0V star with $V_{\text {Johnson }}=0.00$ and $E(B-V)=0.00$.

For $\alpha$ Lyr: $(b-y)=0.004, m_{1}=0.157, c_{1}=1.089, \beta=2.903$. [187]

\section{SYSTEM ANALYSIS}

COLOR INDICES AND PARAMETERS [117], pg 182-190

$(b-y):$ temperature indicator.

$c_{1}=(u-v)-(v-b)$ : measures the Balmer discontinuity; $\mathrm{T}_{\text {eff }}$ indicator in O-A stars, and luminosity indicator in A-F stars.

$m_{1}=(v-b)-(b-y):$ measures the blocking by metallic lines.

$\beta=H \beta_{\text {wide }}-H \beta_{\text {narrow }}$ : luminosity indicator in $\mathrm{O}-\mathrm{A}$ stars and $\mathrm{T}_{\text {eff }}$ indicator in $\mathrm{A}-\mathrm{G}$ stars.

REDDENING-FREE PARAMETERS [117],pg. 183

$$
\begin{aligned}
& {\left[c_{1}\right]=c_{1}-\frac{E\left(c_{1}\right)}{E(b-y)}(b-y)=c_{1}-0.20(b-y)} \\
& {\left[m_{1}\right]=m_{1}-\frac{E\left(m_{1}\right)}{E(b-y)}(b-y)=m_{1}+0.18(b-y)}
\end{aligned}
$$

Fig. 10. The photometric system uvbyH $\beta$ - Strömgren and Crawford - 1956 


$$
\begin{aligned}
& {[u-b]=(u-b)-\frac{E(u-b)}{E(b-v)}(b-y)=(u-b)-1.84(b-y)} \\
& {[u-b]=\left[c_{1}\right]+2\left[m_{1}\right]}
\end{aligned}
$$

REDDENING RATIOS [294]

$$
E(b-y)=0.7 E(B-V)_{J o h n s o n}
$$

RELATIONS WITH OTHER SYSTEMS

$$
\text { UBV - Johnson and Morgan - } 1953 \text { [307] }
$$

\begin{tabular}{lll|}
$(U-B)=0.675( \pm 0.002)(u-b)-0.938( \pm 0.002)$ & for stars O-B I-V, A-F III-V \\
$(B-V)=1.584( \pm 0.007)(b-y)+0.681( \pm 0.014) m_{1}-0.116( \pm 0.002)$ & for stars O-B I-V, A III-V
\end{tabular}

\section{$U_{C} B V-\operatorname{Arp}-1958$ [76]}

\begin{tabular}{|rrrr}
\hline$(B-V)=1.662(b-y)-0.860 m_{1}-0.134-0.027 E(B-V)$ & $(B-V)<0.05$ \\
\hline$(B-V)=1.520(b-y)+0.604 m_{1}-0.092-0.015 E(B-V)$ & $0.05<(B-V)<0.70$ \\
\hline
\end{tabular}

\section{TRANSMISSION CURVES [211]}

$H_{\beta}^{w}$ and $H_{\beta}^{n}$ are derived from Fig 1 of [79].

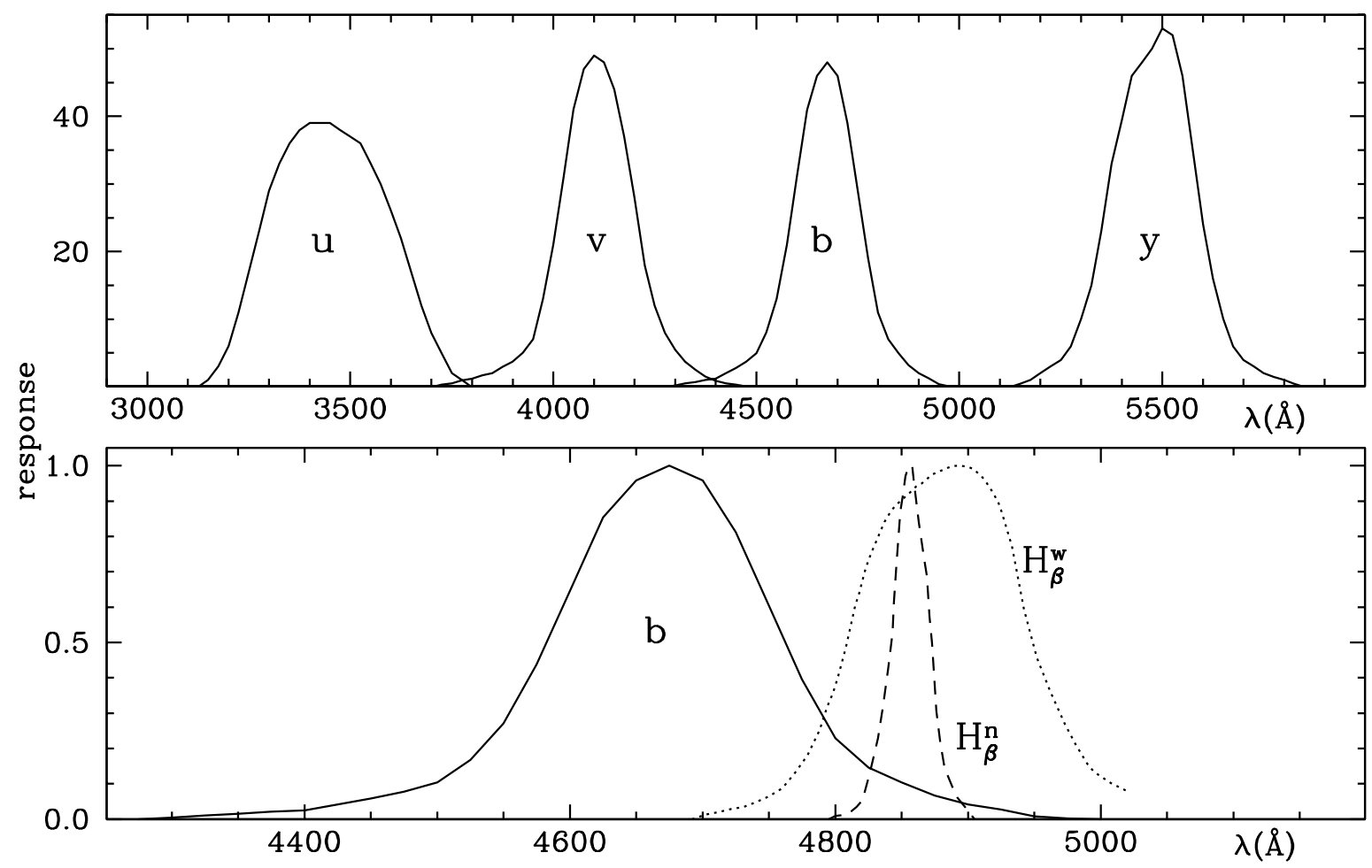

Fig. 10. continued 


\begin{tabular}{|c|c|c|c|c|c|c|c|c|c|c|c|}
\hline \multicolumn{2}{|c|}{$u$} & \multicolumn{2}{|c|}{ V } & \multicolumn{2}{|c|}{$b$} & \multicolumn{2}{|c|}{ y } & \multicolumn{2}{|c|}{$H \beta_{\text {wide }}$} & \multicolumn{2}{|c|}{$H \beta_{\text {narrow }}$} \\
\hline$\lambda(\AA)$ & $\Upsilon(\%)$ & $\lambda(\AA)$ & $\Upsilon(\%)$ & $\lambda(\AA)$ & $\Upsilon(\%)$ & $\lambda(\AA)$ & $\Upsilon(\%)$ & $\lambda(\AA)$ & $\Upsilon$ & $\lambda(\AA)$ & $\Upsilon$ \\
\hline 3125 & 0.0 & 3700 & 0.0 & 4275 & 0.0 & 5125 & 0.0 & 4692 & 0.000 & 4795 & 0.000 \\
\hline 3150 & 1.0 & 3725 & 0.3 & 4300 & 0.2 & 5150 & 0.5 & 4702 & 0.013 & 4800 & 0.009 \\
\hline 3175 & 3.0 & 3750 & 0.5 & 4325 & 0.5 & 5175 & 1.0 & 4712 & 0.020 & 4806 & 0.012 \\
\hline 3200 & 6.0 & 3775 & 1.0 & 4350 & 0.7 & 5200 & 2.0 & 4721 & 0.028 & 4812 & 0.023 \\
\hline 3225 & 11.0 & 3800 & 1.2 & 4375 & 1.0 & 5225 & 3.0 & 4731 & 0.034 & 4816 & 0.034 \\
\hline 3250 & 17.0 & 3825 & 1.7 & 4400 & 1.2 & 5250 & 4.0 & 4740 & 0.047 & 4820 & 0.053 \\
\hline 3275 & 23.0 & 3850 & 2.0 & 4425 & 2.0 & 5275 & 6.0 & 4750 & 0.064 & 4822 & 0.076 \\
\hline 3300 & 29.0 & 3875 & 3.0 & 4450 & 2.8 & 5300 & 10.0 & 4760 & 0.087 & 4826 & 0.130 \\
\hline 3325 & 33.0 & 3900 & 3.7 & 4475 & 3.7 & 5325 & 15.0 & 4769 & 0.128 & 4832 & 0.229 \\
\hline 3350 & 36.0 & 3925 & 5.0 & 4500 & 5.0 & 5350 & 23.0 & 4779 & 0.181 & 4836 & 0.321 \\
\hline 3375 & 38.0 & 3950 & 7.0 & 4525 & 8.0 & 5375 & 33.0 & 4788 & 0.252 & 4840 & 0.428 \\
\hline 3400 & 39.0 & 3975 & 13.0 & 4550 & 13.0 & 5400 & 41.0 & 4794 & 0.319 & 4843 & 0.527 \\
\hline 3425 & 39.0 & 4000 & 21.0 & 4575 & 21.0 & 5425 & 46.0 & 4798 & 0.356 & 4844 & 0.611 \\
\hline 3450 & 39.0 & 4025 & 31.0 & 4600 & 31.0 & 5450 & 48.0 & 4802 & 0.403 & 4846 & 0.719 \\
\hline 3475 & 38.0 & 4050 & 41.0 & 4625 & 41.0 & 5475 & 50.0 & 4808 & 0.484 & 4849 & 0.870 \\
\hline 3500 & 37.0 & 4075 & 47.0 & 4650 & 46.0 & 5500 & 53.0 & 4815 & 0.604 & 4851 & 0.916 \\
\hline 3525 & 36.0 & 4100 & 49.0 & 4675 & 48.0 & 5525 & 52.0 & 4818 & 0.638 & 4853 & 0.970 \\
\hline 3550 & 33.0 & 4125 & 48.0 & 4700 & 46.0 & 5550 & 46.0 & 4820 & 0.672 & 4855 & 0.996 \\
\hline 3575 & 30.0 & 4150 & 44.0 & 4725 & 39.0 & 5575 & 35.0 & 4823 & 0.712 & 4857 & 1.000 \\
\hline 3600 & 26.0 & 4175 & 37.0 & 4750 & 29.0 & 5600 & 24.0 & 4827 & 0.752 & 4858 & 0.996 \\
\hline 3625 & 22.0 & 4200 & 28.0 & 4775 & 19.0 & 5625 & 16.0 & 4831 & 0.789 & 4859 & 0.962 \\
\hline 3650 & 17.0 & 4225 & 18.0 & 4800 & 11.0 & 5650 & 10.0 & 4837 & 0.840 & 4861 & 0.901 \\
\hline 3675 & 12.0 & 4250 & 12.0 & 4825 & 7.0 & 5675 & 6.0 & 4842 & 0.873 & 4863 & 0.840 \\
\hline 3700 & 8.0 & 4275 & 8.0 & 4850 & 5.0 & 5700 & 4.0 & 4846 & 0.890 & 4867 & 0.733 \\
\hline 3725 & 5.0 & 4300 & 5.5 & 4875 & 3.2 & 5725 & 3.0 & 4856 & 0.923 & 4869 & 0.687 \\
\hline 3750 & 2.0 & 4325 & 3.7 & 4900 & 2.0 & 5750 & 2.0 & 4861 & 0.941 & 4871 & 0.573 \\
\hline 3775 & 1.0 & 4350 & 2.5 & 4925 & 1.3 & 5775 & 1.5 & 4865 & 0.950 & 4873 & 0.496 \\
\hline 3800 & 0.0 & 4375 & 1.5 & 4950 & 0.4 & 5800 & 1.0 & 4873 & 0.975 & 4875 & 0.382 \\
\hline & & 4400 & 0.9 & 4975 & 0.1 & 5825 & 0.5 & 4885 & 0.994 & 4876 & 0.305 \\
\hline & & 4425 & 0.5 & 5000 & 0.0 & 5850 & 0.0 & 4888 & 0.999 & 4879 & 0.218 \\
\hline & & 4450 & 0.3 & & & & & 4894 & 1.000 & 4882 & 0.153 \\
\hline & & 4475 & 0.1 & & & & & 4900 & 0.997 & 4884 & 0.130 \\
\hline & & 4500 & 0.1 & & & & & 4904 & 0.990 & 4888 & 0.092 \\
\hline & & 4525 & 0.0 & & & & & 4910 & 0.970 & 4892 & 0.061 \\
\hline & & & & & & & & 4913 & 0.957 & 4900 & 0.024 \\
\hline & & & & & & & & 4916 & 0.940 & 4905 & 0.000 \\
\hline & & & & & & & & 4919 & 0.923 & & \\
\hline & & & & & & & & 4923 & 0.893 & & \\
\hline & & & & & & & & 4925 & 0.873 & & \\
\hline & & & & & & & & 4933 & 0.772 & & \\
\hline & & & & & & & & 4942 & 0.594 & & \\
\hline & & & & & & & & 4952 & 0.457 & & \\
\hline & & & & & & & & 4962 & 0.362 & & \\
\hline & & & & & & & & 4973 & 0.269 & & \\
\hline & & & & & & & & 4981 & 0.212 & & \\
\hline & & & & & & & & 4990 & 0.155 & & \\
\hline & & & & & & & & 4995 & 0.134 & & \\
\hline & & & & & & & & 5000 & 0.121 & & \\
\hline & & & & & & & & 5008 & 0.101 & & \\
\hline & & & & & & & & 5019 & 0.081 & & \\
\hline
\end{tabular}

Fig. 10. continued 


\section{Aerobee UV-57 - 1957}

Ultraviolet observations from an Aerobee rocket flown on March 28, 1957.

\section{GENERAL INFORMATION}

DETECTOR UV photon counter

MAIN ARTICLE Boggess, A., Dunkelmann, L. 1958, AJ 63, 303

SYSTEM DESCRIPTION

\begin{tabular}{|c|cc|}
\hline \multicolumn{3}{|c|}{ BANDS DESCRIPTION [39] } \\
\hline band & $\lambda_{c}(\AA)$ & bandwidth $(\AA)$ \\
\hline 270 & 2700 & 350 \\
\hline
\end{tabular}

A chemical filter has been used to isolate the band.

Fig. 11. The photometric system Aerobee UV-57 - 1957 


$$
U_{c} B V-\operatorname{Arp}-1958
$$

Realization of the UBV - Johnson and Morgan - 1953 system with a refractor at Cape Town.

\section{GENERAL INFORMATION}

$\begin{array}{ll}\text { AUTHORS } & \text { H. C. Arp } \\ \text { TELESCOPE } & \text { 0.60m Mc Clean (refractor), Royal Observatory } \\ \text { DETECTOR } & \text { EMI photocell } \\ \text { MAIN ARTICLE } & \text { Arp, H. C. 1958, AJ 63, } 118\end{array}$

\section{SYSTEM DESCRIPTION}

\begin{tabular}{|l|l|cc|}
\hline \multicolumn{2}{|c|}{ BANDS DESCRIPTION [12] } & \\
\hline band & \multicolumn{1}{|c|}{ lter } & WHM (A) & $\lambda_{c}(\mathrm{~A})$ \\
\hline$U_{C}$ & 2mm Corning 9863 & 320 & 3920 \\
\hline$B$ & 2mm GG 13+2mm BG 12 & 765 & 4320 \\
\hline$V$ & 2mm Omag 302 & 580 & 5300 \\
\hline
\end{tabular}

ZERO POINT: Colors set to 0.00 for unreddened A0V stars. [178], pg. 74

\section{SYSTEM ANALYSIS}

REDDENING-FREE PARAMETERS [285], pg. 290

$Q_{U c B V}=\left(U_{C}-B\right)-0.36(B-V)$

\section{TRANSMISSION CURVES [211]}

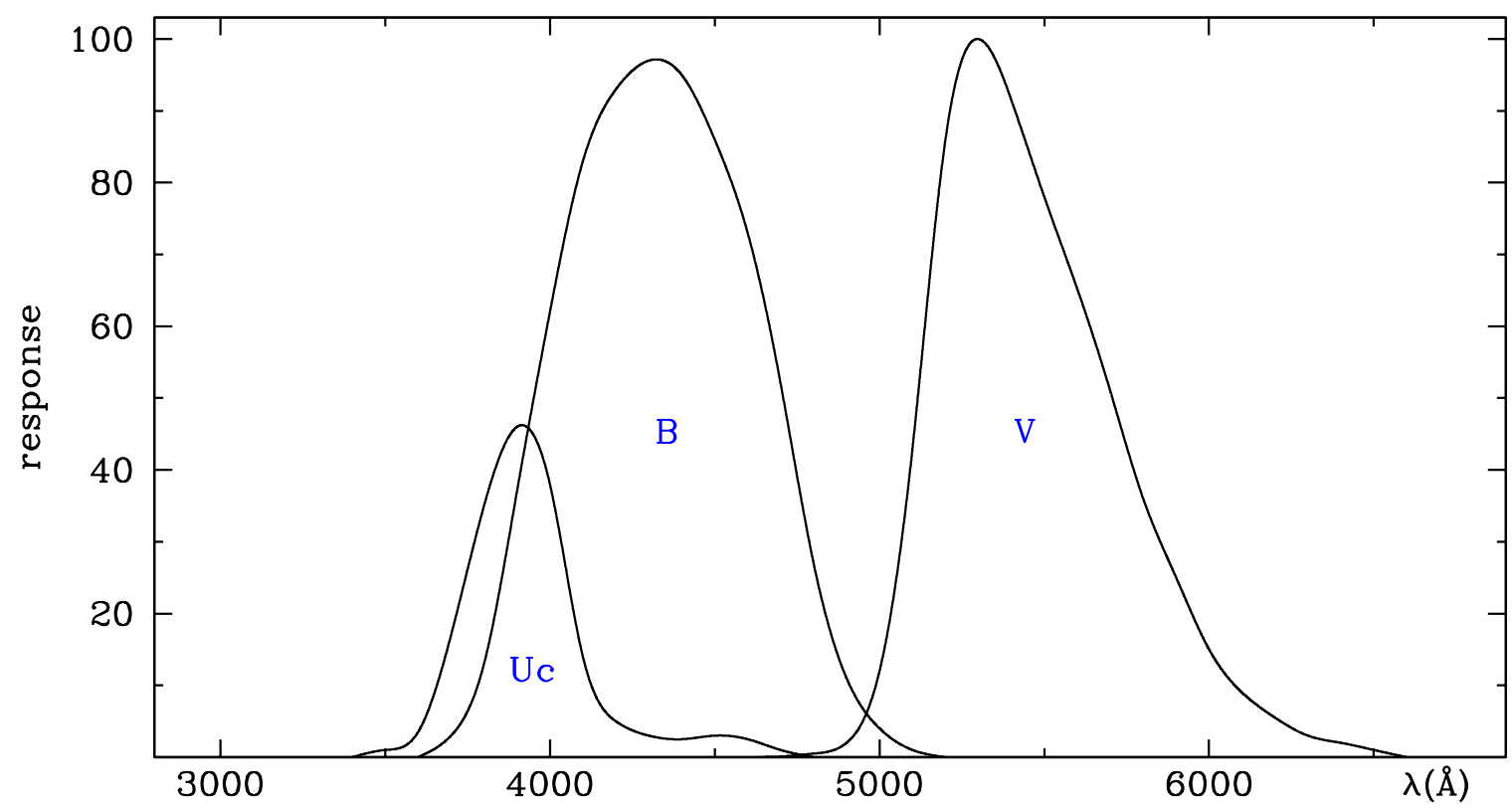

Fig. 12. The photometric system $U_{c} B V-\operatorname{Arp}-1958$ 


\begin{tabular}{|c|c|c|c|c|c|}
\hline \multicolumn{2}{|c|}{$U_{C}$} & \multicolumn{2}{|c|}{$B$} & \multicolumn{2}{|c|}{$V$} \\
\hline$\lambda(\AA)$ & $\Upsilon(\%)$ & $\lambda(\AA)$ & $\Upsilon(\%)$ & $\lambda(\AA)$ & $\Upsilon(\%)$ \\
\hline 3400 & 0.000 & 3600 & 0.000 & 4600 & 0.000 \\
\hline 3450 & 0.606 & 3650 & 1.162 & 4650 & 0.023 \\
\hline 3500 & 1.000 & 3700 & 3.000 & 4700 & 0.100 \\
\hline 3550 & 1.369 & 3750 & 6.387 & 4750 & 0.279 \\
\hline 3600 & 3.500 & 3800 & 13.000 & 4800 & 0.500 \\
\hline 3650 & 8.998 & 3850 & 23.927 & 4850 & 0.832 \\
\hline 3700 & 17.000 & 3900 & 37.000 & 4900 & 2.000 \\
\hline 3750 & 26.127 & 3950 & 49.788 & 4950 & 5.073 \\
\hline 3800 & 35.000 & 4000 & 62.000 & 5000 & 12.000 \\
\hline 3850 & 42.170 & 4050 & 73.539 & 5050 & 24.565 \\
\hline 3900 & 46.000 & 4100 & 83.000 & 5100 & 43.000 \\
\hline 3950 & 44.901 & 4150 & 89.183 & 5150 & 65.799 \\
\hline 4000 & 38.000 & 4200 & 93.000 & 5200 & 86.000 \\
\hline 4050 & 25.860 & 4250 & 95.600 & 5250 & 97.117 \\
\hline 4100 & 14.000 & 4300 & 97.000 & 5300 & 100.000 \\
\hline 4150 & 7.539 & 4350 & 96.918 & 5350 & 97.294 \\
\hline 4200 & 5.000 & 4400 & 95.000 & 5400 & 91.500 \\
\hline 4250 & 3.736 & 4450 & 91.125 & 5450 & 84.770 \\
\hline 4300 & 3.000 & 4500 & 86.000 & 5500 & 78.000 \\
\hline 4350 & 2.583 & 4550 & 80.202 & 5550 & 71.590 \\
\hline 4400 & 2.500 & 4600 & 73.000 & 5600 & 65.200 \\
\hline 4450 & 2.745 & 4650 & 63.544 & 5650 & 58.381 \\
\hline 4500 & 3.000 & 4700 & 52.000 & 5700 & 51.000 \\
\hline 4550 & 2.934 & 4750 & 39.120 & 5750 & 43.213 \\
\hline 4600 & 2.500 & 4800 & 27.000 & 5800 & 36.000 \\
\hline 4650 & 1.768 & 4850 & 17.604 & 5850 & 30.168 \\
\hline 4700 & 1.000 & 4900 & 11.000 & 5900 & 25.000 \\
\hline 4750 & 0.432 & 4950 & 6.713 & 5950 & 19.740 \\
\hline 4800 & 0.000 & 5000 & 4.000 & 6000 & 15.000 \\
\hline & & 5050 & 2.169 & 6050 & 11.495 \\
\hline & & 5100 & 1.000 & 6100 & 9.000 \\
\hline & & 5150 & 0.360 & 6150 & 7.090 \\
\hline & & 5200 & 0.000 & 6200 & 5.500 \\
\hline & & & & 6250 & 4.082 \\
\hline & & & & 6300 & 3.000 \\
\hline & & & & 6350 & 2.394 \\
\hline & & & & 6400 & 2.000 \\
\hline & & & & 6450 & 1.529 \\
\hline & & & & 6500 & 1.000 \\
\hline & & & & 6550 & 0.490 \\
\hline & & & & 6600 & 0.000 \\
\hline
\end{tabular}

Fig. 12. continued 


\section{ubgyri - Bahng - 1958}

General purpose system. Photometry of stars with composite spectra.

\section{GENERAL INFORMATION}

$\begin{array}{ll}\text { AUTHORS } & \text { J. D. R. Bahng } \\ \text { TELESCOPE } & \text { 0.30m (reflector), Washburn Observatory } \\ \text { DETECTOR } & \text { 1P21 ( for } u, b, g \text { bands) } \\ & \text { C7160 (for } y, r, i \text { bands) } \\ \text { MAIN ARTICLE } & \text { Bahng, J.D.R. 1958, MNRAS 14, 572 }\end{array}$

SYSTEM DESCRIPTION

\begin{tabular}{|c|lc|rc|}
\hline \multicolumn{3}{|c|}{ BANDS DESCRIPTION [16] } & \\
\hline band & \multicolumn{1}{|c|}{ filter } & $\lambda_{\text {eff }}(\AA)$ & $W H M(\AA)$ & $\lambda_{c}(\AA)$ \\
\hline$u$ & 3mm Corning 9863 + 1mm clear glass (\#), cemented & 3521 & 480 & 3578 \\
\hline$b$ & 2mm Schott BG 12+2mm Schott GG 13, cemented & 4184 & 830 & 4232 \\
\hline$g$ & 3mm Corning 3384 & 5405 & 825 & 5385 \\
\hline$y$ & 2mm Corning 3385 + 2.5mm Corning 9788, cemented & 5587 & 1360 & 5588 \\
\hline$r$ & 2mm Schott RG 2 + G125 interference (\#) & 7246 & 1785 & 7725 \\
\hline$i$ & $3.5 m m$ Schott RG 10 & 8772 & 1565 & 8805 \\
\hline
\end{tabular}

(\#) Bausch \& Lomb

ZERO POINT: Defined by $10 \mathrm{Lac}(\mathrm{O} 9 \mathrm{~V})$ and $\alpha$ Vir (B1V):

$10 \mathrm{Lac}: \quad(u-g)=-0.88, \quad(b-g)=-0.92, \quad(r-y)=+0.38, \quad(i-y)=+1.38$

$\alpha$ Vir: $\quad(u-g)=-0.82, \quad(b-g)=-0.95, \quad(r-y)=+0.40, \quad(i-y)=+1.44$

\section{SYSTEM ANALYSIS}

RELATIONS WITH OTHER SYSTEMS [16]

UBV - Johnson and Morgan - 1953

\begin{tabular}{|l}
\hline$(B-V)=+0.834( \pm 0.046)(b-g)+0.585( \pm 0.007)$ \\
$(U-V)=+1.028( \pm 0.049)(u-g)-0.308( \pm 0.055)$ \\
\hline
\end{tabular}

UVBGRI - Stebbins and Whitford - 1943

\begin{tabular}{|l}
\hline$(I-G)=+1.107( \pm 0.087)(i-y)-0.192( \pm 0.038)$ \\
\hline$(R-G)=+0.856( \pm 0.088)(r-y)+0.321( \pm 0.029)$ \\
\hline$(V-G)=+1.086( \pm 0.075)(b-g)-0.122( \pm 0.006)$ \\
\hline$(U-G)=+1.046( \pm 0.148)(u-g)-1.159( \pm 0.114)$ \\
\hline
\end{tabular}

Fig. 13. The photometric system ubgyri - Bahng - 1958 


\section{TRANSMISSION CURVES [211]}

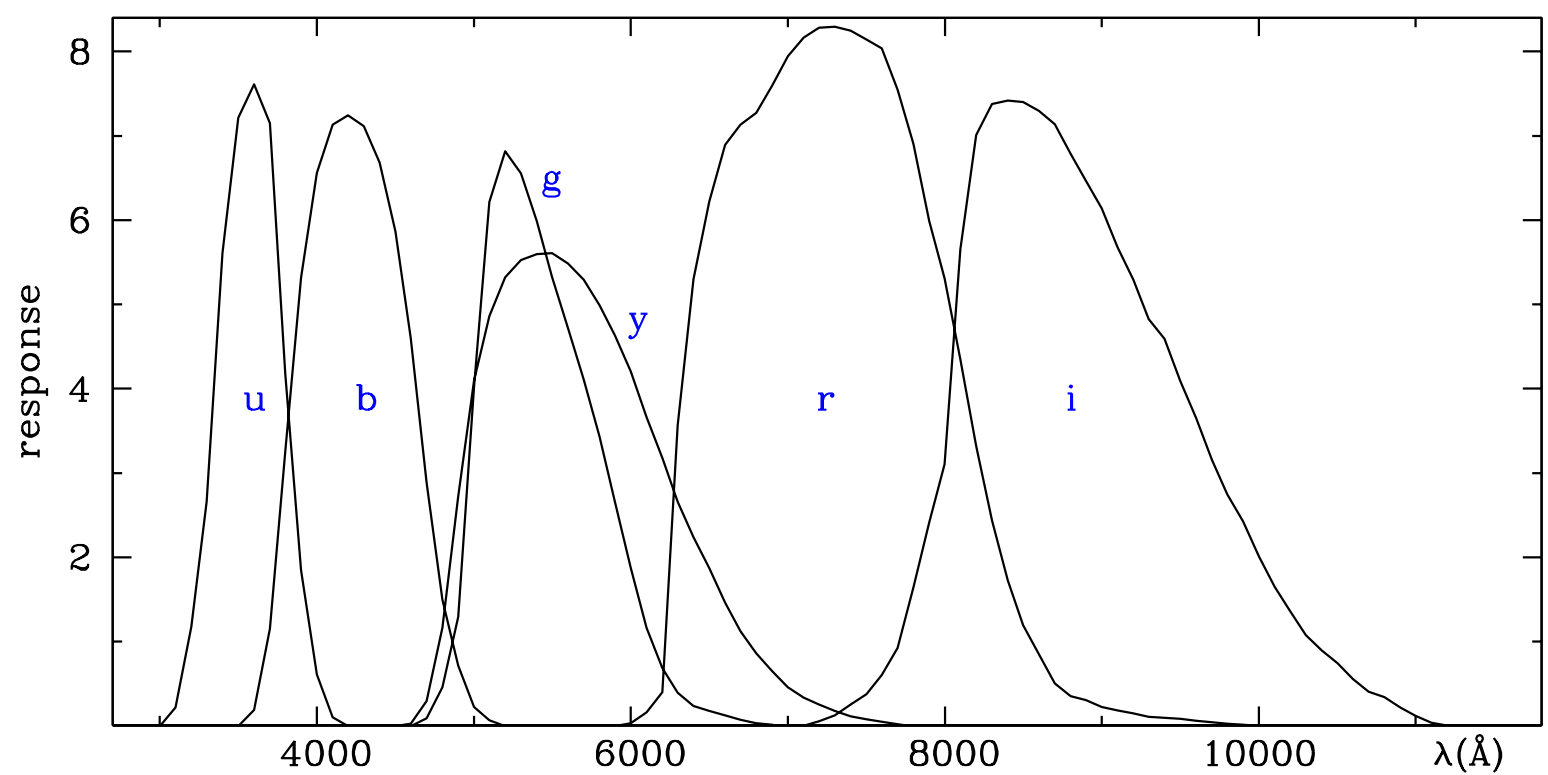

\begin{tabular}{|c|c|c|c|c|c|c|c|c|c|c|c|c|c|c|c|}
\hline \multicolumn{2}{|c|}{$u$} & \multicolumn{2}{|c|}{$b$} & \multicolumn{2}{|c|}{$g$} & \multicolumn{2}{|c|}{$y$} & \multicolumn{4}{|c|}{$r$} & \multicolumn{4}{|c|}{ i } \\
\hline$\lambda(\mathrm{A})$ & $\Upsilon$ & $\lambda(\mathrm{A})$ & $\Upsilon$ & $\lambda(\mathrm{A})$ & $\Upsilon$ & $\lambda(\mathrm{A})$ & $\Upsilon$ & $\lambda(\mathrm{A})$ & $\Upsilon$ & $\lambda(\mathrm{A})$ & $\Upsilon$ & $\lambda(\mathrm{A})$ & $\Upsilon$ & $\lambda(\mathrm{A})$ & $\Upsilon$ \\
\hline 3000 & 0.000 & 3500 & 0.000 & 4600 & 0.000 & 4500 & 0.000 & 5900 & 0.000 & 9300 & 0.108 & 6900 & 0.000 & 10300 & 1.076 \\
\hline 3100 & 0.218 & 3600 & 0.189 & 4700 & 0.092 & 4600 & 0.028 & 6000 & 0.034 & 9400 & 0.099 & 7000 & 0.001 & 10400 & 0.897 \\
\hline 3200 & 1.177 & 3700 & 1.154 & 4800 & 0.459 & 4700 & 0.292 & 6100 & 0.160 & 9500 & 0.084 & 7100 & 0.004 & 10500 & 0.743 \\
\hline 3300 & 2.659 & 3800 & 3.247 & 4900 & 1.295 & 4800 & 1.162 & 6200 & 0.401 & 9600 & 0.060 & 7200 & 0.055 & 10600 & 0.559 \\
\hline 3400 & 5.606 & 3900 & 5.316 & 5000 & 4.022 & 4900 & 2.723 & 6300 & 3.563 & 9700 & 0.042 & 7300 & 0.124 & 10700 & 0.406 \\
\hline 3500 & 7.215 & 4000 & 6.560 & 5100 & 6.214 & 5000 & 4.098 & 6400 & 5.296 & 9800 & 0.029 & 7400 & 0.253 & 10800 & 0.340 \\
\hline 3600 & 7.609 & 4100 & 7.134 & 5200 & 6.815 & 5100 & 4.858 & 6500 & 6.220 & 9900 & 0.016 & 7500 & 0.375 & 10900 & 0.217 \\
\hline 3700 & 7.152 & 4200 & 7.241 & 5300 & 6.555 & 5200 & 5.322 & 6600 & 6.895 & 10000 & 0.000 & 7600 & 0.606 & 11000 & 0.119 \\
\hline 3800 & 4.184 & 4300 & 7.113 & 5400 & 5.986 & 5300 & 5.524 & 6700 & 7.133 & & & 7700 & 0.928 & 11100 & 0.038 \\
\hline 3900 & 1.850 & 4400 & 6.685 & 5500 & 5.320 & 5400 & 5.594 & 6800 & 7.275 & & & 7800 & 1.642 & 11200 & 0.006 \\
\hline 4000 & 0.610 & 4500 & 5.867 & 5600 & 4.721 & 5500 & 5.610 & 6900 & 7.592 & & & 7900 & 2.417 & 11300 & 0.000 \\
\hline 4100 & 0.101 & 4600 & 4.601 & 5700 & 4.108 & 5600 & 5.486 & 7000 & 7.942 & & & 8000 & 3.105 & & \\
\hline 4200 & 0.000 & 4700 & 2.887 & 5800 & 3.428 & 5700 & 5.292 & 7100 & 8.167 & & & 8100 & 5.657 & & \\
\hline & & 4800 & 1.497 & 5900 & 2.657 & 5800 & 4.989 & 7200 & 8.284 & & & 8200 & 7.009 & & \\
\hline & & 4900 & 0.715 & 6000 & 1.875 & 5900 & 4.634 & 7300 & 8.293 & & & 8300 & 7.376 & & \\
\hline & & 5000 & 0.227 & 6100 & 1.163 & 6000 & 4.206 & 7400 & 8.247 & & & 8400 & 7.418 & & \\
\hline & & 5100 & 0.069 & 6200 & 0.685 & 6100 & 3.665 & 7500 & 8.140 & & & 8500 & 7.399 & & \\
\hline & & 5200 & 0.000 & 6300 & 0.392 & 6200 & 3.178 & 7600 & 8.038 & & & 8600 & 7.294 & & \\
\hline & & & & 6400 & 0.238 & 6300 & 2.659 & 7700 & 7.544 & & & 8700 & 7.140 & & \\
\hline & & & & 6500 & 0.176 & 6400 & 2.241 & 7800 & 6.907 & & & 8800 & 6.786 & & \\
\hline & & & & 6600 & 0.123 & 6500 & 1.869 & 7900 & 5.987 & & & 8900 & 6.465 & & \\
\hline & & & & 6700 & 0.074 & 6600 & 1.461 & 8000 & 5.308 & & & 9000 & 6.138 & & \\
\hline & & & & 6800 & 0.031 & 6700 & 1.125 & 8100 & 4.345 & & & 9100 & 5.677 & & \\
\hline & & & & 6900 & 0.012 & 6800 & 0.862 & 8200 & 3.319 & & & 9200 & 5.295 & & \\
\hline & & & & 7000 & 0.000 & 6900 & 0.649 & 8300 & 2.439 & & & 9300 & 4.827 & & \\
\hline & & & & & & 7000 & 0.458 & 8400 & 1.723 & & & 9400 & 4.593 & & \\
\hline & & & & & & 7100 & 0.337 & 8500 & 1.196 & & & 9500 & 4.092 & & \\
\hline & & & & & & 7200 & 0.255 & 8600 & 0.848 & & & 9600 & 3.655 & & \\
\hline & & & & & & 7300 & 0.176 & 8700 & 0.502 & & & 9700 & 3.157 & & \\
\hline & & & & & & 7400 & 0.116 & 8800 & 0.354 & & & 9800 & 2.750 & & \\
\hline & & & & & & 7500 & 0.080 & 8900 & 0.308 & & & 9900 & 2.427 & & \\
\hline & & & & & & 7600 & 0.052 & 9000 & 0.225 & & & 10000 & 2.012 & & \\
\hline & & & & & & 7700 & 0.018 & 9100 & 0.181 & & & 10100 & 1.651 & & \\
\hline & & & & & & 7800 & 0.000 & 9200 & 0.150 & & & 10200 & 1.361 & & \\
\hline
\end{tabular}

Fig. 13. continued 


\section{UN BG R - Tifft - 1958}

General purpose photographic and photoelectric system.

\section{GENERAL INFORMATION}

AUTHORS

TELESCOPE

DETECTOR

MAIN ARTICLE
W. G. Tifft

$1.52 \mathrm{~m}$ and $2.54 \mathrm{~m}$, Mount Wilson Obs. eleven-stage EMI 6094 (unrefrigerated), 103a-O and 103-E

Tifft, W. G. 1958, AJ 63, 127

\section{SYSTEM DESCRIPTION}

\begin{tabular}{|l|l|rc|}
\hline \multicolumn{3}{|c|}{ BANDS DESCRIPTION [302] } & \\
\hline band & \multicolumn{1}{|c|}{ filter/ filter + plate } & $W H M(\AA)$ & $\lambda_{c}(\AA)$ \\
\hline \multicolumn{4}{|c|}{ photoelectric } \\
\hline$U N$ & Corning 5970 & 533 & 3700 \\
\hline$B G$ & Corning 5030 + Corning 3387 & 537 & 4715 \\
\hline$O$ & Corning 2434 & 283 & 5915 \\
\hline \multicolumn{4}{|c|}{ photographic } \\
\hline$U V$ & Corning 5970 + Kodak 103a-O & 503 & 3672 \\
\hline$B G$ & Schott GG9 + Kodak 103a-O & 536 & 4723 \\
\hline$R$ & Corning 2418 + Kodak 103a-E & 430 & 6338 \\
\hline
\end{tabular}

ZERO POINT: Unreddened A0V stars have zero color indices. [302]

PHOTOGRAPHIC VERSION: Tifft, W. G. 1958, AJ 63, 127

\section{SYSTEM ANALYSIS}

REDDENING PARAMETERS [302]

$$
\begin{aligned}
& A(B G)=3.14 E(B G-R) \\
& (B G-R)_{0}=0.320 Q-0.014, \text { with } Q=(U V-B G)-[E(U V-B G) / E(B G-R)](B G-R)
\end{aligned}
$$

Fig. 14. The photometric system $W$ BG $R$ - Tifft -1958 


\section{TRANSMISSION CURVES [302]}

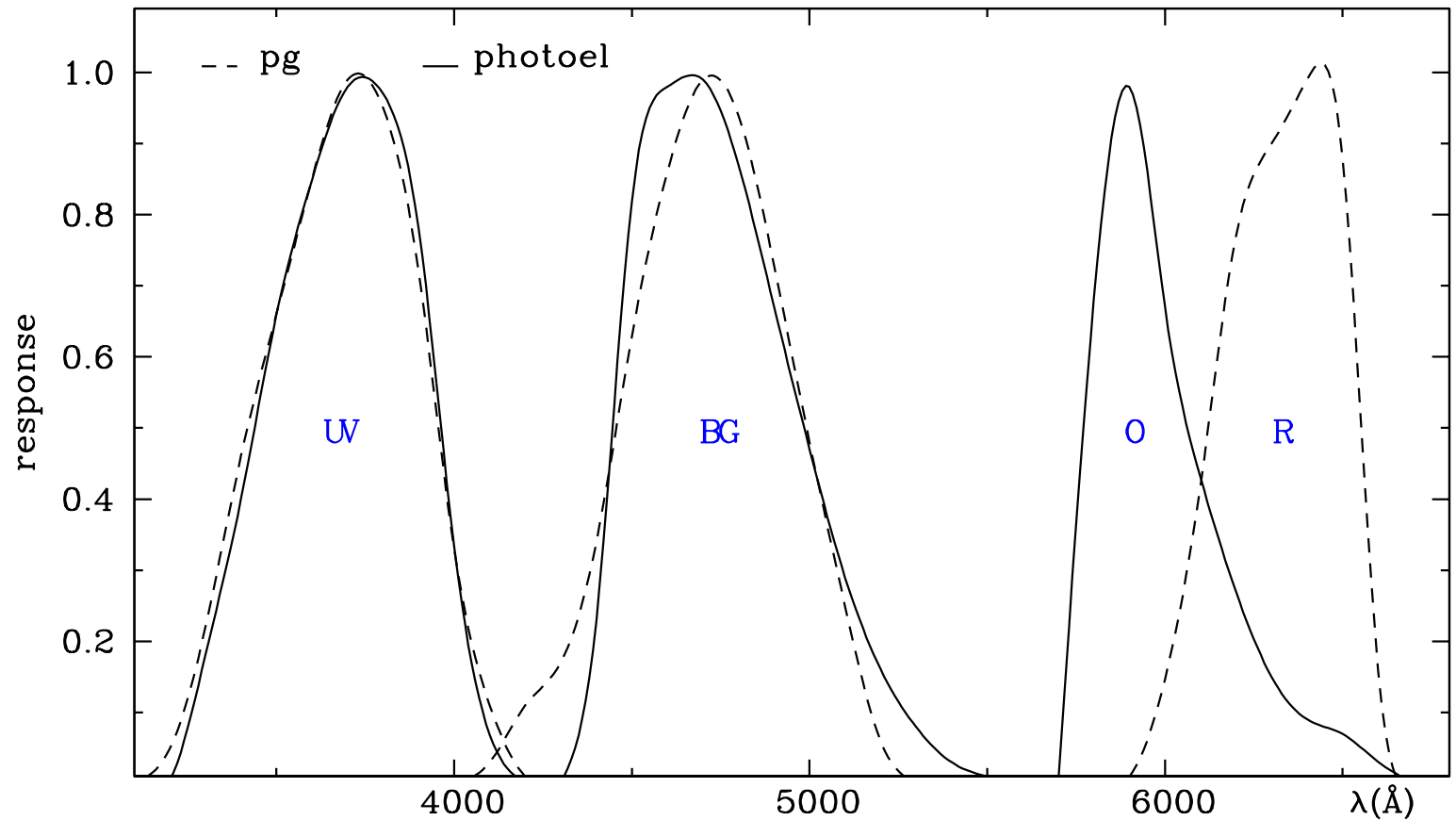

\begin{tabular}{|c|c|c|c|c|c|c|c|c|c|c|c|}
\hline \multicolumn{2}{|c|}{$U N_{p g}$} & \multicolumn{2}{|c|}{ UN photoel } & \multicolumn{2}{|c|}{$B G_{p g}$} & \multicolumn{2}{|c|}{$B G_{\text {photoel }}$} & \multicolumn{2}{|c|}{$R$} & \multicolumn{2}{|c|}{$O$} \\
\hline$\lambda(\mathrm{A})$ & $\Upsilon$ & $\lambda(\mathrm{A})$ & $\Upsilon$ & $\lambda(\mathrm{A})$ & $\Upsilon$ & $\lambda(\mathrm{A})$ & $\Upsilon$ & $\lambda(\mathrm{A})$ & $\Upsilon$ & $\lambda(\mathrm{A})$ & $\Upsilon$ \\
\hline 3100 & 0.00 & 3100 & 0.00 & 4000 & 0.00 & 4300 & 0.00 & 5800 & 0.00 & 5600 & 0.00 \\
\hline 3200 & 0.05 & 3200 & 0.05 & 4100 & 0.03 & 4400 & 0.23 & 5900 & 0.01 & 5700 & 0.01 \\
\hline 3300 & 0.22 & 3300 & 0.18 & 4200 & 0.11 & 4500 & 0.82 & 6000 & 0.14 & 5800 & 0.68 \\
\hline 3400 & 0.46 & 3400 & 0.42 & 4300 & 0.17 & 4600 & 0.98 & 6100 & 0.42 & 5900 & 0.98 \\
\hline 3500 & 0.66 & 3500 & 0.66 & 4400 & 0.34 & 4700 & 0.99 & 6200 & 0.77 & 6000 & 0.67 \\
\hline 3600 & 0.85 & 3600 & 0.85 & 4500 & 0.63 & 4800 & 0.87 & 6300 & 0.90 & 6100 & 0.43 \\
\hline 3700 & 0.99 & 3700 & 0.98 & 4600 & 0.86 & 4900 & 0.67 & 6400 & 0.99 & 6200 & 0.27 \\
\hline 3800 & 0.95 & 3800 & 0.97 & 4700 & 0.99 & 5000 & 0.47 & 6500 & 0.88 & 6300 & 0.15 \\
\hline 3900 & 0.72 & 3900 & 0.78 & 4800 & 0.94 & 5100 & 0.29 & 6600 & 0.16 & 6400 & 0.09 \\
\hline 4000 & 0.33 & 4000 & 0.39 & 4900 & 0.73 & 5200 & 0.16 & 6700 & 0.00 & 6500 & 0.07 \\
\hline 4100 & 0.11 & 4100 & 0.07 & 5000 & 0.48 & 5300 & 0.08 & & & 6600 & 0.03 \\
\hline 4200 & 0.01 & 4200 & 0.00 & 5100 & 0.25 & 5400 & 0.03 & & & 6700 & 0.01 \\
\hline 4300 & 0.00 & & & 5200 & 0.06 & 5500 & 0.01 & & & 6800 & 0.00 \\
\hline & & & & 5300 & 0.00 & 5600 & 0.00 & & & & \\
\hline
\end{tabular}

Fig. 14. continued 


\section{5 colors - Borgman - 1959}

Separates different stellar populations via ultraviolet excess.

\section{GENERAL INFORMATION}

\section{AUTHORS J. Borgman \\ TELESCOPE $\quad 0.91 \mathrm{~m}$, Mc Donald Observatory \\ DETECTOR RCA 1P21 (unrefrigerated) \\ MAIN ARTICLE Borgman, J. 1959, ApJ 129, 362}

SYSTEM DESCRIPTION

\begin{tabular}{|l|lcc|}
\hline \multicolumn{4}{|c|}{ BANDS DESCRIPTION } \\
\hline band & \multicolumn{1}{|c|}{ lter } & $\lambda_{\text {peak }}(\mathrm{A})$ & FWHM (A) \\
\hline$U_{1}$ & glass + silver & 3200 & 208 \\
\hline$U_{2}$ & glass (\#) & 3660 & 325 \\
\hline 404 & interference & 4035 & 94 \\
\hline 453 & interference & 4527 & 92 \\
\hline 503 & interference & 5029 & 87 \\
\hline
\end{tabular}

(\#) The $U_{1}$ is a sandwich of two Corning 9836 lters, each covered by a thin layer of silver.

ZERO POINT: The mean colors of the six A0V stars dening the zero point of the UBV - Johnson and Morgan - 1953 system is taken to be 0.00 here too. [42]

\section{SYSTEM ANALYSIS}

COLOR INDICES AND PARAMETERS [42]

$m=(503-453)-(453-404)=503+404-2(453):$ spectral type and metallicity indicator.

\section{TRANSMISSION CURVES}

As derived from Fig. 1 of [42]

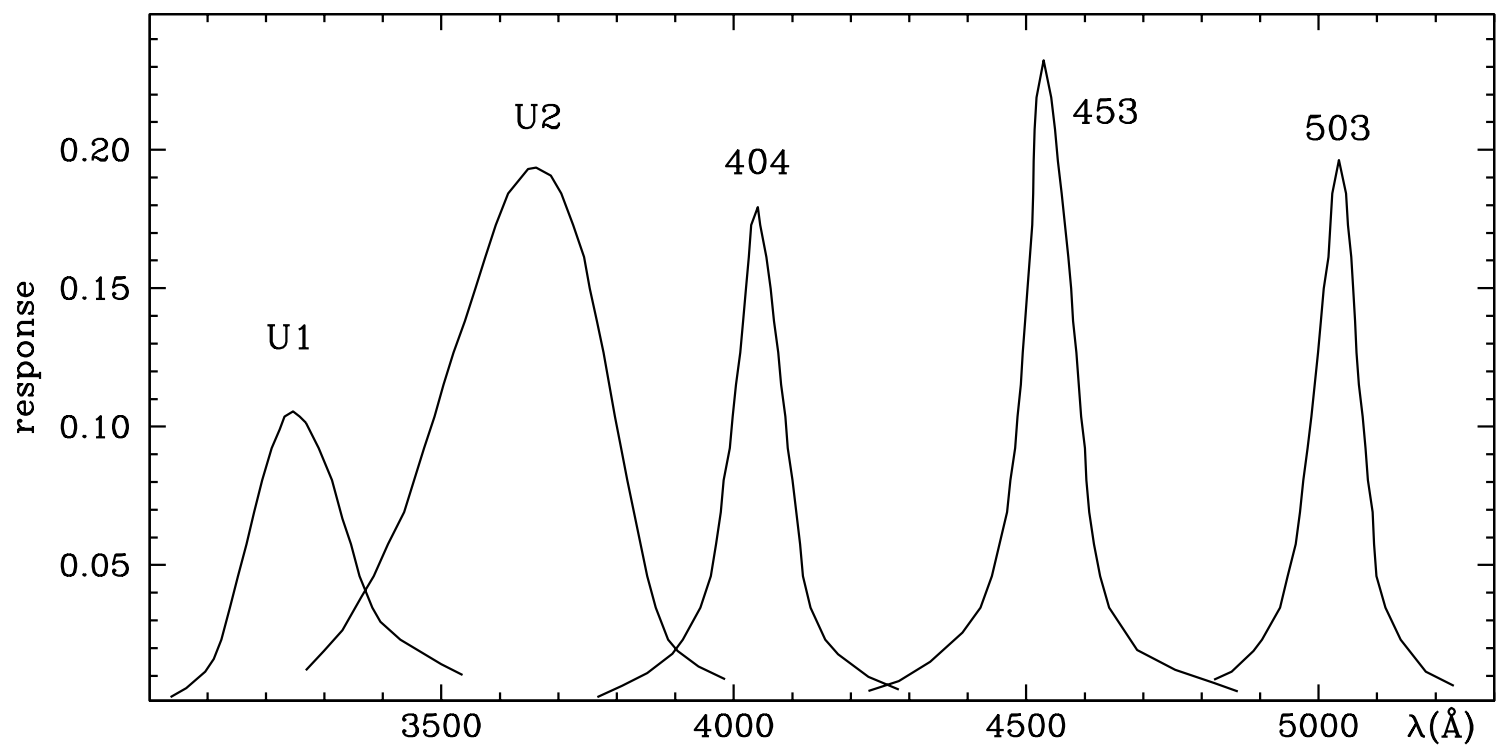

Fig. 15. The photometric system 5 colors - Borgman - 1959 


\begin{tabular}{|c|c|c|c|c|c|c|c|c|c|}
\hline \multicolumn{2}{|c|}{ U1 } & \multicolumn{2}{|c|}{$U 2$} & \multicolumn{2}{|c|}{404} & \multicolumn{2}{|c|}{453} & \multicolumn{2}{|c|}{503} \\
\hline$\lambda(\AA)$ & $\Upsilon$ & $\lambda(\AA)$ & $\Upsilon$ & $\lambda(\AA)$ & $\Upsilon$ & $\lambda(\AA)$ & $\Upsilon$ & $\lambda(\AA)$ & $\Upsilon$ \\
\hline 3025 & 0.0010 & 3225 & 0.0023 & 3780 & 0.0035 & 4200 & 0.0030 & 4760 & 0.0030 \\
\hline 3050 & 0.0037 & 3250 & 0.0080 & 3810 & 0.0065 & 4230 & 0.0046 & 4790 & 0.0057 \\
\hline 3075 & 0.0072 & 3275 & 0.0137 & 3840 & 0.0097 & 4260 & 0.0064 & 4820 & 0.0084 \\
\hline 3100 & 0.0126 & 3300 & 0.0191 & 3870 & 0.0134 & 4290 & 0.0088 & 4850 & 0.0114 \\
\hline 3125 & 0.0235 & 3325 & 0.0248 & 3900 & 0.0192 & 4320 & 0.0125 & 4880 & 0.0164 \\
\hline 3150 & 0.0447 & 3350 & 0.0335 & 3930 & 0.0295 & 4350 & 0.0175 & 4910 & 0.0246 \\
\hline 3175 & 0.0650 & 3375 & 0.0425 & 3960 & 0.0444 & 4380 & 0.0234 & 4940 & 0.0398 \\
\hline 3200 & 0.0851 & 3400 & 0.0534 & 3990 & 0.0887 & 4410 & 0.0298 & 4970 & 0.0725 \\
\hline 3225 & 0.0998 & 3425 & 0.0640 & 4000 & 0.1079 & 4440 & 0.0456 & 4980 & 0.0905 \\
\hline 3250 & 0.1051 & 3450 & 0.0787 & 4010 & 0.1245 & 4470 & 0.0745 & 4990 & 0.1077 \\
\hline 3275 & 0.0986 & 3475 & 0.0953 & 4020 & 0.1469 & 4500 & 0.1437 & 5000 & 0.1292 \\
\hline 3300 & 0.0881 & 3500 & 0.1123 & 4030 & 0.1730 & 4510 & 0.1743 & 5010 & 0.1517 \\
\hline 3325 & 0.0714 & 3525 & 0.1291 & 4040 & 0.1808 & 4520 & 0.2214 & 5020 & 0.1711 \\
\hline 3350 & 0.0538 & 3550 & 0.1447 & 4050 & 0.1672 & 4530 & 0.2323 & 5030 & 0.1955 \\
\hline 3375 & 0.0374 & 3575 & 0.1611 & 4060 & 0.1559 & 4540 & 0.2236 & 5040 & 0.1965 \\
\hline 3400 & 0.0285 & 3600 & 0.1769 & 4070 & 0.1364 & 4550 & 0.2045 & 5050 & 0.1722 \\
\hline 3425 & 0.0238 & 3625 & 0.1884 & 4080 & 0.1178 & 4560 & 0.1857 & 5060 & 0.1446 \\
\hline 3450 & 0.0205 & 3650 & 0.1933 & 4090 & 0.0984 & 4570 & 0.1651 & 5070 & 0.1135 \\
\hline 3475 & 0.0174 & 3675 & 0.1926 & 4100 & 0.0805 & 4580 & 0.1389 & 5080 & 0.0909 \\
\hline 3500 & 0.0143 & 3700 & 0.1866 & 4110 & 0.0628 & 4590 & 0.1152 & 5090 & 0.0731 \\
\hline 3525 & 0.0115 & 3725 & 0.1729 & 4140 & 0.0301 & 4600 & 0.0916 & 5120 & 0.0324 \\
\hline 3550 & 0.0089 & 3750 & 0.1540 & 4170 & 0.0194 & 4610 & 0.0663 & 5150 & 0.0192 \\
\hline 3575 & 0.0063 & 3775 & 0.1293 & 4200 & 0.0140 & 4640 & 0.0356 & 5180 & 0.0121 \\
\hline 3600 & 0.0036 & 3800 & 0.0995 & 4230 & 0.0096 & 4670 & 0.0236 & 5210 & 0.0083 \\
\hline 3625 & 0.0010 & 3825 & 0.0727 & 4260 & 0.0067 & 4700 & 0.0177 & 5240 & 0.0057 \\
\hline & & 3850 & 0.0478 & 4290 & 0.0045 & 4730 & 0.0142 & 5270 & 0.0031 \\
\hline & & 3875 & 0.0298 & 4320 & 0.0024 & 4760 & 0.0118 & & \\
\hline & & 3900 & 0.0197 & & & 4790 & 0.0096 & & \\
\hline & & 3925 & 0.0154 & & & 4820 & 0.0074 & & \\
\hline & & 3950 & 0.0122 & & & 4850 & 0.0053 & & \\
\hline & & 3975 & 0.0097 & & & 4880 & 0.0030 & & \\
\hline & & 4000 & 0.0074 & & & & & & \\
\hline & & 4025 & 0.0052 & & & & & & \\
\hline & & 4050 & 0.0030 & & & & & & \\
\hline & & 4075 & 0.0007 & & & & & & \\
\hline
\end{tabular}

Fig. 15. continued 


\section{KLMNPQR - Borgman - 1960}

Classification of early-type stars.

\section{GENERAL INFORMATION}

$\begin{array}{ll}\text { AUTHORS } & \text { J. Borgman } \\ \text { TELESCOPE } & 0.53 \mathrm{~m} \text { (reflector), Lowell Obs.; 0.91m (reflector), McDonald Obs. } \\ \text { DETECTOR } & \text { 1P21 (refrigerated) } \\ \text { MAIN ARTICLE } & \text { Borgman, J. 1960, BAN 15, 255 }\end{array}$

\section{SYSTEM DESCRIPTION}

\begin{tabular}{|c|ccc|}
\hline \multicolumn{4}{|c|}{ BANDS DESCRIPTION } \\
\hline band & $\lambda_{\text {peak }}(\AA)[43]$ & FWHM $(\AA)[43]$ & $\lambda_{\text {eff }}(\AA)(\#)[44]$ \\
\hline$R$ & 3295 & 80 & 3328 \\
\hline$Q$ & 3560 & 90 & 3601 \\
\hline$P$ & 3750 & 110 & 3731 \\
\hline$N$ & 4055 & 200 & 4009 \\
\hline$M$ & 4550 & 200 & 4431 \\
\hline$L$ & 5240 & 220 & 5189 \\
\hline$K$ & 5880 & 215 & 5784 \\
\hline
\end{tabular}

(\#) For a $30000 \mathrm{~K}$ black body.

Interference filters.

ZERO POINT: The color indices of $15 \mathrm{Mon}=\mathrm{S}$ Mon $(\mathrm{O} 7 \mathrm{Ve})$ are 0.00. [43]

\section{SYSTEM ANALYSIS}

\section{COLOR INDICES AND PARAMETERS [44]}

$(P-N)$ : temperature index.

REDDENING-FREE PARAMETERS [45], [47], [117], pg 232

$\alpha=(M-L)-1.69(N-M):$ chemical composition parameter.

$\beta=(P-N)-0.701(N-M):$ measures the absorption by higher Balmer lines around $3750 \AA$.

$\gamma=(Q-N)-1.086(N-M):$ measures the Balmer discontinuity.

$\delta=(R-Q)-0.882(N-M)$ : difference between the gradients on both sides of the hydrogen Balmer discontinuity. $\varepsilon=(P-N)-1.91(Q-P):$ luminosity parameter for late-type stars.

REDDENING RATIOS [44], [45]

$$
\begin{array}{lll}
E(L-K) / E(N-M)=1.117 & E(M-L) / E(N-M)=1.69 & E(P-N) / E(N-M)=0.701 \\
E(Q-N) / E(N-M)=1.068 & E(R-Q) / E(N-M)=0.882
\end{array}
$$

Fig. 16. The photometric system KLMNPQR - Borgman - 1960 


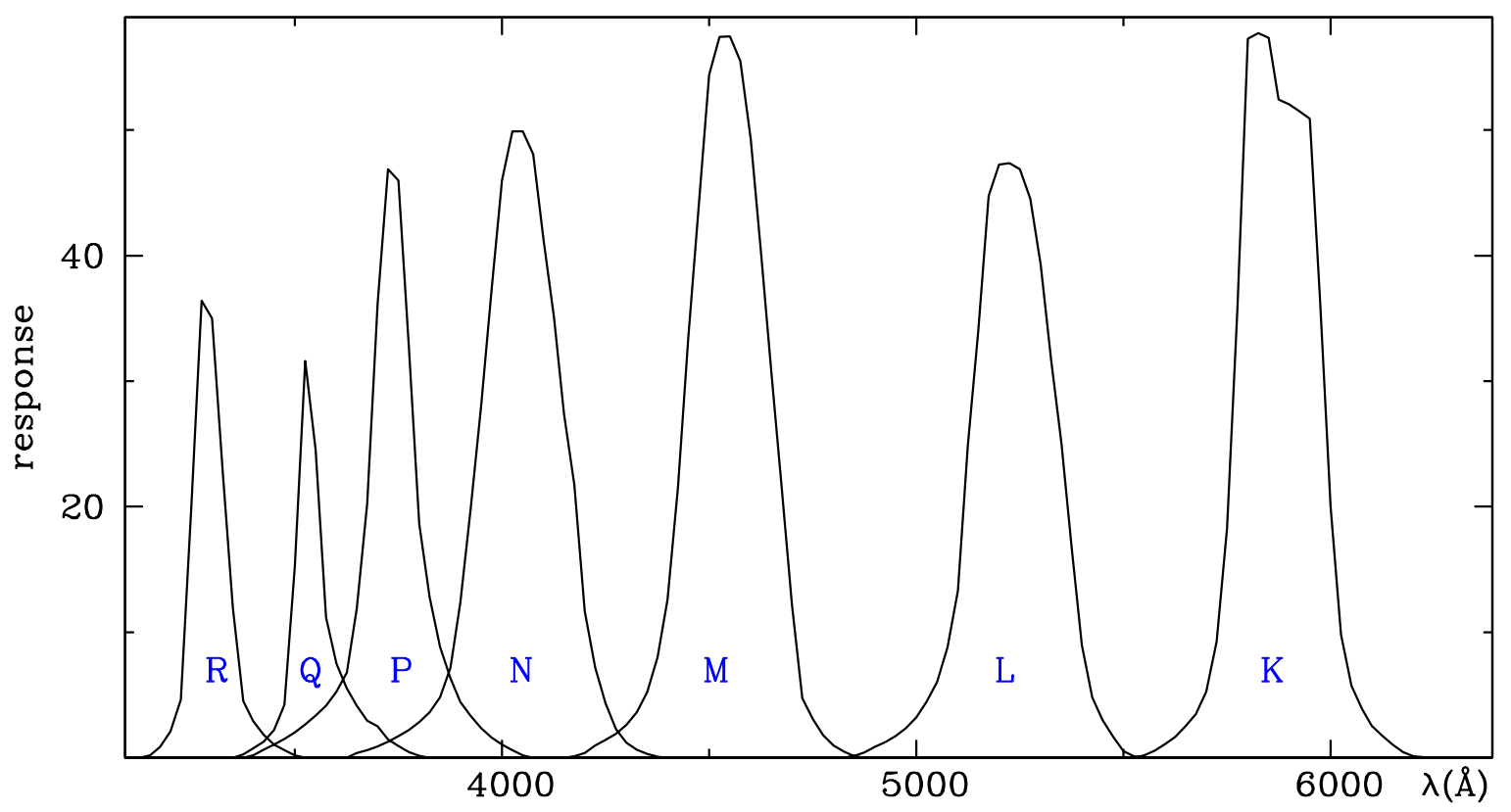

\begin{tabular}{|c|c|c|c|c|c|c|c|c|c|c|c|c|c|}
\hline \multicolumn{2}{|c|}{$R$} & \multicolumn{2}{|c|}{$Q$} & \multicolumn{2}{|c|}{$P$} & \multicolumn{2}{|c|}{$N$} & \multicolumn{2}{|c|}{$M$} & \multicolumn{2}{|c|}{$L$} & \multicolumn{2}{|c|}{$K$} \\
\hline$\lambda(\mathrm{A})$ & $\Upsilon(\%)$ & $\lambda(\mathrm{A})$ & $\Upsilon(\%)$ & $\lambda(\mathrm{A})$ & $\Upsilon(\%)$ & $\lambda(\mathrm{A})$ & $\Upsilon(\%)$ & $\lambda(\mathrm{A})$ & $\Upsilon(\%)$ & $\lambda(\mathrm{A})$ & $\Upsilon(\%)$ & $\lambda(\mathrm{A})$ & $\Upsilon(\%)$ \\
\hline 3125 & 0.00 & 3350 & 0.00 & 3375 & 0.00 & 3625 & 0.00 & 4150 & 0.00 & 4825 & 0.00 & 5525 & 0.00 \\
\hline 3150 & 0.18 & 3375 & 0.27 & 3400 & 0.20 & 3650 & 0.38 & 4175 & 0.11 & 4850 & 0.15 & 5550 & 0.18 \\
\hline 3175 & 0.86 & 3400 & 0.76 & 3425 & 0.65 & 3675 & 0.60 & 4200 & 0.39 & 4875 & 0.42 & 5575 & 0.55 \\
\hline 3200 & 2.10 & 3425 & 1.28 & 3450 & 1.05 & 3700 & 0.90 & 4225 & 0.96 & 4900 & 0.86 & 5600 & 1.07 \\
\hline 3225 & 4.62 & 3450 & 2.21 & 3475 & 1.48 & 3725 & 1.28 & 4250 & 1.41 & 4925 & 1.22 & 5625 & 1.64 \\
\hline 3250 & 19.86 & 3475 & 4.23 & 3500 & 2.02 & 3750 & 1.71 & 4275 & 1.92 & 4950 & 1.72 & 5650 & 2.49 \\
\hline 3275 & 36.41 & 3500 & 15.25 & 3525 & 2.65 & 3775 & 2.20 & 4300 & 2.62 & 4975 & 2.35 & 5675 & 3.47 \\
\hline 3300 & 35.00 & 3525 & 31.60 & 3550 & 3.35 & 3800 & 2.84 & 4325 & 3.62 & 5000 & 3.20 & 5700 & 5.24 \\
\hline 3325 & 23.08 & 3550 & 24.52 & 3575 & 4.15 & 3825 & 3.63 & 4350 & 5.22 & 5025 & 4.46 & 5725 & 9.26 \\
\hline 3350 & 11.93 & 3575 & 11.20 & 3600 & 5.25 & 3850 & 4.80 & 4375 & 8.00 & 5050 & 6.03 & 5750 & 18.25 \\
\hline 3375 & 4.52 & 3600 & 7.50 & 3625 & 6.80 & 3875 & 7.17 & 4400 & 12.60 & 5075 & 8.80 & 5775 & 35.83 \\
\hline 3400 & 2.90 & 3625 & 5.53 & 3650 & 11.90 & 3900 & 12.37 & 4425 & 21.50 & 5100 & 13.31 & 5800 & 57.27 \\
\hline 3425 & 1.82 & 3650 & 4.14 & 3675 & 20.25 & 3925 & 19.90 & 4450 & 33.50 & 5125 & 24.88 & 5825 & 57.75 \\
\hline 3450 & 1.034 & 3675 & 2.95 & 3700 & 36.15 & 3950 & 28.05 & 4475 & 43.98 & 5150 & 33.98 & 5850 & 57.37 \\
\hline 3475 & 0.59 & 3700 & 2.51 & 3725 & 46.90 & 3975 & 37.27 & 4500 & 54.42 & 5175 & 44.75 & 5875 & 52.43 \\
\hline 3500 & 0.19 & 3725 & 1.47 & 3750 & 46.00 & 4000 & 45.98 & 4525 & 57.42 & 5200 & 47.26 & 5900 & 52.06 \\
\hline 3525 & 0.00 & 3750 & 0.94 & 3775 & 32.80 & 4025 & 49.92 & 4550 & 57.48 & 5225 & 47.38 & 5925 & 51.50 \\
\hline & & 3775 & 0.46 & 3800 & 18.65 & 4050 & 49.91 & 4575 & 55.48 & 5250 & 46.87 & 5950 & 50.90 \\
\hline & & 3800 & 0.15 & 3825 & 12.77 & 4075 & 48.09 & 4600 & 49.26 & 5275 & 44.55 & 5975 & 36.23 \\
\hline & & 3825 & 0.00 & 3850 & 8.85 & 4100 & 41.22 & 4625 & 40.10 & 5300 & 39.30 & 6000 & 19.97 \\
\hline & & 3850 & 0.00 & 3875 & 6.33 & 4125 & 35.06 & 4650 & 30.93 & 5325 & 31.70 & 6025 & 9.80 \\
\hline & & & & 3900 & 4.45 & 4150 & 27.44 & 4675 & 21.70 & 5350 & 25.00 & 6050 & 5.75 \\
\hline & & & & 3925 & 3.30 & 4175 & 21.72 & 4700 & 12.21 & 5375 & 16.92 & 6075 & 3.92 \\
\hline & & & & 3950 & 2.35 & 4200 & 11.68 & 4725 & 4.75 & 5400 & 8.98 & 6100 & 2.53 \\
\hline & & & & 3975 & 1.60 & 4225 & 7.18 & 4750 & 3.08 & 5425 & 4.80 & 6125 & 1.75 \\
\hline & & & & 4000 & 1.05 & 4250 & 4.32 & 4775 & 1.78 & 5450 & 3.00 & 6150 & 1.03 \\
\hline & & & & 4025 & 0.60 & 4275 & 2.27 & 4800 & 0.96 & 5475 & 1.68 & 6175 & 0.46 \\
\hline & & & & 4050 & 0.20 & 4300 & 1.21 & 4825 & 0.48 & 5500 & 0.54 & 6200 & 0.095 \\
\hline & & & & 4075 & 0.00 & 4325 & 0.65 & 4850 & 0.12 & 5525 & 0.12 & 6225 & 0.02 \\
\hline & & & & & & 4350 & 0.30 & 4875 & 0.01 & 5550 & 0.00 & 6250 & 0.00 \\
\hline & & & & & & 4375 & 0.08 & 4900 & 0.00 & 5575 & 0.00 & & \\
\hline & & & & & & 4400 & 0.00 & & & & & & \\
\hline
\end{tabular}

Fig. 16. continued 


\section{Deeming - 1960}

Three square-band measurement of $\mathrm{Mgb}$ on spectra of $\mathrm{G}$ and $\mathrm{K}$ stars.

\section{GENERAL INFORMATION}

$\begin{array}{ll}\text { AUTHORS } & \text { T. J. Deeming } \\ \text { TELESCOPE } & 0.90 \mathrm{~m} \text { (reflector), Cambridge Obs. } \\ \text { DETECTOR } & \text { EMI 9502 (refrigerated) } \\ \text { MAIN ARTICLE } & \text { Deeming, T. J. 1960, MNRAS 121, 52 }\end{array}$

SYSTEM DESCRIPTION

\begin{tabular}{|l|c|}
\hline \multicolumn{2}{|c|}{ BANDS DESCRIPTION [84] } \\
\hline band & bandpass $(\AA)$ \\
\hline$A$ & $5110.4-5139.8$ \\
\hline$B$ & $5160.8-5189.0$ \\
\hline$C$ & $5214.4-5243.0$ \\
\hline
\end{tabular}

On the focal plane of a $18.4 \AA / \mathrm{mm}$ dispersion spectrograph three diaphragms transmit to photomultipliers the above three $A, B$ and $C$ portions of the spectrum.

\section{SYSTEM ANALYSIS}

\section{COLOR INDICES AND PARAMETERS [84]}

$r_{M g}=\frac{A+C}{B}:$ measures the strength of 5167-5184 Å MgI aborption lines.

$r_{M g}$ is a spectral type indicator between G8 and K5, and a luminosity indicator for stars with $0.70<(B-V)<1.30$.

Fig. 17. The photometric system Deeming - 1960 


\section{$U B V$ - Eggen and Sandage - 1960}

A general purpose realization of the UBV - Johnson and Morgan - 1953 photometric system. Used on LMC and SMC.

\section{GENERAL INFORMATION}

$\begin{array}{ll}\begin{array}{l}\text { AUTHORS } \\ \text { TELESCOPE }\end{array} & \begin{array}{l}\text { O. Eggen and A. Sandage } \\ \text { aluminized } 0.46 \mathrm{~m} \text { (reflector), Cape Town; } \\ \text { silvered } 1.93 \mathrm{~m} \text { Radcliffe (reflector), Pretoria }\end{array} \\ \text { MAIN ARTICLE } & \text { Eggen, O., Sandage, A. 1960, MNRAS 120, 79 }\end{array}$

\section{NOTES}

A set of $U B V$ primary sequence stars are established in both LMC and SMC with the aluminized $0.46 \mathrm{~m}$ reflector. They are used on the silvered $1.88 \mathrm{~m}$ reflector to calibrate secondary standards in both LMC and SMC.

No details are provided for the photometer at the $0.46 \mathrm{~m}$ reflector, while at the $1.93 \mathrm{~m}$ reflector a refrigerated $1 \mathrm{P} 21$ is used together with a $2 \mathrm{~mm}$ GG11 filter for the $V$ band and $2 \mathrm{~mm}$ GG13 + 1mm BG12 for the $B$ band.

Fig. 18. The photometric system $U B V$ - Eggen and Sandage - 1960 


\title{
Griffin and Redman - 1960
}

Three square-band measurement of $\mathrm{CN}$ and $G$ bands on spectra of $\mathrm{G}$ and $\mathrm{K}$ stars.

\section{GENERAL INFORMATION}

\author{
AUTHORS R. F. Griffin and R. O. Redman \\ TELESCOPE $\quad 0.90 \mathrm{~m}$ (reflector), Cambridge Obs. \\ DETECTOR EMI 9502 (refrigerated) \\ MAIN ARTICLE Griffin, R. F., Redman, R. O. 1960, MNRAS 120, 287
}

\section{SYSTEM DESCRIPTION}

\begin{tabular}{|l|c|}
\hline \multicolumn{2}{|c|}{ BANDS DESCRIPTION [124] } \\
\hline band & bandpass $(\AA)$ \\
\hline \multicolumn{2}{|c|}{ CN } \\
\hline$A$ & $4097-4149$ \\
\hline$B$ & $4164-4214$ \\
\hline$C$ & $4230-4283$ \\
\hline & $G$ band \\
\hline$A$ & $4230-4270$ \\
\hline$B$ & $4285-4315$ \\
\hline$C$ & $4342-4380$ \\
\hline
\end{tabular}

On the focal plane of a $18.4 \AA / \mathrm{mm}$ dispersion spectrograph three diaphragms transmit to photomultipliers the above three $A, B$ and $C$ portions of the spectrum.

\section{SYSTEM ANALYSIS}

\section{COLOR INDICES AND PARAMETERS [124]}

$r_{C N}=\frac{A_{C N}+C_{C N}}{B_{C N}}:$ measures the strength of the $4200 \AA \mathrm{CN}$ band.

$r_{C N}$ offers a good separation of luminosity classes and correlates with space velocities and spectral types for stars between F0 and K5. [248]

$r_{G}=\frac{A_{G}+C_{G}}{B_{G}}:$ measures the strength of the $4300 \AA G$ band.

$r_{G}$ poorly separates luminosity classes and does not to correlate with space velocities; it offers a fairly good spectral type classification for G and K stars. [248]

Fig. 19. The photometric system Griffin and Redman - 1960 


\section{USNO - Kron and Mayall - 1960}

Optical and near-infrared photometry with a single detector.

Used for integral photometry of globular clusters.

\section{GENERAL INFORMATION}
AUTHORS
G. E. Kron, and N. U. Mayall
TELESCOPE
0.91 Crossley (reflector), Mount Hamilton
DETECTORS
1P21, Dumont 6291, CE25A/B, CE23A/B, Lallemand photomultipliers
MAIN ARTICLE Kron, G. E., Mayall, N. U. 1960, AJ 65, 581

\section{SYSTEM DESCRIPTION}

\begin{tabular}{|c|c|c|c|c|}
\hline \multicolumn{5}{|c|}{ BANDS DESCRIPTION [174] } \\
\hline obs. mode & cell & $P$ & V & I \\
\hline \multicolumn{5}{|c|}{ small-field photometer } \\
\hline PV, I separated & $\begin{array}{l}\text { 1P21 } \\
\text { CE25A/B }\end{array}$ & $0.4 \mathrm{~mm} \mathrm{C} 3060+1 \mathrm{~mm} \mathrm{BG12}$ & 2mm GG14 + glass & $\mathrm{W} 88 \mathrm{~A}+$ cover glasses \\
\hline PVI simultanously & Lallemand II & $\begin{array}{l}2 \mathrm{~mm} \mathrm{BG} 23+1 \mathrm{~mm} \mathrm{BG} 12 \\
+2 \mathrm{~mm} \text { WG1 cryolite }\end{array}$ & $2 \mathrm{~mm} \mathrm{GG} 11+2 \mathrm{~mm}$ BG18 & $2 \mathrm{~mm} \mathrm{BG} 21+2 \mathrm{~mm} \mathrm{RG} 8$ \\
\hline I & Lallemand II & & & $2 \mathrm{~mm}$ RG2 coated \\
\hline \multicolumn{5}{|c|}{ large-field photometer } \\
\hline PV, I separated & $\begin{array}{l}\text { Dumont } 6291 \\
\text { CE23A/B }\end{array}$ & $2 \mathrm{~mm} \mathrm{GG} 13$ + 1mm BG12 & 2mm GG14 & W88A + cover glasses \\
\hline PVI simultanously & Lallemand I & $\begin{array}{l}2 \mathrm{~mm} \mathrm{BG} 23+1 \mathrm{~mm} \mathrm{BG} 12 \\
+2 \mathrm{~mm} \mathrm{GG} 13\end{array}$ & $\begin{array}{l}2 \mathrm{~mm} \mathrm{GG14}+2 \mathrm{~mm} \mathrm{BG18} \\
\text { not cemented }\end{array}$ & $\begin{array}{l}\text { C3966 + W89 } \\
+ \text { cover glass }\end{array}$ \\
\hline
\end{tabular}

\section{SYSTEM ANALYSIS}

\section{REDDENING RATIOS [174]}

$A_{V}=2.91( \pm 0.22) E(P-V)=2.33( \pm 0.14) E(V-I)$

\section{RELATIONS WITH OTHER SYSTEMS [174]}

\section{UBV - Johnson and Morgan - 1953}

\begin{tabular}{|lrr|}
\hline$V_{\text {Johnson }}$ & $=V-0.075(P-V)-0.027$ & \\
$(B-V)$ & $=0.10+0.96(P-V)$ & $-0.4<(P-V)<1.0$ \\
\hline$(B-V)$ & $=1.06(P-V)$ & $1.0<(P-V)<1.5$
\end{tabular}

$P V$ - Eggen - 1955

$V_{\text {Eggen }}=V-0.115(P-V)-0.026$

RI - Kron and Smith - 1951

$$
I_{U S N O}=I_{K S}
$$

Fig. 20. The photometric system USNO - Kron and Mayall - 1960 


\section{VBLUW - Walraven and Walraven - 1960}

Classification of early-type stars.

\section{GENERAL INFORMATION}

AUTHORS

TELESCOPE

DETECTOR MAIN ARTICLE
T. Walraven and J. H. Walraven

$0.91 \mathrm{~m}$ (reflector), Leiden Southern Station, Union Obs., South-Africa

1P21 (for $V, B, L$ and $U$ bands) and Lallemand cell (for the $W$ band), refrigerated Walraven, Th., Walraven J. H. 1960, BAN 15, 67

\begin{tabular}{|c|c|c|c|c|}
\hline \multicolumn{5}{|c|}{ SYSTEM DESCRIPTION } \\
\hline \multicolumn{4}{|c|}{ BANDS DESCRIPTION } & FLUX CALIBRATION [112] \\
\hline band & $\lambda_{\text {peak }}(\AA)[178]$, pg. 78 & $\lambda_{\text {eff }}(\AA)[178]$, pg. 78 & FWHM $(\AA)[191]$ & 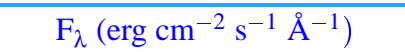 \\
\hline$W$ & 3270 & 3255 & 150 & $2.1210^{-11}$ \\
\hline$U$ & 3670 & 3633 & 260 & $1.6110^{-11}$ \\
\hline$L$ & 3900 & 3838 & 140 & $1.5210^{-11}$ \\
\hline$B$ & 4295 & 4325 & 420 & $1.2310^{-11}$ \\
\hline$V$ & 5450 & 5467 & 850 & $6.7310^{-12}$ \\
\hline
\end{tabular}

The $W, U, B$ and $V$ bands are obtained in a spectro-photometer by redirecting to separate photomultipliers portions of the spectrum geometrically selected by a filter of quartz and Iceland spar (which transmits a spectrum of bright bands at regular intervals separated by dark regions. Quartz prisms are used as a cross-disperser to separate the bright bands and redirect them via a quartz collector lens to four individual photomultipliers).

The L band is more conventionally obtained via standard filter photometry with UG2 (2mm) + WG2 (2mm) placed in front of a fifth photomultiplier (1P21) [313].

The $0.91 \mathrm{~m}$ telescope and the Walraven photometrer were moved in 1979 from the Leiden Station in South Africa (Harteheespoortdam) to ESO - La Silla. As a result the passbands of the system slighly changed [306]

ZERO POINT: Magnitudes and colors of HD $144470=\omega^{1}$ Sco $(B 1 V)$ are:

$V=1.1760,(V-B)=-0.0025,(B-U)=+0.0052,(U-W)=-0.0020,(B-L)=+0.0039$. [191]

\section{SYSTEM ANALYSIS}

\section{COLOR INDICES AND PARAMETERS [50]}

$(V-B):$ sensitive to reddening.

$(B-U)$ : measures the Balmer jump. Temperature indicator for $\mathrm{O}$ and $\mathrm{B}$ stars.

$(B-L)$ : depends mainly on gravity.

$(U-W)$ : measures the slope of the Balmer continuum. Both gravity and temperature dependent.

REDDENING-FREE PARAMETERS [50]

$$
\begin{aligned}
& {[B-U]=(B-U)-0.61(V-B) \quad[U-W]=(U-W)-0.45(V-B)} \\
& {[B-L]=(B-L)-0.39(V-B)}
\end{aligned}
$$

REDDENING RATIOS [191], [306], [50]

$$
\begin{array}{lll}
E(B-U) / E(V-B)=0.62 & E(U-W) / E(V-B)=0.45 & E(B-L) / E(V-B)=0.41 \\
E(L-U) / E(V-B)=0.22 & E(B-V)_{\text {Johnson }} / E(V-B)=2.255 \\
A(V) / E(V-B)=3.16-0.12 E(V-B)
\end{array}
$$

Fig. 21. The photometric system VBLUW - Walraven and Walraven - 1960 
RELATIONS WITH OTHER SYSTEMS [50], [306]

UBV - Johnson and Morgan - 1953
$R G U$ - Trefzger - 1983

$\begin{aligned} V & =0.963 G-0.448(G-R)+0.455 \\ (B-V) & =0.451(G-R)+0.196(U-G)-0.092\end{aligned}$

TRANSMISSION CURVES [191]

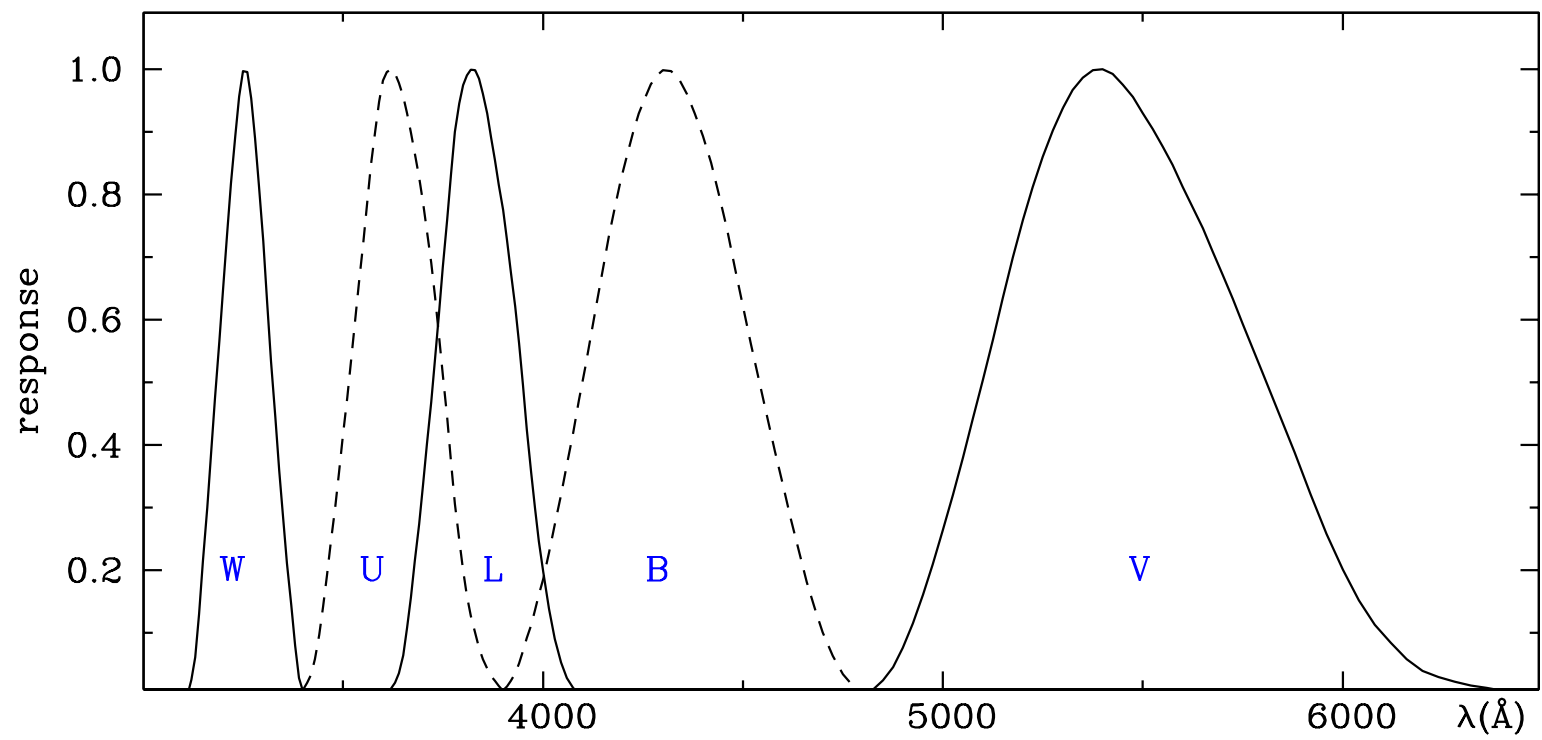

\begin{tabular}{|c|c|c|c|c|c|c|c|c|c|c|c|c|c|c|c|c|c|}
\hline \multicolumn{2}{|c|}{$W$} & \multicolumn{4}{|c|}{$U$} & \multicolumn{4}{|c|}{$L$} & \multicolumn{4}{|c|}{$B$} & \multicolumn{4}{|c|}{ V } \\
\hline$\lambda(\mathrm{A})$ & $r$ & $\lambda(\mathrm{A})$ & $r$ & $\lambda(\mathrm{A})$ & $r$ & $\lambda(\mathrm{A})$ & $r$ & $\lambda(\mathrm{A})$ & $r$ & $\lambda(\mathrm{A})$ & $r$ & $\lambda(\mathrm{A})$ & $r$ & $\lambda(\mathrm{A})$ & $r$ & $\lambda(\mathrm{A})$ & $r$ \\
\hline 3100 & 0.000 & 3380 & 0.000 & 3700 & 0.787 & 3590 & 0.000 & 3910 & 0.727 & 3870 & 0.000 & 4285 & 0.990 & 4775 & 0.000 & 5575 & 0.847 \\
\hline 3110 & 0.000 & 3390 & 0.004 & 3710 & 0.742 & 3600 & 0.003 & 3920 & 0.676 & 3880 & 0.001 & 4300 & 0.998 & 4800 & 0.001 & 5600 & 0.812 \\
\hline 3120 & 0.025 & 3400 & 0.010 & 3720 & 0.691 & 3610 & 0.006 & 3930 & 0.621 & 3890 & 0.004 & 4320 & 0.997 & 4825 & 0.009 & 5625 & 0.779 \\
\hline 3130 & 0.061 & 3410 & 0.020 & 3730 & 0.636 & 3620 & 0.011 & 3940 & 0.562 & 3900 & 0.008 & 4340 & 0.985 & 4850 & 0.024 & 5650 & 0.746 \\
\hline 3140 & 0.130 & 3420 & 0.033 & 3740 & 0.575 & 3630 & 0.020 & 3950 & 0.496 & 3910 & 0.014 & 4360 & 0.960 & 4875 & 0.045 & 5675 & 0.709 \\
\hline 3150 & 0.215 & 3430 & 0.058 & 3750 & 0.512 & 3640 & 0.035 & 3960 & 0.425 & 3920 & 0.023 & 4380 & 0.929 & 4900 & 0.076 & 5700 & 0.671 \\
\hline 3160 & 0.300 & 3440 & 0.097 & 3760 & 0.444 & 3650 & 0.064 & 3970 & 0.356 & 3930 & 0.035 & 4400 & 0.893 & 4925 & 0.115 & 5725 & 0.633 \\
\hline 3170 & 0.388 & 3450 & 0.142 & 3770 & 0.373 & 3660 & 0.108 & 3980 & 0.299 & 3940 & 0.051 & 4420 & 0.850 & 4950 & 0.161 & 5750 & 0.594 \\
\hline 3180 & 0.475 & 3460 & 0.192 & 3780 & 0.306 & 3670 & 0.157 & 3990 & 0.246 & 3950 & 0.071 & 4440 & 0.800 & 4975 & 0.209 & 5775 & 0.554 \\
\hline 3190 & 0.565 & 3470 & 0.245 & 3790 & 0.249 & 3680 & 0.215 & 4000 & 0.198 & 3960 & 0.092 & 4460 & 0.744 & 5000 & 0.264 & 5800 & 0.514 \\
\hline 3200 & 0.652 & 3480 & 0.300 & 3800 & 0.200 & 3690 & 0.274 & 4015 & 0.138 & 3970 & 0.112 & 4480 & 0.683 & 5025 & 0.320 & 5840 & 0.451 \\
\hline 3210 & 0.736 & 3490 & 0.356 & 3810 & 0.159 & 3700 & 0.336 & 4030 & 0.090 & 3980 & 0.136 & 4500 & 0.621 & 5050 & 0.380 & 5880 & 0.388 \\
\hline 3220 & 0.817 & 3500 & 0.415 & 3820 & 0.127 & 3710 & 0.401 & 4045 & 0.053 & 3990 & 0.161 & 4520 & 0.562 & 5075 & 0.441 & 5920 & 0.321 \\
\hline 3230 & 0.893 & 3510 & 0.475 & 3830 & 0.100 & 3720 & 0.468 & 4060 & 0.028 & 4000 & 0.186 & 4540 & 0.504 & 5100 & 0.504 & 5960 & 0.258 \\
\hline 3240 & 0.956 & 3520 & 0.535 & 3840 & 0.076 & 3730 & 0.538 & 4075 & 0.012 & 4015 & 0.228 & 4560 & 0.445 & 5125 & 0.568 & 6000 & 0.201 \\
\hline 3250 & 0.997 & 3530 & 0.596 & 3850 & 0.058 & 3740 & 0.609 & 4090 & 0.004 & 4030 & 0.273 & 4580 & 0.390 & 5150 & 0.634 & 6040 & 0.152 \\
\hline 3260 & 0.995 & 3540 & 0.657 & 3860 & 0.043 & 3750 & 0.684 & 4105 & 0.000 & 4045 & 0.320 & 4600 & 0.335 & 5175 & 0.699 & 6080 & 0.113 \\
\hline 3270 & 0.953 & 3550 & 0.721 & 3870 & 0.031 & 3760 & 0.759 & & & 4060 & 0.368 & 4620 & 0.282 & 5200 & 0.759 & 6120 & 0.084 \\
\hline 3280 & 0.889 & 3560 & 0.784 & 3880 & 0.023 & 3770 & 0.832 & & & 4075 & 0.419 & 4640 & 0.230 & 5225 & 0.811 & 6160 & 0.058 \\
\hline 3290 & 0.814 & 3570 & 0.846 & 3890 & 0.015 & 3780 & 0.900 & & & 4090 & 0.471 & 4660 & 0.181 & 5250 & 0.860 & 6200 & 0.039 \\
\hline 3300 & 0.725 & 3580 & 0.901 & 3900 & 0.007 & 3790 & 0.945 & & & 4105 & 0.523 & 4680 & 0.138 & 5275 & 0.902 & 6240 & 0.029 \\
\hline 3310 & 0.628 & 3590 & 0.948 & 3910 & 0.002 & 3800 & 0.974 & & & 4120 & 0.575 & 4700 & 0.101 & 5300 & 0.938 & 6280 & 0.022 \\
\hline 3320 & 0.534 & 3600 & 0.982 & 3920 & 0.000 & 3810 & 0.990 & & & 4135 & 0.627 & 4725 & 0.063 & 5325 & 0.967 & 6320 & 0.016 \\
\hline 3330 & 0.444 & 3610 & 0.995 & & & 3820 & 0.999 & & & 4150 & 0.682 & 4750 & 0.034 & 5350 & 0.986 & 6360 & 0.012 \\
\hline 3340 & 0.359 & 3620 & 1.000 & & & 3830 & 0.998 & & & 4165 & 0.733 & 4775 & 0.015 & 5375 & 0.998 & 6400 & 0.008 \\
\hline 3350 & 0.285 & 3630 & 0.993 & & & 3840 & 0.985 & & & 4180 & 0.781 & 4800 & 0.003 & 5400 & 1.000 & 6440 & 0.004 \\
\hline 3360 & 0.211 & 3640 & 0.978 & & & 3850 & 0.961 & & & 4195 & 0.824 & 4825 & 0.000 & 5425 & 0.992 & 6480 & 0.000 \\
\hline 3370 & 0.144 & 3650 & 0.956 & & & 3860 & 0.929 & & & 4210 & 0.863 & & & 5450 & 0.975 & & \\
\hline 3380 & 0.080 & 3660 & 0.929 & & & 3870 & 0.891 & & & 4225 & 0.898 & & & 5475 & 0.955 & & \\
\hline 3390 & 0.028 & 3670 & 0.898 & & & 3880 & 0.855 & & & 4240 & 0.929 & & & 5500 & 0.930 & & \\
\hline 3400 & 0.005 & 3680 & 0.865 & & & 3890 & 0.815 & & & 4255 & 0.954 & & & 5525 & 0.904 & & \\
\hline 3410 & 0.000 & 3690 & 0.827 & & & 3900 & 0.775 & & & 4270 & 0.976 & & & 5550 & 0.876 & & \\
\hline
\end{tabular}

Fig. 21. continued 


\section{Griffin - 1961}

Three square-band measurement of 5247-5255 $\AA$ FeI triplet and 5890-5896 A NaI doublet on spectra of G5-K5 stars.

\section{GENERAL INFORMATION}

$\begin{array}{ll}\text { AUTHORS } & \text { R. F. Griffin } \\ \text { TELESCOPE } & 0.90 \mathrm{~m} \text { (reflector), Cambridge Obs. } \\ \text { DETECTOR } & \text { EMI 9502 (refrigerated) } \\ \text { MAIN ARTICLE } & \text { Griffin, R. F. 1961, MNRAS 122, 18 }\end{array}$

SYSTEM DESCRIPTION

\begin{tabular}{|l|c|}
\hline \multicolumn{2}{|c|}{ BANDS DESCRIPTION [123] } \\
\hline band & bandpass $(\AA)$ \\
\hline \multicolumn{2}{|c|}{ Fe I } \\
\hline$A$ & $5190.0-5224.0$ \\
\hline$B$ & $5245.5-5256.5$ \\
\hline$C$ & $5278.0-5312.0$ \\
\hline \multicolumn{2}{|c|}{ Na I } \\
\hline$A$ & $5818.8-5850.0$ \\
\hline$B$ & $5885.0-5900.6$ \\
\hline$C$ & $5935.6-5966.8$ \\
\hline
\end{tabular}

On the focal plane of a $5.0 \AA \AA \mathrm{mm}$ dispersion spectrograph, three diaphragms transmit to photomultipliers the above three $A, B$ and $C$ portions of the spectrum.

[247] has performed similar observations with slightly modified passbands, namely 5830.0-5850.1 $\AA$ for $A, 5885.0-5901.2$ $\AA$ for $B$ and 5936.1-5956.1 $\AA$ for $C$.

\section{SYSTEM ANALYSIS}

\section{COLOR INDICES AND PARAMETERS [123]}

$r_{F e I}=\frac{A_{F e I}+C_{F e I}}{B_{F e I}}:$ measures the strength of the 5247-5255 $\AA$ FeI triplet.

$r_{F e I}$ is found to offer an excellent separation of luminosity classes in G-K stars.

$r_{N a I}=\frac{A_{N a I}+C_{N a I}}{B_{N a I}}:$ measures the strength of the 5890-5896 $\AA$ NaI doublet.

$r_{N a I}$ does not correlate well with spectral type probably due to contamination by interstellar lines.

Fig. 22. The photometric system Griffin - 1961 


\section{8 colors - Tifft - 1961}

Multicolor photometry of galaxies.

\section{GENERAL INFORMATION}

AUTHORS

TELESCOPE

DETECTOR
W. G. Tifft

$1.52 \mathrm{~m}$ and $2.54 \mathrm{~m}$ (reectors), Mount Wilson Obs. 6094 EMI photocell (unrefrigerated) for bands 1-4,

16 PM-1 Farnsworth photocell (refrigerated) for bands 5-8

MAIN ARTICLE Tifft, W. G. 1961, AJ 66, 390

\section{SYSTEM DESCRIPTION}

\begin{tabular}{|c|l|rc|}
\hline \multicolumn{2}{|c|}{ BANDS DESCRIPTION [303] } & & \\
\hline band & \multicolumn{1}{|c|}{ 1ter (\#) } & WHM (A) & $\lambda_{c}(\mathrm{~A})$ \\
\hline 1 & Corning 9863 + approximately Corning 7380 & 260 & 3760 \\
\hline 2 & Schott BG12 + Schott GG13 & 780 & 4165 \\
\hline 3 & Corning 5030 (1.6mm) + Corning 3387 & 750 & 4710 \\
\hline 4 & Corning 3480 & 350 & 5880 \\
\hline 5 & interference & 860 & 6900 \\
\hline 6 & interference + Corning 2434 & 1390 & 8325 \\
\hline 7 & interference + Schott RG-10 & 1487 & 8700 \\
\hline 8 & Heimann 205 & 1430 & 9820 \\
\hline
\end{tabular}

(\#) Thickness quoted when different from stock values. The interference lters have been custom made by Federal Engineering.

\section{TRANSMISSION CURVES [303]}

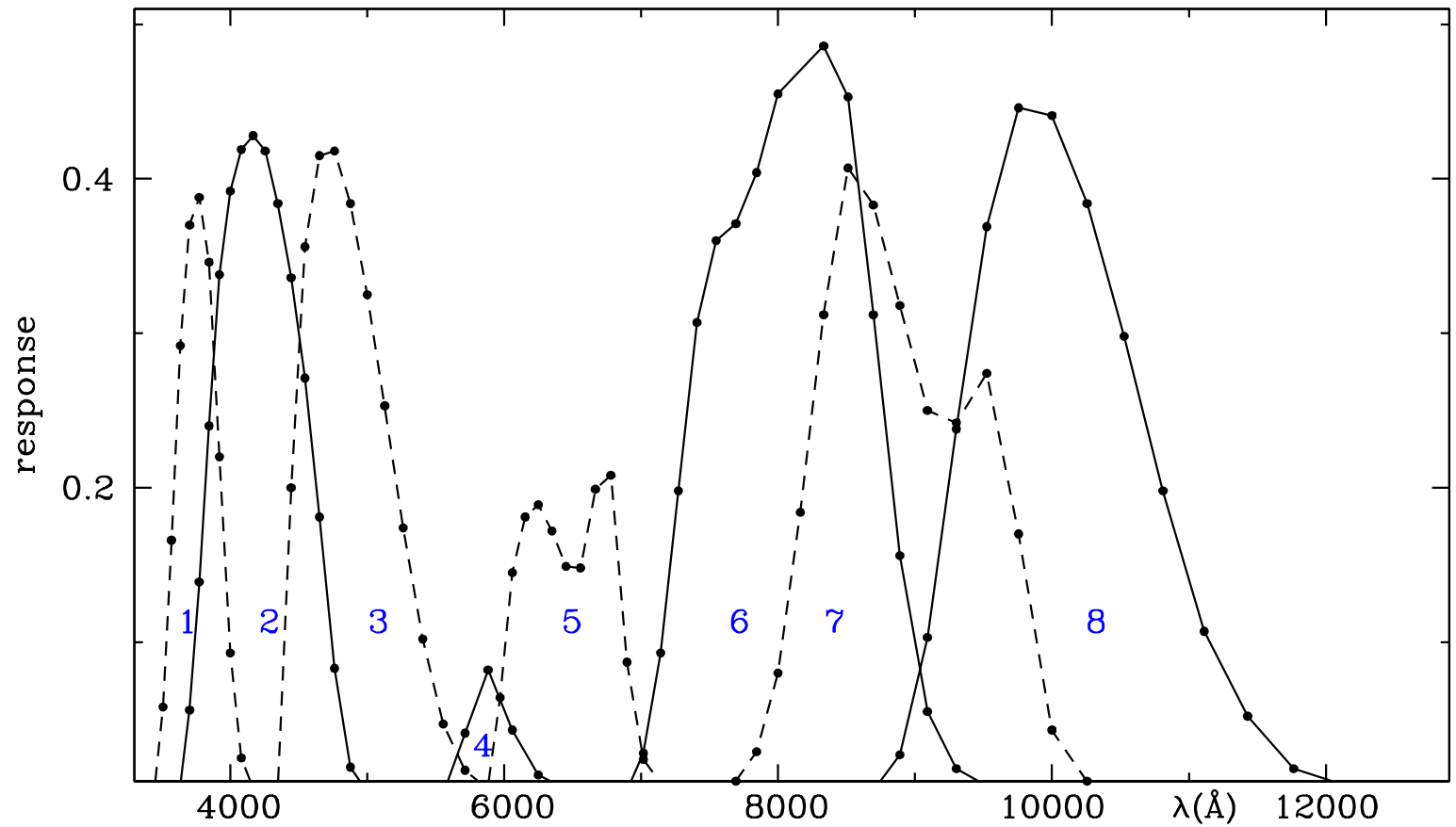

Fig. 23. The photometric system 8 colors - Tifft -1961 
As originally published

\begin{tabular}{|c|c|c|c|c|c|c|c|c|c|c|c|c|c|c|c|}
\hline \multicolumn{2}{|c|}{1} & \multicolumn{2}{|c|}{2} & \multicolumn{2}{|c|}{3} & \multicolumn{2}{|c|}{4} & \multicolumn{2}{|c|}{5} & \multicolumn{2}{|c|}{6} & \multicolumn{2}{|c|}{7} & \multicolumn{2}{|c|}{8} \\
\hline $1 / \lambda(\AA)$ & $r$ & $1 / \lambda(\AA)$ & $\Upsilon$ & $1 / \lambda(\AA)$ & $\Upsilon$ & $1 / \lambda(\AA)$ & $\Upsilon$ & $1 / \lambda(\AA)$ & $\Upsilon$ & $1 / \lambda(\AA)$ & $\Upsilon$ & $1 / \lambda(\AA)$ & $r$ & $1 / \lambda(\AA)$ & $r$ \\
\hline 2.90 & 0.007 & 2.75 & 0.009 & 2.30 & 0.007 & 1.80 & 0.002 & 1.700 & 0.007 & 1.450 & 0.004 & 1.350 & 0.001 & 1.150 & 0.004 \\
\hline 2.85 & 0.058 & 2.70 & 0.056 & 2.25 & 0.200 & 1.75 & 0.041 & 1.675 & 0.064 & 1.425 & 0.028 & 1.325 & 0.003 & 1.125 & 0.027 \\
\hline 2.80 & 0.166 & 2.65 & 0.139 & 2.20 & 0.356 & 1.70 & 0.082 & 1.650 & 0.145 & 1.400 & 0.093 & 1.300 & 0.010 & 1.100 & 0.103 \\
\hline 2.75 & 0.292 & 2.60 & 0.240 & 2.15 & 0.415 & 1.65 & 0.043 & 1.625 & 0.181 & 1.375 & 0.198 & 1.275 & 0.029 & 1.075 & 0.238 \\
\hline 2.70 & 0.370 & 2.55 & 0.338 & 2.10 & 0.418 & 1.60 & 0.014 & 1.600 & 0.189 & 1.350 & 0.307 & 1.250 & 0.080 & 1.050 & 0.369 \\
\hline 2.65 & 0.388 & 2.50 & 0.392 & 2.05 & 0.384 & 1.55 & 0.005 & 1.575 & 0.172 & 1.325 & 0.360 & 1.225 & 0.184 & 1.025 & 0.446 \\
\hline 2.60 & 0.346 & 2.45 & 0.419 & 2.00 & 0.325 & 1.50 & 0.001 & 1.550 & 0.149 & 1.300 & 0.371 & 1.200 & 0.312 & 1.000 & 0.441 \\
\hline 2.55 & 0.220 & 2.40 & 0.428 & 1.95 & 0.253 & & & 1.525 & 0.148 & 1.275 & 0.404 & 1.175 & 0.407 & 0.975 & 0.384 \\
\hline 2.50 & 0.093 & 2.35 & 0.418 & 1.90 & 0.174 & & & 1.500 & 0.199 & 1.250 & 0.455 & 1.150 & 0.383 & 0.950 & 0.298 \\
\hline 2.45 & 0.025 & 2.30 & 0.384 & 1.85 & 0.102 & & & 1.475 & 0.208 & 1.200 & 0.486 & 1.125 & 0.318 & 0.925 & 0.198 \\
\hline 2.40 & 0.006 & 2.25 & 0.336 & 1.80 & 0.047 & & & 1.450 & 0.087 & 1.175 & 0.453 & 1.100 & 0.250 & 0.900 & 0.107 \\
\hline 2.35 & 0.002 & 2.20 & 0.271 & 1.75 & 0.017 & & & 1.425 & 0.024 & 1.150 & 0.312 & 1.075 & 0.242 & 0.875 & 0.052 \\
\hline & & 2.15 & 0.181 & 1.70 & 0.005 & & & 1.400 & 0.009 & 1.125 & 0.156 & 1.050 & 0.274 & 0.850 & 0.018 \\
\hline & & 2.10 & 0.083 & & & & & 1.375 & 0.004 & 1.100 & 0.055 & 1.025 & 0.170 & 0.825 & 0.008 \\
\hline & & 2.05 & 0.019 & & & & & 1.350 & 0.002 & 1.075 & 0.018 & 1.000 & 0.043 & 0.800 & 0.002 \\
\hline & & 2.00 & 0.003 & & & & & 1.325 & 0.002 & 1.050 & 0.007 & 0.975 & 0.010 & & \\
\hline & & & & & & & & 1.300 & 0.001 & 1.025 & 0.003 & 0.950 & 0.002 & & \\
\hline & & & & & & & & 1.275 & 0.001 & 1.000 & 0.002 & & & & \\
\hline & & & & & & & & 1.250 & 0.001 & 0.975 & 0.001 & & & & \\
\hline & & & & & & & & 1.200 & 0.001 & & & & & & \\
\hline & & & & & & & & 1.150 & 0.002 & & & & & & \\
\hline & & & & & & & & 1.100 & 0.006 & & & & & & \\
\hline & & & & & & & & 1.050 & 0.007 & & & & & & \\
\hline & & & & & & & & 1.000 & 0.004 & & & & & & \\
\hline & & & & & & & & 0.950 & 0.002 & & & & & & \\
\hline & & & & & & & & 0.900 & 0.001 & & & & & & \\
\hline
\end{tabular}

and retabulated in $\lambda(\AA)$

\begin{tabular}{|c|c|c|c|c|c|c|c|c|c|c|c|c|c|c|c|}
\hline \multicolumn{2}{|c|}{1} & \multicolumn{2}{|c|}{2} & \multicolumn{2}{|c|}{3} & \multicolumn{2}{|c|}{4} & \multicolumn{2}{|c|}{5} & \multicolumn{2}{|c|}{6} & \multicolumn{2}{|c|}{7} & \multicolumn{2}{|c|}{8} \\
\hline$\lambda(\AA)$ & $\Upsilon$ & $\lambda(\AA)$ & $\Upsilon$ & $\lambda(\AA)$ & $\Upsilon$ & $\lambda(\AA)$ & $\Upsilon$ & $\lambda(\AA)$ & $\Upsilon$ & $\lambda(\AA)$ & $\Upsilon$ & $\lambda(\AA)$ & $\Upsilon$ & $\lambda(\AA)$ & $\Upsilon$ \\
\hline 3448 & 0.007 & 3636 & 0.009 & 4348 & 0.007 & 5556 & 0.002 & 5882 & 0.007 & 6897 & 0.004 & 7407 & 0.001 & 8696 & 0.004 \\
\hline 3509 & 0.058 & 3704 & 0.056 & 4444 & 0.200 & 5714 & 0.041 & 5970 & 0.064 & 7018 & 0.028 & 7547 & 0.003 & 8889 & 0.027 \\
\hline 3571 & 0.166 & 3774 & 0.139 & 4545 & 0.356 & 5882 & 0.082 & 6061 & 0.145 & 7143 & 0.093 & 7692 & 0.010 & 9091 & 0.103 \\
\hline 3636 & 0.292 & 3846 & 0.240 & 4651 & 0.415 & 6061 & 0.043 & 6154 & 0.181 & 7273 & 0.198 & 7843 & 0.029 & 9302 & 0.238 \\
\hline 3704 & 0.370 & 3922 & 0.338 & 4762 & 0.418 & 6250 & 0.014 & 6250 & 0.189 & 7407 & 0.307 & 8000 & 0.080 & 9524 & 0.369 \\
\hline 3774 & 0.388 & 4000 & 0.392 & 4878 & 0.384 & 6452 & 0.005 & 6349 & 0.172 & 7547 & 0.371 & 8163 & 0.184 & 9756 & 0.446 \\
\hline 3846 & 0.346 & 4082 & 0.419 & 5000 & 0.325 & 6667 & 0.001 & 6452 & 0.149 & 7692 & 0.404 & 8333 & 0.312 & 10000 & 0.441 \\
\hline 3922 & 0.220 & 4167 & 0.428 & 5128 & 0.253 & & & 6557 & 0.148 & 7843 & 0.455 & 8511 & 0.407 & 10260 & 0.384 \\
\hline 4000 & 0.093 & 4255 & 0.418 & 5263 & 0.174 & & & 6667 & 0.199 & 8000 & 0.486 & 8696 & 0.383 & 10530 & 0.298 \\
\hline 4082 & 0.025 & 4348 & 0.384 & 5405 & 0.102 & & & 6780 & 0.208 & 8333 & 0.453 & 8889 & 0.318 & 10810 & 0.198 \\
\hline 4167 & 0.006 & 4444 & 0.336 & 5556 & 0.047 & & & 6897 & 0.087 & 8511 & 0.312 & 9091 & 0.250 & 11100 & 0.107 \\
\hline 4255 & 0.002 & 4545 & 0.271 & 5714 & 0.017 & & & 7018 & 0.024 & 8696 & 0.156 & 9302 & 0.242 & 11430 & 0.052 \\
\hline & & 4651 & 0.181 & 5882 & 0.005 & & & 7143 & 0.009 & 8889 & 0.055 & 9524 & 0.274 & 11760 & 0.018 \\
\hline & & 4762 & 0.083 & & & & & 7273 & 0.004 & 9091 & 0.018 & 9756 & 0.170 & 12120 & 0.008 \\
\hline & & 4878 & 0.019 & & & & & 7407 & 0.002 & 9302 & 0.007 & 10000 & 0.043 & 12500 & 0.002 \\
\hline & & 5000 & 0.003 & & & & & 7547 & 0.002 & 9524 & 0.003 & 10260 & 0.010 & & \\
\hline & & & & & & & & 7692 & 0.001 & 9756 & 0.002 & 10530 & 0.002 & & \\
\hline & & & & & & & & 7843 & 0.001 & 10000 & 0.001 & & & & \\
\hline & & & & & & & & 8000 & 0.001 & & & & & & \\
\hline & & & & & & & & 8333 & 0.001 & & & & & & \\
\hline & & & & & & & & 8696 & 0.002 & & & & & & \\
\hline & & & & & & & & 9091 & 0.006 & & & & & & \\
\hline & & & & & & & & 9524 & 0.007 & & & & & & \\
\hline & & & & & & & & 10000 & 0.004 & & & & & & \\
\hline & & & & & & & & 10530 & 0.002 & & & & & & \\
\hline & & & & & & & & 11110 & 0.001 & & & & & & \\
\hline
\end{tabular}

Fig. 23. continued 


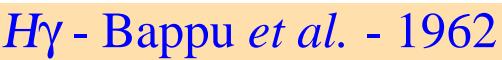

Luminosity classification of early-type stars.

\section{GENERAL INFORMATION}

AUTHORS

TELESCOPE

DETECTOR

MAIN ARTICLE
M. K. V. Bappu, S. Chandra, N. B. Sanwal, and S. D. Sinvhal

$0.25 \mathrm{~m}$ (refractor), Uttar Pradesh State Observatory

1P21 (unrefrigerated)

Bappu, M.K.V., Chandra, S., Sanwal, N.B., Sinvhal, S.D. 1962, MNRAS 123, 521

\section{SYSTEM DESCRIPTION}

\begin{tabular}{|c|ccc|}
\hline \multicolumn{4}{|c|}{ BANDS DESCRIPTION [21] } \\
\hline band & filter $(\#)$ & $\lambda_{\text {peak }}(\AA)$ & FWHM $(\AA)$ \\
\hline$S$ & interference & 4280 & 45 \\
\hline$\gamma$ & interference & 4340 & 45 \\
\hline$L$ & interference & 4410 & 45 \\
\hline
\end{tabular}

(\#) Filters by Baird Atomic.

ZERO POINT: The system zero point is defined by 15 stars of spectral types B0-A0, Ia-V. [21]

\section{SYSTEM ANALYSIS}

\section{COLOR INDICES AND PARAMETERS [21]}

$\Gamma=1000 \cdot\left[-2.5 \log \frac{\sqrt{L \cdot S}}{\gamma}\right]:$ measures the intensity of $\mathrm{H} \gamma$. Strongly dependent upon luminosity.

[21] provides a $\Gamma, \mathrm{M}_{V}$ calibration in tabular form.

RELATIONS WITH OTHER SYSTEMS [21]

H $\gamma$ equivalent width from spectra

\begin{tabular}{|rccc|}
\hline$E W_{\text {Petrie }}(\AA)$ & $=22.653-0.0762 \Gamma$ & {$[244]$} \\
\hline$E W_{\text {Gunther }}(\AA)$ & $=24.734-0.0863 \Gamma$ & {$[126]$} \\
\hline$E W_{\text {Williams }}(\AA)$ & $=27.67-0.0983 \Gamma$ & {$[324]$} \\
\hline$E W_{\text {Stock }}(\AA)$ & $=27.949-0.09616 \Gamma$ & {$[283]$} \\
\hline
\end{tabular}

uvby $H \beta$ - Strömgren and Crawford - 1956

$H \beta=3.1658-0.00227 \Gamma$

Fig. 24. The photometric system $H \gamma-$ Bappu et al. - 1962 


\title{
Geneva - Golay - 1962
}

General purpose system. Photometry of stars in clusters, associations and double systems.

\section{GENERAL INFORMATION}

\author{
AUTHORS M. Golay \\ TELESCOPE $1 \mathrm{~m}$ (reflector), Haute-Provence Obs. ; \\ 0.40m (reflector), Jungfraujoch Obs; ESO. \\ DETECTOR northern emisphere: Victor VI [211] \\ southern emisphere: EMI 9502-AM \\ MAIN ARTICLE Golay, M. 1962 Pub. Obs. Genève No 15 (série A), 29
}

\section{SYSTEM DESCRIPTION}

\begin{tabular}{|l|lcc|}
\hline \multicolumn{4}{|c|}{ BANDS DESCRIPTION [285], pg 369 } \\
\hline band & \multicolumn{1}{|c|}{ filter } & $\lambda_{0}(\AA)$ & half-width $(\AA)$ \\
\hline$U$ & 4mm Schott UG11 & 3456 & 465 \\
\hline$B_{1}$ & 2mm Schott GG 13 + 4mm Schott UG 3 + 1mm Schott BG 23 & 4024 & 395 \\
\hline$B$ & 2mm Schott BG 12+ 2mm Schott GG 13 & 4245 & 810 \\
\hline$B_{2}$ & 3mm Schott BG 25 + 4mm Schott GG 3 & 4480 & 415 \\
\hline$V_{1}$ & 2mm Schott OG 4 + 3mm Schott BG 18 & 5405 & 465 \\
\hline$V$ & 2mm Schott OG 4 & 5500 & 725 \\
\hline$G$ & 1.5mm Corning 3-67 & 5805 & 440 \\
\hline
\end{tabular}

\section{SYSTEM ANALYSIS}

REDDENING RATIOS [118]

$E\left(U-B_{2}\right)=1.186 E\left(B_{2}-V_{1}\right)$ for reddened O-stars.

$E(B-V)_{J o h n s o n}=1.14 E\left(B_{2}-V_{1}\right)$

\section{REDDENING-FREE PARAMETERS}

Golay reddening-free parameters: [285], pg 371

$d=\left(U-B_{1}\right)-1.430\left(B_{1}-B_{2}\right):$ measures the Balmer jump. Almost blanketing-free.

$\Delta=\left(U-B_{2}\right)-0.832\left(B_{2}-G\right)$ : measures the Balmer jump. Also sensitive to metallic line-blocking.

$g=\left(B_{1}-B_{2}\right)-1.357\left(V_{1}-G\right):$ measures the violet line-blocking. Sensitive to blanketing.

$m_{2}=\left(B_{1}-B_{2}\right)-0.457\left(B_{2}-V_{1}\right)=0.550 \Delta-0.550 d-0.337 g:$ measures the violet line-blocking.

Cramer and Maeder reddening-free parameters: [78]

$$
\begin{aligned}
& X=0.3788+1.3764 U-1.2162 B_{1}-0.8498 B_{2}-0.1554 V_{1}+0.8450 G: \text { temperature indicator. } \\
& Y=-0.8288+0.3235 U-2.3228 B_{1}+2.3363 B_{2}+0.7495 V_{1}-1.0865 G \text { : gravity indicator. } \\
& Z=-0.4572+0.0255 U-0.1740 B_{1}+0.4696 B_{2}-1.1205 V_{1}+0.7994 G:
\end{aligned}
$$

related to intensity of the surface magnetic fields (Ap phenomenon).

Fig. 25. The photometric system Geneva - Golay - 1962 


\section{RELATIONS WITH OTHER SYSTEMS [116]}

\section{UBV - Johnson and Morgan - 1953}

for A0-G8 stars:

\begin{tabular}{|ll|}
\hline$(B-V)$ & $=1.164( \pm 0.005)\left(B_{2}-V_{1}\right)+0.189( \pm 0.004)$ \\
\hline$\left(B_{2}-V_{1}\right)=$ & $0.858( \pm 0.004)(B-V)-0.162( \pm 0.004)$ \\
\hline
\end{tabular}

uvby H $\mathrm{H}$ - Strömgren and Crawford - 1956

for $-0.087<\left(B_{2}-V_{1}\right)<+0.440(\mathrm{~A} 3-\mathrm{G} 5$ stars $)$ :
\begin{tabular}{|ll|}
\hline$\left(B_{2}-V_{1}\right)$ & $=1.273( \pm 0.008)(b-y)-0.142( \pm 0.006)$ \\
\hline$(b-y)$ & $=0.784( \pm 0.004)\left(B_{2}-V_{1}\right)+0.112( \pm 0.006)$
\end{tabular}

\section{TRANSMISSION CURVES [117],pg. 142}

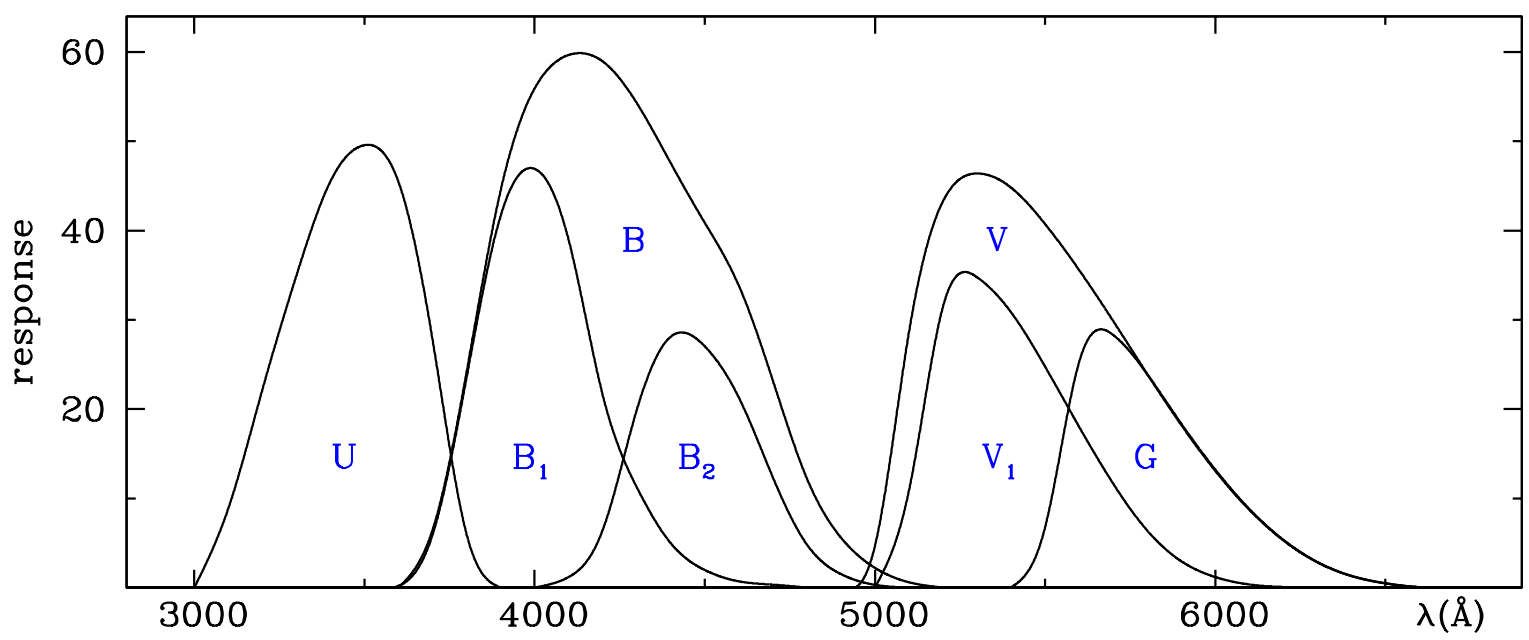

\begin{tabular}{|rr|rr|rr|rr|rr|rr|rr|rr|}
\hline \multicolumn{2}{|c|}{$U$} & \multicolumn{2}{|c|}{$B_{1}$} & \multicolumn{2}{c|}{$B$} & \multicolumn{2}{c|}{$B_{2}$} & \multicolumn{2}{c|}{$V_{1}$} & \multicolumn{2}{|c|}{$V$} & \multicolumn{2}{c|}{$G$} \\
\hline$\lambda(\mathrm{A})$ & $\Upsilon(\%)$ & $\lambda(\mathrm{A})$ & $\Upsilon(\%)$ & $\lambda(\mathrm{A})$ & $\Upsilon(\%)$ & $\lambda(\mathrm{A})$ & $\Upsilon(\%)$ & $\lambda(\mathrm{A})$ & $\Upsilon(\%)$ & $\lambda(\mathrm{A})$ & $\Upsilon(\%)$ & $\lambda(\mathrm{A})$ & $\Upsilon(\%)$ \\
\hline 3000 & 0.00 & 3500 & 0.00 & 3600 & 0.00 & 4000 & 0.00 & 5000 & 0.00 & 4900 & 0.00 & 5400 & 0.00 \\
3100 & 8.89 & 3600 & 0.22 & 3700 & 6.49 & 4100 & 1.20 & 5100 & 10.84 & 5000 & 4.64 & 5500 & 6.73 \\
3200 & 22.16 & 3700 & 5.93 & 3800 & 24.11 & 4200 & 6.45 & 5200 & 31.46 & 5100 & 28.58 & 5600 & 25.25 \\
3300 & 34.95 & 3800 & 23.51 & 3900 & 44.44 & 4300 & 19.88 & 5300 & 34.77 & 5200 & 43.29 & 5700 & 28.30 \\
3400 & 45.50 & 3900 & 41.59 & 4000 & 55.91 & 4400 & 28.10 & 5400 & 30.85 & 5300 & 46.38 & 5800 & 23.97 \\
3500 & 49.56 & 4000 & 46.91 & 4100 & 59.66 & 4500 & 27.04 & 5500 & 24.75 & 5400 & 44.82 & 5900 & 18.38 \\
3600 & 45.47 & 4100 & 38.96 & 4200 & 58.94 & 4600 & 21.30 & 5600 & 18.00 & 5500 & 40.73 & 6000 & 13.23 \\
3700 & 27.82 & 4200 & 21.60 & 4300 & 54.34 & 4700 & 12.38 & 5700 & 11.65 & 5600 & 35.56 & 6100 & 8.79 \\
3800 & 5.99 & 4300 & 11.24 & 4400 & 47.55 & 4800 & 4.85 & 5800 & 6.41 & 5700 & 29.85 & 6200 & 5.35 \\
3900 & 0.00 & 4400 & 4.99 & 4500 & 40.87 & 4900 & 1.51 & 5900 & 2.97 & 5800 & 23.94 & 6300 & 2.87 \\
& 4500 & 1.97 & 4600 & 33.86 & 5000 & 0.32 & 6000 & 1.17 & 5900 & 18.25 & 6400 & 1.31 \\
& 4600 & 0.74 & 4700 & 23.21 & 5100 & 0.00 & 6100 & 0.36 & 6000 & 13.06 & 6500 & 0.47 \\
& 4700 & 0.35 & 4800 & 12.24 & & & 6200 & 0.10 & 6100 & 8.72 & 6600 & 0.07 \\
& 4800 & 0.00 & 4900 & 5.61 & & & 6300 & 0.03 & 6200 & 5.28 & 6700 & 0.00 \\
& & & 5000 & 2.24 & & & 6400 & 0.00 & 6300 & 2.83 & & \\
& & & 5100 & 0.61 & & & & & 6400 & 1.29 & & \\
& & & 5200 & 0.00 & & & & & 6500 & 0.47 & & \\
& & & & & & & & & 6600 & 0.07 & & \\
& & & & & & & & & & & &
\end{tabular}

Fig. 25. continued 


\title{
ubvr $_{20}$ - Sandage and Smith - 1963
}

Line blanketing in subdwarfs.

\section{GENERAL INFORMATION}

\author{
AUTHORS A. Sandage, and L. L. Smith \\ TELESCOPE $\quad 0.50 \mathrm{~m}$ (reflector), Palomar Obs. \\ DETECTOR RCA C7237 (S-20 cathode, refrigerated) \\ MAIN ARTICLE Sandage, A., Smith, L. L. 1963, ApJ 137, 1057
}

\section{SYSTEM DESCRIPTION}

\begin{tabular}{|c|c|c|c|c|}
\hline \multicolumn{3}{|c|}{ BANDS DESCRIPTION [256] } & \multirow[b]{2}{*}{$W H M(\AA)$} & \multirow[b]{2}{*}{$\lambda_{c}(\AA)$} \\
\hline band & filter & $\lambda_{e f f}(\AA)$ & & \\
\hline$u$ & 2.8mm Corning $9863+\mathrm{CuSO}_{4}(\#)$ & 3620 & 657 & 3595 \\
\hline$b$ & $0.7 \mathrm{~mm}$ Schott BG $12+2 \mathrm{~mm} \mathrm{GG} 13+\mathrm{CuSO}_{4}(\#)$ & 4420 & 1000 & 4350 \\
\hline V & 2.2mm Schott GG $11+1.6 \mathrm{~mm}$ Schott BG 18 & 5400 & 842 & 5370 \\
\hline$r_{20}$ & 2mm Schott RG 1 & 6700 & 763 & 6505 \\
\hline
\end{tabular}

(\#) Red-leak suppressor. A $2.5 \mathrm{~mm}$ thick liquid camber filled with an $80 \%$ saturated solution of $\mathrm{CuSO}_{4} \cdot 5 \mathrm{H}_{2} \mathrm{O}$.

ZERO POINT: $\left(v-r_{20}\right)=0.00$ for $(U-B)_{\text {Johnson }}=(B-V)_{\text {Johnson }}=0.00$ stars. $\quad[256]$

\section{SYSTEM ANALYSIS}

RELATIONS WITH OTHER SYSTEMS [256]

UBV - Johnson and Morgan - 1953

\begin{tabular}{|l}
$(B-V)=1.100( \pm 0.004)(b-v)+1.023( \pm 0.003)$ \\
\hline$(U-B)=1.015( \pm 0.003)(u-b)-1.575( \pm 0.006)$
\end{tabular}

Fig. 26. The photometric system $u b v r_{20}$ - Sandage and Smith - 1963 


\section{TRANSMISSION CURVES [211]}

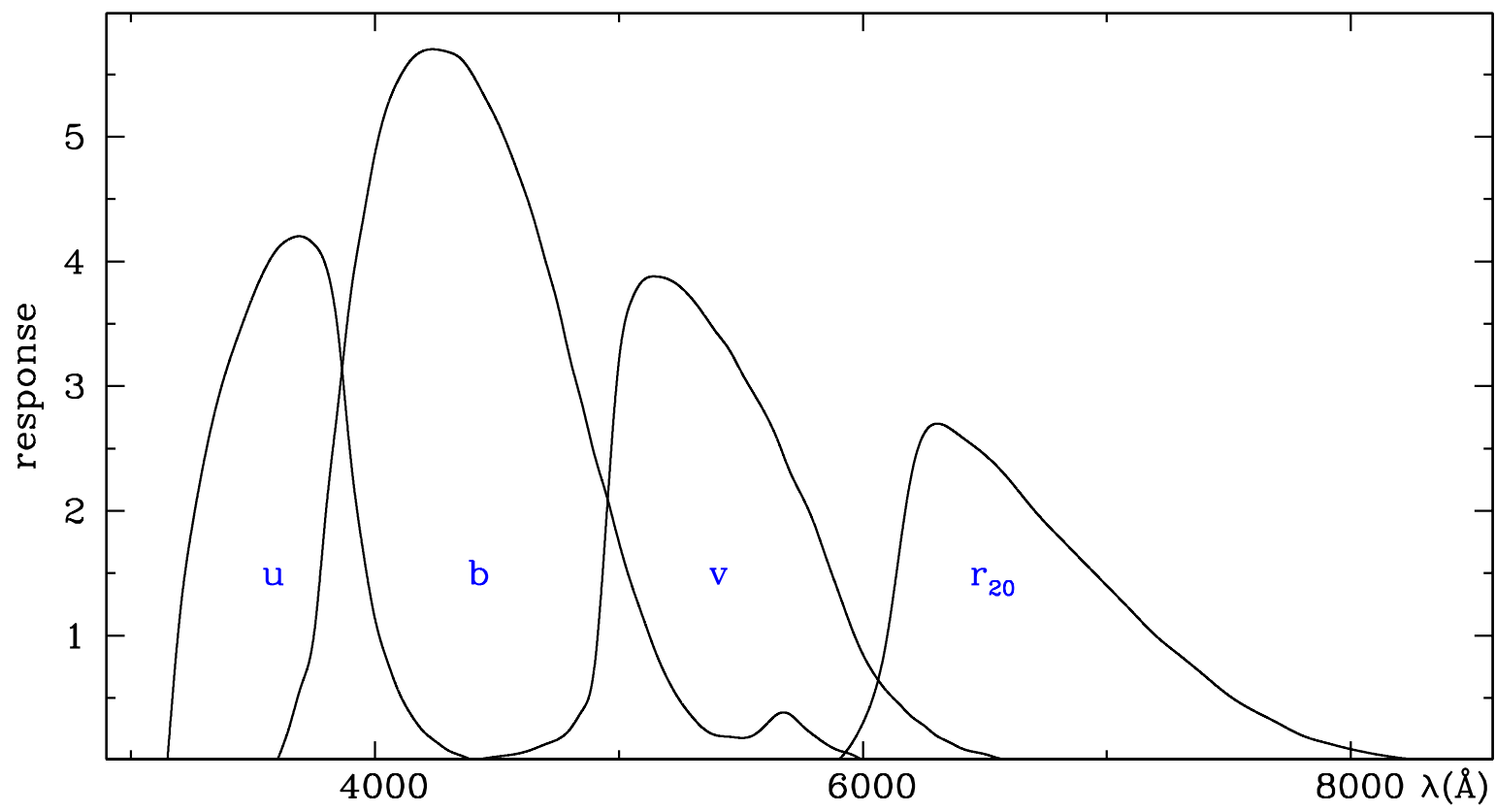

\begin{tabular}{|c|c|c|c|c|c|c|c|c|c|c|c|c|c|}
\hline \multicolumn{2}{|c|}{$u$} & \multicolumn{4}{|c|}{$b$} & \multicolumn{4}{|c|}{$V$} & \multicolumn{4}{|c|}{$r_{20}$} \\
\hline$\lambda(\mathrm{A})$ & $\Upsilon$ & $\lambda(\mathrm{A})$ & $\Upsilon$ & $\lambda(\mathrm{A})$ & $\Upsilon$ & $\lambda(\mathrm{A})$ & $\Upsilon$ & $\lambda(\mathrm{A})$ & $\Upsilon$ & $\lambda(\mathrm{A})$ & $\Upsilon$ & $\lambda(\mathrm{A})$ & $\Upsilon$ \\
\hline 3150 & 0.000 & 3600 & 0.000 & 4900 & 2.450 & 4400 & 0.000 & 5700 & 2.320 & 5900 & 0.000 & 7200 & 1.000 \\
\hline 3200 & 1.140 & 3650 & 0.270 & 4950 & 2.120 & 4450 & 0.016 & 5750 & 2.120 & 5950 & 0.122 & 7250 & 0.917 \\
\hline 3250 & 1.860 & 3700 & 0.607 & 5000 & 1.750 & 4500 & 0.030 & 5800 & 1.900 & 6000 & 0.300 & 7300 & 0.840 \\
\hline 3300 & 2.400 & 3750 & 1.000 & 5050 & 1.420 & 4550 & 0.042 & 5850 & 1.620 & 6050 & 0.557 & 7350 & 0.761 \\
\hline 3350 & 2.840 & 3800 & 2.000 & 5100 & 1.140 & 4600 & 0.060 & 5900 & 1.350 & 6100 & 1.000 & 7400 & 0.680 \\
\hline 3400 & 3.180 & 3850 & 2.860 & 5150 & 0.870 & 4650 & 0.091 & 5950 & 1.080 & 6150 & 1.680 & 7450 & 0.597 \\
\hline 3450 & 3.460 & 3900 & 3.720 & 5200 & 0.650 & 4700 & 0.130 & 6000 & 0.850 & 6200 & 2.300 & 7500 & 0.520 \\
\hline 3500 & 3.720 & 3950 & 4.320 & 5250 & 0.480 & 4750 & 0.169 & 6050 & 0.680 & 6250 & 2.617 & 7550 & 0.455 \\
\hline 3550 & 3.940 & 4000 & 4.860 & 5300 & 0.350 & 4800 & 0.250 & 6100 & 0.550 & 6300 & 2.700 & 7600 & 0.400 \\
\hline 3600 & 4.100 & 4050 & 5.240 & 5350 & 0.250 & 4850 & 0.400 & 6150 & 0.450 & 6350 & 2.670 & 7650 & 0.350 \\
\hline 3650 & 4.180 & 4100 & 5.470 & 5400 & 0.200 & 4900 & 0.750 & 6200 & 0.350 & 6400 & 2.600 & 7700 & 0.300 \\
\hline 3700 & 4.200 & 4150 & 5.620 & 5450 & 0.190 & 4950 & 1.970 & 6250 & 0.280 & 6450 & 2.528 & 7750 & 0.247 \\
\hline 3750 & 4.140 & 4200 & 5.690 & 5500 & 0.180 & 5000 & 3.200 & 6300 & 0.200 & 6500 & 2.450 & 7800 & 0.200 \\
\hline 3800 & 3.960 & 4250 & 5.700 & 5550 & 0.200 & 5050 & 3.680 & 6350 & 0.150 & 6550 & 2.358 & 7850 & 0.166 \\
\hline 3850 & 3.420 & 4300 & 5.680 & 5600 & 0.280 & 5100 & 3.850 & 6400 & 0.100 & 6600 & 2.250 & 7900 & 0.140 \\
\hline 3900 & 2.440 & 4350 & 5.630 & 5650 & 0.370 & 5150 & 3.880 & 6450 & 0.070 & 6650 & 2.130 & 7950 & 0.114 \\
\hline 3950 & 1.700 & 4400 & 5.500 & 5700 & 0.370 & 5200 & 3.860 & 6500 & 0.050 & 6700 & 2.010 & 8000 & 0.090 \\
\hline 4000 & 1.140 & 4450 & 5.320 & 5750 & 0.280 & 5250 & 3.800 & 6550 & 0.020 & 6750 & 1.901 & 8050 & 0.069 \\
\hline 4050 & 0.800 & 4500 & 5.130 & 5800 & 0.200 & 5300 & 3.700 & 6600 & 0.000 & 6800 & 1.800 & 8100 & 0.050 \\
\hline 4100 & 0.540 & 4550 & 4.900 & 5850 & 0.130 & 5350 & 3.570 & & & 6850 & 1.700 & 8150 & 0.034 \\
\hline 4150 & 0.360 & 4600 & 4.640 & 5900 & 0.080 & 5400 & 3.430 & & & 6900 & 1.600 & 8200 & 0.020 \\
\hline 4200 & 0.230 & 4650 & 4.350 & 5950 & 0.050 & 5450 & 3.300 & & & 6950 & 1.500 & 8250 & 0.009 \\
\hline 4250 & 0.150 & 4700 & 4.000 & 6000 & 0.000 & 5500 & 3.120 & & & 7000 & 1.400 & 8300 & 0.000 \\
\hline 4300 & 0.080 & 4750 & 3.650 & & & 5550 & 2.950 & & & 7050 & 1.301 & & \\
\hline 4350 & 0.040 & 4800 & 3.220 & & & 5600 & 2.780 & & & 7100 & 1.200 & & \\
\hline 4400 & 0.000 & 4850 & 2.850 & & & 5650 & 2.570 & & & 7150 & 1.097 & & \\
\hline
\end{tabular}

Fig. 26. continued 


\section{Barbier and Morguleff - 1964}

Narrow band photometry of early-type stars.

\section{GENERAL INFORMATION}

AUTHORS D. Barbier and N. Morguleff

TELESCOPE $\quad 0.80 \mathrm{~m}$ (reflector), Haute Provence Obs.

DETECTOR Lallemand cell

MAIN ARTICLE Barbier, D., Morguleff, N. 1964, Compt. Rend. Acad. Sci. Paris 258, 4925

\section{SYSTEM DESCRIPTION}

\begin{tabular}{|c|cc|}
\hline \multicolumn{3}{|c|}{ BANDS DESCRIPTION [22] } \\
\hline band & bandpass $(\AA)$ & feature \\
\hline 361 & $3575-3650$ & Balmer Discontinuity \\
\hline 375 & $3720-3780$ & \\
\hline 395 & $3925-3965$ & Ca II (K) \\
\hline 405 & $4010-4070$ & \\
\hline 435 & $4310-4380$ & H $\gamma$ \\
\hline 486 & $4815-4905$ & H $\beta$ \\
\hline 495 & $4900-5000$ & \\
\hline 595 & $5900-6000$ & \\
\hline
\end{tabular}

A mask-wheel is placed on the focal plane of a two fused silica prism spectrograph at the Coudé focus, and upon rotation trasmits in turn to the detector (a Lallemand cell) selected portions of the spectrum (in form of square bands).

Fig. 27. The photometric system Barbier and Morguleff - 1964 


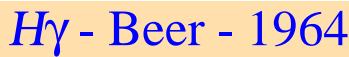

Three square-band measurement of $\mathrm{H} \gamma$ on spectra of early type stars.

\section{GENERAL INFORMATION}

$\begin{array}{ll}\text { AUTHORS } & \text { A. Beer } \\ \text { TELESCOPE } & 0.90 \mathrm{~m} \text { (reflector), Cambridge Obs. } \\ \text { DETECTOR } & \text { EMI 9502 photomultiplier (refrigerated) } \\ \text { MAIN ARTICLE } & \text { Beer, A. 1964, MNRAS 128, 261 }\end{array}$

\section{SYSTEM DESCRIPTION}

\begin{tabular}{|l|c|}
\hline \multicolumn{2}{|c|}{ BANDS DESCRIPTION [27] } \\
\hline band & bandpass $(\AA)$ \\
\hline$A$ & $4255-4289$ \\
\hline$B$ & $4325-4357$ \\
\hline$C$ & $4393-4427$ \\
\hline
\end{tabular}

On the focal plane of a $5.0 \AA / \mathrm{mm}$ dispersion spectrograph, three diaphragms transmit to photomultipliers the above three $A, B$ and $C$ portions of the spectrum.

\section{SYSTEM ANALYSIS}

\section{COLOR INDICES AND PARAMETERS [27]}

Light from the comparison bands $A+C$ is fed to one photomultiplier, that from $B$ to a second one. To normalize the readings, the spectrum of a comparison lamp is recorded in a similar manner. Then the following ratio is obtained:

$$
R_{\gamma}=\left[\frac{(A+C)_{\text {star }}}{(A+C)_{\text {lamp }}}\right] /\left[\frac{(B)_{\text {star }}}{(B)_{\text {lamp }}}\right]
$$

It is found to be a good luminosity indicator.

Fig. 28. The photometric system $H \gamma-$ Beer - 1964 


\section{$H \alpha$ - Peat - 1964}

Three square-band measurement of $\mathrm{H} \alpha$ on spectra of $\mathrm{F}, \mathrm{G}$ and $\mathrm{K}$ stars.

\section{GENERAL INFORMATION}

$\begin{array}{ll}\text { AUTHORS } & \text { D. W. Peat } \\ \text { TELESCOPE } & 0.90 \mathrm{~m} \text { (reflector), Cambridge Obs. } \\ \text { DETECTOR } & \text { EMI 9558 (cooled at }-20^{\circ} \text { C) } \\ \text { MAIN ARTICLE } & \text { Peat, D. W. 1964, MNRAS } 128,435\end{array}$

SYSTEM DESCRIPTION

\begin{tabular}{|l|c|}
\hline \multicolumn{2}{|c|}{ BANDS DESCRIPTION [240] } \\
\hline band & bandpass $(\AA)$ \\
\hline$A$ & $6477.5-6495.0$ \\
\hline$B$ & $6545.0-6580.0$ \\
\hline$C$ & $6630.0-6647.5$ \\
\hline
\end{tabular}

On the focal plane of a $5.0 \AA / \mathrm{mm}$ dispersion spectrograph, three diaphragms transmit to photomultipliers the above three $A, B$ and $C$ portions of the spectrum.

\section{SYSTEM ANALYSIS}

\section{COLOR INDICES AND PARAMETERS [240]}

Light from the comparison bands $A+C$ is fed to one photomultiplier, that from $B$ to a second one. To normalize the readings, the spectrum of a comparison lamp is recorded in an identical way. Then the following ratio is a measure of $\mathrm{H} \alpha$ intensity:

$$
R_{\alpha}=\left[\frac{(A+C)_{\text {star }}}{(A+C)_{\text {lamp }}}\right] /\left[\frac{(B)_{\text {star }}}{(B)_{\text {lamp }}}\right]
$$

It is found to be a good luminosity indicator.

Fig. 29. The photometric system $H \alpha-$ Peat -1964 


\section{Aerobee UV-64 -1964}

Ultraviolet observations by an Aerobee rocket flown on September 2, 1964.

\section{GENERAL INFORMATION}

DETECTOR photoelectric photometers

MAIN ARTICLE $\quad$ Bless, R. C., Code, A. D., Houck, T. E., McNall, J. F., Taylor, D. J. 1965, AJ 70, 666

SYSTEM DESCRIPTION

\begin{tabular}{|c|cc|}
\hline \multicolumn{3}{|c|}{ BANDS DESCRIPTION [37] } \\
\hline band & $\lambda_{c}(\AA)$ & half-width $(\AA)$ \\
\hline 230 & 2100 & 400 \\
\hline 270 & 2500 & 400 \\
\hline 300 & 2800 & 400 \\
\hline
\end{tabular}

The ultraviolet bands were isolated by five-layer $\mathrm{Al}-\mathrm{MgF}_{2}$ interference filters.

Measurements were also taken in a fourth unspecified "blue" optical band. [37]

Fig. 30. The photometric system Aerobee UV-64 - 1964 


\section{$H \alpha, \beta, \gamma, \delta$ - Abt and Golson - 1965}

Luminosity classification of early-type stars with emission lines.

\section{GENERAL INFORMATION}

$\begin{array}{ll}\text { AUTHORS } & \text { H. A. Abt and J. C. Golson } \\ \text { TELESCOPE } & \text { 0.41m and 0.91m (reflectors), KPNO } \\ \text { DETECTOR } & \text { RCA 1P21, or 931-A, or EMI 9558 } \\ \text { MAIN ARTICLE } & \text { Abt, H.A., Golson, J.C. 1965, ApJ 143, } 106\end{array}$

\section{SYSTEM DESCRIPTION}

\begin{tabular}{|ll|lccc|}
\hline \multicolumn{5}{|c|}{ BANDS DESCRIPTION } & [2] \\
\hline \multicolumn{2}{|c|}{ band } & filter (\#) & $\lambda_{\text {peak }}(\AA)$ & half-width $(\AA)$ & $\Upsilon_{\text {peak }}(\%)$ \\
\hline$H \alpha$ & narrow & KPNO 71 & 6567 & 54 & 56.7 \\
$H \alpha$ & wide & KPNO 210 & 6595 & 159 & 87.3 \\
\hline$H \beta$ & narrow & KPNO 216 & 4868 & 26 & 60.8 \\
$H \beta$ & wide & KPNO 225 & 4880 & 210 & 84.5 \\
\hline$H \gamma$ & narrow & KPNO 48 & 4340 & 31 & 56.0 \\
$H \gamma$ & wide & KPNO 49 & 4385 & 161 & 70.6 \\
\hline$H \delta$ & narrow & KPNO 200 & 4115 & 64 & 44.1 \\
$H \delta$ & wide & KPNO 199 & 4115 & 130 & 53.2 \\
\hline
\end{tabular}

\section{SYSTEM ANALYSIS}

\section{COLOR INDICES PARAMETERS [2]}

$$
\begin{aligned}
& \alpha=-2.5 \log I(H \alpha \text { wide }) / I(H \alpha \text { narrow }) \\
& \beta=-2.5 \log I(H \beta \text { wide }) / I(H \beta \text { narrow }) \\
& \gamma=-2.5 \log I(H \gamma \text { wide }) / I(H \gamma \text { narrow }) \\
& \delta=-2.5 \log I(H \delta \text { wide }) / I(H \delta \text { narrow })
\end{aligned}
$$

A set of 12 standard stars without emission lines from G4 to B1 defines the reference value for $\alpha, \beta, \gamma$ and $\delta$.

Fig. 31. The photometric system $H \alpha, \beta, \gamma, \delta$ - Abt and Golson - 1965 


\section{RI - Eggen - 1965}

Red and near-infrared photometry. Visual binaries.

\section{GENERAL INFORMATION}

AUTHORS O. J. Eggen

TELESCOPE $\quad 5 \mathrm{~m}$ and $0.5 \mathrm{~m}$ (reflectors), Palomar Obs.;

$2.5 \mathrm{~m}$ and $1.52 \mathrm{~m}$ (reflectors), Mount Wilson Obs.;

$0.41 \mathrm{~m}$ (reflector) and $0.61 \mathrm{~m}$ (refractor), Cape Observatory

DETECTOR RCA 7102

MAIN ARTICLE Eggen, O.J. 1965, AJ 70, 19

SYSTEM DESCRIPTION

\begin{tabular}{|c|cc|}
\hline \multicolumn{3}{|c|}{ BANDS DESCRIPTION [31] } \\
\hline band & $\lambda_{\text {eff }}(\AA)$ & bandpass $(\AA)$ \\
\hline$R$ & 6350 & $5500-7200$ \\
\hline$I$ & 7900 & $7200-8650$ \\
\hline
\end{tabular}

Fig. 32. The photometric system $R I-$ Eggen - 1965 


\section{UBVRI(JHKLMN) - Johnson - 1965}

Extention toward the infrared of the UBV - Johnson and Morgan - 1953 system.

The system is also often called "The Arizona system".

\section{GENERAL INFORMATION}
AUTHORS
H. L. Johnson
TELESCOPE
$0.71 \mathrm{~cm}$ (reflector), Lunar and Planetary Lab.
DETECTOR
1P21 (for $U B V$ ),
ITT FW-118 (S-1 cathode, for RI),
$\mathrm{PbS}$ or InSb cells (for $J H K L$ ),
liquid He cooled Ge bolometer (for $M$ and $N$ )
MAIN ARTICLE Johnson, H. L. 1965, ApJ 141, 923

\section{SYSTEM DESCRIPTION}

\begin{tabular}{|l|cc|cc|}
\hline \multicolumn{3}{|c|}{ BANDS DESCRIPTION } & \multicolumn{2}{c|}{ FLUX CALIBRATION (\#) [152], [153] } \\
\hline band & $\lambda_{0}(\mu \mathrm{m})[152]$ & half-width $(\mu \mathrm{m}) \quad[285], \mathrm{pg} .292$ & $\mathrm{~F}(\lambda)\left(\mathrm{W} \mathrm{cm}^{-2} \mu \mathrm{m}^{-1}\right)$ & $\mathrm{F}(\lambda)\left(\mathrm{W} \mathrm{m}^{-2} \mathrm{~Hz}^{-1}\right)$ \\
\hline$U$ & 0.36 & 0.04 & $4.3510^{-12}$ & $1.8810^{-23}$ \\
\hline$B$ & 0.44 & 0.10 & $7.2010^{-12}$ & $4.4410^{-23}$ \\
\hline$V$ & 0.55 & 0.08 & $3.9210^{-12}$ & $3.8110^{-23}$ \\
\hline$R$ & 0.70 & 0.21 & $1.7610^{-12}$ & $3.0110^{-23}$ \\
\hline$I$ & 0.90 & 0.22 & $8.310^{-13}$ & $2.4310^{-23}$ \\
\hline$J$ & 1.25 & 0.3 & $3.410^{-13}$ & $1.7710^{-23}$ \\
\hline$H(\# \#)$ & 1.62 & 0.2 & $1.2610^{-13}$ & \\
\hline$K$ & 2.2 & 0.6 & $3.910^{-14}$ & $6.310^{-24}$ \\
\hline$L$ & 3.4 & 0.9 & $8.110^{-15}$ & $3.110^{-24}$ \\
\hline $\boldsymbol{M}$ & 5.0 & 1.1 & $2.210^{-15}$ & $1.810^{-24}$ \\
\hline $\boldsymbol{N}$ & 10.2 & 6.0 & $1.2310^{-16}$ & $4.310^{-25}$ \\
\hline
\end{tabular}

(\#) Fluxes for a 0.0 mag star.

(\#\#) The $\mathrm{H}$ band appeared later in 1967 as part of the Arizona system, and its transmission profile has been a subject of debate. A profile for it is tabulated in VJHKLL'M - Bessell and Brett - 1988 [34].

$O\left(\lambda_{0}=11.5 \mu \mathrm{m}\right), P\left(\lambda_{0}=13.1 \mu \mathrm{m}\right)$ and $Q\left(\lambda_{0}=20.0 \mu \mathrm{m}\right)$ bands have been sometimes associated with this system.

The UBV bands are essentially those of UBV - Johnson and Morgan - 1953.

In the table with the band transmission profiles, the $L$ band is indicated with $L^{\prime}\left(\lambda_{e f f}=3.5 \mu \mathrm{m}\right)$ when realized with a InSb detector, $L "\left(\lambda_{e f f}=3.4 \mu \mathrm{m}\right)$ for a PbS detector.

ZERO POINT: Color indices are zero for unreddened A0 V stars. [151]

\section{SYSTEM ANALYSIS}

REDDENING RATIOS [258]
$A_{R}=2.32 E(B-V)$
$A_{I}=1.50 E(B-V)$
$A_{J}=0.87 E(B-V)$
$A_{K}=0.38 E(B-V)$
$A_{L}=0.16 E(B-V)$

Fig. 33. The photometric system UBVRI(JHKLMN) - Johnson - 1965 


\section{TRANSMISSION CURVES [151]}

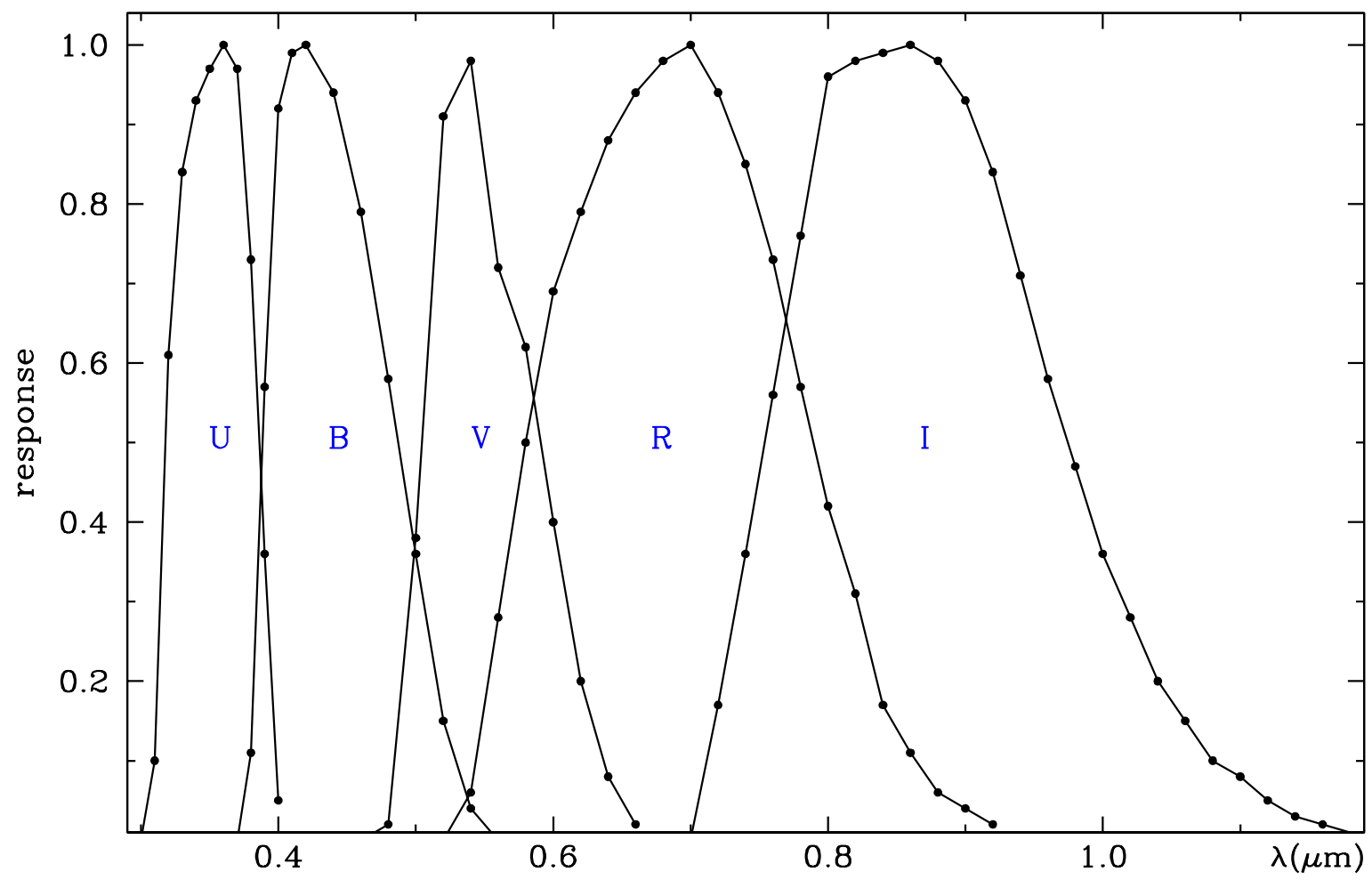

\begin{tabular}{|cc|cc|cc|cc|ccc|cc|c|c|}
\hline \multicolumn{2}{|c|}{$U$} & \multicolumn{3}{c|}{$B$} & \multicolumn{3}{c|}{$V$} & \multicolumn{3}{c|}{$R$} & \multicolumn{3}{c|}{$I$} \\
\hline$\lambda(\mu \mathrm{m})$ & $\Upsilon$ & $\lambda(\mu \mathrm{m})$ & $\Upsilon$ & $\lambda(\mu \mathrm{m})$ & $\Upsilon$ & $\lambda(\mu \mathrm{m})$ & $\Upsilon$ & $\lambda(\mu \mathrm{m})$ & $\Upsilon$ & $\lambda(\mu \mathrm{m})$ & $\Upsilon$ & $\lambda(\mu \mathrm{m})$ & $\Upsilon$ \\
\hline 0.30 & 0.00 & 0.37 & 0.00 & 0.46 & 0.00 & 0.52 & 0.00 & 0.82 & 0.31 & 0.68 & 0.00 & 0.98 & 0.47 \\
0.31 & 0.10 & 0.38 & 0.11 & 0.48 & 0.02 & 0.54 & 0.06 & 0.84 & 0.17 & 0.70 & 0.01 & 1.00 & 0.36 \\
0.32 & 0.61 & 0.40 & 0.92 & 0.50 & 0.38 & 0.56 & 0.28 & 0.86 & 0.11 & 0.72 & 0.17 & 1.02 & 0.28 \\
0.33 & 0.84 & 0.42 & 1.00 & 0.52 & 0.91 & 0.58 & 0.50 & 0.88 & 0.06 & 0.74 & 0.36 & 1.04 & 0.20 \\
0.34 & 0.93 & 0.44 & 0.94 & 0.54 & 0.98 & 0.60 & 0.69 & 0.90 & 0.04 & 0.76 & 0.56 & 1.06 & 0.15 \\
0.35 & 0.97 & 0.46 & 0.79 & 0.56 & 0.72 & 0.62 & 0.79 & 0.92 & 0.02 & 0.78 & 0.76 & 1.08 & 0.10 \\
0.36 & 1.00 & 0.48 & 0.58 & 0.58 & 0.62 & 0.64 & 0.88 & 0.94 & 0.01 & 0.80 & 0.96 & 1.10 & 0.08 \\
0.37 & 0.97 & 0.50 & 0.36 & 0.60 & 0.40 & 0.66 & 0.94 & 0.96 & 0.00 & 0.82 & 0.98 & 1.12 & 0.05 \\
0.38 & 0.73 & 0.52 & 0.15 & 0.62 & 0.20 & 0.68 & 0.98 & & & 0.84 & 0.99 & 1.14 & 0.03 \\
0.39 & 0.36 & 0.54 & 0.04 & 0.64 & 0.08 & 0.70 & 1.00 & & & 0.86 & 1.00 & 1.16 \\
0.40 & 0.05 & 0.56 & 0.00 & 0.66 & 0.02 & 0.72 & 0.94 & & & 0.88 & 0.98 & 1.20 \\
0.41 & 0.01 & & & 0.68 & 0.01 & 0.74 & 0.85 & & & 0.90 & 0.00 \\
0.42 & 0.00 & & & 0.70 & 0.01 & 0.76 & 0.73 & & & 0.92 & 0.84 & \\
& & & & 0.72 & 0.01 & 0.78 & 0.57 & & & 0.94 & 0.71 & \\
\end{tabular}

Fig. 33. continued 

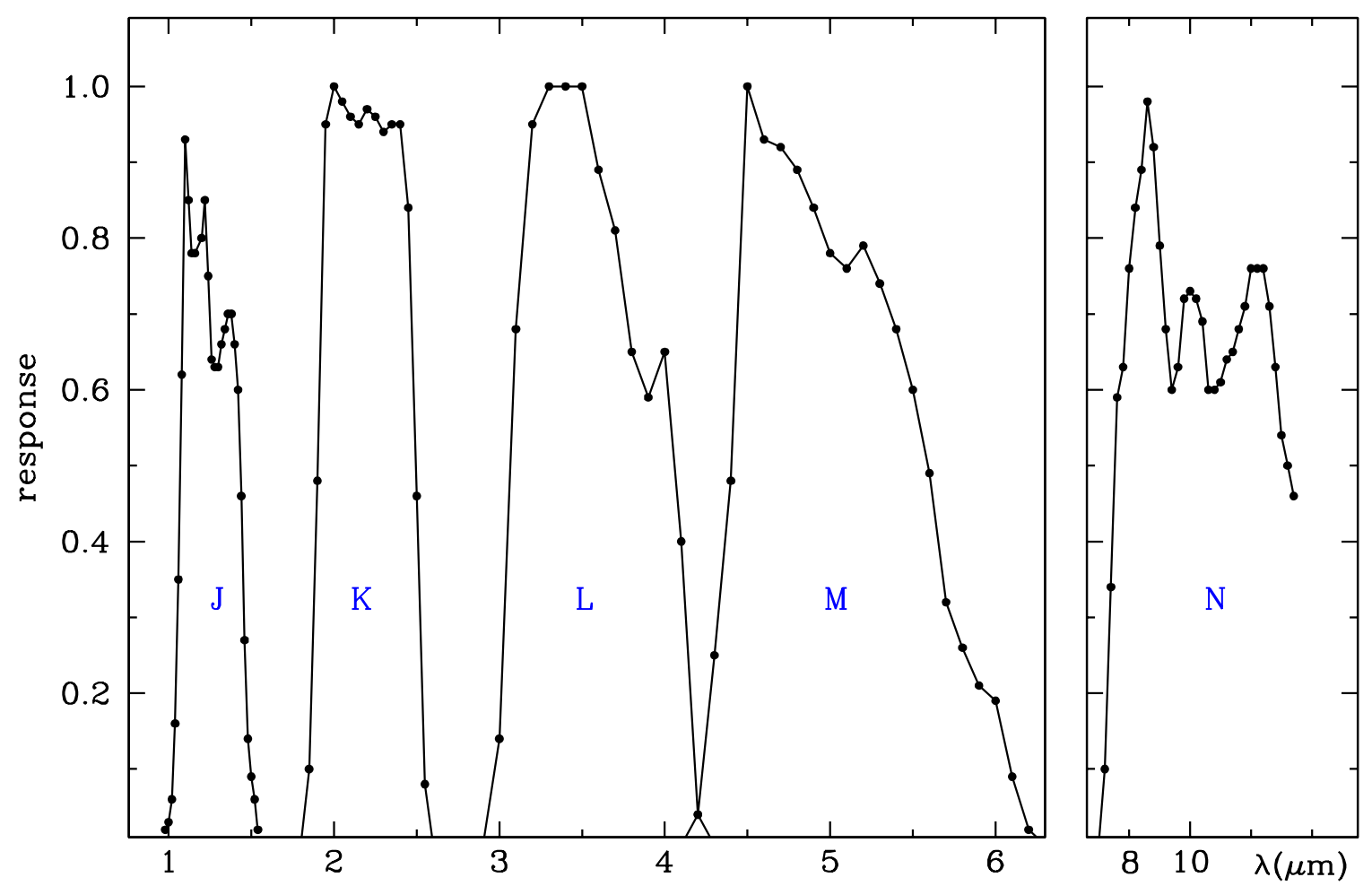

\begin{tabular}{|c|c|c|c|c|c|c|c|c|c|c|c|c|c|c|c|}
\hline \multicolumn{4}{|c|}{$J$} & \multicolumn{2}{|c|}{$K$} & \multicolumn{2}{|c|}{$L^{\prime}$} & \multicolumn{2}{|c|}{$L^{\prime \prime}$} & \multicolumn{2}{|c|}{$M$} & \multicolumn{4}{|c|}{$N$} \\
\hline$\lambda(\mu \mathrm{m})$ & $\Upsilon$ & $\lambda(\mu \mathrm{m})$ & $\Upsilon$ & $\lambda(\mu \mathrm{m})$ & $\Upsilon$ & $\lambda(\mu \mathrm{m})$ & $\Upsilon$ & $\lambda(\mu \mathrm{m})$ & $\Upsilon$ & $\lambda(\mu \mathrm{m})$ & $\Upsilon$ & $\lambda(\mu \mathrm{m})$ & $\Upsilon$ & $\lambda(\mu \mathrm{m})$ & $\Upsilon$ \\
\hline 0.96 & 0.00 & 1.44 & 0.46 & 1.80 & 0.00 & 2.90 & 0.00 & 2.90 & 0.00 & 4.10 & 0.00 & 7.00 & 0.00 & 11.8 & 0.71 \\
\hline 0.98 & 0.02 & 1.46 & 0.27 & 1.85 & 0.10 & 3.00 & 0.14 & 3.00 & 0.14 & 4.20 & 0.04 & 7.20 & 0.10 & 12.0 & 0.76 \\
\hline 1.00 & 0.03 & 1.48 & 0.14 & 1.90 & 0.48 & 3.10 & 0.68 & 3.10 & 0.68 & 4.30 & 0.25 & 7.40 & 0.34 & 12.2 & 0.76 \\
\hline 1.02 & 0.06 & 1.50 & 0.09 & 1.95 & 0.95 & 3.20 & 0.95 & 3.20 & 0.95 & 4.40 & 0.48 & 7.60 & 0.59 & 12.4 & 0.76 \\
\hline 1.04 & 0.16 & 1.52 & 0.06 & 2.00 & 1.00 & 3.30 & 1.00 & 3.30 & 1.00 & 4.50 & 1.00 & 7.80 & 0.70 & 12.6 & 0.71 \\
\hline 1.06 & 0.35 & 1.54 & 0.02 & 2.05 & 0.98 & 3.40 & 1.00 & 3.40 & 1.00 & 4.60 & 0.93 & 8.00 & 0.76 & 12.8 & 0.63 \\
\hline 1.08 & 0.62 & 1.56 & 0.00 & 2.10 & 0.96 & 3.50 & 1.00 & 3.50 & 0.98 & 4.70 & 0.92 & 8.20 & 0.84 & 13.0 & 0.54 \\
\hline 1.10 & 0.93 & & & 2.15 & 0.95 & 3.60 & 0.89 & 3.60 & 0.85 & 4.80 & 0.89 & 8.40 & 0.89 & 13.2 & 0.50 \\
\hline 1.12 & 0.85 & & & 2.20 & 0.97 & 3.70 & 0.81 & 3.70 & 0.69 & 4.90 & 0.84 & 8.60 & 0.98 & 13.4 & 0.46 \\
\hline 1.14 & 0.78 & & & 2.25 & 0.96 & 3.80 & 0.65 & 3.80 & 0.39 & 5.00 & 0.78 & 8.80 & 0.92 & 13.6 & 0.43 \\
\hline 1.16 & 0.78 & & & 2.30 & 0.94 & 3.90 & 0.59 & 3.90 & 0.21 & 5.10 & 0.76 & 9.00 & 0.79 & & \\
\hline 1.18 & 0.80 & & & 2.35 & 0.95 & 4.00 & 0.65 & 4.00 & 0.10 & 5.20 & 0.79 & 9.20 & 0.68 & & \\
\hline 1.20 & 0.85 & & & 2.40 & 0.95 & 4.10 & 0.40 & 4.10 & 0.02 & 5.30 & 0.74 & 9.40 & 0.60 & & \\
\hline 1.22 & 0.93 & & & 2.45 & 0.84 & 4.20 & 0.04 & 4.20 & 0.00 & 5.40 & 0.68 & 9.60 & 0.63 & & \\
\hline 1.24 & 0.75 & & & 2.50 & 0.46 & 4.30 & 0.00 & & & 5.50 & 0.60 & 9.80 & 0.69 & & \\
\hline 1.26 & 0.64 & & & 2.55 & 0.08 & & & & & 5.60 & 0.49 & 10.0 & 0.73 & & \\
\hline 1.28 & 0.63 & & & 2.60 & 0.00 & & & & & 5.70 & 0.32 & 10.2 & 0.72 & & \\
\hline 1.30 & 0.63 & & & & & & & & & 5.80 & 0.26 & 10.4 & 0.65 & & \\
\hline 1.32 & 0.66 & & & & & & & & & 5.90 & 0.21 & 10.6 & 0.60 & & \\
\hline 1.34 & 0.68 & & & & & & & & & 6.00 & 0.19 & 10.8 & 0.60 & & \\
\hline 1.36 & 0.70 & & & & & & & & & 6.10 & 0.09 & 11.0 & 0.61 & & \\
\hline 1.38 & 0.70 & & & & & & & & & 6.20 & 0.02 & 11.2 & 0.64 & & \\
\hline 1.40 & 0.66 & & & & & & & & & 6.30 & 0.00 & 11.4 & 0.65 & & \\
\hline 1.42 & 0.60 & & & & & & & & & & & 11.6 & 0.68 & & \\
\hline
\end{tabular}

Fig. 33. continued 


\title{
Miner - 1965
}

Temperature and metallicity of F5-K5 stars via narrow-band photometry.

\section{GENERAL INFORMATION}

\author{
AUTHORS \\ E. D. Miner \\ TELESCOPE \\ $0.61 \mathrm{~m}$ (reflector), Brigham Young University \\ MAIN ARTICLE Miner, E.D. 1965, ApJ 144, 1101
}

\section{SYSTEM DESCRIPTION}

\begin{tabular}{|c|lccc|}
\hline \multicolumn{5}{|c|}{ BANDS DESCRIPTION [212] } \\
\hline band & filter manufacturer & $\lambda_{\text {peak }}(\AA)$ & half-width $(\AA)$ & $\Upsilon(\%)$ \\
\hline 416 & Baird B-1 & 4160 & 60 & 60 \\
\hline 426 & Baird B-3 + film & 4260 & 160 & 80 \\
\hline 431 & Spectrolab & 4305 & 60 & 35 \\
\hline 439 & Baird B-1 & 4390 & 70 & 80 \\
\hline 452 & Baird B-1 & 4520 & 70 & 60 \\
\hline 486 & Baird B-3 + film & 4861 & 220 & 80 \\
\hline 560 & Baird B-9 & 5600 & 50 & 60 \\
\hline
\end{tabular}

All filters are interference ones.

\section{SYSTEM ANALYSIS}

\section{COLOR INDICES AND PARAMETERS [212]}

$g^{\prime}=$ cost $+2.5[\log I(439)-\log I(431)]:$ measures the G-band.

$n^{\prime}=$ cost $+2.5[\log I(426)-\log I(416)]:$ measures the CN-band.

$m^{\prime}=\cos t+2.5[2 \log I(452)-\log I(431)-\log I(486)]:$ metallicity indicator.

$b v^{\prime}=\operatorname{cost}+2.5[\log I(560)-\log I(439)]:$ temperature indicator.

RELATIONS WITH OTHER SYSTEMS [212]

UBV - Johnson and Morgan - 1953

\begin{tabular}{|llll|}
\hline$(B-V)$ & $=0.208+0.897 b v^{\prime}$ & & for $b v^{\prime}<1.000$ \\
\hline$(B-V)$ & $=0.272+0.815 b v^{\prime}$ & & for $b v^{\prime}>1.000$ \\
\hline$(B-V)$ & $=-0.72+1.93 g^{\prime}+0.14 n^{\prime}+0.06 m^{\prime}-0.6 \Delta m^{\prime}$ & & for $g^{\prime}<0.680$ \\
\hline$(B-V)$ & $=-2.15+5.63 g^{\prime}+0.01 n^{\prime}-1.22 m^{\prime}-1.9 \Delta m^{\prime}$ & & for $g^{\prime} \geq 0.680$ \\
\hline
\end{tabular}

The $\Delta m^{\prime}$ correction (to eliminate chemical abundance effects) generally varies between +0.05 and -0.10 . Numerical values for $\Delta m^{\prime}$ are given in graphical form by [212].

Fig. 34. The photometric system Miner - 1965 


\section{Vilnius - Straižys et al. - 1965}

General purpose system.

\section{GENERAL INFORMATION}
AUTHORS
V. Straižys, J. Sŭdžius, and K. Zdanavičius
TELESCOPE
$0.63 \mathrm{~m}$ (reflector), Moletai Obs.
DETECTOR
FEU-79 (S-20 cathode)

MAIN ARTICLE Straižys, V., Zdanavičius, K. 1965 Bull. Vilnius Obs. 14, 1

\begin{tabular}{|c|c|c|c|c|c|c|c|}
\hline \multicolumn{8}{|c|}{ SYSTEM DESCRIPTION } \\
\hline \multicolumn{4}{|c|}{ BANDS DESCRIPTION [178], pg 66} & \multicolumn{4}{|c|}{ FLUX CALIBRATION } \\
\hline band & filter & $\lambda_{0}(\AA)$ & FWHM $(\AA)$ & $\mathrm{A} 0 \mathrm{~V}(\#)$ & O V (\#) & $\mathrm{A} 0 \mathrm{~V}(*)$ & $\mathrm{O} V(*)$ \\
\hline$U$ & $1.5 \mathrm{~mm}$ BS5 + 10mm UFS2 & 3450 & 400 & 3.23 & 18.97 & 3.34 & 19.22 \\
\hline$P$ & $2 \mathrm{~mm}$ SZS22 + 2.7mm UFS6 & 3740 & 260 & 4.47 & 14.39 & 4.53 & 14.58 \\
\hline$X$ & $\begin{array}{l}3.2 \mathrm{~mm} \mathrm{ZhS} 4+6.2 \mathrm{~mm} 3 \mathrm{~S} 7+ \\
1.8 \mathrm{~mm} \mathrm{SZS} 21+1.0 \mathrm{FS} 7\end{array}$ & 4050 & 220 & 7.41 & 11.12 & 7.51 & 11.27 \\
\hline$Y$ & $5.2 \mathrm{~mm} \mathrm{ZhS} 12+2.6 \mathrm{~mm} \mathrm{SZS} 21+2 \mathrm{~mm} \mathrm{SS} 15$ & 4660 & 260 & 5.68 & 6.52 & 5.76 & 6.60 \\
\hline$Z$ & $2 \mathrm{~mm} \mathrm{ZhS17}+3.1 \mathrm{~mm}$ ZS7 $+10 \mathrm{~mm} \mathrm{SZS} 22$ & 5160 & 210 & 4.39 & 4.59 & 4.45 & 4.65 \\
\hline$V$ & $1.5 \mathrm{~mm}$ OS $11+5 \mathrm{~mm} \mathrm{SZS} 22+2 \mathrm{~mm}$ PS7 & 5440 & 260 & 3.75 & 3.75 & 3.80 & 3.80 \\
\hline$S$ & interference & 6550 & 200 & 2.16 & 1.90 & 2.19 & 1.92 \\
\hline$T$ & interference & 6250 & 200 & & & & \\
\hline
\end{tabular}

(\#) Fluxes for a $V=0.0 \mathrm{star}$ (units of $10^{-9} \mathrm{erg} \mathrm{cm}^{-2} \mathrm{~s}^{-1} \AA^{-1}$ ). [178], pg. 66

(*) Fluxes for a $V=0.0 \mathrm{star}$ (units of $10^{-12} \mathrm{~W} \mathrm{~cm}^{-2} \mu \mathrm{m}^{-1}$ ). [285], pg. 427

ZERO POINT: Color indices are zero for unreddened O-type stars. [178], pg. 66

\section{SYSTEM ANALYSIS}

REDDENING-FREE PARAMETERS [285], pg. 431-454

A general expression for reddening-free parameters (involving four or three bands) is:

$Q_{A B C D}=(A-B)-\frac{E(A-B)}{E(C-D)} \cdot(C-D) \quad Q_{A B C}=(A-B)-\frac{E(A-B)}{E(B-C)} \cdot(B-C)$

$\mathrm{Q}_{U P Y}$ vs $\mathrm{Q}_{P Y V}$ : classification of B stars.

$\mathrm{Q}_{U P Y}$ vs $\mathrm{Q}_{X Y V}$ : classification of B8 to early-G stars $\quad \mathrm{Q}_{U X Y}$ vs $\mathrm{Q}_{U P Y V}$ : classification of A5-G0 stars.

$\mathrm{Q}_{U P Y}$ vs $\mathrm{Q}_{X Z S}$ : classification of G-K type stars. $\quad \mathrm{Q}_{X Z S}$ vs $\mathrm{Q}_{X Y Z}$ : classification of K-M type stars.

RELATIONS WITH OTHER SYSTEMS [285], pg. 488-493

UBV - Johnson and Morgan - 1953

$(B-V)=-0.33+0.50[(X-V)+(Y-V)] \quad$ for stars with $(B-V) \leq 0.8$

uvby $H \beta$ - Strömgren and Crawford - 1956

\begin{tabular}{rlr|}
$(U-Y)$ & $=(u-b)+0.30-0.024(b-y)_{0}+c E(b-y)$ & excluding M-giants and supergiants \\
$(Y-V)$ & $=0.16+0.09(b-y)$ & for O-K stars \\
\hline
\end{tabular}

where $c$ increases from 0.005 for early-type stars to 0.016 for F-supergiants and goes to zero for late-type stars.

Fig. 35. The photometric system Vilnius - Straižys et al. - 1965 


\section{TRANSMISSION CURVES [287]}

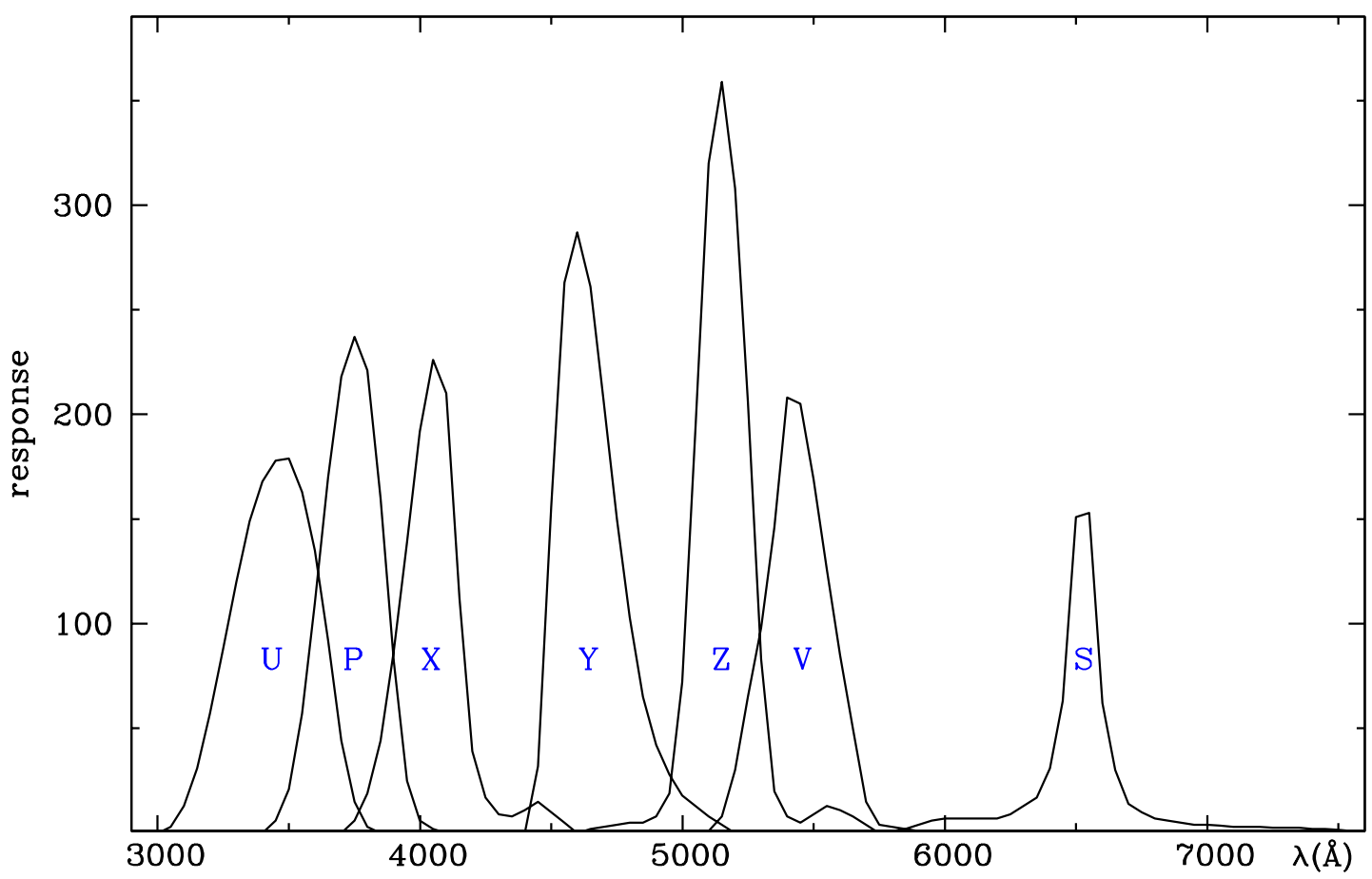

\begin{tabular}{|c|c|c|c|c|c|c|c|c|c|c|c|c|c|c|c|}
\hline \multicolumn{2}{|c|}{$U$} & \multicolumn{2}{|c|}{$P$} & \multicolumn{2}{|c|}{$X$} & \multicolumn{2}{|c|}{$Y$} & \multicolumn{2}{|c|}{$Z$} & \multicolumn{2}{|c|}{$V$} & \multicolumn{4}{|c|}{$S$} \\
\hline$\lambda(\mathrm{A})$ & $\Upsilon$ & $\lambda(\mathrm{A})$ & $\Upsilon$ & $\lambda(\mathrm{A})$ & $\Upsilon$ & $\lambda(\mathrm{A})$ & $\Upsilon$ & $\lambda(\mathrm{A})$ & $\Upsilon$ & $\lambda(\mathrm{A})$ & $\Upsilon$ & $\lambda(\mathrm{A})$ & $\Upsilon$ & $\lambda(\mathrm{A})$ & $\Upsilon$ \\
\hline 3000 & 0 & 3400 & 0 & 3700 & 0 & 4400 & 0 & 4600 & 0 & 5050 & 0 & 5750 & 0 & 6950 & 4 \\
\hline 3050 & 3 & 3450 & 6 & 3750 & 6 & 4450 & 32 & 4650 & 2 & 5100 & 1 & 5800 & 1 & 7000 & 4 \\
\hline 3100 & 13 & 3500 & 21 & 3800 & 19 & 4500 & 156 & 4700 & 3 & 5150 & 8 & 5850 & 2 & 7050 & 3.5 \\
\hline 3150 & 31 & 3550 & 57 & 3850 & 44 & 4550 & 263 & 4750 & 4 & 5200 & 30 & 5900 & 4 & 7100 & 3 \\
\hline 3200 & 57 & 3600 & 110 & 3900 & 87 & 4600 & 287 & 4800 & 5 & 5250 & 65 & 5950 & 6 & 7150 & 3 \\
\hline 3250 & 88 & 3650 & 170 & 3950 & 139 & 4650 & 261 & 4850 & 5 & 5300 & 98 & 6000 & 7 & 7200 & 3 \\
\hline 3300 & 120 & 3700 & 218 & 4000 & 192 & 4700 & 206 & 4900 & 8 & 5350 & 146 & 6050 & 7 & 7300 & 2.5 \\
\hline 3350 & 149 & 3750 & 237 & 4050 & 226 & 4750 & 150 & 4950 & 19 & 5400 & 208 & 6100 & 7 & 7350 & 2.5 \\
\hline 3400 & 168 & 3800 & 221 & 4100 & 210 & 4800 & 103 & 5000 & 72 & 5450 & 205 & 6150 & 7 & 7400 & 2 \\
\hline 3450 & 178 & 3850 & 160 & 4150 & 112 & 4850 & 65 & 5050 & 193 & 5500 & 169 & 6200 & 7 & 7450 & 2 \\
\hline 3500 & 179 & 3900 & 82 & 4200 & 39 & 4900 & 42 & 5100 & 320 & 5550 & 126 & 6250 & 9 & 7500 & 1.5 \\
\hline 3550 & 163 & 3950 & 25 & 4250 & 17 & 4950 & 28 & 5150 & 359 & 5600 & 85 & 6300 & 13 & 7550 & 1 \\
\hline 3600 & 135 & 4000 & 6 & 4300 & 9 & 5000 & 18 & 5200 & 308 & 5650 & 50 & 6350 & 17 & 7600 & 1 \\
\hline 3650 & 92 & 4050 & 2 & 4350 & 8 & 5050 & 13 & 5250 & 206 & 5700 & 15 & 6400 & 31 & 7650 & 0.5 \\
\hline 3700 & 44 & 4100 & 0 & 4400 & 11 & 5100 & 8 & 5300 & 83 & 5750 & 4 & 6450 & 63 & 7700 & 0 \\
\hline 3750 & 15 & & & 4450 & 15 & 5150 & 4 & 5350 & 20 & 5800 & 3 & 6500 & 151 & & \\
\hline 3800 & 3 & & & 4500 & 10 & 5200 & 0 & 5400 & 8 & 5850 & 2 & 6550 & 153 & & \\
\hline 3850 & 0 & & & 4550 & 5 & & & 5450 & 5 & 5900 & 1 & 6600 & 62 & & \\
\hline & & & & 4600 & 0 & & & 5500 & 9 & 5950 & 0 & 6650 & 30 & & \\
\hline & & & & & & & & 5550 & 13 & & & 6700 & 14 & & \\
\hline & & & & & & & & 5600 & 11 & & & 6750 & 10 & & \\
\hline & & & & & & & & 5650 & 8 & & & 6800 & 7 & & \\
\hline & & & & & & & & 5700 & 4 & & & 6850 & 6 & & \\
\hline & & & & & & & & 5750 & 0 & & & 6900 & 5 & & \\
\hline
\end{tabular}

Fig. 35. continued 


\section{UVBY - Kruszewski - 1966}

General purpose system.

\section{GENERAL INFORMATION}

AUTHORS

TELESCOPE

DETECTOR

MAIN ARTICLE

\section{A. Kruszewski}

$0.35 \mathrm{~m}$ (reflector), Ostrowik station, Warsaw University Observatory 1P21 (uncooled)

Kruszewski, A. 1966, Acta Astronomica 16, 285

\section{SYSTEM DESCRIPTION}

\begin{tabular}{|c|cccc|}
\hline \multicolumn{5}{|c|}{ BANDS DESCRIPTION [175] } \\
\hline band & filter & $\lambda_{\text {peak }}(\AA)$ & $\lambda_{\text {eff }}(\AA)$ & half-width $(\AA)$ \\
\hline$U$ & 2mm UG 2 + 2mm UG 11 & 3600 & 3521 & 460 \\
\hline$V$ & 1mm UG 2 + 1mm BG 12 + 1mm GG 18 & 3820 & 3781 & 290 \\
\hline$B$ & 2mm BG 12 + 1mm GG 5 + 2mm GG 13 & 4700 & 4614 & 330 \\
\hline$Y$ & $1 \mathrm{~mm} \mathrm{BG} \mathrm{18} \mathrm{+} \mathrm{1mm} \mathrm{OG} \mathrm{4}$ & 5230 & 5480 & 620 \\
\hline
\end{tabular}

ZERO POINT: Unreddened A0 V stars have 0.00 color indices [175]

\section{SYSTEM ANALYSIS}

REDDENING RATIOS [175]

$$
\frac{E(U-V)}{E(B-Y)}=0.35 \quad \frac{E(V-B)}{E(B-Y)}=0.78
$$

\section{REDDENING-FREE PARAMETERS [175]}

$[V-B]=(V-B)-\frac{E(V-B)}{E(B-Y)}(B-Y):$ metallicity indicator.

$[U-V]=(U-V)-\frac{E(U-V)}{E(B-Y)}(B-Y):$ luminosity indicator.

$L=[V-B]-0.5[U-V]:$ correlates with absolute magnitude.

$H=[V-B]-0.2[U-V]+0.4[U-V]^{2}:$ measures the intensity of Balmer lines.

RELATIONS WITH OTHER SYSTEMS [175]

\section{UBV - Johnson and Morgan - 1953}

$Y_{K r}=V_{J}+0.001( \pm 0.008)+0.09( \pm 0.02)(B-Y)_{K r}$

Fig. 36. The photometric system $U V B Y-$ Kruszewski - 1966 


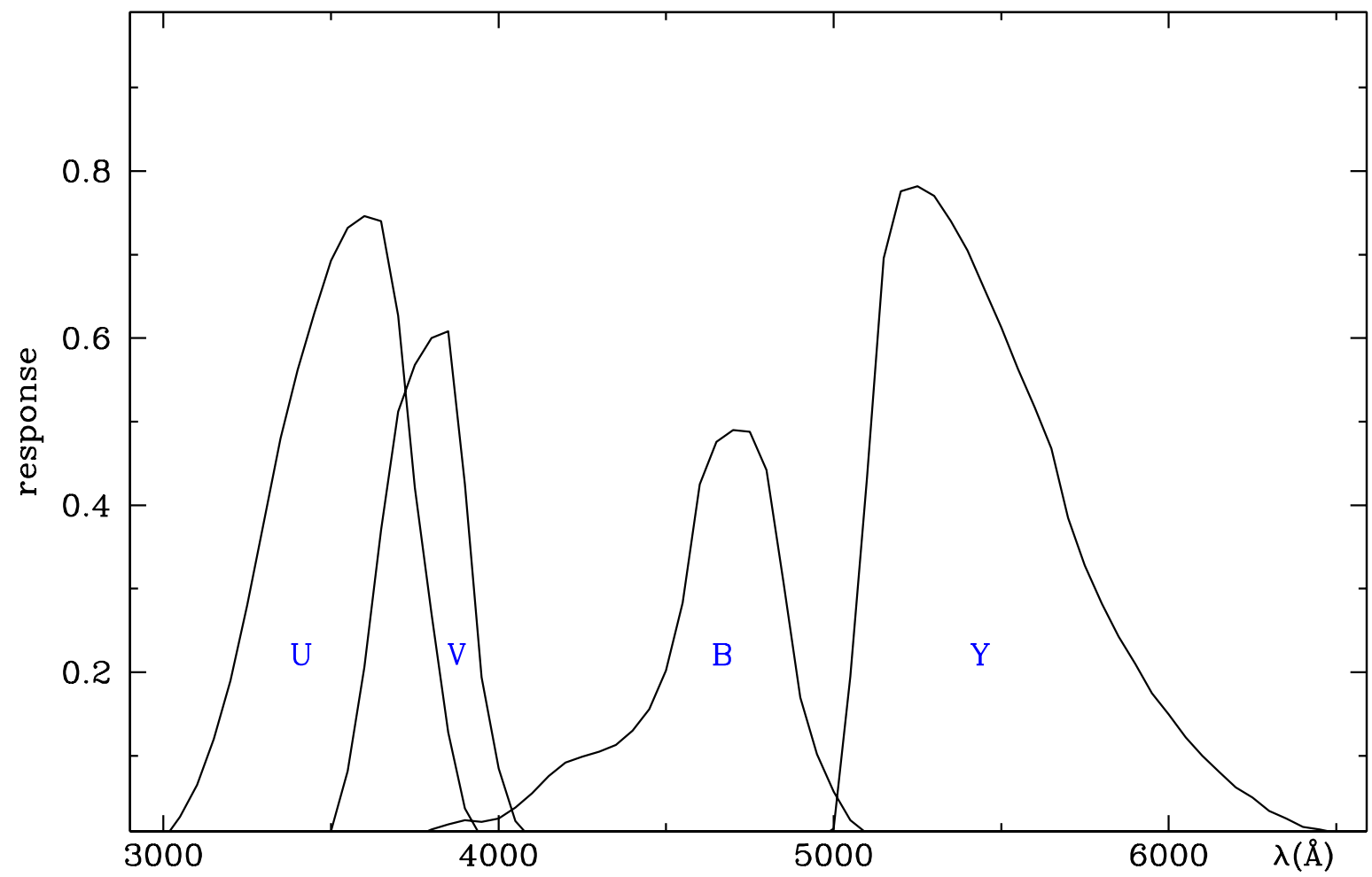

\begin{tabular}{|cc|cc|cc|cc|cc|cc|}
\hline \multicolumn{2}{|c|}{$U$} & \multicolumn{3}{|c|}{$V$} & \multicolumn{3}{c|}{$B$} & \multicolumn{4}{c|}{$Y$} \\
\hline$\lambda(\mathrm{A})$ & $\Upsilon$ & $\lambda(\mathrm{A})$ & $\Upsilon$ & $\lambda(\mathrm{A})$ & $\Upsilon$ & $\lambda(\mathrm{A})$ & $\Upsilon$ & $\lambda(\mathrm{A})$ & $\Upsilon$ & $\lambda(\mathrm{A})$ & $\Upsilon$ \\
\hline 3000 & 0.000 & 3450 & 0.000 & 3700 & 0.000 & 4750 & 0.488 & 4950 & 0.000 & 6000 & 0.150 \\
3050 & 0.027 & 3500 & 0.009 & 3750 & 0.003 & 4800 & 0.442 & 5000 & 0.013 & 6050 & 0.122 \\
3100 & 0.065 & 3550 & 0.082 & 3800 & 0.012 & 4850 & 0.307 & 5050 & 0.195 & 6100 & 0.100 \\
3150 & 0.120 & 3600 & 0.206 & 3850 & 0.018 & 4900 & 0.170 & 5100 & 0.433 & 6150 & 0.081 \\
3200 & 0.190 & 3650 & 0.370 & 3900 & 0.023 & 4950 & 0.102 & 5150 & 0.696 & 6200 & 0.062 \\
3250 & 0.280 & 3700 & 0.512 & 3950 & 0.021 & 5000 & 0.057 & 5200 & 0.776 & 6250 & 0.050 \\
3300 & 0.380 & 3750 & 0.568 & 4000 & 0.025 & 5050 & 0.023 & 5250 & 0.782 & 6300 & 0.034 \\
3350 & 0.480 & 3800 & 0.600 & 4050 & 0.038 & 5100 & 0.007 & 5300 & 0.770 & 6350 & 0.025 \\
3400 & 0.562 & 3850 & 0.608 & 4100 & 0.055 & 5150 & 0.000 & 5350 & 0.740 & 6400 & 0.015 \\
3450 & 0.630 & 3900 & 0.425 & 4150 & 0.076 & & & 5400 & 0.705 & 6450 & 0.012 \\
3500 & 0.693 & 3950 & 0.194 & 4200 & 0.092 & & & 5450 & 0.658 & 6500 & 0.008 \\
3550 & 0.732 & 4000 & 0.085 & 4250 & 0.099 & & & 5500 & 0.613 & 6550 & 0.006 \\
3600 & 0.746 & 4050 & 0.022 & 4300 & 0.105 & & & 5550 & 0.563 & 6600 & 0.004 \\
3650 & 0.740 & 4100 & 0.000 & 4350 & 0.113 & & & 5600 & 0.517 & 6650 & 0.003 \\
3700 & 0.627 & & & 4400 & 0.130 & & & 5650 & 0.468 & 6700 & 0.000 \\
3750 & 0.421 & & & 4450 & 0.156 & & & 5700 & 0.385 & & \\
3800 & 0.270 & & & 4500 & 0.202 & & & 5750 & 0.328 & & \\
3850 & 0.128 & & & 4550 & 0.283 & & & 5800 & 0.282 & & \\
3900 & 0.037 & & & 4600 & 0.425 & & & 5850 & 0.243 & & \\
3950 & 0.002 & & & 4650 & 0.476 & & & 5900 & 0.210 & & \\
4000 & 0.000 & & & 4700 & 0.490 & & & 5950 & 0.175 & & \\
\hline
\end{tabular}

Fig. 36. continued 


\section{4 colors - Neff and Travis - 1966}

General purpose system, aimed in particular to late type stars.

\section{GENERAL INFORMATION}

$\begin{array}{ll}\text { AUTHORS } & \text { J. S. Neff and L. D. Travis } \\ \text { TELESCOPE } & 0.91 \mathrm{~m} \text { and } 2.08 \mathrm{~m} \text { (reectors), Mc Donald Obs. } \\ \text { DETECTOR } & 1 \mathrm{P} 21 \text { (refrigerated) }\end{array}$

MAIN ARTICLE Neff, J. S., Travis, L. D. 1967, AJ 72, 48

\section{SYSTEM DESCRIPTION}

\begin{tabular}{|c|l|cc|}
\hline \multicolumn{3}{|c|}{ BANDS DESCRIPTION [224], [225] } & \\
\hline band & interference lter manufacturer & $W H M(\mathrm{~A})$ & $\lambda_{c}(\mathrm{~A})$ \\
\hline 33 & Space Astr. Lab., Univ. Wisconsin & 330 & 3330 \\
\hline 37 & Fish-Schurman & 140 & 3700 \\
\hline 47 & Baird Atomic & 190 & 4680 \\
\hline 55 & Baird Atomic & 220 & 5500 \\
\hline
\end{tabular}

ZERO POINT: Unreddened A0 V stars have 0.00 color indices. [225]

\section{TRANSMISSION CURVES [225]}

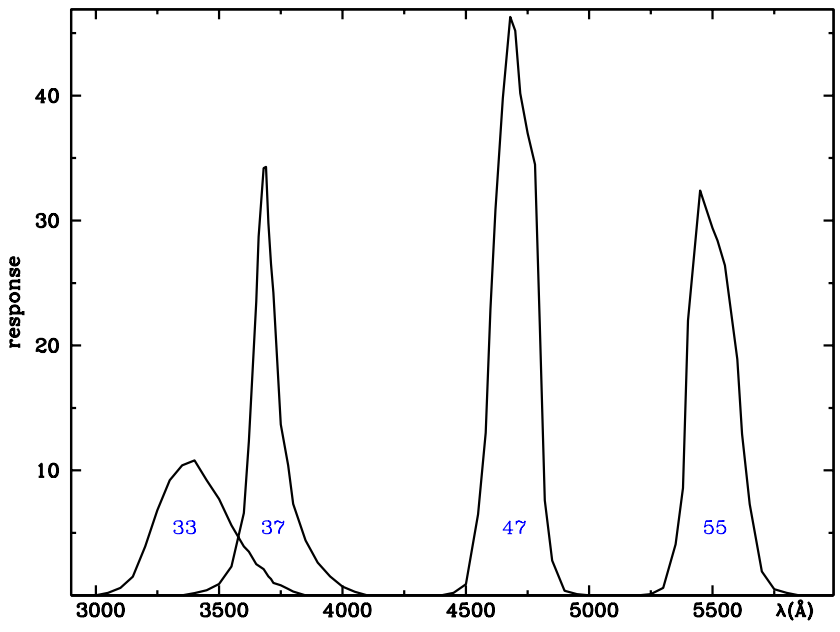

\begin{tabular}{|rr|rr|rr|rr|}
\hline \multicolumn{2}{|c|}{33} & \multicolumn{2}{|c|}{37} & \multicolumn{2}{c|}{47} & \multicolumn{2}{c|}{55} \\
\hline$\lambda(\mathrm{A})$ & $\Upsilon$ & $\lambda(\mathrm{A})$ & $\Upsilon$ & $\lambda(\mathrm{A})$ & $\Upsilon$ & $\lambda(\mathrm{A})$ & $\Upsilon$ \\
\hline 3000 & 0.0 & 3350 & 0.0 & 4400 & 0.0 & 5200 & 0.0 \\
3050 & 0.2 & 3400 & 0.2 & 4450 & 0.2 & 5250 & 0.1 \\
3100 & 0.6 & 3450 & 0.4 & 4500 & 0.9 & 5300 & 0.6 \\
3150 & 1.5 & 3500 & 0.9 & 4550 & 6.5 & 5350 & 4.1 \\
3200 & 3.9 & 3550 & 2.3 & 4580 & 13.0 & 5380 & 8.6 \\
3250 & 6.8 & 3600 & 6.6 & 4600 & 22.9 & 5400 & 22.0 \\
3300 & 9.2 & 3620 & 12.3 & 4620 & 30.9 & 5450 & 32.4 \\
3350 & 10.4 & 3650 & 23.5 & 4650 & 39.8 & 5500 & 29.4 \\
3400 & 10.8 & 3660 & 28.7 & 4680 & 46.3 & 5520 & 28.4 \\
3450 & 9.2 & 3680 & 34.2 & 4700 & 45.2 & 5550 & 26.4 \\
3500 & 7.7 & 3690 & 34.3 & 4720 & 40.2 & 5600 & 18.9 \\
3550 & 5.6 & 3700 & 29.8 & 4750 & 37.0 & 5620 & 12.9 \\
3600 & 3.9 & 3710 & 26.5 & 4780 & 34.5 & 5650 & 7.3 \\
3620 & 3.5 & 3720 & 24.2 & 4800 & 21.7 & 5700 & 1.9 \\
3650 & 2.5 & 3750 & 13.7 & 4820 & 7.6 & 5750 & 0.5 \\
3660 & 2.4 & 3780 & 10.4 & 4850 & 2.8 & 5800 & 0.2 \\
3680 & 2.1 & 3800 & 7.3 & 4900 & 0.4 & 5850 & 0.0 \\
3690 & 1.8 & 3850 & 4.4 & 4950 & 0.1 & & \\
3700 & 1.5 & 3900 & 2.6 & 5000 & 0.0 & & \\
3710 & 1.3 & 3950 & 1.5 & & & & \\
3720 & 1.0 & 4000 & 0.7 & & & & \\
3750 & 0.8 & 4050 & 0.3 & & & & \\
3780 & 0.5 & 4100 & 0.0 & & & & \\
3800 & 0.3 & & & & & & \\
3850 & 0.0 & & & & & & \\
\hline
\end{tabular}

Fig. 37. The photometric system 4 colors - Neff and Travis - 1966 


\section{Scarfe - 1966}

Square-band measurement of FeI lines in G-K stars.

\section{GENERAL INFORMATION}

AUTHORS TELESCOPE

DETECTOR

MAIN ARTICLE
C. D. Scarfe

0.90m (reflector), Cambridge Obs.

EMI 9502 (refrigerated)

Scarfe, C. D. 1966, MNRAS 133, 99

\section{SYSTEM DESCRIPTION}

\begin{tabular}{|l|cll|}
\hline \multicolumn{4}{|c|}{ BANDS DESCRIPTION [259] } \\
\hline band & bandpass $(\AA)$ & \multicolumn{1}{c|}{ feature } \\
\hline$a_{c}$ & $5289.5-5296.0$ & continuum \\
\hline$b_{c}$ & $5303.5-5321.5$ & continuum \\
\hline$c_{c}$ & $5317.0-5322.5$ & continuum \\
\hline$d_{c}$ & $5333.5-5339.0$ & continuum \\
\hline$e_{c}$ & $5350.5-5363.5$ & continuum \\
\hline$f_{l}$ & $5363.5-5369.0$ & FeI multp. 1146 (5364.9, 5367.5 $)$ \\
\hline$g_{c}$ & $5373.0-5379.0$ & continuum \\
\hline$h_{l}$ & $5382.0-5385.0$ & FeI multp. $1146(5383.4 \AA)$ \\
\hline$i_{l}$ & $5388.0-5391.0$ & FeI multp. $1145(5389.5 \AA)$ \\
\hline$j_{l}$ & $5395.8-5398.8$ & FeI multp. $15(5397.1 \AA)$ \\
\hline$k_{l}$ & $5413.5-5416.5$ & FeI multp. $1165(5415.2 \AA)$ \\
\hline$l_{l}$ & $5422.5-5425.5$ & FeI multp. $1146(5424.1 \AA)$ \\
\hline$m_{l}$ & $5428.2-5431.2$ & FeI multp. $15(5429.7 \AA)$ \\
\hline$n_{l}$ & $5433.0-5436.0$ & FeI multp. $15(5434.5 \AA)$ \\
\hline$O_{l}$ & $5445.7-5448.7$ & FeI multp. $15(5446.9 \AA)$ \\
\hline$p_{l}$ & $5462.0-5465.0$ & FeI multp. 1163 (5463.0, 5463.3 $\AA)$ \\
\hline$q_{c}$ & $5490.0-5496.0$ & continuum \\
\hline$r_{l}$ & $5496.0-5502.5$ & FeI multp. $15(5497.5,5501.6 \AA)$ \\
\hline$s_{l}$ & $5505.3-5508.3$ & FeI multp. $15(5506.8 \AA)$ \\
\hline$t_{c}$ & $5508.0-5526.0$ & continuum \\
\hline$u_{c}$ & $5532.5-5538.5$ & continuum \\
\hline$v_{c}$ & $5538.0-5568.0$ & continuum \\
\hline & & \\
\hline
\end{tabular}

On the focal plane of a $6.2 \AA / \mathrm{mm}$ dispersion spectrograph diaphragms transmit to three separate photomultipliers three portions of the spectrum (a line band and two surrounding continuum bands).

The system includes also the bands from Griffin - 1961 .

\section{SYSTEM ANALYSIS}

\section{COLOR INDICES AND PARAMETERS [259]}

If $A$ and $C$ are the continuum bands, and $B$ is the line band, then:

$R=\frac{A+C}{B}:$ measures the strength of a given FeI line.

Fig. 38. The photometric system Scarfe - 1966 


\title{
$u b V t$ - Smak - 1966
}

Broad and narrow band photometry of late-type stars.

\section{GENERAL INFORMATION}

\author{
AUTHORS J. Smak \\ TELESCOPE $\quad 0.60 \mathrm{~m}$ (reector), Lick Obs. \\ DETECTOR 1P21 (refrigerated) \\ MAIN ARTICLE Smak, J. 1966, Acta Astron. 16, 109
}

\section{SYSTEM DESCRIPTION}

\begin{tabular}{|c|c|c|c|c|c|c|}
\hline \multicolumn{5}{|c|}{ BANDS DESCRIPTION [271] } & \multirow[b]{2}{*}{$W H M(A)$} & \multirow[b]{2}{*}{$\lambda_{c}(\mathrm{~A})$} \\
\hline band & lter & $\lambda_{c}(\mathrm{~A})$ & width (A) & $\Upsilon_{c}(\%)$ & & \\
\hline$u$ & 2mm UG11 & & & & $\sim 1000$ & $\sim 3275$ \\
\hline$b$ & 2mm GG5 + 2mm Corning 5030 & & & & 700 & 4790 \\
\hline 490 & Bausch \& Lomb 33-78-51 (interf.) & 5100 & 80 & 35 & & \\
\hline 500 & Bausch \& Lomb 33-78-50 (interf.) & 5000 & 80 & 35 & & \\
\hline 510 & Bausch \& Lomb 33-78-49 (interf.) & 4900 & 80 & 35 & & \\
\hline$V$ & 2mm GG11 & & & & 870 & 5465 \\
\hline
\end{tabular}

The $V$ band is identical to the corresponding band of the 1953 - Johnson and Morgan - UBV system [271].

\section{SYSTEM ANALYSIS}

\section{COLOR INDICES AND PARAMETERS [271]}

$t=0.5[510+490]-500:$ measures the strength of the TiO absorption band at 4955A.

$T=9.25-21.67 t$ : gives the spectral type for $\mathrm{M}$ giants $(T=0$ at $\mathrm{M} 0, T=8$ at M8)

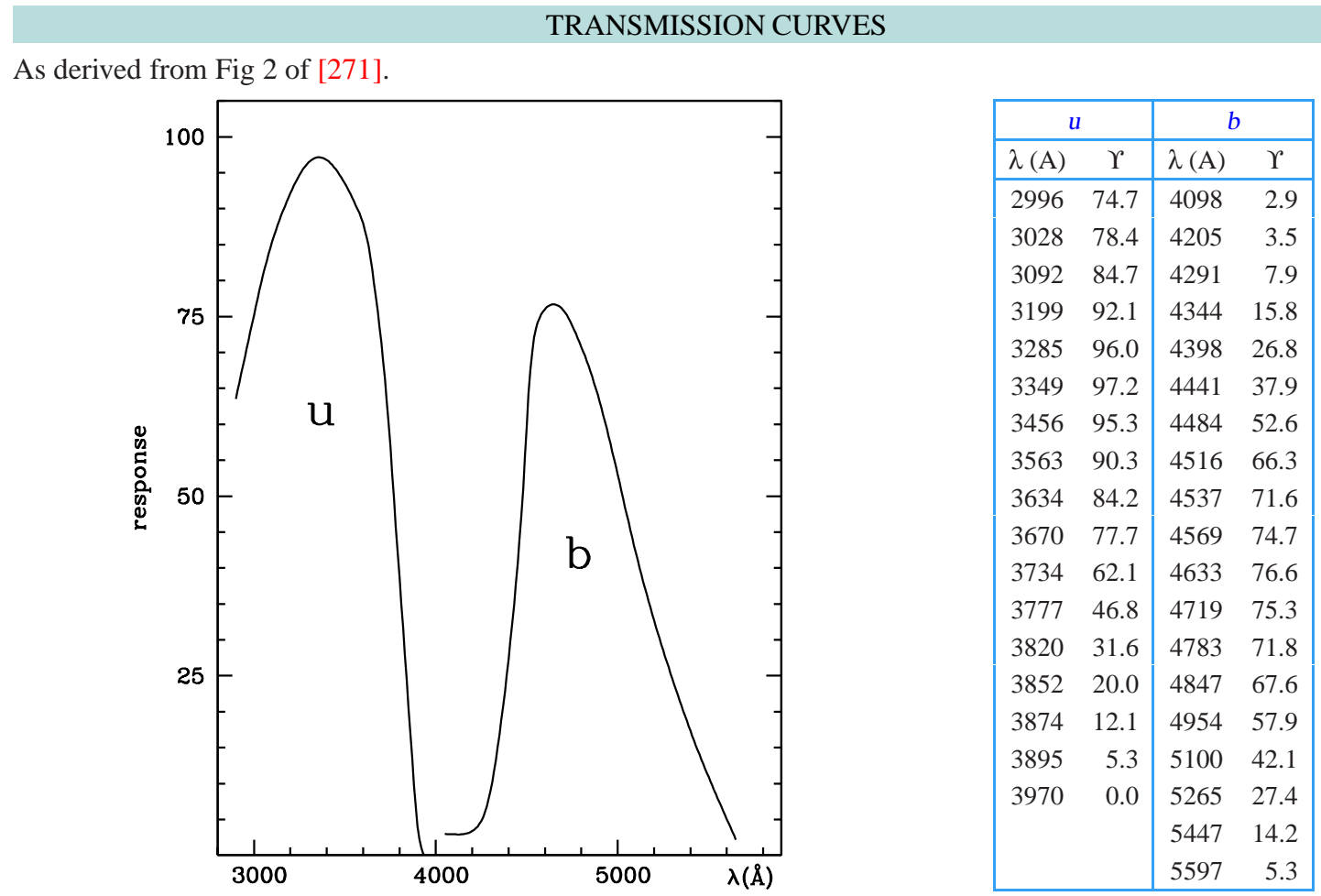

Fig. 39. The photometric system $u b V t-$ Smak -1966 


\section{ubvr - Westerlund - 1966}

Narrow band measurements of the continuum in Wolf-Rayet stars.

\section{GENERAL INFORMATION}

AUTHORS

TELESCOPE

DETECTOR

MAIN ARTICLE
B. E. Westerlund

$0.91 \mathrm{~m}$ (reflector), Kitt Peak National Observatory

1P21 (refrigerated)

Westerlund, B. E. 1966, ApJ 145, 724

\section{SYSTEM DESCRIPTION}

\begin{tabular}{|c|lcc|}
\hline \multicolumn{4}{|c|}{ BANDS DESCRIPTION $[320]$} \\
\hline band & $\lambda_{\text {eff }}(\AA)$ & FWHM $(\AA)$ & $\Upsilon_{\text {peak }}(\%)$ \\
\hline$u$ & 3650 & 100 & 52 \\
\hline$b$ & 4280 & 65 & 70 \\
\hline$V$ & 5190 & 100 & 70 \\
\hline$r$ & 5990 & 80 & 67 \\
\hline
\end{tabular}

Interference filters by Baird Atomic.

\section{SYSTEM ANALYSIS}

\section{REDDENING-FREE PARAMETERS [320]}

$\Delta=u-b-0.68(b-v)$ : intensity of the Balmer discontinuity.

$\delta=b-v-1.43(v-r)$ : deviation of the continuum at $b$ relative to an extrapolation from the $v, r$ bands.

Fig. 40. The photometric system ubvr - Westerlund - 1966 


\section{2 colors - Wood - 1966}

Wide wavelength range photometric system for studies of galaxies.

\section{GENERAL INFORMATION}
AUTHORS
D. B. Wood
TELESCOPE
$0.91 \mathrm{~m}$ and $0.40 \mathrm{~m}$ (reflectors), Kitt Peak National Observatory
DETECTOR
EMI 9558A tri-alkali
MAIN ARTICLE Wood, D. B. 1966, ApJ 145, 36

\section{SYSTEM DESCRIPTION}

\begin{tabular}{|c|c|c|c|c|c|c|}
\hline \multicolumn{7}{|c|}{ BANDS DESCRIPTION [332] } \\
\hline & \multicolumn{3}{|c|}{ only filter } & \multicolumn{2}{|c|}{ filter plus photomultiplier } & \\
\hline band & $\lambda_{\text {peak }}$ & half-width & $\Upsilon_{\text {peak }}(\%)$ & $\lambda_{\text {peak }}$ & $\Upsilon_{\text {peak }}(\%)$ & spectral feature \\
\hline$u$ & 3435 & 375 & 37 & 3459 & 22 & Balmer jump \\
\hline V & 4100 & 195 & 46 & 4102 & 33 & metals \\
\hline$b$ & 4675 & 180 & 46 & 4667 & 42 & continuum \\
\hline$g$ & 5165 & 56 & 75 & 5169 & 56 & $\mathrm{Mg}, \mathrm{MgH}$ \\
\hline$y$ & 5510 & 233 & 52 & 5470 & 33 & continuum \\
\hline a & 5894 & 64 & 64 & 5901 & 33 & $\mathrm{Na} \mathrm{D}, \mathrm{TiO}$ \\
\hline $\mathrm{O}$ & 6060 & 145 & 83 & 6029 & 38 & continuum \\
\hline$t_{1}$ & 6220 & 79 & 78 & 6220 & 31 & $\mathrm{TiO}$ \\
\hline$h$ & 6568 & 65 & 83 & 6571 & 22 & $\mathrm{H} \alpha$ \\
\hline$r$ & 6706 & 155 & 85 & 6696 & 19 & continuum \\
\hline$t_{2}$ & 7123 & 96 & 81 & 7118 & 8 & $\mathrm{TiO}$ \\
\hline$i$ & 7338 & 100 & 84 & 7331 & 5 & continuum \\
\hline
\end{tabular}

\section{SYSTEM ANALYSIS}

\section{COLOR INDICES AND PARAMETERS [332]}

Denoting with $\langle M\rangle=\frac{M_{b}+M_{y}+M_{o}+M_{r}}{4}$, color indices for the bands covering continuum portion of the spectrum are obtained as:

$$
\begin{array}{llll}
\mathrm{C}_{35}=M_{u}-<M> & C_{41}=M_{v}-<M> & C_{47}=M_{b}-<M> & C_{52}=M_{g}-<M> \\
\mathrm{C}_{55}=M_{y}-<M> & C_{59}=M_{a}-<M> & C_{60}=M_{o}-<M> & C_{62}=M_{t 1}-<M> \\
\mathrm{C}_{66}=M_{h}-<M> & C_{67}=M_{r}-<M> & C_{71}=M_{t 2}-<M> & C_{73}=M_{i}-<M>
\end{array}
$$

$I_{41}=\left(C_{41}-C_{47}\right)-\left(C_{47}-C_{55}\right):$ analogous to Strömgren's $m_{1}$ index. Measures metallic line strength.

$I_{35}=\left(C_{35}-C_{41}\right)-\left(C_{41}-C_{47}\right):$ analogous to Strömgren's $c_{1}$ index. Measures Balmer discontinuity.

Fig. 41. The photometric system 12 colors - Wood - 1966 
Line indices are obtained by subtraction of mean continuum indices as:

$$
\begin{array}{ll}
L_{52}=C_{52}-\frac{1}{2}\left(C_{47}+C_{55}\right) & L_{59}=C_{59}-\frac{1}{2}\left(C_{55}+C_{60}\right) \\
\mathrm{L}_{66}=C_{66}-\frac{1}{2}\left(C_{60}+C_{67}\right) & L_{71}=C_{71}-\frac{1}{2}\left(C_{67}+C_{73}\right)
\end{array}
$$

REDDENING RATIOS [332]

$E\left(C_{47}-C_{55}\right)=0.74 E(B-V)$

RELATIONS WITH OTHER SYSTEMS [332]

UVBGRI - Stebbin and Whitford - 1943

\begin{tabular}{|lll|}
\hline$U_{S t-W}=-1.87+1.00 C_{35}$ & \\
\hline$V_{S t-W}=-0.67+0.77 C_{41}$ & \\
\hline$R_{S t-W}=0.30+0.92 C_{73}$ & for $C_{73} \geq-0.78$ \\
\hline$R_{S t-W}$ & $=-0.05+0.47 C_{73}$ & for $C_{73}<-0.78$ \\
\hline
\end{tabular}

UBV - Johnson and Morgan - 1953

\begin{tabular}{|lll|}
\hline$(U-B)=-0.92+0.73\left(C_{35}-C_{47}\right)$ & for $C_{35}-C_{47} \leq 3.60$ \\
\hline$(U-B)=-0.30+0.56\left(C_{35}-C_{47}\right)$ & for $C_{35}-C_{47}>3.60$ \\
\hline$(B-V)=0.16+1.66\left(C_{47}-C_{55}\right)$ & for $C_{47}-C_{55} \leq 0.75$ \\
\hline$(B-V)=0.61+1.06\left(C_{47}-C_{55}\right)$ & for $C_{47}-C_{55}>0.75$ \\
\hline
\end{tabular}

\begin{tabular}{|rc|}
\hline$(b-y)$ & $=0.116( \pm 0.002)+0.979( \pm 0.005)\left(C_{47}-C_{55}\right)$ \\
\hline$m_{1}$ & $=0.076( \pm 0.003)+1.052( \pm 0.016) I_{41}$ \\
\hline$c_{1}=-0.253( \pm 0.010)+0.998( \pm 0.011) I_{35}$ \\
\hline
\end{tabular}

Fig. 41. continued 


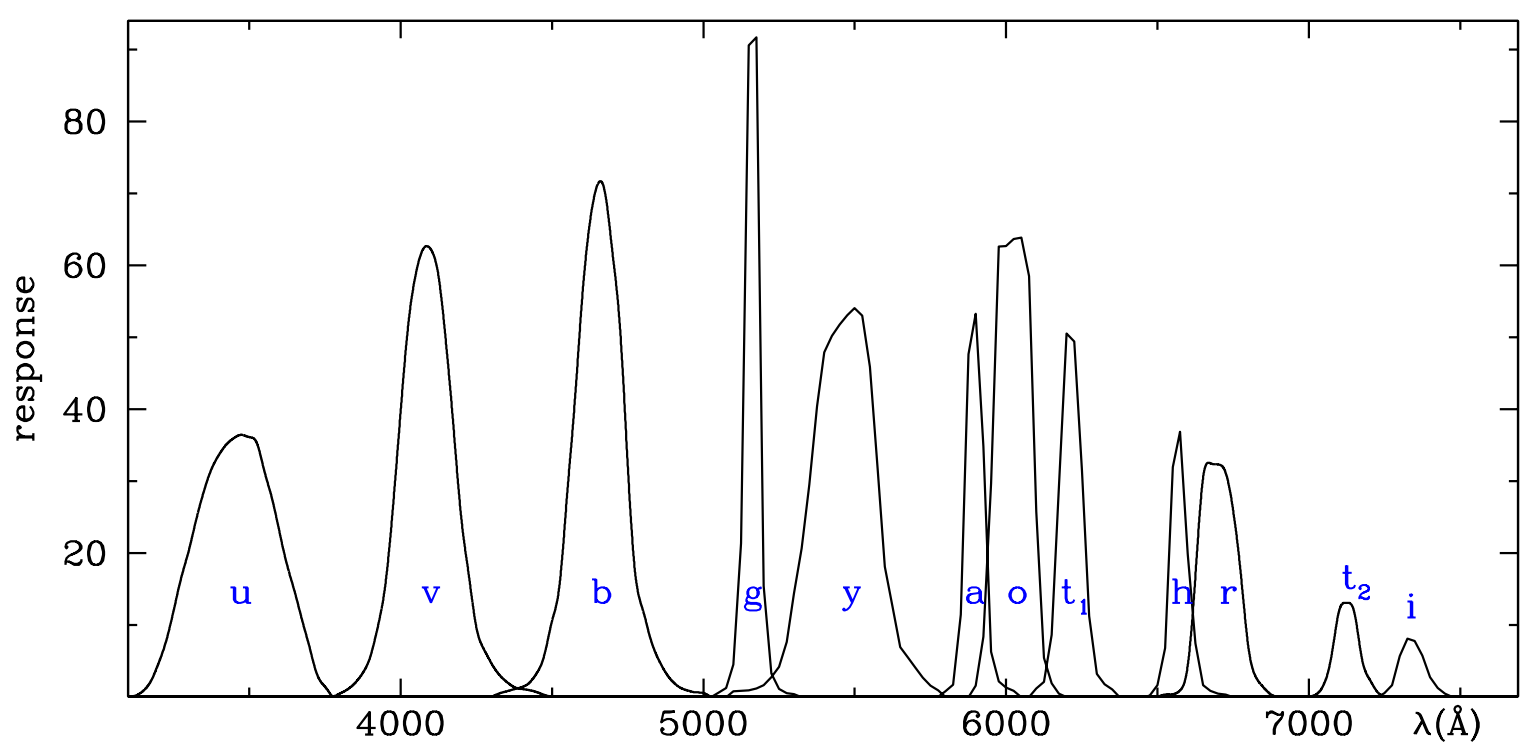

\begin{tabular}{|c|c|c|c|c|c|c|c|c|c|c|c|c|c|c|c|}
\hline \multicolumn{4}{|c|}{$u$} & \multicolumn{4}{|c|}{$V$} & \multicolumn{4}{|c|}{$b$} & \multicolumn{2}{|c|}{$g$} & \multicolumn{2}{|c|}{$y$} \\
\hline$\lambda(\mathrm{A})$ & $\Upsilon(\%)$ & $\lambda(\mathrm{A})$ & $\Upsilon(\%)$ & $\lambda(\mathrm{A})$ & $\Upsilon(\%)$ & $\lambda(\mathrm{A})$ & $\Upsilon(\%)$ & $\lambda(\mathrm{A})$ & $\Upsilon(\%)$ & $\lambda(\mathrm{A})$ & $\Upsilon(\%)$ & $\lambda(\mathrm{A})$ & $\Upsilon(\%)$ & $\lambda(\mathrm{A})$ & $\Upsilon(\%)$ \\
\hline 3100 & 0.000 & 3475 & 36.457 & 3775 & 0.000 & 4150 & 49.340 & 4300 & 0.000 & 4675 & 70.217 & 5025 & 0.000 & 5075 & 0.000 \\
\hline 3125 & 0.207 & 3500 & 36.137 & 3800 & 0.498 & 4175 & 37.537 & 4325 & 0.483 & 4700 & 61.802 & 5050 & 0.595 & 5100 & 0.815 \\
\hline 3150 & 0.849 & 3525 & 35.349 & 3825 & 1.064 & 4200 & 25.169 & 4350 & 0.793 & 4725 & 51.273 & 5075 & 1.279 & 5125 & 0.842 \\
\hline 3175 & 2.177 & 3550 & 31.461 & 3850 & 1.975 & 4225 & 17.129 & 4375 & 1.088 & 4750 & 32.614 & 5100 & 4.500 & 5150 & 0.923 \\
\hline 3200 & 4.612 & 3575 & 27.696 & 3875 & 3.323 & 4250 & 9.797 & 4400 & 1.369 & 4775 & 17.260 & 5125 & 21.373 & 5175 & 1.229 \\
\hline 3225 & 7.664 & 3600 & 22.992 & 3900 & 5.588 & 4275 & 6.681 & 4425 & 1.970 & 4800 & 12.184 & 5150 & 90.577 & 5200 & 1.679 \\
\hline 3250 & 11.891 & 3625 & 18.412 & 3925 & 9.782 & 4300 & 4.707 & 4450 & 3.336 & 4825 & 8.190 & 5175 & 91.705 & 5225 & 2.778 \\
\hline 3275 & 16.463 & 3650 & 14.706 & 3950 & 15.666 & 4325 & 3.158 & 4475 & 5.832 & 4850 & 5.431 & 5200 & 15.454 & 5250 & 4.185 \\
\hline 3300 & 20.246 & 3675 & 10.697 & 3975 & 26.387 & 4350 & 2.135 & 4500 & 10.514 & 4875 & 3.454 & 5225 & 3.278 & 5275 & 7.640 \\
\hline 3325 & 24.631 & 3700 & 6.847 & 4000 & 39.493 & 4375 & 1.392 & 4525 & 15.528 & 4900 & 2.104 & 5250 & 1.145 & 5300 & 14.395 \\
\hline 3350 & 28.530 & 3725 & 3.080 & 4025 & 51.534 & 4400 & 1.129 & 4550 & 28.003 & 4925 & 1.287 & 5275 & 0.525 & 5325 & 20.669 \\
\hline 3375 & 31.623 & 3750 & 1.471 & 4050 & 58.712 & 4425 & 0.967 & 4575 & 40.749 & 4950 & 0.885 & 5300 & 0.423 & 5350 & 29.550 \\
\hline 3400 & 33.646 & 3775 & 0.000 & 4075 & 62.296 & 4450 & 0.683 & 4600 & 55.460 & 4975 & 0.657 & 5325 & 0.000 & 5375 & 40.389 \\
\hline 3425 & 35.120 & & & 4100 & 62.252 & 4475 & 0.297 & 4625 & 65.834 & 5000 & 0.564 & & & 5400 & 47.936 \\
\hline 3450 & 36.013 & & & 4125 & 58.637 & 4500 & 0.000 & 4650 & 71.149 & 5025 & 0.000 & & & 5425 & 50.230 \\
\hline
\end{tabular}

\begin{tabular}{|c|c|c|c|c|c|c|c|c|c|c|c|c|c|c|c|}
\hline \multicolumn{2}{|c|}{$y$} & \multicolumn{2}{|c|}{$a$} & \multicolumn{2}{|c|}{$O$} & \multicolumn{2}{|c|}{$t_{1}$} & \multicolumn{2}{|c|}{$h$} & \multicolumn{2}{|c|}{$r$} & \multicolumn{2}{|c|}{$t_{2}$} & \multicolumn{2}{|c|}{1} \\
\hline$\lambda(\mathrm{A})$ & $\Upsilon(\%)$ & $\lambda(\mathrm{A})$ & $\Upsilon(\%)$ & $\lambda(\mathrm{A})$ & $\Upsilon(\%)$ & $\lambda(\mathrm{A})$ & $\Upsilon(\%)$ & $\lambda(\mathrm{A})$ & $\Upsilon(\%)$ & $\lambda(\mathrm{A})$ & $\Upsilon(\%)$ & $\lambda(\mathrm{A})$ & $\Upsilon(\%)$ & $\lambda(\mathrm{A})$ & $\Upsilon(\%)$ \\
\hline 5450 & 51.753 & 5775 & 0.000 & 5875 & 0.000 & 6075 & 0.000 & 6450 & 0.000 & 6500 & 0.000 & 6975 & 0.000 & 7225 & 0.000 \\
\hline 5475 & 53.052 & 5800 & 0.775 & 5900 & 1.564 & 6100 & 1.179 & 6475 & 0.184 & 6525 & 0.360 & 7000 & 0.063 & 7250 & 0.542 \\
\hline 5500 & 54.093 & 5825 & 1.714 & 5925 & 8.377 & 6125 & 2.134 & 6500 & 1.651 & 6550 & 0.393 & 7025 & 0.391 & 7275 & 1.625 \\
\hline 5525 & 53.045 & 5850 & 11.486 & 5950 & 29.715 & 6150 & 8.654 & 6525 & 6.833 & 6575 & 0.943 & 7050 & 1.827 & 7300 & 5.675 \\
\hline 5550 & 45.888 & 5875 & 47.646 & 5975 & 62.626 & 6175 & 29.071 & 6550 & 31.954 & 6600 & 4.402 & 7075 & 5.814 & 7325 & 8.090 \\
\hline 5575 & 31.756 & 5900 & 53.257 & 6000 & 62.715 & 6200 & 50.514 & 6575 & 36.876 & 6625 & 16.671 & 7100 & 12.007 & 7350 & 7.823 \\
\hline 5600 & 18.145 & 5925 & 34.845 & 6025 & 63.678 & 6225 & 49.420 & 6600 & 19.873 & 6650 & 30.292 & 7125 & 13.094 & 7375 & 5.791 \\
\hline 5625 & 12.584 & 5950 & 6.274 & 6050 & 63.889 & 6250 & 31.530 & 6625 & 7.499 & 6675 & 32.488 & 7150 & 11.782 & 7400 & 2.754 \\
\hline 5650 & 6.945 & 5975 & 2.156 & 6075 & 58.496 & 6275 & 11.236 & 6650 & 1.631 & 6700 & 32.337 & 7175 & 5.375 & 7425 & 1.272 \\
\hline 5675 & 5.537 & 6000 & 1.306 & 6100 & 25.781 & 6300 & 3.194 & 6675 & 0.839 & 6725 & 31.199 & 7200 & 2.501 & 7450 & 0.457 \\
\hline 5700 & 4.137 & 6025 & 0.874 & 6125 & 5.431 & 6325 & 1.764 & 6700 & 0.492 & 6750 & 25.900 & 7225 & 0.776 & 7475 & 0.000 \\
\hline 5725 & 2.686 & 6050 & 0.000 & 6150 & 1.934 & 6350 & 1.084 & 6725 & 0.359 & 6775 & 17.212 & 7250 & 0.000 & & \\
\hline 5750 & 1.599 & & & 6175 & 0.497 & 6375 & 0.000 & 6750 & 0.000 & 6800 & 6.994 & & & & \\
\hline 5775 & 0.901 & & & 6200 & 0.000 & & & & & 6825 & 2.773 & & & & \\
\hline 5800 & 0.000 & & & & & & & & & 6850 & 1.353 & & & & \\
\hline & & & & & & & & & & 6875 & 0.345 & & & & \\
\hline & & & & & & & & & & 6900 & 0.000 & & & & \\
\hline
\end{tabular}

Fig. 41. continued 


\section{ri - Argue - 1967}

General photometry of F-G-K stars.

\section{GENERAL INFORMATION}

$\begin{array}{ll}\text { AUTHORS } & \text { A. N. Argue } \\ \text { TELESCOPE } & 0.40 \mathrm{~m} \text { (reflector), Kitt Peak National Observatory } \\ \text { DETECTOR } & \text { RCA 7102 (refrigerated) } \\ \text { MAIN ARTICLE } & \text { Argue A.N. 1967, MNRAS 135, } 23\end{array}$

SYSTEM DESCRIPTION

\begin{tabular}{|l|l|cr|}
\hline \multicolumn{3}{|c|}{ BANDS DESCRIPTION [11] } & \\
\hline band & \multicolumn{1}{|c|}{ filter } & WHM $(\AA)$ & $\lambda_{c}(\AA)$ \\
\hline$r$ & multilayer dielectric & 1400 & 6800 \\
\hline$r 8$ & 2mm Schott RG8 & 2700 & 8100 \\
\hline$r 9$ & 2mm Schott RG9 & 1900 & 8200 \\
\hline$i$ & interference & 300 & 10200 \\
\hline
\end{tabular}

\section{SYSTEM ANALYSIS}

RELATIONS WITH OTHER SYSTEMS [11]

UBVRI(JHKLMN) - Johnson - 1965

$(R-I)=0.7411(r-i)-2.373$

Fig. 42. The photometric system ri - Argue - 1967 
TRANSMISSION CURVES

As derived from Fig 1 of [11]

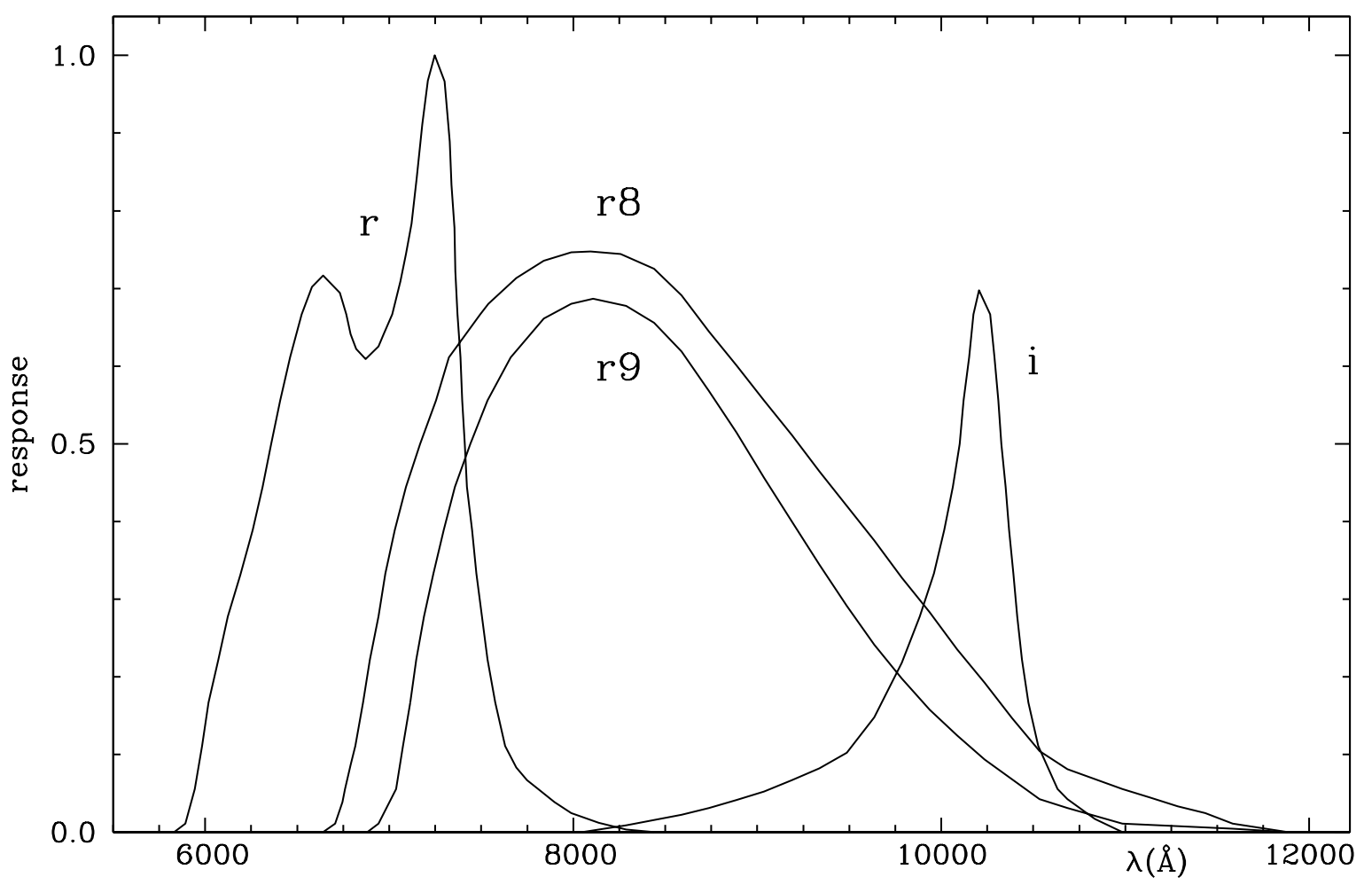

\begin{tabular}{|c|c|c|c|c|c|c|c|c|c|c|c|c|c|c|c|}
\hline \multicolumn{4}{|c|}{$r$} & \multicolumn{4}{|c|}{ r8 } & \multicolumn{4}{|c|}{ r9 } & \\
\hline$\lambda(\AA)$ & $\Upsilon$ & $\lambda(\AA)$ & $\Upsilon$ & $\lambda(\AA)$ & $\Upsilon$ & $\lambda(\AA)$ & $\Upsilon$ & $\lambda(\AA)$ & $\Upsilon$ & $\lambda(\AA)$ & $\Upsilon$ & $\lambda(\AA)$ & $\Upsilon$ & $\lambda(\AA)$ & $\Upsilon$ \\
\hline 5850 & 0.0008 & 7000 & 0.6567 & 6680 & 0.0032 & 9210 & 0.5041 & 6790 & 0.0000 & 9320 & 0.3507 & 8090 & 0.0015 & 9850 & 0.2568 \\
\hline 5900 & 0.0155 & 7050 & 0.6975 & 6790 & 0.0859 & 9320 & 0.4697 & 6900 & 0.0039 & 9430 & 0.3111 & 8170 & 0.0044 & 9890 & 0.2829 \\
\hline 5950 & 0.0643 & 7100 & 0.7558 & 6900 & 0.2278 & 9430 & 0.4366 & 7010 & 0.0316 & 9540 & 0.2727 & 8250 & 0.0074 & 9930 & 0.3104 \\
\hline 6000 & 0.1401 & 7150 & 0.8416 & 7010 & 0.3686 & 9540 & 0.4045 & 7120 & 0.1759 & 9650 & 0.2370 & 8330 & 0.0105 & 9970 & 0.3416 \\
\hline 6050 & 0.2013 & 7200 & 0.9508 & 7120 & 0.4678 & 9650 & 0.3713 & 7230 & 0.3228 & 9760 & 0.2051 & 8410 & 0.0138 & 10010 & 0.3808 \\
\hline 6100 & 0.2532 & 7250 & 1.0000 & 7230 & 0.5377 & 9760 & 0.3360 & 7340 & 0.4298 & 9870 & 0.1748 & 8490 & 0.0173 & 10050 & 0.4306 \\
\hline 6150 & 0.3010 & 7300 & 0.9667 & 7340 & 0.6212 & 9870 & 0.3030 & 7450 & 0.5056 & 9980 & 0.1475 & 8570 & 0.0213 & 10090 & 0.4784 \\
\hline 6200 & 0.3375 & 7350 & 0.8008 & 7450 & 0.6567 & 9980 & 0.2694 & 7560 & 0.5693 & 10090 & 0.1236 & 8650 & 0.0257 & 10130 & 0.5725 \\
\hline 6250 & 0.3816 & 7400 & 0.5371 & 7560 & 0.6858 & 10090 & 0.2343 & 7670 & 0.6149 & 10200 & 0.1002 & 8730 & 0.0306 & 10170 & 0.6544 \\
\hline 6300 & 0.4319 & 7450 & 0.3886 & 7670 & 0.7099 & 10200 & 0.2025 & 7780 & 0.6481 & 10310 & 0.0805 & 8810 & 0.0358 & 10210 & 0.6996 \\
\hline 6350 & 0.4888 & 7500 & 0.2843 & 7780 & 0.7279 & 10310 & 0.1700 & 7890 & 0.6694 & 10420 & 0.0614 & 8890 & 0.0413 & 10250 & 0.6897 \\
\hline 6400 & 0.5473 & 7550 & 0.1977 & 7890 & 0.7407 & 10420 & 0.1360 & 8000 & 0.6809 & 10530 & 0.0429 & 8970 & 0.0469 & 10290 & 0.6101 \\
\hline 6450 & 0.6000 & 7600 & 0.1391 & 8000 & 0.7470 & 10530 & 0.1056 & 8110 & 0.6867 & 10640 & 0.0336 & 9050 & 0.0533 & 10330 & 0.4873 \\
\hline 6500 & 0.6468 & 7650 & 0.0982 & 8110 & 0.7477 & 10 & 0.0865 & 8220 & 0.6837 & $10^{\circ}$ & 0.0268 & 9130 & 0.0608 & 10370 & 0.3819 \\
\hline 6550 & 0.6851 & 7700 & 0.0801 & 8220 & 0.7460 & 10750 & 0.0743 & 8330 & 0.6729 & 10860 & 0.0187 & 9210 & 0.0690 & 10410 & 0.2839 \\
\hline 6600 & 0.7094 & 7800 & 0.0571 & 8330 & 0.7395 & 10860 & 0.0648 & 8440 & 0.6552 & 10970 & 0.0118 & 9290 & 0.0774 & 10450 & 0.2030 \\
\hline 6650 & 0.7169 & 7900 & 0.0387 & 8440 & 0.7253 & 10970 & 0.0566 & 8550 & 0.6298 & 11080 & 0.0084 & 9370 & 0.0855 & 10530 & 0.1083 \\
\hline 6700 & 0.7085 & 8000 & 0.0231 & 8550 & 0.7015 & 11190 & 0.0401 & 8660 & 0.5953 & 11410 & 0.0069 & 9450 & 0.0956 & 10610 & 0.0631 \\
\hline 6750 & 0.6818 & 8100 & 0.0147 & 8660 & 0.6691 & 11300 & 0.0324 & 8770 & 0.5565 & 11630 & 0.0037 & 9490 & 0.1029 & 10690 & 0.0412 \\
\hline 6800 & 0.6337 & 8200 & 0.0083 & 8770 & 0.6349 & 11520 & 0.0168 & 8880 & 0.5173 & 11850 & 0.0004 & 9570 & 0.1243 & 10770 & 0.0264 \\
\hline 6850 & 0.6121 & 8300 & 0.0029 & 8880 & 0.6034 & 11630 & 0.0079 & 8990 & 0.4751 & & & 9650 & 0.1530 & 10850 & 0.0147 \\
\hline 6900 & 0.6113 & & & 8990 & 0.5700 & 11740 & 0.0030 & 9100 & 0.4328 & & & 9730 & 0.1881 & 10930 & 0.0056 \\
\hline 6950 & 0.6303 & & & 9100 & 0.5370 & & & 9210 & 0.3915 & & & 9810 & 0.2317 & & \\
\hline
\end{tabular}

Fig. 42. continued 


\title{
Boyce et al. - 1967
}

Classification of early-type $\mathrm{M}$ giants via TiO bands.

\section{GENERAL INFORMATION}

\author{
AUTHORS P. B. Boyce, E. H. Olsen and B. E. Helt \\ TELESCOPE $\quad 1.82 \mathrm{~m}$ Perkins (reflector), Lowell Observatory \\ DETECTOR EMR 514R (refrigerated) \\ MAIN ARTICLE $\quad$ Boyce, P. B., Olsen, E. H., Helt, B. E. 1967, PASP 79, 473
}

\section{SYSTEM DESCRIPTION}

Spectral scans (exit slit of $20 \AA$ ) over the 4430-5575 and 6900-7400 $\AA$ spectral intervals are used to synthesize square band filters of $150 \AA$ width centered at 4510, 4970, 5500 and 6975, 7140, $7315 \AA$. [49]

\section{SYSTEM ANALYSIS}

\section{COLOR INDICES AND PARAMETERS [49]}

$m_{5}=[m(6975)-m(7140)]-[m(7140)-m(7315)]:$ spectral type index.

$m_{4}=[m(4510)-m(4970)]-[m(4970)-m(5500)]:$ luminosity class index.

$S=-2.97-26.74 m_{5}-6.03 m_{5}^{2}$ : spectral type in units of 0.25 of a subtype starting with $S=0$ at K5.

Fig. 43. The photometric system Boyce -1967 


\section{2 - Eggen - 1967}

Narrow-band photometry for K-M giants. TiO intensity.

\section{GENERAL INFORMATION}

$\begin{array}{ll}\text { AUTHORS } & \text { O. J. Eggen } \\ \text { TELESCOPE } & \text { 0.50m and 1.52m (reflectors), Mount Wilson Obs. } \\ \text { DETECTOR } & \text { RCA 7102 } \\ \text { MAIN ARTICLE } & \text { Eggen, O 1967, ApJS 14, } 307\end{array}$

SYSTEM DESCRIPTION

\begin{tabular}{|l|rl|cr|}
\hline \multicolumn{3}{|l|}{ BANDS DESCRIPTION [93] } & & \\
\hline band & $\lambda_{c}(\AA)$ & feature & WHM $(\AA)$ & $\lambda_{c}(\AA)$ \\
\hline 62 & 6250 & TiO & 266 & 6235 \\
\hline 65 & 6500 & continuum & 272 & 6505 \\
\hline 102 & 10200 & continuum & 320 & 10185 \\
\hline
\end{tabular}

All filter are interference Baird Atomic type "B".

\section{SYSTEM ANALYSIS}

REDDENING RATIOS [93]

$E(102-65) / E(B-V)=1.25 \quad A(102) / E(102-65)=1.5$

where $E(B-V)$ is the color excess in the $U B V$ - Johnson and Morgan - 1953 system.

RELATIONS WITH OTHER SYSTEMS [93]

UBVRI(JHKLMN) - Johnson - 1965

$102=I+0.15$

Fig. 44. The photometric system 6265102 - Eggen - 1967 


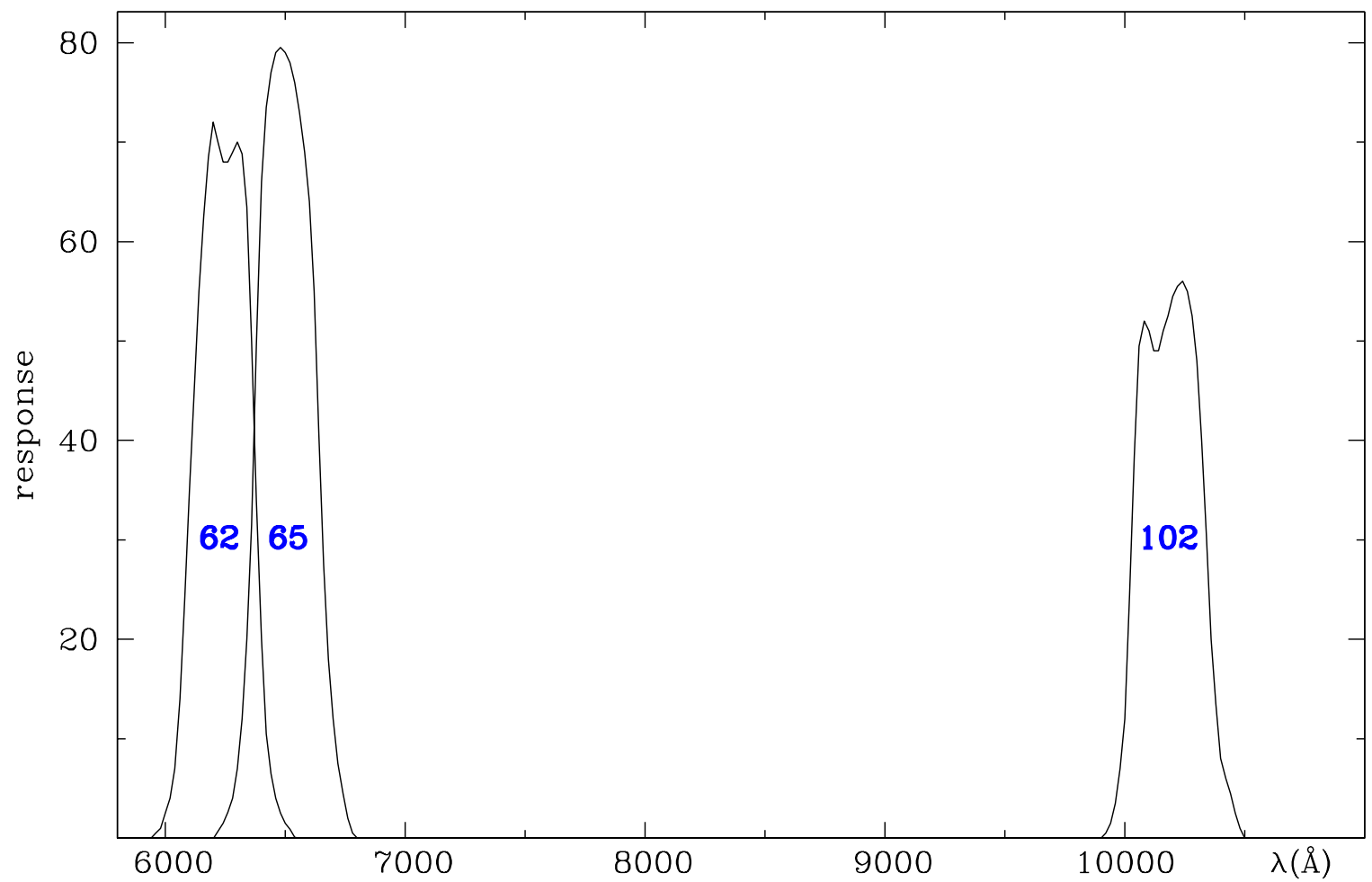

\begin{tabular}{|c|c|c|c|c|c|c|c|c|c|c|c|}
\hline \multicolumn{4}{|c|}{62} & \multicolumn{4}{|c|}{65} & \multicolumn{4}{|c|}{102} \\
\hline$\lambda(\AA)$ & $\Upsilon(\%)$ & $\lambda(\AA)$ & $\Upsilon(\%)$ & $\lambda(\AA)$ & $\Upsilon(\%)$ & $\lambda(\AA)$ & $\Upsilon(\%)$ & $\lambda(\AA)$ & $\Upsilon(\%)$ & $\lambda(\AA)$ & $\Upsilon(\%)$ \\
\hline 5940 & 0.0 & 6260 & 68.0 & 6200 & 0.0 & 6520 & 78.0 & 9900 & 0.0 & 10220 & 55.5 \\
\hline 5960 & 0.5 & 6280 & 69.0 & 6220 & 0.7 & 6540 & 76.0 & 9920 & 0.5 & 10240 & 56.0 \\
\hline 5980 & 1.0 & 6300 & 70.0 & 6240 & 1.5 & 6560 & 73.0 & 9940 & 1.5 & 10260 & 55.0 \\
\hline 6000 & 2.5 & 6320 & 68.8 & 6260 & 2.6 & 6580 & 69.0 & 9960 & 3.5 & 10280 & 52.5 \\
\hline 6020 & 4.0 & 6340 & 63.5 & 6280 & 4.0 & 6600 & 64.0 & 9980 & 7.0 & 10300 & 48.0 \\
\hline 6040 & 7.0 & 6360 & 50.0 & 6300 & 7.0 & 6620 & 55.0 & 10000 & 12.0 & 10320 & 40.0 \\
\hline 6060 & 14.0 & 6380 & 34.0 & 6320 & 12.0 & 6640 & 40.5 & 10020 & 24.5 & 10340 & 30.5 \\
\hline 6080 & 24.0 & 6400 & 20.0 & 6340 & 20.0 & 6660 & 27.5 & 10040 & 38.5 & 10360 & 20.0 \\
\hline 6100 & 35.0 & 6420 & 10.5 & 6360 & 31.5 & 6680 & 18.0 & 10060 & 49.5 & 10380 & 13.5 \\
\hline 6120 & 45.0 & 6440 & 6.5 & 6380 & 49.5 & 6700 & 12.0 & 10080 & 52.0 & 10400 & 8.0 \\
\hline 6140 & 55.0 & 6460 & 4.0 & 6400 & 66.0 & 6720 & 7.5 & 10100 & 51.0 & 10420 & 6.0 \\
\hline 6160 & 62.5 & 6480 & 2.5 & 6420 & 73.5 & 6740 & 4.5 & 10120 & 49.0 & 10440 & 4.5 \\
\hline 6180 & 68.5 & 6500 & 1.5 & 6440 & 77.0 & 6760 & 2.0 & 10140 & 49.0 & 10460 & 2.5 \\
\hline 6200 & 72.0 & 6520 & 0.9 & 6460 & 79.0 & 6780 & 0.5 & 10160 & 51.0 & 10480 & 1.0 \\
\hline 6220 & 70.0 & 6540 & 0.1 & 6480 & 79.5 & 6800 & 0.0 & 10180 & 52.5 & 10500 & 0.0 \\
\hline 6260 & 68.0 & 6560 & 0.0 & 6500 & 79.0 & & & 10200 & 54.5 & & \\
\hline
\end{tabular}

Fig. 44. continued 


\section{LPL - Johnson et al. - 1967}

General purpose system.

\section{GENERAL INFORMATION}

AUTHORS

TELESCOPE

DETECTOR

MAIN ARTICLE
H. L. Johnson, R. I. Mitchell, and A. S. Latham

$0.53 \mathrm{~m}$ and $0.71 \mathrm{~m}$, Catalina observing station of the Lun.Plan.Lab.

RCA 1P21 (S-4 cathode)

Johnson, H. L., Mitchell, R. I., Lathan, A. S. 1967, Com. L.P.L. 6, No 92, 85

\section{SYSTEM DESCRIPTION}

\begin{tabular}{|c|c|rc|cc|}
\hline \multicolumn{2}{|c|}{ BANDS DESCRIPTION [154] } & & \multicolumn{2}{|c|}{ FLUX CALIBRATION (\#) [154] } \\
\hline band & $\lambda_{0}(\mu \mathrm{m})$ & WHM $(\AA)$ & $\lambda_{c}(\AA)$ & $\left(\mathrm{W} \mathrm{cm}^{-2} \mu \mathrm{m}^{-1}\right)$ & $\left(\mathrm{W} \mathrm{m}^{-2} \mathrm{~Hz}^{-1}\right)$ \\
\hline 33 & 0.337 & 92 & 3368 & $3.3310^{-12}$ & $1.1310^{-23}$ \\
\hline 35 & 0.353 & 95 & 3563 & $3.5110^{-12}$ & $1.4610^{-23}$ \\
\hline 37 & 0.375 & 90 & 3754 & $5.0410^{-12}$ & $2.3610^{-23}$ \\
\hline 40 & 0.402 & 200 & 4064 & $7.7310^{-12}$ & $4.1710^{-23}$ \\
\hline 45 & 0.459 & 234 & 4556 & $6.3110^{-12}$ & $4.4310^{-23}$ \\
\hline 52 & 0.518 & 232 & 5193 & $4.6110^{-12}$ & $4.1310^{-23}$ \\
\hline 58 & 0.583 & 197 & 5834 & $3.3210^{-12}$ & $3.7610^{-23}$ \\
\hline 63 & 0.635 & 266 & 6300 & $2.4910^{-12}$ & $3.3510^{-23}$ \\
\hline
\end{tabular}

(\#) Fluxes for a mag. 0.0 star.

Fig. 45. The photometric system LPL - Johnson et al. - 1967 


\section{TRANSMISSION CURVES [154]}

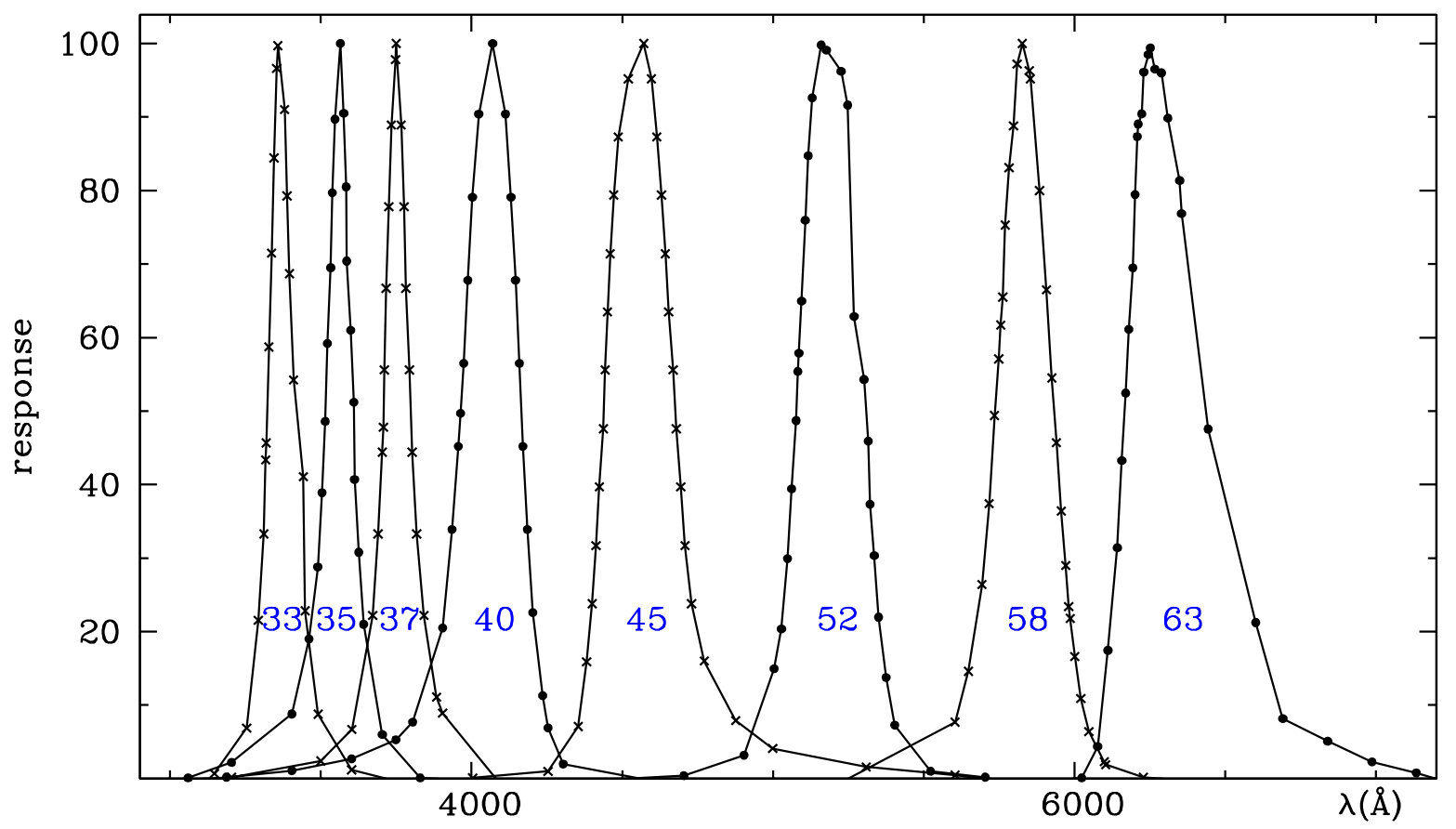

\begin{tabular}{|c|c|c|c|c|c|c|c|c|c|c|c|c|c|c|c|}
\hline \multicolumn{2}{|c|}{33} & \multicolumn{2}{|c|}{35} & \multicolumn{2}{|c|}{37} & \multicolumn{2}{|c|}{40} & \multicolumn{2}{|c|}{45} & \multicolumn{2}{|c|}{52} & \multicolumn{2}{|c|}{58} & \multicolumn{2}{|c|}{63} \\
\hline$\lambda(\mathrm{A})$ & $\Upsilon(\%)$ & $\lambda(\mathrm{A})$ & $\Upsilon(\%)$ & $\lambda(\mathrm{A})$ & $\Upsilon(\%)$ & $\lambda(\mathrm{A})$ & $\Upsilon(\%)$ & $\lambda(\mathrm{A})$ & $\Upsilon(\%)$ & $\lambda(\mathrm{A})$ & $\Upsilon(\%)$ & $\lambda(\mathrm{A})$ & $\Upsilon(\%)$ & $\lambda(\mathrm{A})$ & $\Upsilon(\%)$ \\
\hline 3157 & 0.7 & 3060 & 0.1 & 3204 & 0.2 & 3187 & 0.2 & 4004 & 0.1 & 4704 & 0.4 & 5249 & 0.0 & 6024 & 0.1 \\
\hline 3254 & 6.9 & 3204 & 2.2 & 3500 & 2.4 & 3404 & 1.1 & 4254 & 1.0 & 4904 & 3.2 & 5604 & 7.7 & 6077 & 4.4 \\
\hline 3293 & 21.5 & 3404 & 8.8 & 3603 & 6.7 & 3602 & 2.7 & 4354 & 7.1 & 5004 & 15.0 & 5648 & 14.6 & 6111 & 17.5 \\
\hline 3311 & 33.3 & 3461 & 19.0 & 3672 & 22.2 & 3749 & 5.3 & 4382 & 15.9 & 5028 & 20.4 & 5693 & 26.4 & 6142 & 31.5 \\
\hline 3317 & 43.4 & 3490 & 28.8 & 3690 & 33.3 & 3805 & 7.7 & 4400 & 23.8 & 5048 & 30.0 & 5717 & 37.4 & 6157 & 43.3 \\
\hline 3319 & 45.7 & 3504 & 38.9 & 3704 & 44.4 & 3904 & 20.5 & 4413 & 31.7 & 5062 & 39.5 & 5735 & 49.4 & 6170 & 52.5 \\
\hline 3328 & 58.7 & 3515 & 48.6 & 3708 & 47.8 & 3935 & 33.9 & 4424 & 39.7 & 5077 & 48.8 & 5749 & 57.1 & 6180 & 61.2 \\
\hline 3337 & 71.5 & 3522 & 59.2 & 3711 & 55.6 & 3956 & 45.2 & 4437 & 47.6 & 5082 & 55.5 & 5755 & 61.7 & 6194 & 69.6 \\
\hline 3345 & 84.5 & 3533 & 69.5 & 3717 & 66.7 & 3964 & 49.7 & 4443 & 55.6 & 5086 & 58.0 & 5762 & 65.5 & 6201 & 79.6 \\
\hline 3354 & 96.6 & 3538 & 79.7 & 3726 & 77.8 & 3974 & 56.5 & 4451 & 63.5 & 5095 & 65.1 & 5770 & 75.3 & 6208 & 87.5 \\
\hline 3358 & 99.7 & 3547 & 89.7 & 3734 & 88.9 & 3988 & 67.8 & 4460 & 71.4 & 5107 & 76.1 & 5783 & 83.1 & 6211 & 89.2 \\
\hline 3380 & 91.4 & 3565 & 100.0 & 3748 & 97.8 & 4003 & 79.1 & 4472 & 79.4 & 5117 & 84.9 & 5798 & 88.8 & 6223 & 90.6 \\
\hline 3388 & 79.3 & 3576 & 90.5 & 3750 & 100.0 & 4024 & 90.4 & 4487 & 87.3 & 5130 & 92.8 & 5810 & 97.2 & 6230 & 96.3 \\
\hline 3396 & 68.7 & 3584 & 80.5 & 3767 & 88.9 & 4070 & 100.0 & 4520 & 95.2 & 5160 & 99.8 & 5827 & 100.0 & 6245 & 98.7 \\
\hline 3399 & 66.9 & 3586 & 70.4 & 3776 & 77.8 & 4113 & 90.4 & 4571 & 100.0 & 5177 & 99.2 & 5850 & 96.3 & 6252 & 99.7 \\
\hline 3410 & 54.2 & 3599 & 61.0 & 3782 & 66.7 & 4131 & 79.1 & 4597 & 95.2 & 5226 & 96.3 & 5854 & 95.2 & 6267 & 96.7 \\
\hline 3422 & 41.1 & 3610 & 51.2 & 3794 & 55.6 & 4146 & 67.8 & 4615 & 87.3 & 5248 & 91.7 & 5884 & 80.0 & 6288 & 96.2 \\
\hline 3442 & 27.7 & 3612 & 40.7 & 3803 & 44.4 & 4159 & 56.5 & 4630 & 79.4 & 5269 & 81.1 & 5907 & 66.5 & 6310 & 90.0 \\
\hline 3448 & 22.8 & 3626 & 30.8 & 3818 & 33.3 & 4170 & 45.2 & 4643 & 71.4 & 5284 & 70.9 & 5925 & 54.5 & 6349 & 81.5 \\
\hline 3491 & 8.8 & 3642 & 21.0 & 3842 & 22.2 & 4185 & 33.9 & 4654 & 63.5 & 5292 & 62.5 & 5940 & 45.7 & 6355 & 77.0 \\
\hline 3602 & 1.2 & 3704 & 6.0 & 3884 & 11.1 & 4203 & 22.6 & 4669 & 55.6 & 5302 & 54.3 & 5956 & 36.4 & 6443 & 47.6 \\
\hline 3719 & 0.1 & 3830 & 0.1 & 3904 & 8.9 & 4236 & 11.3 & 4679 & 47.6 & 5316 & 46.0 & 5971 & 29.0 & 6601 & 21.2 \\
\hline & & & & 4082 & 0.0 & 4254 & 6.9 & 4694 & 39.7 & 5322 & 37.4 & 5981 & 23.4 & 6691 & 8.2 \\
\hline & & & & & & 4304 & 2.0 & 4708 & 31.7 & 5336 & 30.4 & 5986 & 21.8 & 6840 & 5.1 \\
\hline & & & & & & 4564 & 0.0 & 4730 & 23.8 & 5350 & 22.0 & 6001 & 16.6 & 6987 & 2.3 \\
\hline & & & & & & & & 4772 & 16.0 & 5375 & 13.8 & 6021 & 10.9 & 7134 & 0.8 \\
\hline & & & & & & & & 4876 & 7.9 & 5404 & 7.3 & 6048 & 6.4 & & \\
\hline & & & & & & & & 4999 & 4.1 & 5523 & 1.0 & 6099 & 2.3 & & \\
\hline & & & & & & & & 5309 & 1.6 & 5704 & 0.2 & 6104 & 1.9 & & \\
\hline & & & & & & & & 5604 & 0.5 & & & 6229 & 0.2 & & \\
\hline
\end{tabular}

Fig. 45. continued 


\section{7 colors - Wing - 1967}

Continuum energy distribution and molecular band photometry in late-type stars.

\section{GENERAL INFORMATION}
AUTHORS
R. F. Wing
TELESCOPE
0.91 Crossley (reflector), Lick Obs.
DETECTOR
RCA 7102 (refrigerated)

MAIN ARTICLE Wing, R. F. 1967, proc. Colloquium on Late-Type Stars (ed. M. Hack), pg. 205 [189]

\section{SYSTEM DESCRIPTION}

The system is defined by 27 wavelength intervals (30 A width) distributed over the $0.78-1.1 \mu \mathrm{m}$, and covering the major molecular bands in the region, namely TiO, $\mathrm{VO}, \mathrm{H}_{2} \mathrm{O}, \mathrm{ZrO}$ and $\mathrm{CN}$. The 27 bands are obtained from photoelectric scans at the Coudé spectrograph. [329]

ZERO POINT: The fluxes in all the bands are linked to the system of absolute fluxes given by [135] and the zero point of the whole system is set by requiring that the magnitude of $\alpha$ Lyr in the 10400 band is 0.00 . [329]

\section{SYSTEM ANALYSIS}

\section{COLOR INDICES AND PARAMETERS [329]}

If $C(\lambda)$ are the counts/sec received through a $30 \AA$ band centered at $\lambda$, then $m(\lambda)=-2.5 \log C(\lambda)$

$T=m(10400)-m(8834)-B+1.200:$ temperature index where

$B=m(9190)-0.339[m(9190)-m(8140)]-m(8834)$ is the blanketing correction.

TiO $=m(8834)-m(8880)+B+f(T):$ index of TiO band strength where

$f(T)=0.045 T-0.028$ is the correction to compensate for the spectral slope when combining a band and a reference continuum measured at different wavelengths.

$V O=m(10400)-0.372[m(10400)-m(10834)]-m(10564)+0.054$ : index of VO band strength.

REDDENING RATIOS [329]

$E(T)=0.33 E(B-V)_{J o h n s o n}$

Fig. 46. The photometric system 27 colors - Wing - 1967 


\section{$H \alpha$ - Andrews - 1968}

Three square-band measurement of the $H \alpha$ intensity in early type stars.

\section{GENERAL INFORMATION}

AUTHORS

TELESCOPE

DETECTOR

MAIN ARTICLE
P.J. Andrews

0.91m (reflector), Cambridge; $1.93 \mathrm{~m}$ (reflector), Pretoria

EMI 9558A

Andrews, P. J. 1968, MemRAS 72, 35

\begin{tabular}{|l|rl|}
\hline \multicolumn{4}{|c|}{ BANDS DESCRIPTION [9] } \\
\hline band & bandpass $(\AA)$ & \multicolumn{1}{c|}{ feature } \\
\hline \multicolumn{3}{|c|}{ Cambridge } \\
\hline$A$ & $6477.5-6497.0$ & $\mathrm{H} \alpha_{\text {blue cont. }}$ \\
\hline$B$ & $6546.4-6582.5$ & $\mathrm{H} \alpha$ \\
\hline$C$ & $6634.3-6653.5$ & $\mathrm{H} \alpha_{\text {red cont }}$ \\
\hline \multicolumn{3}{|c|}{ Pretoria } \\
\hline$A$ & $6469.0-6505.0$ & $\mathrm{H} \alpha_{\text {blue cont. }}$ \\
\hline$B$ & $6546.5-6582.4$ & $\mathrm{H} \alpha$ \\
\hline$C$ & $6623.8-6659.6$ & $\mathrm{H} \alpha_{\text {red cont }}$ \\
\hline
\end{tabular}

On a focal plane of a $5.0 \AA \mathrm{mm}$ dispersion spectrograph, three diaphrams transmit to separate photomultipliers the $A$, $B, C$ portions of the spectrum.

In the Cambridge setup the light from both $A$ and $C$ bands are brought onto a single photometer, whereas at Pretoria two separate photomultipliers are used.

\section{SYSTEM ANALYSIS}

\section{COLOR INDICES AND PARAMETERS [9]}

Subscript " $L$ " denotes readings on a comparison lamp.

$$
\begin{aligned}
& R_{\alpha}=\frac{(A+C) /\left(C_{L}+A_{L}\right)}{B / B_{L}}: \text { for Cambridge observations. } \\
& R_{\alpha}=\frac{1}{2} \frac{A / A_{L}+C / C_{L}}{B / B_{L}}: \text { for Pretoria observations. }
\end{aligned}
$$

RELATIONS WITH OTHER SYSTEMS [9]

$$
R_{\alpha}=0.830 \beta-1.069 \text { for } \beta>2.600
$$

Fig. 47. The photometric system $\mathrm{H} \alpha$ - Andrews - 1968 


\section{DDO - McClure and Van den Bergh - 1968}

General purpose system.

\section{GENERAL INFORMATION}

AUTHORS

TELESCOPE

R. D. McClure and S. Van den Bergh

$1.88 \mathrm{~m}$ (reflector), DDO; $0.5 \mathrm{~m}$ (reflector), Yale's Bethany obs. station

$0.41 \mathrm{~m}$ and $0.91 \mathrm{~m}$ (reflectors), KPNO; $0.41 \mathrm{~m}$ and $1.0 \mathrm{~m}$ (reflectors), CTIO

DETECTOR

1P21, EMI 9502 (refrigerated), FW 130 (refrigerated)

MAIN ARTICLE McClure R. and Van den Bergh, S. 1968, AJ 73, 313

\section{SYSTEM DESCRIPTION}

\begin{tabular}{|l|lcccc|}
\hline \multicolumn{7}{|c|}{ BANDS DESCRIPTION [201] } \\
\hline band & filter & $\lambda_{\text {peak }}(\AA)$ & FWHM $(\AA)$ & $\lambda_{\text {eff }}(\AA)(\#)$ & FWHM $(\AA)(\#)$ \\
\hline 35 & 8mm Schott UG11 + 1mm Schott WG345 & 3460 & 383 & 3531 & 280 \\
\hline 38 & 5mm Corning 7-51 + 2mm Schott GG375 & 3815 & 330 & 3816 & 287 \\
\hline 41 & interference & $4166 \pm 2.5$ & $83 \pm 5$ & 4164 & 76 \\
\hline 42 & interference & $4257 \pm 2.5$ & $73 \pm 5$ & 4258 & 79 \\
\hline 45 & interference & $4517 \pm 2.5$ & $76 \pm 5$ & 4521 & 68 \\
\hline 48 & interference & $4886 \pm 2.5$ & $186 \pm 10$ & 4880 \\
\hline \multicolumn{5}{|c|}{$\lambda_{c}(\AA)$} & FWHM $(\AA)$ \\
\hline band & BANDS DESCRIPTION $[69]$ \\
\hline 51 & 5130 & 154 \\
\hline
\end{tabular}

(\#) As realized at the South Africal Astron. Obs. [77]

\section{SYSTEM ANALYSIS}

COLOR INDICES AND PARAMETERS [203]

$C(35-38)=2.5 \log [I(38) / I(35)]:$ measures the Balmer discontinuity.

$C(38-41)=2.5 \log [I(41) / I(38)]$ : measures the line-blanketing discontinuity near $4000 \AA$.

$C(41-42)=2.5 \log [I(42) / I(41)]:$ measures the $\mathrm{CN}$ intensity.

$C(42-45)=2.5 \log [I(45) / I(42)]:$ measures the strength of the G-band break.

$C(48-51)=2.5 \log [I(51) / I(48)]:$ measures the $\mathrm{MgH}+\mathrm{Mg} b$ complex.

\section{REDDENING-FREE PARAMETERS [203]}

Obtained by adding $(B-V)$ from $U B V-$ Johnson and Morgan - 1953:

$$
\begin{array}{ll}
C^{*}(35-38)=C(35-38)-0.306(B-V) & C^{*}(38-41)=C(38-41)-0.318(B-V) \\
C^{*}(41-42)=C(41-42)-0.066(B-V) & C^{*}(42-45)=C(42-45)-0.234(B-V)
\end{array}
$$

REDDENING RATIOS [202]

$$
\begin{aligned}
& E[C(35-38)]=0.32 E(B-V)_{J o h n s o n} \\
& E[C(38-41)]=0.33 E(B-V)_{J o h n s o n} \\
& E[C(41-42)]=0.07 E(B-V)_{J o h n s o n} \\
& E[C(42-45)]=0.23 E(B-V)_{J o h n s o n} \\
& E[C(45-48)]=0.31 E(B-V)_{J o h n s o n} \\
& E[C(48-51)]=0.22 E(B-V)_{J o h n s o n}
\end{aligned}
$$

Fig. 48. The photometric system DDO - McClure and Van den Bergh - 1968 


\section{TRANSMISSION CURVES}

As from the original paper [201]. Transmission curve for the 51 band is from [69]

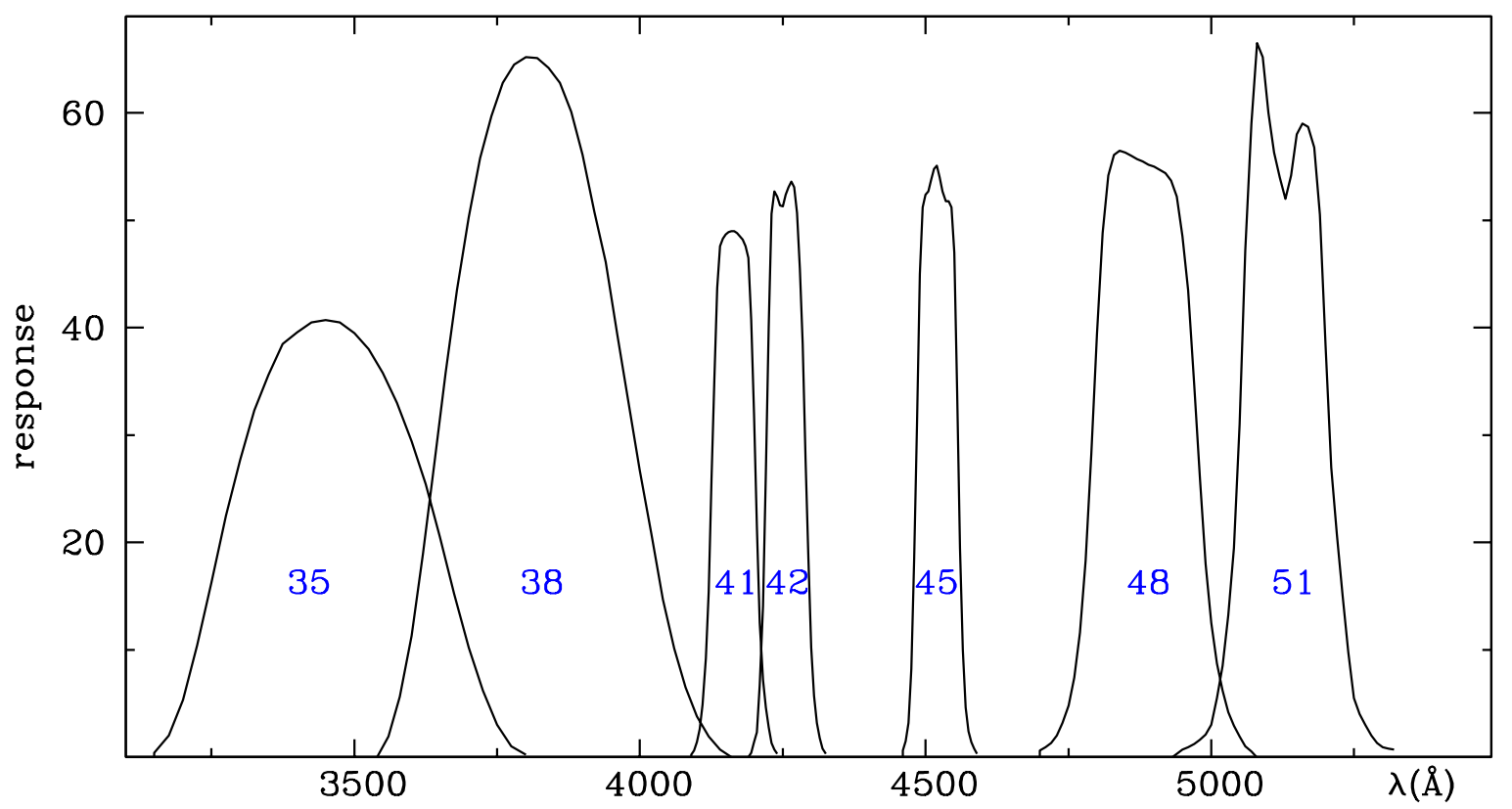

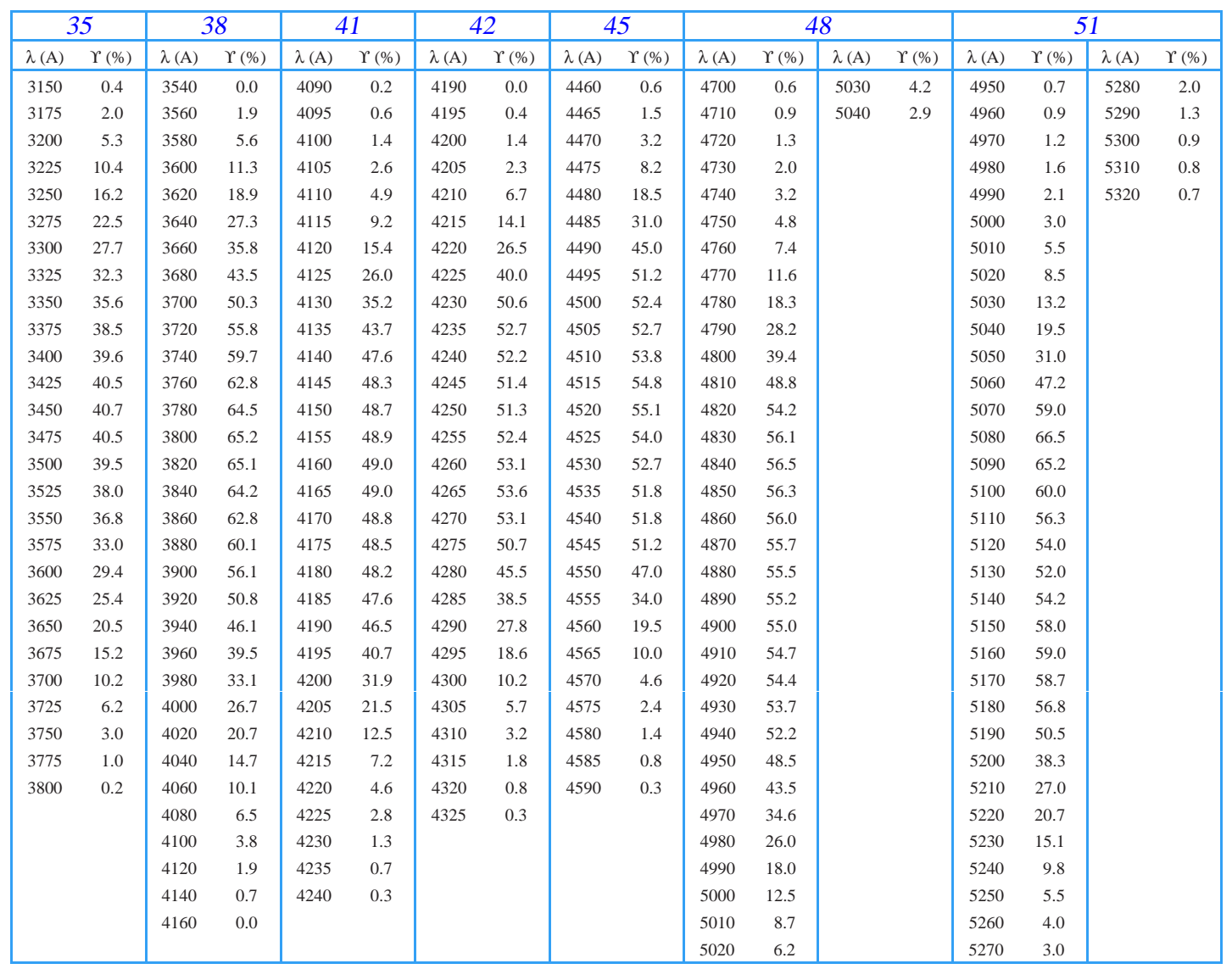

Fig. 48. continued 
As realized at the South Africal Astron. Obs. [77].

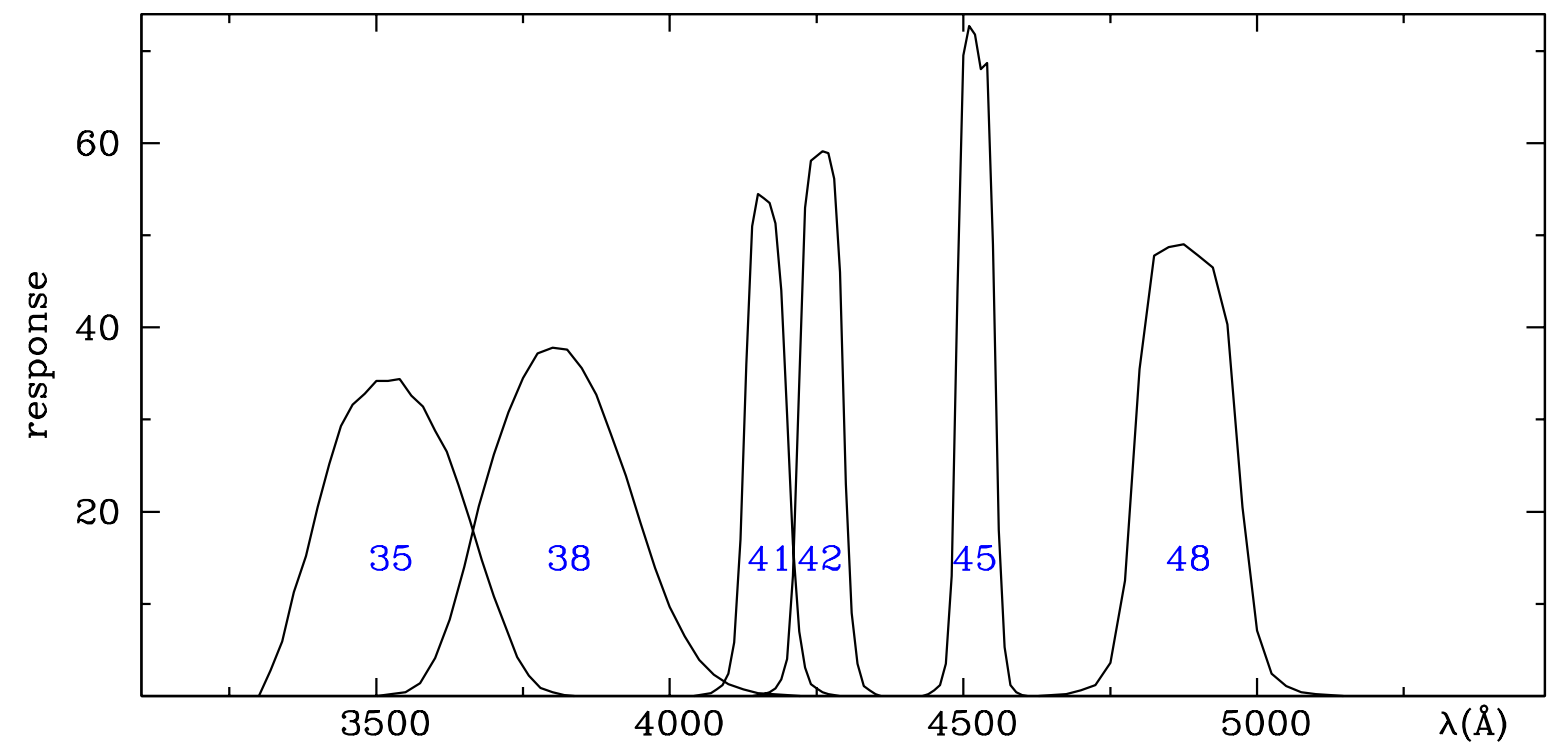

\begin{tabular}{|c|c|c|c|c|c|c|c|c|c|c|c|}
\hline \multicolumn{2}{|c|}{35} & \multicolumn{2}{|c|}{38} & \multicolumn{2}{|c|}{41} & \multicolumn{2}{|c|}{42} & \multicolumn{2}{|c|}{45} & \multicolumn{2}{|c|}{48} \\
\hline$\lambda(\mathrm{A})$ & $\Upsilon(\%)$ & $\lambda(\mathrm{A})$ & $\Upsilon(\%)$ & $\lambda(\mathrm{A})$ & $\Upsilon(\%)$ & $\lambda(\mathrm{A})$ & $\Upsilon(\%)$ & $\lambda(\mathrm{A})$ & $\Upsilon(\%)$ & $\lambda(\mathrm{A})$ & $\Upsilon(\%)$ \\
\hline 3300 & 0.0 & 3500 & 0.0 & 4040 & 0.0 & 4140 & 0.0 & 4430 & 0.0 & 4625 & 0.0 \\
\hline 3320 & 2.8 & 3525 & 0.2 & 4050 & 0.1 & 4150 & 0.1 & 4440 & 0.2 & 4650 & 0.1 \\
\hline 3340 & 5.9 & 3550 & 0.4 & 4060 & 0.2 & 4160 & 0.2 & 4450 & 0.6 & 4675 & 0.2 \\
\hline 3360 & 11.3 & 3575 & 1.4 & 4070 & 0.3 & 4170 & 0.4 & 4460 & 1.2 & 4700 & 0.6 \\
\hline 3380 & 15.2 & 3600 & 4.1 & 4080 & 0.7 & 4180 & 0.8 & 4470 & 3.5 & 4725 & 1.2 \\
\hline 3400 & 20.5 & 3625 & 8.3 & 4090 & 1.2 & 4190 & 1.8 & 4480 & 13.0 & 4750 & 3.6 \\
\hline 3420 & 25.2 & 3650 & 14.0 & 4100 & 2.4 & 4200 & 4.0 & 4490 & 44.0 & 4775 & 12.5 \\
\hline 3440 & 29.3 & 3675 & 20.6 & 4110 & 5.8 & 4210 & 13.0 & 4500 & 69.5 & 4800 & 35.5 \\
\hline 3460 & 31.6 & 3700 & 26.2 & 4120 & 17.0 & 4220 & 34.0 & 4510 & 72.7 & 4825 & 47.8 \\
\hline 3480 & 32.8 & 3725 & 30.8 & 4130 & 36.0 & 4230 & 53.0 & 4520 & 71.8 & 4850 & 48.7 \\
\hline 3500 & 34.2 & 3750 & 34.5 & 4140 & 51.0 & 4240 & 58.1 & 4530 & 68.0 & 4875 & 49.0 \\
\hline 3520 & 34.2 & 3775 & 37.2 & 4150 & 54.5 & 4250 & 58.6 & 4540 & 68.7 & 4900 & 47.8 \\
\hline 3540 & 34.4 & 3800 & 37.8 & 4160 & 54.0 & 4260 & 59.1 & 4550 & 49.0 & 4925 & 46.5 \\
\hline 3560 & 32.6 & 3825 & 37.6 & 4170 & 53.5 & 4270 & 58.9 & 4560 & 18.0 & 4950 & 40.3 \\
\hline 3580 & 31.4 & 3850 & 35.6 & 4180 & 51.3 & 4280 & 56.1 & 4570 & 5.3 & 4975 & 20.5 \\
\hline 3600 & 28.8 & 3875 & 32.7 & 4190 & 44.0 & 4290 & 46.0 & 4580 & 1.2 & 5000 & 7.1 \\
\hline 3620 & 26.5 & 3900 & 28.4 & 4200 & 30.5 & 4300 & 23.0 & 4590 & 0.4 & 5025 & 2.4 \\
\hline 3640 & 22.9 & 3925 & 23.9 & 4210 & 16.5 & 4310 & 9.0 & 4600 & 0.1 & 5050 & 1.1 \\
\hline 3660 & 19.0 & 3950 & 18.8 & 4220 & 7.0 & 4320 & 3.5 & 4610 & 0.0 & 5075 & 0.4 \\
\hline 3680 & 14.8 & 3975 & 13.9 & 4230 & 3.1 & 4330 & 1.1 & & & 5100 & 0.2 \\
\hline 3700 & 10.8 & 4000 & 9.7 & 4240 & 1.3 & 4340 & 0.6 & & & 5125 & 0.1 \\
\hline 3720 & 7.5 & 4025 & 6.5 & 4250 & 0.8 & 4350 & 0.2 & & & 5150 & 0.0 \\
\hline 3740 & 4.2 & 4050 & 3.9 & 4260 & 0.4 & 4360 & 0.0 & & & & \\
\hline 3760 & 2.2 & 4075 & 2.3 & 4270 & 0.2 & & & & & & \\
\hline 3780 & 0.9 & 4100 & 1.3 & 4280 & 0.1 & & & & & & \\
\hline 3800 & 0.4 & 4125 & 0.7 & 4290 & 0.0 & & & & & & \\
\hline 3820 & 0.1 & 4150 & 0.3 & & & & & & & & \\
\hline 3840 & 0.0 & 4175 & 0.2 & & & & & & & & \\
\hline & & 4200 & 0.1 & & & & & & & & \\
\hline & & 4225 & 0.0 & & & & & & & & \\
\hline
\end{tabular}

Fig. 48. continued 


\title{
$H \beta, \gamma$ - Sinnerstad et al. - 1968
}

$\mathrm{H} \beta, \mathrm{H} \gamma$ and HeI $4471 \AA$ narrow band photometry for early type stars.

\section{GENERAL INFORMATION}

\author{
AUTHORS U. Sinnerstad, J. Arkling, S. H. Alm, P. Brattlund \\ TELESCOPE $\quad 1.0 \mathrm{~m}$ (reflector), Stockholm Observatory \\ DETECTOR EMI $6256 \mathrm{~S}$ (uncooled)
}

MAIN ARTICLE Sinnerstad U., Arkling J., Alm S.H., Brattlund P. 1968, Ark. Astr. 5, 105

\section{SYSTEM DESCRIPTION}

The light coming into the photometer is split in two parts by a beam-splitting mirror and each of the two beams proceeds through a filter and falls on a separate photomultiplier.

\begin{tabular}{|ll|lcc|}
\hline \multicolumn{5}{|c|}{ BANDS DESCRIPTION [270] } \\
\hline & band & filter manufacturer & $\lambda_{\text {peak }}(\AA)$ & half-width $(\AA)$ \\
\hline$H \gamma$ & narrow & Baird Atomic & 4340 & 20 \\
\hline$H \gamma$ & wide & Baird Atomic & 4340 & 165 \\
\hline HeI & narrow & Baird Atomic & 4471 & 23 \\
\hline HeI & interm & Baird Atomic & 4471 & 88 \\
\hline$H e I$ & wide & Baird Atomic & 4471 & 140 \\
\hline$H \beta$ & narrow & Spectrolab & 4861 & 20 \\
\hline$H \beta$ & narrow & Spectrolab & 4861 & 26 \\
\hline$H \beta$ & wide & Spectrolab & 4861 & 164 \\
\hline & & & & 6 \\
\hline$H \beta$ & narrow (\#) & Baird Atomic & 4861 & 28 \\
\hline$H \beta$ & interm (\#) & Baird Atomic & 4861 & 195 \\
\hline$H \beta$ & wide (\#) & Baird Atomic & 4861 & \\
\hline
\end{tabular}

Interference filters.

(\#) Filter set used in April and May 1967.

\section{SYSTEM ANALISYS}

\section{COLOR INDICES AND PARAMETERS [270]}

Line indices are obtained as the magnitude difference corresponding to the ratio of the intensities measured simultaneously through the narrow and wide filters. The simultaneity directly accounts for variability of the atmosphere transmission which is cancelled out. The difference in sensitivity between the direct and reflected beams is accounted for by interchanging their light paths.

RELATIONS WITH OTHER SYSTEMS [270]

$$
\text { uvby } H \beta \text { - Strömgren and Crawford - } 1956
$$

$\mathrm{H} \beta=2.088 \beta_{\text {Stromgren }}-5.260$

Fig. 49. The photometric system $H \beta, \gamma$ - Sinnerstad et al. - 1968 


\section{TRANSMISSION CURVES}

As derived from Fig 1a,b,c of [270].

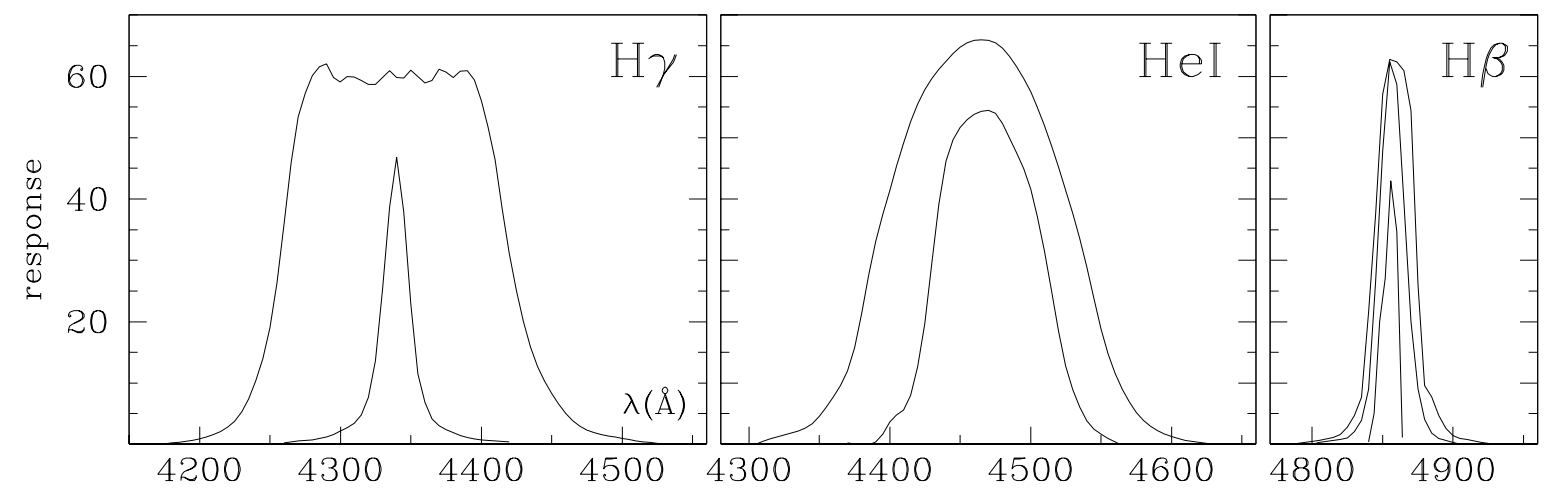

\begin{tabular}{|c|c|c|c|c|c|c|c|c|c|c|c|c|c|}
\hline \multicolumn{2}{|c|}{$H \gamma 20 \AA$} & \multicolumn{2}{|c|}{$H \gamma 165 \AA$} & \multicolumn{2}{|c|}{ HeI $88 \AA$} & \multicolumn{2}{|c|}{ HeI $140 \AA$} & \multicolumn{2}{|c|}{$H \beta 6 \AA$} & \multicolumn{2}{|c|}{$H \beta 20 \AA$} & \multicolumn{2}{|c|}{$H \beta 26 \AA$} \\
\hline$\lambda(\AA)$ & $\Upsilon$ & $\lambda(\AA)$ & $\Upsilon$ & $\lambda(\AA)$ & $\Upsilon$ & $\lambda(\AA)$ & $\Upsilon$ & $\lambda(\AA)$ & $\Upsilon$ & $\lambda(\AA)$ & $\Upsilon$ & $\lambda(\AA)$ & $\Upsilon$ \\
\hline 4252.7 & 0.0 & 4167.7 & 0.0 & 4377.0 & 0.0 & 4304.1 & 0.0 & 4825.5 & 0.0 & 4799.0 & 0.0 & 4785.8 & 0.0 \\
\hline 4269.7 & 0.5 & 4203.4 & 1.1 & 4394.9 & 1.7 & 4327.1 & 1.7 & 4833.3 & 0.8 & 4820.4 & 0.8 & 4811.8 & 0.9 \\
\hline 4288.3 & 1.1 & 4219.7 & 2.7 & 4401.5 & 4.1 & 4345.1 & 3.4 & 4842.1 & 1.7 & 4829.6 & 1.9 & 4824.0 & 2.5 \\
\hline 4305.3 & 2.7 & 4230.6 & 5.4 & 4412.8 & 6.6 & 4361.5 & 8.3 & 4843.7 & 4.2 & 4835.7 & 4.2 & 4831.7 & 5.4 \\
\hline 4316.4 & 5.4 & 4240.7 & 10.9 & 4420.5 & 13.2 & 4375.9 & 16.6 & 4846.3 & 15.0 & 4839.8 & 8.3 & 4836.8 & 10.8 \\
\hline 4323.9 & 12.0 & 4252.6 & 22.6 & 4425.6 & 20.7 & 4386.1 & 29.0 & 4854.1 & 34.1 & 4842.3 & 17.5 & 4841.9 & 28.3 \\
\hline 4329.0 & 22.3 & 4259.4 & 34.8 & 4431.7 & 33.1 & 4401.5 & 42.6 & 4856.7 & 44.9 & 4847.4 & 35.7 & 4847.0 & 44.5 \\
\hline 4330.7 & 27.2 & 4269.6 & 53.0 & 4439.4 & 45.5 & 4416.9 & 53.8 & 4859.3 & 38.2 & 4852.5 & 58.2 & 4850.5 & 58.6 \\
\hline 4334.1 & 35.9 & 4274.7 & 57.1 & 4445.0 & 49.7 & 4438.4 & 62.1 & 4861.9 & 19.1 & 4856.1 & 62.7 & 4853.1 & 61.1 \\
\hline 4337.5 & 45.1 & 4279.1 & 59.8 & 4456.3 & 53.2 & 4452.8 & 65.2 & 4863.0 & 7.5 & 4860.2 & 58.2 & 4857.2 & 64.0 \\
\hline 4340.2 & 46.7 & 4283.9 & 61.3 & 4469.1 & 54.5 & 4467.1 & 66.0 & 4867.1 & 3.7 & 4865.3 & 38.2 & 4860.2 & 62.3 \\
\hline 4342.5 & 44.3 & 4293.4 & 60.9 & 4475.8 & 53.8 & 4478.4 & 65.0 & 4872.4 & 1.7 & 4870.4 & 18.9 & 4863.8 & 62.3 \\
\hline 4345.9 & 35.1 & 4303.6 & 59.8 & 4485.5 & 49.7 & 4488.7 & 62.1 & 4877.6 & 0.8 & 4875.5 & 8.3 & 4865.3 & 60.7 \\
\hline 4349.3 & 25.0 & 4328.1 & 59.2 & 4494.7 & 45.1 & 4504.1 & 55.4 & 4882.8 & 0.0 & 4880.6 & 3.7 & 4868.9 & 59.8 \\
\hline 4352.7 & 15.2 & 4335.9 & 60.9 & 4500.3 & 41.4 & 4524.6 & 41.8 & & & 4885.7 & 1.7 & 4869.9 & 54.8 \\
\hline 4356.1 & 10.3 & 4344.4 & 59.5 & 4510.1 & 31.4 & 4540.0 & 29.0 & & & 4891.8 & 0.8 & 4872.5 & 39.9 \\
\hline 4362.2 & 5.4 & 4351.2 & 60.9 & 4515.2 & 24.8 & 4550.2 & 18.6 & & & 4906.1 & 0.0 & 4875.0 & 25.8 \\
\hline 4372.4 & 2.7 & 4364.8 & 59.2 & 4525.4 & 12.4 & 4566.6 & 8.3 & & & & & 4877.6 & 14.1 \\
\hline 4390.0 & 1.1 & 4371.6 & 61.4 & 4531.1 & 8.3 & 4575.9 & 5.0 & & & & & 4882.7 & 8.7 \\
\hline 4412.0 & 0.5 & 4378.3 & 59.8 & 4539.2 & 4.1 & 4595.3 & 1.7 & & & & & 4887.8 & 6.0 \\
\hline 4432.4 & 0.0 & 4385.1 & 60.9 & 4550.0 & 1.7 & 4637.4 & 0.0 & & & & & 4898.0 & 1.8 \\
\hline & & 4395.3 & 59.2 & 4563.8 & 0.0 & & & & & & & 4908.2 & 0.8 \\
\hline & & 4398.7 & 57.1 & & & & & & & & & 4928.6 & 0.0 \\
\hline & & 4402.1 & 54.1 & & & & & & & & & & \\
\hline & & 4408.9 & 47.8 & & & & & & & & & & \\
\hline & & 4415.7 & 37.2 & & & & & & & & & & \\
\hline & & 4422.5 & 28.0 & & & & & & & & & & \\
\hline & & 4434.4 & 16.3 & & & & & & & & & & \\
\hline & & 4450.4 & 8.2 & & & & & & & & & & \\
\hline & & 4458.5 & 5.4 & & & & & & & & & & \\
\hline & & 4497.3 & 1.1 & & & & & & & & & & \\
\hline & & 4510.8 & 0.0 & & & & & & & & & & \\
\hline
\end{tabular}

Fig. 49. continued 


\section{u'ubvv' - Smith - 1968}

Narrow-band photometry of Wolf-Rayet stars.

\section{GENERAL INFORMATION}

$\begin{array}{ll}\text { AUTHORS } & \text { L. F. Smith } \\ \text { TELESCOPE } & 1.0 \mathrm{~m} \text { (reflector), Siding Spring Observatory; } \\ & 1.27 \mathrm{~m} \text { (reflector), Mount Stromlo Observatory } \\ \text { DETECTOR } & 1 \mathrm{P} 21 \text { (refrigerated) } \\ \text { MAIN ARTICLE } & \text { Smith, L. F. 1968, MNRAS 140, } 409\end{array}$

\section{SYSTEM DESCRIPTION}

\begin{tabular}{|l|lccc|}
\hline \multicolumn{5}{|c|}{ BANDS DESCRIPTION [272] } \\
\hline band & filter manufacturer & $\lambda_{c}(\AA)$ & FWHM $(\AA)$ & $\Upsilon_{\text {peak }}(\%)$ \\
\hline$u^{\prime}$ & Schott & 3500 & 80 & 43 \\
\hline$u$ & Schott & 3650 & 100 & 39 \\
\hline$b$ & Spectrolab & 4270 & 70 & 60 \\
\hline$v$ & Spectrolab & 5160 & 130 & 50 \\
\hline$v^{\prime}$ & Schott & 5500 & 230 & 64 \\
\hline
\end{tabular}

Interference filters.

A slightly different set of filters (and a refrigerated EMI 6256 photomultiplier) have been used at ESO by [193].

The passbands have been reproduced with a spectral scanner by [197].

\section{SYSTEM ANALYSIS}

\section{REDDENING-FREE PARAMETERS [272]}

$\xi=-\left(u^{\prime}-u\right)+0.24(b-v)$ : intensity of N IV $3480 \AA$ (in WN stars).

$\Delta=(u-b)-0.69(b-v)$ : measures the non-linearity on the continuum in the region covered by the $u, b, v$ bands.

$\phi=\left(b-v^{\prime}\right)-1.29(b-v):$ measures HeII $5412 \AA$ in WN stars; in WC stars it measures the intensity of the HeII 5412, CIV 5469 and OV $5592 \AA$ blend.

REDDENING RATIOS [272]

$E(B-V) / E(b-v)=1.20 \quad E\left(v-v^{\prime}\right) / E(b-v)=0.29$

Fig. 50. The photometric system u'ubvv' - Smith - 1968 


\section{jhk - Bahng - 1969}

General purpose system. Cool stars.

\section{GENERAL INFORMATION}

$\begin{array}{ll}\text { AUTHORS } & \text { J. Bahng } \\ \text { TELESCOPE } & 0.91 \mathrm{~m} \text { (reflector), KPNO } \\ \text { DETECTOR } & \text { PbS, operating at dry-ice temperatures } \\ \text { MAIN ARTICLE } & \text { Bahng, J. 1969, MNRAS 143, 73 }\end{array}$

SYSTEM DESCRIPTION

\begin{tabular}{|c|ccc|}
\hline \multicolumn{4}{|c|}{ BANDS DESCRIPTION [17] } \\
\hline band & $\lambda_{c}(\mu \mathrm{m})$ & half-width $(\mu \mathrm{m})$ & $\Upsilon_{c}(\%)$ \\
\hline$j$ & 1.21 & 0.077 & 58 \\
\hline$h$ & 1.59 & 0.086 & 49 \\
\hline$k$ & 2.15 & 0.098 & 61 \\
\hline
\end{tabular}

Filters by Infrared Industries

SYSTEM ANALYSIS

\section{RELATIONS WITH OTHER SYSTEMS [17]}

UBVRI(JHKLMN) - Johnson - 1965

$(J-K)_{J o h n s o n}=0.923(j-k)+1.446$

Fig. 51. The photometric system jhk - Bahng - 1969 


\section{Ca II K - Henry - 1969}

Square band spectrophotometric measurement of Ca II K in A-type stars.

\section{GENERAL INFORMATION}

$\begin{array}{ll}\text { AUTHORS } & \text { R. C. Henry } \\ \text { TELESCOPE } & \text { 0.40m (reflector), KPNO } \\ \text { DETECTOR } & \text { EMR 641-A } \\ \text { MAIN ARTICLE } & \text { Henry, R. C. 1969, ApJS 18, 47 }\end{array}$

SYSTEM DESCRIPTION

\begin{tabular}{|l|ccc|}
\hline \multicolumn{4}{|c|}{ BANDS DESCRIPTION [137] } \\
\hline band & $\lambda_{c}(\AA)$ & width $(\AA)$ & feature \\
\hline$K$ & 3933.68 & 8.50 & Ca II K line \\
\hline$C$ & 3915.30 & 8.23 & continuum \\
\hline
\end{tabular}

Two exit slits direct to two separate photomultipliers the $K$ and $C$ portions of the spectrum.

\section{SYSTEM ANALYSIS}

COLOR INDICES AND PARAMETERS [137]

$k=-2.5 \log \frac{K}{C}+$ const

Fig. 52. The photometric system Ca II K - Henry - 1969 


\section{Newell et al. - 1969}

Narrow-band system. Horizontal-branch stars.

\section{GENERAL INFORMATION}

AUTHORS

TELESCOPE

DETECTOR

E. B. Newell, A. W. Rodgers, and L. Searle

$0.41 \mathrm{~m}$ and $1.02 \mathrm{~m}$, Siding Spring Observatory

1P21 (refrigerated)

MAIN ARTICLE Newell, E. B., Rodgers, A. W., Searle, L. 1969, ApJ 156, 597

\section{SYSTEM DESCRIPTION}

\begin{tabular}{|c|lccc|}
\hline \multicolumn{5}{|c|}{ BANDS DESCRIPTION[229] } \\
\hline band & interference filter manufacturer & $\lambda_{\text {eff }}(\AA)$ & FWHM $(\AA)$ & $\Upsilon_{\text {peak }}(\%)$ \\
\hline $354[229]$ & Thin Film Products & 3535 & 78 & 43 \\
\hline $427[229]$ & Thin Film Products & 4272 & 84 & 68 \\
\hline $531[229]$ & Thin Film Products & 5305 & 155 & 40 \\
\hline$H \gamma[228]$ & & 4348 & 50 & 53 \\
\hline
\end{tabular}

Additional observations in the UBV bands of UBV - Johnson and Morgan - 1953 system have been obtained with the following filters: $2 \mathrm{~mm}$ Corning 9863 (U), 3mm Corning $5030+2 \mathrm{~mm}$ Schott 6613 (B), $2 \mathrm{~mm}$ Corning $3384(V)$.

\section{SYSTEM ANALYSIS}

\section{COLOR INDICES AND PARAMETERS [228]}

353 - 427 : measures the Balmer discontinuity.

427 - 531 : measures the continuum.

$\gamma=427-0.074(427-531)-H \gamma$ : measures $\mathrm{H} \gamma$.

Fig. 53. The photometric system Newell et al. - 1969 


\section{TRANSMISSION CURVES}

As derived from Fig 4 of [228].

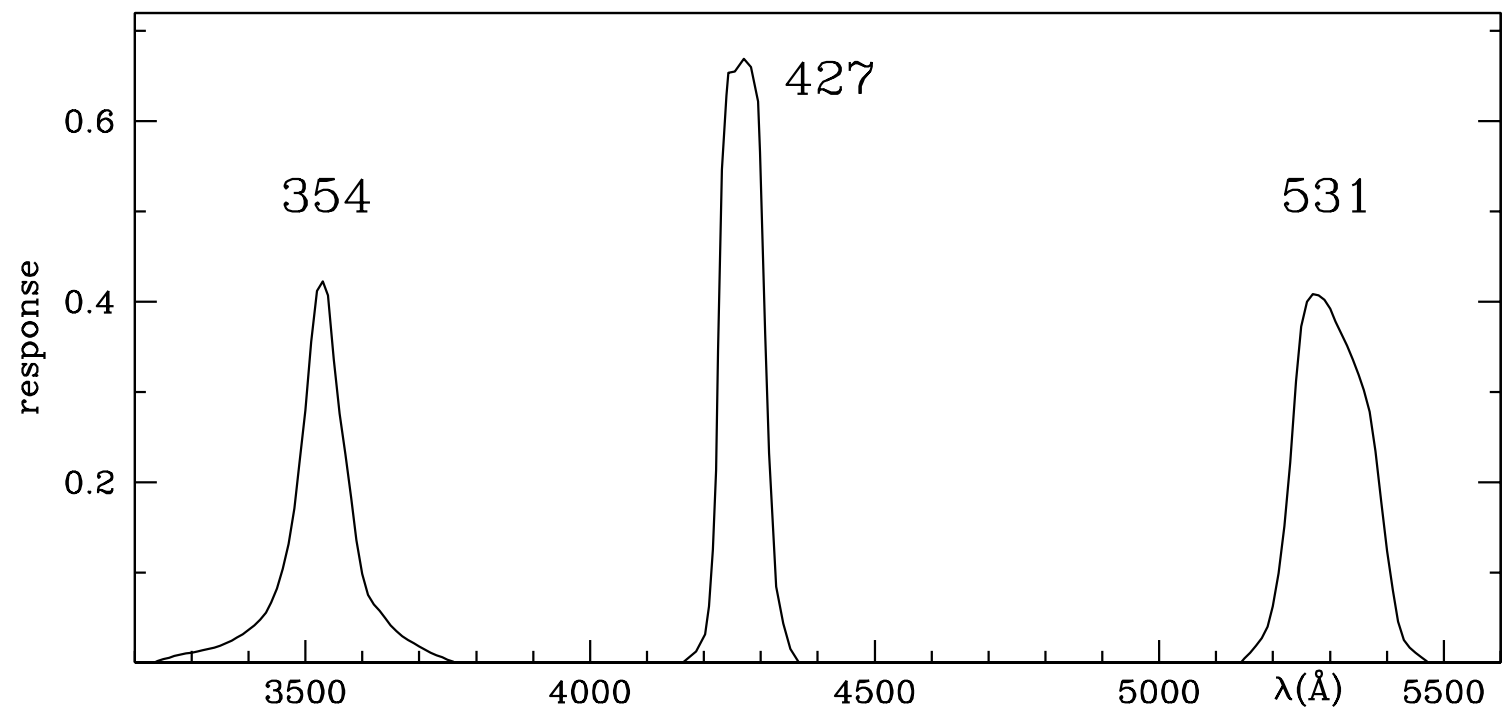

\begin{tabular}{|cc|cc|cc|cc|cc|cc|}
\hline \multicolumn{6}{|c|}{354} & \multicolumn{4}{c|}{427} & \multicolumn{4}{c|}{531} \\
\hline$\lambda(\mathrm{A})$ & $\Upsilon$ & $\lambda(\mathrm{A})$ & $\Upsilon$ & $\lambda(\mathrm{A})$ & $\Upsilon$ & $\lambda(\mathrm{A})$ & $\Upsilon$ & $\lambda(\mathrm{A})$ & $\Upsilon$ & $\lambda(\mathrm{A})$ & $\Upsilon$ \\
\hline 3230 & 0.000 & 3530 & 0.423 & 4161 & 0.000 & 4282 & 0.660 & 5143 & 0.000 & 5334 & 0.346 \\
3269 & 0.008 & 3543 & 0.393 & 4187 & 0.013 & 4295 & 0.622 & 5168 & 0.018 & 5353 & 0.314 \\
3309 & 0.013 & 3549 & 0.342 & 4202 & 0.031 & 4299 & 0.565 & 5184 & 0.031 & 5372 & 0.270 \\
3348 & 0.019 & 3568 & 0.239 & 4210 & 0.063 & 4301 & 0.515 & 5200 & 0.063 & 5385 & 0.207 \\
3388 & 0.031 & 3587 & 0.148 & 4216 & 0.126 & 4308 & 0.364 & 5215 & 0.126 & 5398 & 0.135 \\
3422 & 0.049 & 3606 & 0.082 & 4221 & 0.214 & 4314 & 0.232 & 5226 & 0.188 & 5410 & 0.079 \\
3437 & 0.063 & 3623 & 0.063 & 4225 & 0.346 & 4327 & 0.085 & 5232 & 0.239 & 5423 & 0.038 \\
3460 & 0.104 & 3651 & 0.041 & 4231 & 0.547 & 4340 & 0.044 & 5238 & 0.298 & 5442 & 0.016 \\
3479 & 0.166 & 3689 & 0.022 & 4240 & 0.628 & 4352 & 0.016 & 5251 & 0.377 & 5474 & 0.000 \\
3492 & 0.232 & 3728 & 0.009 & 4243 & 0.653 & 4368 & 0.000 & 5273 & 0.408 & & \\
3505 & 0.311 & 3766 & 0.000 & 4254 & 0.655 & & & 5302 & 0.390 & & \\
3515 & 0.390 & & & 4271 & 0.669 & & & 5311 & 0.377 & & \\
\hline
\end{tabular}

Fig. 53. continued 


\section{Spinrad and Taylor - 1969}

Square-band measurement of line and molecular absorptions in late-type stars.

\section{GENERAL INFORMATION}

\begin{tabular}{|c|c|}
\hline AUTH & H. \\
\hline TELI & ors), Li \\
\hline DETH & $\begin{array}{l}\text { FW-130 (S-20 cathode, for } 3800-7400 \AA) \\
\text { RCA } 7102(\text { S-1 cathode, for } 7400-10700 \AA)\end{array}$ \\
\hline & Spinrad, H., Taylor, B. J. 1969, ApJ 157 \\
\hline
\end{tabular}

\section{SYSTEM DESCRIPTION}

\begin{tabular}{|cc|cc|}
\hline \multicolumn{4}{|c|}{ BANDS DESCRIPTION $[275]$} \\
\hline$\lambda_{c}(\AA)$ & feature & $\lambda_{c}(\AA)$ & feature \\
\hline 3880 & $\mathrm{CN}(3883 \AA)$ & 6180 & $\mathrm{TiO}(6152 \AA)$ \\
\hline 4040 & continuum & 6386 & $\mathrm{CaH}(6382 \AA)$ \\
\hline 4100 & $\mathrm{H} \delta$ & 6564 & $\mathrm{H} \alpha$ \\
\hline 4200 & $\mathrm{CN}(4215 \AA)$ & 6620 & continuum \\
\hline 4227 & $\mathrm{CaI}$ & 7000 & continuum \\
\hline 4300 & $\mathrm{CH}(4313 \AA)$ & 7100 & $\mathrm{TiO}(7054 \AA)$ \\
\hline 4340 & H $\gamma$ & 7400 & continuum \\
\hline 4500 & continuum & 7980 & continuum \\
\hline 4715 & continuum & 8190 & NaI \\
\hline 4900 & continuum & 8400 & continuum \\
\hline 5000 & continuum & 8662 & $\mathrm{Ca}$ II \\
\hline 5175 & MgI $+\mathrm{MgH}$ & 8800 & continuum \\
\hline 5300 & continuum & 8900 & TiO $(8859 \AA)$ \\
\hline 5360 & continuum & 9200 & $\mathrm{CN}$ \\
\hline 5864 & continuum & 10300 & continuum \\
\hline 5892 & NaI D & 10700 & continuum \\
\hline 6110 & continuum & & \\
\hline
\end{tabular}

A spectrum scanner feeds the photomultipliers.

A set of 12 stars given in [275] set the zero points of the system.

[275] prescribes that to accurately reproduce the system the observations must be obtained at $15 \AA$ resolution shortward of $5360 \AA$ and $30 \AA$ resolution longward.

\section{SYSTEM ANALYSIS}

\section{COLOR INDICES AND PARAMETERS [275]}

$T=I(7000)+I(7400):$ red temperature index.

$T_{I R}=I(8800)+I(10300)+I(10700):$ infrared temperature index.

Fig. 54. The photometric system Spinrad and Taylor - 1969 


\section{$H \alpha, \beta$ - Tebbe - 1969}

Separation between B supergiants and Be stars by means of $\mathrm{H} \alpha, \mathrm{H} \beta$ narrow band photometry.

\section{GENERAL INFORMATION}
AUTHORS
P. L. Tebbe
TELESCOPE
0.30m (refractor), Georgetown College Observatory
DETECTOR
RCA C-31000 B (S-20 cathode, refrigerated)
MAIN ARTICLE Tebbe, P.L. 1969, AJ 74, 920

\section{SYSTEM DESCRIPTION}

\begin{tabular}{|l|lccc|}
\hline \multicolumn{5}{|c|}{ BANDS DESCRIPTION [298] } \\
\hline band & comments & $\lambda_{\text {peak }}(\AA)$ & half-width $(\AA)$ & $\Upsilon_{\text {peak }}(\%)$ \\
\hline$N \alpha$ & H $\alpha$ narrow & 6570 & 45 & 41 \\
\hline$W \alpha$ & H $\alpha$ wide & 6550 & 157 & 57 \\
\hline$N \beta$ & H $\beta$ narrow & 4863 & 39 & 59 \\
\hline$W \beta$ & H $\beta$ wide & 4837 & 184 & 63 \\
\hline
\end{tabular}

Interference filters by Baird Atomic.

\section{SYSTEM ANALYSIS}

COLOR INDICES AND PARAMETERS [298]

$\alpha=2.5 \log \left(W_{\alpha}\right)-2.5 \log \left(N_{\alpha}\right)+$ const. $\quad \beta=2.5 \log \left(W_{\beta}\right)-2.5 \log \left(N_{\beta}\right)+$ const.

RELATIONS WITH OTHER SYSTEMS [298]

uvbyH $\beta$ - Strömgren and Crawford - 1956

least-square fit relations

\begin{tabular}{|lll|}
\hline$\beta_{\text {Strom }}=+0.246+1.415 \beta$ & for B stars \\
\hline$\beta_{\text {Strom }}=-0.225+1.667 \beta$ & for A-F stars \\
\hline
\end{tabular}

Fig. 55. The photometric system $H \alpha, \beta$ - Tebbe -1969 


\section{gnkmfu - Dickow et al. - 1970}

Narrow band photometry of late-type stars.

\section{GENERAL INFORMATION}

AUTHORS P. Dickow, K. Gyldenkerne, L. Hansen, P.-U. Jacobsen, K. T. Johansen, P. Kjaeregaard, E. H. Olsen

TELESCOPE $\quad 0.61 \mathrm{~m}$ Morgan (reflector), Lowell Observatory, Arizona

DETECTOR EMI 6265 S/A (CsSb cathode, unrefrigerated)

MAIN ARTICLE Dickow, P., Gyldenkerne, K., Hansen, L., Jacobsen, P.-U.,

Johansen, K. T., Kjaergaard, P., Olsen, E. H. 1970, A\&AS 2, 1

\section{SYSTEM DESCRIPTION}

\begin{tabular}{|l|lcc|}
\hline \multicolumn{4}{|c|}{ BANDS DESCRIPTION [86] } \\
\hline band & \multicolumn{1}{|c|}{ filter } & $\lambda_{\text {peak }}(\AA)$ & FWHM $(\AA)$ \\
\hline 344 & Schott UG 11 + WG 3 & 3440 & 389 \\
\hline 391 & Schott UV - PIL (tilted by 5 $\left.{ }^{\circ}\right)$ & 3910 & 55 \\
\hline 406 & Schott PIL & 4057 & 73 \\
\hline 417 & Baird B9 & 4174 & 40 \\
\hline 427 & Baird B9 & 4271 & 27 \\
\hline 439 & Baird B9 & 4389 & 47 \\
\hline 452 & Schott PIL & 4517 & 80 \\
\hline 497 & Schott PIL & 4973 & 95 \\
\hline
\end{tabular}

Except for the 344 band, all filters are of interference type.

\section{SYSTEM ANALYSIS}

\section{COLOR INDICES AND PARAMETERS [86]}

$g=2.5\left(\log I_{439}-\log I_{427}\right):$ measures the break around the G-band.

$n=2.5\left(\log I_{427}-\log I_{417}\right):$ measures the $\mathrm{CN}$ absorption.

$k=2.5\left(\log I_{391}-\log I_{406}\right):$ aimed to the iron discontinuity shortward of $4000 \AA$, including the Ca II K line.

$m=2.5\left(2 \log I_{452}-\log I_{406}-\log I_{497}\right):$ metallicity index, similar to $m_{1}$ Strömgren index.

$f=2.5\left(\log I_{497}-\log I_{406}\right):$ given the wide $\Delta \lambda$ baseline, it is an useful tool to evaluate the reddening.

$u=2.5\left(\log I_{406}-\log I_{344}\right):$ ultraviolet color index.

$1.00[\mathrm{Fe} / \mathrm{H}]=-16.39( \pm 1.05) g+9.80( \pm 0.78) m+8.14( \pm 1.25):$ for giant with $-0.8 \leq[\mathrm{Fe} / \mathrm{H}] \leq 0.3$. [166]

REDDENING RATIOS [86]

$$
\begin{array}{lll}
\text { for } \mathrm{A}_{V}=1.0 \mathrm{mag} & & \\
E(g)=0.035 & E(n)=0.029 & E(k)=-0.037 \\
E(m)=-0.029 & E(f)=0.305 & E(u)=0.194
\end{array}
$$

Fig. 56. The photometric system gnkmfu - Dickow et al. - 1970 


\section{Uppsala - Haggkvist and Oja - 1970}

Narrow band photometry of late-type stars.

\section{GENERAL INFORMATION}

$\begin{array}{ll}\text { AUTHORS } & \text { L. Häggkvist, and T. Oja } \\ \text { TELESCOPE } & \text { 0.33m (photographic refractor), Uppsala Obs.; } \\ & \text { 0.15m Zeiss-Heyde astrograph } \\ \text { DETECTOR } & \text { EMI 9502B } \\ \text { MAIN ARTICLE } & \text { Häggkvist, L., Oja, T. 1970, A\&AS 1, 199 }\end{array}$

\section{SYSTEM DESCRIPTION}

\begin{tabular}{|c|cc|}
\hline \multicolumn{3}{|c|}{ BANDS DESCRIPTION [132] } \\
\hline band & $\lambda_{\text {peak }}(\AA)$ & half-width $(\AA)$ \\
\hline 418 & 4176 & 40 \\
\hline 427 & 4269 & 43 \\
\hline 451 & 4508 & 50 \\
\hline
\end{tabular}

Interference filters by Baird Atomic.

\section{SYSTEM ANALYSIS}

\section{COLOR INDICES AND PARAMETERS [132]}

$g_{e}=(427)-(451)$ : measures the break at the G-band.

$c_{e}=(418)-(427)$ : measures the $\mathrm{CN}$ absorption.

\section{RELATIONS WITH OTHER SYSTEMS [132]}

gnkmfu - Dickow et. al - 1970

\begin{tabular}{|rr|}
\hline$g$ & $=0.416( \pm 0.005) g_{e}+0.105( \pm 0.007) c_{e}-0.257( \pm 0.006)$ \\
\hline$n$ & $=-0.489( \pm 0.063) g_{e}+0.241( \pm 0.023) g_{e}^{2}+0.454( \pm 0.008) c_{e}+0.332( \pm 0.042)$ \\
\hline$g_{e}=$ & $2.504( \pm 0.048) g-0.504( \pm 0.051) n+0.638( \pm 0.008)$ \\
\hline$c_{e}$ & $=0.995( \pm 0.196) g-2.605( \pm 0.267) g^{2}+2.180( \pm 0.052) n-0.286( \pm 0.029)$
\end{tabular}

DDO - McClure and Van den Bergh - 1968

\begin{tabular}{|rrr|}
\hline$C(42-45)=$ & $0.846( \pm 0.040) g_{e}+0.085( \pm 0.016) g_{e}^{2}+0.070( \pm 0.013) c_{e}-0.329( \pm 0.024)$ \\
\hline$C(41-42)=$ & $0.831( \pm 0.010) c_{e}+0.041( \pm 0.002)$ \\
\hline$g_{e}=$ & $1.069( \pm 0.024) C(42-45)-0.072( \pm 0.013) C(42-45)^{2}+$ \\
& $-0.070( \pm 0.015) C(41-42)+0.386( \pm 0.009)$ \\
\hline$c_{e}=$ & $1.187( \pm 0.014) C(41-42)-0.047( \pm 0.003)$ \\
\hline
\end{tabular}

Fig. 57. The photometric system Uppsala - Häggkvist and Oja - 1970 


\section{$R I$ - Jacobsen - 1970}

Photometry in the red and near-infrared.

\section{GENERAL INFORMATION}

$\begin{array}{ll}\text { AUTHORS } & \text { P. U. Jacobsen } \\ \text { TELESCOPE } & 0.61 \mathrm{~m} \text { Morgan reflector, Lowell Obs. } \\ \text { DETECTOR } & \text { FW118 (S-1 cathode, refrigerated) } \\ \text { MAIN ARTICLE } & \text { Jacobsen, P. U. 1969, A\&A 4, 302 }\end{array}$

\section{SYSTEM DESCRIPTION}

\begin{tabular}{|l|l|cc|}
\hline \multicolumn{2}{|c|}{ BANDS DESCRIPTION [146] } & \\
\hline band & \multicolumn{1}{|c|}{ filter (\#) } & $W H M(\AA)$ & $\lambda_{c}(\AA)$ \\
\hline$R$ & 2mm RG 610/1 + Corning 3965 $(150 \%)+2 \mathrm{~mm} \mathrm{KG} \mathrm{3}$ & 980 & 6290 \\
\hline$I$ & Corning 2540 (75\%) & 1960 & 9430 \\
\hline
\end{tabular}

(\#) The filter thickness is given in percentage of the standard optical thickness in the Corning Catalogue [146].

\section{SYSTEM ANALYSIS}

RELATIONS WITH OTHER SYSTEMS [146]

UVBGRI - Stebbin and Withford - 1943

$(R-I)_{S-W}=0.924( \pm 0.015)(R-I)_{\text {Jacobsen }}-0.703( \pm 0.014) \quad$ for 44 stars in common

UBVRI(JHKLMN) - Johnson - 1965

$(R-I)_{\text {Johnson }}=0.761( \pm 0.004)(R-I)_{\text {Jacobsen }}-0.196( \pm 0.005) \quad$ for 90 stars in common

ri - Argue - 1967

$(r-i)_{\text {Argue }}=0.758( \pm 0.009)(R-I)_{\text {Jacobsen }}-0.181( \pm 0.009) \quad$ for 46 stars in common

Fig. 58. The photometric system $R I-$ Jacobsen -1970 


\section{TRANSMISSION CURVE}

As derived from Fig 1 of [146].

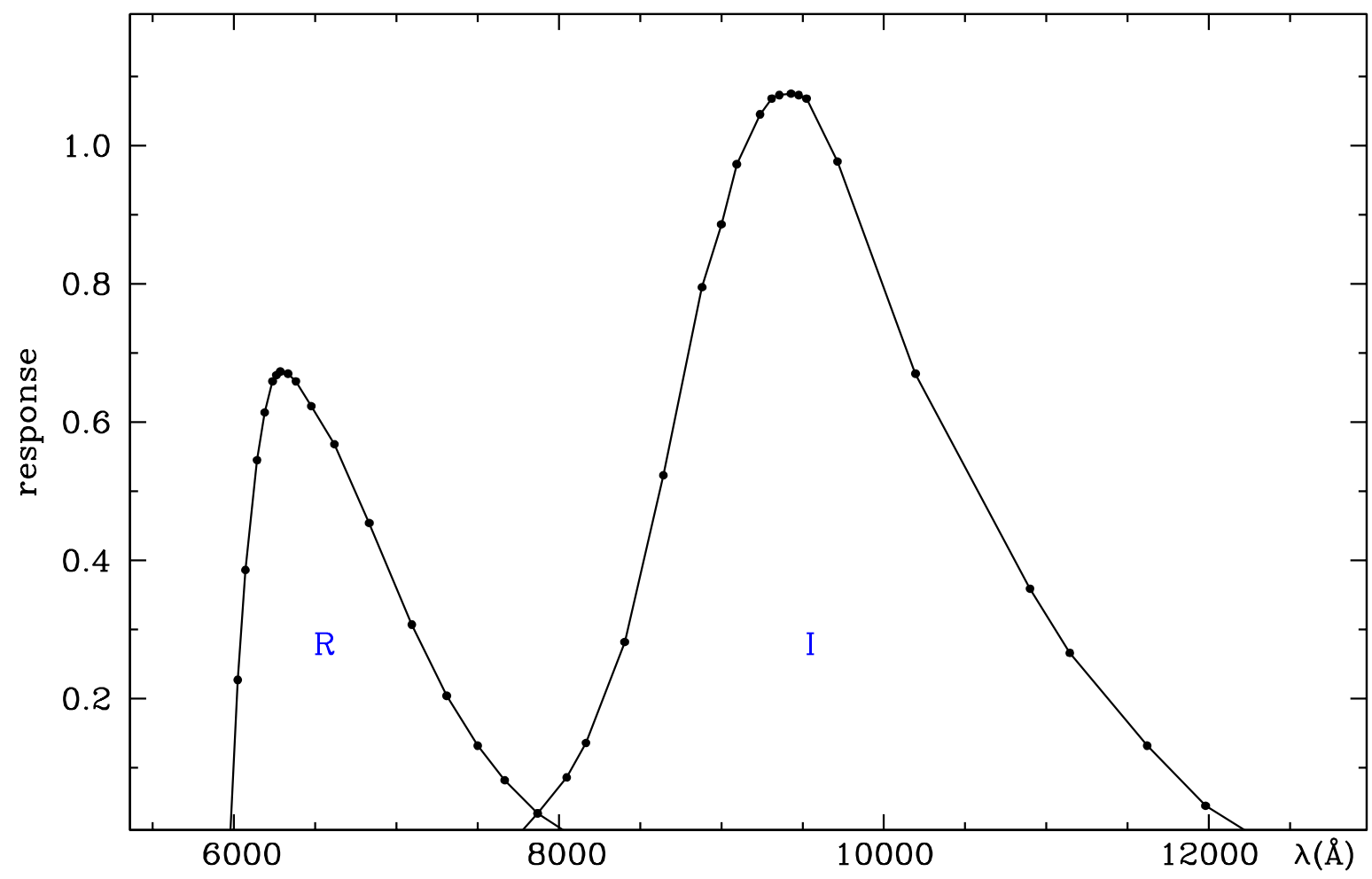

\begin{tabular}{|cc|cc|cc|rc|}
\hline \multicolumn{4}{|c|}{$R$} & \multicolumn{4}{c|}{$I$} \\
\hline$\lambda(\mathrm{A})$ & $\Upsilon$ & $\lambda(\mathrm{A})$ & $\Upsilon$ & $\lambda(\mathrm{A})$ & $\Upsilon$ & $\lambda(\mathrm{A})$ & $\Upsilon$ \\
\hline 5976 & 0.000 & 6619 & 0.568 & 7738 & 0.000 & 9357 & 1.073 \\
6024 & 0.227 & 6833 & 0.454 & 7869 & 0.034 & 9429 & 1.075 \\
6071 & 0.386 & 7095 & 0.307 & 8048 & 0.086 & 9476 & 1.073 \\
6143 & 0.545 & 7310 & 0.204 & 8167 & 0.136 & 9524 & 1.068 \\
6190 & 0.614 & 7500 & 0.132 & 8405 & 0.282 & 9714 & 0.977 \\
6238 & 0.659 & 7667 & 0.082 & 8643 & 0.523 & 10195 & 0.670 \\
6262 & 0.668 & 7869 & 0.034 & 8881 & 0.795 & 10900 & 0.359 \\
6286 & 0.673 & 8095 & 0.000 & 9000 & 0.886 & 11145 & 0.266 \\
6333 & 0.670 & & & 9095 & 0.973 & 11620 & 0.132 \\
6381 & 0.659 & & & 9238 & 1.045 & 11980 & 0.045 \\
6476 & 0.623 & & & 9310 & 1.068 & 12000 & 0.000 \\
\hline
\end{tabular}

Fig. 58. continued 


\section{nh - Landolt - 1970}

Luminosity class of $\mathrm{O}$ stars.

\section{GENERAL INFORMATION}

$\begin{array}{ll}\text { AUTHORS } & \text { A. U. Landolt } \\ \text { TELESCOPE } & 0.40 \mathrm{~m} \text { and } 0.91 \mathrm{~m} \text { (reflectors), KPNO } \\ \text { DETECTOR } & \text { RCA 1P21 }\end{array}$

MAIN ARTICLE Landolt, A. U. 1970, AJ 75, 337

SYSTEM DESCRIPTION

\begin{tabular}{|ll|ccc|}
\hline \multicolumn{4}{|c|}{ BANDS DESCRIPTION [180] } \\
\hline \multicolumn{2}{|c|}{ band } & $\lambda_{\text {eff }}(\AA)$ & half-width $(\AA)$ & $\Upsilon(\%)$ \\
\hline N III wide & $4633.9^{a}$ & $182.0^{a}$ & $65.0^{a}$ \\
& & $4637.5^{b}$ & $178.5^{b}$ & $64.2^{b}$ \\
& & $4635.5^{c}$ & $180.6^{c}$ & $62.6^{c}$ \\
\hline N III & narrow & $4624.2^{a}$ & $48.8^{a}$ & $60.5^{a}$ \\
& & $4627.9^{b}$ & $47.0^{b}$ & $59.0^{b}$ \\
& & $4628.3^{c}$ & $47.7^{c}$ & $59.0^{c}$ \\
\hline \multirow{2}{*}{ He II } & \multirow{2}{*}{ wide } & $4678.8^{b}$ & $191.1^{b}$ & $48.0^{b}$ \\
& & $4679.7^{c}$ & $193.0^{c}$ & $47.0^{c}$ \\
\hline \multirow{2}{*}{ He II } & \multirow{2}{*}{ narrow } & $4687.1^{b}$ & $41.6^{b}$ & $47.0^{b}$ \\
& & $4686.9^{c}$ & $41.6^{c}$ & $47.2^{c}$ \\
\hline
\end{tabular}

$\mathrm{a}=$ June $9,1965 \quad \mathrm{~b}=$ October $10,1965 \quad \mathrm{c}=$ October 14,1966

The zero point of the system is defined by the $n$ and $h$ values of seven standard stars, all supergiants of spectral types between B9 and A5.

\section{SYSTEM ANALYSIS}

\section{COLOR INDICES AND PARAMETERS [180]}

$n=2.5 \log (N$ III narrow $)-2.5 \log (N$ III wide $)$

$h=2.5 \log ($ He II narrow $)-2.5 \log ($ He II wide $)$

Absolute magnitude from a least-square fit on 20 stars of spectral type $\mathrm{O}$ :

$M_{V}=-67.927+43.756 n$
$M_{V}=-21.645+10.661 h$

Fig. 59. The photometric system $n h-$ Landolt -1970 


\section{Morguleff et al - 1970}

Classification and metallicity of cool stars.

\section{GENERAL INFORMATION}

AUTHORS N. Morguleff, M. P. Véron and D. Barbier

TELESCOPE $\quad 0.80 \mathrm{~m}$ (reflector), Haute-Provence Obs.

DETECTOR Lallemand photomultiplier

MAIN ARTICLE Morguleff, N., Véron, M. P. 1970, A\&A 4, 391

SYSTEM DESCRIPTION

\begin{tabular}{|c|c|c|}
\hline \multicolumn{3}{|c|}{ BANDS DESCRIPTION [214] } \\
\hline band & bandpass $(\AA)$ & feature \\
\hline$B j$ & $3575-3650$ & Balmer jump \\
\hline$K$ & $3925-3965$ & K Ca II \\
\hline$C N_{c}$ & $4010-4070$ & continuum \\
\hline$C N_{l}$ & $4170-4215$ & $\mathrm{CN}$ \\
\hline $\mathrm{CaI}$ & $4220-4260$ & $\mathrm{Ca} \mathrm{I}$ \\
\hline$H \beta_{l}$ & $4815-4905$ & $\mathrm{H} \beta$ \\
\hline$H \beta_{c}$ & $4920-5000$ & continuum \\
\hline$M g I_{l}$ & $5150-5200$ & Mg I \\
\hline$M g I_{c}$ & $5300-5350$ & continuum \\
\hline $\mathrm{Na}_{l}$ & $5840-5900$ & $\mathrm{Na} \mathrm{I}$ \\
\hline $\mathrm{Na}_{c}$ & $5900-6000$ & continuum \\
\hline
\end{tabular}

A mask-wheel is placed on the focal plane of a two fused silica prism spectrograph at the Coudé focus, and upon rotation trasmits in turn to the detector (a Lallemand cell) selected portions of the spectrum (in form of square bands).

Fig. 60. The photometric system Morguleff et al. - 1970 


\section{OAO2 WEP - 1970}

Winsconsin Experiment Package for ultraviolet photometry on board the OAO-2 satellite.

\section{GENERAL INFORMATION}

TELESCOPE $\quad 0.40 \mathrm{~m}$ and $0.20 \mathrm{~m}$ (reflectors)

DETECTOR photometer 1 and 2: EMI 6256B

photometer 3: Ascop 541F cesium telluride

photometer 4: Ascop 541G cesium iodide

MAIN ARTICLE Code, A. D., Houck, T. E., McNall, J. F., Bless, R. C., Lillie, C. F. 1970, ApJ 161, 377

\section{SYSTEM DESCRIPTION}

\begin{tabular}{|c|cc|}
\hline \multicolumn{3}{|c|}{ BANDS DESCRIPTION [70] } \\
\hline band & $\lambda_{\text {eff }}(\AA)(\#)$ & $\lambda$ at $50 \%$ trans. $(\AA)$ \\
\hline 133 & 1330 & $1185-1370$ \\
\hline 143 & 1430 & $1260-1500$ \\
\hline 155 & 1550 & $1410-1680$ \\
\hline 168 & 1680 & $1460-1730$ \\
\hline 191 & 1910 & $1760-2020$ \\
\hline 203 & 2030 & $1690-2180$ \\
\hline 213 & 2130 & $1930-2230$ \\
\hline 239 & 2390 & $2220-2550$ \\
\hline 246 & 2460 & $2290-2650$ \\
\hline 256 & 2560 & $2350-2770$ \\
\hline 294 & 2940 & $2740-3170$ \\
\hline 297 & 2970 & $2740-3170$ \\
\hline 298 & 2980 & $2760-3170$ \\
\hline 332 & 3320 & $3040-3560$ \\
\hline 333 & 3330 & $3050-3570$ \\
\hline 425 & 4250 & $3810-4670$ \\
\hline
\end{tabular}

Interference filters. (\#) For an Equal Energy Source.

\section{SYSTEM ANALISYS}

The magnitudes are defined by $m(\lambda)=-2.5 \log F_{\lambda}-21.10$

RELATIONS WITH OTHER SYSTEMS [71]

13 colors - Johnson and Mitchell - 1976

$m(332)=1.028( \pm 0.046) 33_{J-M}-0.0385( \pm 0.188)$

UBV - Johnson and Morgan - 1953

$m(425)=0.986( \pm 0.033) B+0.178( \pm 0.107)(B-V)-0.606( \pm 0.186)$

Fig. 61. The photometric system OAO2 WEP - 1970 


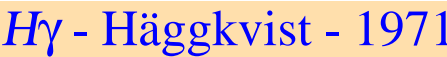

$\mathrm{H} \gamma$ in early-type stars.

\section{GENERAL INFORMATION}
AUTHORS
L. Häggkvist
TELESCOPE
$0.33 \mathrm{~m}$ refractor, Uppsala Obs.
MAIN ARTICLE
Häggkvist L. 1971, A\&A 12, 5

\section{SYSTEM DESCRIPTION}

\begin{tabular}{|c|ccc|}
\hline \multicolumn{4}{|c|}{ BANDS DESCRIPTION [131] } \\
\hline band & $\lambda_{\text {peak }}(\AA)$ & half-width $(\AA)$ & feature \\
\hline 41 & 4059 & 56 & continuum \\
\hline 44 & 4346 & 30 & $\mathrm{H} \gamma$ \\
\hline 45 & 4508 & 50 & continuum \\
\hline
\end{tabular}

Interference filters.

\section{SYSTEM ANALYSIS}

COLOR INDICES AND PARAMETERS [131]

$\gamma=44-0.666 .45-0.334 .41$ : measures the $\mathrm{H} \gamma$ absorption.

RELATIONS WITH OTHER SYSTEMS [131]

uvby $H \beta$ - Strömgren and Crawford - 1956

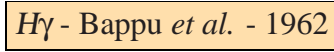

$\gamma=3.300-2.878 \beta+0.744 \beta^{2}$

$\beta=1.348+1.947 \gamma-0.553 \gamma^{2}$

$\gamma=1.455-0.00244 \Gamma$

$H \gamma$ - Beer - 1964

H $\gamma$ equivalent width from spectra

$\gamma=-0.025+0.803 R_{\gamma}$

\begin{tabular}{|rrr|}
\hline$\gamma=0.717+0.0385 E W_{\text {Petrie }}+0.00060 E W_{\text {Petrie }}^{2}$ & {$[244]$} \\
\hline$\gamma=0.749+0.0289 E W_{\text {Gunther }}$ & {$[126]$} \\
\hline$\gamma=0.757+0.0239 E W_{\text {Williams }}$ & {$[324]$} \\
\hline$\gamma=0.757+0.0246 E W_{\text {Stock }}$ & {$[283]$} \\
\hline
\end{tabular}

Fig. 62. The photometric system $\mathrm{H} \gamma$ - Häggkvist - 1971 


\section{DAO - Hill et al. - 1971}

General purpose system.

\section{GENERAL INFORMATION}

$\begin{array}{ll}\text { AUTHORS } & \text { G. Hill, S. C. Morris and G. A. H. Walker } \\ \text { TELESCOPE } & \text { 0.40m (reflector), Mt. Kobau, British Columbia; } \\ & \text { 0.40m (reflector), KPNO } \\ \text { DETECTOR } & \text { EMI 6256 SA (filter 35), EMI 6094 SA (filters 38,42,44), } \\ & \text { EMI 9558 QA (filters 54, 56) [211] } \\ \text { MAIN ARTICLE } & \text { Hill, G., Morris, S. C., Walker, G. A. H. 1971, AJ 76, 246 }\end{array}$

SYSTEM DESCRIPTION

\begin{tabular}{|c|c|}
\hline \multicolumn{2}{|c|}{ BANDS DESCRIPTION [140] } \\
\hline band & bandpass $(\AA)$ \\
\hline 35 & $3400-3600$ \\
\hline 38 & $3700-3850$ \\
\hline 42 & $3900-4400$ \\
\hline 44 & $3900-4900$ \\
\hline 54 & $5050-5770$ \\
\hline 56 & $5410-5770$ \\
\hline
\end{tabular}

The photometer consists of a small spectrograph that isolates four spectral bands $(35,38,44,54)$. The radiation from each region is diverted to a separate photomultiplier. A mask cutting-off the blue side of the the 54 band and the red side of the 44 band allows the realization of further two bands, 42 and 56.

ZERO POINT: $(44-54)=(42-56)=0.0$ for unreddened A0 V stars. $[140]$

\section{SYSTEM ANALYSIS}

RELATIONS WITH OTHER SYSTEMS [140]

UBV - Johnson and Morgan - 1953

$(B-V)_{\text {Johnson }}=1.270( \pm 0.007)(44-54)+0.000( \pm 0.003)$

Fig. 63. The photometric system DAO - Hill et al. - 1971 


\section{5 colors - Lockwood and Wing - 1971}

Measurements of $\mathrm{TiO}$ and $\mathrm{VO}$ bands in M stars. It is a partial realization with interference filters at KPNO of the 27 colors - Wing - 1967 system used at Lick on digitized spectra.

\section{GENERAL INFORMATION}

$\begin{array}{ll}\text { AUTHORS } & \text { G. W. Lockwood and R. F. Wing } \\ \text { TELESCOPE } & 0.91 \mathrm{~m} \text { and } 1.27 \mathrm{~m} \text { (reflectors), KPNO } \\ \text { DETECTOR } & \text { ITT FW-118 } \\ \text { MAIN ARTICLE } & \text { Lockwood, G. W., Wing, R. F. 1971, ApJ 169, 63 }\end{array}$

\section{SYSTEM DESCRIPTION}

\begin{tabular}{|r|cccl|}
\hline \multicolumn{5}{|c|}{ BANDS DESCRIPTION [189] } \\
\hline band & $\lambda_{c}(\AA)$ & FWHM $(\AA)$ & $\Upsilon_{c}(\%)$ & feature $[188]$ \\
\hline 78 & 7817 & 90 & 66 & TiO \\
\hline 87 & 8778 & 82 & 69 & Continuum \\
\hline 88 & 8884 & 114 & 50 & TiO \\
\hline 104 & 10351 & 125 & 51 & Continuum \\
\hline 105 & 10506 & 100 & 45 & VO \\
\hline
\end{tabular}

ZERO POINT: The fluxes in all the bands are linked to the system of absolute fluxes given by [135] and the zero point of the whole system is set by requiring that the magnitude of $\alpha$ Lyr in the 10400 band is 0.00 . [329]

\section{SYSTEM ANALYSIS}

\section{COLOR INDICES AND PARAMETERS [188]}

(78-87) : increases with $\mathrm{TiO}$ absorption and decreasing photospheric temperature.

(87-104) : temperature indicator.

$T_{1}=(78-87)-0.6(87-104):$ temperature independent index measuring TiO absorption in the 78 band.

$T_{2}=(88-87)+0.07(87-104):$ temperature independent index measuring TiO absorption in the 88 band.

$V_{1}=(105-104)+0.1(87-104):$ temperature independent index measuring VO absorption in the 105 band.

$D=0.18 T_{1}+T_{2}+V_{1}$ : increases monotonically from M0 to M10 (cfr. Fig 1 of [188]).

Fig. 64. The photometric system 5 colors - Lockwood and Wing - 1971 


\section{Mendoza - 1971}

Intensity of the OI $7774 \mathrm{~A}$ line.

\section{GENERAL INFORMATION}

AUTHORS

TELESCOPE

DETECTOR

E. V. Mendoza

$1.52 \mathrm{~m}$ (reector), University of Arizona

ITT FW-118 (S-1 cathode) [208]

MAIN ARTICLE Mendoza, E.V. 1971, Boletin de los observatorios de Tonantzintla y Tacubaya No 37, 6

\section{SYSTEM DESCRIPTION}

\begin{tabular}{|c|cc|}
\hline \multicolumn{3}{|c|}{ BANDS DESCRIPTION [209] } \\
\hline band & $\lambda_{\text {peak }}(\mathrm{A})$ & half-width (A) \\
\hline F1 & 7755 & 20 \\
\hline F2 & 7772 & 25 \\
\hline F3 & 7792 & 25 \\
\hline
\end{tabular}

Interference lters by Oriel Optics Corporation.

SYSTEM ANALYSIS

COLOR INDICES AND PARAMETERS [209]

$\Lambda=1 / 2[F 1+F 3]-F 2:$ OI intensity. Luminosity indicator.

TRANSMISSION CURVES

As derived from Fig 2 of [209].

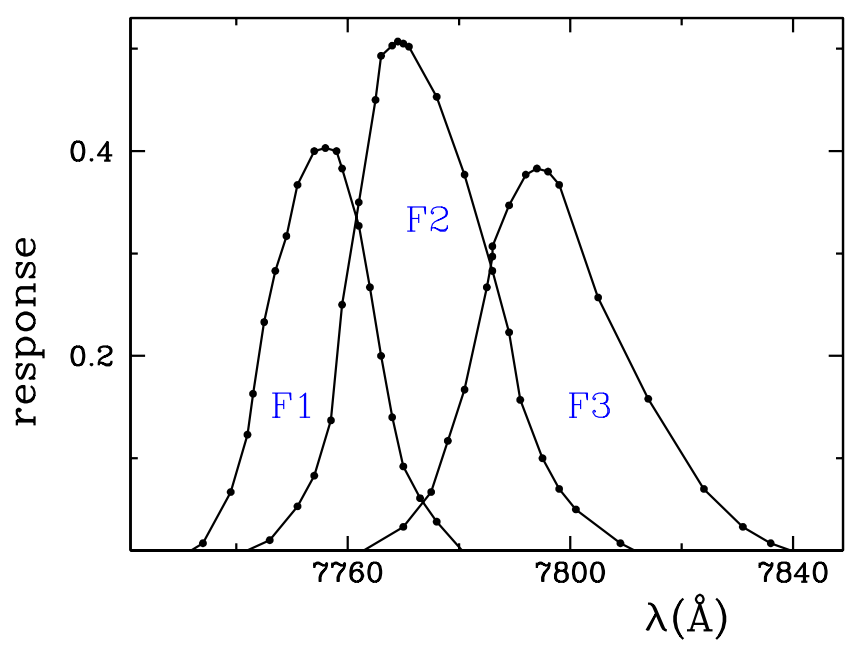

\begin{tabular}{|cc|cc|cc|}
\hline \multicolumn{2}{|c|}{$F 1$} & \multicolumn{2}{c|}{$F 2$} & \multicolumn{2}{c|}{$F 3$} \\
\hline$\lambda(\mathrm{A})$ & $\Upsilon$ & $\lambda(\mathrm{A})$ & $\Upsilon$ & $\lambda(\mathrm{A})$ & $\Upsilon$ \\
\hline 7729 & 0.000 & 7738 & 0.000 & 7754 & 0.000 \\
7734 & 0.017 & 7746 & 0.020 & 7762 & 0.007 \\
7739 & 0.067 & 7751 & 0.053 & 7770 & 0.033 \\
7742 & 0.123 & 7754 & 0.083 & 7775 & 0.067 \\
7743 & 0.163 & 7757 & 0.137 & 7778 & 0.117 \\
7745 & 0.233 & 7759 & 0.250 & 7781 & 0.167 \\
7747 & 0.283 & 7762 & 0.350 & 7785 & 0.267 \\
7749 & 0.317 & 7765 & 0.450 & 7786 & 0.297 \\
7751 & 0.367 & 7766 & 0.493 & 7786 & 0.307 \\
7754 & 0.400 & 7768 & 0.503 & 7789 & 0.347 \\
7756 & 0.403 & 7769 & 0.507 & 7792 & 0.377 \\
7758 & 0.400 & 7770 & 0.505 & 7794 & 0.383 \\
7759 & 0.383 & 7771 & 0.502 & 7796 & 0.380 \\
7762 & 0.327 & 7776 & 0.453 & 7798 & 0.367 \\
7764 & 0.267 & 7781 & 0.377 & 7805 & 0.257 \\
7766 & 0.200 & 7786 & 0.283 & 7814 & 0.158 \\
7768 & 0.140 & 7789 & 0.223 & 7824 & 0.070 \\
7770 & 0.092 & 7791 & 0.157 & 7831 & 0.033 \\
7773 & 0.061 & 7795 & 0.100 & 7836 & 0.017 \\
7776 & 0.038 & 7798 & 0.070 & 7841 & 0.007 \\
7781 & 0.007 & 7801 & 0.050 & 7892 & 0.000 \\
7783 & 0.000 & 7809 & 0.017 & & \\
& & 7814 & 0.003 & & \\
& & 7816 & 0.000 & & \\
\hline
\end{tabular}

Fig. 65. The photometric system Mendoza - 1971 


\section{Williams et al. - 1971}

Square-band photometry of FeII, CaI and BaII lines in late-type stars.

\section{GENERAL INFORMATION}

$\begin{array}{ll}\text { AUTHORS } & \text { P. M. Williams, D. W. Peat and M. E. Rego } \\ \text { TELESCOPE } & 0.91 \mathrm{~m} \text { (reflector), Cambridge Obs. } \\ \text { DETECTOR } & \text { EMI 9502 (refrigerated) } \\ \text { MAIN ARTICLE } & \text { Williams, P. M. 1971, MNRAS 153, 171 } \\ & \text { Rego, M. E., Williams, P. M., Peat, D. W. 1972, MNRAS 160, } 129 \\ & \text { Williams, P. M. 1975, MNRAS 170, } 343\end{array}$

\section{SYSTEM DESCRIPTION}

\begin{tabular}{|l|c|c|c|}
\hline \multicolumn{4}{|c|}{ BANDS DESCRIPTION } \\
\hline \multirow{2}{*}{ band } & \multicolumn{3}{|c|}{ bandpass $(\AA)$} \\
\cline { 2 - 4 } & FeII [325] & CaI [249] & BaII [326] \\
\hline$A$ & $5354.3-5368.3$ & $6106.4-6115.6$ & $6067.0-6077.0$ \\
\hline$B$ & $5427.8-5435.7$ & $6160.1-6168.0$ & $6140.4-6143.6$ \\
\hline$C$ & $5485.2-5496.3$ & $6201.6-6209.9$ & $6202.0-6212.0$ \\
\hline
\end{tabular}

On the focal plane of a $5.0 \AA \mathrm{Amm}$ dispersion spectrograph three diaphragms transmit to three photomultipliers the $A, B$ and $C$ portions of the spectrum.

Readings on a tungsten lamp are obtained to monitor the relative response of the three channels.

\section{SYSTEM ANALYSIS}

COLOR INDICES AND PARAMETERS [249], [325], [326]

If subscripts $s$ and 1 denotes observations of the stars and the lamp respectively, and $a, b, c$ are three constants, the line strength ratio is then:

$$
\begin{aligned}
& R=\frac{a A_{s} / A_{l}+c C_{s} / C_{l}}{b B_{s} / B_{l}}=\frac{a \Omega+c \Lambda}{b \Psi} \\
& R(F e)=\frac{0.45 \Omega_{F e}+0.55 \Lambda_{F e}}{\Psi_{F e}}: \text { measures the strength of FeII } 5435 \AA . \\
& R(C a)=\frac{0.44 \Omega_{C a}+0.56 \Lambda_{C a}}{\Psi_{C a}}: \text { measures the strength of CaI } 6162 \AA . \\
& R(B a)=0.5 \frac{\Omega_{B a}+\Lambda_{B a}}{\Psi_{B a}}: \text { measures the strength of BaII } 6141.7 \AA .
\end{aligned}
$$

Fig. 66. The photometric system Williams et al. - 1971 


\title{
8 colors - Wing - 1971
}

Measurements of TiO, $\mathrm{CN}$ and $\mathrm{VO}$ bands in late-type stars. It is a partial realization with interference filters at KPNO of the 27 colors - Wing - 1967 system devised at Lick on photoelectric spectral scans.

\section{GENERAL INFORMATION}

\author{
AUTHORS \\ TELESCOPE \\ R. F. Wing \\ DETECTOR \\ KPNO telescopes \\ MAIN ARTICLE Wing, R. F. 1971, in Proc. of the Conf. on Late-Type Stars, \\ (G. W. Lockwood and H. M. Dyck ed.s), KPNO Contr. 554, 145
}

\section{SYSTEM DESCRIPTION}

\begin{tabular}{|r|rcll|}
\hline \multicolumn{5}{|c|}{ BANDS DESCRIPTION [328] } \\
\hline band & $\lambda_{c}(\AA)$ & FWHM $(\AA)$ & \multicolumn{1}{c|}{ feature } & contaminants \\
\hline 71 & 7117 & 53 & TiO & CN \\
\hline 75 & 7545 & 50 & continuum (M0-M7) & CN, VO \\
\hline 78 & 7806 & 42 & continuum $(\mathrm{G}, \mathrm{K}, \mathrm{C})$ & $\mathrm{TiO}$ \\
\hline 81 & 8122 & 43 & $\mathrm{CN}$ & $\mathrm{TiO}$ \\
\hline 104 & 10392 & 55 & continuum & \\
\hline 105 & 10544 & 58 & VO & \\
\hline 108 & 10800 & 74 & continuum $(\mathrm{C})$ & HeI $(10830 \AA)$ \\
\hline 109 & 10968 & 73 & CN & \\
\hline
\end{tabular}

\begin{tabular}{|l|llll|}
\hline $65(\#)$ & 6510 & 40 & $\mathrm{ZrO}$ & $\mathrm{TiO}, \mathrm{CN}$ \\
\hline $79(\#)$ & 7945 & 50 & $\mathrm{LaO}$ & $\mathrm{TiO}, \mathrm{VO}, \mathrm{CN}$ \\
\hline
\end{tabular}

Interference filters by Infrared Industries.

(\#) These two bands have been introduced by [245] in a later variant of the system. [245] replaced the original 71 and 81 with the 65 and 79 bands to measure the strength of the $\mathrm{ZrO}$ and $\mathrm{LaO}$ absorptions in S stars.

Fig. 67. The photometric system 8 colors - Wing - 1971 


\section{Aerobee IR-71 - 1971}

Far-Infrared observations of the Galactic Center by an Aerobee rocket flown on July 16, 1971.

GENERAL INFORMATION

TELESCOPE $\quad 0.18 \mathrm{~m}$, far-infrared telescope (helium cooled)

MAIN ARTICLE Houck, J. R., Soifer, B. T., Pipher, J. L., Harwit, M. 1971, ApJ 169, L31

SYSTEM DESCRIPTION

\begin{tabular}{|c|rc|}
\hline \multicolumn{3}{|c|}{ BANDS DESCRIPTION [143] } \\
\hline band & $\lambda_{c}(\mu \mathrm{m})$ & half-width $(\mu \mathrm{m})$ \\
\hline$A$ & 5.5 & 1 \\
\hline$B$ & 13 & 2 \\
\hline$C$ & 19 & 7 \\
\hline$D$ & 100 & 30 \\
\hline
\end{tabular}

The radiation has been fully chopped by a turning-fork chopper placed in the telescope focal plane, and has been directed to the individual detectors (one for each band) through separate light pipes.

Fig. 68. The photometric system Aerobee IR-71 - 1971 


\section{Jones and Dixon - 1972}

Luminosity and metallicity of late-type stars.

\section{GENERAL INFORMATION}

$\begin{array}{ll}\text { AUTHORS } & \text { D.H. P. Jones and M. E. Dixon } \\ \text { TELESCOPE } & 0.41 \mathrm{~m} \text { and 1.02m (reflectors), Siding Spring Obs. } \\ \text { DETECTOR } & \text { 1P21 (refrigerated) and FW130 (S-20 cathode) } \\ \text { MAIN ARTICLE } & \text { Jones, D. H. P., Dixon, M. E. 1972, ApJ 177, } 665\end{array}$

SYSTEM DESCRIPTION

\begin{tabular}{|l|cc|}
\hline \multicolumn{3}{|c|}{ BANDS DESCRIPTION [158] } \\
\hline \multicolumn{1}{|c|}{ band } & $\lambda_{\text {peak }}(\AA)$ & half width $(\AA)$ \\
\hline$C N_{\text {narrow }}$ & 4189 & 50 \\
\hline$C N_{\text {wide }}$ & 4189 & 200 \\
\hline$M g_{\text {narrow }}$ & 5174 & 30 \\
\hline$M g_{\text {wide }}$ & 5174 & 140 \\
\hline
\end{tabular}

Interference filters.

\section{SYSTEM ANALYSIS}

COLOR INDICES AND PARAMETERS [158]

$$
[M g]=M g_{\text {wide }} / M g_{\text {narrow }} \quad[C N]=C N_{\text {wide }} / C N_{\text {narrow }}
$$

RELATIONS WITH OTHER SYSTEMS [158]

$$
\text { Griffin and Redman - } 1960
$$

$r_{C N}=1.16[C N]-1.94 \quad$ for 36 stars in common

Deeming - 1960

$r_{M g}=1.13[M g]-3.09 \quad$ for 36 stars in common

Fig. 69. The photometric system Jones and Dixon - 1972 


\section{H $\alpha, \beta$ - Peton et al. - 1972}

Narrow-band photometry of Be stars.

\section{GENERAL INFORMATION}

$\begin{array}{ll}\text { AUTHORS } & \text { A. Peton, J. H. Bigay, R. Garnier, and G. Paturel } \\ \text { TELESCOPE } & \text { 0.60m (reflector), Haute Provence Obs. } \\ \text { DETECTOR } & \text { EMI three-alkaline } \\ \text { MAIN ARTICLE } & \text { Peton, A., Bigay, J. H., Garnier, R., Paturel, G. 1972, A\&A 17, } 47\end{array}$

SYSTEM DESCRIPTION

\begin{tabular}{|c|ccc|}
\hline \multicolumn{4}{|c|}{ BANDS DESCRIPTION $[243]$} \\
\hline band & $\lambda_{c}(\AA)$ & bandpass $(\AA)$ & feature \\
\hline 1 & 4861 & 29 & $\mathrm{H} \beta$ narrow \\
\hline 2 & 4861 & 112 & $\mathrm{H} \beta$ wide \\
\hline 5 & 6350 & 164 & $\mathrm{H} \alpha$ continuum \\
\hline 3 & 6563 & 158 & $\mathrm{H} \alpha$ line \\
\hline 4 & 6645 & 89 & $\mathrm{H} \alpha$ continuum \\
\hline
\end{tabular}

Interference filters by Baird Atomic.

COLOR INDICES AND PARAMETERS [243]

$\beta=m_{1}-m_{2} \quad \alpha=m_{3}-1 / 2\left(m_{4}+m_{5}\right)$

Fig. 70. The photometric system $H \alpha, \beta$ - Peton -1972 


\section{TRANSMISSION CURVES}

As derived from Fig 1 of [243].
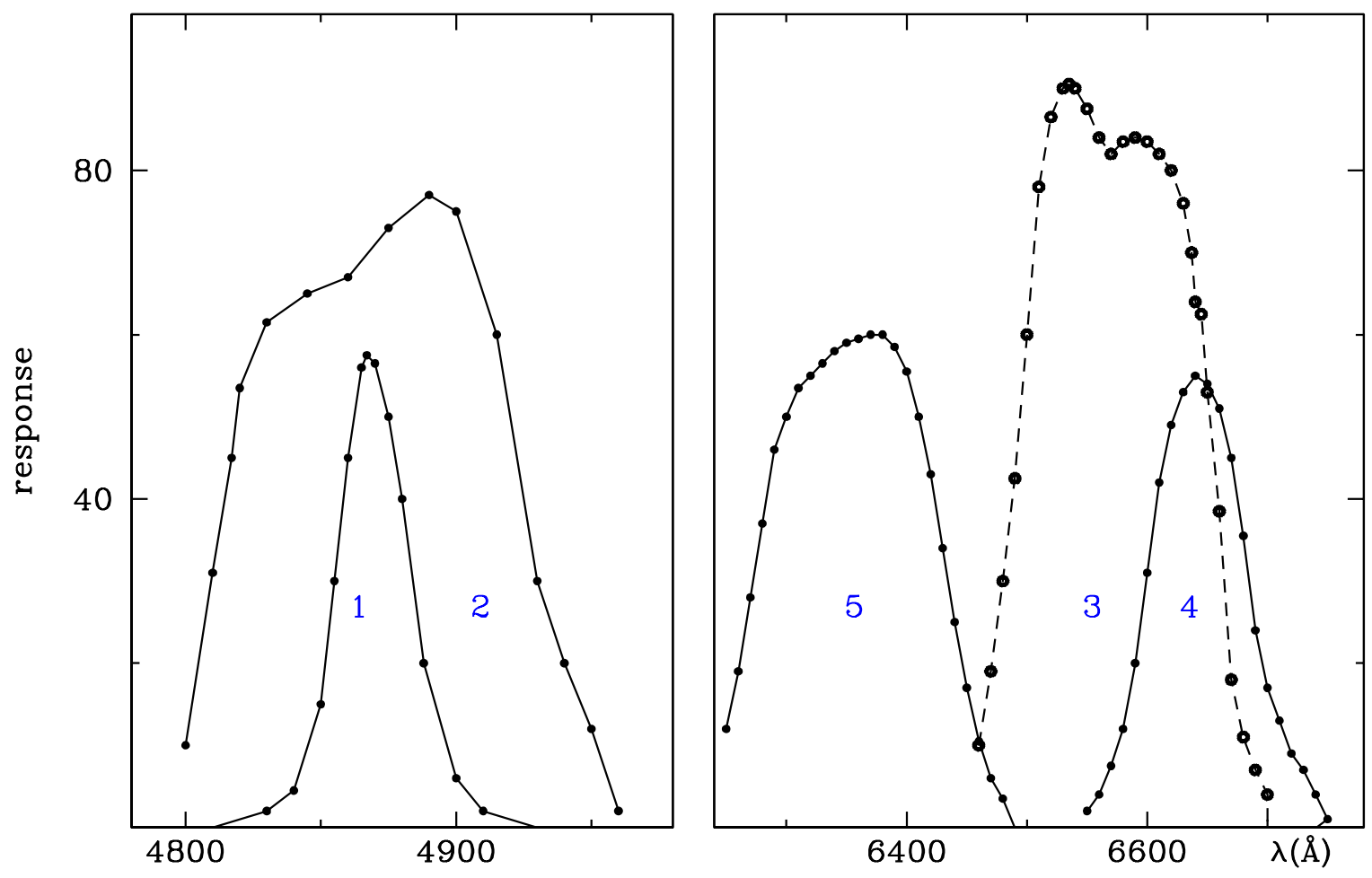

\begin{tabular}{|cc|cc|cc|cc|c|cc|cc|cc|c|c|}
\hline \multicolumn{2}{|c|}{1} & \multicolumn{3}{|c|}{2} & \multicolumn{3}{|c|}{5} & \multicolumn{4}{|c|}{4} & \multicolumn{4}{c|}{3} \\
\hline$\lambda(\mathrm{A})$ & $\Upsilon(\%)$ & $\lambda(\mathrm{A})$ & $\Upsilon(\%)$ & $\lambda(\mathrm{A})$ & $\Upsilon(\%)$ & $\lambda(\mathrm{A})$ & $\Upsilon(\%)$ & $\lambda(\mathrm{A})$ & $\Upsilon(\%)$ & $\lambda(\mathrm{A})$ & $\Upsilon(\%)$ & $\lambda(\mathrm{A})$ & $\Upsilon(\%)$ & $\lambda(\mathrm{A})$ & $\Upsilon(\%)$ \\
\hline 4810 & 0.0 & 4800 & 10.0 & 6250 & 12.0 & 6400 & 55.5 & 6550 & 2.0 & 6700 & 17.0 & 6460 & 10.0 & 6600 & 83.5 \\
4830 & 2.0 & 4810 & 31.0 & 6260 & 19.0 & 6410 & 50.0 & 6560 & 4.0 & 6710 & 13.0 & 6470 & 19.0 & 6610 & 82.0 \\
4840 & 4.5 & 4817 & 45.0 & 6270 & 28.0 & 6420 & 43.0 & 6570 & 7.5 & 6720 & 9.0 & 6480 & 30.0 & 6620 & 80.0 \\
4850 & 15.0 & 4820 & 53.5 & 6280 & 37.0 & 6430 & 34.0 & 6580 & 12.0 & 6730 & 7.0 & 6490 & 42.5 & 6630 & 76.0 \\
4855 & 30.0 & 4830 & 61.5 & 6290 & 46.0 & 6440 & 25.0 & 6590 & 20.0 & 6740 & 4.0 & 6500 & 60.0 & 6637 & 70.0 \\
4860 & 45.0 & 4845 & 65.0 & 6300 & 50.0 & 6450 & 17.0 & 6600 & 31.0 & 6750 & 1.0 & 6510 & 78.0 & 6640 & 64.0 \\
4865 & 56.0 & 4860 & 67.0 & 6310 & 53.5 & 6460 & 10.5 & 6610 & 42.0 & & & 6520 & 86.5 & 6645 & 62.5 \\
4867 & 57.5 & 4875 & 73.0 & 6320 & 55.0 & 6470 & 6.0 & 6620 & 49.0 & & & 6530 & 90.0 & 6650 & 53.0 \\
4870 & 56.5 & 4890 & 77.0 & 6330 & 56.5 & 6480 & 3.5 & 6630 & 53.0 & & & 6535 & 90.5 & 6660 & 38.5 \\
4875 & 50.0 & 4900 & 75.0 & 6340 & 58.0 & 6490 & 0.0 & 6640 & 55.0 & & & 6540 & 90.0 & 6670 & 18.0 \\
4880 & 40.0 & 4915 & 60.0 & 6350 & 59.0 & & & 6650 & 54.0 & & & 6550 & 87.5 & 6680 & 11.0 \\
4888 & 20.0 & 4930 & 30.0 & 6360 & 59.5 & & & 6660 & 51.0 & & & 6560 & 84.0 & 6690 & 7.0 \\
4900 & 6.0 & 4940 & 20.0 & 6370 & 60.0 & & & 6670 & 45.0 & & & 6570 & 82.0 & 6700 & 4.0 \\
4910 & 2.0 & 4950 & 12.0 & 6380 & 60.0 & & & 6680 & 35.5 & & & 6580 & 83.5 & 6708 & 4.0 \\
4930 & 0.0 & 4960 & 2.0 & 6390 & 58.5 & & & 6690 & 24.0 & & & 6590 & 84.0 & \\
\hline
\end{tabular}

Fig. 70. continued 


\section{$u_{A} b_{A} \beta_{A} y_{A}-$ Wickramasinghe and Strittmatter - 1972}

Temperature and surface gravity of DA white dwarfs.

\section{GENERAL INFORMATION}

AUTHORS D. T. Wickramasinghe, and P. A. Strittmatter

MAIN ARTICLE Wickramasinghe D. T., Strittmatter, P. A. 1972, MNRAS 160, 421

\section{SYSTEM DESCRIPTION}

\begin{tabular}{|c|cc|}
\hline \multicolumn{3}{|c|}{ BANDS DESCRIPTION [323] } \\
\hline band & $\lambda_{c}(\AA)$ & FWHM $(\AA)$ \\
\hline$u_{A}$ & 3500 & 300 \\
\hline$b_{A}$ & 4500 & 200 \\
\hline$\beta_{A}$ & 4860 & 400 \\
\hline$y_{A}$ & 5400 & 300 \\
\hline
\end{tabular}

Conceptual design for an optimized photometric system.

\section{SYSTEM ANALYSIS}

REDDENING FREE PARAMETERS [323]

$w_{A}=\left(b_{A}-\beta_{A}\right)-0.63\left(\beta_{A}-y_{A}\right) \quad d_{A}=\left(u_{A}-b_{A}\right)-1.89\left(b_{A}-y_{A}\right)$

Fig. 71. The photometric system $u_{A} b_{A} \beta_{A} y_{A}-$ Wickramasinghe and Strittmatter -1972 


\title{
TD1 - 1972
}

Ultraviolet photometry with the TD-1 satellite.

\section{GENERAL INFORMATION}

\author{
TELESCOPE $\quad 0.275 \mathrm{~m}$ \\ DETECTOR EMR 542 F-08-16 and EMR 542 G-08 ASCOP photomultipliers \\ MAIN ARTICLE Boksenberg, A., Evans, R.G., Fowler, R. G., Gardner, I. S. K., Honziaux, L., \\ Humphries, C. M., Jamar, C., Macau, D., Malaise, D., Monfils, A., Nandy, K., \\ Wroe, H. 1973, MNRAS 163, 291
}

\section{SYSTEM DESCRIPTION}

\begin{tabular}{|c|cc|c|cc|}
\hline \multicolumn{5}{|c|}{ BANDS DESCRIPTION $[60]$} \\
\hline band & $\lambda_{c}(\AA)$ & width $(\AA)$ & band & $\lambda_{c}(\AA)$ & width $(\AA)$ \\
\hline 140 & 1400 & 100 & 200 & 2000 & 100 \\
\hline 146 & 1455 & 110 & 208 & 2075 & 110 \\
\hline 150 & 1500 & 100 & 210 & 2100 & 100 \\
\hline 157 & 1565 & 110 & 220 & 2200 & 100 \\
\hline 157 & 1565 & 330 & 226 & 2255 & 110 \\
\hline 160 & 1600 & 100 & 230 & 2300 & 100 \\
\hline 168 & 1675 & 110 & 237 & 2365 & 110 \\
\hline 170 & 1700 & 100 & 237 & 2365 & 330 \\
\hline 180 & 1800 & 100 & 240 & 2400 & 100 \\
\hline 186 & 1855 & 110 & 247 & 2465 & 110 \\
\hline 190 & 1900 & 100 & 250 & 2500 & 100 \\
\hline 197 & 1965 & 110 & 274 & 2740 & 310 \\
\hline 197 & 1965 & 330 & & & \\
\hline
\end{tabular}

An ultraviolet telescope feeds in turn a spectrophotometer which gives low dispersion spectra over the range $1350-2550 \AA$ and a broad band photometer for measurements centered at 1505, 1965, 2365 and $2740 \AA$.

As the satellite spins a set of slits on the telescope focal plane realizes the spectral scanning and band delimitation.

The 21 narrow bands are synthesized from the $10 \AA$ resolution spectra.

\section{SYSTEM ANALYSIS}

\section{REDDENING-FREE PARAMETERS [60]}

The reddening-free parameter $\Phi$ makes use of the $V$ magnitude of $U B V$ - Johnson and Morgan - 1953.

$\Phi=(274-V)-1.57(237-274)$

RELATIONS and REDDENING RATIOS [60]

for $(157-237)_{0}<-0.70$, i.e. $\mathrm{O}$ to $\mathrm{B} 8 \mathrm{~V}$ stars:

$E(B-V)=0.49(157-274)-1.07(157-237)-0.34$

$(157-274)_{0}=0.09(157-274)+1.97(157-237)+0.63$

$(157-237)_{0}=0.04(157-274)+0.92(157-237)-0.03$ for $(157-237)_{0}>-0.70$, i.e. B8.5V to F2V stars:

$E(B-V)=0.51(157-274)-0.61(157-237)$

$(157-274)_{0}=0.05(157-274)+1.13(157-237)$

$(157-237)_{0}=0.04(157-274)+0.95(157-237)$

Fig. 72. The photometric system TD1 - 1972 


\section{4 colors - Chapman et al. - 1973}

Taxonomy of asteroids.

\section{GENERAL INFORMATIONS}

AUTHORS

TELESCOPE

DETECTOR

MAIN ARTICLE

\section{R. Chapman, B. McCord, and T. V. Johnson}

$5 \mathrm{~m}$ (reflector), Palomar Obs.; 0.61m and 1.52m (reflectors), Mt Wilson; $0.91 \mathrm{~m}$ (reflector), KPNO

S-1 and S-20 dry-ice-cooled ITT photomultipliers

Chapman, C. R., McCord, B., Johnson, T. V. 1973, AJ 78, 126

\section{SYSTEM DESCRIPTION}

\begin{tabular}{|r|ccccc|}
\hline \multicolumn{7}{|c|}{ BANDS DESCRIPTION [66] } \\
\hline band & $\lambda(\mu \mathrm{m})$ & bandpass $(\mu \mathrm{m})$ & $\Upsilon_{\text {peak }}$ & \multicolumn{2}{c|}{$\lambda_{\text {eff }}(\mu \mathrm{m})$} \\
\cline { 4 - 6 } & & & & $\mathrm{S}-20$ & $\mathrm{~S}-1$ \\
\hline (\#) 1 & 0.3010 & 0.019 & 0.13 & & 0.3100 \\
\hline 2 & 0.3196 & 0.016 & 0.19 & 0.3216 & 0.3229 \\
\hline 3 & 0.3383 & 0.022 & 0.14 & 0.3406 & 0.3412 \\
\hline 4 & 0.3590 & 0.014 & 0.18 & 0.3598 & 0.3593 \\
\hline 5 & 0.3831 & 0.013 & 0.23 & 0.3833 & 0.3825 \\
\hline 6 & 0.4019 & 0.028 & 0.48 & 0.4021 & 0.4015 \\
\hline 7 & 0.4344 & 0.028 & 0.42 & 0.4344 & 0.4340 \\
\hline 8 & 0.4687 & 0.033 & 0.49 & 0.4684 & 0.4685 \\
\hline 9 & 0.5001 & 0.029 & 0.57 & 0.4998 & 0.5001 \\
\hline 10 & 0.5336 & 0.033 & 0.46 & 0.5331 & 0.5336 \\
\hline 11 & 0.5662 & 0.029 & 0.55 & 0.5658 & 0.5663 \\
\hline 12 & 0.5993 & 0.032 & 0.54 & 0.5987 & 0.5994 \\
\hline 13 & 0.6328 & 0.032 & 0.56 & 0.6322 & 0.6326 \\
\hline 14 & 0.6649 & 0.027 & 0.50 & 0.6645 & 0.6649 \\
\hline 15 & 0.6991 & 0.026 & 0.54 & 0.6986 & 0.6992 \\
\hline 16 & 0.7299 & 0.033 & 0.51 & 0.7287 & 0.7300 \\
\hline 17 & 0.7640 & 0.031 & 0.57 & 0.7628 & 0.7640 \\
\hline 18 & 0.8078 & 0.049 & 0.46 & 0.8016 & 0.8072 \\
\hline 19 & 0.8551 & 0.047 & 0.48 & 0.8499 & 0.8551 \\
\hline 20 & 0.9063 & 0.048 & 0.58 & & 0.9058 \\
\hline 21 & 0.9475 & 0.051 & 0.59 & & 0.9469 \\
\hline 22 & 1.0036 & 0.050 & 0.53 & & 1.0025 \\
\hline 23 & 1.0548 & 0.050 & 0.55 & & 1.0525 \\
\hline 24 & 1.1033 & 0.049 & 0.44 & & 1.1009 \\
\hline & & & & & \\
\hline
\end{tabular}

(\#) Not used after November 1970.

Interference filters used in a double-beam photometer.

Fig. 73. The photometric system 24 colors - Chapman et al. - 1973 


\section{0 colors - Faber - 1973}

Old stellar populations in globular clusters and galaxies.

Combines bands from DDO - McClure and Van den Bergh - 1968 and 12 colors - Wood - 1966 systems.

\section{GENERAL INFORMATION}
AUTHORS
S. M. Faber
TELESCOPE
$0.40 \mathrm{~m}$ and $0.91 \mathrm{~m}$ (reflectors), KPNO
DETECTOR
ITT FW-130 (S-20 cathode, refrigerated)
MAIN ARTICLE Faber, S. M. 1973, A\&AS 10, 201

\section{SYSTEM DESCRIPTION}

\begin{tabular}{|l|lccl|}
\hline \multicolumn{5}{|c|}{ BANDS DESCRIPTION [101] } \\
\hline band & source & $\lambda_{\text {eff }}(\AA)$ & half-width $(\AA)$ & feature \\
\hline 35 & DDO, Wood & 3490 & 372 & Balmer jump \\
\hline 38 & DDO & 3800 & 172 & Balmer head \\
\hline 41 & DDO & 4166 & 83 & $(\#)$ \\
\hline 42 & DDO & 4257 & 73 & $(\#)$ \\
\hline 45 & DDO & 4517 & 76 & $(\#)$ \\
\hline 52 & Wood & 5169 & 56 & $\mathrm{MgH}+\mathrm{Mg}_{b}$ \\
\hline 55 & Wood & 5470 & 233 & continuum \\
\hline 62 & Wood & 6220 & 79 & TiO \\
\hline 67 & Wood & 6696 & 155 & continuum \\
\hline 74 & Wood & 7331 & 100 & continuum \\
\hline
\end{tabular}

(\#) These three bands are aimed to measure the line blanketing discontinuity, CN absorption and the G-band.

\section{SYSTEM ANALYSIS}

RELATIONS WITH OTHER SYSTEMS [101]

For stars with $(B-V)_{\text {Johnson }}<1.30$ :

DDO - McClure and Van den Bergh - 1968

\begin{tabular}{|l}
\hline$(35-41)_{D D O}=0.977( \pm 0.007)(35-41)_{\text {Faber }}-0.815( \pm 0.003)$ \\
\hline$(38-41)_{D D O}=-0.067( \pm 0.017)(38-41)_{\text {Faber }}^{2}+1.048( \pm 0.035)(38-41)_{\text {Faber }}-0.116( \pm 0.004)$ \\
\hline$(41-42)_{D D O}=+0.139( \pm 0.222)(41-42)_{\text {Faber }}^{2}+0.879( \pm 0.017)(41-42)_{\text {Faber }}+0.040( \pm 0.000)$ \\
\hline$(42-45)_{D D O}=-0.009( \pm 0.005)(42-45)_{\text {Faber }}^{2}+0.971( \pm 0.007)(42-45)_{\text {Faber }}+0.321( \pm 0.000)$ \\
\hline$(45-48)_{D D O}=+0.007( \pm 0.010)(45-55)_{\text {Faber }}^{2}+0.486( \pm 0.007)(45-55)_{\text {Faber }}+0.859( \pm 0.001)$ \\
\hline
\end{tabular}

12 colors - Wood - 1966

\begin{tabular}{|l}
\hline$(35-55)_{\text {Wood }}=-0.001( \pm 0.000)(35-55)_{\text {Faber }}^{2}+1.001( \pm 0.014)(35-55)_{\text {Faber }}+0.092( \pm 0.018)$ \\
\hline$(48-55)_{\text {Wood }}=+0.050( \pm 0.023)(45-55)_{\text {Faber }}^{2}+0.715( \pm 0.029)(45-55)_{\text {Faber }}+0.032( \pm 0.002)$ \\
\hline$(52-55)_{\text {Wood }}=-0.028( \pm 0.117)(52-55)_{\text {Faber }}^{2}+0.877( \pm 0.052)(52-55)_{\text {Faber }}+0.005( \pm 0.001)$ \\
\hline$(62-55)_{\text {Wood }}=-0.214( \pm 0.002)(62-55)_{\text {Faber }}^{2}+1.100( \pm 0.046)(62-55)_{\text {Faber }}-0.001( \pm 0.001)$ \\
\hline$(67-55)_{\text {Wood }}=+0.052( \pm 0.084)(67-55)_{\text {Faber }}^{2}+1.118( \pm 0.028)(67-55)_{\text {Faber }}-0.009( \pm 0.001)$ \\
\hline$(74-55)_{\text {Wood }}=-0.040( \pm 0.018)(74-55)_{\text {Faber }}^{2}+1.006( \pm 0.021)(74-55)_{\text {Faber }}-0.078( \pm 0.002)$ \\
\hline
\end{tabular}

Fig. 74. The photometric system 10 colors - Faber - 1973 


\section{JHKL SAAO - Glass - 1973}

Realization at South African Astronomical Observatory of the infrared bands of the UBVRI(JHKLMN) - Johnson - 1965 system.

\section{GENERAL INFORMATION}

AUTHORS

TELESCOPE

DETECTOR

MAIN ARTICLE

\section{S. Glass}

1.0m (reector), Sutherland; $1.93 \mathrm{~m}$ Radcliffe reector , Pretoria $\mathrm{PbS}$ cell

Glass, I. S. 1973, MNRAS 164, 155

\begin{tabular}{|c|cc|}
\hline \multicolumn{3}{|c|}{ BANDS DESCRIPTION } \\
\hline band & $W H M(\mu \mathrm{m})$ & $\lambda_{c}(\mu \mathrm{m})$ \\
\hline$J$ & 0.286 & 1.229 \\
\hline$H$ & 0.323 & 1.657 \\
\hline$K$ & 0.380 & 2.227 \\
\hline$L$ & 0.613 & 3.442 \\
\hline$M$ & 0.531 & 5.076 \\
\hline$N$ & 7.11 & 11.88 \\
\hline$Q$ & 10.31 & 20.84 \\
\hline
\end{tabular}

\section{SYSTEM ANALYSIS}

RELATIONS WITH OTHER SYSTEMS [114]

MSSO - Thomas et al. - 1973

\begin{tabular}{|lll|}
\hline$J_{S A A O}$ & $=J_{M S S O}+0.05( \pm 0.01)$ & 2 stars in common \\
\hline$H_{S A A O}$ & $=H_{M S S O}+0.13( \pm 0.01)$ & 2 stars in common,$\alpha$ Cet excluded \\
\hline$K_{S A A O}$ & $=K_{M S S O}+0.06( \pm 0.01)$ & 4 stars in common \\
\hline$L_{S A A O}$ & $=L_{M S S O}+0.01( \pm 0.03)$ & 4 stars in common \\
\hline
\end{tabular}

\section{TRANSMISSION CURVES}

As derived from Fig 3 of [113].
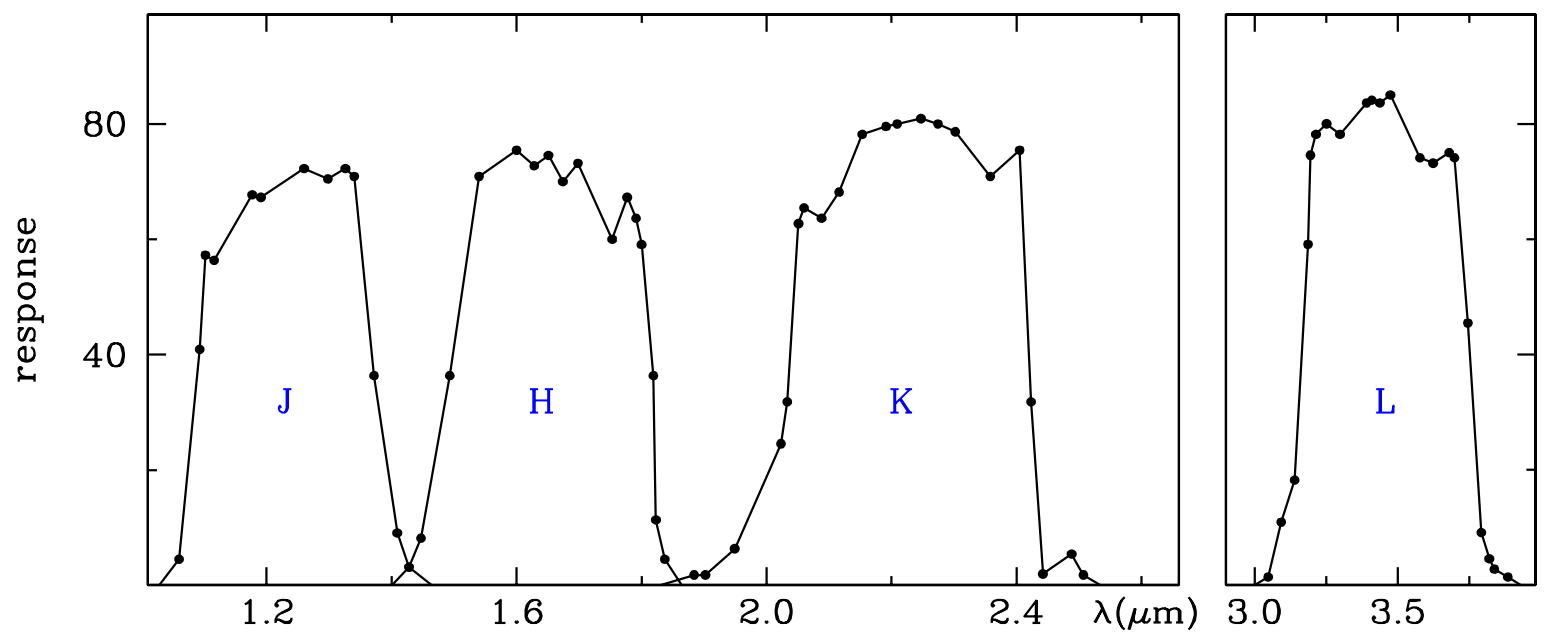

Fig. 75. The photometric system JHKL SAAO - Glass - 1973 

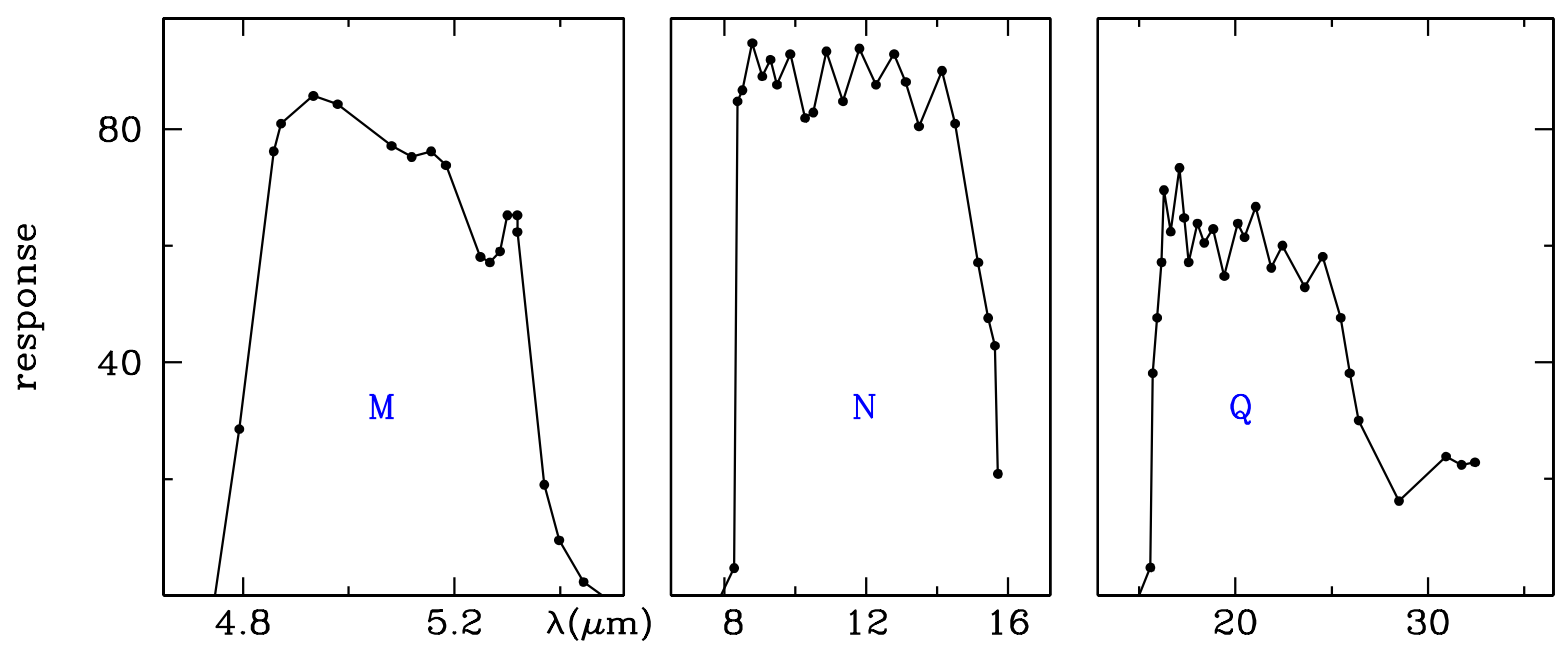

\begin{tabular}{|c|c|c|c|c|c|c|c|c|c|c|c|c|c|}
\hline \multicolumn{2}{|c|}{$J$} & \multicolumn{2}{|c|}{$H$} & \multicolumn{2}{|c|}{$K$} & \multicolumn{2}{|c|}{$L$} & \multicolumn{2}{|c|}{$M$} & \multicolumn{2}{|c|}{$N$} & \multicolumn{2}{|c|}{$Q$} \\
\hline$\lambda(\mu \mathrm{m})$ & $\Upsilon$ & $\lambda(\mu \mathrm{m})$ & $\Upsilon$ & $\lambda(\mu \mathrm{m})$ & $\Upsilon$ & $\lambda(\mu \mathrm{m})$ & $\Upsilon$ & $\lambda(\mu \mathrm{m})$ & $\Upsilon$ & $\lambda(\mu \mathrm{m})$ & $\Upsilon$ & $\lambda(\mu \mathrm{m})$ & $\Upsilon$ \\
\hline 1.028 & 0.00 & 1.400 & 0.00 & 1.828 & 0.00 & 3.000 & 0.00 & 4.747 & 0.00 & 7.907 & 0.00 & 15.00 & 0.00 \\
\hline 1.060 & 4.54 & 1.428 & 3.18 & 1.884 & 1.82 & 3.047 & 1.36 & 4.793 & 28.57 & 8.279 & 4.76 & 15.58 & 4.76 \\
\hline 1.093 & 40.91 & 1.447 & 8.18 & 1.902 & 1.82 & 3.093 & 10.91 & 4.858 & 76.19 & 8.372 & 84.76 & 15.70 & 38.10 \\
\hline 1.102 & 57.27 & 1.493 & 36.36 & 1.949 & 6.36 & 3.140 & 18.18 & 4.872 & 80.95 & 8.512 & 86.67 & 15.93 & 47.62 \\
\hline 1.116 & 56.36 & 1.540 & 70.91 & 2.023 & 24.55 & 3.186 & 59.09 & 4.933 & 85.71 & 8.791 & 94.76 & 16.16 & 57.14 \\
\hline 1.177 & 67.73 & 1.600 & 75.45 & 2.033 & 31.82 & 3.195 & 74.55 & 4.979 & 84.29 & 9.070 & 89.05 & 16.28 & 69.52 \\
\hline 1.191 & 67.27 & 1.628 & 72.73 & 2.051 & 62.73 & 3.214 & 78.18 & 5.081 & 77.14 & 9.302 & 91.90 & 16.63 & 62.38 \\
\hline 1.260 & 72.27 & 1.651 & 74.55 & 2.060 & 65.45 & 3.251 & 80.00 & 5.119 & 75.24 & 9.488 & 87.62 & 17.09 & 73.33 \\
\hline 1.298 & 70.45 & 1.674 & 70.00 & 2.088 & 63.64 & 3.298 & 78.18 & 5.156 & 76.19 & 9.860 & 92.86 & 17.33 & 64.76 \\
\hline 1.326 & 72.27 & 1.698 & 73.18 & 2.116 & 68.18 & 3.391 & 83.64 & 5.184 & 73.81 & 10.28 & 81.90 & 17.56 & 57.14 \\
\hline 1.340 & 70.91 & 1.753 & 60.00 & 2.153 & 78.18 & 3.409 & 84.09 & 5.249 & 58.10 & 10.51 & 82.86 & 18.02 & 63.81 \\
\hline 1.372 & 36.36 & 1.777 & 67.27 & 2.191 & 79.55 & 3.437 & 83.64 & 5.267 & 57.14 & 10.88 & 93.33 & 18.37 & 60.48 \\
\hline 1.409 & 9.09 & 1.791 & 63.64 & 2.209 & 80.00 & 3.474 & 85.00 & 5.286 & 59.05 & 11.35 & 84.76 & 18.84 & 62.86 \\
\hline 1.428 & 3.18 & 1.800 & 59.09 & 2.247 & 80.91 & 3.577 & 74.09 & 5.300 & 65.24 & 11.81 & 93.81 & 19.42 & 54.76 \\
\hline 1.465 & 0.00 & 1.819 & 36.36 & 2.274 & 80.00 & 3.623 & 73.18 & 5.319 & 65.24 & 12.28 & 87.62 & 20.12 & 63.81 \\
\hline & & 1.823 & 11.36 & 2.302 & 78.64 & 3.679 & 75.00 & 5.319 & 62.38 & 12.79 & 92.86 & 20.47 & 61.43 \\
\hline & & 1.837 & 4.54 & 2.358 & 70.91 & 3.698 & 74.09 & 5.370 & 19.05 & 13.12 & 88.10 & 21.05 & 66.67 \\
\hline & & 1.865 & 0.00 & 2.405 & 75.45 & 3.744 & 45.45 & 5.398 & 9.52 & 13.49 & 80.48 & 21.86 & 56.19 \\
\hline & & & & 2.423 & 31.82 & 3.791 & 9.09 & 5.444 & 2.38 & 14.14 & 90.00 & 22.44 & 60.00 \\
\hline & & & & 2.442 & 2.00 & 3.819 & 4.54 & 5.481 & 0.00 & 14.51 & 80.95 & 23.60 & 52.86 \\
\hline & & & & 2.488 & 5.45 & 3.837 & 2.73 & & & 15.16 & 57.14 & 24.53 & 58.10 \\
\hline & & & & 2.507 & 1.82 & 3.884 & 1.36 & & & 15.44 & 47.62 & 25.47 & 47.62 \\
\hline & & & & 2.535 & 0.00 & 3.930 & 0.00 & & & 15.63 & 42.86 & 25.93 & 38.10 \\
\hline & & & & & & & & & & 15.72 & 20.95 & 26.40 & 30.00 \\
\hline & & & & & & & & & & & & 28.49 & 16.19 \\
\hline & & & & & & & & & & & & 30.93 & 23.81 \\
\hline & & & & & & & & & & & & 31.74 & 22.38 \\
\hline & & & & & & & & & & & & 32.44 & 22.86 \\
\hline
\end{tabular}

Fig. 75. continued 


\section{WBVR - Straižys - 1973}

Generale purpose system.

\section{GENERAL INFORMATION}

$\begin{array}{ll}\text { AUTHORS } & \text { V. Straižys } \\ \text { TELESCOPE } & 0.25 m \text { (reflector), Moletai Obs. } \\ \text { DETECTOR } & \text { FZU 106 (S-20 cathode) [178], pg. 64 } \\ \text { MAIN ARTICLE } & \text { Straižys, V. 1973, A\&A 28, 349 }\end{array}$

SYSTEM DESCRIPTION

\begin{tabular}{|l|lrr|rr|}
\hline \multicolumn{5}{|c|}{ BANDS DESCRIPTION [178], pg. 64 } & \\
\hline band & \multicolumn{1}{|c|}{ filter } & $\lambda_{0}(\AA)$ & half-width $(\AA)$ & $W H M(\AA)$ & $\lambda_{c}(\AA)$ \\
\hline$W$ & $3.0 \mathrm{~mm} \mathrm{UFS2}+1.5 \mathrm{~mm}$ BS 5 (\#) & 3500 & 510 & 480 & 3545 \\
\hline$B$ & $2.0 \mathrm{~mm} \mathrm{ZhS} \mathrm{10+2.0mm} \mathrm{SS} \mathrm{5} \mathrm{+} \mathrm{1.7mm} \mathrm{SZS} \mathrm{21}$ & 4435 & 950 & 885 & 4355 \\
\hline$V$ & $3.0 \mathrm{~mm} \mathrm{ZhS} \mathrm{18+1.7mm} \mathrm{SZS} \mathrm{21}$ & 5540 & 800 & 860 & 5435 \\
\hline$R$ & $5.0 \mathrm{~mm} \mathrm{KS} \mathrm{14}$ & 6930 & 900 & 1160 & 6945 \\
\hline
\end{tabular}

(\#) Filter for suppressing the red leak: $\mathrm{CuSO}_{4} \cdot 5 \mathrm{H}_{2} \mathrm{O}$ crystal plate. Alternative filter for $W$ : Schott UG11 + BG32 or Corning 5840.

ZERO POINT: Color indices are 0.00 for unreddened O-type stars. [207]

Fig. 76. The photometric system $W B V R-$ Straižys -1973 


\section{TRANSMISSION CURVES [211]}

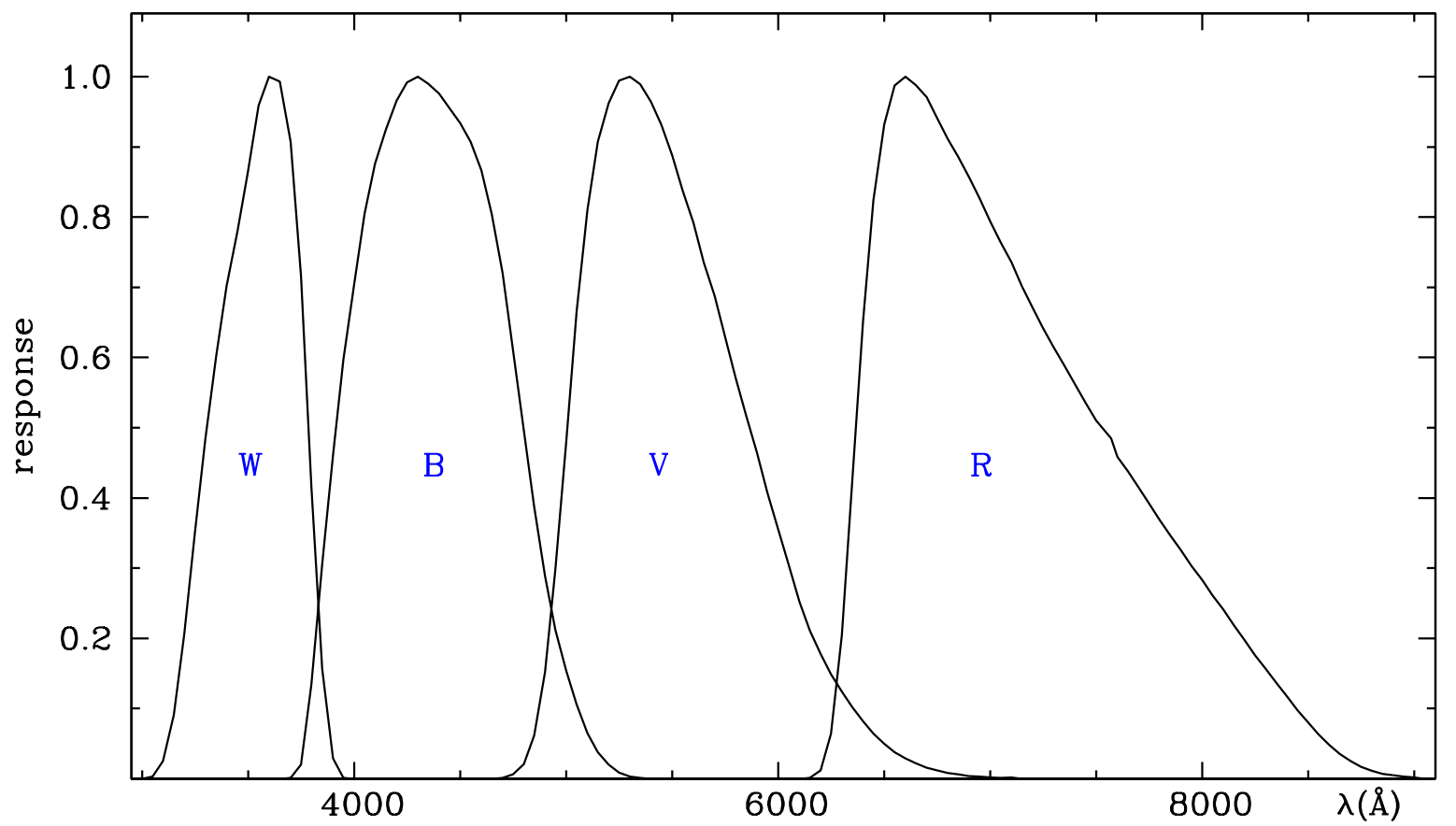

\begin{tabular}{|c|c|c|c|c|c|c|c|c|c|c|c|c|c|c|c|}
\hline \multicolumn{2}{|c|}{$W$} & \multicolumn{4}{|c|}{$B$} & \multicolumn{4}{|c|}{$V$} & \multicolumn{6}{|c|}{$R$} \\
\hline$\lambda(\mathrm{A})$ & $\Upsilon$ & $\lambda(\mathrm{A})$ & $\Upsilon$ & $\lambda(\mathrm{A})$ & $\Upsilon$ & $\lambda(\mathrm{A})$ & $\Upsilon$ & $\lambda(\mathrm{A})$ & $\Upsilon$ & $\lambda(\mathrm{A})$ & $\Upsilon$ & $\lambda(\mathrm{A})$ & $\Upsilon$ & $\lambda(\mathrm{A})$ & $\Upsilon$ \\
\hline 3000 & 0.000 & 3600 & 0.000 & 5000 & 0.154 & 4650 & 0.000 & 6050 & 0.305 & 6050 & 0.000 & 7450 & 0.536 & 8850 & 0.007 \\
\hline 3100 & 0.025 & 3700 & 0.001 & 5100 & 0.065 & 4750 & 0.006 & 6150 & 0.211 & 6150 & 0.001 & 7570 & 0.485 & 8950 & 0.003 \\
\hline 3150 & 0.091 & 3750 & 0.020 & 5150 & 0.038 & 4800 & 0.021 & 6200 & 0.178 & 6200 & 0.012 & 7600 & 0.458 & 9000 & 0.002 \\
\hline 3300 & 0.484 & 3900 & 0.461 & 5300 & 0.003 & 4950 & 0.299 & 6350 & 0.102 & 6350 & 0.423 & 7750 & 0.392 & & \\
\hline 3350 & 0.602 & 3950 & 0.597 & 5350 & 0.001 & 5000 & 0.480 & 6400 & 0.082 & 6400 & 0.651 & 7800 & 0.368 & & \\
\hline 3400 & 0.702 & 4000 & 0.705 & 5400 & 0.000 & 5050 & 0.665 & 6450 & 0.064 & 6450 & 0.824 & 7850 & 0.347 & & \\
\hline 3450 & 0.779 & 4050 & 0.805 & 5450 & 0.000 & 5100 & 0.810 & 6500 & 0.050 & 6500 & 0.932 & 7900 & 0.325 & & \\
\hline 3500 & 0.863 & 4100 & 0.876 & & & 5150 & 0.907 & 6550 & 0.038 & 6550 & 0.987 & 7950 & 0.303 & & \\
\hline 3700 & 0.907 & 4300 & 1.000 & & & 5350 & 0.989 & 6750 & 0.012 & 6750 & 0.941 & 8150 & 0.219 & & \\
\hline 3750 & 0.716 & 4350 & 0.990 & & & 5400 & 0.964 & 6800 & 0.008 & 6800 & 0.911 & 8200 & 0.197 & & \\
\hline 3800 & 0.416 & 4400 & 0.976 & & & 5450 & 0.931 & 6850 & 0.006 & 6850 & 0.886 & 8250 & 0.176 & & \\
\hline 3850 & 0.156 & 4450 & 0.955 & & & 5500 & 0.888 & 6900 & 0.004 & 6900 & 0.857 & 8300 & 0.156 & & \\
\hline 3900 & 0.029 & 4500 & 0.934 & & & 5550 & 0.838 & 6950 & 0.003 & 6950 & 0.827 & 8350 & 0.137 & & \\
\hline 3950 & 0.001 & 4550 & 0.907 & & & 5600 & 0.792 & 7000 & 0.002 & 7000 & 0.794 & 8400 & 0.117 & & \\
\hline 4000 & 0.000 & 4600 & 0.866 & & & 5650 & 0.735 & 7050 & 0.001 & 7050 & 0.764 & 8450 & 0.098 & & \\
\hline 4050 & 0.000 & 4650 & 0.804 & & & 5700 & 0.688 & 7100 & 0.002 & 7100 & 0.736 & 8500 & 0.080 & & \\
\hline & & 4700 & 0.720 & & & 5750 & 0.630 & 7150 & 0.000 & 7150 & 0.702 & 8550 & 0.063 & & \\
\hline
\end{tabular}

Fig. 76. continued 


\section{MSSO - Thomas et al. - 1973}

General purpose infrared system.

\section{GENERAL INFORMATION}

\section{AUTHORS \\ J. A. Thomas, A. R. Hyland, and G. Robinson}

TELESCOPE

$1.25 \mathrm{~m}$ (reflector), Mount Stromlo and Siding Spring Obs.

MAIN ARTICLE Thomas, J. A., Hyland, A. R., Robinson, G. 1973, MNRAS 165, 201

\section{SYSTEM DESCRIPTION}

\begin{tabular}{|c|c|c|}
\hline \multicolumn{2}{|c|}{ BANDS DESCRIPTION [300] } & FLUX CALIBRATION [300] \\
\hline band & $\lambda_{\text {eff }}(\mu \mathrm{m})$ & $\mathrm{F}_{\lambda}\left(\mathrm{W} \mathrm{cm}^{-2} \mu \mathrm{m}^{-1}\right) \quad(\#)$ \\
\hline$V$ & 0.548 & $3.6410^{-12}$ \\
\hline$R$ & 0.7 & $1.7410^{-12}$ \\
\hline$I$ & 0.9 & $8.4910^{-13}$ \\
\hline$J$ & 1.25 & $3.0310^{-13}$ \\
\hline$H$ & 1.65 & $1.1710^{-13}$ \\
\hline$K$ & 2.2 & $4.0210^{-14}$ \\
\hline$L$ & 3.6 & $6.1810^{-15}$ \\
\hline$M$ & 4.8 & $2.1310^{-15}$ \\
\hline 8.4 & 8.4 & $2.3110^{-16}$ \\
\hline$N$ & 10.2 & $1.0510^{-16}$ \\
\hline 11.2 & 11.2 & $7.4610^{-17}$ \\
\hline
\end{tabular}

(\#) For the model by [260] of $\alpha$ Lyr normalized to the $V$ flux measured by [236].

ZERO POINT: The colors are 0.00 for the [260] model of $\alpha$ Lyr.

colors of the Sun: $(V-R)=0.52, \quad(V-I)=0.80, \quad(V-J)=1.17$,

$$
\begin{aligned}
& (V-H)=1.52, \quad(V-K)=1.52, \quad(V-L)=1.55, \\
& (V-M)=1.53, \quad(V-8.4)=1.55,(V-N)=1,57, \quad(V-11.2)=1.56 . \quad[300]
\end{aligned}
$$

Fig. 77. The photometric system MSSO - Thomas et al. - 1973 


\section{Alexander and Branch - 1974}

Narrow-band measurement of $\mathrm{C}_{2}$ absorption bands in G-K stars.

\section{GENERAL INFORMATION}

$\begin{array}{ll}\text { AUTHORS } & \text { J. B. Alexander and D. Branch } \\ \text { TELESCOPE } & \text { 0.91m Yapp reflector, Herstmonceux, UK } \\ \text { DETECTOR } & \text { dual-channel photometer } \\ \text { MAIN ARTICLE } & \text { Alexander, J. B., Branch, D. 1974, MNRAS 167, 539 }\end{array}$

SYSTEM DESCRIPTION

\begin{tabular}{|l|ccc|}
\hline \multicolumn{4}{|c|}{ BANDS DESCRIPTION [3] } \\
\hline band & $\lambda_{\text {peak }}(\AA)$ & half width $(\AA)$ & $\Upsilon_{\text {peak }}(\%)$ \\
\hline$A$ & 4564 & 66 & 55 \\
\hline$B$ & 4682 & 74 & 60 \\
\hline$C$ & 4797 & 72 & 57 \\
\hline
\end{tabular}

Interference filters.

A beam splitter divides the incident starlight into two beams: a main beam in which measurements are made using the three filters $A, B$ and $C$ in turn, and a monitor beam to record the sky transparency with a broad band blue filter.

\section{SYSTEM ANALYSIS}

\section{COLOR INDICES AND PARAMETERS [3]}

If $I_{M}$ is the intensity from the monitor beam, and $r_{i}=I_{i} / I_{M}$, then:

$c=2 r_{B} /\left(r_{A}+r_{C}\right):$ measures the absorption by the $\mathrm{C}_{2}$ band.

Fig. 78. The photometric system Alexander and Branch - 1974 


\section{UBVR - Cathey - 1974}

General purpose system. Photometry in globular clusters.

\section{GENERAL INFORMATION}

AUTHORS

TELESCOPE

L. R. Cathey

$0.91 \mathrm{~m}$ Crossley (reflector), Lick Obs.;

$1.50 \mathrm{~m}$ and $0.91 \mathrm{~m}$ (reflectors), CTIO; $1.27 \mathrm{~m}$ (reflector), KPNO

DETECTOR

FW-129 (S-11 cathode), FW-130 (S-20 cathode), and 1P21 (S-4 cathode)

MAIN ARTICLE

\section{SYSTEM DESCRIPTION}

\begin{tabular}{|c|c|c|}
\hline \multicolumn{3}{|c|}{ BANDS DESCRIPTION [63] } \\
\hline band & photocathode & filter \\
\hline \multicolumn{3}{|c|}{ Lick Obs. } \\
\hline$U$ & FW-129 (S-11) & $\begin{array}{l}1.0 \mathrm{~mm} \mathrm{UG} 1 \\
6.5 \mathrm{~mm} \mathrm{CuSO}_{4}\end{array}$ \\
\hline$B$ & FW-129 (S-11) & $\begin{array}{l}\text { 2.0mm GG-13 } \\
1.0 \mathrm{~mm} \text { BG-12 } \\
4.5 \mathrm{~mm} \text { Quartz }\end{array}$ \\
\hline V & FW-129 (S-11) & $\begin{array}{l}\text { 2.0mm GG-14 } \\
5.5 \mathrm{~mm} \mathrm{GG}-13\end{array}$ \\
\hline V & FW-130 (S-20) & $\begin{array}{l}\text { 2.0mm GG-14 } \\
\text { 2.0mm BG-18 }\end{array}$ \\
\hline$R$ & FW-130 (S-20) & $\begin{array}{l}2.0 \mathrm{~mm} \text { RG-2 } \\
2.0 \mathrm{~mm} \text { GG-13 }\end{array}$ \\
\hline \multicolumn{3}{|c|}{ KPNO and CTIO } \\
\hline $\begin{array}{l}U \\
B\end{array}$ & $\begin{array}{l}\text { 1P21 (S-4) (\#) } \\
\text { 1P21 (S-4) (\#) }\end{array}$ & $\begin{array}{l}\text { 2.0mm Corning } 9863 \\
\text { 2.0mm Corning } 5030 \\
\text { 2.0mm GG-13 }\end{array}$ \\
\hline V & 1P21 (S-4) (\#) & 2.0mm Corning 3384 \\
\hline Red-leak of $U(\# \#)$ & 1P21 (S-4) (\#) & 2.0mm Corning $9863+$ RG-1 \\
\hline
\end{tabular}

(\#) A FW-129 (S-11) photocathode can be used instead.

(\#\#) To measure the red-leak of the $U$ band that in the KPNO/CTIO version has no red-leak suppressor.

Fig. 79. The photometric system UBVR - Cathey - 1974 


\section{H $\alpha, \beta, \gamma$ - Feinstein - 1974}

Balmer lines in early-type stars.

\section{GENERAL INFORMATION}

$\begin{array}{ll}\text { AUTHORS } & \text { A. Feinstein } \\ \text { TELESCOPE } & 0.41 \mathrm{~m} \text { (reflector), KPNO; 0.41m and 0.91m (reflectors), CTIO } \\ \text { DETECTOR } & 1 \mathrm{P} 21 \\ \text { MAIN ARTICLE } & \text { Feinstein, A. 1974, MNRAS 169, 171 }\end{array}$

SYSTEM DESCRIPTION

\begin{tabular}{|c|c|c|c|c|}
\hline \multicolumn{5}{|c|}{ BANDS DESCRIPTION [105] } \\
\hline band & filter & $\lambda_{\text {peak }}(\AA)$ & half-width $(\AA)$ & $\Upsilon_{\text {peak }}(\%)$ \\
\hline \multicolumn{5}{|c|}{ Filters at KPNO } \\
\hline Ho wide & KPNO 210 & 6595 & 159 & 88 \\
\hline Ho narrow & KPNO 71 & 6575 & 54 & 47 \\
\hline$H \beta$ wide & KPNO 214 & 4900 & 150 & 70 \\
\hline H $\beta$ narrow & KPNO 212 & 4861 & 31 & 62 \\
\hline$H \gamma$ wide & KPNO 49 & 4385 & 160 & 70 \\
\hline$H \gamma$ narrow & KPNO 48 & 4340 & 31 & 56 \\
\hline \multicolumn{5}{|c|}{ Filters at CTIO } \\
\hline Ho wide & KPNO 210 & 6595 & 159 & 88 \\
\hline Ho narrow & KPNO 71 & 6575 & 54 & 47 \\
\hline$H \beta$ wide & LP & 4880 & 200 & 51 \\
\hline H $\beta$ narrow & LP & 4865 & 45 & 32 \\
\hline 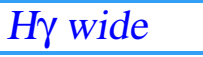 & LP & 4340 & 170 & 60 \\
\hline$H \gamma$ narrow & LP & 4340 & 50 & 25 \\
\hline
\end{tabular}

Interference filters.

\section{SYSTEM ANALYSIS}

COLOR INDICES AND PARAMETERS [105]

$$
\alpha=-2.5 \log \left(\frac{H \alpha \text { narrow }}{H \alpha \text { wide }}\right) \quad \beta=-2.5 \log \left(\frac{H \beta \text { narrow }}{H \beta \text { wide }}\right) \quad \gamma=-2.5 \log \left(\frac{H \gamma \text { narrow }}{H \gamma \text { wide }}\right)
$$

RELATIONS WITH OTHER SYSTEMS [105]

$$
\begin{gathered}
H \gamma \text { - Beer - } 1964 \\
R_{\gamma}=1.40 \gamma-1.86 \\
\hline
\end{gathered}
$$

$$
\begin{array}{rl|}
H \alpha \text { - Andrews - } 1968 \\
\hline R_{\alpha}=3.08 \alpha-3.42 \\
\hline
\end{array}
$$

$$
\text { Ho, } \beta \text { - Tebbe - } 1969
$$$$
\alpha_{\text {Tebbe }}=1.15 \alpha+0.085
$$

Fig. 80. The photometric system $H \alpha, \beta, \gamma-$ Feinstein - 1974 


\section{UBVRI - Fernie - 1974}

Realization with a single photocathode of the optical bands of the UBVRI(JHKLM) - Johnson - 1965 system. Later revised by Moffett and Barnes [213].

\section{GENERAL INFORMATION}

$\begin{array}{ll}\text { AUTHORS } & \text { J. D. Fernie } \\ \text { TELESCOPE } & 0.61 \mathrm{~m} \text { (reflector), David Dunlap Observatory } \\ \text { DETECTOR } & \text { EMI 9658R (refrigerated) } \\ \text { MAIN ARTICLE } & \text { Fernie, J. D. 1974, PASP 86, 837 }\end{array}$

SYSTEM DESCRIPTION

\begin{tabular}{|c|c|}
\hline \multicolumn{2}{|c|}{ BANDS DESCRIPTION [106] } \\
\hline band & filter \\
\hline$U$ & $2 \mathrm{~mm} \mathrm{UG} 2+2 \mathrm{~mm}$ BG18 \\
\hline$B$ & $4 \mathrm{~mm} \mathrm{BG} 12+2 \mathrm{~mm} \mathrm{BG18}+1 \mathrm{~mm} \mathrm{GG} 4$ \\
\hline$V$ & $3 \mathrm{~mm} \mathrm{GG} 14+2 \mathrm{~mm} \mathrm{BG18}$ \\
\hline$R$ & $2 \mathrm{~mm} \mathrm{OG} 550+1 \mathrm{~mm} \mathrm{RG} 6$ \\
\hline$I$ & $1 \mathrm{~mm} \mathrm{BG} 3+2 \mathrm{~mm} \mathrm{RG610}$ \\
\hline
\end{tabular}

ZERO POINT: Color indices are 0.00 for unreddened A0 V stars. [213]

\section{SYSTEM ANALYSIS}

RELATIONS WITH OTHER SYSTEMS [213]

UBVRI(JHKLMN) - Johnson - 1965

\begin{tabular}{|l}
$(B-V)=-0.0044+1.0702(B-V)_{\text {Johnson }}-0.0553(B-V)_{\text {Johnson }}^{2}$ \\
$(V-R)=+0.05+0.82(V-R)_{\text {Johnson }}$
\end{tabular}

Fig. 81. The photometric system UBVRI - Fernie - 1974 


\section{H $\alpha, \beta$ - Guinan and McCook - 1974}

Narrow-band photometry of Balmer lines.

GENERAL INFORMATION

$\begin{array}{ll}\text { AUTHORS } & \text { E. F. Guinan and G. P. McCook } \\ \text { TELESCOPE } & \text { 0.38m Villanova University } \\ \text { DETECTOR } & \text { EMI 9558 QB (unrefrigerated) } \\ \text { MAIN ARTICLE } & \text { Guinan, E.F., McCook, G. P. 1974, PASP 86, } 947\end{array}$

SYSTEM DESCRIPTION

\begin{tabular}{|l|ccc|}
\hline \multicolumn{4}{|c|}{ BANDS DESCRIPTION $[125]$} \\
\hline \multicolumn{1}{|c|}{ band } & $\lambda_{\text {peak }}(\AA)$ & half-width $(\AA)$ & $\Upsilon_{\text {peak }}(\%)$ \\
\hline$H \alpha$ wide & 6575 & 295 & 83 \\
\hline$H \alpha$ narrow & 6565 & 36 & 74 \\
\hline$H \beta$ wide & 4870 & 180 & 77 \\
\hline$H \beta$ narrow & 4863 & 25 & 70 \\
\hline
\end{tabular}

Interference filters.

\section{SYSTEM ANALYSIS}

COLOR INDICES AND PARAMETERS [20]

$H_{\alpha}=-2.5 \log \left(\frac{H \alpha \text { narrow }}{H \alpha \text { wide }}\right)+$ const.

$H_{\beta}=-2.5 \log \left(\frac{H \beta \text { narrow }}{H \beta \text { wide }}\right)+$ const.

Fig. 82. The photometric system $H \alpha, \beta$ - Guinan and McCook - 1974 


\title{
NQ - Low and Rieke - 1974
}

General purpose infrared photometric system.

\section{GENERAL INFORMATION}

\author{
AUTHORS F. J. Low and G. H. Rieke \\ TELESCOPE $\quad 1.54 \mathrm{~m}$ (reector), Catalina station of the Lun. Plan. Lab. \\ DETECTOR Ge:Ga bolometer \\ MAIN ARTICLE Low, F. J., Rieke, G. H. 1794, Methods of Experimental Physics \\ Vol. 12, Part A (N. Carleton ed., Academic, New York), pg. 456
}

\section{SYSTEM DESCRIPTION}

\begin{tabular}{|c|cc|cc|}
\hline \multicolumn{3}{|c|}{ BANDS DESCRIPTION [251] } & \multicolumn{2}{c|}{ FLUX CALIBRATION (\#) } \\
\hline band & $\lambda_{\text {eff }}(\mu \mathrm{m})$ & bandpass $(\mu \mathrm{m})$ & $\mathrm{F}_{\lambda, 0}\left(\mathrm{~W} \mathrm{~cm}^{-2} \mu \mathrm{m}^{-1}\right)$ & $\mathrm{F}_{\mathrm{v}, 0}(\mathrm{Jy})$ \\
\hline$Q$ & 10.6 & $8-13$ & $9.610^{-17}$ & $36.0 \pm 1.2$ \\
\hline$N$ & 21 & $17-25$ & $6.410^{-18}$ & $9.4 \pm 0.5$ \\
\hline
\end{tabular}

(\#) For a 0.0 mag star. [251]

ZERO POINT: $K_{\text {Johnson }}-N=K_{\text {Johnson }}-Q=0.00$ for $\alpha$ Lyr. [251]

\section{TRANSMISSION CURVES}

As derived from Fig 1 and 2 of [251].

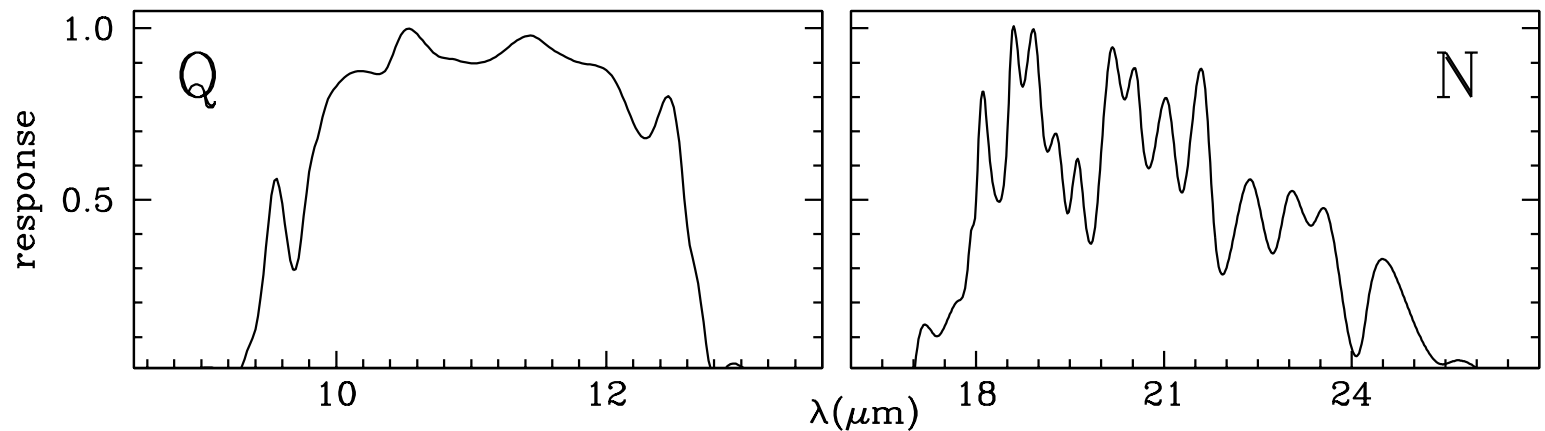

\begin{tabular}{|c|c|c|c|c|c|c|c|c|c|c|c|c|c|c|c|}
\hline \multicolumn{8}{|c|}{$Q$} & \multicolumn{8}{|c|}{$N$} \\
\hline$\lambda(\mu \mathrm{m})$ & $\Upsilon$ & $\lambda(\mu \mathrm{m})$ & $\Upsilon$ & $\lambda(\mu \mathrm{m})$ & $\Upsilon$ & $\lambda(\mu \mathrm{m})$ & $\Upsilon$ & $\lambda(\mu \mathrm{m})$ & $\Upsilon$ & $\lambda(\mu \mathrm{m})$ & $\Upsilon$ & $\lambda(\mu \mathrm{m})$ & $\Upsilon$ & $\lambda(\mu \mathrm{m})$ & $\Upsilon$ \\
\hline 9.17 & 0.000 & 10.16 & 0.875 & 11.55 & 0.952 & 12.72 & 0.149 & 17.01 & 0.000 & 18.45 & 0.562 & 20.16 & 0.941 & 22.77 & 0.348 \\
\hline 9.34 & 0.060 & 10.39 & 0.893 & 11.79 & 0.902 & 12.89 & 0.013 & 17.35 & 0.102 & 18.56 & 0.946 & 20.58 & 0.838 & 23.36 & 0.424 \\
\hline 9.40 & 0.119 & 10.45 & 0.952 & 11.90 & 0.893 & 13.07 & 0.000 & 17.71 & 0.204 & 18.72 & 0.838 & 20.64 & 0.716 & 23.57 & 0.475 \\
\hline 9.69 & 0.292 & 10.77 & 0.917 & 12.49 & 0.789 & & & 18.00 & 0.511 & 19.31 & 0.680 & 21.28 & 0.521 & 24.58 & 0.317 \\
\hline 9.81 & 0.595 & 10.85 & 0.911 & 12.54 & 0.655 & & & 18.03 & 0.613 & 19.47 & 0.460 & 21.65 & 0.838 & 24.80 & 0.235 \\
\hline 9.86 & 0.685 & 10.94 & 0.902 & 12.57 & 0.536 & & & 18.13 & 0.803 & 19.62 & 0.619 & 21.81 & 0.409 & 25.44 & 0.020 \\
\hline 9.92 & 0.774 & 11.20 & 0.923 & 12.60 & 0.417 & & & 18.19 & 0.685 & 19.73 & 0.460 & 22.02 & 0.317 & 25.65 & 0.031 \\
\hline 9.98 & 0.821 & 11.47 & 0.976 & 12.66 & 0.298 & & & 18.35 & 0.496 & 19.89 & 0.409 & 22.40 & 0.557 & 26.08 & 0.000 \\
\hline
\end{tabular}

Fig. 83. The photometric system NQ - Low and Rieke - 1974 


\section{H $\alpha$ - Vidal - 1974}

Photometry of diffuse nebulae.

\section{GENERAL INFORMATION}

$\begin{array}{ll}\text { AUTHORS } & \text { J. -L. Vidal } \\ \text { TELESCOPE } & \text { 0.60m (reector), Pic-du-Midi Obs. } \\ \text { DETECTOR } & \text { EMI 9558 B (S-20 cathode, refrigerated) } \\ \text { MAIN ARTICLE } & \text { Vidal, J.-L. 1974, A\&A 34, 401 }\end{array}$

SYSTEM DESCRIPTION

\begin{tabular}{|ll|cccc|}
\hline \multicolumn{6}{|c|}{ BANDS DESCRIPTION [309] } \\
\hline & band & lter & $\lambda_{\text {peak }}(\mathrm{A})$ & FWHM (A) & $\Upsilon_{\text {peak }}(\%)$ \\
\hline $\mathrm{H} \alpha$ & continuum & interference & 6435.7 & 10.7 & 77.4 \\
\hline $\mathrm{H} \alpha$ & line & interference & 6562.2 & 10.5 & 56 \\
\hline
\end{tabular}

The light from the two beams is switched 100 times per second onto the same photomultiplier.

\section{TRASMISSION CURVES}

As derived from description given in [309].

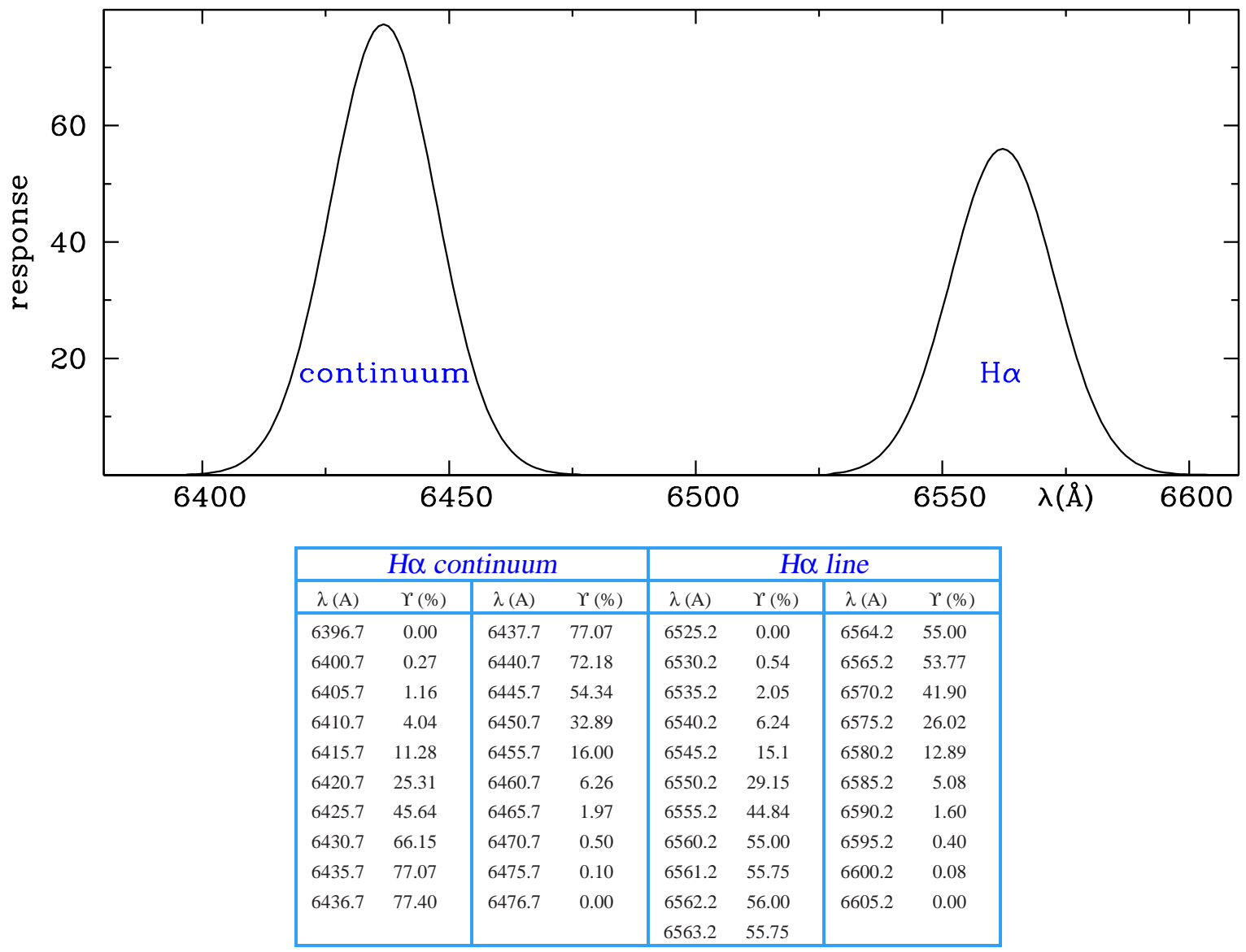

Fig. 84. The photometric system $\mathrm{H} \alpha-$ Vidal -1974 


\section{ANS - 1974}

Ultraviolet photometry from the Astronomical Netherland Satellite.

\section{GENERAL INFORMATION}

\section{TELESCOPE $\quad 0.22 \mathrm{~m}$ (reflector)}

MAIN ARTICLE Wesselius, P. R., van Duinen, R. J., Aalders, J. W.,

Kester, D. 1980, A\&A 85, 221

\section{SYSTEM DESCRIPTION}

\begin{tabular}{|l|cc|c|}
\hline \multicolumn{3}{|c|}{ BANDS DESCRIPTION [318] } & FLUX CALIBRATION (\#) [318] \\
\hline band & $\lambda_{c}(\AA)$ & bandwidth $(\AA)$ & $\mathrm{F}_{\lambda}\left(\mathrm{erg} \mathrm{cm}^{-2} \mathrm{~s}^{-1} \AA^{-1}\right)$ \\
\hline $15 N$ & 1545 & 50 & $16.7010^{-14}$ \\
\hline $15 W$ & 1549 & 149 & $5.9310^{-14}$ \\
\hline 18 & 1799 & 149 & $7.0510^{-14}$ \\
\hline 22 & 2200 & 200 & $5.2310^{-14}$ \\
\hline 25 & 2493 & 150 & $10.4910^{-14}$ \\
\hline 33 & 3294 & 101 & $6.5210^{-14}$ \\
\hline
\end{tabular}

(\#) Corresponding to 1 count in the given band.

Slits on the focal plane of a spectrograph feed light to separate photomultipliers, one for each band.

\section{SYSTEM ANALYSIS}

REDDENING RATIOS [318]

$$
\begin{array}{ll}
E(15 N-V)=5.13 E(B-V) & E(15 W-V)=5.13( \pm 0.48) E(B-V) \\
E(18-V)=4.74( \pm 0.42) E(B-V) & E(22-V)=6.46( \pm 0.47) E(B-V) \\
E(25-V)=3.99( \pm 0.37) E(B-V) & E(33-V)=1.92( \pm 0.24) E(B-V)
\end{array}
$$

RELATIONS WITH OTHER SYSTEMS [46]

\begin{tabular}{rl|}
\hline $33_{A N S}=$ & $R-1.680( \pm 0.007)$ \\
& $\mathrm{TD} 1-1972$ \\
\hline $15_{A N S}=$ & $157_{T D 1}+0.073( \pm 0.007)$ \\
\hline $18_{A N S}=$ & $180_{T D 1}-0.023( \pm 0.005)$ \\
\hline $22_{A N S}=$ & $220_{T D 1}-0.073( \pm 0.007)$ \\
\hline $25_{A N S}=$ & $250_{T D 1}-0.055( \pm 0.005)$ \\
\hline
\end{tabular}




\section{TRANSMISSION CURVES}

As derived from Fig 1 of [318].

Response are in units of photon count ${ }^{-1} \mathrm{~cm}^{-2}$.

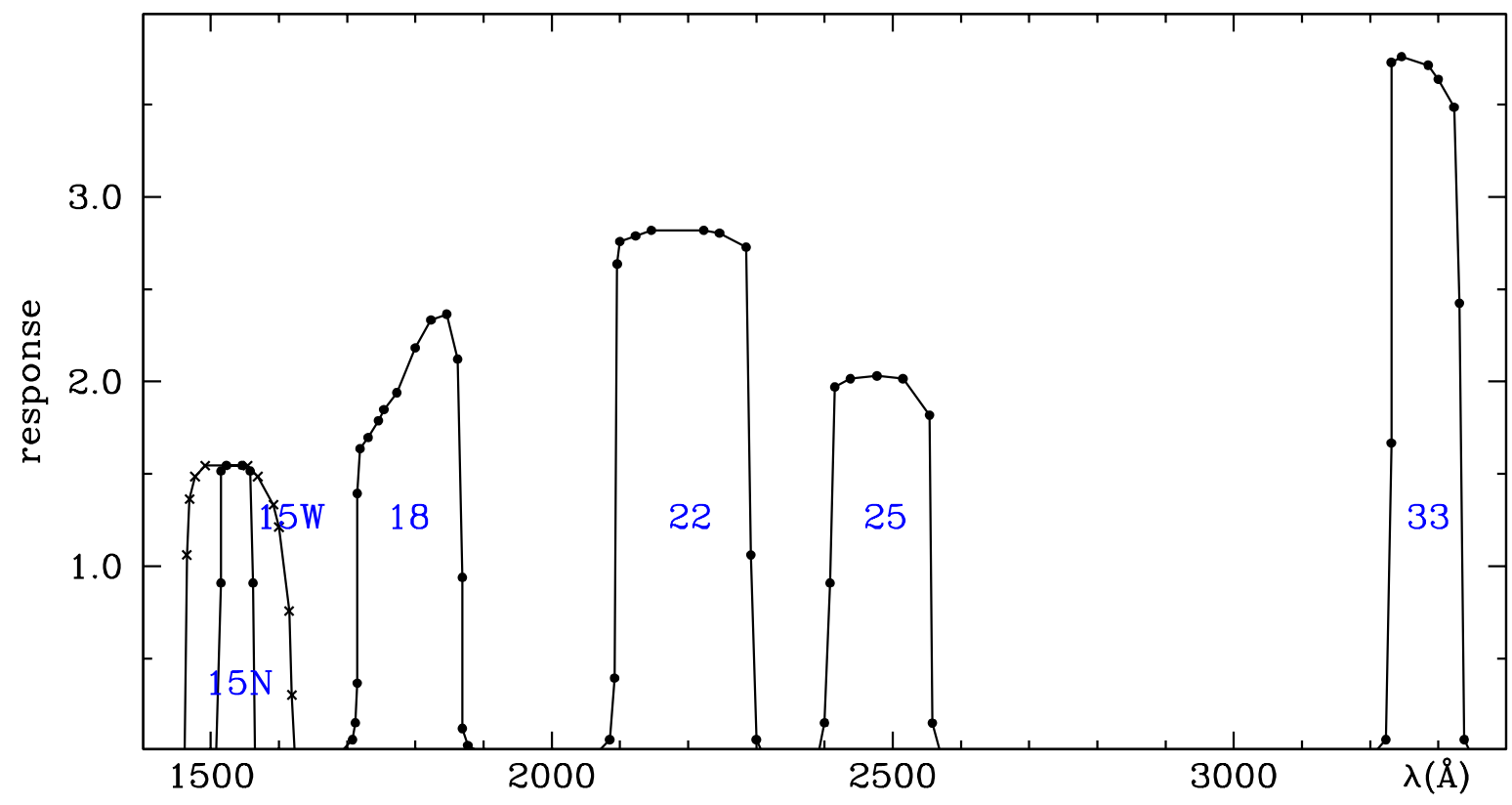

\begin{tabular}{|c|c|c|c|c|c|c|c|c|c|c|c|}
\hline \multicolumn{2}{|c|}{$15 \mathrm{~N}$} & \multicolumn{2}{|c|}{$15 \mathrm{~W}$} & \multicolumn{2}{|c|}{18} & \multicolumn{2}{|c|}{22} & \multicolumn{2}{|c|}{25} & \multicolumn{2}{|c|}{33} \\
\hline$\lambda(\mathrm{A})$ & $\Upsilon$ & $\lambda(\mathrm{A})$ & $\Upsilon$ & $\lambda(\mathrm{A})$ & $\Upsilon$ & $\lambda(\mathrm{A})$ & $\Upsilon$ & $\lambda(\mathrm{A})$ & $\Upsilon$ & $\lambda(\mathrm{A})$ & $\Upsilon$ \\
\hline 1508 & 0.000 & 1462 & 0.000 & 1692 & 0.000 & 2069 & 0.000 & 2392 & 0.000 & 3208 & 0.000 \\
\hline 1515 & 0.909 & 1465 & 1.061 & 1708 & 0.061 & 2085 & 0.061 & 2400 & 0.152 & 3223 & 0.061 \\
\hline 1515 & 1.515 & 1469 & 1.364 & 1712 & 0.152 & 2092 & 0.394 & 2408 & 0.909 & 3231 & 1.667 \\
\hline 1523 & 1.545 & 1477 & 1.485 & 1715 & 0.367 & 2096 & 2.636 & 2415 & 1.970 & 3231 & 3.727 \\
\hline 1546 & 1.545 & 1492 & 1.545 & 1715 & 1.394 & 2100 & 2.758 & 2438 & 2.015 & 3246 & 3.758 \\
\hline 1558 & 1.515 & 1554 & 1.545 & 1719 & 1.636 & 2123 & 2.788 & 2477 & 2.030 & 3285 & 3.712 \\
\hline 1562 & 0.909 & 1569 & 1.485 & 1731 & 1.697 & 2146 & 2.818 & 2515 & 2.015 & 3300 & 3.636 \\
\hline 1565 & 0.000 & 1592 & 1.333 & 1746 & 1.788 & 2223 & 2.818 & 2554 & 1.818 & 3323 & 3.485 \\
\hline & & 1600 & 1.212 & 1754 & 1.848 & 2246 & 2.803 & 2558 & 0.151 & 3331 & 2.424 \\
\hline & & 1615 & 0.758 & 1773 & 1.939 & 2285 & 2.727 & 2569 & 0.000 & 3338 & 0.061 \\
\hline & & 1619 & 0.303 & 1800 & 2.182 & 2292 & 1.061 & & & 3346 & 0.000 \\
\hline & & 1623 & 0.000 & 1823 & 2.333 & 2300 & 0.061 & & & & \\
\hline & & & & 1846 & 2.364 & 2308 & 0.000 & & & & \\
\hline & & & & 1862 & 2.121 & & & & & & \\
\hline & & & & 1869 & 0.939 & & & & & & \\
\hline & & & & 1869 & 0.121 & & & & & & \\
\hline & & & & 1877 & 0.030 & & & & & & \\
\hline & & & & 1892 & 0.000 & & & & & & \\
\hline
\end{tabular}

Fig. 85. continued 


\section{H $\alpha$ - Dachs and Schmidt-Kaler - 1975}

$\mathrm{H} \alpha$ photometry .

\section{GENERAL INFORMATION}

AUTHORS J. Dachs, and T. Schmidt-Kaler

TELESCOPE $\quad 0.61 \mathrm{~m}$ Bochum reflector, ESO

DETECTOR EMI 9558 (refrigerated)

MAIN ARTICLE Dachs, J., Schmidt-Kaler, Th. 1975, A\&AS 21, 81

\section{SYSTEM DESCRIPTION}

\begin{tabular}{|l|lccc|}
\hline \multicolumn{5}{|c|}{ BANDS DESCRIPTION [81] } \\
\hline \multicolumn{1}{|c|}{ band } & filter & $\lambda_{\text {peak }}(\AA)$ & half-width $(\AA)$ & $\Upsilon_{\text {peak }}(\%)$ \\
\hline$H \alpha$ narrow & B11 $(\mathrm{X})$ & 6560.5 & 13 & 46.5 \\
\hline$H \alpha$ wide & B2 & 6566 & 157 & 59 \\
\hline
\end{tabular}

Interference filters by Baird Atomic.

\section{SYSTEM ANALYSIS}

COLOR INDICES AND PARAMETERS [81]

$\alpha_{\text {Bochum }}=-2.5 \log \frac{H \alpha \text { narrow }}{H \alpha \text { wide }}$

RELATIONS WITH OTHER SYSTEMS [81]

uvbyH $\beta$ - Strömgren and Crawford - 1956

$\alpha_{\text {Bochum }}=1.400+0.645 \beta_{C M} \quad \beta>2.65$

$H \alpha$ - Andrews - 1968

$\alpha_{\text {Bochum }}=2.965+1.050(R \alpha-1.00) \quad 0.97<R_{\alpha}<1.20$

$\mathrm{H} \alpha, \beta, \gamma$ - Feinstein - 1974

\begin{tabular}{|lll|}
$\alpha_{\text {Bochum }}=3.196+4.80\left(\alpha_{\text {Feinstein }}-1.500\right)$ & for O-B stars \\
\hline$\alpha_{\text {Bochum }}=3.154+4.80\left(\alpha_{\text {Feinstein }}-1.500\right)$ & for A-F stars \\
\hline
\end{tabular}

Fig. 86. The photometric system $\mathrm{H} \alpha-$ Dachs and Schmidt-Kaler - 1975 


\section{Helt and Gyldenkerne - 1975}

Narrow band photometry of $\mathrm{M}$ giants.

\section{GENERAL INFORMATION}

AUTHORS

B. E. Helt and K. Gyldenkerne

TELESCOPE

$0.61 \mathrm{~m}$ Morgan (reflector), Lowell Observatory, Arizona

DETECTOR

EMI 6265 S/A (S13 cathode, unrefrigerated)

MAIN ARTICLE Helt, B. E., Gyldenkerne, K. 1975, A\&AS 22, 171

\section{SYSTEM DESCRIPTION}

\begin{tabular}{|l|lccc|}
\hline \multicolumn{5}{|c|}{ BANDS DESCRIPTION [136] } \\
\hline band & \multicolumn{1}{|c|}{ filter } & $\lambda_{\text {peak }}(\AA)$ & half-width $(\AA)$ & $\Upsilon_{\text {peak }}(\%)$ \\
\hline 405 & Schott 405 & 4055 & 74 & 37 \\
\hline 437 & Baird 437 & 4380 & 65 & 40 \\
\hline 451 & Schott 451 & 4513 & 80 & 38 \\
\hline 480 & Baird 480 & 4803 & 61 & 70 \\
\hline 494 & Baird 494 (5 tilted) & 4942 & 44 & 69 \\
\hline 497 & Schott 497 & 4973 & 93 & 40 \\
\hline 501 & Baird 501 (5 tilted) & 5006 & 34 & 61 \\
\hline 536 & Baird 536 & 5365 & 62 & 72 \\
\hline 550 & Schott 550 & 5502 & 119 & 39 \\
\hline
\end{tabular}

Interference filters.

SYSTEM ANALYSIS

\section{COLOR INDICES AND PARAMETERS [136]}

$$
\begin{aligned}
& m_{2}=(438-480)-(480-536)=438-2 \cdot 480+536: \text { temperature indicator. } \\
& m_{4}=(451-497)-(497-550)=451-2 \cdot 497+550: \text { luminosity indicator. } \\
& t=(494-501): \text { measures the TiO absorption band at } 4954 \AA .
\end{aligned}
$$

Fig. 87. The photometric system Helt and Gyldenkerne - 1975 


\section{UBViyz - Jennens and Helfer - 1975}

Metal abundances and luminosities of $\mathrm{G}$ and $\mathrm{K}$ stars.

\section{GENERAL INFORMATION}

AUTHORS P.A Jennens, and H. L. Helfer

TELESCOPE KPNO telescopes; 0.90m and 1.50m (reflectors), CTIO [147]

DETECTOR ITT FW 130 or EMI 9658 (S-20 cathode)

MAIN ARTICLE Jennens P.A., Helfer H.L. 1975, MNRAS 172, 667

\section{SYSTEM DESCRIPTION}

\begin{tabular}{|c|lcc|}
\hline \multicolumn{4}{|c|}{ BANDS DESCRIPTION [148] } \\
\hline band & \multicolumn{1}{|c|}{ filter } & $\lambda_{\text {eff }}(\AA)$ & half-width $(\AA)$ \\
\hline$U$ & Corning 9863 + $\mathrm{CuSO}_{4}(\#)$ & 3660 & 600 \\
\hline$B$ & Corning 5030 + 1mm Schott 9913 + $\mathrm{CuSO}_{4}$ & 4400 & 900 \\
\hline$V$ & Corning 3384 + 1.6mm Schott BG18 & 5500 & 700 \\
\hline$i$ & 3mm D RG 780 & 7900 & 600 \\
\hline$y$ & Baird Atomic 12-39-5 & 4600 & 200 \\
\hline$Z$ & Baird Atomic 14-74-4 & 5160 & 200 \\
\hline
\end{tabular}

(\#) 2.5mm of $80 \%$ saturated copper sulphate solution, or a solid sulphate filter are used to block infrared leak [148].

\section{SYSTEM ANALYSIS}

RELATIONS WITH OTHER SYSTEMS [148]

UBVRI(JHKLMN) - Johnson - 1965

$(R-I) \approx 1.333+0.60(V-i)$

Fig. 88. The photometric system UBViyz - Jennens and Helfer - 1975 


\section{8 colors - Morguleff et al. - 1975}

Square, narrow-band photometry of early-type stars.

\section{GENERAL INFORMATION}

AUTHORS TELESCOPE DETECTOR MAIN ARTICLE
N. Morguleff, M. Gerbaldi and D. Barbier $0.80 \mathrm{~m}$ (reflector), Haute-Provence Obs.

Lallemand photomultipliers

Morguleff, N., Gerbaldi, M. 1975, A\&AS 19, 189

\section{SYSTEM DESCRIPTION}

\begin{tabular}{|c|cc|}
\hline \multicolumn{3}{|c|}{ BANDS DESCRIPTION [215] } \\
\hline band & bandpass $(\AA)$ & feature \\
\hline 361 & $3575-3650$ & blue side of the Balmer jump \\
\hline 375 & $3720-3780$ & red side of the Balmer jump \\
\hline 394 & $3925-3965$ & CaII \\
\hline 404 & $4010-4070$ & continuum \\
\hline 434 & $4310-4380$ & H \\
\hline 460 & $4815-4905$ & H $\beta$ \\
\hline 495 & $4900-5000$ & continuum \\
\hline 595 & $5900-6000$ & continuum \\
\hline
\end{tabular}

The bands are isolated on the focal plane of a prism spectrograph (dispersion $35 \AA / \mathrm{mm}$ at 3500, $60 \AA / \mathrm{mm}$ at 4000 and $160 \AA / \mathrm{mm}$ at $5550 \AA$ ) by diaphragms that direct to photomultipliers the selected spectral portions.

Fig. 89. The photometric system 8 colors - Morguleff et al. - 1975 


\section{ri - Weistrop - 1975}

General purpose photometry in the red and near-infrared.

\section{GENERAL INFORMATION}

$\begin{array}{ll}\text { AUTHORS } & \text { D. Weistrop } \\ \text { TELESCOPE } & 0.90 \text { m (reflector), KPNO } \\ \text { DETECTOR } & \text { RCA C31034A } \\ \text { MAIN ARTICLE } & \text { Weistrop, D. 1975 PASP 87, 367 }\end{array}$

SYSTEM DESCRIPTION

\begin{tabular}{|c|c|}
\hline \multicolumn{2}{|c|}{ BANDS DESCRIPTION [317] } \\
\hline band & filter \\
\hline$r$ & 2 mm Schott OG5 + 2mm Schott KG3 \\
\hline$i$ & 2 mm Schott RGN9 \\
\hline
\end{tabular}

SYSTEM ANALYSIS

RELATIONS WITH OTHER SYSTEMS [317]

RI - Kron and Smith - 1951

\begin{tabular}{|cl|}
\hline$R_{K-S}$ & $=r-0.340(r-i)+0.225$ \\
\hline$(R-I)_{K-S}$ & $=0.895(r-i)-0.142$ \\
\hline
\end{tabular}

Fig. 90. The photometric system ri - Weistrop - 1975 


\section{D2B Aura - 1975}

Ultraviolet sky survey with the D2B-Aura satellite.

\section{GENERAL INFORMATION}

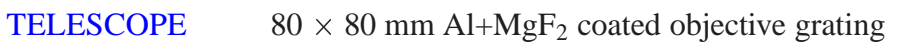

DETECTOR EMR 541 N09 ASCOP photomultiplier

MAIN ARTICLE Maucherat-Joubert, M., Cruvellier, P., Deharveng J. M. 1978, A\&A 70, 467

\section{SYSTEM DESCRIPTION}

\begin{tabular}{|l|cc|}
\hline \multicolumn{3}{|c|}{ BANDS DESCRIPTION [200] } \\
\hline band & $\lambda_{c}$ & bandpass $(\AA)$ \\
\hline 12 & 1250 & 500 \\
\hline $17(\#)$ & 1690 & 330 \\
\hline 22 & 2200 & 410 \\
\hline 31 & 3100 & 500 \\
\hline
\end{tabular}

On the focal plane of a concave (f/3) objective grating of $80 \times 80 \mathrm{~mm}, 610 \mathrm{ln} / \mathrm{mm}$, a series of exit slits are placed just ahead of a single photomultiplier. The different bands are obtained by rotation of the grating and the slit wheel. The bands aproximately have a square profile.

(\#) To further attenuate the disturbance from the geocoronal $\mathrm{Ly} \alpha, \mathrm{a} \mathrm{CaF}_{2}$ window is placed in front of the corresponding slit.

Fig. 91. The photometric system D2B AURA - 1975 


\section{Washington - Canterna - 1976}

General purpose system aimed to $\mathrm{G}$ and $\mathrm{K}$ stars.

\section{GENERAL INFORMATION}

$\begin{array}{ll}\text { AUTHORS } & \text { R. Canterna } \\ \text { TELESCOPE } & \text { 0.42m (reflector), KPNO; 0.77m (reflector), Manastash Obs. } \\ \text { DETECTOR } & \text { RCA C31034 (refrigerated) } \\ \text { MAIN ARTICLE } & \text { Canterna, R. 1976, AJ 81, 228 }\end{array}$

SYSTEM DESCRIPTION

\begin{tabular}{|c|c|c|c|c|c|c|}
\hline \multicolumn{7}{|c|}{ BANDS DESCRIPTION } \\
\hline \multicolumn{4}{|c|}{ photoelectric [57] } & \multicolumn{3}{|c|}{ photographic [111] } \\
\hline band & filter & $\lambda_{0}(\AA)$ & FWHM $(\AA)$ & filter & plate & $\lambda_{\text {eff }}(\AA)$ \\
\hline$C$ & $4 \mathrm{~mm}$ Corning CS-7-59+2.5mm $80 \% \mathrm{CuSO}_{4} \cdot \mathrm{H}_{2} \mathrm{O}$ & 3910 & 1100 & $3 \mathrm{~mm}$ BG1 & $103 a-O$ & 3870 \\
\hline$M$ & 3mm Schott GG455 + 5mm Corning CS-4-96 & 5085 & 1050 & $3 \mathrm{~mm}$ GG455 & IIIa-J & 5119 \\
\hline$T_{1}$ & 1.5mm Schott BG38 + 3mm Schott OG 590 & 6330 & 800 & $3 \mathrm{~mm}$ OG590 & O98-04 & 6533 \\
\hline$T_{2}$ & 3mm Schott RGN9 & 7885 & 1400 & $3 \mathrm{mmm}$ RGN-9 & IVN(IN) & 8056 \\
\hline
\end{tabular}

ZERO POINT: $(C-M)=\left(M-T_{1}\right)=\left(T_{1}-T_{2}\right)=0.0$ for unreddened A0 V stars [57]

CCD VERSION: Geisler, D. 1996, AJ 111, 480

PHOTOGRAPHIC VERSION: Geisler, D., Kapradinis, S. 1983, AJ 88, 461

\section{SYSTEM ANALYSIS}

\section{COLOR INDICES AND PARAMETERS [238]}

$\left(T_{1}-T_{2}\right)$ : temperature index similar to $(R-I)_{\text {Cousins }}$; relatively insensitive to metallicity and surface gravity.

$\left(M-T_{1}\right):$ sensitive to metallicity.

$(M-51)$ : luminosity class indicator (band 51 is from the DDO - McClure and Van den Bergh - 1968 system).

REDDENING RATIOS [57]

$$
\begin{aligned}
& E(C-M)=1.10 E(B-V)_{J o h n s o n} \quad E\left(M-T_{1}\right)=0.95 E(B-V)_{J o h n s o n} \\
& E\left(T_{1}-T_{2}\right)=0.72 E(B-V)_{J o h n s o n}
\end{aligned}
$$

RELATIONS WITH OTHER SYSTEMS

\begin{tabular}{|rc|}
\hline$\left(R-T_{1}\right)$ & $=-0.012+0.046\left(T_{1}-T_{2}\right)-0.082\left(T_{1}-T_{2}\right)^{2}$ \\
\hline$(V-I)$ & $=0.003+1.794\left(T_{1}-T_{2}\right)+0.517\left(T_{1}-T_{2}\right)^{2}-0.402\left(T_{1}-T_{2}\right)^{3}$ \\
\hline$(V-R)$ & $=0.008+1.016\left(V-T_{1}\right)$ \\
\hline$(M-V)$ & $=0.006+0.240(V-I)$ \\
\hline$\left(M-T_{2}\right)$ & $=1.242(V-I)+0.018(V-I)^{2}$ \\
\hline
\end{tabular}

Fig. 92. The photometric system Washington - Canterna - 1976 
UBVRI - Landolt - 1983 [119]

\begin{tabular}{|rll|}
\hline$(V-R)$ & $=1.062( \pm 0.031)\left(V-T_{1}\right)$ & $\left(V-T_{1}\right)<0.30$ \\
\hline$(V-R)$ & $=-0.055( \pm 0.020)+1.141( \pm 0.031)\left(V-T_{1}\right)$ & $\left(V-T_{1}\right)>0.30$ \\
\hline$(R-I)$ & $=0.970( \pm 0.016)\left(T_{1}-T_{2}\right)$ & $\left(T_{1}-T_{2}\right)<0.40$ \\
\hline$(R-I)$ & $=0.064( \pm 0.007)+0.849( \pm 0.010)\left(T_{1}-T_{2}\right)$ & $\left(T_{1}-T_{2}\right)>0.40$ \\
\hline
\end{tabular}

TRANSMISSION CURVES

PHOTOELECTRIC VERSION [58], pg 489

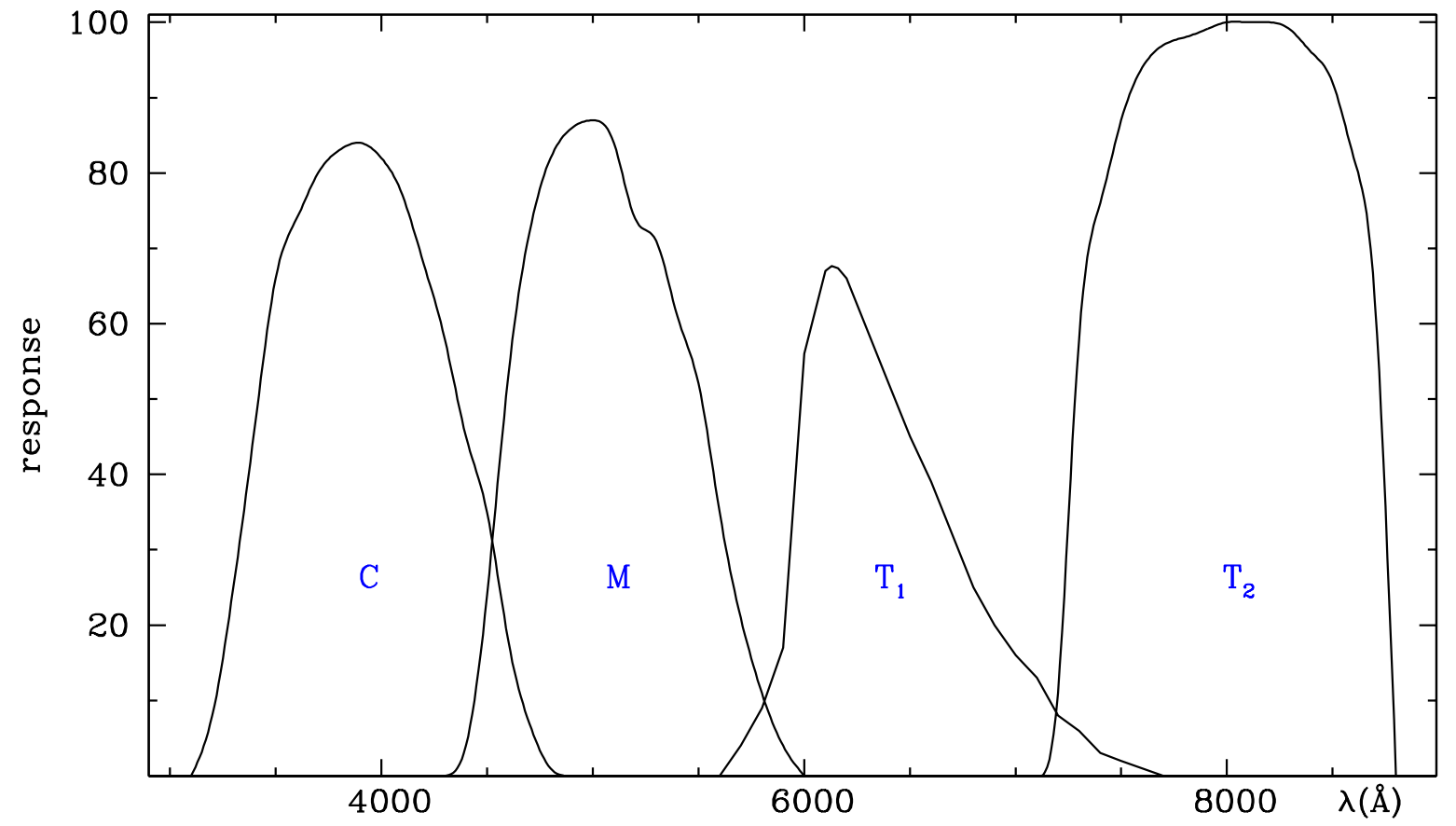

\begin{tabular}{|c|c|c|c|c|c|c|c|c|c|c|c|c|c|c|c|}
\hline \multicolumn{4}{|c|}{$C$} & \multicolumn{4}{|c|}{$M$} & \multicolumn{4}{|c|}{$T_{1}$} & \multicolumn{4}{|c|}{$T_{2}$} \\
\hline$\lambda(\mathrm{A})$ & $\Upsilon(\%)$ & $\lambda(\mathrm{A})$ & $\Upsilon(\%)$ & $\lambda(\mathrm{A})$ & $\Upsilon(\%)$ & $\lambda(\mathrm{A})$ & $\Upsilon(\%)$ & $\lambda(\mathrm{A})$ & $\Upsilon(\%)$ & $\lambda(\mathrm{A})$ & $\Upsilon(\%)$ & $\lambda(\mathrm{A})$ & $\Upsilon(\%)$ & $\lambda(\mathrm{A})$ & $\Upsilon(\%)$ \\
\hline 3100 & 0 & 4200 & 68 & 4300 & 0 & 5400 & 61 & 5600 & 0 & 6700 & 32 & 7100 & 0 & 8200 & 100 \\
\hline 3200 & 8 & 4300 & 58 & 4400 & 4 & 5500 & 52 & 5700 & 4 & 6800 & 25 & 7200 & 11 & 8300 & 99 \\
\hline 3300 & 25 & 4400 & 45 & 4500 & 24 & 5600 & 35 & 5800 & 9 & 6900 & 20 & 7300 & 58 & 8400 & 96 \\
\hline 3400 & 46 & 4500 & 35 & 4600 & 53 & 5700 & 21 & 5900 & 17 & 7000 & 16 & 7400 & 76 & 8500 & 92 \\
\hline 3500 & 66 & 4600 & 18 & 4700 & 72 & 5800 & 11 & 6000 & 56 & 7100 & 13 & 7500 & 87 & 8600 & 82 \\
\hline 3600 & 74 & 4700 & 7 & 4800 & 82 & 5900 & 4 & 6100 & 67 & 7200 & 8 & 7600 & 94 & 8700 & 63 \\
\hline 3700 & 80 & 4800 & 1 & 4900 & 86 & 6000 & 0 & 6200 & 66 & 7300 & 6 & 7700 & 97 & 8800 & 0 \\
\hline 3800 & 83 & 4900 & 0 & 5000 & 87 & & & 6300 & 59 & 7400 & 3 & 7800 & 98 & & \\
\hline 3900 & 84 & & & 5100 & 84 & & & 6400 & 52 & 7500 & 2 & 7900 & 99 & & \\
\hline 4000 & 82 & & & 5200 & 74 & & & 6500 & 45 & 7600 & 1 & 8000 & 100 & & \\
\hline 4100 & 77 & & & 5300 & 71 & & & 6600 & 39 & 7700 & 0 & 8100 & 100 & & \\
\hline
\end{tabular}

Fig. 92. continued 
PHOTOGRAPHIC VERSION [111]

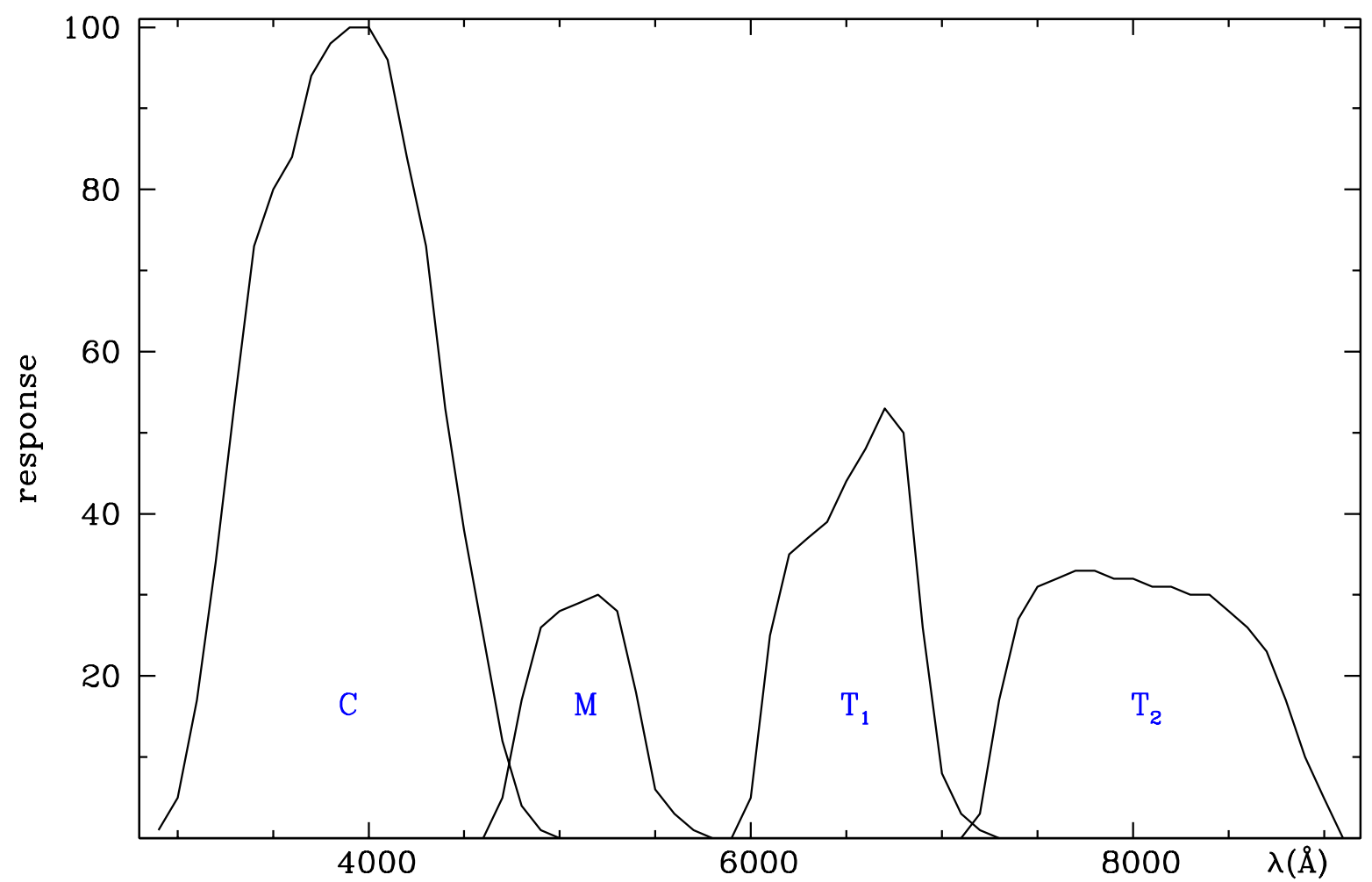

\begin{tabular}{|rc|cc|cc|cc|cc|cc|}
\hline \multicolumn{4}{|c|}{$C$} & \multicolumn{3}{c|}{$M$} & \multicolumn{3}{c|}{$T_{1}$} & \multicolumn{4}{c|}{$T_{2}$} \\
\hline$\lambda(\mathrm{A})$ & $\Upsilon(\%)$ & $\lambda(\mathrm{A})$ & $\Upsilon(\%)$ & $\lambda(\mathrm{A})$ & $\Upsilon(\%)$ & $\lambda(\mathrm{A})$ & $\Upsilon(\%)$ & $\lambda(\mathrm{A})$ & $\Upsilon(\%)$ & $\lambda(\mathrm{A})$ & $\Upsilon(\%)$ \\
\hline 2900 & 1 & 4400 & 53 & 4600 & 0 & 5900 & 0 & 7100 & 0 & 8600 & 26 \\
3000 & 5 & 4500 & 38 & 4700 & 5 & 6000 & 5 & 7200 & 3 & 8700 & 23 \\
3100 & 17 & 4600 & 25 & 4800 & 17 & 6100 & 25 & 7300 & 17 & 8800 & 17 \\
3200 & 34 & 4700 & 12 & 4900 & 26 & 6200 & 35 & 7400 & 27 & 8900 & 10 \\
3300 & 54 & 4800 & 4 & 5000 & 28 & 6300 & 37 & 7500 & 31 & 9000 & 5 \\
3400 & 73 & 4900 & 1 & 5100 & 29 & 6400 & 39 & 7600 & 32 & 9100 & 0 \\
3500 & 80 & 5000 & 0 & 5200 & 30 & 6500 & 44 & 7700 & 33 & & \\
3600 & 84 & & & 5300 & 28 & 6600 & 48 & 7800 & 33 & & \\
3700 & 94 & & & 5400 & 18 & 6700 & 53 & 7900 & 32 & & \\
3800 & 98 & & & 5500 & 6 & 6800 & 50 & 8000 & 32 & & \\
3900 & 100 & & & 5600 & 3 & 6900 & 26 & 8100 & 31 & \\
4000 & 100 & & & 5700 & 1 & 7000 & 8 & 8200 & 31 & \\
4100 & 96 & & & 5800 & 0 & 7100 & 3 & 8300 & 30 & \\
4200 & 84 & & & & & 7200 & 1 & 8400 & 30 & & \\
4300 & 73 & & & & & 7300 & 0 & 8500 & 28 & \\
\hline
\end{tabular}

Fig. 92. continued 


\section{RI - Cousins - 1976}

Photometry in the red and near-infrared.

\section{GENERAL INFORMATION}

$\begin{array}{ll}\text { AUTHORS } & \text { A. W. J. Cousins } \\ \text { TELESCOPE } & \text { 0.45m (reflector), South African Astronomical Obs. } \\ \text { DETECTOR } & \text { RCA C31034A (refrigerated), at Cape Obs. } \\ & \text { EMI 9659A (S-20 cathode), at Sutherland station }\end{array}$

MAIN ARTICLE Cousins A.W.J. 1976, Mem. R. Astr. Soc. 81, 25

SYSTEM DESCRIPTION

\begin{tabular}{|c|c|c|c|c|c|c|c|c|}
\hline \multicolumn{5}{|c|}{ BANDS DESCRIPTION [178], pg. 83} & & & \multicolumn{2}{|c|}{ FLUX CALIBRATION } \\
\hline band & filter $_{\text {Cape }}$ & filter ${ }_{\text {Sutherland }}$ & $\lambda_{0}(\AA)$ & $\lambda_{e f f}(\mu \mathrm{m})$ & WHM $(\AA)$ & $\lambda_{c}(\AA)$ & $\mathrm{F}_{\mathrm{v}}(\#)$ & $\mathrm{F}_{\lambda}(\# \#)$ \\
\hline$R$ & interference & $\begin{array}{l}2 \mathrm{~mm} \text { OG570 + } \\
2 \mathrm{~mm} \mathrm{KG3}\end{array}$ & 6700 & 0.64 & 1515 & 6470 & 3.08 & 2.254 \\
\hline$I$ & 3mm RGN9 & Wratten $88 \mathrm{~A}$ & 8100 & 0.79 & 1090 & 7865 & 2.55 & 1.196 \\
\hline \multicolumn{9}{|c|}{ Added band } \\
\hline V & 2mm Omag 302 & $\begin{array}{l}3 \mathrm{~mm} \text { GG495 + } \\
1 \mathrm{~mm} \mathrm{BG38}\end{array}$ & & 0.55 & & & 3.64 & 3.607 \\
\hline
\end{tabular}

(\#) For a 0.0 mag star, in units of $10^{-23} \mathrm{~W} \mathrm{~m}^{-2} \mathrm{~Hz}^{-1}[31]$

(\#\#) For a $V=0.0 \mathrm{~A} 0 \mathrm{~V}$ star, in units of $10^{-9} \mathrm{erg} \mathrm{cm}^{-2} \mathrm{~s}^{-1} \AA^{-1}$ [178], pg. 82

The $V$ band is a realization with the EMI 9659 photomultiplier of the equivalent band in the $U B V$ - Johnson and Morgan - 1953, originally obtained with a 1P21 tube.

An $U B V R I$ system composed by the UBV bands from UBV - Johnson and Morgan - 1953 and the Cousins' $R I$ is sometimes called Johnson-Cousins in literature.

ZERO POINT: All color indices for unreddened A0 V stars are 0.00. [178], pg. 83

PHOTOGRAPHIC VERSION: Bessell, M. S. 1979, PASP 91, 589

\section{SYSTEM ANALYSIS}

REDDENING RATIOS [258]

$$
A_{R}=2.66 E(B-V) \quad A_{I}=2.10 E(B-V)
$$

RELATIONS WITH OTHER SYSTEMS [31]

UVBGRI - Stebbins and Whitford - 1943

$$
(V-I)_{C}=1.5(G-R)_{S t-W}+0.75
$$

RI - Kron and Smith - 1951

\begin{tabular}{|rrr|}
\hline$(R-I)_{C}=0.118+1.03(R-I)_{K S}$ & $(R-I)_{K S}<0.35$ \\
\hline$(R-I)_{C}$ & $=0.033+1.246(R-I)_{K S}$ & $0.35<(R-I)_{K S}<1.30$ \\
\hline$(V-I)_{C}=0.27+0.936(V-I)_{K S}$ & $(V-I)_{K S}>0.5$ \\
\hline$(V-I)_{C}=0.24+1.00(V-I)_{K S}$ & $(V-I)_{K S}<0.5$ \\
\hline
\end{tabular}

Fig. 93. The photometric system $R I-$ Cousins - 1976 
uvbyH $\beta$ - Strömgren and Crawford - 1956

\begin{tabular}{|rrr|}
\hline$(V-I)_{C}=2.60(b-y)+0.02$ & $(b-y)<-0.05$ \\
\hline$(V-I)_{C}=2.12(b-y)$ & $-0.05<(b-y)<0.12$ \\
\hline$(V-I)_{C}=1.53(b-y)+0.075$ & $0.12<(b-y)<0.60$ \\
\hline
\end{tabular}

USNO - Kron and Mayall - 1960

$\begin{array}{rlr}(V-I)_{C} & =0.188+0.946(V-I)_{K M} & -0.4<(V-I)_{K M}<2.5 \\ (V-I)_{C} & =0.91(V-I)_{K M}+0.26 & 2.2<(V-I)_{K M}<4.3\end{array}$

UBVRI(JHKLMN) - Johnson - 1965

\begin{tabular}{|c|c|c|c|}
\hline$(V-I)_{C}$ & $=$ & $0.713(V-I)_{J}$ & $(V-I)<0$ \\
\hline$(V-I)_{C}$ & $=$ & $0.778(V-I)_{J}$ & $0<(V-I)_{J}<2.0$ \\
\hline$(V-I)_{C}$ & $=$ & $0.835(V-I)_{J}-0.13$ & $2.0<(V-I)_{J}<3.0$ \\
\hline$(R-I)_{C}$ & $=$ & $0.856(R-I)_{J}+0.025+\Delta(R-I)_{J}$ & \\
\hline$(V-R)_{C}$ & $=$ & $0.73(V-R)_{J}-0.03$ & $(V-R)_{J}<1.0$ \\
\hline$(V-R)_{C}$ & $=$ & $0.62(V-R)_{J}-0.08$ & $1.0<(V-R)_{J}<1.7$ \\
\hline$R_{J}$ & $=$ & $0.988 R_{C}-0.002( \pm 0.02)$ & [316] \\
\hline$I_{J}$ & $=$ & $0.990 I_{C}-0.007( \pm 0.02)$ & [316] \\
\hline
\end{tabular}

ri - Argue - 1967

$(V-R)_{C}=0.838( \pm 0.007)\left(V_{\text {Johnson }}-r\right)+0.162( \pm 0.003) \quad-0.25<\left(V_{\text {Johnson }}-r\right)<0.79[296]$

ri - Weistrop - 1975

$(R-I)_{C}=1.053( \pm 0.008)(r-i)-0.077( \pm 0.010) \quad 0.77<(r-i)<2.15$, class V only [296]

Washington - Canterna - 1976

\begin{tabular}{|rrr|}
\hline$(R-I)_{C}$ & $=0.980\left(T_{1}-T_{2}\right)$ & $-0.15<\left(T_{1}-T_{2}\right)<0.40$ \\
\hline$(R-I)_{C}$ & $=0.875\left(T_{1}-T_{2}\right)+0.044$ & $0.40<\left(T_{1}-T_{2}\right)<0.85$ \\
\hline$(V-R)_{C}$ & $=0.98\left(V-T_{1}\right)+0.015$ & $0<\left(V-T_{1}\right)<0.30$ \\
\hline
\end{tabular}

BVRI - Kunkel and Rydgren - 1979

\begin{tabular}{|rrr|}
\hline$(R-I)_{C}$ & $=1.12(r-i)$ & $(r-i)<1.0$ \\
\hline$(R-I)_{C}$ & $=1.233(r-i)-0.133$ & $(r-i)>1.0$ \\
\hline$(V-I)_{C}$ & $=1.02(V-i)+0.03$ & $(V-i)<1.6$ \\
\hline$(V-I)_{C}$ & $=0.96(V-i)+0.13$ & $(V-i)>1.6$ \\
\hline
\end{tabular}

Fig. 93. continued 


\section{TRANSMISSION CURVES [211]}

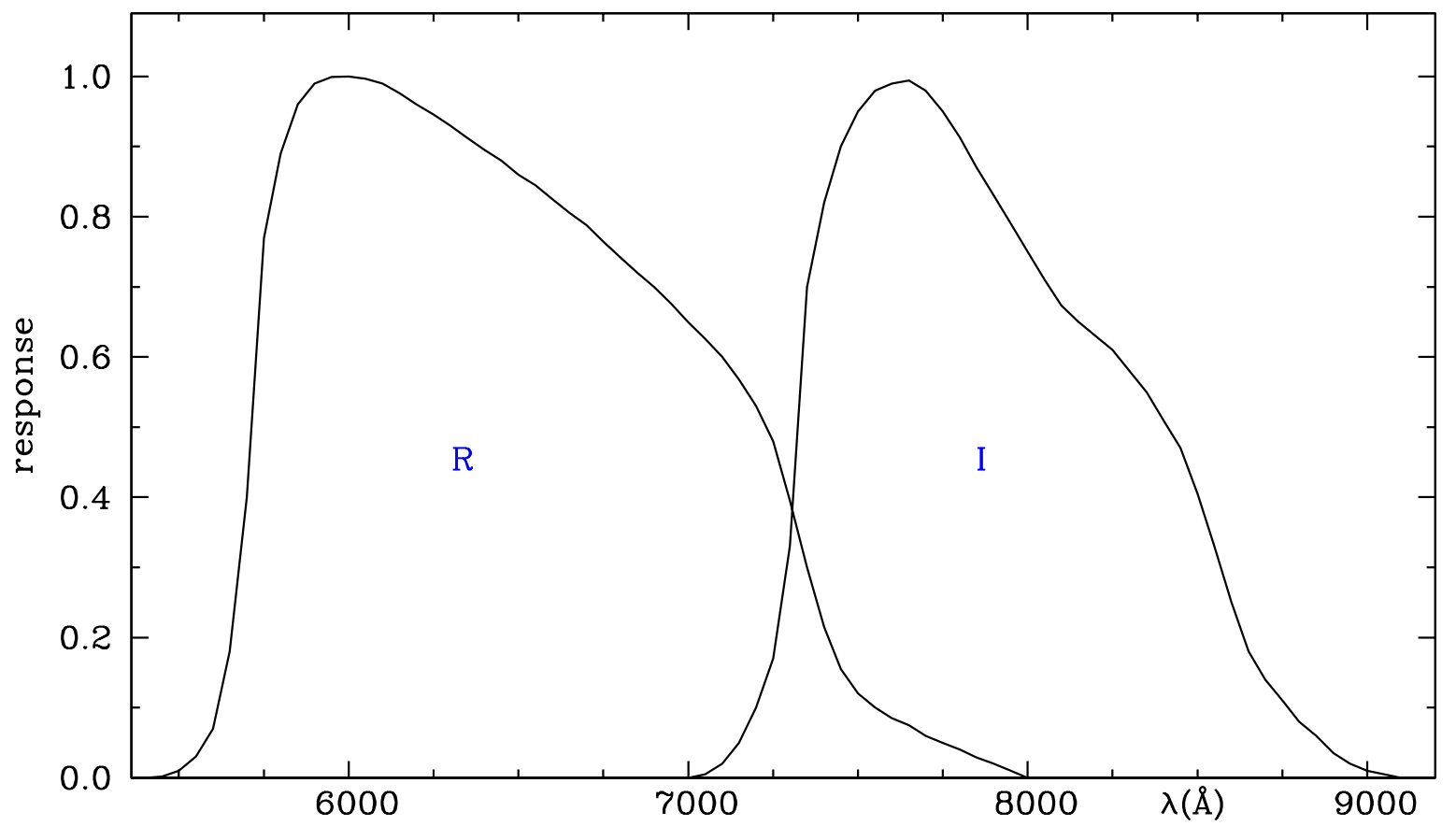

\begin{tabular}{|cc|cc|cc|cc|cc|}
\hline \multicolumn{7}{|c|}{$R$} & \multicolumn{3}{|c|}{$I$} \\
\hline$\lambda(\mathrm{A})$ & $\Upsilon$ & $\lambda(\mathrm{A})$ & $\Upsilon$ & $\lambda(\mathrm{A})$ & $\Upsilon$ & $\lambda(\mathrm{A})$ & $\Upsilon$ & $\lambda(\mathrm{A})$ & $\Upsilon$ \\
\hline 5400 & 0.000 & 6500 & 0.860 & 7600 & 0.085 & 7000 & 0.000 & 8100 & 0.673 \\
5450 & 0.002 & 6550 & 0.845 & 7650 & 0.075 & 7050 & 0.005 & 8150 & 0.650 \\
5500 & 0.010 & 6600 & 0.825 & 7700 & 0.060 & 7100 & 0.020 & 8200 & 0.630 \\
5550 & 0.030 & 6650 & 0.806 & 7750 & 0.050 & 7150 & 0.050 & 8250 & 0.610 \\
5600 & 0.070 & 6700 & 0.788 & 7800 & 0.040 & 7200 & 0.100 & 8300 & 0.580 \\
5650 & 0.180 & 6750 & 0.765 & 7850 & 0.029 & 7250 & 0.170 & 8350 & 0.550 \\
5700 & 0.400 & 6800 & 0.742 & 7900 & 0.020 & 7300 & 0.330 & 8400 & 0.510 \\
5750 & 0.770 & 6850 & 0.720 & 7950 & 0.010 & 7350 & 0.700 & 8450 & 0.470 \\
5800 & 0.890 & 6900 & 0.700 & 8000 & 0.000 & 7400 & 0.820 & 8500 & 0.405 \\
5850 & 0.960 & 6950 & 0.676 & & & 7450 & 0.900 & 8550 & 0.330 \\
5900 & 0.990 & 7000 & 0.650 & & & 7500 & 0.950 & 8600 & 0.250 \\
5950 & 0.999 & 7050 & 0.626 & & & 7550 & 0.980 & 8650 & 0.180 \\
6000 & 1.000 & 7100 & 0.600 & & & 7600 & 0.990 & 8700 & 0.140 \\
6050 & 0.997 & 7150 & 0.568 & & & 7650 & 0.994 & 8750 & 0.110 \\
6100 & 0.990 & 7200 & 0.530 & & & 7700 & 0.980 & 8800 & 0.080 \\
6150 & 0.976 & 7250 & 0.480 & & & 7750 & 0.950 & 8850 & 0.060 \\
6200 & 0.960 & 7300 & 0.395 & & & 7800 & 0.913 & 8900 & 0.035 \\
6250 & 0.946 & 7350 & 0.300 & & & 7850 & 0.870 & 8950 & 0.020 \\
6300 & 0.930 & 7400 & 0.215 & & & 7900 & 0.830 & 9000 & 0.010 \\
6350 & 0.912 & 7450 & 0.155 & & & 7950 & 0.790 & 9050 & 0.005 \\
6400 & 0.895 & 7500 & 0.120 & & & 8000 & 0.750 & 9100 & 0.000 \\
6450 & 0.880 & 7550 & 0.100 & & & 8050 & 0.710 & & \\
\hline
\end{tabular}

Fig. 93. continued 


\title{
uvby - Eggen - 1976
}

Intermediate-band system.

\section{GENERAL INFORMATION}

\author{
AUTHORS \\ O. J. Eggen \\ TELESCOPE \\ $1 \mathrm{~m}$ and $0.61 \mathrm{~m}$ (reflectors), Siding Spring Mountain Observatory \\ MAIN ARTICLE \\ Eggen, O. J. 1976, PASP 88, 732
}

\section{NOTES}

The system originates from an attempt to reproduce the uvby H - Strömgren and Crawford - 1956 system, using filters from a bulk order placed to the manufacturer by Crawford. However, during Eggen's observations it became evident that the set of filters differred from the original one (mainly in the $v$ filter, which FWHM instead of $190 \AA$ was only $100 \AA$ A).

The effect was particularly noteworthy for stars cooler than $(b-v) \quad 0.200$.

Therefore the Eggen's observations at Siding Spring evolved into an independent system.

Fig. 94. The photometric system uvby - Eggen - 1976 


\title{
13 colors - Johnson and Mitchell - 1976
}

General purpose system.

\section{GENERAL INFORMATION}

\begin{abstract}
AUTHORS
TELESCOPE

H. L. Johnson, and R. I. Mitchell

DETECTOR

$\delta>-20: 0.53 \mathrm{~m}$ and $0.71 \mathrm{~m}$, Catalina observing station of the Lun. Plan. Lab. $\delta<-20: 0.41 \mathrm{~m}$ and $0.61 \mathrm{~m}, \mathrm{CTIO}$

RCA-7102 (S-1 cathode) for the 58, 72, 80, 86, 99, 110 bands (6-RC sub-system)

MAIN ARTICLE Johnson, H.L., Mitchell, R.I. 1976, RMex.A.A. 1, 299
\end{abstract}

\section{SYSTEM DESCRIPTION}

The 8-C sub-system is indeed the LPL - Johnson et al - 1967 system [154].

\begin{tabular}{|l|ccc|cc|}
\hline \multicolumn{3}{|c|}{ BANDS DESCRIPTION [155], [178] pg. 70 } & \multicolumn{2}{|c|}{ FLUX CALIBRATION (\#\#) } \\
\hline band & $\lambda_{0}(\AA)$ & ERP $(\%)(\#)$ & bandwidth $(\mu \mathrm{m})$ & $\left(\mathrm{W} \mathrm{cm}^{-2} \mu \mathrm{m}^{-1}\right)[155]$ & $\left(\mathrm{erg} \mathrm{cm}^{-2} \mathrm{~s}^{-1} \AA^{-1} \AA\right)[178], \mathrm{pg} .70$ \\
\hline 33 & 3371 & 3.3 & 0.01 & $3.3710^{-12}$ & $3.6310^{-9}$ \\
\hline 35 & 3536 & 3.6 & 0.01 & $3.2610^{-12}$ & $3.5710^{-9}$ \\
\hline 37 & 3751 & 3.4 & 0.01 & $4.2810^{-12}$ & $4.8910^{-9}$ \\
\hline 40 & 4030 & 5.6 & 0.02 & $7.7510^{-12}$ & $6.4010^{-9}$ \\
\hline 45 & 4571 & 6.1 & 0.023 & $6.1110^{-12}$ & $4.6910^{-9}$ \\
\hline 52 & 5183 & 5.0 & 0.023 & $4.3010^{-12}$ & $3.3610^{-9}$ \\
\hline 58 & 5827 & 3.8 & 0.02 & $2.3210^{-12}$ & $1.7310^{-9}$ \\
\hline 63 & 6356 & 5.1 & 0.02 & $2.3210^{-12}$ & $1.2510^{-9}$ \\
\hline 72 & 7241 & 8.1 & 0.06 & $1.5810^{-12}$ & $1.0210^{-9}$ \\
\hline 80 & 8000 & 5.4 & 0.45 & $1.1510^{-12}$ & $0.7610^{-9}$ \\
\hline 86 & 8584 & 5.6 & 0.51 & $0.9410^{-12}$ & $0.5210^{-9}$ \\
\hline 99 & 9831 & 5.9 & 0.57 & $0.7010^{-12}$ & $0.4810^{-12}$ \\
\hline 110 & 11084 & 7.4 & 0.70 & & \\
\hline
\end{tabular}

(\#) Effective Rectangular Passband. (\#\#) For a 0.00 mag star.

ZERO POINT: The mean color of the following six A0 V unreddened stars is taken to be 0.00: $\alpha$ Lyr, $\gamma \mathrm{UMa}$, 109 Vir, $\alpha \mathrm{CrB}, \gamma$ Oph and HR3314. [155]

\section{SYSTEM ANALYSIS}

REDDENING-FREE PARAMETERS [264]

Luminosity indicators:

$$
\begin{aligned}
& \ell=(37-45)-0.467(40-58) \\
& \lambda=(33-37)-0.307(40-58) \\
& \beta=(37-40)-0.355(40-45)
\end{aligned}
$$

Temperature indicators:

$$
\begin{aligned}
& \tau=(35-40)-0.304(40-58) \\
& \gamma=(35-40)-0.882(40-45) \\
& \delta=(33-35)-0.365(40-45)
\end{aligned}
$$

\section{RELATIONS WITH OTHER SYSTEMS [264]}

UBVRI(JHKLMN) - Johnson - 1965

\begin{tabular}{|ll|}
\hline$(B-V)$ & $=0.814(40-52)+0.015$ \\
\hline$(U-B)$ & $=0.692(35-45)-0.003$ \\
\hline$(R-I)$ & $=1.721(72-86)-0.064$ \\
\hline
\end{tabular}

Fig. 95. The photometric system 13 colors - Johnson and Mitchell - 1976 


\section{TRANSMISSION CURVES [155]}

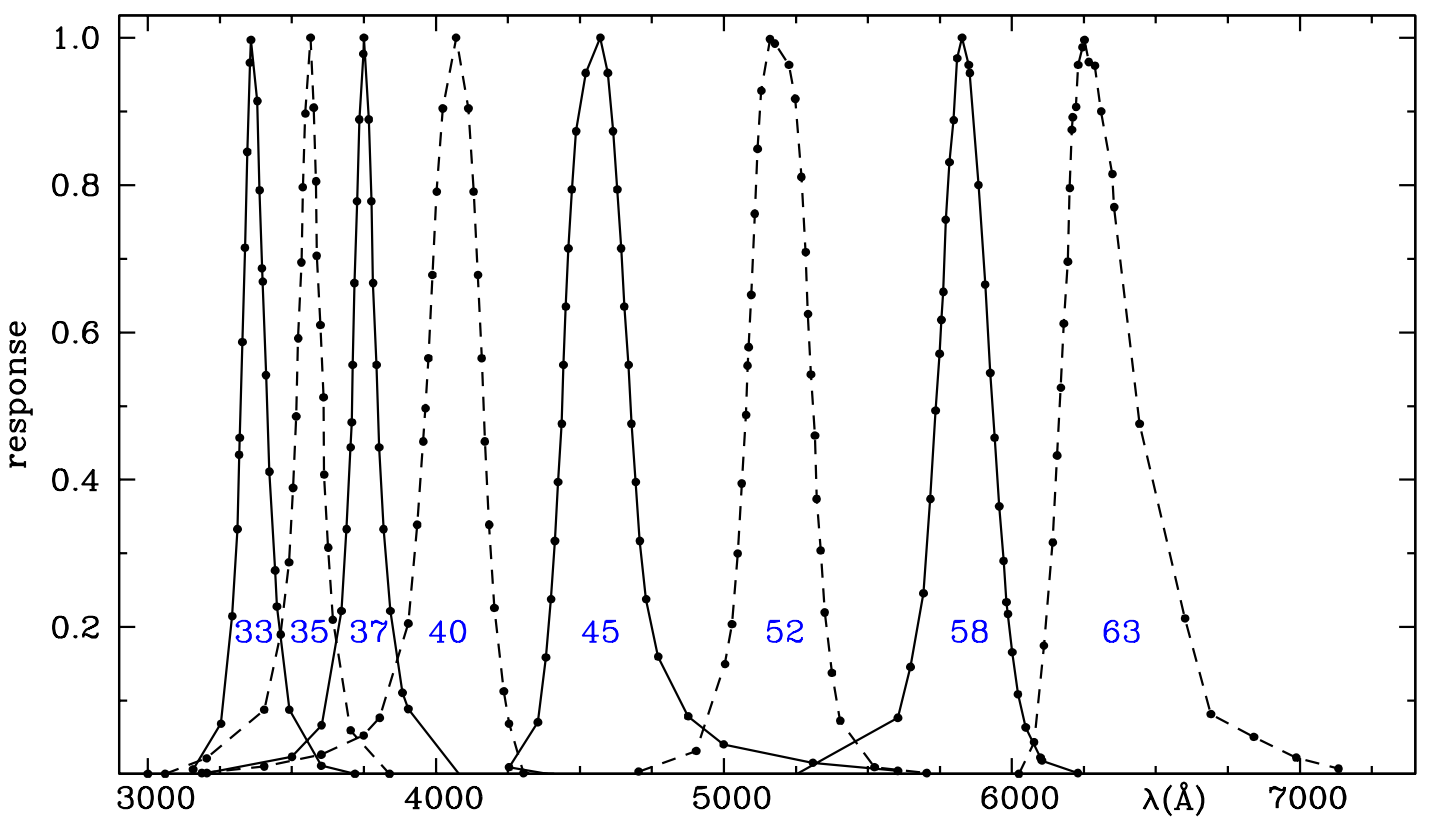

\begin{tabular}{|c|c|c|c|c|c|c|c|c|c|c|c|c|c|c|c|}
\hline \multicolumn{16}{|c|}{ 8-C sub-system } \\
\hline \multicolumn{2}{|c|}{33} & \multicolumn{2}{|c|}{35} & \multicolumn{2}{|c|}{37} & \multicolumn{2}{|c|}{40} & \multicolumn{2}{|c|}{45} & \multicolumn{2}{|c|}{52} & \multicolumn{2}{|c|}{58} & \multicolumn{2}{|c|}{63} \\
\hline$\lambda(\mathrm{A})$ & $\Upsilon$ & $\lambda(\mathrm{A})$ & $\Upsilon$ & $\lambda(\mathrm{A})$ & $\Upsilon$ & $\lambda(\mathrm{A})$ & $\Upsilon$ & $\lambda(\mathrm{A})$ & $\Upsilon$ & $\lambda(\mathrm{A})$ & $\Upsilon$ & $\lambda(\mathrm{A})$ & $\Upsilon$ & $\lambda(\mathrm{A})$ & $\Upsilon$ \\
\hline 3157 & 0.007 & 3060 & 0.001 & 3204 & 0.002 & 3187 & 0.002 & 4004 & 0.001 & 4704 & 0.004 & 5249 & 0.000 & 6024 & 0.001 \\
\hline 3254 & 0.069 & 3204 & 0.022 & 3500 & 0.024 & 3404 & 0.011 & 4254 & 0.010 & 4904 & 0.032 & 5604 & 0.077 & 6077 & 0.044 \\
\hline 3293 & 0.215 & 3404 & 0.088 & 3603 & 0.067 & 3602 & 0.027 & 4354 & 0.071 & 5004 & 0.150 & 5648 & 0.146 & 6111 & 0.175 \\
\hline 3311 & 0.333 & 3461 & 0.190 & 3672 & 0.222 & 3749 & 0.053 & 4382 & 0.159 & 5028 & 0.204 & 5693 & 0.246 & 6142 & 0.315 \\
\hline 3317 & 0.434 & 3490 & 0.288 & 3690 & 0.333 & 3805 & 0.077 & 4400 & 0.238 & 5048 & 0.300 & 5717 & 0.374 & 6157 & 0.433 \\
\hline 3319 & 0.457 & 3504 & 0.389 & 3704 & 0.444 & 3904 & 0.205 & 4413 & 0.317 & 5062 & 0.395 & 5735 & 0.494 & 6170 & 0.525 \\
\hline 3328 & 0.587 & 3515 & 0.486 & 3708 & 0.478 & 3935 & 0.339 & 4424 & 0.397 & 5077 & 0.488 & 5749 & 0.571 & 6180 & 0.612 \\
\hline 3337 & 0.715 & 3522 & 0.592 & 3711 & 0.556 & 3956 & 0.452 & 4437 & 0.476 & 5082 & 0.555 & 5755 & 0.617 & 6194 & 0.696 \\
\hline 3345 & 0.845 & 3533 & 0.695 & 3717 & 0.667 & 3964 & 0.497 & 4443 & 0.556 & 5086 & 0.580 & 5762 & 0.655 & 6201 & 0.796 \\
\hline 3354 & 0.966 & 3538 & 0.797 & 3726 & 0.778 & 3974 & 0.565 & 4451 & 0.635 & 5095 & 0.651 & 5770 & 0.753 & 6208 & 0.875 \\
\hline 3358 & 0.997 & 3547 & 0.897 & 3734 & 0.889 & 3988 & 0.678 & 4460 & 0.714 & 5107 & 0.761 & 5783 & 0.831 & 6211 & 0.892 \\
\hline 3380 & 0.914 & 3565 & 1.000 & 3748 & 0.978 & 4003 & 0.791 & 4472 & 0.794 & 5117 & 0.849 & 5798 & 0.888 & 6223 & 0.906 \\
\hline 3388 & 0.793 & 3576 & 0.905 & 3750 & 1.000 & 4024 & 0.904 & 4487 & 0.873 & 5130 & 0.928 & 5810 & 0.972 & 6230 & 0.963 \\
\hline 3396 & 0.687 & 3584 & 0.805 & 3767 & 0.889 & 4070 & 1.000 & 4520 & 0.952 & 5160 & 0.998 & 5827 & 1.000 & 6245 & 0.987 \\
\hline 3399 & 0.669 & 3586 & 0.704 & 3776 & 0.778 & 4113 & 0.904 & 4571 & 1.000 & 5177 & 0.992 & 5850 & 0.963 & 6252 & 0.997 \\
\hline 3410 & 0.542 & 3599 & 0.610 & 3782 & 0.667 & 4131 & 0.791 & 4597 & 0.952 & 5226 & 0.963 & 5854 & 0.952 & 6267 & 0.967 \\
\hline 3422 & 0.411 & 3610 & 0.512 & 3794 & 0.556 & 4146 & 0.678 & 4615 & 0.873 & 5248 & 0.917 & 5884 & 0.800 & 6288 & 0.962 \\
\hline 3442 & 0.277 & 3612 & 0.407 & 3803 & 0.444 & 4159 & 0.565 & 4630 & 0.794 & 5269 & 0.811 & 5907 & 0.665 & 6310 & 0.900 \\
\hline 3448 & 0.228 & 3626 & 0.308 & 3818 & 0.333 & 4170 & 0.452 & 4643 & 0.714 & 5284 & 0.709 & 5925 & 0.545 & 6349 & 0.815 \\
\hline 3491 & 0.088 & 3642 & 0.210 & 3842 & 0.222 & 4185 & 0.339 & 4654 & 0.635 & 5292 & 0.625 & 5940 & 0.457 & 6355 & 0.770 \\
\hline 3602 & 0.012 & 3704 & 0.060 & 3884 & 0.111 & 4203 & 0.226 & 4669 & 0.556 & 5302 & 0.543 & 5956 & 0.364 & 6443 & 0.476 \\
\hline \multirow[t]{9}{*}{3719} & 0.001 & 3830 & 0.001 & 3904 & 0.089 & 4236 & 0.113 & 4679 & 0.476 & 5316 & 0.460 & 5971 & 0.290 & 6601 & 0.212 \\
\hline & & & & 4082 & 0.000 & 4254 & 0.069 & 4694 & 0.397 & 5322 & 0.374 & 5981 & 0.234 & 6691 & 0.082 \\
\hline & & & & & & 4304 & 0.002 & 4708 & 0.317 & 5336 & 0.304 & 5986 & 0.218 & 6840 & 0.051 \\
\hline & & & & & & 4564 & 0.000 & 4730 & 0.238 & 5350 & 0.220 & 6001 & 0.166 & 6987 & 0.023 \\
\hline & & & & & & & & 4772 & 0.160 & 5375 & 0.138 & 6021 & 0.109 & 7134 & 0.008 \\
\hline & & & & & & & & 4876 & 0.079 & 5404 & 0.073 & 6048 & 0.064 & & \\
\hline & & & & & & & & 4999 & 0.041 & 5523 & 0.010 & 6099 & 0.023 & & \\
\hline & & & & & & & & 5309 & 0.016 & 5704 & 0.002 & 6104 & 0.019 & & \\
\hline & & & & & & & & 5604 & 0.005 & & & 6229 & 0.002 & & \\
\hline
\end{tabular}

Fig. 95. continued 


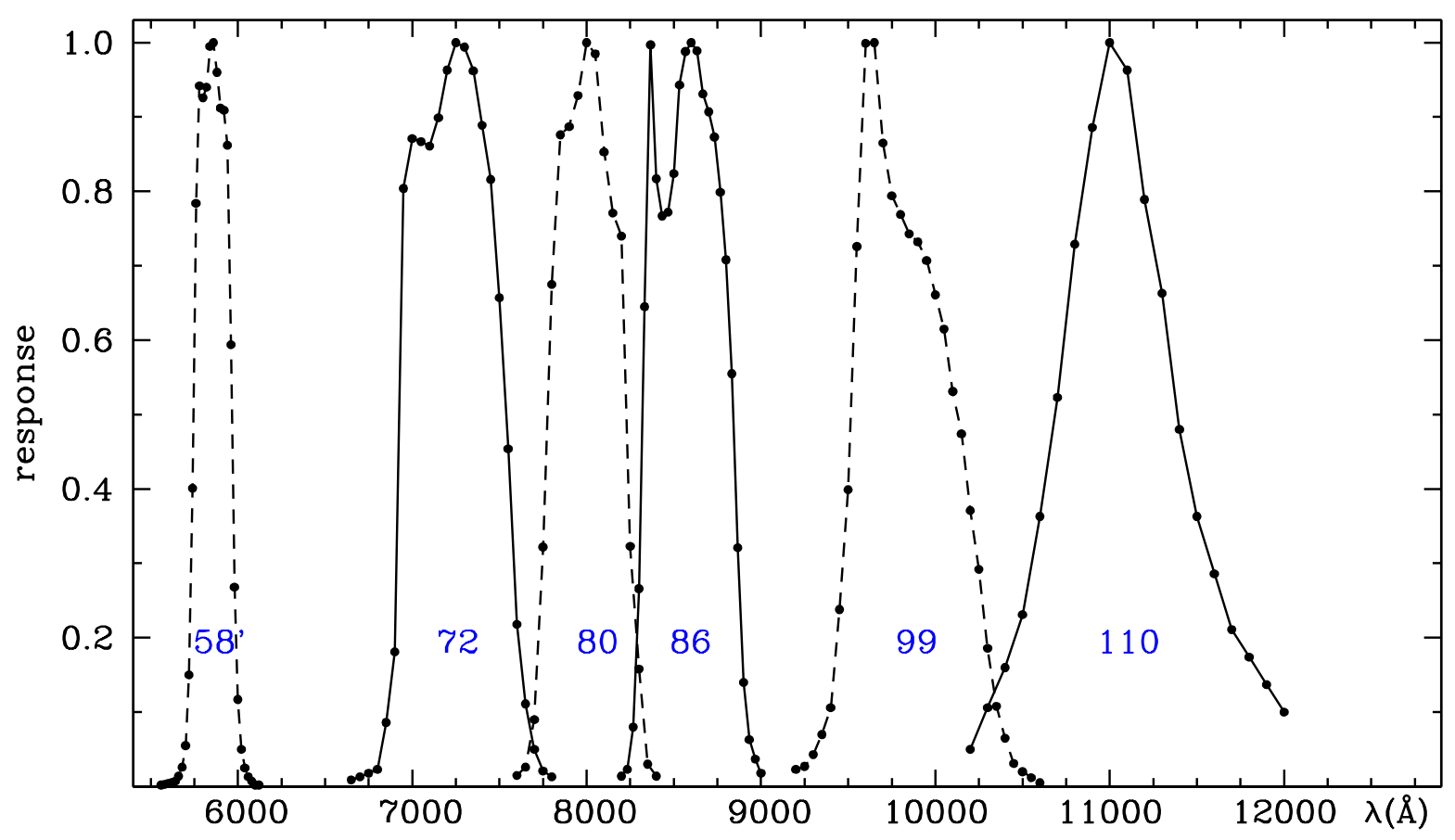

\begin{tabular}{|c|c|c|c|c|c|c|c|c|c|c|c|}
\hline \multicolumn{12}{|c|}{ 6-RC sub-system } \\
\hline \multicolumn{2}{|c|}{$58^{\prime}$} & \multicolumn{2}{|c|}{72} & \multicolumn{2}{|c|}{80} & \multicolumn{2}{|c|}{86} & \multicolumn{2}{|c|}{99} & \multicolumn{2}{|c|}{110} \\
\hline$\lambda(\mathrm{A})$ & $\Upsilon$ & $\lambda(\mathrm{A})$ & $\Upsilon$ & $\lambda(\mathrm{A})$ & $\Upsilon$ & $\lambda(\mathrm{A})$ & $\Upsilon$ & $\lambda(\mathrm{A})$ & $\Upsilon$ & $\lambda(\mathrm{A})$ & $\Upsilon$ \\
\hline 5560 & 0.002 & 6650 & 0.009 & 7600 & 0.015 & 8200 & 0.014 & 9200 & 0.023 & 10200 & 0.050 \\
\hline 5580 & 0.003 & 6700 & 0.013 & 7650 & 0.026 & 8233 & 0.023 & 9250 & 0.027 & 10300 & 0.106 \\
\hline 5600 & 0.004 & 6750 & 0.018 & 7700 & 0.090 & 8267 & 0.080 & 9300 & 0.043 & 10400 & 0.160 \\
\hline 5620 & 0.005 & 6800 & 0.023 & 7750 & 0.322 & 8300 & 0.266 & 9350 & 0.070 & 10500 & 0.231 \\
\hline 5640 & 0.007 & 6850 & 0.086 & 7800 & 0.675 & 8333 & 0.645 & 9400 & 0.106 & 10600 & 0.363 \\
\hline 5660 & 0.014 & 6900 & 0.181 & 7850 & 0.876 & 8367 & 0.997 & 9450 & 0.238 & 10700 & 0.523 \\
\hline 5680 & 0.026 & 6950 & 0.804 & 7900 & 0.887 & 8400 & 0.817 & 9500 & 0.399 & 10800 & 0.729 \\
\hline 5700 & 0.055 & 7000 & 0.871 & 7950 & 0.929 & 8433 & 0.767 & 9550 & 0.726 & 10900 & 0.886 \\
\hline 5720 & 0.150 & 7050 & 0.867 & 8000 & 1.000 & 8467 & 0.772 & 9600 & 0.999 & 11000 & 1.000 \\
\hline 5740 & 0.401 & 7100 & 0.861 & 8050 & 0.985 & 8500 & 0.824 & 9650 & 1.000 & 11100 & 0.963 \\
\hline 5760 & 0.784 & 7150 & 0.899 & 8100 & 0.853 & 8533 & 0.943 & 9700 & 0.865 & 11200 & 0.789 \\
\hline 5780 & 0.942 & 7200 & 0.963 & 8150 & 0.771 & 8567 & 0.988 & 9750 & 0.794 & 11300 & 0.663 \\
\hline 5800 & 0.926 & 7250 & 1.000 & 8200 & 0.740 & 8600 & 1.000 & 9800 & 0.769 & 11400 & 0.480 \\
\hline 5820 & 0.940 & 7300 & 0.994 & 8250 & 0.323 & 8633 & 0.989 & 9850 & 0.743 & 11500 & 0.363 \\
\hline 5840 & 0.995 & 7350 & 0.962 & 8300 & 0.158 & 8667 & 0.931 & 9900 & 0.732 & 11600 & 0.286 \\
\hline 5860 & 1.000 & 7400 & 0.889 & 8350 & 0.030 & 8700 & 0.907 & 9950 & 0.707 & 11700 & 0.211 \\
\hline 5880 & 0.960 & 7450 & 0.816 & 8400 & 0.014 & 8733 & 0.873 & 10000 & 0.661 & 11800 & 0.174 \\
\hline 5900 & 0.912 & 7500 & 0.657 & & & 8767 & 0.799 & 10050 & 0.615 & 11900 & 0.137 \\
\hline 5920 & 0.909 & 7550 & 0.454 & & & 8800 & 0.708 & 10100 & 0.531 & 12000 & 0.100 \\
\hline 5940 & 0.862 & 7600 & 0.218 & & & 8833 & 0.555 & 10150 & 0.474 & & \\
\hline 5960 & 0.594 & 7650 & 0.111 & & & 8867 & 0.321 & 10200 & 0.371 & & \\
\hline 5980 & 0.268 & 7700 & 0.050 & & & 8900 & 0.140 & 10250 & 0.292 & & \\
\hline 6000 & 0.117 & 7750 & 0.021 & & & 8933 & 0.063 & 10300 & 0.186 & & \\
\hline 6020 & 0.050 & 7800 & 0.013 & & & 8967 & 0.037 & 10350 & 0.108 & & \\
\hline 6040 & 0.025 & & & & & 9000 & 0.018 & 10400 & 0.065 & & \\
\hline 6060 & 0.013 & & & & & & & 10450 & 0.031 & & \\
\hline 6080 & 0.007 & & & & & & & 10500 & 0.020 & & \\
\hline 6100 & 0.002 & & & & & & & 10550 & 0.012 & & \\
\hline 6120 & 0.002 & & & & & & & 10600 & 0.005 & & \\
\hline 6140 & 0.000 & & & & & & & & & & \\
\hline
\end{tabular}

Fig. 95. continued 


\section{vbyg $_{1} g_{2}$ - Maitzen - 1976}

Extension of the uvbyH $\beta$-Strömgren and Crawford -1956 system to Ap stars.

\section{GENERAL INFORMATION}

$\begin{array}{ll}\text { AUTHORS } & \text { H. M. Maitzen } \\ \text { TELESCOPE } & 1 \mathrm{~m} \text { (reflector), ESO; 0.61m (reflector), Bochum } \\ \text { DETECTOR } & \text { EMI 6256 and EMI 9502 } \\ \text { MAIN ARTICLE } & \text { Maitzen, H. M. 1976, A\&A 51, 223 }\end{array}$

SYSTEM DESCRIPTION

\begin{tabular}{|c|c|cc|}
\hline \multicolumn{3}{|c|}{ BANDS DESCRIPTION [195] } & \\
\hline band & $\lambda_{0}(\AA)$ & WHM $(\AA)$ & $\lambda_{c}(\AA)$ \\
\hline$g_{1}$ & 5020 & 126 & 5020 \\
\hline$g_{2}$ & 5240 & 135 & 5240 \\
\hline
\end{tabular}

Interference filters.

The system includes also the v,b,y bands of uvbyH - Strömgren and Crawford - 1956 system.

\section{SYSTEM ANALYSIS}

\section{COLOR INDICES AND PARAMETERS [195]}

$a^{1}=g_{2}-\left(g_{1}+y\right) / 2$ : measures the flux depression at $5200 \AA$. Ap stars (but not the Am) show an excess in $a^{1}$ compared to normal A stars.

Fig. 96. The photometric system $\operatorname{vbyg}_{1} g_{2}-$ Maitzen -1976 


\section{TRANSMISSION CURVES}

As derived from Fig 1 of [195].

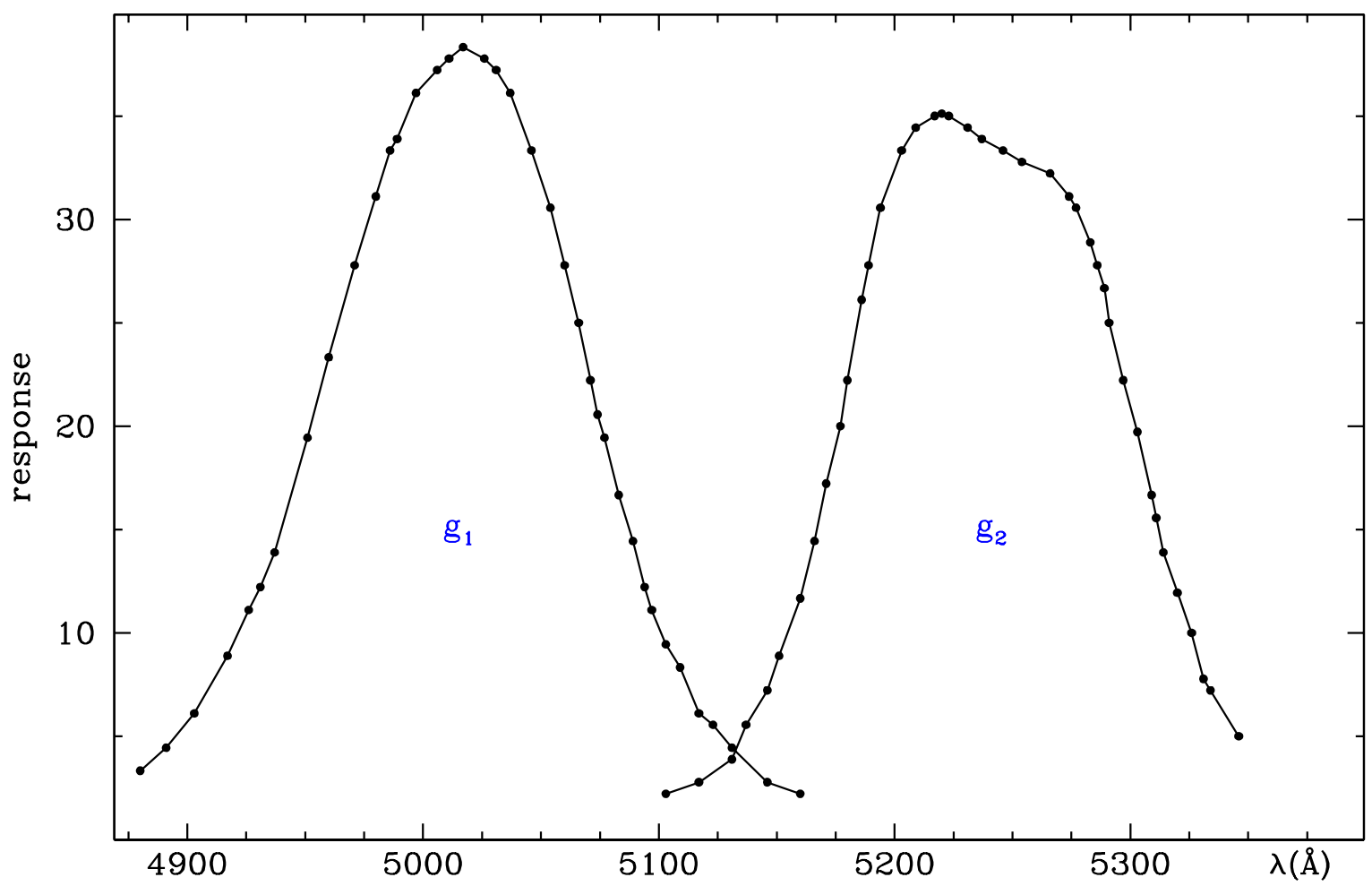

\begin{tabular}{|rr|rl|rr|rr|rr|rr|rrr|r|r|}
\hline \multicolumn{8}{|c|}{$g_{1}$} & \multicolumn{10}{c|}{$g_{2}$} \\
\hline$\lambda(\mathrm{A})$ & $\Upsilon(\%)$ & $\lambda(\mathrm{A})$ & $\Upsilon(\%)$ & $\lambda(\mathrm{A})$ & $\Upsilon(\%)$ & $\lambda(\mathrm{A})$ & $\Upsilon(\%)$ & $\lambda(\mathrm{A})$ & $\Upsilon(\%)$ & $\lambda(\mathrm{A})$ & $\Upsilon(\%)$ & $\lambda(\mathrm{A})$ & $\Upsilon(\%)$ & $\lambda(\mathrm{A})$ & $\Upsilon(\%)$ \\
\hline 4880 & 3.33 & 4980 & 31.11 & 5046 & 33.33 & 5097 & 11.11 & 5103 & 2.22 & 5180 & 22.22 & 5237 & 33.89 & 5297 & 22.22 \\
4891 & 4.44 & 4986 & 33.33 & 5054 & 30.56 & 5103 & 9.44 & 5117 & 2.78 & 5186 & 26.11 & 5246 & 33.33 & 5303 & 19.72 \\
4903 & 6.11 & 4989 & 33.89 & 5060 & 27.78 & 5109 & 8.33 & 5131 & 3.89 & 5189 & 27.78 & 5254 & 32.78 & 5309 & 16.67 \\
4917 & 8.89 & 4997 & 36.11 & 5066 & 25.00 & 5117 & 6.11 & 5137 & 5.56 & 5194 & 30.56 & 5266 & 32.22 & 5311 & 15.56 \\
4926 & 11.11 & 5006 & 37.22 & 5071 & 22.22 & 5123 & 5.56 & 5146 & 7.22 & 5203 & 33.33 & 5274 & 31.11 & 5314 & 13.89 \\
4931 & 12.22 & 5011 & 37.78 & 5074 & 20.56 & 5131 & 4.44 & 5151 & 8.89 & 5209 & 34.44 & 5277 & 30.56 & 5320 & 11.94 \\
4937 & 13.89 & 5017 & 38.33 & 5077 & 19.44 & 5146 & 2.78 & 5160 & 11.67 & 5217 & 35.00 & 5283 & 28.89 & 5326 & 10.00 \\
4951 & 19.44 & 5026 & 37.78 & 5083 & 16.67 & 5160 & 2.22 & 5166 & 14.44 & 5220 & 35.11 & 5286 & 27.78 & 5331 & 7.78 \\
4960 & 23.33 & 5031 & 37.22 & 5089 & 14.44 & & & 5171 & 17.22 & 5223 & 35.00 & 5289 & 26.67 & 5334 & 7.22 \\
4971 & 27.78 & 5037 & 36.11 & 5094 & 12.22 & & & 5177 & 20.00 & 5231 & 34.44 & 5291 & 25.00 & 5346 & 5.00 \\
\hline
\end{tabular}

Fig. 96. continued 


\section{uvgr - Thuan and Gunn - 1976}

Generale purpose system.

\section{GENERAL INFORMATION}

AUTHORS

TELESCOPE

DETECTOR

MAIN ARTICLE
T. X. Thuan and J. E. Gunn $1.52 \mathrm{~m}$ (reflector), Palomar Obs.

ITT FW-130 (S-20 cathode, refrigerated)

Thuan, X. T., and Gunn, J. E. 1976, PASP 88, 543

\section{SYSTEM DESCRIPTION}

\begin{tabular}{|l|lcc|}
\hline \multicolumn{4}{|c|}{ BANDS DESCRIPTION [301] } \\
\hline band & \multicolumn{1}{|c|}{ filter } & $\lambda_{\text {eff }}(\AA)$ & FWHM $(\AA)$ \\
\hline$u$ & $1 \mathrm{~mm}$ Schott BG38 + 4mm Schott UG11 & 3530 & 400 \\
\hline$V$ & $3 \mathrm{~mm}$ Schott BG38 + 4mm Schott UG3 + & \\
\hline & 1mm Schott BG1 + 1mm Schott GG13 & 3980 & 400 \\
\hline$g$ & Corion interference & 4930 & 700 \\
\hline$r$ & 3mm Schott BG610 + Balzer B1 short-pass & 6550 & 900 \\
\hline
\end{tabular}

ZERO POINT: $\mathrm{BD}+17^{\circ} 4708$ (F8) has the following magnitudes and color indices: $g=9.50,(g-r)=0.00,(u-v)=0.00,(v-g)=0.00[301]$

A revision made in 1985 by Kent [162] changed these values to: $g=9.50,(g-r)=0.001,(u-v)=-0.001,(v-g)=0.001$.

\section{SYSTEM ANALYSIS}

REDDENING RATIOS [162]

$$
E(u-v)=0.574 E(v-g) \quad E(v-g)=0.797 E(g-r) \quad E(v-g)=0.89 E(B-V)_{J o h n s o n}
$$

\section{RELATIONS WITH OTHER SYSTEMS}

UBV - Johnson and Morgan - 1953 [301]

\begin{tabular}{|lll|}
\hline$(B-g)$ & $=0.14+0.63(B-V)$ & for $-0.34<(B-V)<1.45,-1.35<(U-B)<1.16$ \\
\hline$(u-b)$ & $=-0.28+0.94(U-B)$ & for $-0.34<(B-V)<1.45,-1.35<(U-B)<1.16$ \\
\hline$(v-V)=-0.69+1.69(B-V)$ & for $-0.34<(B-V)<1.45,-1.35<(U-B)<1.16$ \\
\hline
\end{tabular}

UBVRI(JHKLMN) - Johnson - 1965 [162]

\begin{tabular}{|c|c|c|}
\hline$g$ & $=$ & $V-0.19+0.41(B-V)$ \\
\hline$r$ & $=$ & $R+0.43+0.15(B-V)$ \\
\hline$(v-g)$ & $=$ & $-0.47+1.37(B-V)$ \\
\hline$(g-r)$ & $=$ & $-0.53+0.98(B-V)$ \\
\hline$(g-r)$ & $=$ & $-0.66+1.37(V-R)$ \\
\hline
\end{tabular}

Fig. 97. The photometric system uvgr - Thuan and Gunn - 1976 
TRANSMISSION CURVES

As derived from Fig 1 of [301].

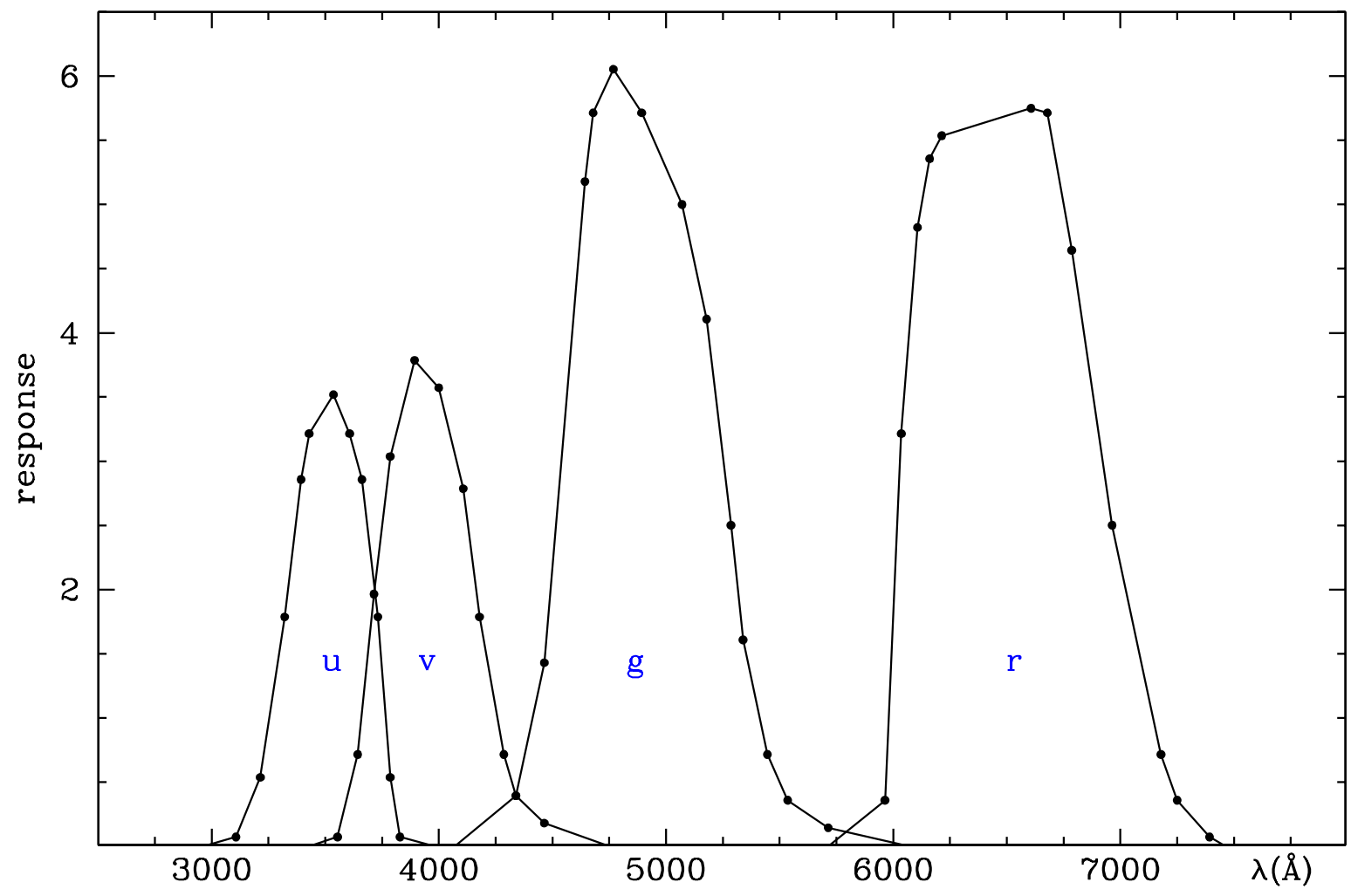

\begin{tabular}{|cc|cc|cc|cc|}
\hline \multicolumn{2}{|c|}{$u$} & \multicolumn{2}{|c|}{$V$} & \multicolumn{2}{c|}{$g$} & \multicolumn{2}{c|}{$r$} \\
\hline$\lambda(\mathrm{A})$ & $\Upsilon$ & $\lambda(\mathrm{A})$ & $\Upsilon$ & $\lambda(\mathrm{A})$ & $\Upsilon$ & $\lambda(\mathrm{A})$ & $\Upsilon$ \\
\hline 2964 & 0.000 & 3429 & 0.000 & 4071 & 0.000 & 5714 & 0.000 \\
3107 & 0.071 & 3554 & 0.071 & 4339 & 0.393 & 5964 & 0.357 \\
3214 & 0.536 & 3643 & 0.714 & 4464 & 1.429 & 6036 & 3.214 \\
3321 & 1.786 & 3714 & 1.964 & 4643 & 5.179 & 6107 & 4.821 \\
3393 & 2.857 & 3786 & 3.036 & 4679 & 5.714 & 6161 & 5.357 \\
3429 & 3.214 & 3893 & 3.786 & 4768 & 6.054 & 6214 & 5.536 \\
3536 & 3.518 & 4000 & 3.571 & 4893 & 5.714 & 6607 & 5.750 \\
3607 & 3.214 & 4107 & 2.786 & 5071 & 5.000 & 6679 & 5.714 \\
3661 & 2.857 & 4179 & 1.786 & 5179 & 4.107 & 6786 & 4.643 \\
3732 & 1.786 & 4286 & 0.714 & 5286 & 2.500 & 6964 & 2.500 \\
3786 & 0.536 & 4339 & 0.393 & 5339 & 1.607 & 7179 & 0.714 \\
3829 & 0.071 & 4464 & 0.179 & 5446 & 0.714 & 7250 & 0.357 \\
3982 & 0.000 & 4750 & 0.000 & 5536 & 0.357 & 7393 & 0.071 \\
& & & & 5714 & 0.143 & 7464 & 0.000 \\
& & & & 6071 & 0.000 & & \\
\hline
\end{tabular}

Fig. 97. continued 


\section{Apollo-Soyuz - 1976}

Extreme ultraviolet photometry during the Apollo-Soyuz mission.

\section{GENERAL INFORMATION}

TELESCOPE $\quad 0.37 \mathrm{~m}$ grazing-incidence mirror

DETECTOR channel electron-multiplier photon detectors

MAIN ARTICLE Lampton, M., Margon, B., Paresce, F., Stern, R., Bowyer, S. 1976, ApJ 203, L71

\section{SYSTEM DESCRIPTION}

\begin{tabular}{|r|ccc|}
\hline \multicolumn{4}{|c|}{ BANDS DESCRIPTION [179] } \\
\hline \multicolumn{1}{|c|}{ band } & filter material & bandpass $(\AA)(\#)$ & bandpass $(\mathrm{eV})(\#)$ \\
\hline 102 & Parylene & $55-150$ & $83-225$ \\
\hline 132 & Beryllium & $114-150$ & $83-109$ \\
\hline 395 & Aluminium & $170-620$ & $20-73$ \\
\hline 640 & Tin & $500-780$ & $16-25$ \\
\hline 1445 & $\mathrm{BaF}_{2}$ & $1350-1540$ & $8.0-9.2$ \\
\hline
\end{tabular}

(\#) bandpasses at $10 \%$ peak transmission.

Fig. 98. The photometric system Apollo-Soyuz - 1976 


\section{Trieste - Cester et al. - 1977}

$\mathrm{H} \alpha$ photometry of O-F stars.

\section{GENERAL INFORMATION}

$\begin{array}{ll}\text { AUTHORS } & \text { B. Cester, G. Giuricin, F. Mardirossian, } \\ & \text { M. Pucillo, F. Castelli, and F. Flora } \\ \text { TELESCOPE } & \text { 0.30m (reflector), Trieste Obs. } \\ \text { DETECTOR } & 9658 \text { B EMI (S-20 cathode) } \\ \text { MAIN ARTICLE } & \text { Cester, B., Giuricin, G., Mardirossian, F., Pucillo, M., } \\ & \text { Castelli, F., Flora, U. 1977, A\&AS 30, 1 }\end{array}$

\section{SYSTEM DESCRIPTION}

\begin{tabular}{|l|cc|}
\hline \multicolumn{3}{|c|}{ BANDS DESCRIPTION } \\
\hline \multicolumn{1}{|c|}{ band } & $\lambda_{c}(\AA)$ & half-width $(\AA)$ \\
\hline$H \alpha$ line & 6563 & 30 \\
\hline$H \alpha$ continuum & 6622 & 29 \\
\hline
\end{tabular}

\section{SYSTEM ANALYSIS}

\section{COLOR INDICES AND PARAMETERS [64]}

$\alpha=-2.5 \log \frac{I(6563)}{I(6622)}-2.5 \log \left(\int F_{\lambda} \Upsilon_{6563} d \lambda\right) /\left(\int F_{\lambda} \Upsilon_{6622} d \lambda\right)$

where $\Upsilon$ is the band response function and $F_{\lambda}$ is the monochromatic stellar flux.

RELATIONS WITH OTHER SYSTEMS [64]

uvbyH $\beta$ - Strömgren and Crawford - 1956

$\alpha_{\text {Trieste }}=0.722 \beta-1.719 \quad$ for $\beta>2.580$

$H \gamma$ - Beer - 1964

$\alpha_{\text {Trieste }}=0.478 \gamma+0.125$ for $\gamma>0.080$, where $\gamma=2.5 \log R_{\gamma}$

$H \alpha$ - Andrews - 1968

$\alpha_{\text {Trieste }}=0.911 \alpha_{\text {Andrews }}+0.075 \quad$ where $\alpha_{\text {Andrews }}=2.5 \log R_{\alpha}$

Fig. 99. The photometric system Trieste - Cester et al. - 1977 


\section{TRANSMISSION CURVES}

As derived from Fig 1 of [64].

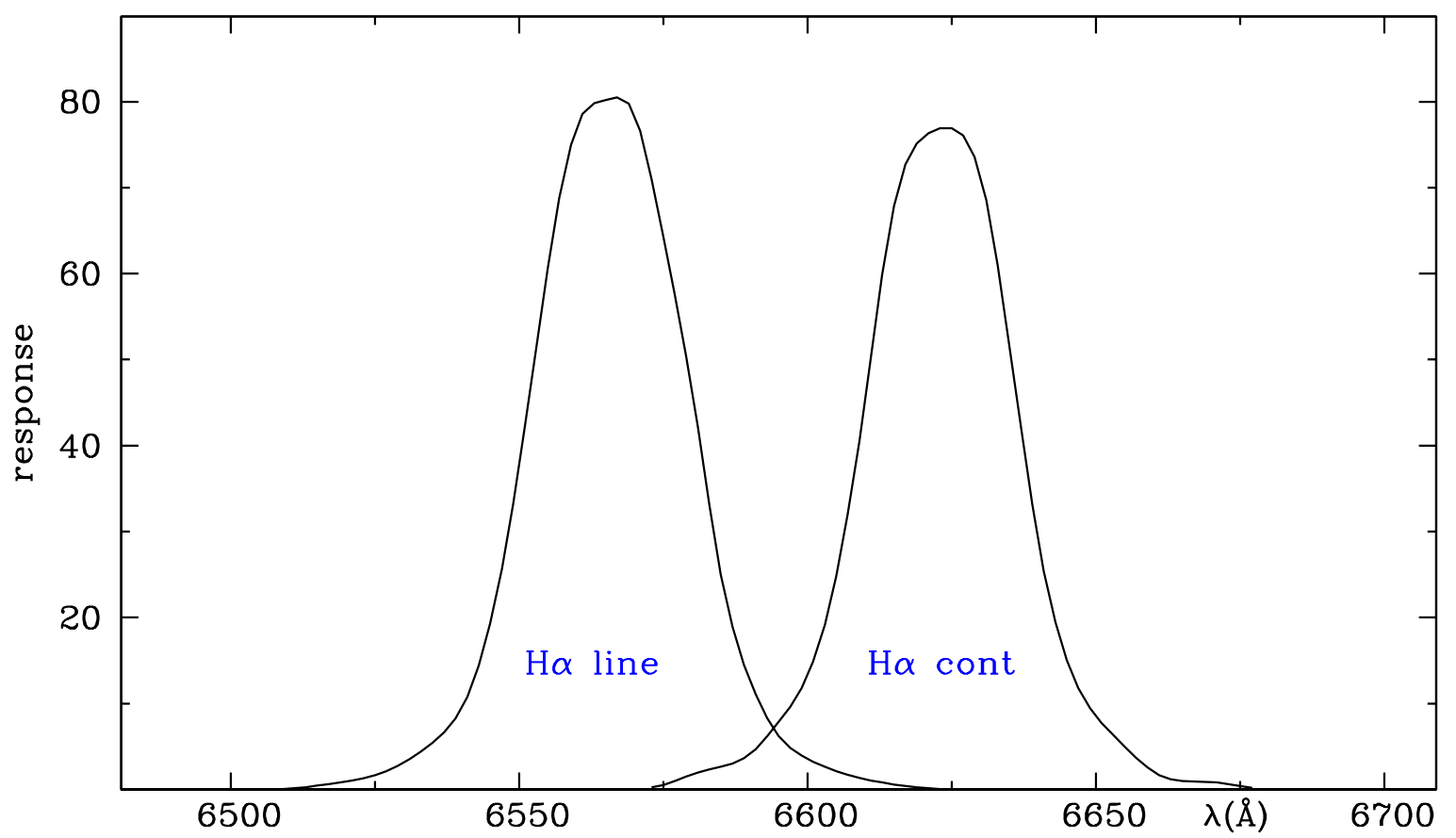

\begin{tabular}{|c|c|c|c|c|c|c|c|c|c|c|c|}
\hline \multicolumn{6}{|c|}{ Ho line } & \multicolumn{6}{|c|}{ Ho continuum } \\
\hline$\lambda(\mathrm{A})$ & $\Upsilon(\%)$ & $\lambda(\mathrm{A})$ & $\Upsilon(\%)$ & $\lambda(\mathrm{A})$ & $\Upsilon(\%)$ & $\lambda(\mathrm{A})$ & $\Upsilon(\%)$ & $\lambda(\mathrm{A})$ & $\Upsilon(\%)$ & $\lambda(\mathrm{A})$ & $\Upsilon(\%)$ \\
\hline 6509 & 0.00 & 6549 & 33.26 & 6589 & 14.52 & 6573 & 0.24 & 6613 & 59.90 & 6653 & 6.31 \\
\hline 6511 & 0.13 & 6551 & 42.06 & 6591 & 11.10 & 6575 & 0.53 & 6615 & 67.86 & 6655 & 5.00 \\
\hline 6513 & 0.28 & 6553 & 51.51 & 6593 & 8.32 & 6577 & 0.97 & 6617 & 72.70 & 6657 & 3.70 \\
\hline 6515 & 0.45 & 6555 & 60.71 & 6595 & 6.22 & 6579 & 1.49 & 6619 & 75.13 & 6659 & 2.53 \\
\hline 6517 & 0.63 & 6557 & 68.79 & 6597 & 4.83 & 6581 & 1.96 & 6621 & 76.34 & 6661 & 1.68 \\
\hline 6519 & 0.81 & 6559 & 74.98 & 6599 & 3.92 & 6583 & 2.34 & 6623 & 76.92 & 6663 & 1.20 \\
\hline 6521 & 1.03 & 6561 & 78.55 & 6601 & 3.24 & 6585 & 2.67 & 6625 & 76.89 & 6665 & 0.97 \\
\hline 6523 & 1.31 & 6563 & 79.83 & 6603 & 2.65 & 6587 & 3.03 & 6627 & 76.06 & 6667 & 0.92 \\
\hline 6525 & 1.67 & 6565 & 80.17 & 6605 & 2.14 & 6589 & 3.64 & 6629 & 73.59 & 6669 & 0.90 \\
\hline 6527 & 2.14 & 6567 & 80.48 & 6607 & 1.71 & 6591 & 4.68 & 6631 & 68.53 & 6671 & 0.81 \\
\hline 6529 & 2.75 & 6569 & 79.77 & 6609 & 1.35 & 6593 & 6.17 & 6633 & 60.93 & 6673 & 0.62 \\
\hline 6531 & 3.52 & 6571 & 76.62 & 6611 & 1.05 & 6595 & 7.86 & 6635 & 51.81 & 6675 & 0.41 \\
\hline 6533 & 4.44 & 6573 & 71.00 & 6613 & 0.80 & 6597 & 9.62 & 6637 & 42.24 & 6677 & 0.22 \\
\hline 6535 & 5.48 & 6575 & 64.29 & 6615 & 0.59 & 6599 & 11.81 & 6639 & 33.15 & & \\
\hline 6537 & 6.65 & 6577 & 57.55 & 6617 & 0.41 & 6601 & 14.88 & 6641 & 25.45 & & \\
\hline 6539 & 8.29 & 6579 & 50.40 & 6619 & 0.25 & 6603 & 19.15 & 6643 & 19.47 & & \\
\hline 6541 & 10.77 & 6581 & 42.12 & 6621 & 0.14 & 6605 & 24.81 & 6645 & 15.00 & & \\
\hline 6543 & 14.38 & 6583 & 33.14 & 6623 & 0.00 & 6607 & 31.98 & 6647 & 11.79 & & \\
\hline 6545 & 19.29 & 6585 & 25.00 & & & 6609 & 40.52 & 6649 & 9.49 & & \\
\hline 6547 & 25.61 & 6587 & 18.91 & & & 6611 & 50.17 & 6651 & 7.75 & & \\
\hline
\end{tabular}

Fig. 99. continued 


\section{JHK $\mathrm{H}_{2} \mathrm{O} \mathrm{CO}$ - Persson et al. - 1977}

Broad and intermediate band infrared photometry.

\section{GENERAL INFORMATION}

AUTHORS

TELESCOPE

DETECTOR

MAIN ARTICLE
S. E. Persson, M. Aaronson, J. A. Frogel and J. R. Baldwin

1.5m Hale (reflector), Mount Wilson Obs.; 1.52m Tillingast reflector, Mt. Hopkins Obs. $\mathrm{InSb}$

Persson, S. E., Aaronson, M., Frogel, J.A. 1977, AJ 82, 729

Baldwin, J. R., Frogel, J. A., Persson, S. E. 1973, ApJ 184, 427

\section{SYSTEM DESCRIPTION}

\begin{tabular}{|cr|cr|}
\hline \multicolumn{4}{|c|}{ BANDS DESCRIPTION } \\
\hline \multicolumn{2}{|c|}{ band } & $\lambda_{\text {eff }}(\mu \mathrm{m})$ & \multicolumn{1}{c|}{ FWHM $(\mu \mathrm{m})$} \\
\hline$J$ & {$[242]$} & $1.25 \pm 0.02$ & $0.24 \pm 0.01$ \\
\hline$H$ & {$[242]$} & $1.65 \pm 0.02$ & $0.30 \pm 0.01$ \\
\hline$K$ & {$[242]$} & $2.20 \pm 0.02$ & $0.40 \pm 0.01$ \\
\hline $\mathrm{H}_{2} \mathrm{O}$ & {$[19]$} & $2.10 \pm 0.01$ & $0.052 \pm 0.005$ \\
\hline 2.20 & {$[19]$} & $2.20 \pm 0.01$ & $0.080 \pm 0.005$ \\
\hline $\mathrm{CO}$ & {$[19]$} & $2.31 \pm 0.01$ & $0.095 \pm 0.005$ \\
\hline
\end{tabular}

ZERO POINT: $\alpha$ Lyr has magnitude 0.00 at all wavelenghts [242].

Later on the $\lambda_{\text {eff }}$ (and the FWHM) of the $\mathrm{H}_{2} \mathrm{O}, 2.20$ and $\mathrm{CO}$ bands have been revised to $2.00(0.080), 2.20(0.110)$ and $2.36(0.080) \mu \mathrm{m}$, respectively [242].

\section{SYSTEM ANALYSIS}

\section{COLOR INDICES AND PARAMETERS [19]}

[ $\left.\mathrm{H}_{2} \mathrm{O}-2.20\right]$ : strength of the $\mathrm{H}_{2} \mathrm{O}$ absorption band.

[ $\mathrm{CO}-2.20]$ : strength of the $\mathrm{CO}$ absorption band.

RELATIONS WITH OTHER SYSTEMS [108]

UBVRI(JHKLMN) - Johnson - 1965

$J_{\text {Johnson }}=1.09(J-H)+H$

Fig. 100. The photometric system $\mathrm{JHK}_{2} \mathrm{O} \mathrm{CO}$ - Persson et al. - 1977 


\section{TRANSMISSION CURVES}

As derived from Fig 1 of [19].

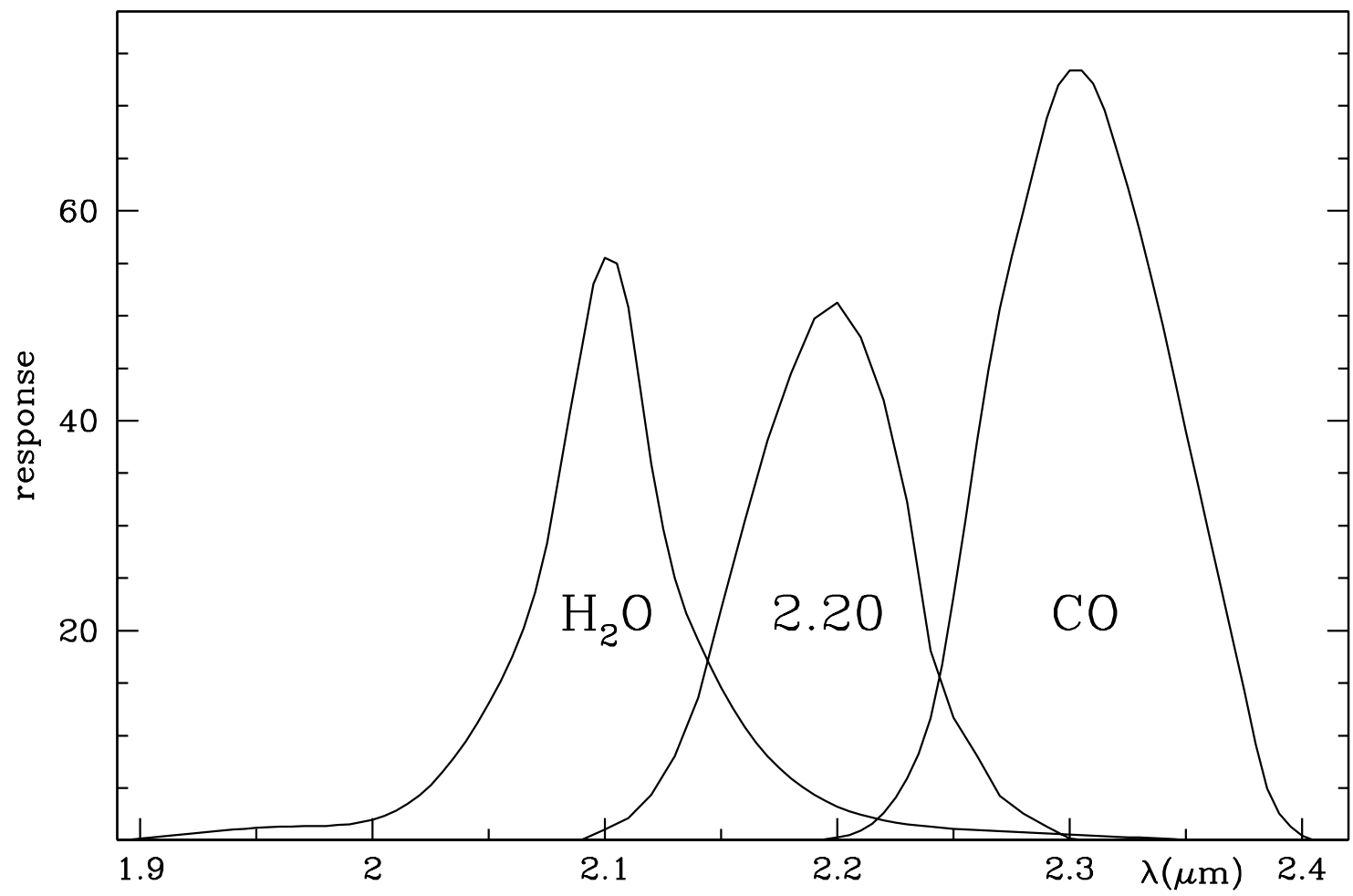

\begin{tabular}{|cc|cc|cc|cc|cc|cc|}
\hline \multicolumn{4}{|c|}{$\mathrm{H}_{2} \mathrm{O}$} & \multicolumn{5}{c|}{2.20} & \multicolumn{5}{c|}{$\mathrm{CO}$} \\
\hline$\lambda(\mu \mathrm{m})$ & $\Upsilon$ & $\lambda(\mu \mathrm{m})$ & \multicolumn{1}{c|}{$\Upsilon$} & $\lambda(\mu \mathrm{m})$ & \multicolumn{1}{c|}{$\Upsilon$} & $\lambda(\mu \mathrm{m})$ & $\Upsilon$ & $\lambda(\mu \mathrm{m})$ & $\Upsilon$ & $\lambda(\mu \mathrm{m})$ & $\Upsilon$ \\
\hline 1.891 & 0.0 & 2.102 & 55.6 & 2.089 & 0.0 & 2.212 & 46.8 & 2.188 & 0.0 & 2.322 & 64.8 \\
1.922 & 0.7 & 2.108 & 53.5 & 2.103 & 1.3 & 2.222 & 40.1 & 2.207 & 0.7 & 2.332 & 56.7 \\
1.964 & 1.3 & 2.113 & 46.5 & 2.116 & 3.3 & 2.233 & 28.7 & 2.216 & 1.7 & 2.349 & 40.1 \\
2.000 & 2.0 & 2.117 & 40.1 & 2.127 & 6.7 & 2.241 & 16.7 & 2.229 & 5.6 & 2.361 & 28.2 \\
2.010 & 2.9 & 2.127 & 27.9 & 2.140 & 13.4 & 2.256 & 10.0 & 2.241 & 12.7 & 2.376 & 13.0 \\
2.028 & 5.9 & 2.140 & 18.9 & 2.151 & 22.6 & 2.263 & 6.7 & 2.250 & 22.9 & 2.383 & 6.3 \\
2.045 & 11.0 & 2.157 & 11.7 & 2.164 & 33.8 & 2.275 & 3.3 & 2.262 & 40.4 & 2.386 & 4.1 \\
2.062 & 18.4 & 2.175 & 7.0 & 2.173 & 40.1 & 2.290 & 1.3 & 2.273 & 53.5 & 2.393 & 1.7 \\
2.075 & 28.7 & 2.195 & 3.7 & 2.185 & 47.1 & 2.304 & 0.0 & 2.284 & 63.4 & 2.407 & 0.0 \\
2.082 & 37.4 & 2.216 & 2.1 & 2.191 & 50.1 & & & 2.292 & 70.2 & & \\
2.089 & 45.8 & 2.250 & 1.1 & 2.197 & 51.3 & & & 2.302 & 73.5 & & \\
2.096 & 53.8 & 2.284 & 0.7 & 2.205 & 50.1 & & & 2.314 & 70.2 & & \\
\hline
\end{tabular}

Fig. 100. continued 


\section{$u b V r$ - Sandage and Visvanathan - 1978}

Photometry of galaxies.

\section{GENERAL INFORMATION}

AUTHORS

A. Sandage and N. Visvanathan

TELESCOPE

$1.5 \mathrm{~m}$ and $5 \mathrm{~m}$ (reflectors), Palomar Obs. ;

$1 \mathrm{~m}$ Swope reflector, Las Campanas Obs.

DETECTOR ITT-FW130 (S-20 cathode)

MAIN ARTICLE Sandage, A., Visvanathan, N. 1978, ApJ 223, 707

\section{SYSTEM DESCRIPTION}

\begin{tabular}{|c|c|c|c|}
\hline \multicolumn{4}{|c|}{ BANDS DESCRIPTION [257] } \\
\hline band & filter & $\lambda_{e f f}(\AA)$ & bandwidth $(\AA)$ [310] \\
\hline$u_{1}$ & interference & 3466 & 200 \\
\hline$u_{2}$ & interference & 3625 & 100 \\
\hline$u$ & $(\#)$ & 3550 & $\approx 250$ \\
\hline$b$ & interference & 4522 & 300 \\
\hline$V$ & GG14 + BG18 & 5400 & $\approx 800$ \\
\hline$r$ & interference & 6738 & 300 \\
\hline
\end{tabular}

(\#) The band $u$ is obtained during data reduction by adding the fluxes collected in the $u_{1}$ and $u_{2}$ bands.

ZERO POINT: The zero point of colors is defined by the 13 standard stars listed in [257].

\section{SYSTEM ANALYSIS}

RELATIONS WITH OTHER SYSTEMS [257]

UBV - Johnson and Morgan - 1953

\begin{tabular}{|l}
$(U-V)_{J}=1.05(u-V)-0.94$ \\
$(B-V)_{J}=1.25(b-V)+0.22$
\end{tabular}

Fig. 101. The photometric system $u b V r-$ Sandage and Visvanathan - 1978 


\section{8 colors - White and Wing - 1978}

Narrow band photometry of M stars.

\section{GENERAL INFORMATION}

$\begin{array}{ll}\text { AUTHORS } & \text { N. M. White and R. F. Wing } \\ \text { TELESCOPE } & \text { telescopes at Lowell Observatory and CTIO } \\ \text { DETECTOR } & \text { ITT FW-118 (S-1 cathode, refrigerated) } \\ \text { MAIN ARTICLE } & \text { White, N. M., Wing, R. F. 1978, ApJ 222, } 209\end{array}$

SYSTEM DESCRIPTION

\begin{tabular}{|c|ccll|}
\hline \multicolumn{5}{|c|}{ BANDS DESCRIPTION [322] } \\
\hline band & $\lambda_{c}(\AA)$ & FWHM $(\AA)$ & feature & contaminants \\
\hline 0.71 & 7120 & 60 & TiO & CN \\
\hline 0.75 & 7540 & 50 & continuum for K4-M6 stars & CN \\
\hline 0.78 & 7810 & 40 & continuum for G,K, Carbon stars & TiO \\
\hline 0.81 & 8120 & 50 & CN & TiO \\
\hline 1.04 & 10395 & 50 & continuum & \\
\hline 1.05 & 10540 & 60 & VO & He I $(10830 \AA)$ \\
\hline 1.08 & 10810 & 60 & continuum \\
\hline 1.10 & 10975 & 70 & CN & \\
\hline
\end{tabular}

Interference filters.

ZERO POINT: $\alpha$ Lyr has $\mathrm{m}(1.04)=0.00$ [322].

For the other bands the measurements are reduced to a system of absolute fluxes per unit wavelength interval defined by the model atmosphere energy distribution of Vega by [260].

Fig. 102. The photometric system 8 colors - White and Wing - 1978 


\title{
U'JF - van der Kruit - 1979
}

Broad-band photographic system for the Palomar-Westerbork survey of galaxies.

\section{GENERAL INFORMATION}

\author{
AUTHORS \\ P. C. van der Kruit \\ TELESCOPE $\quad$ 1.20 Schmidt, Palomar Obs. \\ DETECTOR photographic plates \\ MAIN ARTICLE Wevers, B. M. H. R., van der Kruit, P. C., Allen, R. J. 1986, A\&AS 66, 505
}

\section{SYSTEM DESCRIPTION}

\begin{tabular}{|c|c|c|c|c|c|c|}
\hline \multicolumn{5}{|c|}{ BANDS DESCRIPTION [321] } & \multirow[b]{2}{*}{$W H M(\AA)$} & \multirow[b]{2}{*}{$\lambda_{c}(\AA)$} \\
\hline band & emulsion & filter & wav. range $(\AA)$ & $\lambda_{0}(\AA)$ & & \\
\hline$U^{\prime}$ & IIIa-J & UG 5 & $3400-4250$ & $\approx 3760$ & 510 & 3728 \\
\hline$J$ & IIIa-J & Wr2C & $3950-5450$ & $\approx 4700$ & 1385 & 4675 \\
\hline$F$ & IIIa-F & Wr23A & $5800-6900$ & $\approx 6400$ & 1000 & 6355 \\
\hline
\end{tabular}

\section{SYSTEM ANALYSIS}

\section{RELATIONS WITH OTHER SYSTEMS [321]}

UBV - Johnson and Morgan - 1953

\begin{tabular}{|rll|}
\hline$J$ & $=B-0.24( \pm 0.04)(B-V) \pm 0.01$ \\
\hline$\left(U^{\prime}-J\right)$ & $=1.08( \pm 0.15)(U-B) \pm 0.10 \quad$ for galaxies and F-G-K-M stars \\
\hline$(J-F)$ & $=1.25( \pm 0.10)(B-V) \pm 0.03$ & \\
\hline
\end{tabular}

Fig. 103. The photometric system U'JF - van der Kruit - 1979 


\section{TRANSMISSION CURVES}

As derived from Fig a of [321].

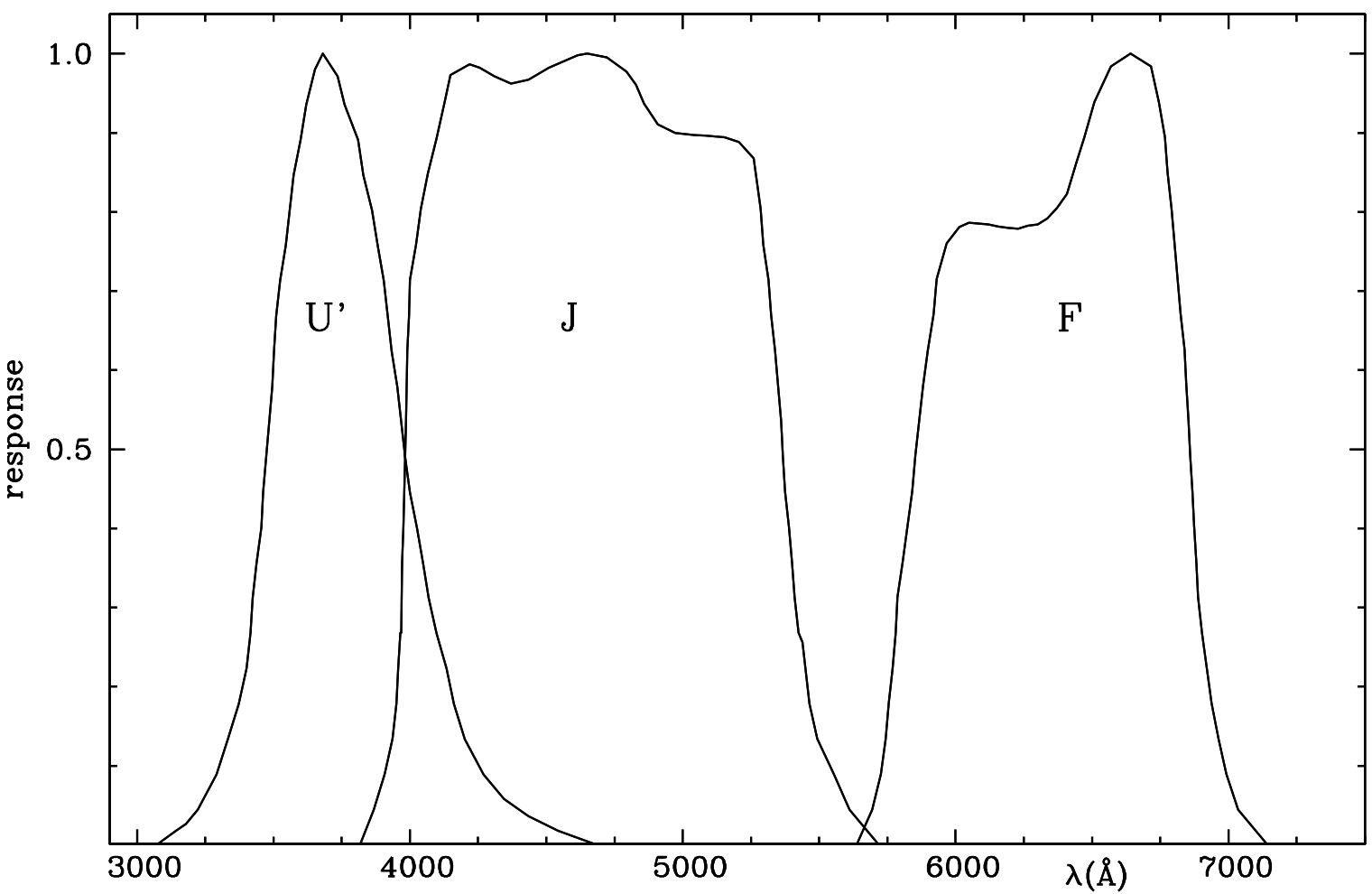

\begin{tabular}{|cc|cc|cc|cc|cc|cc|}
\hline \multicolumn{4}{|c|}{$U^{\prime}$} & \multicolumn{3}{c|}{$J$} & \multicolumn{4}{c|}{$F$} \\
\hline$\lambda(\mathrm{A})$ & $\Upsilon$ & $\lambda(\mathrm{A})$ & $\Upsilon$ & $\lambda(\mathrm{A})$ & $\Upsilon$ & $\lambda(\mathrm{A})$ & $\Upsilon$ & $\lambda(\mathrm{A})$ & $\Upsilon$ & $\lambda(\mathrm{A})$ & $\Upsilon$ \\
\hline 3040 & 0.000 & 3800 & 0.909 & 3800 & 0.000 & 4840 & 0.953 & 5620 & 0.000 & 6540 & 0.968 \\
3080 & 0.002 & 3840 & 0.828 & 3840 & 0.018 & 4880 & 0.923 & 5660 & 0.015 & 6580 & 0.987 \\
3120 & 0.012 & 3880 & 0.763 & 3880 & 0.057 & 4920 & 0.907 & 5700 & 0.051 & 6620 & 0.995 \\
3160 & 0.022 & 3920 & 0.663 & 3920 & 0.105 & 5000 & 0.899 & 5740 & 0.119 & 6660 & 1.000 \\
3200 & 0.034 & 3960 & 0.563 & 3960 & 0.244 & 5080 & 0.896 & 5780 & 0.267 & 6700 & 0.997 \\
3240 & 0.054 & 4000 & 0.448 & 4000 & 0.702 & 5160 & 0.894 & 5820 & 0.394 & 6740 & 0.947 \\
3280 & 0.080 & 4040 & 0.373 & 4040 & 0.802 & 5240 & 0.885 & 5860 & 0.518 & 6780 & 0.841 \\
3320 & 0.119 & 4080 & 0.293 & 4080 & 0.867 & 5280 & 0.823 & 5900 & 0.629 & 6820 & 0.687 \\
3360 & 0.162 & 4120 & 0.241 & 4120 & 0.927 & 5320 & 0.689 & 5940 & 0.742 & 6860 & 0.493 \\
3400 & 0.220 & 4160 & 0.182 & 4160 & 0.986 & 5360 & 0.539 & 5980 & 0.765 & 6900 & 0.275 \\
3440 & 0.360 & 4200 & 0.135 & 4200 & 0.992 & 5400 & 0.357 & 6020 & 0.783 & 6940 & 0.177 \\
3480 & 0.517 & 4240 & 0.106 & 4240 & 0.984 & 5440 & 0.256 & 6100 & 0.785 & 6980 & 0.109 \\
3520 & 0.705 & 4320 & 0.067 & 4320 & 0.969 & 5480 & 0.147 & 6180 & 0.781 & 7020 & 0.058 \\
3560 & 0.803 & 4400 & 0.044 & 4400 & 0.963 & 5520 & 0.114 & 6260 & 0.782 & 7060 & 0.029 \\
3600 & 0.893 & 4480 & 0.028 & 4480 & 0.976 & 5560 & 0.085 & 6340 & 0.793 & 7100 & 0.012 \\
3640 & 0.966 & 4560 & 0.015 & 4560 & 0.992 & 5600 & 0.053 & 6380 & 0.808 & 7140 & 0.000 \\
3680 & 1.000 & 4640 & 0.005 & 4640 & 1.000 & 5680 & 0.012 & 6420 & 0.835 & & \\
3720 & 0.989 & 4720 & 0.000 & 4720 & 0.996 & 5720 & 0.000 & 6460 & 0.881 & & \\
3760 & 0.936 & & & 4800 & 0.976 & & & 6500 & 0.929 & & \\
\hline
\end{tabular}

Fig. 103. continued 


\title{
BVRI - Kunkel and Rydgren - 1979
}

Exports the BVRI bands of the UBVRI(JHKLMN) - Johnson - 1965 system (based on 1P21 and S-1 photomultipliers) to a natural system based on the RCA 31034A.

\section{GENERAL INFORMATION}

\author{
AUTHORS W. E. Kunkel and A. E. Rydgren \\ TELESCOPE CTIO telescopes \\ DETECTOR S-1 and RCA 31034A photomultipliers \\ MAIN ARTICLE Kunkel W.E., Rydgren A.E. 1979, AJ. 84, 633
}

\section{SYSTEM DESCRIPTION}

\begin{tabular}{|c|l|}
\hline \multicolumn{2}{|c|}{ BANDS DESCRIPTION [176] } \\
\hline band & \multicolumn{1}{c|}{ filter } \\
\hline$B$ & Corning 5030 + 2mm BG18 + 2mm GG385 \\
\hline$V$ & Corning 3384 + Corning 9780 \\
\hline$R$ & 3mm KG1 + 2mm OG5 + 1.5mm RG6 \\
\hline$I$ & 3mm RG715 + 1mm RG780 \\
\hline
\end{tabular}

All filters by Schott unless otherwise noted. Corning filters have standard optical thickness. [176]

ZERO POINT: Unreddened A0 V stars have 0.00 colors. [176]

\section{SYSTEM ANALYSIS}

For the 48 faint equatorial standard stars (covering a large range in color) in common with Landolt [181]

$$
\text { S-1 photomultiplier - RCA 31034A photomultiplier }
$$

Transformation relations between the S-1 (natural) and the RCA 31034A (natural) systems

\begin{tabular}{|rll|}
\hline$(V-R)_{S 1 \text { nat }}$ & $=1.067(V-R)_{R C A \text { nat }}+0.054$ & $\sigma= \pm 0.013 \mathrm{mag}$ \\
\hline$\left(V_{I}\right)_{S 1 \text { nat }}$ & $=1.163(V-I)_{R C A \text { nat }}+0.028$ & $\sigma= \pm 0.024 \mathrm{mag}$ \\
\hline
\end{tabular}

\section{RELATIONS WITH OTHER SYSTEMS [176]}

UBVRI(JHKLMN) - Johnson - 1965

with S-1 photomultiplier (S-1 nat)

\begin{tabular}{|lll|}
\hline$(V-R)_{J}$ & $=1.220(V-R)_{S 1 \text { nat }}-0.002$ & $\sigma= \pm 0.023 \mathrm{mag}$ \\
\hline$(V-I)_{J}$ & $=1.136(V-I)_{S 1 \text { nat }}-0.020$ & $\sigma= \pm 0.032 \mathrm{mag}$ \\
\hline
\end{tabular}

with RCA 31034A photomultiplier (RCA nat)

\begin{tabular}{|lll|}
\hline$(V-R)_{J}$ & $=1.303(V-R)_{R C A ~ n a t}+0.064$ & $\sigma= \pm 0.014 \mathrm{mag}$ \\
\hline$(V-I)_{J}$ & $=1.331(V-I)_{R C A ~ n a t}+0.004$ & $\sigma= \pm 0.027 \mathrm{mag}$ \\
\hline
\end{tabular}

Fig. 104. The photometric system BVRI - Kunkel and Rydgren - 1979 


\section{ri - Wade et al. - 1979}

Near-infrared system. Related to photographic photometry with the IV-N emulsion.

\section{GENERAL INFORMATION}

AUTHORS

TELESCOPE

DETECTOR

MAIN ARTICLE
R. A. Wade, J. G. Hoessel, J. H. Elias and J. P. Huchra

$1.5 \mathrm{~m}$ (reflector), Palomar Observatory

ITT FW118 (S-1 cathode, refrigerated)

Wade, R. A., Hoessel, J. G., Elias, J. H.,and Huchra, J. P. 1979, PASP 91, 35

\section{SYSTEM DESCRIPTION}

\begin{tabular}{|c|ccc|}
\hline \multicolumn{4}{|c|}{ BANDS DESCRIPTION [312] } \\
\hline band & filter & $\lambda_{\text {eff }}(\AA)$ & FWHM $(\AA)$ \\
\hline$r$ & 3mm Schott RG610 + Balzers B1 (\#) & 6500 & 1000 \\
\hline$i$ & Wratten 88a gelatin filter + Balzers infrared mirror & 8200 & 1300 \\
\hline
\end{tabular}

(\#) The red leak at $1 \mu m$ in the Balzers filter is blocked with Schott KG1 glass.

ZERO POINT: $i=r=9.50$ for $\mathrm{BD}+17^{\circ} 4708$ (F8). [312]

\section{SYSTEM ANALYSIS}

RELATIONS WITH OTHER SYSTEMS [312]

UBVRI(JHKLMN) - Johnson - 1965

$\begin{aligned} i & =0.690+0.999 I_{J}+0.419(R-I)_{J} & (\sigma=0.033 \mathrm{mag}) & -0.20<(R-I)<+0.70 \\ (r-i) & =-0.252+0.765(R-I)_{J} & (\sigma=0.016 \mathrm{mag}) & -0.20<(R-I)<+0.70\end{aligned}$

Fig. 105. The photometric system ri - Wade et al. - 1979 


\section{$B_{J} R_{F}-$ Couch and Newell - 1980}

Photoelectric porting of the photographic JF system (IIIa-J + GG385 and IIIa-F RG630 combinations).

\section{GENERAL INFORMATION}
AUTHORS
W. J. Couch and E. B. Newell
TELESCOPE
$1 \mathrm{~m}$ and $0.6 \mathrm{~m}$ (reflectors), Siding Spring National Observatory; $1.5 \mathrm{~m}$ and $0.9 \mathrm{~m}$ (reflectors), CTIO
DETECTOR
EMI 9658AM (S-20 cathode, refrigerated) at SSNO
ITT FW130 (S-20 cathode) at CTIO
MAIN ARTICLE Couch, W. J., Newell, E. B. 1980, PASP 92, 746

\section{SYSTEM DESCRIPTION}

\begin{tabular}{|c|c|c|c|c|c|c|c|}
\hline \multicolumn{4}{|c|}{ BANDS DESCRIPTION [74], photoel. ver. } & \multirow{2}{*}{\multicolumn{2}{|c|}{$W H M(\AA)$}} & \multirow{2}{*}{\multicolumn{2}{|c|}{$\lambda_{c}(\AA)$}} \\
\hline band & filter $_{\text {photoel }}$ & $\lambda_{e f f}(\AA)(\#)$ & half-width $(\AA)$ & & & & \\
\hline$B_{J}$ & 2mm GG385 + 2mm BG28 (\#) & 4385 & 1050 & 1055 & 1465 & 4422 & 4588 \\
\hline$R_{F}$ & $3 \mathrm{~mm}$ RG630 + 3mm BG 20 + 1mm BG38 (\#) & 6676 & 500 & 490 & 525 & 6545 & 6685 \\
\hline
\end{tabular}

(\#) 2mm GG 385 + IIIa-J $\left(B_{J}\right)$, and 2mm RG630 + IIIa-F $\left(R_{F}\right)$ for the photographic version.

For $W H M$ and $\lambda_{C}$, left values refer to the photoelectric version while those on the right to the photographic one.

(\#) Based on A0 star model atmosphere. [74]

PHOTOGRAPHIC VERSION Couch, W. J., Newell, E. B. 1980, PASP 92, 746

\section{SYSTEM ANALYSIS}

\section{RELATIONS WITH OTHER SYSTEMS [74]}

\begin{tabular}{c} 
UBVRI(JHKLMN) - Johnson - 1965 and RI - Cousin - 1976 \\
\hline$\left(B_{J}-R_{F}\right)=-0.027(B-R)^{2}+1.059(B-R)-0.017 \quad$ for $0.0 \leq(B-R) \leq 2.5$ \\
\hline
\end{tabular}

\section{TRANSMISSION CURVES [74]}

\begin{tabular}{|cc|cc|cc|cc|cc|cc|c|c|}
\hline \multicolumn{7}{|c|}{ photoelectric } & \multicolumn{5}{c|}{ photographic } \\
\hline \multicolumn{7}{|c|}{$B_{J}$} & \multicolumn{3}{c|}{$R_{F}$} & \multicolumn{4}{c|}{$B_{J}$} \\
\hline$\lambda(\AA)$ & $\Upsilon(\%)$ & $\lambda(\AA)$ & $\Upsilon(\%)$ & $\lambda(\AA)$ & $\Upsilon(\%)$ & $\lambda(\AA)$ & $\Upsilon(\%)$ & $\lambda(\AA)$ & $\Upsilon(\%)$ & $\lambda(\AA)$ & $\Upsilon(\%)$ & $\lambda(\AA)$ & $\Upsilon(\%)$ \\
\hline 3600 & 0 & 4800 & 73 & 6100 & 0 & 7300 & 4 & 3600 & 0 & 4800 & 78 & 6200 & 0 \\
3700 & 10 & 4900 & 56 & 6200 & 1 & 7400 & 0 & 3700 & 4 & 4900 & 78 & 6300 & 6 \\
3800 & 29 & 5000 & 42 & 6300 & 19 & 7500 & 0 & 3800 & 24 & 5000 & 76 & 6400 & 18 \\
3900 & 50 & 5100 & 28 & 6400 & 38 & 7600 & 1 & 3900 & 72 & 5100 & 74 & 6500 & 26 \\
4000 & 67 & 5200 & 16 & 6500 & 39 & 7700 & 2 & 4000 & 92 & 5200 & 68 & 6600 & 29 \\
4100 & 78 & 5300 & 8 & 6600 & 34 & 7800 & 1 & 4100 & 99 & 5300 & 56 & 6700 & 34 \\
4200 & 88 & 5400 & 5 & 6700 & 27 & 7900 & 0 & 4200 & 99 & 5400 & 28 & 6800 & 40 \\
4300 & 93 & 5500 & 5 & 6800 & 19 & & & 4300 & 94 & 5500 & 12 & 6900 & 29 \\
4400 & 96 & 5600 & 4 & 6900 & 16 & & & 4400 & 90 & 5600 & 4 & 7000 & 11 \\
4500 & 96 & 5700 & 3 & 7000 & 15 & & & 4500 & 86 & 5700 & 0 & 7100 & 3 \\
4600 & 93 & 5800 & 1 & 7100 & 12 & & & 4600 & 84 & & & 7200 & 1 \\
4700 & 85 & 5900 & 0 & 7200 & 9 & & & 4700 & 82 & & & 7300 & 0 \\
\hline
\end{tabular}

Fig. 106. The photometric system $B_{J} R_{F}-$ Couch and Newell -1980 


\section{PHOTOELECTRIC VERSION}

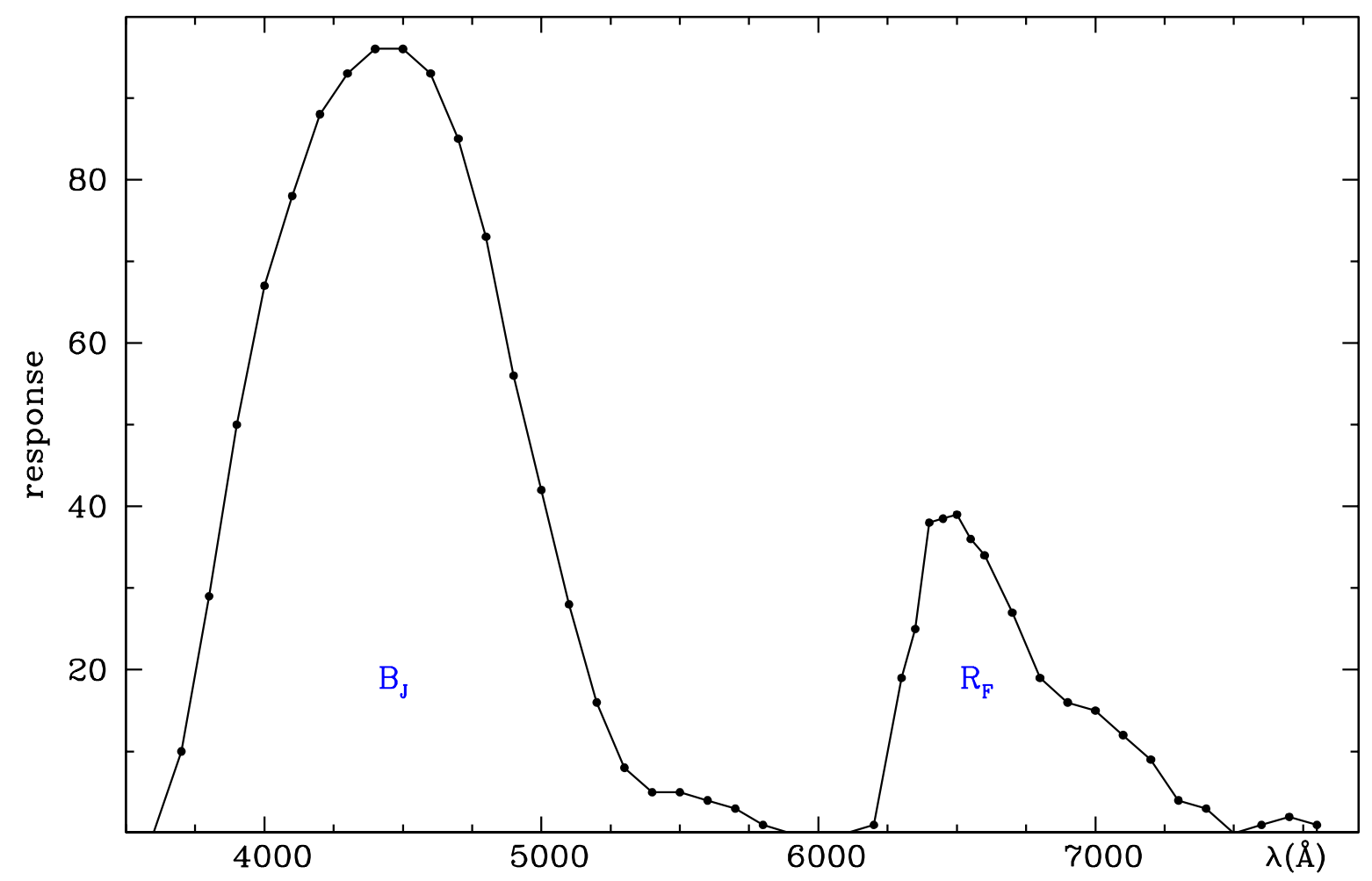

PHOTOGRAPHIC VERSION

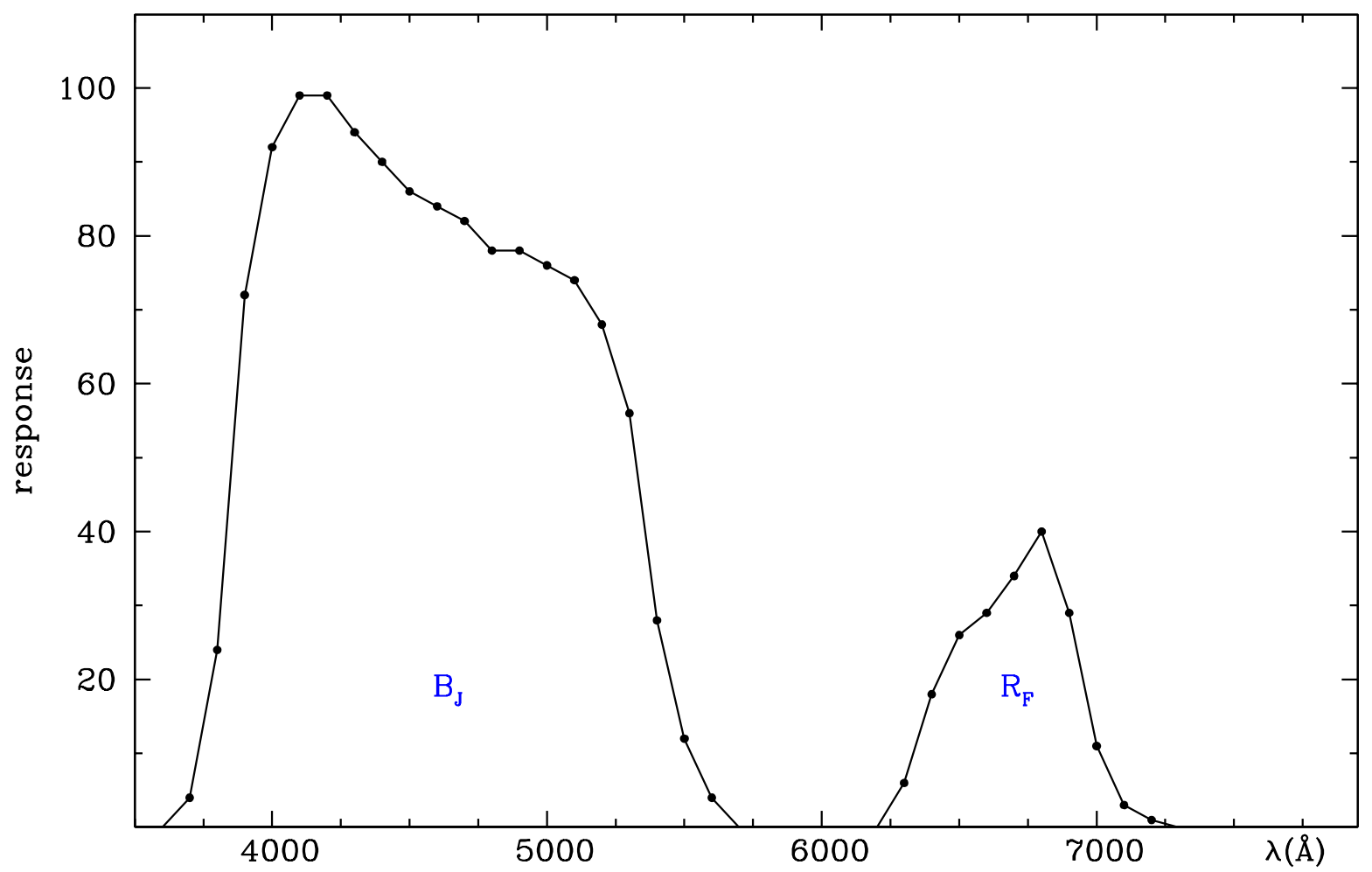

Fig. 106. continued 


\section{UBVRI - Neckel and Chini - 1980}

An attempt to realize UBVRI bands of the UBVRIJHKLMN - Johnson - 1965 system with EMI 6256 and RCA 31034A photomultipliers. Interstellar reddening law.

\section{GENERAL INFORMATION}

$\begin{array}{ll}\text { AUTHORS } & \text { T. Neckel and R. Chini } \\ \text { TELESCOPE } & \text { 0.50m (reflector), Gamsberg, Namibia; } \\ & \text { 1.0m ESO, La Silla; 1.23m Calar Alto, Spain } \\ \text { DETECTOR } & \text { EMI 6256 (UBV bands) and RCA 31034 (VRI bands) } \\ \text { MAIN ARTICLE } & \text { Neckel, Th., Chini, R. 1980, A\&AS 39, 411 }\end{array}$

\section{SYSTEM DESCRIPTION}

\begin{tabular}{|c|ll|}
\hline \multicolumn{3}{|c|}{ BANDS DESCRIPTION [222] } \\
\hline band & photomultiplier & \multicolumn{1}{c|}{ filter } \\
\hline$U$ & EMI 6256 & $1.5 \mathrm{~mm} \mathrm{UG} 2$ \\
\hline$B$ & EMI 6256 & $1.0 \mathrm{~mm}$ BG3 + 2.0mm GG385 \\
\hline$V$ & EMI 6256 & 3.0mm GG495 \\
& RCA 31034 & $3.0 \mathrm{~mm}$ GG495 + 1.0mm BG18 \\
\hline$R$ & RCA 31034 & 3.0mm OG590 + 2mm Calflex C \\
\hline$I$ & RCA 31034 & 3.0mm RG780 \\
\hline
\end{tabular}

SYSTEM ANALYSIS

REDDENING RATIOS [223]

$$
\begin{array}{ll}
\frac{E(V-R)}{E(B-V)}=0.82( \pm 0.01) & \frac{E(V-I)}{E(B-V)}=1.65( \pm 0.02) \\
R(V)=A_{V} / E(B-V)=3.1
\end{array}
$$

RELATIONS WITH OTHER SYSTEMS [297]

only for [222] and [223] data
\begin{tabular}{|cc|}
\hline$(V-R)_{C}=$ & $0.758( \pm 0.004)(V-R)_{N-C}-0.037( \pm 0.003)$ \\
\hline$(R-I)_{C}=$ & $0.794( \pm 0.004)(R-I)_{N-C}+0.039( \pm 0.002)$ \\
\hline
\end{tabular}

Fig. 107. The photometric system UBVRI - Neckel and Chini - 1980 


\section{uvgr $39_{B} 39_{N}-$ Zinn - 1980}

Interstellar reddening and metallicity of globular clusters.

\section{GENERAL INFORMATION}

$\begin{array}{ll}\text { AUTHORS } & \text { R. Zinn } \\ \text { TELESCOPE } & 0.51 \mathrm{~m} \text { and } 1.5 \mathrm{~m} \text { (reflectors), Mount Palomar Obs.; } \\ & \text { 1m and 2.5m (reflectors), Las Campanas Obs. } \\ \text { DETECTOR } & \text { 1P21 (S-20 cathode) } \\ \text { MAIN ARTICLE } & \text { Zinn, R. 1980, ApJS 42, 19 }\end{array}$

\section{SYSTEM DESCRIPTION}

\begin{tabular}{|c|c|c|c|c|}
\hline \multicolumn{5}{|c|}{ BANDS DESCRIPTION [335] } \\
\hline band & filter & $\lambda_{0}(\AA)$ & $\lambda_{\text {peak }}(\AA)$ & FWHM $(\AA)$ \\
\hline$u$ & 1mm Schott BG38 + 4mm Schott UG11 & 3540 & 3600 & 380 \\
\hline \multirow[t]{2}{*}{$V$} & 3mm Schott BG38 + 4mm Schott UG3 + & & & \\
\hline & 1mm Schott BG1 + 1mm Schott GG13 & 3990 & 3940 & 450 \\
\hline \multirow[t]{2}{*}{$g$} & $3 \mathrm{~mm}$ Schott BG38 + 1mm Schott GG460+ & & & \\
\hline & Kodak Wratten 44 & 4910 & 4930 & 700 \\
\hline$r$ & $6 \mathrm{~mm}$ Schott RG2 + 3mm Schott BG20 & 6730 & 6550 & 810 \\
\hline $39_{B}$ & interference & 3910 & 3920 & 180 \\
\hline $39_{N}$ & interference & 3955 & 3950 & 70 \\
\hline
\end{tabular}

ZERO POINT: $\mathrm{BD}+17^{\circ} 4708(\mathrm{~F} 8)$ is taken to have $g=9.500$ and all colors equal to 0.00 . [335]

\section{SYSTEM ANALYSIS}

\section{COLOR INDICES AND PARAMETERS [335]}

$39_{B}$ : centered on $\mathrm{H}$ and $\mathrm{K} \mathrm{CaII}$ lines.

$39_{N}$ : sensitive to line blocking.

$[F E / H]=-1.98( \pm 0.03)+4.40( \pm 0.21) Q 39$

REDDENING-FREE PARAMETERS [335]

$$
\begin{aligned}
& Q(v g r)=(v-g)-0.68(g-r) \quad Q(u g r)=(u-g)-1.08(g-r) \\
& Q\left(39_{B} g r\right)=\left(39_{B}-g\right)-0.76(g-r) \quad Q\left(39_{N} g r\right)=\left(39_{N}-g\right)-0.75(g-r) \\
& Q(39 u v)=\left(39_{B}-v\right)+\left(39_{N}-v\right)-0.36(u-v) \quad Q(39 v g)=\left(39_{B}-v\right)+\left(39_{N}-v\right)-0.20(v-g) \\
& Q 39=1 / 2[Q(39 u v)+Q(39 v g)]
\end{aligned}
$$

Fig. 108. The photometric system uvgr $39_{B} 39_{N}-$ Zinn -1980 
REDDENING RATIOS [335]

Calculated for A0 V stars:

$$
\begin{array}{lll}
\frac{E(u-v)}{E(v-g)}=0.56 & \frac{E(v-g)}{E(g-r)}=0.63 & \frac{E(u-g)}{E(g-r)}=0.98 \\
E\left(39_{B}-g\right) / E(g-r)=0.69 & \frac{E(B-V)_{J}}{E(g-r)}=0.73 & E\left(39_{N}-g\right) / E(g-r)=0.67 \\
{\left[E\left(39_{N}-v\right)+E\left(39_{B}-v\right)\right] / E(u-v)=0.29} & {\left[E\left(39_{N}-v\right)+E\left(39_{B}-v\right)\right] / E(v-g)=0.16}
\end{array}
$$

RELATIONS WITH OTHER SYSTEMS [335]

uvgr - Thuan and Gunn - 1976 (Kent's revision)

\begin{tabular}{|rll|}
\hline$(u-v)_{T-G}$ & $=0.948( \pm 0.006)(u-v)-0.003( \pm 0.002)$ & $\sigma=0.007$ \\
\hline$(v-g)_{T-G}$ & $=1.023( \pm 0.004)(v-g)+0.000( \pm 0.002)$ & $\sigma=0.008$ \\
\hline$(g-r)_{T-G}$ & $=0.930( \pm 0.006)(g-r)+0.006( \pm 0.002)$ & $\sigma=0.008$ \\
\hline$g_{T-G}=g+0.042( \pm 0.011)(g-r)-0.009( \pm 0.004)$ & $\sigma=0.015$ \\
\hline
\end{tabular}

Fig. 108. continued 


\section{VJHKLM ESO - Engels et al. - 1981}

Infrared observations at ESO - La Silla.

\section{GENERAL INFORMATION}

AUTHORS D. Engels, W.A. Sherwood, W. Wamsteker and G. V. Schultz

TELESCOPE $\quad 1 \mathrm{~m}$ (reflector) ESO, La Silla

DETECTOR InSb (liquid $\mathrm{N}_{2}$ refrigerated)

MAIN ARTICLE Engels, D., Sherwood, W. A., Wamsteker, W., Schultz, G. V. 1981, A\&AS 45, 5

\section{SYSTEM DESCRIPTION}

\begin{tabular}{|c|cc|ccc|}
\hline \multicolumn{3}{|c|}{ BANDS DESCRIPTION [314] } & \multicolumn{3}{|c|}{ FLUX CALIBRATION (\#) [314] } \\
\hline band & $\lambda_{0}(\mu \mathrm{m})$ & FWHM $(\mu \mathrm{m})$ & $\mathrm{F}_{\lambda, 0}\left(\mathrm{~W} \mathrm{~m}^{-2} \AA^{-1}\right)$ & $\mathrm{F}_{\mathrm{v}, 0}\left(\mathrm{~W} \mathrm{~m}^{-2} \mathrm{~Hz}^{-1}\right)$ & $\mathrm{F}_{\mathrm{v}, 0}(\mathrm{Jy})$ \\
\hline$V$ & 0.55 & 0.09 & $3.64( \pm 0.02) 10^{-12}$ & $3.6710^{-23}$ & 3670 \\
\hline$J$ & 1.25 & 0.3 & $3.18( \pm 0.11) 10^{-13}$ & $1.6510^{-23}$ & 1650 \\
\hline$H$ & 1.65 & 0.4 & $1.18( \pm 0.04) 10^{-13}$ & $1.0710^{-23}$ & 1070 \\
\hline$K$ & 2.2 & 0.6 & $4.17( \pm 0.13) 10^{-14}$ & $6.7310^{-24}$ & 673 \\
\hline$L$ & 3.6 & 1.2 & $6.23( \pm 0.21) 10^{-15}$ & $2.6910^{-24}$ & 269 \\
\hline$M$ & 4.8 & 0.8 & $2.07( \pm 0.09) 10^{-15}$ & $1.5810^{-24}$ & 158 \\
\hline
\end{tabular}

(\#) For a mag $=0.0$ star.

ZERO POINT: For $J, K, L$ bands the zero point is the same as for the equivalent bands of the $U B V R I(J H K L M N)$ - Johnson - 1965. The zero point of the $H$ band is such that $(J-K)=0.00 \Longrightarrow(H-K)=0.00$. Finally, for the $\mathrm{M}$ band the zero point is set by the condition that $(V-M)=1.54$ for the Sun. [99], [314]

Fig. 109. The photometric system VJHKLM ESO - Engels et al. - 1981 


\section{$\Delta a$ - Joncas and Borra - 1981}

Measurement of the continuum depression at $5200 \mathrm{~A}$ in Ap stars.

\section{GENERAL INFORMATION}

$\begin{array}{ll}\text { AUTHORS } & \text { G. Joncas and E. F. Borra } \\ \text { TELESCOPE } & \begin{array}{l}\text { 1.6m (reector), Mt. Megantic Obs., Canada } \\ \text { reectors at Las Campanas Obs., Chile }\end{array} \\ \text { DETECTOR } & \begin{array}{l}\text { EMI 6256 S (S-11 cathode) at Mt. Megantic Obs } \\ \text { EMI 9658 RF (S-20 red ext.) at Las Campanas }\end{array} \\ \text { MAIN ARTICLE } & \text { Joncas J., Borra E. F. 1981, A\&A 94, 134 }\end{array}$

\section{SYSTEM DESCRIPTION}

\begin{tabular}{|c|c|c|c|c|}
\hline \multicolumn{3}{|c|}{ BANDS DESCRIPTION [157] } & \multirow[b]{2}{*}{ WHM (A) } & \multirow[b]{2}{*}{$\lambda_{c}(\mathrm{~A})$} \\
\hline band & $\lambda_{\text {peak }}(\mathrm{A})$ & FWHM (A) & & \\
\hline$F_{1}$ & 5000 & $\sim 100$ & 110 & 4995 \\
\hline$F_{2}$ & 5220 & $\sim 100$ & 110 & 5223 \\
\hline$F_{3}$ & 5400 & $\sim 100$ & 90 & 5405 \\
\hline
\end{tabular}

Interference lters.

\section{SYSTEM ANALYSIS}

\section{COLOR INDICES AND PARAMETERS [157]}

$\Delta a=\left[F_{2}-0.5\left(F_{1}+F_{3}\right)\right]-a_{o}$ where $a_{o}$ is the $a$ value of a depression-free normal star of the same Stromgren $(b-y)$ index that has to be measured with the same instrumentation.

\section{TRANSMISSION CURVES}

As derived from Fig 1 of [157].

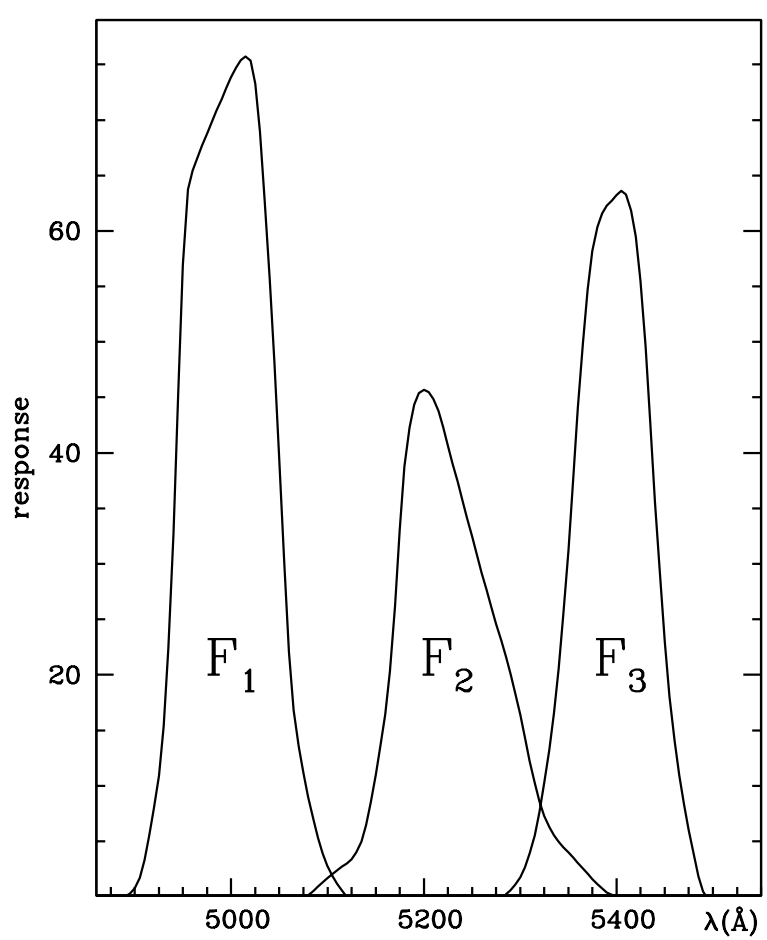

\begin{tabular}{|rr|rr|rr|}
\hline \multicolumn{2}{|c|}{$F_{1}$} & \multicolumn{2}{|c|}{$F_{2}$} & \multicolumn{2}{c|}{$F_{3}$} \\
\hline$\lambda(\mathrm{A})$ & $\Upsilon$ & $\lambda(\mathrm{A})$ & $\Upsilon$ & $\lambda(\mathrm{A})$ & $\Upsilon$ \\
\hline 4889 & 0.0 & 5079 & 0.0 & 5282 & 0.0 \\
4908 & 2.7 & 5116 & 2.7 & 5305 & 2.7 \\
4918 & 6.8 & 5141 & 6.8 & 5318 & 6.8 \\
4928 & 13.6 & 5155 & 13.6 & 5331 & 13.6 \\
4938 & 27.3 & 5166 & 21.8 & 5340 & 20.5 \\
4947 & 49.8 & 5178 & 36.8 & 5351 & 33.0 \\
4954 & 62.7 & 5185 & 42.3 & 5363 & 47.7 \\
4958 & 65.0 & 5201 & 45.7 & 5375 & 58.0 \\
4972 & 68.2 & 5213 & 44.3 & 5384 & 61.4 \\
4995 & 73.0 & 5224 & 40.9 & 5398 & 63.0 \\
5014 & 75.7 & 5245 & 34.1 & 5409 & 63.4 \\
5023 & 74.3 & 5273 & 25.2 & 5416 & 61.4 \\
5030 & 68.9 & 5296 & 18.0 & 5423 & 57.3 \\
5042 & 53.2 & 5319 & 8.9 & 5432 & 46.4 \\
5049 & 42.3 & 5349 & 4.1 & 5444 & 30.3 \\
5058 & 25.2 & 5375 & 1.6 & 5458 & 15.7 \\
5065 & 17.0 & 5402 & 0.0 & 5467 & 9.8 \\
5074 & 11.6 & & & 5476 & 5.5 \\
5086 & 6.8 & & & 5488 & 1.0 \\
5097 & 3.4 & & & 5513 & 0.0 \\
5120 & 0.0 & & & & \\
\hline
\end{tabular}

Fig. 110. The photometric system $\Delta a-$ Joncas and Borra - 1981 


\section{Jones et al. - 1981}

Narrow-band photometry of M stars.

\section{GENERAL INFORMATION}

AUTHORS

TELESCOPE

DETECTOR

MAIN ARTICLE Jones, D.H.P., Sinclair, J.E., Alexander J.B. 1981, MNRAS 194, 403

D.H.P. Jones, J. E. Sinclair and J. B. Alexander

1.0m (reflector), Siding Spring Observatory;

1.0m (reflector), South African Astron. Obs;

$2.5 \mathrm{~m}$ and $0.9 \mathrm{~m}$ (reflectors), Herstmonceux, UK

FW130 (S-20, refrigerated)

SYSTEM DESCRIPTION

\begin{tabular}{|c|cccc|}
\hline \multicolumn{5}{|c|}{ BANDS DESCRIPTION [160] } \\
\hline band & $\lambda_{0}(\AA)$ & $\lambda_{\text {eff }}(\AA)$ & half-width $(\AA)$ & feature \\
\hline 608 & 6076 & 6089 & 44 & continuum \\
\hline 683 & 6830 & 6835 & 41 & $\mathrm{CaH}$ \\
\hline 710 & 7100 & 7101 & 54 & $\mathrm{TiO}$ \\
\hline 746 & 7460 & 7465 & 44 & continnuum \\
\hline
\end{tabular}

Interference filters.

ZERO POINT: 746 is taken to be equivalent to I band of RI - Kron and Smith - 1951 system. [160]

\section{SYSTEM ANALYSIS}

REDDENING-FREE PARAMETERS [160]

$\delta=(710-746)-0.24(608-746)$

$T_{d w a r f s}=-5.07( \pm 1.45) \delta^{2}+10.74( \pm 1.30) \delta+0.13( \pm 0.25):$ to derive the spectral type for dwarfs.

$T_{\text {giants }}=-5.44( \pm 0.72) \delta^{2}+11.11( \pm 0.70) \delta-0.71( \pm 0.13):$ to derive the spectral type for giants.

where it is $T=-6$ at $\mathrm{K} 0, T=-1$ at $\mathrm{K} 5, T=0$ at $\mathrm{M} 0, T=8$ at M8.

Fig. 111. The photometric system Jones et al. - 1981 
TRANSMISSION CURVES [4]

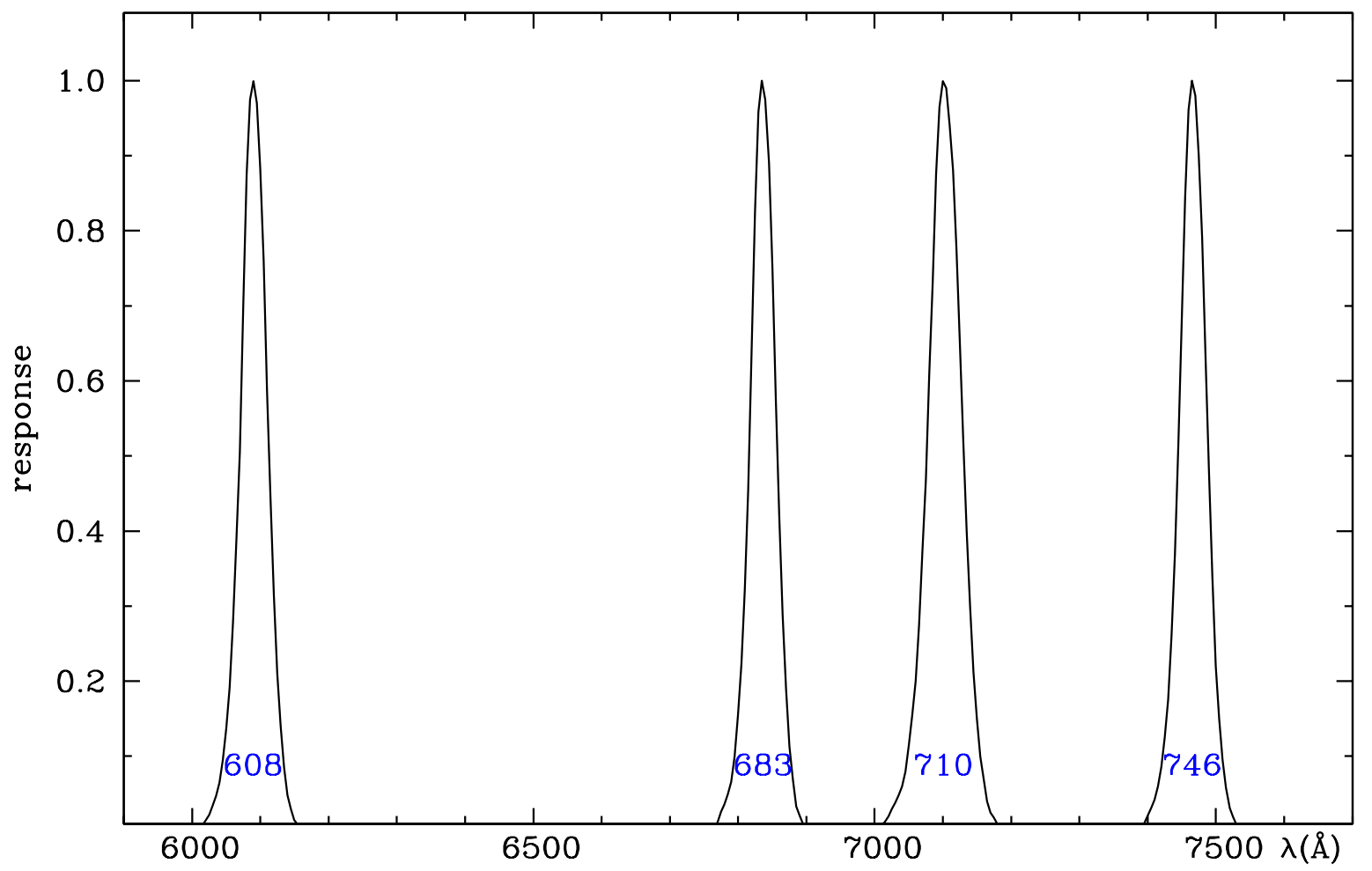

\begin{tabular}{|c|c|c|c|c|c|c|c|c|c|c|c|c|c|c|c|}
\hline \multicolumn{4}{|c|}{608} & \multicolumn{4}{|c|}{683} & \multicolumn{4}{|c|}{710} & \multicolumn{4}{|c|}{746} \\
\hline$\lambda(\mathrm{A})$ & $\Upsilon$ & $\lambda(\mathrm{A})$ & $\Upsilon$ & $\lambda(\mathrm{A})$ & $\Upsilon$ & $\lambda(\mathrm{A})$ & $\Upsilon$ & $\lambda(\mathrm{A})$ & $\Upsilon$ & $\lambda(\mathrm{A})$ & $\Upsilon$ & $\lambda(\mathrm{A})$ & $\Upsilon$ & $\lambda(\mathrm{A})$ & $\Upsilon$ \\
\hline 5995 & 0.000 & 6105 & 0.760 & 6750 & 0.000 & 6860 & 0.420 & 6985 & 0.000 & 7095 & 0.965 & 7370 & 0.000 & 7480 & 0.790 \\
\hline 6000 & 0.001 & 6110 & 0.585 & 6755 & 0.001 & 6865 & 0.288 & 6990 & 0.001 & 7100 & 0.999 & 7375 & 0.001 & 7485 & 0.631 \\
\hline 6005 & 0.002 & 6115 & 0.445 & 6760 & 0.002 & 6870 & 0.190 & 6995 & 0.002 & 7105 & 0.990 & 7380 & 0.002 & 7490 & 0.480 \\
\hline 6010 & 0.005 & 6120 & 0.315 & 6765 & 0.006 & 6875 & 0.113 & 7000 & 0.003 & 7110 & 0.940 & 7385 & 0.003 & 7495 & 0.340 \\
\hline 6015 & 0.008 & 6125 & 0.210 & 6770 & 0.012 & 6880 & 0.070 & 7005 & 0.004 & 7115 & 0.880 & 7390 & 0.008 & 7500 & 0.220 \\
\hline 6020 & 0.015 & 6130 & 0.140 & 6775 & 0.026 & 6885 & 0.033 & 7010 & 0.009 & 7120 & 0.775 & 7395 & 0.011 & 7505 & 0.150 \\
\hline 6025 & 0.022 & 6135 & 0.085 & 6780 & 0.036 & 6890 & 0.021 & 7015 & 0.012 & 7125 & 0.650 & 7400 & 0.021 & 7510 & 0.095 \\
\hline 6030 & 0.034 & 6140 & 0.048 & 6785 & 0.049 & 6895 & 0.010 & 7020 & 0.020 & 7130 & 0.520 & 7405 & 0.031 & 7515 & 0.058 \\
\hline 6035 & 0.047 & 6145 & 0.030 & 6790 & 0.066 & 6900 & 0.005 & 7025 & 0.030 & 7135 & 0.400 & 7410 & 0.042 & 7520 & 0.031 \\
\hline 6040 & 0.065 & 6150 & 0.015 & 6795 & 0.100 & 6905 & 0.000 & 7030 & 0.038 & 7140 & 0.300 & 7415 & 0.060 & 7525 & 0.019 \\
\hline 6045 & 0.095 & 6155 & 0.009 & 6800 & 0.155 & & & 7035 & 0.048 & 7145 & 0.212 & 7420 & 0.086 & 7530 & 0.009 \\
\hline 6050 & 0.137 & 6160 & 0.004 & 6805 & 0.222 & & & 7040 & 0.060 & 7150 & 0.150 & 7425 & 0.125 & 7535 & 0.004 \\
\hline 6055 & 0.192 & 6165 & 0.000 & 6810 & 0.322 & & & 7045 & 0.080 & 7155 & 0.100 & 7430 & 0.175 & 7540 & 0.000 \\
\hline 6060 & 0.280 & & & 6815 & 0.455 & & & 7050 & 0.115 & 7160 & 0.070 & 7435 & 0.261 & & \\
\hline 6065 & 0.390 & & & 6820 & 0.640 & & & 7055 & 0.155 & 7165 & 0.040 & 7440 & 0.370 & & \\
\hline 6070 & 0.505 & & & 6825 & 0.825 & & & 7060 & 0.200 & 7170 & 0.025 & 7445 & 0.515 & & \\
\hline 6075 & 0.700 & & & 6830 & 0.958 & & & 7065 & 0.275 & 7175 & 0.018 & 7450 & 0.680 & & \\
\hline 6080 & 0.875 & & & 6835 & 1.000 & & & 7070 & 0.375 & 7180 & 0.010 & 7455 & 0.845 & & \\
\hline 6085 & 0.975 & & & 6840 & 0.975 & & & 7075 & 0.468 & 7185 & 0.006 & 7460 & 0.961 & & \\
\hline 6090 & 0.999 & & & 6845 & 0.890 & & & 7080 & 0.610 & 7190 & 0.001 & 7465 & 1.000 & & \\
\hline 6095 & 0.970 & & & 6850 & 0.750 & & & 7085 & 0.730 & 7195 & 0.000 & 7470 & 0.980 & & \\
\hline 6100 & 0.882 & & & 6855 & 0.575 & & & 7090 & 0.875 & & & 7475 & 0.900 & & \\
\hline
\end{tabular}

Fig. 111. continued 


\section{$H \alpha$ - Strauss and Ducati - 1981}

$\mathrm{H} \alpha$ photometry.

\section{GENERAL INFORMATION}

$\begin{array}{ll}\text { AUTHORS } & \text { F. M. Strauss and J. R. Ducati } \\ \text { TELESCOPE } & \text { 0.50m (reflector), Universidade Federal do Rio Grande do Sul, Brazil } \\ \text { DETECTOR } & 9658 \text { AR (S-20 cathode, refrigerated) } \\ \text { MAIN ARTICLE } & \text { Strauss, F. M., Ducati, J.R. 1981, A\&AS 44, } 337\end{array}$

SYSTEM DESCRIPTION

\begin{tabular}{|c|c|c|c|}
\hline \multicolumn{4}{|c|}{ BANDS DESCRIPTION [292] } \\
\hline band & $\lambda_{\text {peak }}(\AA)$ & bandwith $(\AA)$ & $\Upsilon_{\text {peak }}(\%)$ \\
\hline Ho narrow & 6563 & 37 & 84 \\
\hline Ho wide & 6563 & 208 & 84 \\
\hline
\end{tabular}

\section{SYSTEM ANALYSIS}

\section{COLOR INDICES AND PARAMETERS [292]}

The $\beta$ index is taken from the uvbyH $\beta$ - Strömgren and Crawford - 1956

$x=\beta-0.4 \alpha$ : depends on spectral type. Separates quite well early $(\leq \mathrm{A} 0)$ from late $(>\mathrm{A} 0)$ supergiants.

$y=\beta-2.18 \alpha$ : correlates with luminosity class for B supergiants. Emission line indicator.

RELATIONS WITH OTHER SYSTEMS [292]

$$
H_{\alpha} \text { - Andrews - } 1968
$$

$R_{\alpha}=1.40( \pm 0.09) \alpha-1.6( \pm 0.2) \quad$ for 17 stars in common

$H \alpha, \beta, \gamma$ - Feinstein - 1974

$\alpha_{\text {Feinstein }}=0.33( \pm 0.03) \alpha+0.8( \pm 0.1) \quad$ for 26 stars in common

H $\alpha$ - Dachs and Schmidt-Kaler - 1975

$\alpha_{D \& S-K}=1.46( \pm 0.04) \alpha+0.3( \pm 0.1) \quad$ for 27 stars in common

Fig. 112. The photometric system $H \alpha$ - Strauss and Ducati - 1981 


\section{JHKL CTIO - Elias et al. - 1982}

Infrared photometry at CTIO.

\section{GENERAL INFORMATION}

AUTHORS J. H. Elias, J. A. Frogel, K. Matthews and G. Neugebauer

TELESCOPE $\quad 4.0 \mathrm{~m}$ and $1.5 \mathrm{~m}$ (reflectors), CTIO

MAIN ARTICLE Elias, J. H, Frogel, J. A., Matthews K., Neugebauer, G. 1982, AJ 87, 1029

\section{SYSTEM DESCRIPTION}

\begin{tabular}{|c|cc|}
\hline \multicolumn{3}{|c|}{ BANDS DESCRIPTION [96] } \\
\hline band & $\lambda_{\text {eff }}(\mu \mathrm{m})$ & bandpass $(\mu \mathrm{m})$ \\
\hline$J$ & 1.2 & $1.13-1.37$ \\
\hline$H$ & 1.6 & $1.50-1.80$ \\
\hline$K$ & 2.2 & $2.01-2.42$ \\
\hline$L$ & 3.5 & $3.22-3.76$ \\
\hline
\end{tabular}

The system also includes the $\mathrm{CO}$ and $\mathrm{H}_{2} \mathrm{O}$ filters defined in the $\mathrm{JHK}_{2} \mathrm{O} \mathrm{CO}$ - Persson et al. - 1977 system.

ZERO POINT: The magnitude of $\alpha$ Lyr is taken as 0.00 at all wavelengths. [96]

\section{SYSTEM ANALYSIS}

RELATIONS WITH OTHER SYSTEMS [97]

JHKL' AAO - Allen and Crag - 1983

\begin{tabular}{|rl|}
\hline$(J-K)_{C T I O}$ & $=0.897( \pm 0.005)(J-K)_{A A O}+0.006( \pm 0.004)$ \\
\hline$(J-H)_{C T I O}$ & $=0.869( \pm 0.005)(J-H)_{A A O}+0.012( \pm 0.003)$ \\
\hline$(H-K)_{C T I O}$ & $=0.954( \pm 0.013)(H-K)_{A A O}-0.04( \pm 0.03)$ \\
\hline$K_{C T I O}$ & $=K_{A A O}-0.011( \pm 0.004)$
\end{tabular}

Fig. 113. The photometric system JHKL CTIO - Elias et al. - 1982 


\section{JHKL MSO - Jones and Hyland - 1982}

Infrared photometry at Mount Stromlo Observatory.

\section{GENERAL INFORMATION}

AUTHORS T. J. Jones and A. R. Hyland

MAIN ARTICLE Jones, T. J. Hyland, A. R. 1982, MNRAS 200, 509

\section{SYSTEM DESCRIPTION}

\begin{tabular}{|c|cc|}
\hline \multicolumn{3}{|c|}{ BANDS DESCRIPTION } \\
\hline band & $W H M(\mu \mathrm{m})$ & $\lambda_{c}(\mu \mathrm{m})$ \\
\hline$J$ & 0.288 & 1.236 \\
\hline$H$ & 0.307 & 1.631 \\
\hline$K$ & 0.377 & 2.176 \\
\hline
\end{tabular}

\section{TRANSMISSION CURVES}

As derived from Fig A3 of [161].

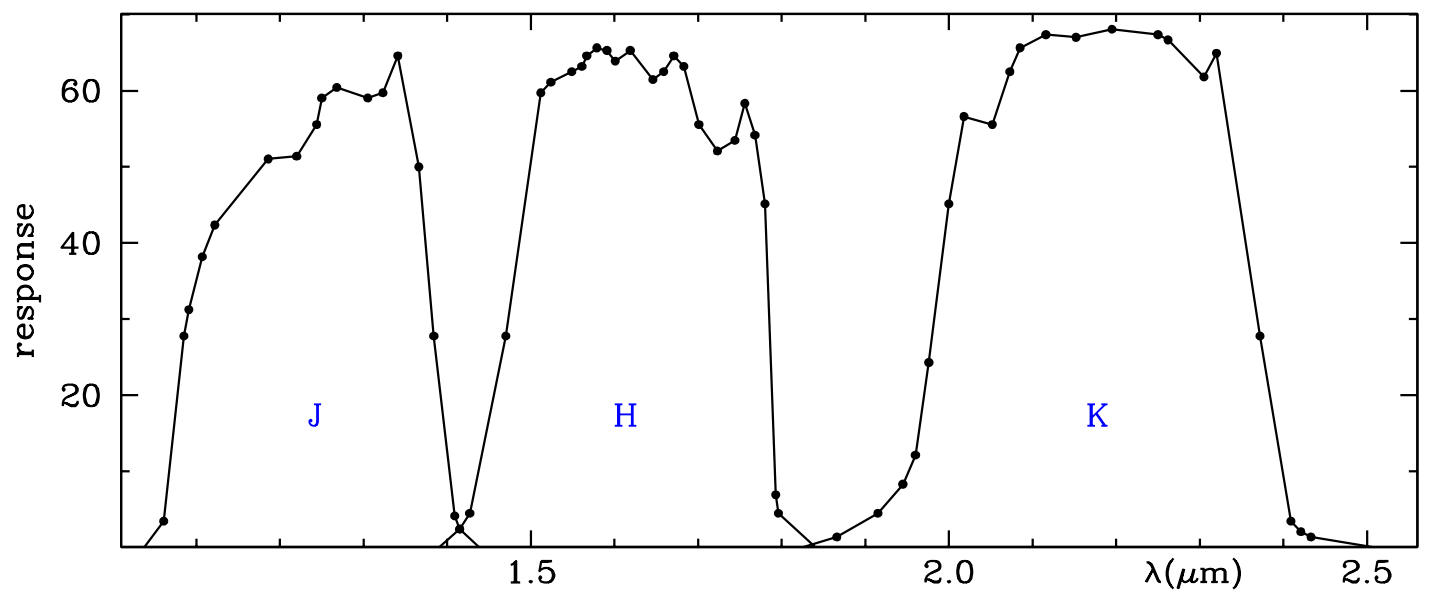

\begin{tabular}{|rr|rr|rr|rrr|rr|rr|r|r|}
\hline \multicolumn{7}{|c|}{$J$} & \multicolumn{4}{c|}{$H$} & \multicolumn{4}{c|}{$K$} \\
\hline$\lambda(\mu \mathrm{m})$ & $\Upsilon(\%)$ & $\lambda(\mu \mathrm{m})$ & $\Upsilon(\%)$ & $\lambda(\mu \mathrm{m})$ & $\Upsilon(\%)$ & $\lambda(\mu \mathrm{m})$ & $\Upsilon(\%)$ & $\lambda(\mu \mathrm{m})$ & $\Upsilon(\%)$ & $\lambda(\mu \mathrm{m})$ & $\Upsilon(\%)$ \\
\hline 1.037 & 0.00 & 1.341 & 64.58 & 1.390 & 0.00 & 1.646 & 61.46 & 1.823 & 0.00 & 2.195 & 68.06 \\
1.061 & 3.47 & 1.366 & 50.00 & 1.415 & 2.43 & 1.659 & 62.50 & 1.866 & 1.39 & 2.250 & 67.36 \\
1.085 & 27.78 & 1.384 & 27.78 & 1.427 & 4.51 & 1.671 & 64.58 & 1.915 & 4.51 & 2.262 & 66.67 \\
1.091 & 31.25 & 1.409 & 4.17 & 1.470 & 27.78 & 1.683 & 63.19 & 1.945 & 8.33 & 2.305 & 61.81 \\
1.107 & 38.19 & 1.415 & 2.43 & 1.512 & 59.72 & 1.701 & 55.56 & 1.960 & 12.15 & 2.320 & 64.93 \\
1.122 & 42.36 & 1.439 & 0.00 & 1.524 & 61.11 & 1.723 & 52.08 & 1.976 & 24.31 & 2.372 & 27.78 \\
1.186 & 51.04 & & & 1.549 & 62.50 & 1.744 & 53.47 & 2.000 & 45.14 & 2.409 & 3.47 \\
1.220 & 51.39 & & & 1.561 & 63.19 & 1.756 & 58.33 & 2.018 & 56.60 & 2.421 & 2.08 \\
1.244 & 55.56 & & & 1.567 & 64.58 & 1.768 & 54.17 & 2.052 & 55.56 & 2.433 & 1.39 \\
1.250 & 59.03 & & & 1.579 & 65.62 & 1.780 & 45.14 & 2.073 & 62.50 & 2.512 & 0.00 \\
1.268 & 60.42 & & & 1.591 & 65.28 & 1.793 & 6.94 & 2.085 & 65.62 & & \\
1.305 & 59.03 & & & 1.601 & 63.89 & 1.796 & 4.51 & 2.116 & 67.36 & & \\
1.323 & 59.72 & & & 1.619 & 65.28 & 1.841 & 0.00 & 2.152 & 67.01 & \\
\hline
\end{tabular}

Fig. 114. The photometric system JHKL MSO - Jones and Hyland - 1982 


\section{VilGen - North et al. - 1982}

Combines bands from the Vilnius - Straižys et al. - 1965 and Geneva - Golay - 1962 systems.

\section{GENERAL INFORMATION}

AUTHORS P. North, B. Hauck and V. Straižys

MAIN ARTICLE North, P., Hauck, B., Straižys, V. 1982, A\&A 108, 373

\begin{tabular}{|c|c|c|c|c|c|c|}
\hline \multicolumn{7}{|c|}{ SYSTEM DESCRIPTION } \\
\hline \multicolumn{7}{|c|}{ BANDS DESCRIPTION [234] } \\
\hline band & old name & Schott filter & Soviet-made filter & $\lambda_{0}(\AA)$ & half-width $(\AA)$ & $\Upsilon_{\text {peak }}(\%)$ \\
\hline 35 & $U$ & WG5 + UG11 & BS5 + UFS2 & 3500 & 510 & 61 \\
\hline 37 & $P$ & $\mathrm{BG} 23$ + UG1 & SZS22 + UFS6 & 3740 & 260 & 40 \\
\hline 40 & $B 1$ & GG395 + UG3 + BG23 & $\mathrm{ZhS} 10+\mathrm{FS} 7+\mathrm{SZS} 21$ & 4020 & 400 & 47 \\
\hline 47 & $Y$ & $\mathrm{GG} 435+\mathrm{BG} 12+\mathrm{BG} 23$ & $\mathrm{ZhS} 12+\mathrm{SS} 15+\mathrm{SZS} 21$ & 4680 & 450 & 48 \\
\hline 52 & $Z$ & GG495 + VG3 + BG23 & $\mathrm{ZhS} 17+\mathrm{ZS} 7+\mathrm{SZS} 22$ & 5160 & 210 & 44 \\
\hline$V$ & V & GG495 + BG23 & ZhS18 + SZS21 & 5500 & 800 & 85 \\
\hline 66 & $S$ & interference & interference & 6560 & 190 & 75 \\
\hline
\end{tabular}

\section{SYSTEM ANALYSIS}

RELATIONS WITH OTHER SYSTEMS [234]

Geneva - Golay - 1962

$(35-V)=(U-V)_{G e n}+1.32$
$(40-V)=(B 1-V)_{G e n}+0.64$

Vilnius - Straižys et al. - 1965

\begin{tabular}{|lll|}
\hline$(37-V)$ & $=(P-V)_{V i l}$ & \\
\hline$(47-V)$ & $=1.01(Y-V)_{\text {Vil }}$ & for luminosity class IV-I \\
\hline$(47-V)$ & $=1.03(Y-V)_{V i l}$ & for luminosity class V \\
\hline$(52-V)$ & $=(Z-V)_{V i l}$ & \\
\hline$(V-66)$ & $=(V-S)_{V i l}$ & \\
\hline
\end{tabular}

Fig. 115. The photometric system VilGen - North et al. - 1982 
As derived from Fig 1 of [234].

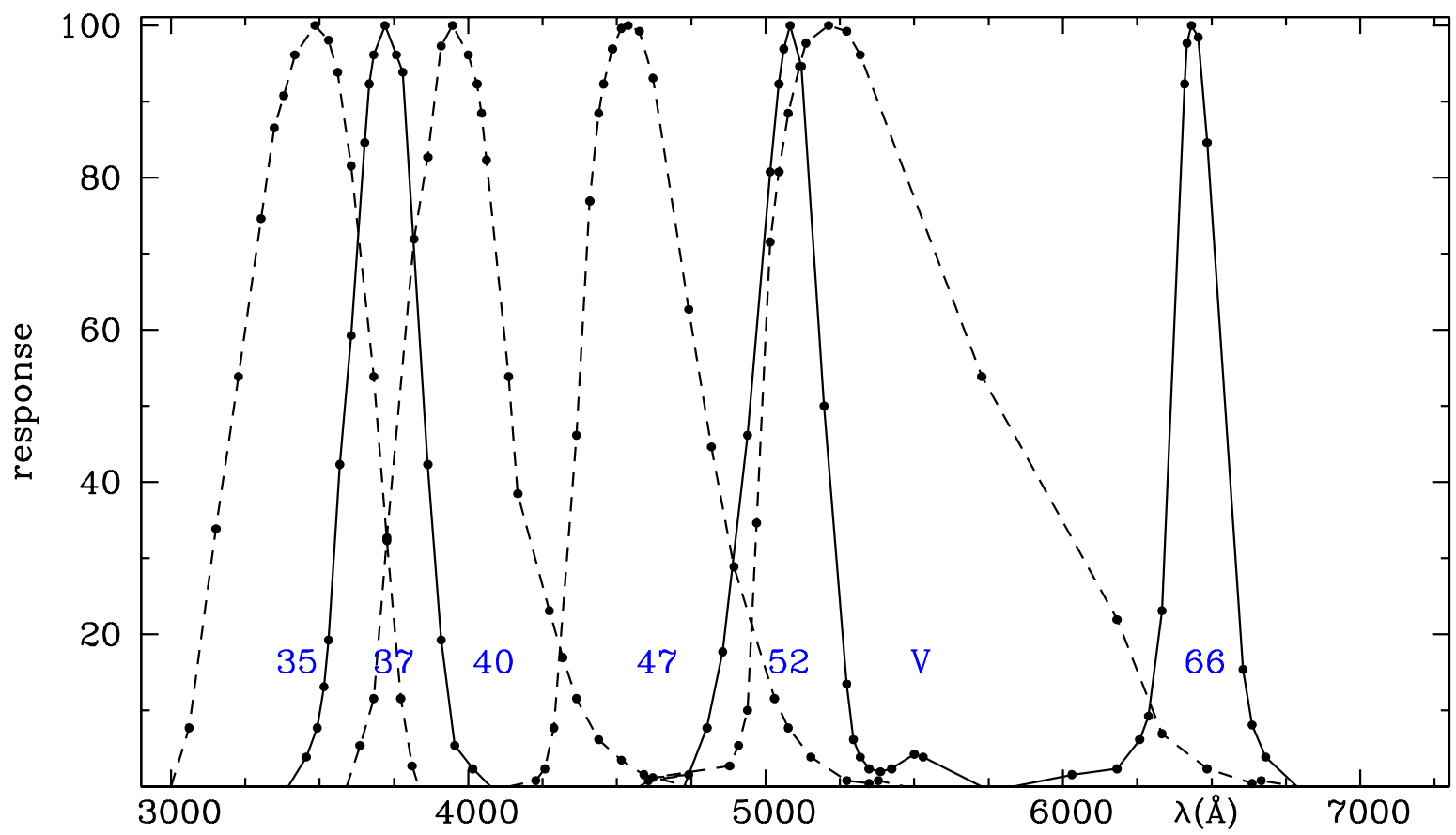

\begin{tabular}{|c|c|c|c|c|c|c|c|c|c|c|c|c|c|}
\hline \multicolumn{2}{|c|}{35} & \multicolumn{2}{|c|}{37} & \multicolumn{2}{|c|}{40} & \multicolumn{2}{|c|}{47} & \multicolumn{2}{|c|}{52} & \multicolumn{2}{|c|}{$V$} & \multicolumn{2}{|c|}{66} \\
\hline$\lambda(\mathrm{A})$ & $\Upsilon$ & $\lambda(\mathrm{A})$ & $\Upsilon$ & $\lambda(\mathrm{A})$ & $\Upsilon$ & $\lambda(\mathrm{A})$ & $\Upsilon$ & $\lambda(\mathrm{A})$ & $\Upsilon$ & $\lambda(\mathrm{A})$ & $\Upsilon$ & $\lambda(\mathrm{A})$ & $\Upsilon$ \\
\hline 3000 & 0.00 & 3394 & 0.00 & 3591 & 0.00 & 4136 & 0.00 & 4727 & 0.00 & 4576 & 0.00 & 5833 & 0.00 \\
\hline 3061 & 7.69 & 3455 & 3.85 & 3636 & 5.38 & 4227 & 0.77 & 4803 & 7.69 & 4606 & 0.77 & 6030 & 1.54 \\
\hline 3152 & 33.85 & 3492 & 7.69 & 3682 & 11.54 & 4258 & 2.31 & 4856 & 17.69 & 4742 & 1.54 & 6182 & 2.31 \\
\hline 3227 & 53.85 & 3515 & 13.08 & 3727 & 32.69 & 4288 & 7.69 & 4939 & 46.15 & 4621 & 1.15 & 6258 & 6.15 \\
\hline 3303 & 74.62 & 3530 & 19.23 & 3818 & 71.92 & 4364 & 46.15 & 5015 & 80.77 & 4879 & 2.69 & 6288 & 9.23 \\
\hline 3348 & 86.54 & 3568 & 42.31 & 3864 & 82.69 & 4409 & 76.92 & 5045 & 92.31 & 4909 & 5.38 & 6333 & 23.08 \\
\hline 3379 & 90.77 & 3606 & 59.23 & 3909 & 97.31 & 4439 & 88.46 & 5061 & 96.92 & 4939 & 10.00 & 6409 & 92.31 \\
\hline 3417 & 96.15 & 3652 & 84.62 & 3947 & 100.00 & 4455 & 92.31 & 5083 & 100.00 & 4970 & 34.62 & 6417 & 97.69 \\
\hline 3485 & 100.00 & 3667 & 92.31 & 4000 & 96.15 & 4485 & 96.92 & 5121 & 94.62 & 5015 & 71.54 & 6432 & 100.00 \\
\hline 3530 & 98.08 & 3682 & 96.15 & 4030 & 92.31 & 4515 & 99.62 & 5197 & 50.00 & 5045 & 80.77 & 6455 & 98.46 \\
\hline 3561 & 93.85 & 3720 & 100.00 & 4045 & 88.46 & 4538 & 100.00 & 5273 & 13.46 & 5076 & 88.46 & 6485 & 84.62 \\
\hline 3606 & 81.54 & 3758 & 96.15 & 4061 & 82.31 & 4576 & 99.23 & 5295 & 6.15 & 5114 & 94.62 & 6606 & 15.38 \\
\hline 3682 & 53.85 & 3780 & 93.85 & 4136 & 53.85 & 4621 & 93.08 & 5318 & 3.85 & 5136 & 97.69 & 6636 & 8.08 \\
\hline 3727 & 32.31 & 3864 & 42.31 & 4167 & 38.46 & 4742 & 62.69 & 5348 & 2.31 & 5212 & 100.00 & 6682 & 3.85 \\
\hline 3773 & 11.54 & 3909 & 19.23 & 4273 & 23.08 & 4818 & 44.62 & 5386 & 1.92 & 5273 & 99.23 & 6788 & 0.00 \\
\hline 3811 & 2.69 & 3955 & 5.38 & 4318 & 16.92 & 4894 & 28.85 & 5424 & 2.31 & 5318 & 96.15 & & \\
\hline 3833 & 0.00 & 4015 & 2.31 & 4364 & 11.54 & 5030 & 11.54 & 5500 & 4.23 & 5727 & 53.85 & & \\
\hline & & 4076 & 0.00 & 4439 & 6.15 & 5076 & 7.69 & 5530 & 3.85 & 6182 & 21.92 & & \\
\hline & & & & 4515 & 3.46 & 5152 & 3.85 & 5727 & 0.00 & 6333 & 6.92 & & \\
\hline & & & & 4591 & 1.54 & 5273 & 0.77 & & & 6485 & 2.31 & & \\
\hline & & & & 4742 & 0.00 & 5348 & 0.38 & & & 6636 & 0.38 & & \\
\hline & & & & & & 5379 & 0.77 & & & 6667 & 0.77 & & \\
\hline & & & & & & 5470 & 0.00 & & & 6788 & 0.00 & & \\
\hline
\end{tabular}

Fig. 115. continued 


\section{Solheim et al. - 1982}

Intermediate band photometry of galaxies.

\section{GENERAL INFORMATION}

AUTHORS

TELESCOPE

DETECTOR

\author{
$0.91 \mathrm{~cm}$ (reector), McDonald Obs.
}

J. -E. Solheim, G. de Vaucouleurs and A. de Vaucouleurs

$1 \mathrm{P} 21$

MAIN ARTICLE Solheim, J. -E., de Vaucouleurs, G., de Vaucouleurs, A. 1982, A\&AS 49, 109

\section{SYSTEM DESCRIPTION}

\begin{tabular}{|l|ccl|c|ccc|}
\hline \multicolumn{7}{|c|}{ BANDS DESCRIPTION $[273]$} \\
\hline band & $\lambda_{\text {eff }}(\mathrm{A})$ & bandwidth (A) & \multicolumn{1}{c|}{ feature } & band & $\lambda_{\text {eff }}(\mathrm{A})$ & bandwidth (A) & feature \\
\hline F34 & 3409 & 119 & continuum & F45 & 4502 & 175 & continuum \\
\hline F37 & 3704 & 103 & {$[\mathrm{OII}]$} & F49 & 4865 & 135 & H $\beta$ \\
\hline F39 & 3894 & 123 & {$[$ NeIII $]$, CN, CaII H \& K } & F50 & 5013 & 224 & [OIII $]$ \\
\hline F41 & 4103 & 135 & H $\delta$ & F53 & 5262 & 122 & Mg, cont. \\
\hline F43 & 4303 & 110 & H $\gamma$, G band & F55 & 5498 & 87 & continuum \\
\hline
\end{tabular}

Interference lters by Film Products Inc.

\section{SYSTEM ANALYSIS}

RELATIONS WITH OTHER SYSTEMS [273]

UBV - Johnson and Morgan - 1953

$m_{55}=V-0.039(B-V)$
$m_{43}=B+0.108(B-V)$

\section{TRANSMISSION CURVES}

As derived from Fig 1 of [273].

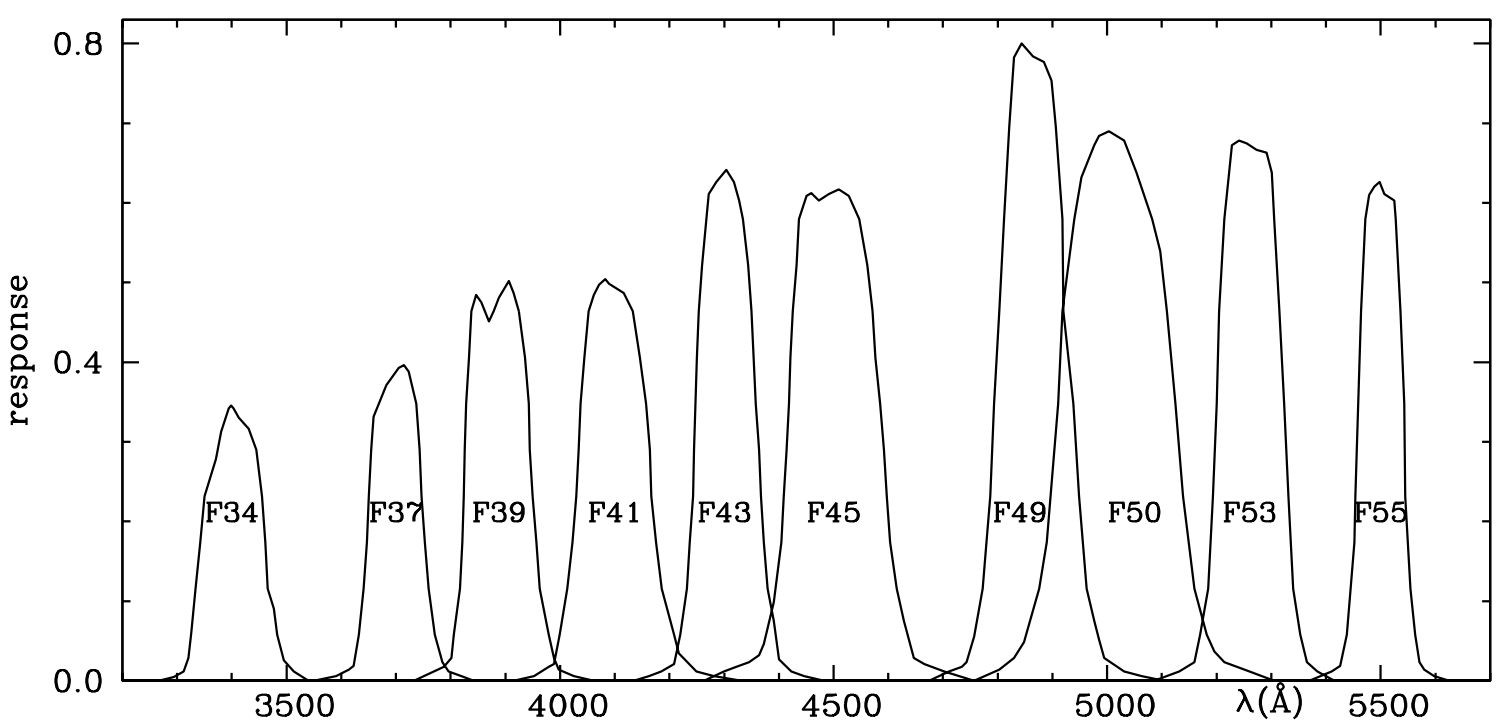

Fig. 116. The photometric system Solheim et al. - 1982 


\begin{tabular}{|c|c|c|c|c|c|c|c|c|c|c|c|c|c|}
\hline \multicolumn{2}{|c|}{ F34 } & \multicolumn{2}{|c|}{ F37 } & \multicolumn{2}{|c|}{ F39 } & \multicolumn{2}{|c|}{ F41 } & \multicolumn{2}{|c|}{$F 43$} & \multicolumn{2}{|c|}{ F45 } & \multicolumn{2}{|c|}{ F49 } \\
\hline$\lambda(\AA)$ & $\Upsilon$ & $\lambda(\AA)$ & $\Upsilon$ & $\lambda(\AA)$ & $\Upsilon$ & $\lambda(\AA)$ & $\Upsilon$ & $\lambda(\AA)$ & $\Upsilon$ & $\lambda(\AA)$ & $\Upsilon$ & $\lambda(\AA)$ & $\Upsilon$ \\
\hline 3266 & 0.000 & 3550 & 0.000 & 3733 & 0.000 & 3915 & 0.000 & 4135 & 0.000 & 4263 & 0.000 & 4675 & 0.000 \\
\hline 3294 & 0.005 & 3591 & 0.006 & 3769 & 0.012 & 3952 & 0.006 & 4162 & 0.006 & 4281 & 0.006 & 4711 & 0.012 \\
\hline 3312 & 0.012 & 3614 & 0.014 & 3787 & 0.017 & 3979 & 0.017 & 4185 & 0.012 & 4300 & 0.012 & 4734 & 0.017 \\
\hline 3321 & 0.029 & 3623 & 0.019 & 3801 & 0.029 & 3989 & 0.021 & 4208 & 0.021 & 4322 & 0.017 & 4743 & 0.023 \\
\hline 3326 & 0.060 & 3632 & 0.058 & 3806 & 0.058 & 4000 & 0.058 & 4220 & 0.058 & 4345 & 0.023 & 4757 & 0.056 \\
\hline 3334 & 0.116 & 3641 & 0.116 & 3817 & 0.116 & 4013 & 0.116 & 4232 & 0.116 & 4364 & 0.032 & 4772 & 0.116 \\
\hline 3342 & 0.174 & 3647 & 0.174 & 3821 & 0.174 & 4022 & 0.174 & 4237 & 0.174 & 4373 & 0.046 & 4780 & 0.174 \\
\hline 3350 & 0.232 & 3650 & 0.232 & 3824 & 0.232 & 4030 & 0.232 & 4243 & 0.232 & 4391 & 0.099 & 4786 & 0.232 \\
\hline 3371 & 0.278 & 3655 & 0.290 & 3826 & 0.290 & 4034 & 0.290 & 4245 & 0.290 & 4405 & 0.174 & 4793 & 0.348 \\
\hline 3380 & 0.313 & 3659 & 0.332 & 3829 & 0.348 & 4037 & 0.348 & 4247 & 0.348 & 4409 & 0.232 & 4803 & 0.464 \\
\hline 3394 & 0.342 & 3682 & 0.371 & 3833 & 0.406 & 4044 & 0.406 & 4250 & 0.406 & 4414 & 0.290 & 4812 & 0.580 \\
\hline 3399 & 0.346 & 3705 & 0.393 & 3838 & 0.464 & 4053 & 0.464 & 4254 & 0.464 & 4418 & 0.348 & 4821 & 0.696 \\
\hline 3403 & 0.342 & 3714 & 0.397 & 3847 & 0.485 & 4062 & 0.485 & 4259 & 0.522 & 4421 & 0.406 & 4830 & 0.783 \\
\hline 3412 & 0.330 & 3723 & 0.388 & 3856 & 0.475 & 4071 & 0.497 & 4268 & 0.580 & 4426 & 0.464 & 4844 & 0.800 \\
\hline 3431 & 0.317 & 3737 & 0.348 & 3870 & 0.451 & 4083 & 0.504 & 4272 & 0.611 & 4432 & 0.522 & 4865 & 0.784 \\
\hline 3444 & 0.290 & 3744 & 0.290 & 3879 & 0.464 & 4089 & 0.499 & 4286 & 0.626 & 4437 & 0.580 & 4885 & 0.777 \\
\hline 3455 & 0.232 & 3747 & 0.232 & 3888 & 0.481 & 4117 & 0.487 & 4304 & 0.641 & 4450 & 0.609 & 4899 & 0.754 \\
\hline 3461 & 0.174 & 3753 & 0.174 & 3906 & 0.502 & 4133 & 0.464 & 4318 & 0.626 & 4460 & 0.612 & 4906 & 0.696 \\
\hline 3466 & 0.116 & 3760 & 0.116 & 3915 & 0.487 & 4146 & 0.406 & 4327 & 0.603 & 4473 & 0.603 & 4919 & 0.580 \\
\hline 3477 & 0.090 & 3771 & 0.058 & 3925 & 0.464 & 4157 & 0.348 & 4334 & 0.580 & 4492 & 0.611 & 4920 & 0.464 \\
\hline 3483 & 0.058 & 3785 & 0.023 & 3936 & 0.406 & 4164 & 0.290 & 4343 & 0.522 & 4510 & 0.617 & 4938 & 0.348 \\
\hline 3495 & 0.026 & 3797 & 0.012 & 3943 & 0.348 & 4167 & 0.232 & 4350 & 0.464 & 4528 & 0.609 & 4949 & 0.232 \\
\hline 3513 & 0.012 & 3819 & 0.006 & 3945 & 0.290 & 4175 & 0.174 & 4358 & 0.348 & 4546 & 0.580 & 4963 & 0.116 \\
\hline 3541 & 0.000 & 3842 & 0.000 & 3950 & 0.232 & 4186 & 0.116 & 4364 & 0.290 & 4562 & 0.522 & 4976 & 0.075 \\
\hline & & & & 3957 & 0.174 & 4208 & 0.058 & 4367 & 0.232 & 4571 & 0.464 & 4985 & 0.052 \\
\hline & & & & 3963 & 0.116 & 4217 & 0.035 & 4373 & 0.174 & 4577 & 0.406 & 4995 & 0.029 \\
\hline & & & & 3979 & 0.058 & 4249 & 0.012 & 4379 & 0.116 & 4585 & 0.348 & 5031 & 0.012 \\
\hline & & & & 3989 & 0.029 & 4281 & 0.006 & 4391 & 0.075 & 4592 & 0.290 & 5063 & 0.006 \\
\hline & & & & 3998 & 0.014 & 4332 & 0.000 & 4400 & 0.027 & 4597 & 0.232 & 5104 & 0.000 \\
\hline & & & & 4025 & 0.006 & & & 4423 & 0.012 & 4603 & 0.174 & & \\
\hline & & & & 4062 & 0.000 & & & 4446 & 0.006 & 4615 & 0.116 & & \\
\hline & & & & & & & & 4482 & 0.000 & 4629 & 0.075 & & \\
\hline & & & & & & & & & & 4647 & 0.029 & & \\
\hline & & & & & & & & & & 4665 & 0.021 & & \\
\hline & & & & & & & & & & 4702 & 0.012 & & \\
\hline & & & & & & & & & & 4757 & 0.000 & & \\
\hline
\end{tabular}

\begin{tabular}{|cc|cc|cc|cc|cc|cc|}
\hline \multicolumn{4}{|c|}{$F 50$} & \multicolumn{4}{c|}{$F 53$} & \multicolumn{4}{c|}{$F 55$} \\
\hline$\lambda(\AA)$ & $\Upsilon$ & $\lambda(\AA)$ & $\Upsilon$ & $\lambda(\AA)$ & $\Upsilon$ & $\lambda(\AA)$ & $\Upsilon$ & $\lambda(\AA)$ & $\Upsilon$ & $\lambda(\AA)$ & $\Upsilon$ \\
\hline 4757 & 0.000 & 5031 & 0.678 & 5086 & 0.000 & 5301 & 0.638 & 5370 & 0.000 & 5507 & 0.611 \\
4803 & 0.014 & 5054 & 0.638 & 5132 & 0.012 & 5306 & 0.580 & 5411 & 0.012 & 5525 & 0.603 \\
4830 & 0.029 & 5082 & 0.580 & 5159 & 0.023 & 5315 & 0.464 & 5426 & 0.019 & 5528 & 0.580 \\
4848 & 0.049 & 5097 & 0.539 & 5170 & 0.058 & 5324 & 0.348 & 5434 & 0.044 & 5536 & 0.464 \\
4876 & 0.116 & 5109 & 0.464 & 5185 & 0.116 & 5331 & 0.232 & 5438 & 0.058 & 5543 & 0.348 \\
4889 & 0.174 & 5124 & 0.348 & 5193 & 0.232 & 5340 & 0.116 & 5445 & 0.116 & 5545 & 0.232 \\
4897 & 0.232 & 5139 & 0.232 & 5200 & 0.348 & 5353 & 0.058 & 5452 & 0.174 & 5554 & 0.116 \\
4910 & 0.348 & 5159 & 0.116 & 5205 & 0.464 & 5365 & 0.023 & 5454 & 0.232 & 5563 & 0.058 \\
4919 & 0.464 & 5182 & 0.058 & 5214 & 0.580 & 5384 & 0.012 & 5459 & 0.348 & 5571 & 0.023 \\
4940 & 0.580 & 5196 & 0.037 & 5228 & 0.672 & 5415 & 0.000 & 5464 & 0.464 & 5580 & 0.014 \\
4953 & 0.632 & 5214 & 0.023 & 5242 & 0.678 & & & 5472 & 0.580 & 5598 & 0.006 \\
4976 & 0.672 & 5260 & 0.012 & 5255 & 0.675 & & & 5479 & 0.610 & 5626 & 0.000 \\
4985 & 0.684 & 5306 & 0.000 & 5274 & 0.667 & & & 5488 & 0.620 & & \\
5004 & 0.690 & & & 5292 & 0.663 & & & 5498 & 0.626 & & \\
\hline
\end{tabular}

Fig. 116. continued 


\section{8colors - Tedesco et al. - 1982}

Spectral reflectance of asteroids and planetary satellites.

\section{GENERAL INFORMATION}
AUTHORS
E. F. Tedesco, D. J. Tholen and B. Zellner
TELESCOPE
$1.54 \mathrm{~m}$, Catalina reflector, Univesity of Arizona
DETECTOR
blue channel: EMI 9789QB (bialkali photocathode, uncooled)
red channel: RCA C31034-O5 (GaAs cathode, thermoelelectrically cooled)
or Varian VPM 159A.12D (InGaAsP cathode, refrigerated)

MAIN ARTICLE Tedesco, E. F., Tholen, D. J., Zellner, B. 1982, AJ 87, 1585

\section{SYSTEM DESCRIPTION}

\begin{tabular}{|c|lcc|}
\hline \multicolumn{4}{|c|}{ BANDS DESCRIPTION [299] } \\
\hline band & \multicolumn{4}{|c|}{ filter } & $\lambda_{\text {eff }}(\mu \mathrm{m})(\#)$ & FWHM $(\mu \mathrm{m})(\#)$ \\
\hline$S$ & $(\# \#)$ & 0.337 & 0.047 \\
\hline$u$ & 3mm Corning 9683 & 0.359 & 0.060 \\
\hline$b$ & 2mm Schott GG13 + 6mm Corning CS 5-57 & 0.437 & 0.090 \\
\hline$V$ & Corion interference & 0.550 & 0.057 \\
& & $(0.552)$ & $(0.058)$ \\
\hline$W$ & Corion interference & 0.701 & 0.058 \\
& & $(0.702)$ & $(0.063)$ \\
\hline$X$ & Corion interference & 0.853 & 0.081 \\
& & $(0.849)$ & $(0.069)$ \\
\hline$p$ & Corion interference & 0.948 & 0.080 \\
\hline$Z$ & Corion interference & 1.041 & 0.067 \\
\hline
\end{tabular}

(\#) for the Sun spectrum.

Upper values refer to Varian phototube, those in brackets to the RCA one.

(\#\#) 3mm Corning CS 7-54 and 1.6mm UV transparent T-12 Optosil grade I glass with

$7.5 \mathrm{~mm}$ of a solution composed by $98 \% \mathrm{NiSO}_{4} \cdot 6 \mathrm{H}_{2} \mathrm{O}(420 \mathrm{~g} / \mathrm{l})$ and $2 \% \mathrm{CuSO}_{4} \cdot 5 \mathrm{H}_{2} \mathrm{O}(260 \mathrm{~g} / \mathrm{l})$.

ZERO POINT: The unweighted mean of color indices for the solar analogs 16 CygB, Hyades VB64, Hyades VB106 and Hyades VB142 is adopted as the zero-point for each of the seven colors. [299]

Fig. 117. The photometric system 8 colors - Tedesco et al. - 1982 


\section{JHKL' AAO - Allen and Crag - 1983}

Infrared photometry at the Anglo Australian Observatory.

\section{GENERAL INFORMATION}

AUTHORS D. A. Allen, and T. A. Cragg

TELESCOPE 3.9m (reflector), Anglo-Australian Obs.

DETECTOR $\mathrm{InSb}($ cooled at $50 \mathrm{~K})$

MAIN ARTICLE Allen, D. A., Cragg, T. A. 1983, MNRAS 203, 777

\section{SYSTEM DESCRIPTION}

\begin{tabular}{|c|cc|cc|}
\hline \multicolumn{3}{|c|}{ BANDS DESCRIPTION [6] } & \multicolumn{2}{c|}{ FLUX CALIBRATION [6] } \\
\hline band & $\lambda_{\text {eff }}(\mu \mathrm{m})$ & FWHM $(\mu \mathrm{m})$ & $\mathrm{F}_{\lambda}\left(\mathrm{W} \mathrm{cm}^{-2} \mu \mathrm{m}\right)(\#)$ & $\mathrm{F}_{\mathrm{v}} \mathrm{Jy}(\#)$ \\
\hline$J$ & 1.20 & 0.30 & $3410^{-14}$ & 1640 \\
\hline$H$ & 1.64 & 0.30 & $11.510^{-14}$ & 1030 \\
\hline$K$ & 2.19 & 0.45 & $4.110^{-14}$ & 650 \\
\hline$L^{\prime}$ & 3.80 & 0.60 & $0.5210^{-14}$ & 250 \\
\hline
\end{tabular}

(\#) Fluxes corresponding to a 0.0 mag star.

ZERO POINT: The star HR 3314 (A0 V) is taken to have

$$
(J-H)=-0.01,(H-K)=0.00,\left(H-L^{\prime}\right)=-0.01 \quad[6]
$$

Fig. 118. The photometric system JHKL' AAO - Allen and Cragg - 1983 


\title{
UBVRI - Landolt - 1983
}

Establishment of a set of equatorial standard stars that realizes the UBV - Johnson and Morgan - 1953 and RI - Cousins - 1976 systems.

\section{GENERAL INFORMATION}

\author{
AUTHORS \\ A. U. Landolt \\ TELESCOPE \\ $0.4 \mathrm{~m}, 0.9 \mathrm{~m}$ and $1.5 \mathrm{~m}$ (reectors), CTIO \\ DETECTOR \\ RCA 31034A (GaAs photocathode, refrigerated) \\ Hamamatsu R943-02 (GaAs photocathode, refrigerated) \\ MAIN ARTICLE Landolt, A. U. 1983, AJ 88, 439
}

\section{SYSTEM DESCRIPTION}

\begin{tabular}{|c|l|cc|}
\hline \multicolumn{3}{|c|}{ BANDS DESCRIPTION [182] } & \\
\hline band & \multicolumn{1}{|c|}{ lters } & WHM (A) & $\lambda_{c}(\mathrm{~A})$ \\
\hline$U$ & Corning 9863 + solid CuSO 4 cristal & 980 & 3400 \\
\hline$B$ & 2mm GG 385 + 2mm BG 18 + 2mm BG 12 & 860 & 4305 \\
\hline$V$ & $2 \mathrm{~mm} \mathrm{GG} \mathrm{495} \mathrm{+} \mathrm{2mm} \mathrm{BG} \mathrm{18}$ & 865 & 5400 \\
\hline$R$ & $2 \mathrm{~mm} \mathrm{KG} \mathrm{3+2mm} \mathrm{OG} \mathrm{570}$ & 1590 & 6360 \\
\hline$I$ & $3 \mathrm{~mm} \mathrm{RG} \mathrm{715+1mm} \mathrm{RG} \mathrm{780}$ & 1320 & 8150 \\
\hline
\end{tabular}

\section{SYSTEM ANALYSIS}

A long term effort initiated by [181] and [182] has culminated with the establishment of 526 equatorial UBVRI standard stars by [183]. The deviations from the original UBV - Johnson and Morgan - 1953 and RI - Cousins - 1976 systems (as realized by the respective standard stars given in [150] and [336]) are discussed in detail by [32], [33], [183], [337] and the references therein.

\section{TRANSMISSION CURVES}

As obtained by combining the transmission curves of the original set of lters with the sensitivity of the RCA 31034A photomultiplier (serial number N49701) given in Tables 6-11 of [183].

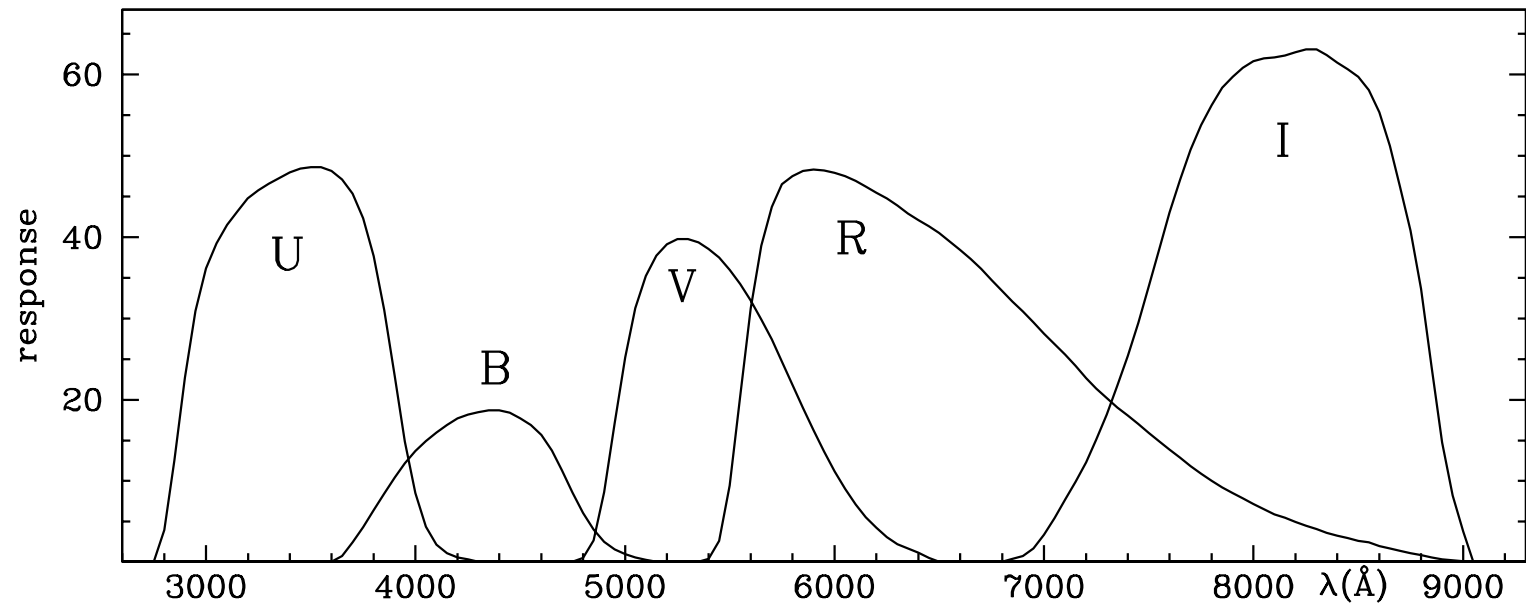

Fig. 119. The photometric system UBVRI - Landolt - 1983 


\begin{tabular}{|c|c|c|c|c|c|c|c|c|c|c|c|}
\hline \multicolumn{2}{|c|}{$U$} & \multicolumn{2}{|c|}{$B$} & \multicolumn{2}{|c|}{ V } & \multicolumn{4}{|c|}{$R$} & \multicolumn{2}{|c|}{$I$} \\
\hline$\lambda(\AA)$ & $\Upsilon(\%)$ & $\lambda(\AA)$ & $\Upsilon(\%)$ & $\lambda(\AA)$ & $\Upsilon(\%)$ & $\lambda(\AA)$ & $\Upsilon(\%)$ & $\lambda(\AA)$ & $\Upsilon(\%)$ & $\lambda(\AA)$ & $\Upsilon(\%)$ \\
\hline 2750 & 0.00 & 3600 & 0.00 & 4750 & 0.00 & 5350 & 0.00 & 7650 & 12.78 & 6800 & 0.00 \\
\hline 2800 & 3.86 & 3650 & 0.71 & 4800 & 0.43 & 5400 & 0.32 & 7700 & 11.77 & 6850 & 0.31 \\
\hline 2850 & 12.43 & 3700 & 2.36 & 4850 & 2.60 & 5450 & 2.52 & 7750 & 10.83 & 6900 & 0.69 \\
\hline 2900 & 22.68 & 3750 & 4.26 & 4900 & 8.57 & 5500 & 9.34 & 7800 & 9.95 & 6950 & 1.63 \\
\hline 2950 & 30.85 & 3800 & 6.33 & 4950 & 17.07 & 5550 & 20.37 & 7850 & 9.13 & 7000 & 3.28 \\
\hline 3000 & 36.11 & 3850 & 8.40 & 5000 & 25.18 & 5600 & 31.22 & 7900 & 8.44 & 7050 & 5.35 \\
\hline 3050 & 39.22 & 3900 & 10.33 & 5050 & 31.22 & 5650 & 38.90 & 7950 & 7.77 & 7100 & 7.62 \\
\hline 3100 & 41.50 & 3950 & 12.08 & 5100 & 35.20 & 5700 & 43.68 & 8000 & 7.09 & 7150 & 9.84 \\
\hline 3150 & 43.15 & 4000 & 13.59 & 5150 & 37.67 & 5750 & 46.47 & 8050 & 6.43 & 7200 & 12.24 \\
\hline 3200 & 44.72 & 4050 & 14.82 & 5200 & 39.06 & 5800 & 47.47 & 8100 & 5.84 & 7250 & 15.02 \\
\hline 3250 & 45.71 & 4100 & 15.88 & 5250 & 39.70 & 5850 & 48.09 & 8150 & 5.40 & 7300 & 18.13 \\
\hline 3300 & 46.53 & 4150 & 16.83 & 5300 & 39.70 & 5900 & 48.30 & 8200 & 4.87 & 7350 & 21.68 \\
\hline 3350 & 47.23 & 4200 & 17.61 & 5350 & 39.33 & 5950 & 48.16 & 8250 & 4.42 & 7400 & 25.34 \\
\hline 3400 & 47.92 & 4250 & 18.09 & 5400 & 38.51 & 6000 & 47.86 & 8300 & 3.98 & 7450 & 29.39 \\
\hline 3450 & 48.41 & 4300 & 18.41 & 5450 & 37.46 & 6050 & 47.50 & 8350 & 3.56 & 7500 & 33.95 \\
\hline 3500 & 48.58 & 4350 & 18.60 & 5500 & 35.96 & 6100 & 46.89 & 8400 & 3.20 & 7550 & 38.54 \\
\hline 3550 & 48.60 & 4400 & 18.64 & 5550 & 34.20 & 6150 & 46.22 & 8450 & 2.87 & 7600 & 43.01 \\
\hline 3600 & 48.12 & 4450 & 18.35 & 5600 & 32.15 & 6200 & 45.44 & 8500 & 2.55 & 7650 & 47.03 \\
\hline 3650 & 47.06 & 4500 & 17.66 & 5650 & 29.84 & 6250 & 44.76 & 8550 & 2.35 & 7700 & 50.72 \\
\hline 3700 & 45.32 & 4550 & 16.83 & 5700 & 27.39 & 6300 & 43.89 & 8600 & 1.92 & 7750 & 53.79 \\
\hline 3750 & 42.28 & 4600 & 15.62 & 5750 & 24.61 & 6350 & 42.87 & 8650 & 1.60 & 7800 & 56.22 \\
\hline 3800 & 37.65 & 4650 & 13.70 & 5800 & 21.76 & 6400 & 42.06 & 8700 & 1.30 & 7850 & 58.37 \\
\hline 3850 & 30.96 & 4700 & 11.20 & 5850 & 18.90 & 6450 & 41.28 & 8750 & 1.04 & 7900 & 59.71 \\
\hline 3900 & 22.81 & 4750 & 8.50 & 5900 & 16.17 & 6500 & 40.45 & 8800 & 0.78 & 7950 & 60.81 \\
\hline 3950 & 14.81 & 4800 & 6.01 & 5950 & 13.55 & 6550 & 39.45 & 8850 & 0.50 & 8000 & 61.63 \\
\hline 4000 & 8.45 & 4850 & 3.94 & 6000 & 11.10 & 6600 & 38.36 & 8900 & 0.28 & 8050 & 61.97 \\
\hline 4050 & 4.31 & 4900 & 2.43 & 6050 & 8.93 & 6650 & 37.29 & 8950 & 0.14 & 8100 & 62.11 \\
\hline 4100 & 2.07 & 4950 & 1.48 & 6100 & 7.06 & 6700 & 36.04 & 9000 & 0.06 & 8150 & 62.32 \\
\hline 4150 & 1.01 & 5000 & 0.90 & 6150 & 5.45 & 6750 & 34.67 & 9050 & 0.00 & 8200 & 62.72 \\
\hline 4200 & 0.53 & 5050 & 0.50 & 6200 & 4.15 & 6800 & 33.30 & & & 8250 & 63.10 \\
\hline 4250 & 0.30 & 5100 & 0.25 & 6250 & 3.04 & 6850 & 32.03 & & & 8300 & 63.08 \\
\hline 4300 & 0.01 & 5150 & 0.00 & 6300 & 2.16 & 6900 & 30.82 & & & 8350 & 62.42 \\
\hline 4350 & 0.00 & & & 6350 & 1.60 & 6950 & 29.44 & & & 8400 & 61.44 \\
\hline & & & & 6400 & 1.08 & 7000 & 28.04 & & & 8450 & 60.64 \\
\hline & & & & 6450 & 0.44 & 7050 & 26.80 & & & 8500 & 59.68 \\
\hline & & & & 6500 & 0.00 & 7100 & 25.51 & & & 8550 & 58.06 \\
\hline & & & & & & 7150 & 24.09 & & & 8600 & 55.32 \\
\hline & & & & & & 7200 & 22.61 & & & 8650 & 51.18 \\
\hline & & & & & & 7250 & 21.32 & & & 8700 & 46.21 \\
\hline & & & & & & 7300 & 20.13 & & & 8750 & 40.75 \\
\hline & & & & & & 7350 & 18.99 & & & 8800 & 33.59 \\
\hline & & & & & & 7400 & 17.98 & & & 8850 & 24.01 \\
\hline & & & & & & 7450 & 16.94 & & & 8900 & 14.66 \\
\hline & & & & & & 7500 & 15.86 & & & 8950 & 8.13 \\
\hline & & & & & & 7550 & 14.80 & & & 9000 & 3.70 \\
\hline & & & & & & 7600 & 13.77 & & & 9050 & 0.00 \\
\hline
\end{tabular}

Fig. 119. continued 


\section{griz - Schneider et al. - 1983}

Generale purpose system.

GENERAL INFORMATION

$\begin{array}{ll}\text { AUTHORS } & \text { D. P. Schneider, J. E. Gunn, J. G. Hoessel } \\ \text { TELESCOPE } & 1.52 \mathrm{~m} \text { (reector), Palomar Obs. } \\ \text { DETECTOR } & 800 \times 800 \mathrm{CCD} \text { (Texas Instruments) }\end{array}$

MAIN ARTICLE D. P. Schneider, J. E. Gunn, J. G. Hoessel 1983, ApJ 264, 337

SYSTEM DESCRIPTION
\begin{tabular}{|c|lcr|rr|}
\hline \multicolumn{2}{|c|}{ BANDS DESCRIPTION } & & \\
\hline band & lter & $\lambda_{e f f}(\mathrm{~A})$ & FWHM (A) & WHM $(\mathrm{A})$ & $\lambda_{c}(\mathrm{~A})$ \\
\hline $\boldsymbol{g}[301]$ & Corion interference & 4930 & 700 & 977 & 5140 \\
\hline$r[301]$ & 3mm Schott BG610 + Balzer B1 short-pass & 6550 & 900 & 905 & 6530 \\
\hline $\boldsymbol{i}[312]$ & Wratten 88a gelatin lter + Blazers infrared mirror & 8200 & 1300 & 1300 & 7860 \\
\hline$Z[261]$ & 3mm RG 850 & & 1165 & 9015 \\
\hline
\end{tabular}

Filters $g, r$ are from the $u v g r$ - Thuan and Gunn - 1976 system,

lter $i$ is equivalent to $i$ lter of the $r i$-Wade et al. - 1979 system.

SYSTEM ANALYSIS

REDDENING RATIOS [261]
$A(g)=3.29 E(B-V)_{J o h n s o n}$
$A(r)=2.23 E(B-V)_{J o h n s o n}$
$A(i)=1.63 E(B-V)_{J o h n s o n}$
$A(z)=1.22 E(B-V)_{\text {Johnson }}$

\section{TRANSMISSION CURVES}

As derived from Fig 4 of [261].

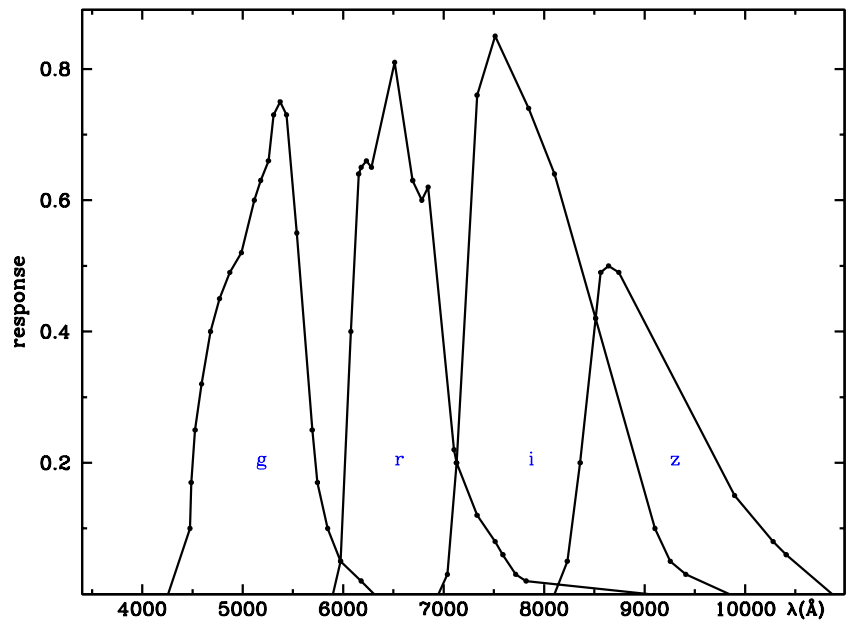

\begin{tabular}{|cc|cc|cc|cc|}
\hline \multicolumn{2}{|c|}{$g$} & \multicolumn{2}{c|}{$r$} & \multicolumn{2}{c|}{$i$} & \multicolumn{2}{c|}{$Z$} \\
\hline$\lambda(\mathrm{A})$ & $\Upsilon$ & $\lambda(\mathrm{A})$ & $\Upsilon$ & $\lambda(\mathrm{A})$ & $\Upsilon$ & $\lambda(\mathrm{A})$ & $\Upsilon$ \\
\hline 4256 & 0.00 & 5897 & 0.00 & 6949 & 0.00 & 8103 & 0.00 \\
4474 & 0.10 & 5974 & 0.05 & 7038 & 0.03 & 8231 & 0.05 \\
4487 & 0.17 & 6077 & 0.40 & 7128 & 0.20 & 8359 & 0.20 \\
4526 & 0.25 & 6154 & 0.64 & 7333 & 0.76 & 8513 & 0.42 \\
4590 & 0.32 & 6179 & 0.65 & 7513 & 0.85 & 8564 & 0.49 \\
4679 & 0.40 & 6231 & 0.66 & 7846 & 0.74 & 8641 & 0.50 \\
4769 & 0.45 & 6282 & 0.65 & 8103 & 0.64 & 8744 & 0.49 \\
4872 & 0.49 & 6513 & 0.81 & 8513 & 0.42 & 9897 & 0.15 \\
4987 & 0.52 & 6692 & 0.63 & 9103 & 0.10 & 10280 & 0.08 \\
5115 & 0.60 & 6782 & 0.60 & 9256 & 0.05 & 10410 & 0.06 \\
5179 & 0.63 & 6846 & 0.62 & 9410 & 0.03 & 10870 & 0.00 \\
5256 & 0.66 & 7103 & 0.22 & 9846 & 0.00 & & \\
5308 & 0.73 & 7128 & 0.20 & & & & \\
5372 & 0.75 & 7333 & 0.12 & & & & \\
5436 & 0.73 & 7513 & 0.08 & & & & \\
5538 & 0.55 & 7590 & 0.06 & & & & \\
5692 & 0.25 & 7718 & 0.03 & & & & \\
5744 & 0.17 & 7821 & 0.02 & & & & \\
5846 & 0.10 & 9051 & 0.00 & & & & \\
5974 & 0.05 & & & & & & \\
6179 & 0.02 & & & & & & \\
6308 & 0.00 & & & & & & \\
\hline
\end{tabular}

Fig. 120. The photometric system griz - Schneider et al. - 1983 


\section{RGU - Trefzger et al. - 1983}

Photoelectric version of the $R G U$ - Becker - 1946 photographic system. [25]

\section{GENERAL INFORMATION}

$\begin{array}{ll}\text { AUTHORS } & \text { C. F. Trefzger, L. M. Cameron, A. Spaenhauer and U. W. Steinlin } \\ \text { TELESCOPE } & 1 \mathrm{~m} \text { (reector), Gornergrat Obs. } \\ \text { DETECTOR } & \text { RCA 7265 (S-20 cathode, refrigerated) } \\ \text { MAIN ARTICLE } & \text { Trefzger, C. F., Cameron, L. M., Spaenhauer, }\end{array}$

A., Steinlin, U.W. 1983, A\&A 117, 347

\section{SYSTEM DESCRIPTION}

\begin{tabular}{|c|cccc|}
\hline \multicolumn{5}{|c|}{ BANDS DESCRIPTION [305] } \\
\hline band & lter & $\lambda_{\max }(\mathrm{A})(\#)$ & FWHM (A) \\
\hline$U$ & K-36 ltrae & $\mathrm{x}$ & 3650 & 440 \\
\hline$G$ & custom lter & $(\#)$ & 4650 & 500 \\
\hline$R$ & K-65 ltrae & $\mathrm{x}$ & 6550 & 490 \\
\hline
\end{tabular}

(\#) in addition 2mm BG13 is needed to suppress the red-leak. [305]

ZERO POINT: As for the photographic version unreddened A0 V stars have:

$G=V_{\text {Johnson }},(G-R)=0.32$ and $(U-G)=1.240 \quad$ [305]

\section{TRANSMISSION CURVES [305]}

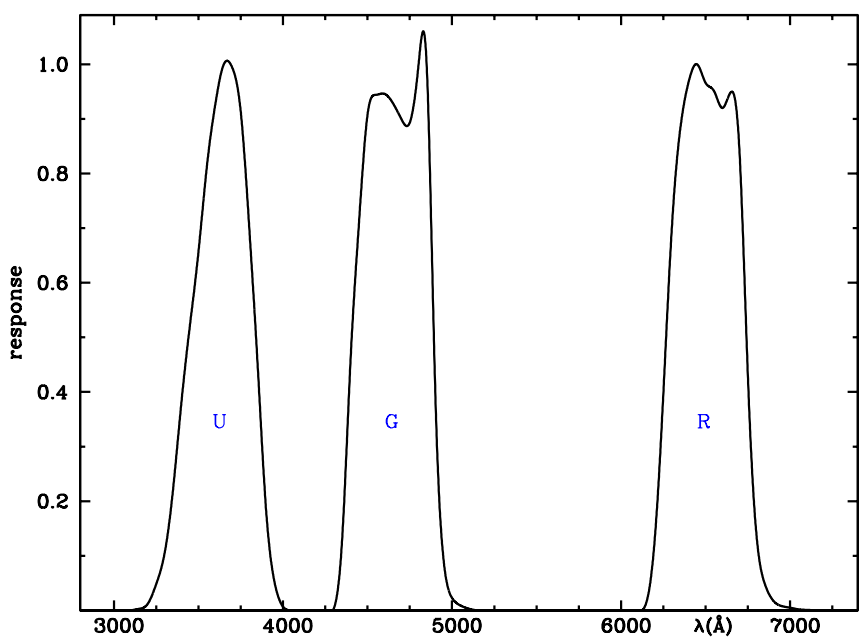

\begin{tabular}{|cc|cc|cc|}
\hline \multicolumn{2}{|c|}{$U$} & \multicolumn{2}{c|}{$G$} & \multicolumn{2}{c|}{$R$} \\
\hline$\lambda(\mathrm{A})$ & $\Upsilon$ & $\lambda(\mathrm{A})$ & $\Upsilon$ & $\lambda(\mathrm{A})$ & $\Upsilon$ \\
\hline 3100 & 0.000 & 4250 & 0.000 & 6100 & 0.000 \\
3150 & 0.003 & 4300 & 0.003 & 6150 & 0.015 \\
3200 & 0.012 & 4350 & 0.130 & 6200 & 0.133 \\
3250 & 0.048 & 4400 & 0.459 & 6250 & 0.396 \\
3300 & 0.105 & 4450 & 0.711 & 6300 & 0.698 \\
3350 & 0.222 & 4500 & 0.907 & 6350 & 0.881 \\
3400 & 0.383 & 4550 & 0.944 & 6400 & 0.968 \\
3450 & 0.523 & 4600 & 0.946 & 6450 & 1.000 \\
3500 & 0.656 & 4650 & 0.929 & 6500 & 0.966 \\
3550 & 0.813 & 4700 & 0.900 & 6550 & 0.951 \\
3600 & 0.927 & 4750 & 0.893 & 6600 & 0.920 \\
3650 & 1.000 & 4800 & 0.993 & 6650 & 0.949 \\
3700 & 0.914 & 4850 & 1.000 & 6700 & 0.844 \\
3750 & 0.994 & 4900 & 0.412 & 6750 & 0.432 \\
3800 & 0.704 & 4950 & 0.093 & 6800 & 0.142 \\
3850 & 0.451 & 5000 & 0.023 & 6850 & 0.049 \\
3900 & 0.185 & 5050 & 0.009 & 6900 & 0.018 \\
3950 & 0.052 & 5100 & 0.003 & 6950 & 0.008 \\
4000 & 0.007 & 5250 & 0.000 & 7000 & 0.005 \\
4050 & 0.000 & & & 7050 & 0.002 \\
& & & & 7100 & 0.001 \\
& & & & 7150 & 0.000 \\
\hline
\end{tabular}

Fig. 121. The photometric system RGU - Trefzger et al. - 1983 


\section{IRAS - 1983}

Infrared photometry by the IRAS satellite.

\section{GENERAL INFORMATION}

TELESCOPE

DETECTOR

MAIN ARTICLE

$0.57 \mathrm{~m}$ (reflector)

Si:As (12), Si:Sb (25), Ge:Ga (60, 100)

Beichman, C. A., Neugebauer, G., Habing, H. J., Clegg, P. E., Chester, T. J.

1988, Infrared Astronomical Satellite (IRAS) Catalogs and Atlases, NASA RP-1190, Vol. 1

\section{SYSTEM DESCRIPTION}

\begin{tabular}{|c|c|c|c|c|c|c|}
\hline \multicolumn{3}{|c|}{ BANDS DESCRIPTION [227] } & \multirow[b]{2}{*}{$W H M(\mu \mathrm{m})$} & \multirow[b]{2}{*}{$\lambda_{c}(\mu \mathrm{m})$} & \multicolumn{2}{|c|}{ FLUX CALIBRATION [185] } \\
\hline band & $\lambda_{c}(\mu \mathrm{m})$ & bandpass $(\mu \mathrm{m})$ & & & $\mathrm{F}_{\lambda}\left(\mathrm{W} \mathrm{m}^{-2} \mu \mathrm{m}^{-1}\right)$ & $\mathrm{F}_{\mathrm{V}}(\mathrm{Jy})$ \\
\hline 12 & 12 & $8.5-15$ & 6.93 & 11.43 & $5.8410^{-13} \pm 4 \%$ & $28.0 \pm 4 \%$ \\
\hline 25 & 25 & $19-30$ & 11.35 & 23.92 & $3.8110^{-14} \pm 6 \%$ & $7.94 \pm 6 \%$ \\
\hline 60 & 60 & $40-80$ & 33.00 & 62.00 & $5.9710^{-15} \pm 6 \%$ & $7.17 \pm 6 \%$ \\
\hline 100 & 100 & $83-120$ & 32.10 & 101.00 & $2.2810^{-15} \pm 10 \%$ & $7.61 \pm 10 \%$ \\
\hline
\end{tabular}

\section{TRANSMISSION CURVES [28], pg II-18}

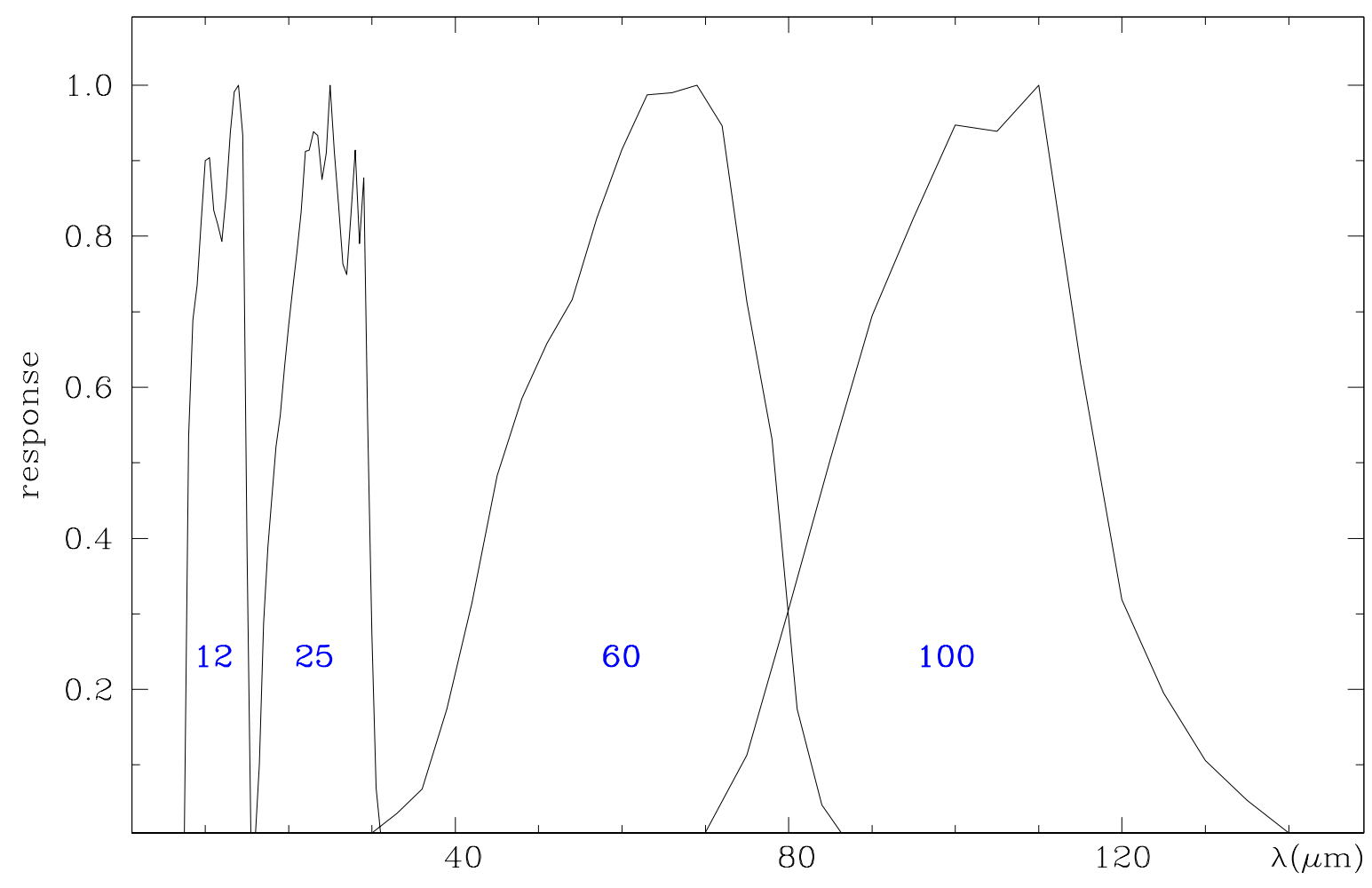

Fig. 122. The photometric system IRAS - 1983 
D. Moro and U. Munari: The Asiago Database on Photometric Systems (ADPS). I.

\begin{tabular}{|rc|rc|rc|rc|rc|}
\hline \multicolumn{2}{|c|}{12} & \multicolumn{3}{|c|}{25} & \multicolumn{2}{c|}{60} & \multicolumn{2}{c|}{100} \\
\hline$\lambda(\mu \mathrm{m})$ & $\Upsilon$ & $\lambda(\mu \mathrm{m})$ & $\Upsilon$ & $\lambda(\mu \mathrm{m})$ & $\Upsilon$ & $\lambda(\mu \mathrm{m})$ & $\Upsilon$ & $\lambda(\mu \mathrm{m})$ & $\Upsilon$ \\
\hline 7.0 & 0.000 & 16.0 & 0.007 & 26.5 & 0.763 & 27.0 & 0.000 & 65.0 & 0.000 \\
7.5 & 0.008 & 16.5 & 0.101 & 27.0 & 0.749 & 30.0 & 0.01 & 70.0 & 0.01 \\
8.0 & 0.535 & 17.0 & 0.288 & 27.5 & 0.829 & 33.0 & 0.036 & 75.0 & 0.113 \\
8.5 & 0.689 & 17.5 & 0.388 & 28.0 & 0.914 & 36.0 & 0.068 & 80.0 & 0.306 \\
9.0 & 0.735 & 18.0 & 0.452 & 28.5 & 0.790 & 39.0 & 0.174 & 85.0 & 0.505 \\
9.5 & 0.815 & 18.5 & 0.521 & 29.0 & 0.877 & 42.0 & 0.315 & 90.0 & 0.695 \\
10.0 & 0.900 & 19.0 & 0.562 & 29.5 & 0.558 & 45.0 & 0.483 & 95.0 & 0.824 \\
10.5 & 0.904 & 19.5 & 0.626 & 30.0 & 0.274 & 48.0 & 0.585 & 100.0 & 0.947 \\
11.0 & 0.834 & 20.0 & 0.683 & 30.5 & 0.069 & 51.0 & 0.658 & 105.0 & 0.939 \\
11.5 & 0.816 & 20.5 & 0.729 & 31.0 & 0.012 & 54.0 & 0.716 & 110.0 & 1.000 \\
12.0 & 0.793 & 21.0 & 0.778 & 31.5 & 0.000 & 57.0 & 0.824 & 115.0 & 0.631 \\
12.5 & 0.854 & 21.5 & 0.832 & & & 60.0 & 0.915 & 120.0 & 0.319 \\
13.0 & 0.938 & 22.0 & 0.912 & & & 63.0 & 0.987 & 125.0 & 0.195 \\
13.5 & 0.991 & 22.5 & 0.914 & & & 66.0 & 0.990 & 130.0 & 0.106 \\
14.0 & 1.000 & 23.0 & 0.938 & & & 69.0 & 1.000 & 135.0 & 0.053 \\
14.5 & 0.934 & 23.5 & 0.933 & & & 72.0 & 0.946 & 140.0 & 0.01 \\
15.0 & 0.388 & 24.0 & 0.875 & & & 75.0 & 0.713 & & \\
15.5 & 0.000 & 24.5 & 0.910 & & & 78.0 & 0.531 & & \\
& & 25.0 & 1.000 & & & 81.0 & 0.174 & & \\
& & 25.5 & 0.911 & & & 84.0 & 0.047 & & \\
& & 26.0 & 0.840 & & & 87.0 & 0.000 & & \\
\hline
\end{tabular}

Fig. 122. continued 


\section{VWFSC Spacelab 1-1983}

Ultraviolet photometry with the Very Wide Field Survey Camera on board of Spacelab-1.

\section{GENERAL INFORMATION}

TELESCOPE all reection f/1.9 Schmidt Camera

DETECTOR ITT image intensier (CsTe photocathode) + Kodak IIa-O

MAIN ARTICLE Viton, M., Burgarella, D., Cassatella, A., Prévot, L. 1988, A\&A 205, 147

\section{SYSTEM DESCRIPTION}

\begin{tabular}{|c|ccc|cr|}
\hline \multicolumn{7}{|c|}{ BANDS DESCRIPTION [311] } & & \\
\hline band & lter & $\lambda_{c}(\mathrm{~A})$ & half-width (A) & WHM (A) & $\lambda_{c}(\mathrm{~A})$ \\
\hline$F 2$ & interference & 1680 & 410 & 430 & 1690 \\
\hline$F 1$ & interference & 1950 & 230 & 243 & 1963 \\
\hline$F 3$ & interference & 2540 & 230 & 253 & 2556 \\
\hline
\end{tabular}

\section{TRANSMISSION CURVES}

As derived from Fig 1 of [304].

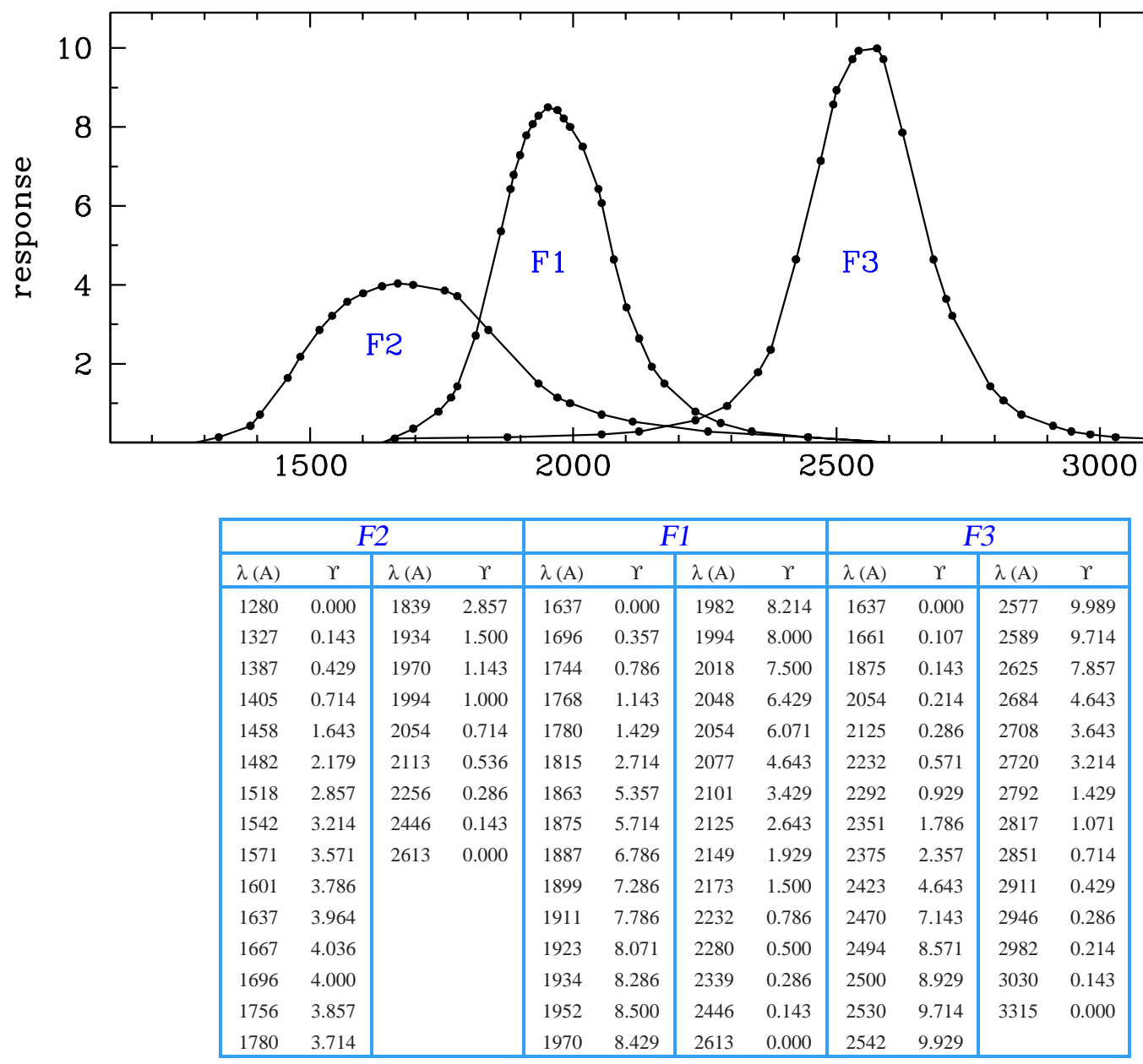

Fig. 123. The photometric system VWFSC Spacelab $1-1983$ 


\section{JHKLMNQ OAN - Roth et al. - 1984}

Infrared photometry at the San Pedro Màrtir National Observatory.

\section{GENERAL INFORMATION}

AUTHORS

TELESCOPE

DETECTOR

MAIN ARTICLE
M. Roth, A. Iriarte, M. Tapia and G. Resendiz

2.1m (reflector), San Pedro Mártir National Obs., Mexico

InSb cell $(1-5 \mu \mathrm{m})$ and Ge bolometer $(5-20 \mu \mathrm{m})$,

Roth, M., Iriarte, M., Tapia, M., Resendiz, G. 1984, RMxAA 9, 25

\section{SYSTEM DESCRIPTION}

\begin{tabular}{|c|ccc|cc|}
\hline \multicolumn{5}{|c|}{ BANDS DESCRIPTION [252] } & \multicolumn{2}{|c|}{ FLUX CALIBRATION (\#) [61] } \\
\hline band & $\lambda_{0}(\mu \mathrm{m})$ & width $(\mu \mathrm{m})$ & $\lambda_{\text {eff }}(\mu \mathrm{m})[61]$ & $\mathrm{F}_{\lambda, 0}\left(\mathrm{~W} \mathrm{~cm}^{-2} \mu \mathrm{m}^{-1}\right)$ & $\mathrm{F}_{\mathrm{v}, 0}(\mathrm{Jy})$ \\
\hline$J$ & 1.25 & 0.32 & 1.198 & $3.42010^{-13}$ & 1642 \\
\hline$H$ & 1.65 & 0.28 & 1.580 & $1.29510^{-13}$ & 1105 \\
\hline$K$ & 2.23 & 0.35 & 2.210 & $3.92010^{-14}$ & 632 \\
\hline$L^{\prime}$ & 3.80 & 0.70 & & & \\
\hline$M(\mathrm{InSb})$ & 4.70 & 0.6 & & & \\
\hline$M(\mathrm{Ge})$ & 4.84 & 0.9 & & & \\
\hline$N_{1}$ & 8.00 & 0.6 & & & \\
\hline$N_{2}$ & 8.60 & 0.91 & & & \\
\hline$N$ & 10.39 & 5.34 & & & \\
\hline$N_{3}$ & 10.92 & 0.58 & & \\
\hline$N_{4}$ & 11.31 & 1.95 & & \\
\hline$Q_{1}$ & 18.00 & 3.63 & & \\
\hline$Q$ & 19.60 & 8.6 & & \\
\hline
\end{tabular}

(\#) For a 0.0 mag star.

ZERO POINT: $J=H=K=0.0$. for $\alpha$ Lyr. [61]

\section{SYSTEM ANALYSIS}

RELATIONS WITH OTHER SYSTEMS [61]

$$
\text { UBVRI(JHKLMN) - Johnson - } 1965
$$

\begin{tabular}{rl|}
\hline$K_{J}$ & $=K_{O A N}+0.029-0.011(V-K)_{O A N}$ \\
\hline$(J-K)_{J}$ & $=0.038+0.974(J-K)_{O A N}$ \\
\hline$(H-K)_{J}$ & $=0.014+0.834(H-K)_{O A N}$ \\
\hline
\end{tabular}

JHKL CTIO - Elias et al - 1982
VJHKLM ESO - Engels et al - 1981

\begin{aligned} & \hline$K_{E S O}=0.0321( \pm 0.0157)+0.9989( \pm 0.0040) K_{\text {OAN }} \\ &$\hline$(J-K)_{E S O}=0.0422( \pm 0.0058)+0.9591( \pm 0.0216)(J-K)_{\text {OAN }} \\ &$\hline$(H-K)_{E S O}=0.0322( \pm 0.0047)+0.8057( \pm 0.0922)(H-K)_{\text {OAN }} \\ &$\hline\end{aligned}

JHKL' AAO - Allen and Cragg - 1983

\begin{tabular}{|rcc|rcr|}
\hline$K_{C T I O}$ & $=-0.0176( \pm 0.0141)+1.0047( \pm 0.0030) K_{\text {OAN }}$ & $K_{A A O}$ & $=$ & $-0.0044( \pm 0.0186)+1.0047( \pm 0.0046) K_{O A N}$ \\
\hline$(J-K)_{C T I O}$ & $=0.0356( \pm 0.0048)+0.8962( \pm 0.0083)(J-K)_{\text {OAN }}$ & $(J-K)_{A A O}$ & $=0.0429( \pm 0.0052)+1.0010( \pm 0.0126)(J-K)_{\text {OAN }}$ \\
\hline$(H-K)_{C T I O}$ & $=0.0138( \pm 0.0042)+0.8593( \pm 0.0264)(H-K)_{\text {OAN }}$ & $(H-K)_{A A O}$ & $=0.0176( \pm 0.0046)+0.8196( \pm 0.0614)(H-K)_{\text {OAN }}$ \\
\hline
\end{tabular}

Fig. 124. The photometric system JHKLMNQ OAN - Roth et al. - 1984 


\section{WBVR - Khaliullin et al. - 1985}

General purpose system. Evolved from the WBVR -Straižys - 1973

\section{GENERAL INFORMATION}

AUTHORS K. H. Khaliullin, A. V. Mironov and V. G. Moshkalyov

TELESCOPE $\quad 0.48 \mathrm{~m}$ (reflector), Thien-Shan High Altitude Obs.

DETECTOR S-20 photomultiplier

MAIN ARTICLE Khaliullin, K. H., Mironov, A. V., Moshkalyov, V. G. 1984, Ap\&SS 111, 291

\section{SYSTEM DESCRIPTION}

\begin{tabular}{|l|c|cc|}
\hline \multicolumn{3}{|c|}{ BANDS DESCRIPTION [164] } & \\
\hline band & $\lambda_{\text {peak }}(\AA)$ & WHM $(\AA)$ & $\lambda_{c}(\AA)$ \\
\hline$W$ & 3500 & 930 & 3490 \\
\hline$B$ & 4400 & 905 & 4400 \\
\hline$V$ & 5300 & 805 & 5455 \\
\hline$R$ & 6600 & 1085 & 6925 \\
\hline
\end{tabular}

ZERO POINT: HD 221525 (A7 IV) defines the zero point of magnitudes as follows:

$W=5.922 \quad B=5.800 \quad V=5.574 \quad R=5.346$

Unreddened A0 V stars have 0.00 colors. [164]

SYSTEM ANALYSIS

RELATIONS WITH OTHER SYSTEMS [169]

UBV - Johnson and Morgan - 1953

$V_{J}-V_{S}=-0.0019-0.061(B-V)+0.072(V-R)$

Fig. 125. The photometric system $W B V R-$ Khalliullin et al. -1985 


\section{NORMALIZED TRASMISSION CURVES [164]}

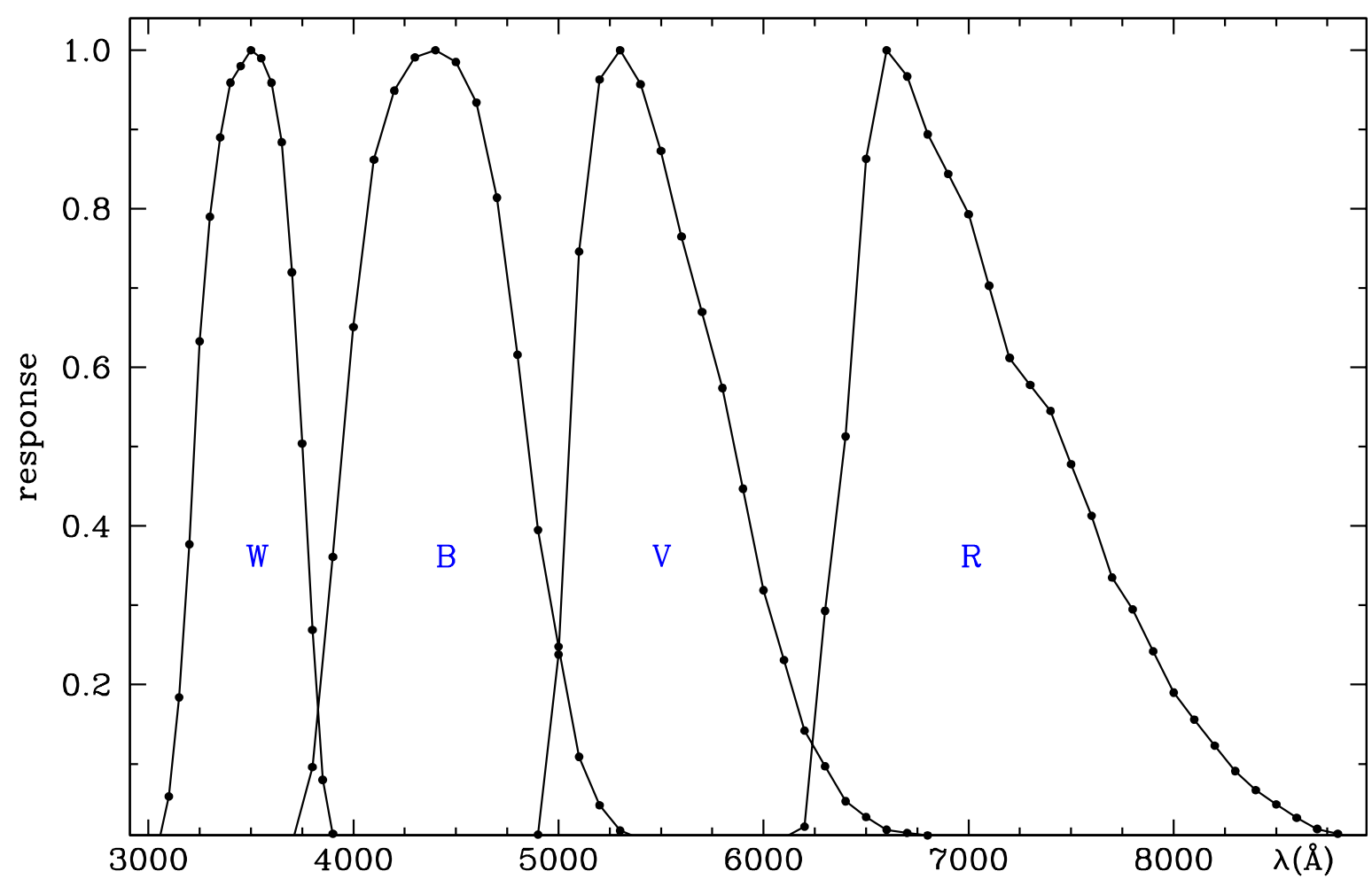

\begin{tabular}{|cc|cc|cc|cc|cc|cc|}
\hline \multicolumn{3}{|c|}{$W$} & \multicolumn{3}{|c|}{$B$} & \multicolumn{3}{c|}{$V$} & \multicolumn{4}{c|}{$R$} \\
\hline$\lambda(\mathrm{A})$ & $\Upsilon$ & $\lambda(\mathrm{A})$ & $\Upsilon$ & $\lambda(\mathrm{A})$ & $\Upsilon$ & $\lambda(\mathrm{A})$ & $\Upsilon$ & $\lambda(\mathrm{A})$ & $\Upsilon$ & $\lambda(\mathrm{A})$ & $\Upsilon$ \\
\hline 3050 & 0.000 & 3700 & 0.000 & 4600 & 0.000 & 6600 & 0.017 & 5900 & 0.000 & 7900 & 0.242 \\
3100 & 0.059 & 3800 & 0.096 & 4700 & 0.001 & 6700 & 0.013 & 6000 & 0.002 & 8000 & 0.190 \\
3150 & 0.184 & 3900 & 0.361 & 4800 & 0.003 & 6800 & 0.010 & 6100 & 0.007 & 8100 & 0.156 \\
3200 & 0.377 & 4000 & 0.651 & 4900 & 0.011 & 6900 & 0.007 & 6200 & 0.021 & 8200 & 0.123 \\
3250 & 0.633 & 4100 & 0.862 & 5000 & 0.238 & 7000 & 0.004 & 6300 & 0.293 & 8300 & 0.091 \\
3300 & 0.790 & 4200 & 0.949 & 5100 & 0.746 & 7100 & 0.003 & 6400 & 0.513 & 8400 & 0.067 \\
3350 & 0.890 & 4300 & 0.991 & 5200 & 0.963 & 7200 & 0.001 & 6500 & 0.863 & 8500 & 0.049 \\
3400 & 0.959 & 4400 & 1.000 & 5300 & 1.000 & 7300 & 0.000 & 6600 & 1.000 & 8600 & 0.032 \\
3450 & 0.980 & 4500 & 0.985 & 5400 & 0.957 & & & 6700 & 0.967 & 8700 & 0.018 \\
3500 & 1.000 & 4600 & 0.934 & 5500 & 0.873 & & & 6800 & 0.894 & 8800 & 0.012 \\
3550 & 0.990 & 4700 & 0.814 & 5600 & 0.765 & & & 6900 & 0.844 & 8900 & 0.005 \\
3600 & 0.959 & 4800 & 0.616 & 5700 & 0.670 & & & 7000 & 0.793 & 9000 & 0.000 \\
3650 & 0.884 & 4900 & 0.395 & 5800 & 0.574 & & & 7100 & 0.703 & & \\
3700 & 0.720 & 5000 & 0.248 & 5900 & 0.447 & & & 7200 & 0.612 & & \\
3750 & 0.504 & 5100 & 0.109 & 6000 & 0.319 & & & 7300 & 0.578 & & \\
3800 & 0.269 & 5200 & 0.048 & 6100 & 0.231 & & & 7400 & 0.545 & & \\
3850 & 0.080 & 5300 & 0.016 & 6200 & 0.142 & & & 7500 & 0.478 & & \\
3900 & 0.012 & 5400 & 0.006 & 6300 & 0.097 & & & 7600 & 0.413 & & \\
3950 & 0.002 & 5500 & 0.002 & 6400 & 0.053 & & & 7700 & 0.335 & & \\
4000 & 0.000 & 5600 & 0.000 & 6500 & 0.033 & & & 7800 & 0.295 & & \\
\hline
\end{tabular}

Fig. 125. continued 


\section{$J_{n} K_{n} L_{n} M_{n}$ - Leggett et al. - 1986}

Narrow band version of the common JKLM bands.

\section{GENERAL INFORMATION}

AUTHORS S. K. Leggett, M. Bartholomew, C. M. Mountain and M. J. Selby

TELESCOPE $\quad 1.5 \mathrm{~m}$ Sanchez-Magro (reflector), Teide Obs.;

3.8m UKIRT (reflector), Mauna Kea

DETECTOR InSb (liquid $\mathrm{N}_{2}$ cooled)

MAIN ARTICLE Leggett, S. K., Bartholomew, M., Mountain, C. M., Selby, M. J. 1986 MNRAS 223, 443

\section{SYSTEM DESCRIPTION}

\begin{tabular}{|l|cc|}
\hline \multicolumn{3}{|c|}{ BANDS DESCRIPTION [186] } \\
\hline band & $\lambda_{\text {eff }}(\mu \mathrm{m})$ & FWHM $(\mu \mathrm{m})$ \\
\hline$J_{n}$ & 1.24 & 0.03 \\
\hline $\boldsymbol{K}_{n}$ & 2.20 & 0.05 \\
\hline$L_{n}$ & 3.80 & 0.14 \\
\hline $\boldsymbol{M}_{n}$ & 4.70 & 0.21 \\
\hline
\end{tabular}

ZERO POINT: $J n=K n=L n=M n=0.0$ for $\alpha$ Lyr. [186], [266]

\section{SYSTEM ANALYSIS}

RELATIONS WITH OTHER SYSTEMS [266]

UBVRI(JKHLMN) - Johnson - 1965

\begin{tabular}{|rll|}
\hline$(V-J)$ & $=-0.044+0.988(V-J n)$ & for 103 stars in common \\
\hline$(V-K)$ & $=-0.020+0.989(V-K n)$ & for 103 stars in common \\
\hline$(V-L)$ & $=-0.038+0.981(V-L n)$ & for 37 stars in common \\
\hline
\end{tabular}

Fig. 126. The photometric system $J_{n} K_{n} L_{n} M_{n}$ - Leggett et al. - 1986 
TRANSMISSION CURVES [266]

$M_{n}$ band is derived from Fig 1 of [186].

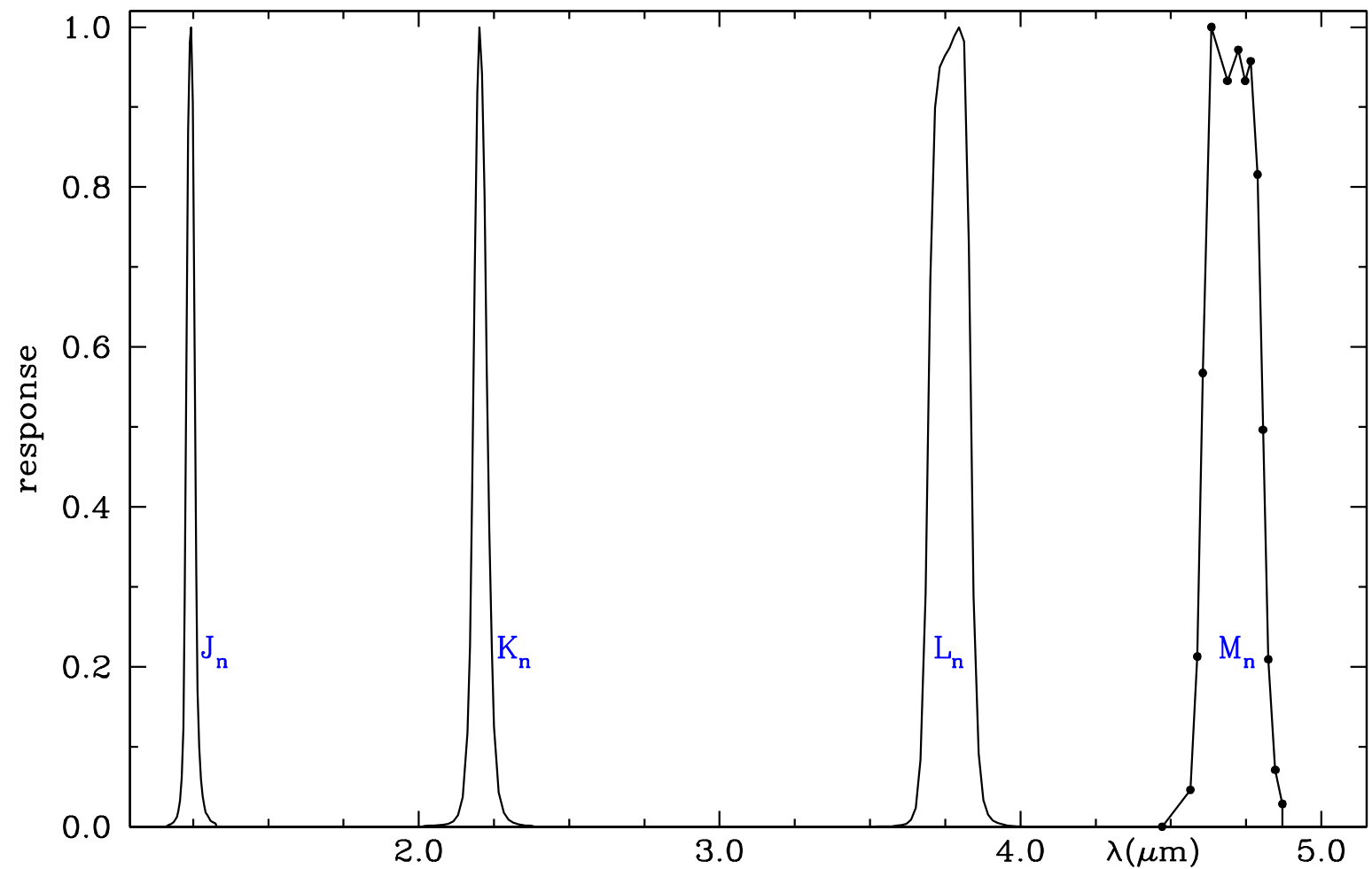

\begin{tabular}{|c|c|c|c|c|c|c|c|c|c|c|c|c|c|}
\hline \multicolumn{4}{|c|}{$J_{n}$} & \multicolumn{4}{|c|}{$K_{n}$} & \multicolumn{4}{|c|}{$L_{n}$} & \multicolumn{2}{|c|}{$M_{n}$} \\
\hline$\lambda(\mu \mathrm{m})$ & $\Upsilon$ & $\lambda(\mu \mathrm{m})$ & $\Upsilon$ & $\lambda(\mu \mathrm{m})$ & $\Upsilon$ & $\lambda(\mu \mathrm{m})$ & $\Upsilon$ & $\lambda(\mu \mathrm{m})$ & $\Upsilon$ & $\lambda(\mu \mathrm{m})$ & $\Upsilon$ & $\lambda(\mu \mathrm{m})$ & $\Upsilon$ \\
\hline 1.1587 & 0.000 & 1.2494 & 0.905 & 2.0102 & 0.000 & 2.2184 & 0.789 & 3.5081 & 0.000 & 3.7803 & 0.989 & 4.471 & 0.0000 \\
\hline 1.1640 & 0.000909 & 1.2548 & 0.611 & 2.0182 & 0.000928 & 2.2264 & 0.574 & 3.5241 & 0.000297 & 3.7963 & 1.000 & 4.565 & 0.0461 \\
\hline 1.1694 & 0.00267 & 1.2601 & 0.322 & 2.0343 & 0.00129 & 2.2344 & 0.372 & 3.5401 & 0.000374 & 3.8123 & 0.982 & 4.588 & 0.2128 \\
\hline 1.1747 & 0.00304 & 1.2654 & 0.170 & 2.0503 & 0.00147 & 2.2424 & 0.223 & 3.5562 & 0.000377 & 3.8283 & 0.729 & 4.606 & 0.5673 \\
\hline 1.1800 & 0.00426 & 1.2708 & 0.0986 & 2.0663 & 0.00183 & 2.2504 & 0.125 & 3.5722 & 0.000607 & 3.8444 & 0.289 & 4.635 & 1.0000 \\
\hline 1.1854 & 0.00542 & 1.2761 & 0.0595 & 2.0823 & 0.00228 & 2.2664 & 0.0428 & 3.5882 & 0.00122 & 3.8604 & 0.0915 & 4.688 & 0.9327 \\
\hline 1.1907 & 0.00857 & 1.2815 & 0.0383 & 2.0983 & 0.00335 & 2.2824 & 0.0179 & 3.6042 & 0.00185 & 3.8764 & 0.0332 & 4.724 & 0.9716 \\
\hline 1.1961 & 0.0124 & 1.2868 & 0.0257 & 2.1143 & 0.00650 & 2.2984 & 0.00885 & 3.6202 & 0.00372 & 3.8924 & 0.0150 & 4.747 & 0.9327 \\
\hline 1.2014 & 0.0192 & 1.2921 & 0.0178 & 2.1303 & 0.0147 & 2.3144 & 0.00504 & 3.6362 & 0.00860 & 3.9084 & 0.00774 & 4.765 & 0.9575 \\
\hline 1.2067 & 0.0322 & 1.2975 & 0.0144 & 2.1463 & 0.0373 & 2.3305 & 0.00318 & 3.6522 & 0.0235 & 3.9244 & 0.00448 & 4.788 & 0.8156 \\
\hline 1.2121 & 0.0606 & 1.3028 & 0.0109 & 2.1623 & 0.117 & 2.3465 & 0.00212 & 3.6682 & 0.0838 & 3.9404 & 0.00289 & 4.806 & 0.4964 \\
\hline 1.2174 & 0.124 & 1.3081 & 0.00795 & 2.1703 & 0.2261 & 2.3545 & 0.00168 & 3.6842 & 0.292 & 3.9564 & 0.00155 & 4.824 & 0.2092 \\
\hline 1.2227 & 0.286 & 1.3135 & 0.00621 & 2.1783 & 0.422 & 2.3625 & 0.00133 & 3.7003 & 0.684 & 3.9724 & 0.000826 & 4.847 & 0.0709 \\
\hline 1.2281 & 0.582 & 1.3188 & 0.00532 & 2.1864 & 0.693 & 2.3705 & 0.00142 & 3.7163 & 0.899 & 3.9884 & 0.000463 & 4.871 & 0.0284 \\
\hline 1.2334 & 0.871 & 1.3241 & 0.00393 & 2.1944 & 0.918 & 2.3785 & 0.00106 & 3.7323 & 0.950 & 4.0044 & 0.000134 & 4.941 & 0.0000 \\
\hline 1.2388 & 0.981 & 1.3294 & 0.0000 & 2.2024 & 1.000 & 2.3860 & 0.000 & 3.7483 & 0.964 & 4.0200 & 0.000 & & \\
\hline 1.2441 & 1.000 & & & 2.2104 & 0.942 & & & 3.7643 & 0.974 & & & & \\
\hline
\end{tabular}

Fig. 126. continued 


\section{Park and Lee - 1986}

Conceptual study of a broad-band system optimized for G-K dwarfs.

\section{GENERAL INFORMATION}

AUTHORS N.-K. Park and S. W. Lee

MAIN ARTICLE Park, N.-K., and Lee, S.W. 1986, Journal of the Korean Astron. Soc. 19, 1

\section{SYSTEM DESCRIPTION}

\begin{tabular}{|c|cc|}
\hline \multicolumn{3}{|c|}{ BANDS DESCRIPTION [239] } \\
\hline band & $\lambda_{0}(\AA)$ & FWHM $(\AA)$ \\
\hline 34 & 3400 & 200 \\
\hline 38 & 3850 & 200 \\
\hline 42 & 4190 & 200 \\
\hline 46 & 4600 & 200 \\
\hline
\end{tabular}

The band profiles are gaussians truncated to 0.0 when the transmission reaches half of the peak value.

\section{SYSTEM ANALYSIS}

\section{COLOR INDICES AND PARAMETERS [239]}

34, 38 : bands sensitive to temperature, gravity and metallicity.

46 : band mainly sensitive to metallicity and gravity.

42 : band mainly sensitive to effective temperature.

Fig. 127. The photometric system Park and Lee - 1986 


\section{TRANSMISSION CURVES [239]}

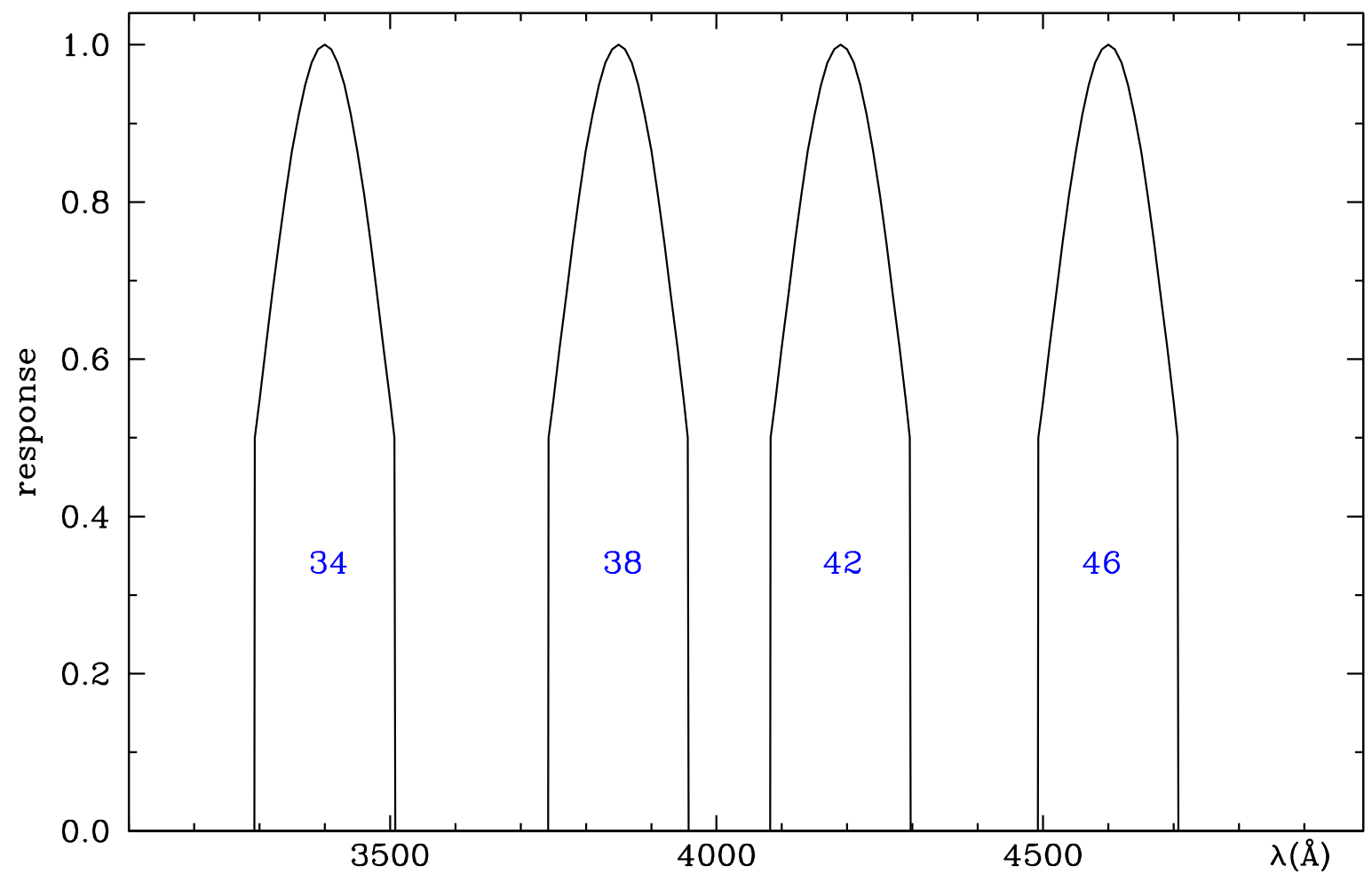

\begin{tabular}{|cc|cc|cc|cc|cc|cc|cc|cc|c|}
\hline \multicolumn{6}{|c|}{34} & \multicolumn{6}{|c|}{38} & \multicolumn{4}{c|}{42} & \multicolumn{4}{c|}{46} \\
\hline$\lambda(\mathrm{A})$ & $\Upsilon$ & $\lambda(\mathrm{A})$ & $\Upsilon$ & $\lambda(\mathrm{A})$ & $\Upsilon$ & $\lambda(\mathrm{A})$ & $\Upsilon$ & $\lambda(\mathrm{A})$ & $\Upsilon$ & $\lambda(\mathrm{A})$ & $\Upsilon$ & $\lambda(\mathrm{A})$ & $\Upsilon$ & $\lambda(\mathrm{A})$ & $\Upsilon$ \\
\hline 3292 & 0.000 & 3410 & 0.994 & 3742 & 0.000 & 3860 & 0.994 & 4082 & 0.000 & 4200 & 0.994 & 4492 & 0.000 & 4610 & 0.994 \\
3293 & 0.500 & 3420 & 0.977 & 3743 & 0.500 & 3870 & 0.977 & 4083 & 0.500 & 4210 & 0.977 & 4493 & 0.500 & 4620 & 0.977 \\
3300 & 0.547 & 3430 & 0.949 & 3750 & 0.546 & 3880 & 0.949 & 4090 & 0.546 & 4220 & 0.949 & 4500 & 0.546 & 4630 & 0.949 \\
3310 & 0.616 & 3440 & 0.911 & 3760 & 0.615 & 3890 & 0.911 & 4100 & 0.615 & 4230 & 0.911 & 4510 & 0.615 & 4640 & 0.911 \\
3320 & 0.684 & 3450 & 0.865 & 3770 & 0.683 & 3900 & 0.865 & 4110 & 0.683 & 4240 & 0.865 & 4520 & 0.683 & 4650 & 0.865 \\
3330 & 0.750 & 3460 & 0.810 & 3780 & 0.749 & 3910 & 0.809 & 4120 & 0.749 & 4250 & 0.809 & 4530 & 0.749 & 4660 & 0.809 \\
3340 & 0.810 & 3470 & 0.750 & 3790 & 0.809 & 3920 & 0.749 & 4130 & 0.809 & 4260 & 0.749 & 4540 & 0.809 & 4670 & 0.749 \\
3350 & 0.865 & 3480 & 0.684 & 3800 & 0.865 & 3930 & 0.683 & 4140 & 0.865 & 4270 & 0.683 & 4550 & 0.865 & 4680 & 0.683 \\
3360 & 0.911 & 3490 & 0.616 & 3810 & 0.911 & 3940 & 0.615 & 4150 & 0.911 & 4280 & 0.615 & 4560 & 0.911 & 4690 & 0.615 \\
3370 & 0.949 & 3500 & 0.547 & 3820 & 0.949 & 3950 & 0.546 & 4160 & 0.949 & 4290 & 0.546 & 4570 & 0.949 & 4700 & 0.546 \\
3380 & 0.977 & 3507 & 0.500 & 3830 & 0.977 & 3956 & 0.500 & 4170 & 0.977 & 4296 & 0.500 & 4580 & 0.977 & 4706 & 0.500 \\
3390 & 0.994 & 3508 & 0.000 & 3840 & 0.994 & 3957 & 0.000 & 4180 & 0.994 & 4297 & 0.000 & 4590 & 0.994 & 4707 & 0.000 \\
3400 & 1.000 & & & 3850 & 1.000 & & & 4190 & 1.000 & & & 4600 & 1.000 & \\
\hline
\end{tabular}

Fig. 127. continued 


\section{Kenyon and Fernandez-Castro - 1987}

Classification of M stars.

\section{GENERAL INFORMATION}

AUTHORS

TELESCOPE

DETECTOR

MAIN ARTICLE

S. J. Kenyon and T. Fernandez-Castro $2.9 \mathrm{~m}$ (reflector), KPNO

dual-beam intensified Reticon scanner

Kenyon, S. J., Fernandez-Castro, T. 1987, AJ 93, 938

\section{SYSTEM DESCRIPTION}

\begin{tabular}{|c|c|c|c|}
\hline \multicolumn{4}{|c|}{ BANDS DESCRIPTION [163] } \\
\hline band & $\lambda_{\text {peak }}(\AA)$ & bandpass $(\AA)$ & feature $(\AA)$ \\
\hline 613 & 6125 & 30 & continuum \\
\hline 618 & 6180 & 30 & $\mathrm{TiO}$ \\
\hline 637 & 6370 & 30 & continuum \\
\hline 703 & 7025 & 30 & continuum \\
\hline 710 & 7100 & 30 & $\mathrm{TiO}$ \\
\hline 740 & 7400 & 30 & continuum \\
\hline 786 & 7865 & 30 & VO \\
\hline 805 & 8050 & 30 & continuum \\
\hline
\end{tabular}

Square bands obtained from direct measurement on fluxed spectra.

\section{SYSTEM ANALYSIS}

\section{COLOR INDICES AND PARAMETERS [163]}

$[\mathrm{TiO}]_{1}=-2.5 \log \left[F_{618} /\left(F_{613}+0.22\left(F_{637}-F_{613}\right)\right]:\right.$ measures the strength of the TiO absorption band at $6180 \AA$.

$[\mathrm{TiO}]_{2}=-2.5 \log \left(F_{710} /\left[F_{703}+0.2\left(F_{740}-F_{703}\right)\right]:\right.$ measures the strength of the TiO absorption band at $7100 \AA$.

$[V O]=-2.5 \log \left(F_{787} /\left[F_{740}+0.29\left(F_{805}-F_{740}\right)\right]:\right.$ measures the strength of the VO absorption band at $7865 \AA$.

$[N a I]=-2.5 \log \left[F_{819} / F_{805}+0.6\left(F_{840}-F_{805}\right)\right]:$ measures the Na I doublet at 8181-95.

If $T$ denotes the spectral type ( $T=-6$ at $\mathrm{K} 0, T=0$ at $\mathrm{M} 0, T=+6$ at $\mathrm{M} 6$ ), than the following relations hold true for giants and supergiants:

$$
\begin{aligned}
& T=1.75+9.31[\mathrm{TiO}]_{1} \\
& T=1.83+10.37[\mathrm{TiO}]_{2}-3.28[\mathrm{TiO}]_{2}^{2} \\
& T=0.74+19.3[\mathrm{VO}]-17.22[\mathrm{VO}]_{2}^{2}
\end{aligned}
$$

Fig. 128. The photometric system Kenyon and Fernandez-Castro - 1987 


\section{$\psi(25)$ - Mendoza - 1987}

Intensity of the HeI $10830 \mathrm{~A}$ line.

\section{GENERAL INFORMATION}

$\begin{array}{ll}\text { AUTHORS } & \text { E. V. Mendoza } \\ \text { TELESCOPE } & 1.5 \mathrm{~m} \text { and 0.84m (reectors), OAN San Pedro Martir, Mexico } \\ \text { DETECTOR } & \text { RCA 7102 (S-1 cathode) } \\ \text { MAIN ARTICLE } & \text { Mendoza, E.V. 1987, Rev.Mex.Astron.Astrophys. 14, 385 }\end{array}$

\section{SYSTEM DESCRIPTION}

\begin{tabular}{|c|cc|}
\hline \multicolumn{3}{|c|}{ BANDS DESCRIPTION [210] } \\
\hline band & $\lambda_{\text {peak }}(\mathrm{A})$ & FWHM (A) \\
\hline$a$ & 10771.5 & 31 \\
\hline$b$ & 10832.5 & 25 \\
\hline$c$ & 10893.0 & 28 \\
\hline
\end{tabular}

Interference lters.

A set of 89 stars of spectral types from $\mathrm{O}$ to M denes the system [210].

\section{SYSTEM ANALYSIS}

REDDENING-FREE PARAMETERS [210]

$\psi(25)=0.167+b-0.5(a+c)$ : intensity of HeI 10830 A. Stars with no HeI 10830 A absorption have $\psi(25)=0.00$

\section{TRANSMISSION CURVES}

As derived from Fig 1 of [210].

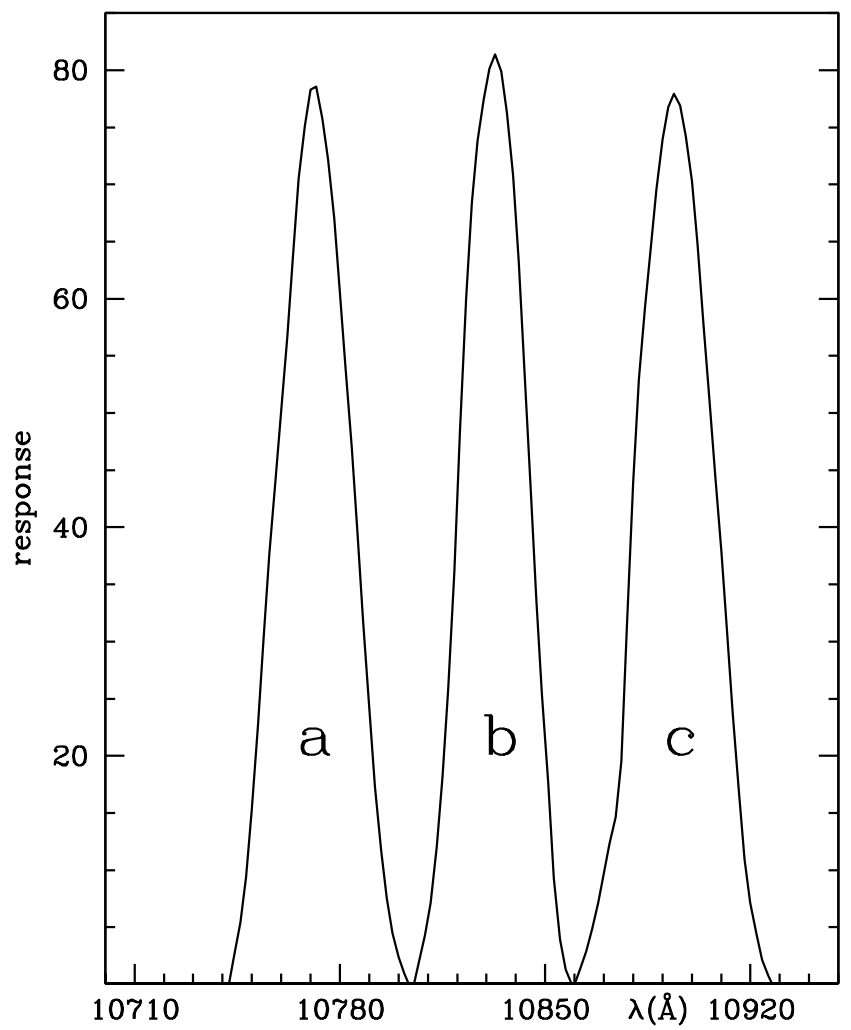

\begin{tabular}{|rr|rr|rr|}
\hline \multicolumn{2}{|c|}{$a$} & \multicolumn{2}{|c|}{$b$} & \multicolumn{2}{c|}{$c$} \\
\hline$\lambda(\mathrm{A})$ & $\Upsilon$ & $\lambda(\mathrm{A})$ & \multicolumn{1}{c|}{$\Upsilon$} & $\lambda(\mathrm{A})$ & $\Upsilon$ \\
\hline 10742 & 0.0 & 10805 & 0.0 & 10860 & 0.0 \\
10745 & 3.4 & 10808 & 3.4 & 10865 & 3.4 \\
10747 & 6.9 & 10811 & 6.9 & 10868 & 6.9 \\
10750 & 13.7 & 10814 & 13.7 & 10873 & 13.7 \\
10753 & 26.8 & 10816 & 22.7 & 10876 & 20.6 \\
10757 & 41.9 & 10820 & 39.8 & 10879 & 35.7 \\
10761 & 52.2 & 10822 & 55.6 & 10882 & 52.9 \\
10764 & 63.9 & 10825 & 67.3 & 10886 & 64.9 \\
10767 & 72.1 & 10828 & 75.9 & 10890 & 73.1 \\
10768 & 75.5 & 10830 & 79.0 & 10891 & 75.9 \\
10771 & 79.0 & 10833 & 81.4 & 10894 & 77.9 \\
10774 & 75.5 & 10836 & 79.0 & 10897 & 75.5 \\
10777 & 70.7 & 10837 & 75.5 & 10901 & 69.0 \\
10780 & 60.4 & 10841 & 64.5 & 10906 & 52.5 \\
10783 & 49.1 & 10845 & 43.9 & 10909 & 41.5 \\
10786 & 39.8 & 10848 & 28.2 & 10913 & 26.8 \\
10789 & 26.8 & 10852 & 15.1 & 10917 & 12.7 \\
10793 & 15.2 & 10854 & 6.9 & 10919 & 8.6 \\
10796 & 6.9 & 10855 & 3.4 & 10921 & 6.2 \\
10799 & 3.4 & 10859 & 0.0 & 10923 & 3.4 \\
10804 & 0.0 & & & 10927 & 0.0 \\
\hline
\end{tabular}

Fig. 129. The photometric system $\psi(25)$ - Mendoza - 1987 


\section{VJHKLL'M - Bessell and Brett - 1988}

Homogenization of infrared systems.

GENERAL INFORMATION
AUTHORS
M. S. Bessell and J. M. Brett
MAIN ARTICLE Bessell, M.S., Brett, J.M. 1988, PASP 100, 1134

\section{SYSTEM DESCRIPTION}

\begin{tabular}{|c|c|cc|}
\hline \multicolumn{2}{|c|}{ BANDS DESCRIPTION [34] } & \multicolumn{2}{|c|}{ ABSOLUTE CALIBRATION (\#) [34] } \\
\hline band & $\lambda_{\text {eff }}(\mu \mathrm{m})(\#)$ & $\mathrm{F}_{\lambda}\left(\mathrm{W} \mathrm{cm}^{-2} \mu \mathrm{m}^{-1}\right)$ & $\mathrm{F}_{\mathrm{v}}\left(\mathrm{W} \mathrm{cm}^{-2} \mathrm{~Hz}^{-1}\right)$ \\
\hline$V$ & 0.545 & $359010^{-15}$ & $360010^{-30}$ \\
\hline$J$ & 1.22 & $31210^{-15}$ & $157010^{-30}$ \\
\hline$H$ & 1.63 & $11410^{-15}$ & $102010^{-30}$ \\
\hline$K$ & 2.19 & $39.410^{-15}$ & $63610^{-30}$ \\
\hline$L$ & 3.45 & $6.9910^{-15}$ & $28110^{-30}$ \\
\hline$L^{\prime}$ & 3.80 & $4.8310^{-15}$ & $23510^{-30}$ \\
\hline$M$ & 4.75 & $2.0410^{-15}$ & $15410^{-30}$ \\
\hline
\end{tabular}

(\#) Calculated for a 0.03 mag star from Dreiling and Bell (1980) ApJ 241, 736 model of $\alpha$ Lyr.

ZERO POINT: $\alpha$ Lyr has colors equal to 0.00 [34]

\section{SYSTEM ANALYSIS}

REDDENING RATIOS [34]

$$
\begin{array}{lll}
E(V-K)=2.78 E(B-V) & E(J-H)=0.37 E(B-V) & E(H-K)=0.19 E(B-V) \\
E(K-L)=0.15 E(B-V) & E(K-M)=0.24 E(B-V) & A(K)=0.34 E(B-V)
\end{array}
$$

RELATIONS WITH OTHER SYSTEMS [34]

JHKL SAAO - Glass - 1973

\begin{tabular}{|rcr|}
\hline$(V-K)$ & $=-0.005+(V-K)_{\text {SAAO }}$ \\
\hline$(J-K)$ & $=-0.005+(J-K)_{\text {SAAO }}$ \\
\hline$(H-K)$ & $=-0.021+(H-K)_{\text {SAAO }}$ \\
\hline$(J-H)$ & $=0.016+(J-H)_{S A A O}$ \\
\hline$(K-L)$ & $=(K-L)_{\text {SAAO }}$ \\
\hline
\end{tabular}

VJHKLM ESO - Engels et al - 1981

\begin{tabular}{|rcr|}
\hline$(V-K)$ & $=0.015+(V-K)_{E S O}$ \\
\hline$(J-K)$ & $=$ & $-0.010+1.025(J-K)_{E S O}$ \\
\hline$(H-K)$ & $=0.005+0.87(H-K)_{E S O}$ \\
\hline$(J-H)$ & $=-0.028+1.105(J-H)_{E S O}$ \\
\hline$(K-L)$ & $=-0.03+(K-L)_{E S O}$ \\
\hline
\end{tabular}

\begin{tabular}{|rcc|}
\hline$(V-K)$ & $=0.01+0.993(V-K)_{J}$ \\
\hline$(J-K)$ & $=0.01+0.99(J-K)_{J}$ \\
\hline$(H-K)$ & $\approx 0.01+0.91(H-K)_{J}$ \\
\hline$(J-H)$ & $=-0.004+1.01(J-H)_{J}$ \\
\hline$(K-L)$ & $=-0.03+(K-L)_{J}$ \\
\hline
\end{tabular}

JHKL MSO - Jones and Hyland - 1982

\begin{tabular}{|rcr|}
\hline$(V-K)$ & $=$ & $-0.012+0.997(V-K)_{M S O}$ \\
\hline$(J-K)$ & $=$ & $0.010+1.008(J-K)_{M S O}$ \\
\hline$(H-K)$ & $=$ & $-0.007+0.97(H-K)_{M S O}$ \\
\hline$(J-H)$ & $=0.017+1.016(J-H)_{M S O}$ \\
\hline$(K-L)$ & $=$ & $-0.02+(K-L)_{M S O}$ \\
\hline
\end{tabular}

Fig. 130. The photometric system VJHKLL'M - Bessell and Brett - 1988 


\begin{tabular}{|rcr|}
\hline$(V-K)$ & $=-0.02+1.001(V-K)_{\text {CTIO }}$ \\
\hline$(J-K)$ & $=-0.002+1.086(J-K)_{\text {CTIO }}$ \\
\hline$(J-H)$ & $=0.002+1.098(J-H)_{\text {CTIO }}$ \\
\hline$(K-L)$ & $=(K-L)_{\text {CTIO }}$ \\
\hline$(H-K)$ & $=1.03(H-K)_{\text {CTIO }}$ \\
\hline
\end{tabular}

\begin{tabular}{|rl|}
\hline$(V-K)$ & $=1.002(V-K)_{A A O}$ \\
\hline$(J-K)$ & $=0.974(J-K)_{A A O}$ \\
\hline$(H-K)$ & $=-0.003+0.98(H-K)_{A A O}$ \\
\hline$(J-H)$ & $=0.005+0.963(J-H)_{A A O}$ \\
\hline$\left(K-L^{\prime}\right)$ & $=\left(K-L^{\prime}\right)_{A A O}$
\end{tabular}

\section{TRANSMISSION CURVES [34]}

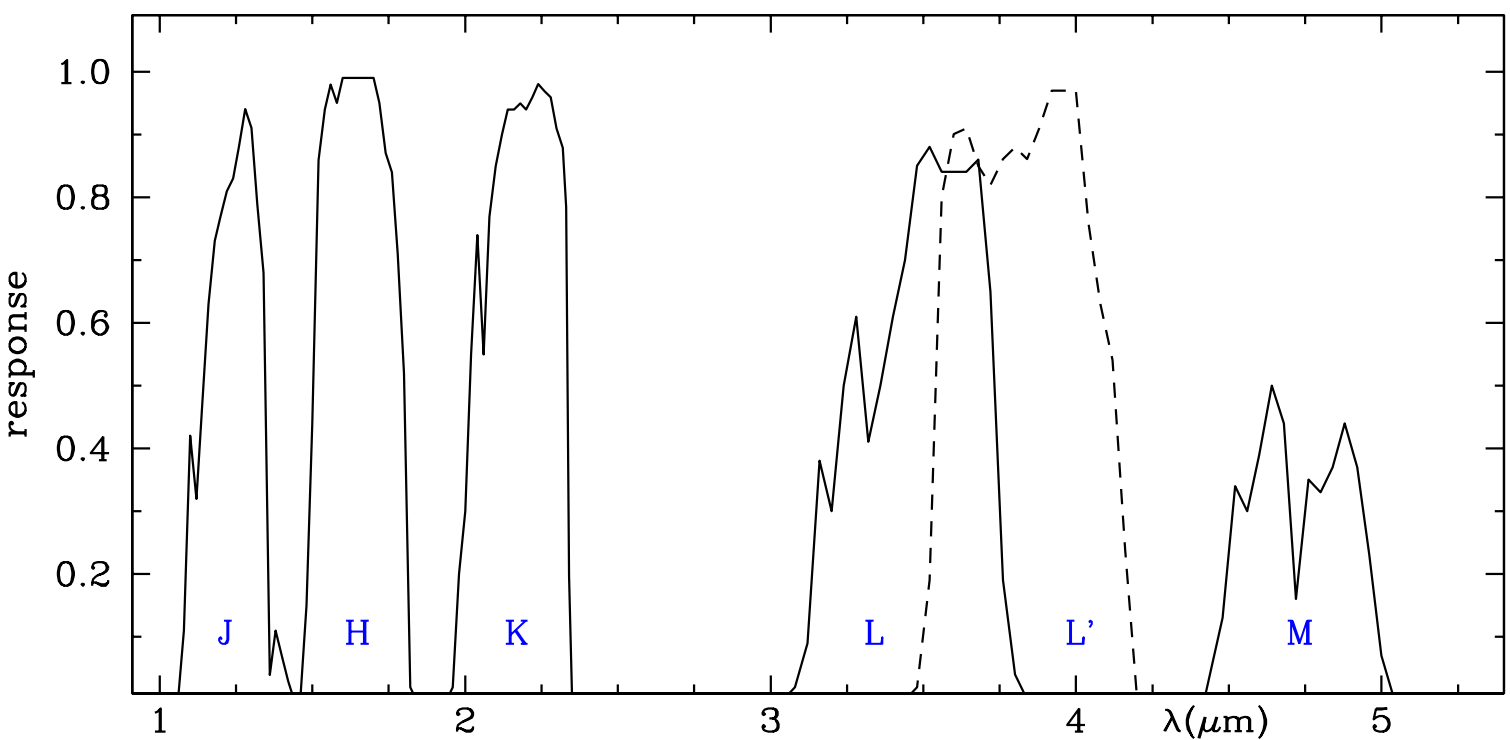

\begin{tabular}{|c|c|c|c|c|c|c|c|c|c|c|c|c|c|}
\hline \multicolumn{2}{|c|}{$J$} & \multicolumn{2}{|c|}{$H$} & \multicolumn{4}{|c|}{$K$} & \multicolumn{2}{|c|}{$L$} & \multicolumn{2}{|c|}{$L^{\prime}$} & \multicolumn{2}{|c|}{$M$} \\
\hline$\lambda(\mu \mathrm{m})$ & $\Upsilon$ & $\lambda(\mu \mathrm{m})$ & $\Upsilon$ & $\lambda(\mu \mathrm{m})$ & $\Upsilon$ & $\lambda(\mu \mathrm{m})$ & $\Upsilon$ & $\lambda(\mu \mathrm{m})$ & $\Upsilon$ & $\lambda(\mu \mathrm{m})$ & $\Upsilon$ & $\lambda(\mu \mathrm{m})$ & $\Upsilon$ \\
\hline 1.040 & 0.00 & 1.460 & 0.00 & 1.940 & 0.00 & 2.380 & 0.75 & 3.040 & 0.00 & 3.440 & 0.00 & 4.440 & 0.00 \\
\hline 1.060 & 0.02 & 1.480 & 0.15 & 1.960 & 0.12 & 2.400 & 0.64 & 3.080 & 0.02 & 3.480 & 0.02 & 4.480 & 0.13 \\
\hline 1.080 & 0.11 & 1.500 & 0.44 & 1.980 & 0.20 & 2.440 & 0.10 & 3.120 & 0.09 & 3.520 & 0.19 & 4.520 & 0.34 \\
\hline 1.100 & 0.42 & 1.520 & 0.86 & 2.000 & 0.30 & 2.480 & 0.00 & 3.160 & 0.38 & 3.560 & 0.80 & 4.560 & 0.30 \\
\hline 1.120 & 0.32 & 1.540 & 0.94 & 2.020 & 0.55 & & & 3.200 & 0.30 & 3.600 & 0.90 & 4.600 & 0.39 \\
\hline 1.140 & 0.47 & 1.560 & 0.98 & 2.040 & 0.74 & & & 3.240 & 0.50 & 3.640 & 0.91 & 4.640 & 0.50 \\
\hline 1.160 & 0.63 & 1.580 & 0.95 & 2.060 & 0.55 & & & 3.280 & 0.61 & 3.680 & 0.85 & 4.680 & 0.44 \\
\hline 1.180 & 0.73 & 1.600 & 0.99 & 2.080 & 0.77 & & & 3.320 & 0.41 & 3.720 & 0.82 & 4.720 & 0.16 \\
\hline 1.200 & 0.77 & 1.620 & 0.99 & 2.100 & 0.85 & & & 3.360 & 0.50 & 3.760 & 0.86 & 4.760 & 0.35 \\
\hline 1.220 & 0.81 & 1.640 & 0.99 & 2.120 & 0.90 & & & 3.400 & 0.61 & 3.800 & 0.88 & 4.800 & 0.33 \\
\hline 1.240 & 0.83 & 1.660 & 0.99 & 2.140 & 0.94 & & & 3.440 & 0.70 & 3.840 & 0.86 & 4.840 & 0.37 \\
\hline 1.260 & 0.88 & 1.680 & 0.99 & 2.160 & 0.94 & & & 3.480 & 0.85 & 3.880 & 0.91 & 4.880 & 0.44 \\
\hline 1.280 & 0.94 & 1.700 & 0.99 & 2.180 & 0.95 & & & 3.520 & 0.88 & 3.920 & 0.97 & 4.920 & 0.37 \\
\hline 1.300 & 0.91 & 1.720 & 0.95 & 2.200 & 0.94 & & & 3.560 & 0.84 & 3.960 & 0.97 & 4.960 & 0.23 \\
\hline 1.320 & 0.79 & 1.740 & 0.87 & 2.220 & 0.96 & & & 3.600 & 0.84 & 4.000 & 0.97 & 5.000 & 0.07 \\
\hline 1.340 & 0.68 & 1.760 & 0.84 & 2.240 & 0.98 & & & 3.640 & 0.84 & 4.040 & 0.76 & 5.040 & 0.03 \\
\hline 1.360 & 0.04 & 1.780 & 0.71 & 2.260 & 0.97 & & & 3.680 & 0.86 & 4.080 & 0.63 & 5.080 & 0.00 \\
\hline 1.380 & 0.11 & 1.800 & 0.52 & 2.280 & 0.96 & & & 3.720 & 0.65 & 4.120 & 0.54 & & \\
\hline 1.400 & 0.07 & 1.820 & 0.02 & 2.300 & 0.91 & & & 3.760 & 0.19 & 4.160 & 0.24 & & \\
\hline 1.420 & 0.03 & 1.840 & 0.00 & 2.320 & 0.88 & & & 3.800 & 0.04 & 4.200 & 0.00 & & \\
\hline 1.440 & 0.00 & & & 2.340 & 0.84 & & & 3.840 & 0.00 & & & & \\
\hline
\end{tabular}

The original table in [34] appears wrongly formatted.

Fig. 130. continued 


\section{1 - Cook and Aaronson - 1989}

Intermediate-band photometry of M stars.

\section{GENERAL INFORMATION}

$\begin{array}{ll}\text { AUTHORS } & \text { K. H. Cook and M. Aaronson } \\ \text { TELESCOPE } & \text { 0.4m (reflector), KPNO } \\ \text { DETECTOR } & \text { RCA 31034A (refrigerated) }\end{array}$

MAIN ARTICLE Cook, K.H., Aaronson, M. 1989, AJ 97, 923

\section{SYSTEM DESCRIPTION}

\begin{tabular}{|l|cc|}
\hline \multicolumn{3}{|c|}{ BANDS DESCRIPTION [73] } \\
\hline band & $\lambda_{c}(\AA)$ & FWHM $(\AA)$ \\
\hline 77 & 7752 & 284 \\
\hline 81 & 8104 & 365 \\
\hline
\end{tabular}

Interference filters by Spectro-Optics.

ZERO POINT: $(77-81)=0.00$ for unreddened A0 stars. [73]

\section{SYSTEM ANALYSIS}

\section{COLOR INDICES AND PARAMETERS [73]}

77 : measures the $\mathrm{TiO}$ band at $7750 \AA$.

81 : measures $\mathrm{CN}$ bands around $8100 \AA$.

If $M$ indicates the spectal type ( $M=0$ at M0, $M=5$ at M5 ,etc..) then the following relations hold for stars later than M0.5:

$(77-81)=0.099+0.053 M:$ for giants.

$(77-81)=0.180+0.043 M:$ for dwarfs.

RELATIONS WITH OTHER SYSTEMS [73]

UBVRI(JHKLMN) - Johnson - 1965

\begin{tabular}{|lll|}
\hline$(V-I)$ & $=1.180+3.796(77-81)$ & for field giants with $(V-I)>1.6$ \\
\hline$(V-I)$ & $=1.196+3.761(77-81)$ & for sample stars with $(V-I)>1.6$ \\
\hline
\end{tabular}

Fig. 131. The photometric system 7781 - Cook and Aaronson - 1989 


\section{$g_{4} r_{4} i_{4} Z_{4}-$ Schneider et al. - 1989}

Photometry of quasars.

\section{GENERAL INFORMATION}

AUTHORS D. P. Schneider, M. Schmidt J. E. Gunn,

TELESCOPE $5 \mathrm{~m}$ Hale (reector), Palomar Obs.

DETECTOR $\quad 800$ X 800 UV-ooded CCDs (Texas Instruments)

MAIN ARTICLE Schneider, D. P., Schmidt, M., Gunn, J. E. 1989, AJ 98, 1507

\section{SYSTEM DESCRIPTION}

\begin{tabular}{|l|cc|rc|}
\hline \multicolumn{3}{|c|}{ BANDS DESCRIPTION [262] } & & \\
\hline band & \multicolumn{1}{|c|}{ lter } & $\lambda_{\text {eff }}(\mathrm{A})$ & WHM (A) & $\lambda_{c}(\mathrm{~A})$ \\
\hline$g_{4}$ & Corion interference & 5090 & 877 & 4690 \\
\hline$r_{4}$ & 3mm Schott BG610 + Balzer B1 short-pass & 6610 & 870 & 6105 \\
\hline$i_{4}$ & & & 1375 & 7290 \\
\hline$Z_{4}$ & & & & 8385 \\
\hline
\end{tabular}

ZERO POINT: $\mathrm{BD}+17^{\circ} 4708$ (F8 IV) is taken to have to have $g_{4}=r_{4}=i_{4}=z_{4}=9.50$ mag. [262]

\section{TRANSMISSION CURVES}

As derived from Fig 3 of [262].

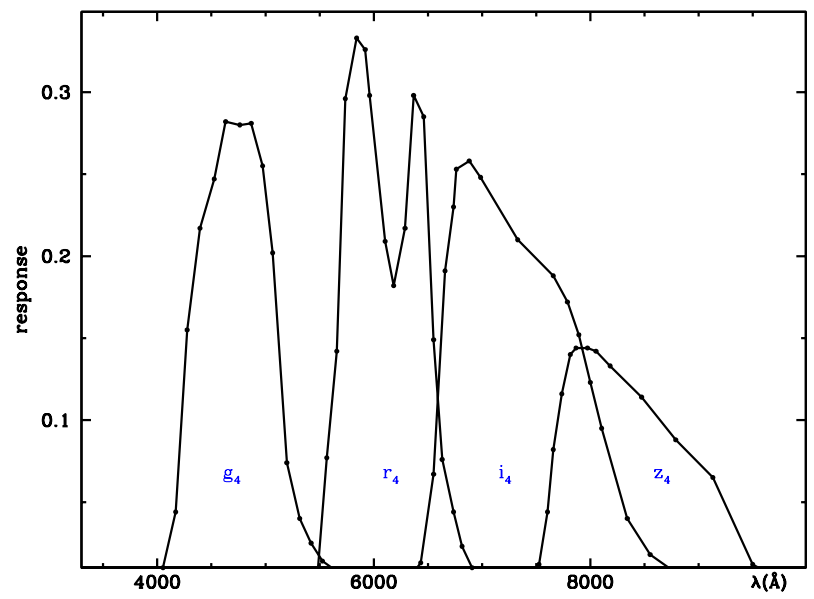

\begin{tabular}{|cc|cc|cc|cc|}
\hline \multicolumn{2}{|c|}{$g 4$} & \multicolumn{2}{c|}{$r 4$} & \multicolumn{2}{c|}{ i4 } & \multicolumn{2}{c|}{$Z 4$} \\
\hline$\lambda(\mathrm{A})$ & $\Upsilon$ & $\lambda(\mathrm{A})$ & $\Upsilon$ & $\lambda(\mathrm{A})$ & $\Upsilon$ & $\lambda(\mathrm{A})$ & $\Upsilon$ \\
\hline 3474 & 0.000 & 5474 & 0.000 & 6237 & 0.000 & 7421 & 0.000 \\
3605 & 0.002 & 5566 & 0.077 & 6316 & 0.003 & 7526 & 0.012 \\
3974 & 0.007 & 5658 & 0.142 & 6434 & 0.013 & 7605 & 0.044 \\
4053 & 0.010 & 5737 & 0.296 & 6553 & 0.067 & 7658 & 0.082 \\
4171 & 0.044 & 5842 & 0.333 & 6658 & 0.191 & 7737 & 0.116 \\
4276 & 0.155 & 5921 & 0.326 & 6737 & 0.230 & 7816 & 0.140 \\
4395 & 0.217 & 5961 & 0.298 & 6763 & 0.253 & 7868 & 0.144 \\
4526 & 0.247 & 6105 & 0.209 & 6882 & 0.258 & 7974 & 0.144 \\
4632 & 0.282 & 6184 & 0.182 & 6987 & 0.248 & 8053 & 0.142 \\
4763 & 0.280 & 6289 & 0.217 & 7329 & 0.210 & 8184 & 0.133 \\
4868 & 0.281 & 6368 & 0.298 & 7658 & 0.188 & 8474 & 0.114 \\
4974 & 0.255 & 6461 & 0.285 & 7789 & 0.172 & 8789 & 0.088 \\
5066 & 0.202 & 6553 & 0.149 & 7895 & 0.152 & 9132 & 0.065 \\
5197 & 0.074 & 6632 & 0.076 & 8000 & 0.123 & 9500 & 0.012 \\
5316 & 0.040 & 6737 & 0.044 & 8105 & 0.095 & 9842 & 0.000 \\
5421 & 0.025 & 6816 & 0.023 & 8342 & 0.040 & & \\
5526 & 0.014 & 6908 & 0.010 & 8553 & 0.018 & & \\
5763 & 0.003 & 7000 & 0.007 & 8789 & 0.007 & & \\
5868 & 0.002 & 7079 & 0.004 & 9000 & 0.002 & & \\
6316 & 0.000 & 7263 & 0.007 & 9342 & 0.000 & & \\
& & 7368 & 0.000 & & & & \\
\hline
\end{tabular}

Fig. 132. The photometric system $g_{4} r_{4} i_{4} z_{4}-$ Schneider et al. -1989 


\section{FOC HST - 1989}

Photometric bands of the FOC camera on board HST.

\section{GENERAL INFORMATIONS}

TELESCOPE 2.4m Hubble Space Telescope

MAIN ARTICLE Nota, A., et al 1996, FOC Intrument Handbook, Version 7.0 (Baltimore, STScI)

\section{SYSTEM DESCRIPTION}

\begin{tabular}{|c|c|c|c|c|c|c|c|c|c|}
\hline \multicolumn{10}{|c|}{ BANDS DESCRIPTION [235], pg. 25} \\
\hline band & $\lambda_{0}(\mathrm{~A})$ & FWHM (A) & $\Upsilon$ & Notes & band & $\lambda_{0}(\mathrm{~A})$ & FWHM (A) & $\Upsilon$ & Notes \\
\hline \multicolumn{5}{|c|}{$\mathrm{F} / 96$} & \multicolumn{5}{|c|}{$\mathrm{F} / 96$} \\
\hline F120M & 1230 & 82 & 0.10 & & F372M & 3710 & 406 & 0.73 & \\
\hline F130M & 1280 & 88 & 0.10 & & F430W & 3940 & 832 & 0.74 & $B$ \\
\hline F140W & 1370 & 298 & 0.21 & & F410M & 4100 & 194 & 0.58 & $b$ \\
\hline F140M & 1400 & 178 & 0.08 & & F437M & 4290 & 438 & 0.71 & \\
\hline F152M & 1500 & 184 & 0.08 & & F470M & 4710 & 212 & 0.79 & V \\
\hline F165W & 1700 & 910 & 0.28 & & F486N & 4870 & 34 & 0.63 & $H \beta$ \\
\hline F170M & 1760 & 184 & 0.18 & & F502M & 4940 & 530 & 0.82 & \\
\hline F175W & 1730 & 716 & 0.24 & & F501N & 5010 & 74 & 0.68 & [OIII] \\
\hline F190M & 2000 & 276 & 0.15 & & F550M & 5460 & 188 & 0.77 & y \\
\hline F195W & 2110 & 946 & 0.42 & & F600M & 5800 & 410 & 0.80 & \\
\hline$F 210 M$ & 2156 & 214 & 0.18 & & F630M & 6382 & 208 & 0.67 & \\
\hline F220W & 2280 & 480 & 0.39 & & \multicolumn{5}{|c|}{$\mathrm{F} / 48$} \\
\hline$F 231 M$ & 2330 & 228 & 0.18 & & F140W & 1320 & 300 & 0.20 & \\
\hline$F 253 M$ & 2550 & 236 & 0.18 & & F150W & 1400 & 628 & 0.23 & \\
\hline F275W & 2770 & 594 & 0.40 & & $\mathrm{~F} 175 \mathrm{~W}$ & 1730 & 678 & 0.18 & \\
\hline$F 278 M$ & 2800 & 316 & 0.26 & & F195W & 2110 & 1076 & 0.36 & \\
\hline F307M & 3080 & 328 & 0.26 & & F220W & 2250 & 480 & 0.36 & \\
\hline F320W & 3360 & 844 & 0.89 & & $\mathrm{~F} 275 \mathrm{~W}$ & 2750 & 656 & 0.29 & \\
\hline F342W & 3410 & 702 & 0.81 & $U$ & F342W & 3400 & 706 & 0.83 & $U$ \\
\hline F346M & 3480 & 434 & 0.58 & $u$ & F430W & 3950 & 938 & 0.75 & $B$ \\
\hline
\end{tabular}

The long-pass lters (F130LP, F370LP, F480LP for the f/96 mode and F130LP, F180LP, F305LP for the f/48 mode) are not listed.

\section{TRANSMISSION CURVES [235]}

To save space the transmission proles of the bands are plotted but not tabulated. The proles can be obtained in tabular form via STSDAS (inside Iraf) or from the ADPS $w w w$ site.

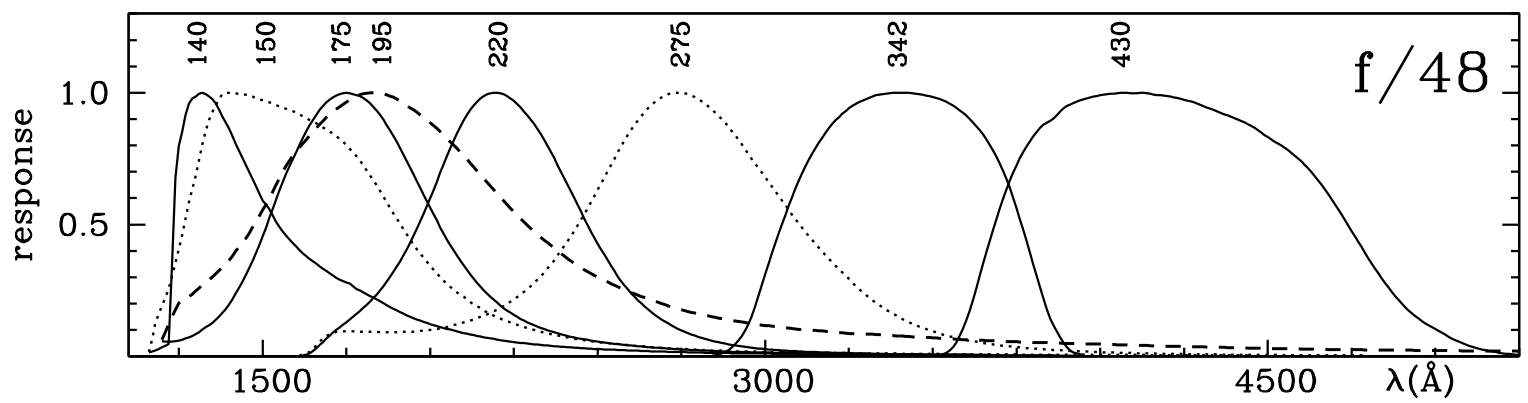

Fig. 133. The photometric system FOC HST - 1990 

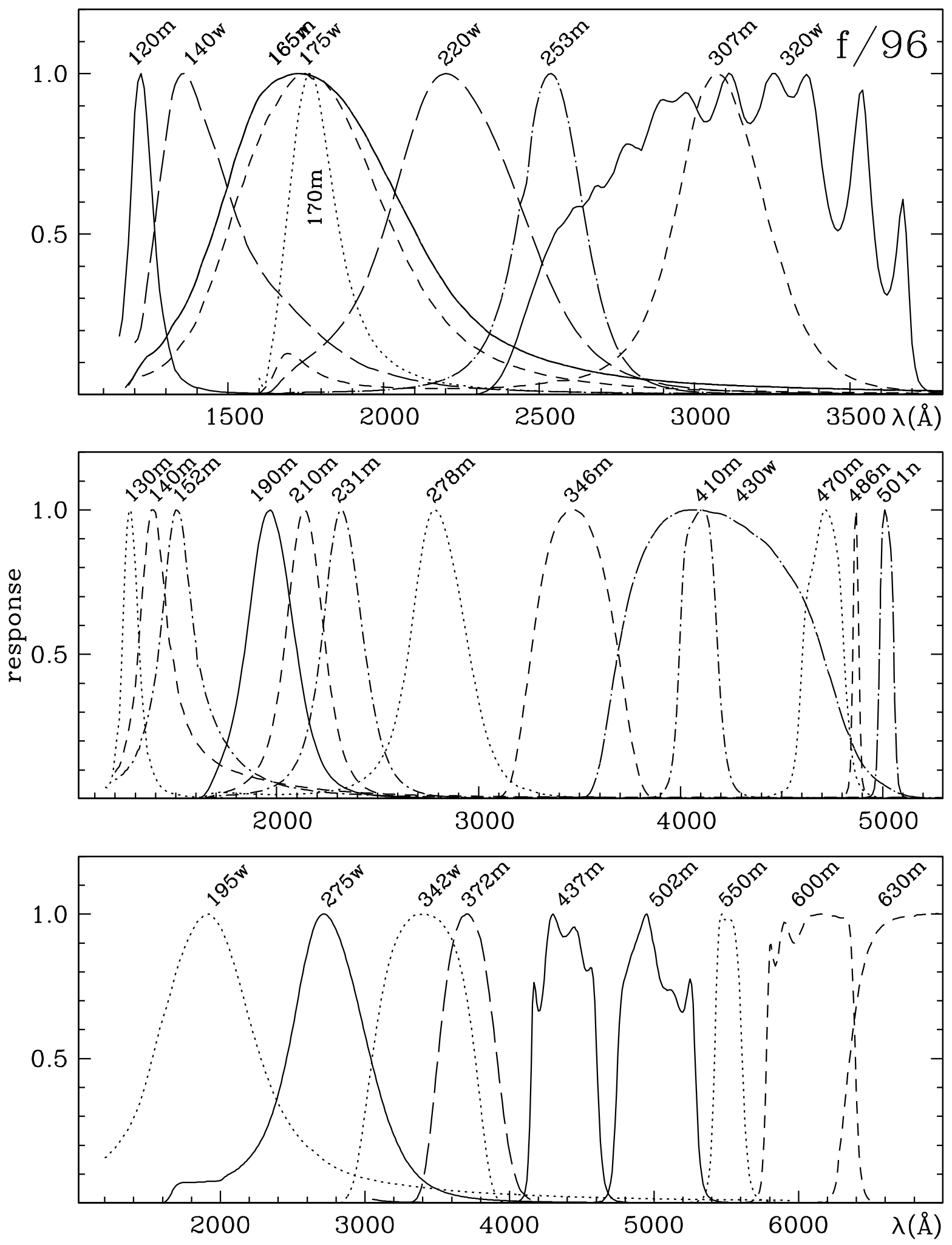

Fig. 133. continued 


\section{WFPC1 HST - 1989}

Photometric bands of the Wide Field and Planetary Camera 1 on the Hubble Space Telescope.

GENERAL INFORMATION

$\begin{array}{ll}\text { TELESCOPE } & 2.4 \mathrm{~m} \text { Hubble Space Telescope } \\ \text { DETECTOR } & \text { Texas Intrument CCD } \\ \text { MAIN ARTICLE } & \text { HST Manuals }\end{array}$

\section{SYSTEM DESCRIPTION}

\begin{tabular}{|c|rc|c|rc|r|rr|}
\hline \multicolumn{10}{|c|}{ BANDS DESCRIPTION [90] } \\
\hline band & $\lambda_{0}(\mathrm{~A})$ & FWHM (A) & band & $\lambda_{0}(\mathrm{~A})$ & FWHM (A) & band & $\lambda_{0}(\mathrm{~A})$ & FWHM (A) \\
\hline $122 \mathrm{M}$ & 1185 & 160 & $492 \mathrm{M}$ & 4913 & 505 & $658 \mathrm{~N}$ & 6576 & 15 \\
\hline $157 \mathrm{~W}$ & 1445 & 270 & $502 \mathrm{~N}$ & 5015 & 25 & $664 \mathrm{~N}$ & 6630 & 165 \\
\hline $194 \mathrm{~W}$ & 1880 & 430 & $517 \mathrm{~N}$ & 5180 & 110 & $673 \mathrm{~N}$ & 6718 & 40 \\
\hline $230 \mathrm{~W}$ & 2300 & 400 & $547 \mathrm{M}$ & 5459 & 630 & $675 \mathrm{~W}$ & 6721 & 1300 \\
\hline $284 \mathrm{~W}$ & 2795 & 450 & $555 \mathrm{~W}$ & 5471 & 1500 & $702 \mathrm{~W}$ & 6989 & 2000 \\
\hline $336 \mathrm{~W}$ & 3374 & 480 & $569 \mathrm{~W}$ & 5631 & 1120 & $718 \mathrm{M}$ & 7173 & 760 \\
\hline $368 \mathrm{M}$ & 3690 & 280 & $588 \mathrm{~N}$ & 5877 & 40 & $791 \mathrm{~W}$ & 7953 & 1865 \\
\hline $375 \mathrm{~N}$ & 3740 & 100 & $606 \mathrm{~W}$ & 5937 & 2275 & $814 \mathrm{~W}$ & 8221 & 2400 \\
\hline $413 \mathrm{M}$ & 4131 & 300 & $622 \mathrm{~W}$ & 6180 & 1340 & $875 \mathrm{M}$ & 8769 & 755 \\
\hline $437 \mathrm{~N}$ & 4366 & 20 & $631 \mathrm{~N}$ & 6305 & 25 & $889 \mathrm{~N}$ & 8885 & 45 \\
\hline $439 \mathrm{~W}$ & 4375 & 635 & $648 \mathrm{M}$ & 6476 & 495 & $1042 \mathrm{M}$ & 10172 & 275 \\
\hline $469 \mathrm{~N}$ & 4688 & 25 & $656 \mathrm{~N}$ & 6559 & 15 & $1083 \mathrm{~N}$ & 10840 & 100 \\
\hline $487 \mathrm{~N}$ & 4868 & 30 & & & & & & \\
\hline
\end{tabular}

The long-pass lters (F725LP, F785LP, F850LP) are not listed.

\section{SYSTEM ANALYSIS}

RELATIONS WITH OTHER SYSTEMS [90]

UBVRI(JHKLMN) - Johnson - 1965, RI - Cousins - 1976

\begin{tabular}{|c|c|}
\hline$(B-V)_{\text {Johnson }}$ & $=0.995( \pm 0.025)(439 W-555 W)$ \\
\hline$(V-I)_{\text {Cousins }}$ & $=0.89( \pm 0.01)(555 W-785 L P)$
\end{tabular}

\section{TRANSMISSION CURVES}

To save space the transmission proles of the bands are plotted but not tabulated. The proles can be obtained in tabular form via STSDAS (inside Iraf) or from the ADPS $w w w$ site.

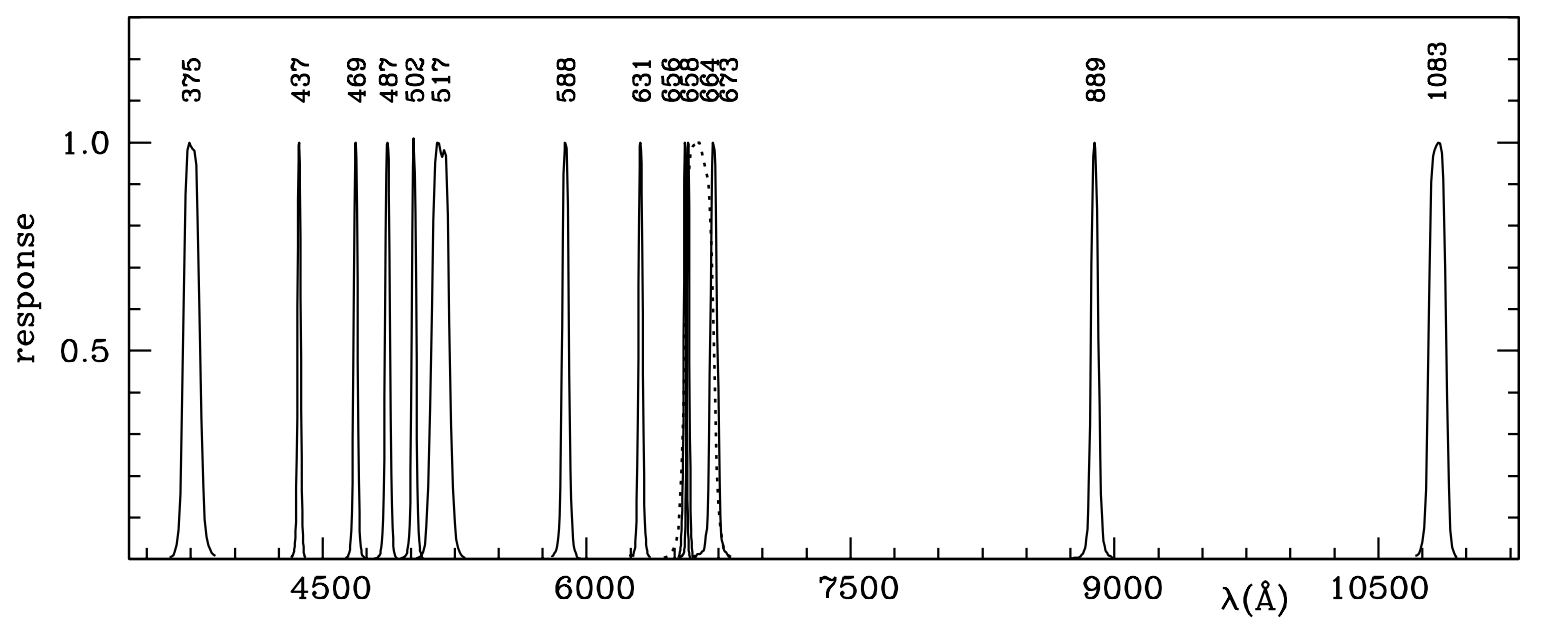

Fig. 134. The photometric system WFPC1 HST - 1989 

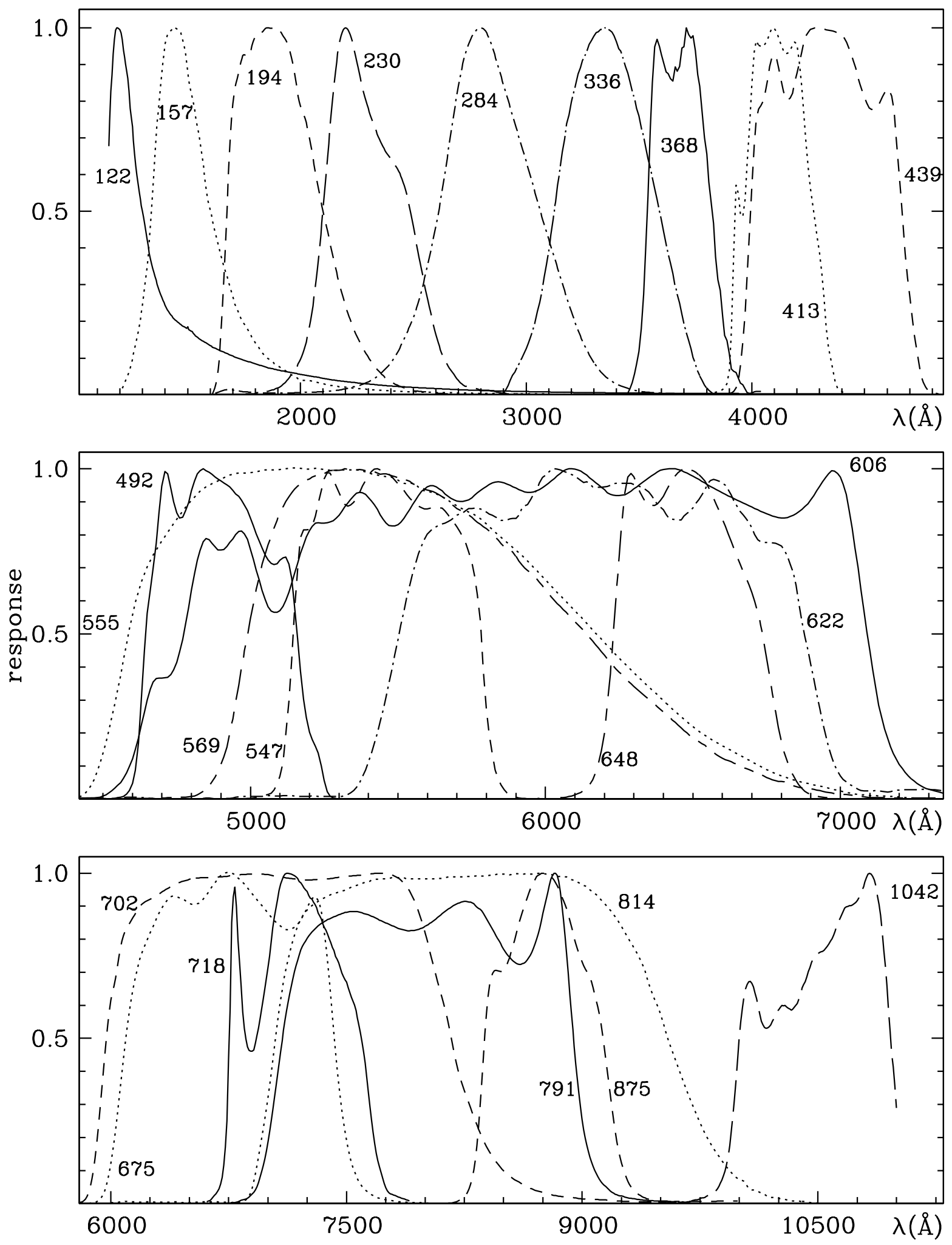

Fig. 134. continued 


\section{Hipparcos - 1989}

Photometric bands of the Hipparcos-Tycho mission.

\section{GENERAL INFORMATION}

TELESCOPE $\quad 0.29 \mathrm{~m}$ (reector)

DETECTOR Hipparcos: image dissector tube, (S-20 cathode)

Tycho: photomultiplier (Bi-alkali cathode)

MAIN ARTICLE Hipparcos and Tycho catalogues 1997, ESA pub SP-1200, Volume 1, pg. 39

\section{SYSTEM DESCRIPTION}

\begin{tabular}{|c|c|cc|}
\hline \multicolumn{3}{|c|}{ BANDS DESCRIPTION [141], pg. 42 } & \\
\hline band & $\lambda_{\text {peak }}(\mathrm{A})$ & WHM (A) & $\lambda_{c}(\mathrm{~A})$ \\
\hline$B_{T}$ & 4350 & 710 & 4190 \\
\hline$H_{p}$ & 4525 & 2220 & 5045 \\
\hline$V_{T}$ & 5050 & 950 & 5230 \\
\hline
\end{tabular}

ZERO POINT: $H_{p}=B_{T}=V_{T}=0.00$ for stars with $V_{\text {Johnson }}=0$ and $(B-V)=0$. [141], pg. 58

SYSTEM ANALYSIS

RELATIONS WITH OTHER SYSTEMS [141],pg. 57

$U B V$ - Johnson and Morgan - 1953

\begin{tabular}{|rrrr|}
\hline$V_{\text {Johnson }}$ & $=$ & $V_{\text {Tycho }}-0.090(B-V)_{\text {Tycho }}$ & for $-0.2<(B-V)_{\text {Tycho }}<1.8$ \\
\hline$(B-V)_{\text {Johnson }}$ & $=$ & $0.850(B-V)_{\text {Tycho }}$ & for $-0.2<(B-V)_{\text {Tycho }}<1.8$ \\
\hline
\end{tabular}

TRANSMISSION CURVES [141], pg. 42

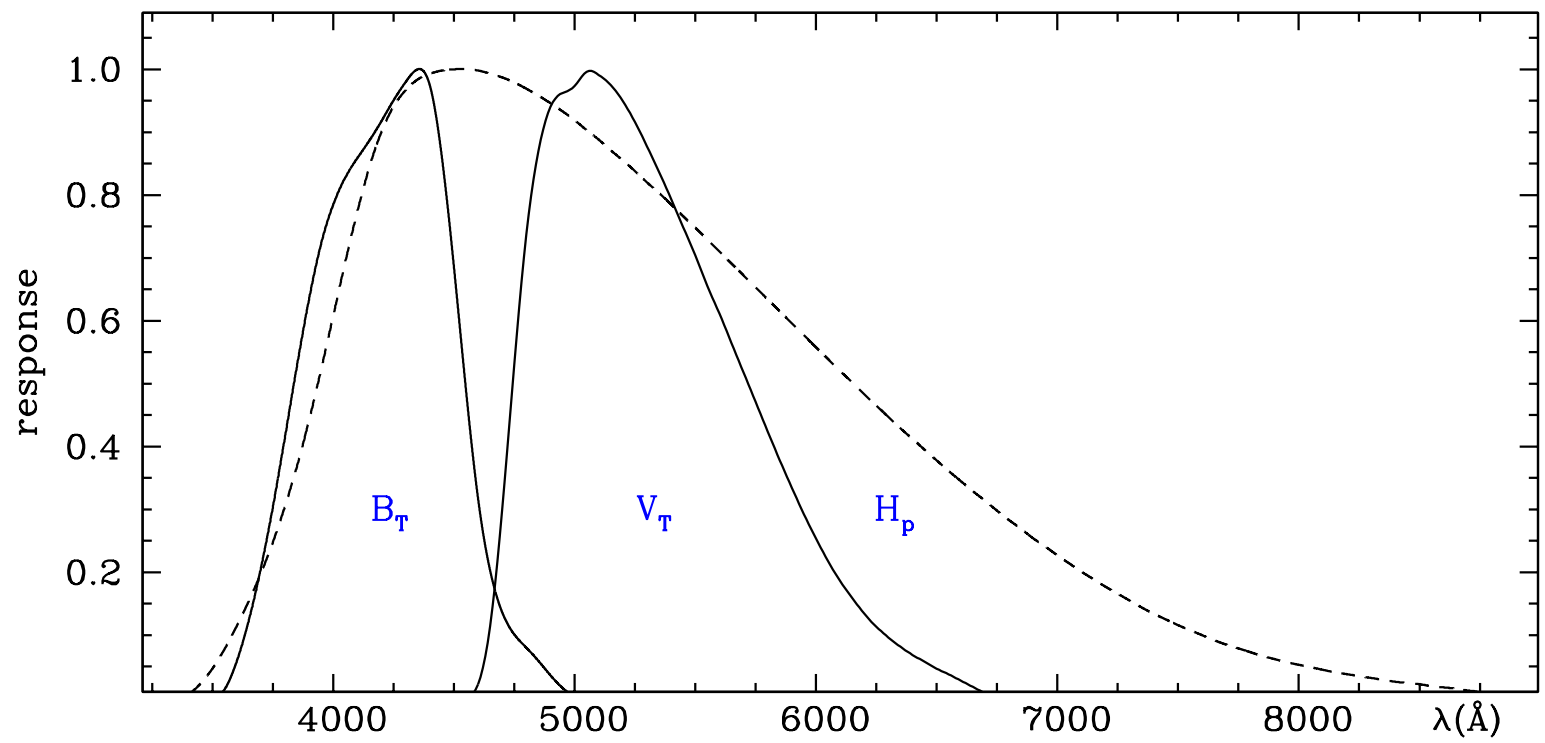

Fig. 135. The photometric system Hipparcos - 1989 


\begin{tabular}{|c|c|c|c|c|c|c|c|c|c|}
\hline \multicolumn{6}{|c|}{$H_{p}$} & \multicolumn{2}{|c|}{$B_{T}$} & \multicolumn{2}{|c|}{$V_{T}$} \\
\hline$\lambda(\AA)$ & $\Upsilon$ & $\lambda(\AA)$ & $\Upsilon$ & $\lambda(\AA)$ & $\Upsilon$ & $\lambda(\AA)$ & $\Upsilon$ & $\lambda(\AA)$ & $\Upsilon$ \\
\hline 3350 & 0.000 & 5600 & 0.710 & 7850 & 0.067 & 3500 & 0.000 & 4550 & 0.000 \\
\hline 3400 & 0.006 & 5650 & 0.691 & 7900 & 0.062 & 3550 & 0.014 & 4600 & 0.022 \\
\hline 3450 & 0.023 & 5700 & 0.672 & 7950 & 0.057 & 3600 & 0.058 & 4650 & 0.115 \\
\hline 3500 & 0.047 & 5750 & 0.653 & 8000 & 0.053 & 3650 & 0.123 & 4700 & 0.301 \\
\hline 3550 & 0.078 & 5800 & 0.634 & 8050 & 0.049 & 3700 & 0.206 & 4750 & 0.530 \\
\hline 3600 & 0.114 & 5850 & 0.615 & 8100 & 0.045 & 3750 & 0.305 & 4800 & 0.737 \\
\hline 3650 & 0.154 & 5900 & 0.596 & 8150 & 0.041 & 3800 & 0.416 & 4850 & 0.870 \\
\hline 3700 & 0.198 & 5950 & 0.577 & 8200 & 0.038 & 3850 & 0.530 & 4900 & 0.940 \\
\hline 3750 & 0.248 & 6000 & 0.558 & 8250 & 0.035 & 3900 & 0.636 & 4950 & 0.973 \\
\hline 3800 & 0.305 & 6050 & 0.539 & 8300 & 0.032 & 3950 & 0.724 & 5000 & 0.990 \\
\hline 3850 & 0.369 & 6100 & 0.520 & 8350 & 0.029 & 4000 & 0.787 & 5050 & 0.996 \\
\hline 3900 & 0.442 & 6150 & 0.502 & 8400 & 0.026 & 4050 & 0.830 & 5100 & 0.991 \\
\hline 3950 & 0.523 & 6200 & 0.483 & 8450 & 0.024 & 4100 & 0.861 & 5150 & 0.975 \\
\hline 4000 & 0.608 & 6250 & 0.465 & 8500 & 0.022 & 4150 & 0.889 & 5200 & 0.949 \\
\hline 4050 & 0.694 & 6300 & 0.447 & 8550 & 0.019 & 4200 & 0.920 & 5250 & 0.916 \\
\hline 4100 & 0.774 & 6350 & 0.429 & 8600 & 0.017 & 4250 & 0.953 & 5300 & 0.878 \\
\hline 4150 & 0.845 & 6400 & 0.412 & 8650 & 0.015 & 4300 & 0.982 & 5350 & 0.837 \\
\hline 4200 & 0.901 & 6450 & 0.395 & 8700 & 0.012 & 4350 & 1.002 & 5400 & 0.794 \\
\hline 4250 & 0.941 & 6500 & 0.378 & 8750 & 0.010 & 4400 & 0.976 & 5450 & 0.749 \\
\hline 4300 & 0.967 & 6550 & 0.361 & 8800 & 0.007 & 4450 & 0.861 & 5500 & 0.704 \\
\hline 4350 & 0.984 & 6600 & 0.345 & 8850 & 0.005 & 4500 & 0.685 & 5550 & 0.658 \\
\hline 4400 & 0.993 & 6650 & 0.329 & 8900 & 0.002 & 4550 & 0.489 & 5600 & 0.612 \\
\hline 4450 & 0.998 & 6700 & 0.314 & 8950 & 0.000 & 4600 & 0.317 & 5650 & 0.565 \\
\hline 4500 & 1.000 & 6750 & 0.298 & & & 4650 & 0.202 & 5700 & 0.518 \\
\hline 4550 & 1.000 & 6800 & 0.283 & & & 4700 & 0.136 & 5750 & 0.471 \\
\hline 4600 & 0.998 & 6850 & 0.269 & & & 4750 & 0.101 & 5800 & 0.424 \\
\hline 4650 & 0.993 & 6900 & 0.254 & & & 4800 & 0.080 & 5850 & 0.379 \\
\hline 4700 & 0.987 & 6950 & 0.241 & & & 4850 & 0.059 & 5900 & 0.335 \\
\hline 4750 & 0.979 & 7000 & 0.227 & & & 4900 & 0.036 & 5950 & 0.293 \\
\hline 4800 & 0.969 & 7050 & 0.214 & & & 4950 & 0.016 & 6000 & 0.254 \\
\hline 4850 & 0.958 & 7100 & 0.201 & & & 5000 & 0.003 & 6050 & 0.218 \\
\hline 4900 & 0.946 & 7150 & 0.189 & & & 5050 & 0.000 & 6100 & 0.186 \\
\hline 4950 & 0.933 & 7200 & 0.177 & & & & & 6150 & 0.159 \\
\hline 5000 & 0.919 & 7250 & 0.166 & & & & & 6200 & 0.135 \\
\hline 5050 & 0.903 & 7300 & 0.155 & & & & & 6250 & 0.114 \\
\hline 5100 & 0.888 & 7350 & 0.144 & & & & & 6300 & 0.097 \\
\hline 5150 & 0.871 & 7400 & 0.134 & & & & & 6350 & 0.082 \\
\hline 5200 & 0.855 & 7450 & 0.125 & & & & & 6400 & 0.069 \\
\hline 5250 & 0.838 & 7500 & 0.116 & & & & & 6450 & 0.058 \\
\hline 5300 & 0.820 & 7550 & 0.108 & & & & & 6500 & 0.047 \\
\hline 5350 & 0.803 & 7600 & 0.100 & & & & & 6550 & 0.038 \\
\hline 5400 & 0.785 & 7650 & 0.092 & & & & & 6600 & 0.028 \\
\hline 5450 & 0.766 & 7700 & 0.085 & & & & & 6650 & 0.018 \\
\hline 5500 & 0.748 & 7750 & 0.079 & & & & & 6700 & 0.008 \\
\hline 5550 & 0.729 & 7800 & 0.073 & & & & & 6750 & 0.000 \\
\hline
\end{tabular}

Fig. 135. continued 


\section{UBVRI - Bessell - 1990}

Standarization of the optical band profiles of the UBVRI(JHKLMN) - Johnson - 1965 and RI - Cousins - 1976 systems.

\section{GENERAL INFORMATION}

AUTHORS M. B. Bessell

MAIN ARTICLE Bessell, M. S. 1990, PASP 102, 1181

\section{SYSTEM DESCRIPTION}

Recommended filters to match the standard system with various photomultipliers and CCDs.

\begin{tabular}{|c|c|}
\hline \multicolumn{2}{|r|}{ BANDS DESCRIPTION [32] } \\
\hline band & filters \\
\hline \multicolumn{2}{|r|}{ glass filters for $S-11 / S-4$ phototubes } \\
\hline$U$ & 1mm UG1 + (2mm WG320 with UV tubes) \\
\hline$B$ & 2mm GG395 + 1mm BG12 \\
\hline$V$ & 3mm GG515 \\
\hline \multicolumn{2}{|r|}{ glass filters for GaAs / S-20R phototubes } \\
\hline$U$ & 1mm UG1 + 1mm BG39 \\
\hline$B$ & $2 \mathrm{~mm}$ GG385 + 1mm BG12 + 1mm BG 18 \\
\hline$V$ & $2 \mathrm{~mm} \mathrm{GG} 495+1 \mathrm{~mm}$ BG18 + (1mm BG $38 \mathrm{GaAs})$ \\
\hline$R$ & $2 \mathrm{~mm}$ OG570 + 2mm KG3 \\
\hline$I$ & 3mm RG9 \\
\hline \multicolumn{2}{|r|}{ glass filters for $U V$ coated $C C D$} \\
\hline$U$ & 1mm UG1 + 1mm BG39 + (3mm WG305 fill) \\
\hline$B$ & 2mm GG385 + 1mm BG12 + 2mm BG39 \\
\hline V & 2mm GG495 + 3mm BG39 \\
\hline$R$ & $2 \mathrm{~mm}$ OG570 + 3mm KG3 \\
\hline$I$ & 3mm RG9 + (2mm WG305 fill) \\
\hline BJ & $2 \mathrm{~mm} \mathrm{BG} 28+3 \mathrm{~mm}$ BG39 \\
\hline$Z$ & $3 \mathrm{~mm}$ RG1000 \\
\hline
\end{tabular}

Fig. 136. The photometric system UBVRI - Bessell - 1990 


\section{TRANSMISSION CURVES [32]}

$U X$ and $B X$ should be used for computing standard ( $U-B)$ color.

$B, V, R, I$ should be used for the other colors and magnitudes. [32]

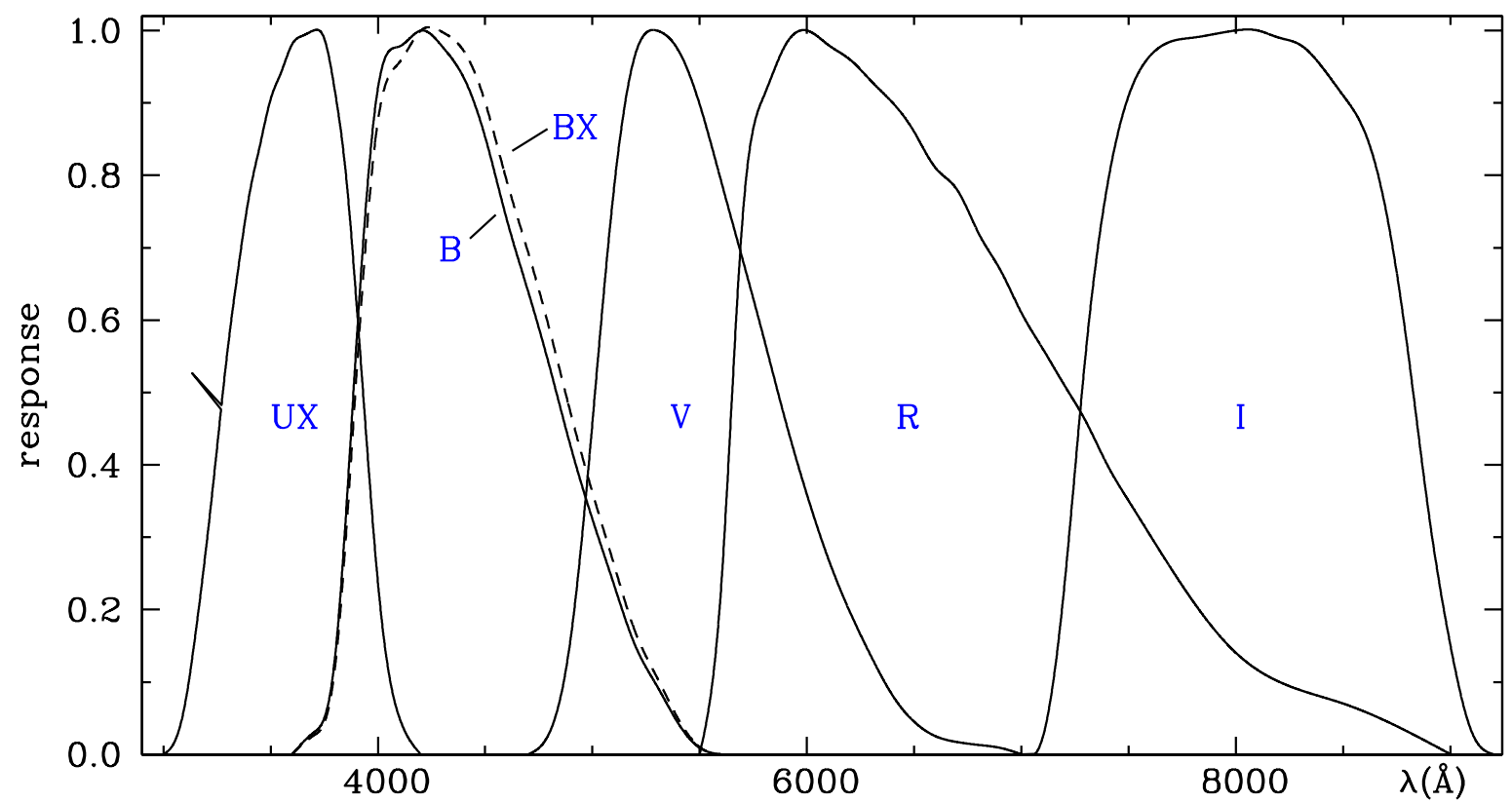

\begin{tabular}{|lc|cc|cc|cc|cc|cc|}
\hline \multicolumn{2}{|c|}{$U X$} & \multicolumn{2}{|c|}{$B X$} & \multicolumn{2}{c|}{$B$} & \multicolumn{2}{c|}{$V$} & \multicolumn{2}{c|}{$R$} & \multicolumn{3}{c|}{$I$} \\
\hline$\lambda(\mathrm{A})$ & $\Upsilon$ & $\lambda(\mathrm{A})$ & $\Upsilon$ & $\lambda(\mathrm{A})$ & $\Upsilon$ & $\lambda(\mathrm{A})$ & $\Upsilon$ & $\lambda(\mathrm{A})$ & $\Upsilon$ & $\lambda(\mathrm{A})$ & $\Upsilon$ \\
\hline 3000 & 0.000 & 3600 & 0.000 & 3600 & 0.000 & 4700 & 0.000 & 5500 & 0.00 & 7000 & 0.000 \\
3050 & 0.016 & 3700 & 0.026 & 3700 & 0.030 & 4800 & 0.030 & 5600 & 0.23 & 7100 & 0.024 \\
3100 & 0.068 & 3800 & 0.120 & 3800 & 0.134 & 4900 & 0.163 & 5700 & 0.74 & 7200 & 0.232 \\
3150 & 0.167 & 3900 & 0.523 & 3900 & 0.567 & 5000 & 0.458 & 5800 & 0.91 & 7300 & 0.555 \\
3200 & 0.287 & 4000 & 0.875 & 4000 & 0.920 & 5100 & 0.780 & 5900 & 0.98 & 7400 & 0.785 \\
3250 & 0.423 & 4100 & 0.956 & 4100 & 0.978 & 5200 & 0.967 & 6000 & 1.00 & 7500 & 0.910 \\
3300 & 0.560 & 4200 & 1.000 & 4200 & 1.000 & 5300 & 1.000 & 6100 & 0.98 & 7600 & 0.965 \\
3350 & 0.673 & 4300 & 0.998 & 4300 & 0.978 & 5400 & 0.973 & 6200 & 0.96 & 7700 & 0.985 \\
3400 & 0.772 & 4400 & 0.972 & 4400 & 0.935 & 5500 & 0.898 & 6300 & 0.93 & 7800 & 0.990 \\
3450 & 0.841 & 4500 & 0.901 & 4500 & 0.853 & 5600 & 0.792 & 6400 & 0.90 & 7900 & 0.995 \\
3500 & 0.905 & 4600 & 0.793 & 4600 & 0.740 & 5700 & 0.684 & 6500 & 0.86 & 8000 & 1.000 \\
3550 & 0.943 & 4700 & 0.694 & 4700 & 0.640 & 5800 & 0.574 & 6600 & 0.81 & 8100 & 1.000 \\
3600 & 0.981 & 4800 & 0.587 & 4800 & 0.536 & 5900 & 0.461 & 6700 & 0.78 & 8200 & 0.990 \\
3650 & 0.993 & 4900 & 0.470 & 4900 & 0.424 & 6000 & 0.359 & 6800 & 0.72 & 8300 & 0.980 \\
3700 & 1.000 & 5000 & 0.362 & 5000 & 0.325 & 6100 & 0.270 & 6900 & 0.67 & 8400 & 0.950 \\
3750 & 0.989 & 5100 & 0.263 & 5100 & 0.235 & 6200 & 0.197 & 7000 & 0.61 & 8500 & 0.910 \\
3800 & 0.916 & 5200 & 0.169 & 5200 & 0.150 & 6300 & 0.135 & 7100 & 0.56 & 8600 & 0.860 \\
3850 & 0.804 & 5300 & 0.107 & 5300 & 0.095 & 6400 & 0.081 & 7200 & 0.51 & 8700 & 0.750 \\
3900 & 0.625 & 5400 & 0.049 & 5400 & 0.043 & 6500 & 0.045 & 7300 & 0.46 & 8800 & 0.560 \\
3950 & 0.423 & 5500 & 0.010 & 5500 & 0.009 & 6600 & 0.025 & 7400 & 0.40 & 8900 & 0.330 \\
4000 & 0.238 & 5600 & 0.000 & 5600 & 0.000 & 6700 & 0.017 & 7500 & 0.35 & 9000 & 0.150 \\
4050 & 0.114 & & & & & 6800 & 0.013 & 8000 & 0.14 & 9100 & 0.030 \\
4100 & 0.051 & & & & & 6900 & 0.009 & 8500 & 0.03 & 9200 & 0.000 \\
4150 & 0.019 & & & & & 7000 & 0.000 & 9000 & 0.00 & & \\
4200 & 0.000 & & & & & & & & & & \\
\hline
\end{tabular}

Fig. 136. continued 


\section{JHKL SAAO - Carter - 1990}

Definition of the SAAO infrared photometry by fixing the standard stars.

\section{GENERAL INFORMATION}

$\begin{array}{ll}\text { AUTHORS } & \text { B. S. Carter } \\ \text { TELESCOPE } & 0.75 \mathrm{~m} \text { (reflector), Sutherland } \\ \text { DETECTOR } & \text { InSb }\end{array}$

MAIN ARTICLE Carter, B. S. 1990, MNRAS 242, 1

\section{SYSTEM DESCRIPTION}

ZERO POINT: The basis of the South African Astronomical Observatory infrared photometric system are defined through colors and magnitudes of 230 standard stars.

The zero-points are set by 25 standards in the B1-A7 spectral-type range. [62]

\section{SYSTEM ANALYSIS}

RELATIONS WITH OTHER SYSTEMS [62]

VJHKLM ESO - Engels et al - 1981

\begin{tabular}{|r|}
\hline$J_{E S O}=J-0.062(J-K)+0.053$ \\
\hline$H_{E S O}=H+0.059(J-K)+0.034$ \\
\hline$K_{E S O}=K+0.031$ \\
\hline$L_{E S O}=L+0.013(J-K)-0.019$ \\
\hline
\end{tabular}

JHKL CTIO - Elias et al - 1982

\begin{tabular}{|l}
$J_{C T I O}=J-0.134(J-K)-0.001$ \\
\hline$H_{C T I O}=H-0.022(J-K)+0.004$ \\
\hline$K_{C T I O}=K-0.027(J-K)-0.003$ \\
\hline$L_{C T I O}=L-0.023(J-K)-0.002$ \\
\hline
\end{tabular}

JHKL MSO - Jones and Hyland - 1982

\begin{tabular}{|rc|}
\hline$J_{M S S O}$ & $=J-0.055(J-K)+0.005$ \\
\hline$H_{M S S O}$ & $=H+0.001(J-K)+0.024$ \\
\hline$K_{M S S O}$ & $=K-0.014(J-K)+0.009$ \\
\hline
\end{tabular}

JHKL' $\quad$ AAO - Allen and Cragg - 1983

\begin{tabular}{|c|c|}
\hline$J_{A A O}$ & $=J+0.005(J-K)+0.013$ \\
\hline$H_{A A O}$ & $=H+0.015(J-K)+0.024$ \\
\hline$K_{A A O}$ & $=K+0.006(J-K)+0.012$ \\
\hline$L_{A A O}$ & $=L-0.051(J-K)+0.018$ \\
\hline
\end{tabular}

Fig. 137. The photometric system JHKL SAAO - Carter - 1990 


\section{Guide Star Catalogue - Lasker et al. - 1990}

Input catalogue for HST pointing and guiding.

\begin{tabular}{ll} 
& \multicolumn{1}{c}{ GENERAL INFORMATION } \\
AUTHORS & B. M. Lasker, C. R. Sturch, B. J. McClean, \\
& J. L. Russell, H. Jenkner and M. M. Shara \\
TELESCOPE & 1.2m UK Schmidt, Siding Spring Obs. ; 1.2m Oschin Schmidt, Palomar Obs. \\
DETECTOR & Kodak IIIa-J, IIa-D photographic plates \\
MAIN ARTICLE & Lasker, B. M., Sturch, C.R., McClean, B. J., Russell, J. L., \\
& Jenkner, H., and Shara, M. M. 1990, AJ 99, 2019
\end{tabular}

\section{SYSTEM DESCRIPTION}

\begin{tabular}{|l|cccc|}
\hline \multicolumn{5}{|c|}{ BANDS DESCRIPTION [184] } \\
\hline band & plate & filter & $\lambda_{0}(\AA)$ & FWHM $(\AA)$ \\
\hline$J(\#)$ & IIIaJ & GG395 & 4500 & 1500 \\
\hline$D$ & IIaD & none & 5610 & 3200 \\
\hline$V 495$ & IIaD & GG495 & 5650 & 1400 \\
\hline$V 12$ & IIaD & W12 & 5760 & 1140 \\
\hline
\end{tabular}

(\#) For UK Schmidt plates (southern emisphere).

Fig. 138. The photometric system Guide Star Catalogue - Lasker et al. - 1990 


\section{UIT - 1990}

Photometric bands of the Ultraviolet Imaging Telescope o w on Astro-1 (1990) and Astro-2 (1995) missions (Shuttle).

\section{GENERAL INFORMATION}

\section{TELESCOPE $\quad 0.38 \mathrm{~m}$ (reector)}

DETECTOR $\quad \mathrm{Cs}_{2}$ Te cathode (Near-UV camera), CsI cathode (Far-UV camera) + Kodak IIa-O lms.

MAIN ARTICLE Stecher et al. 1992, ApJ 395, L1

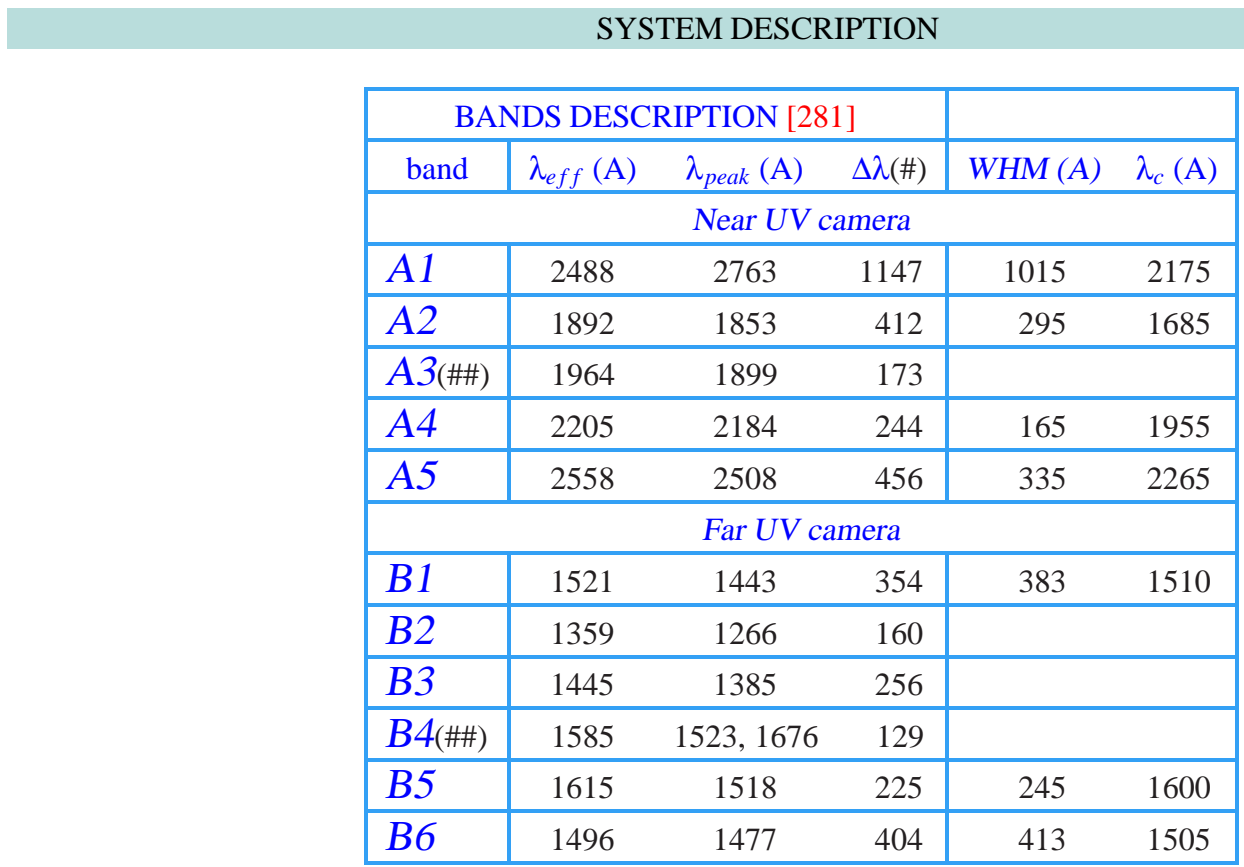

(\#) Equivalent width. (\#\#) Saddle-shaped bands.

\section{TRANSMISSION CURVES}

As derived from Fig 1 of [281]

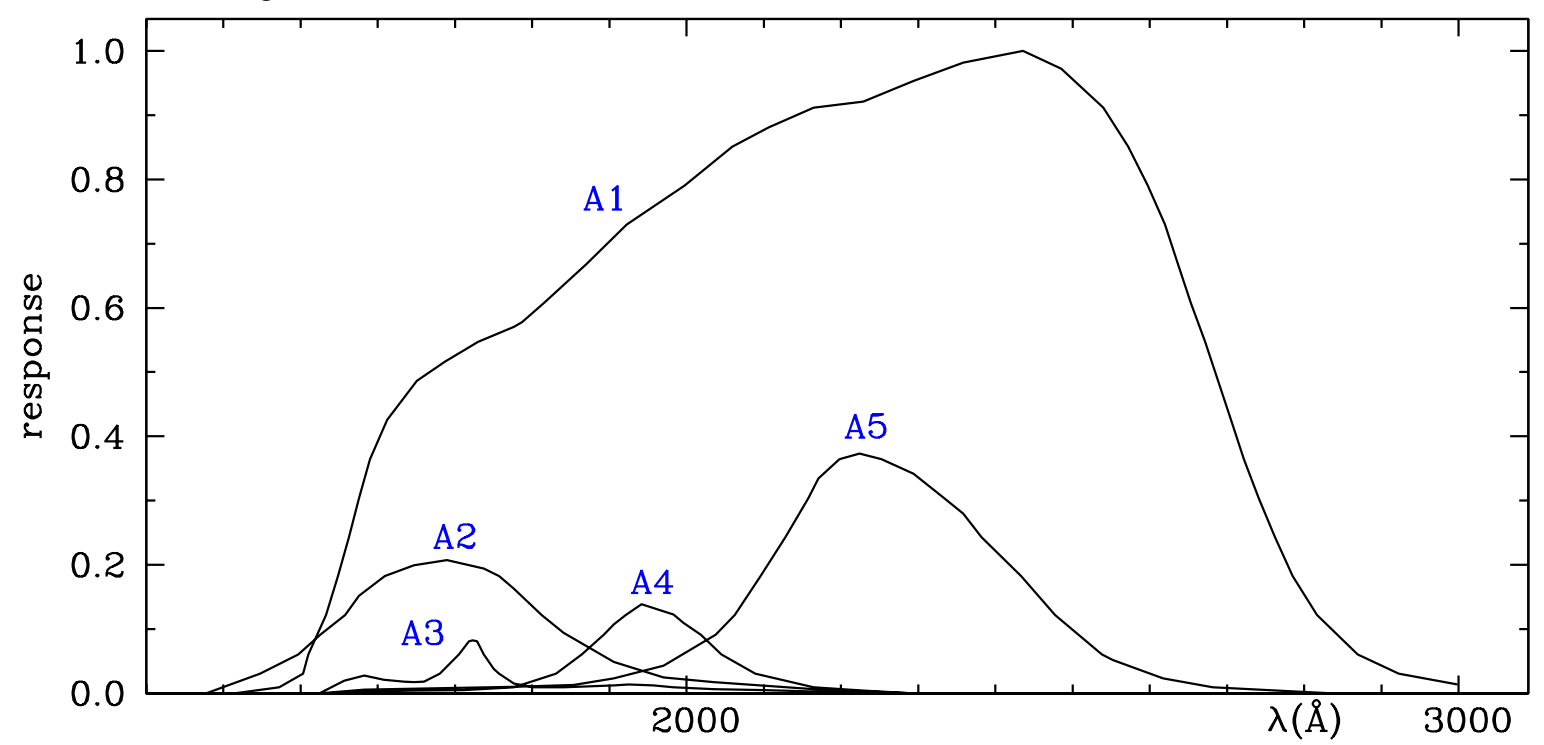

Fig. 139. The photometric system UIT - 1990 


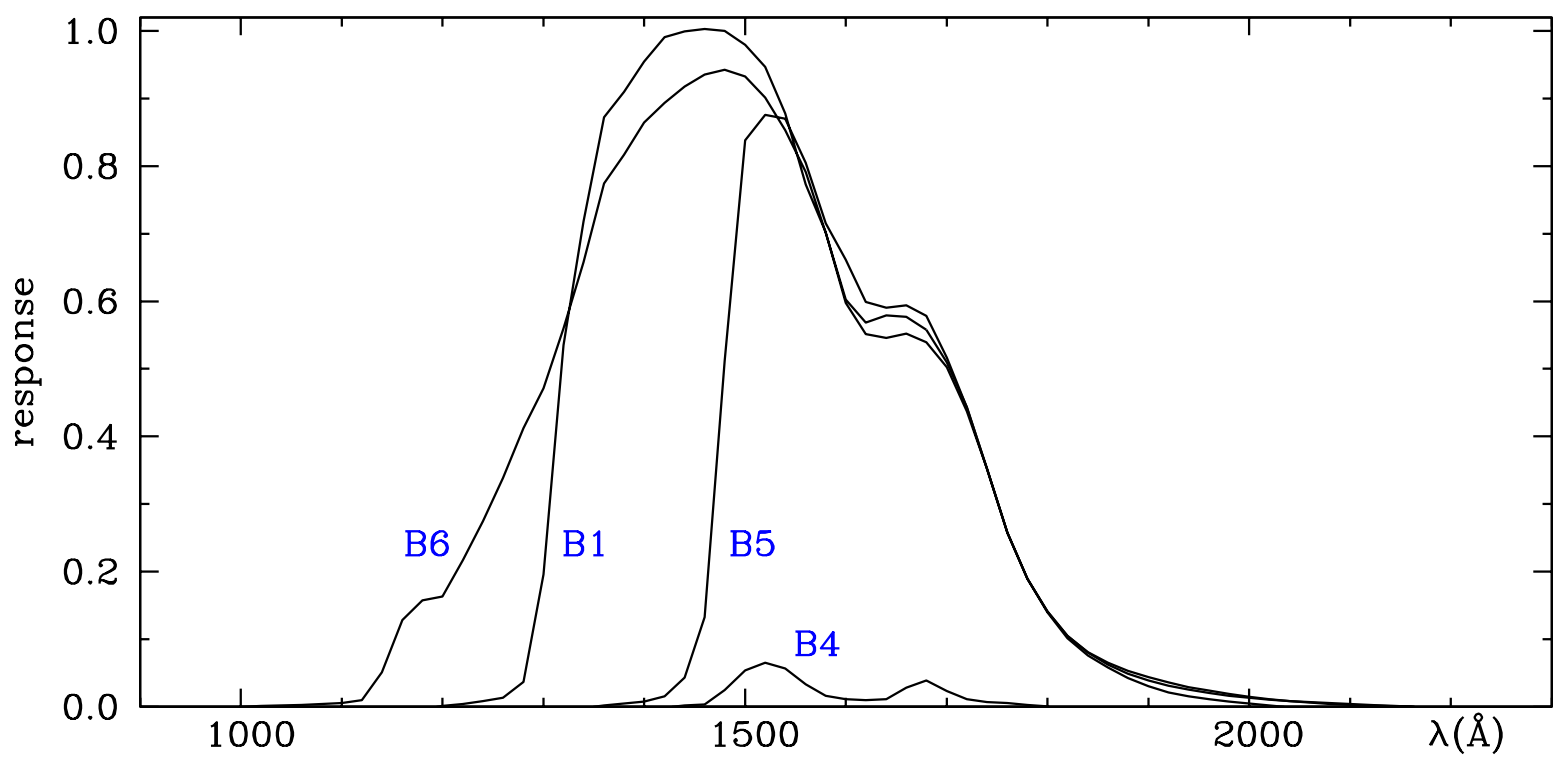

\begin{tabular}{|c|c|c|c|c|c|c|c|c|c|c|c|c|c|c|c|c|c|}
\hline \multicolumn{2}{|c|}{ A1 } & \multicolumn{2}{|c|}{ A2 } & \multicolumn{2}{|c|}{ A3 } & \multicolumn{2}{|c|}{ A4 } & \multicolumn{2}{|c|}{$A 5$} & \multicolumn{2}{|c|}{ B1 } & \multicolumn{2}{|c|}{$B 4$} & \multicolumn{2}{|c|}{ B5 } & \multicolumn{2}{|c|}{ B6 } \\
\hline$\lambda(\mathrm{A})$ & $\Upsilon$ & $\lambda(\mathrm{A})$ & $\Upsilon$ & $\lambda(\mathrm{A})$ & $r$ & $\lambda(\mathrm{A})$ & $\Upsilon$ & $\lambda(\mathrm{A})$ & $\Upsilon$ & $\lambda(\mathrm{A})$ & $\Upsilon$ & $\lambda(\mathrm{A})$ & $\Upsilon$ & $\lambda(\mathrm{A})$ & $\Upsilon$ & $\lambda(\mathrm{A})$ & $\Upsilon$ \\
\hline 1414 & 0.000 & 1378 & 0.000 & 1524 & 0.000 & 1530 & 0.000 & 1524 & 0.000 & 1194 & 0.000 & 1427 & 0.000 & 1349 & 0.000 & 1000 & 0.000 \\
\hline 1472 & 0.009 & 1448 & 0.030 & 1556 & 0.019 & 1582 & 0.003 & 1582 & 0.005 & 1265 & 0.015 & 1453 & 0.003 & 1388 & 0.006 & 1065 & 0.003 \\
\hline 1503 & 0.030 & 1497 & 0.061 & 1582 & 0.027 & 1647 & 0.004 & 1647 & 0.007 & 1278 & 0.030 & 1479 & 0.021 & 1414 & 0.012 & 1116 & 0.009 \\
\hline 1510 & 0.061 & 1525 & 0.091 & 1608 & 0.021 & 1712 & 0.005 & 1712 & 0.009 & 1285 & 0.061 & 1483 & 0.030 & 1433 & 0.030 & 1135 & 0.030 \\
\hline 1533 & 0.122 & 1558 & 0.122 & 1634 & 0.018 & 1776 & 0.009 & 1776 & 0.010 & 1291 & 0.122 & 1492 & 0.046 & 1449 & 0.061 & 1142 & 0.061 \\
\hline 1549 & 0.182 & 1576 & 0.152 & 1647 & 0.017 & 1831 & 0.030 & 1854 & 0.013 & 1298 & 0.182 & 1511 & 0.061 & 1459 & 0.122 & 1158 & 0.122 \\
\hline 1563 & 0.243 & 1609 & 0.182 & 1660 & 0.018 & 1864 & 0.061 & 1906 & 0.023 & 1304 & 0.243 & 1524 & 0.066 & 1466 & 0.243 & 1175 & 0.155 \\
\hline 1576 & 0.304 & 1647 & 0.199 & 1680 & 0.030 & 1893 & 0.091 & 1970 & 0.043 & 1307 & 0.304 & 1536 & 0.061 & 1472 & 0.365 & 1194 & 0.158 \\
\hline 1590 & 0.365 & 1690 & 0.207 & 1705 & 0.061 & 1906 & 0.108 & 2038 & 0.091 & 1310 & 0.365 & 1550 & 0.046 & 1479 & 0.486 & 1210 & 0.182 \\
\hline 1612 & 0.426 & 1737 & 0.195 & 1718 & 0.081 & 1921 & 0.122 & 2062 & 0.122 & 1313 & 0.426 & 1563 & 0.030 & 1485 & 0.608 & 1228 & 0.243 \\
\hline 1651 & 0.486 & 1757 & 0.182 & 1723 & 0.082 & 1942 & 0.139 & 2096 & 0.182 & 1317 & 0.486 & 1582 & 0.015 & 1492 & 0.729 & 1251 & 0.304 \\
\hline 1687 & 0.517 & 1776 & 0.163 & 1728 & 0.081 & 1983 & 0.123 & 2128 & 0.243 & 1321 & 0.547 & 1614 & 0.010 & 1498 & 0.821 & 1266 & 0.365 \\
\hline 1730 & 0.547 & 1812 & 0.122 & 1737 & 0.061 & 1996 & 0.109 & 2158 & 0.304 & 1329 & 0.608 & 1647 & 0.015 & 1502 & 0.851 & 1285 & 0.426 \\
\hline 1776 & 0.570 & 1841 & 0.094 & 1750 & 0.038 & 2018 & 0.091 & 2171 & 0.334 & 1334 & 0.669 & 1662 & 0.030 & 1523 & 0.874 & 1304 & 0.486 \\
\hline 1787 & 0.578 & 1906 & 0.049 & 1757 & 0.030 & 2045 & 0.061 & 2198 & 0.365 & 1342 & 0.729 & 1677 & 0.040 & 1550 & 0.851 & 1317 & 0.547 \\
\hline 1815 & 0.608 & 1970 & 0.024 & 1776 & 0.015 & 2089 & 0.030 & 2224 & 0.373 & 1348 & 0.790 & 1692 & 0.030 & 1563 & 0.790 & 1342 & 0.669 \\
\hline 1871 & 0.669 & 2035 & 0.017 & 1802 & 0.009 & 2164 & 0.009 & 2252 & 0.365 & 1356 & 0.851 & 1712 & 0.015 & 1576 & 0.729 & 1349 & 0.729 \\
\hline 1922 & 0.729 & 2164 & 0.006 & 1841 & 0.009 & 2229 & 0.004 & 2294 & 0.342 & 1382 & 0.912 & 1750 & 0.006 & 1598 & 0.669 & 1369 & 0.790 \\
\hline 1997 & 0.790 & 2294 & 0.000 & 1873 & 0.011 & 2294 & 0.000 & 2334 & 0.304 & 1408 & 0.973 & 1802 & 0.000 & 1616 & 0.608 & 1393 & 0.851 \\
\hline 2060 & 0.851 & & & 1906 & 0.012 & & & 2358 & 0.280 & 1445 & 1.000 & & & 1640 & 0.591 & 1411 & 0.881 \\
\hline 2106 & 0.881 & & & 1925 & 0.013 & & & 2382 & 0.243 & 1485 & 0.997 & & & 1660 & 0.594 & 1435 & 0.912 \\
\hline 2164 & 0.912 & & & 1957 & 0.012 & & & 2433 & 0.182 & 1505 & 0.973 & & & 1680 & 0.578 & 1479 & 0.942 \\
\hline 2229 & 0.921 & & & 1983 & 0.009 & & & 2477 & 0.122 & 1524 & 0.942 & & & 1692 & 0.547 & 1515 & 0.912 \\
\hline 2294 & 0.953 & & & 2035 & 0.006 & & & 2538 & 0.061 & 1536 & 0.912 & & & 1708 & 0.486 & 1541 & 0.851 \\
\hline 2358 & 0.982 & & & 2100 & 0.005 & & & 2552 & 0.052 & 1543 & 0.851 & & & 1724 & 0.426 & 1560 & 0.790 \\
\hline 2436 & 1.000 & & & 2164 & 0.003 & & & 2617 & 0.023 & 1556 & 0.790 & & & 1737 & 0.365 & 1572 & 0.729 \\
\hline 2485 & 0.973 & & & 2294 & 0.000 & & & 2682 & 0.009 & 1569 & 0.729 & & & 1750 & 0.304 & 1589 & 0.669 \\
\hline 2539 & 0.912 & & & & & & & 2830 & 0.000 & 1589 & 0.669 & & & 1763 & 0.243 & 1599 & 0.608 \\
\hline 2572 & 0.851 & & & & & & & & & 1598 & 0.608 & & & 1783 & 0.182 & 1631 & 0.576 \\
\hline 2598 & 0.790 & & & & & & & & & 1608 & 0.578 & & & 1810 & 0.122 & 1655 & 0.579 \\
\hline 2620 & 0.729 & & & & & & & & & 1621 & 0.550 & & & 1867 & 0.061 & 1673 & 0.568 \\
\hline 2636 & 0.669 & & & & & & & & & 1656 & 0.553 & & & 1906 & 0.041 & 1686 & 0.547 \\
\hline 2653 & 0.608 & & & & & & & & & 1669 & 0.547 & & & 1970 & 0.021 & 1708 & 0.486 \\
\hline 2671 & 0.547 & & & & & & & & & 1695 & 0.517 & & & 2035 & 0.009 & 1724 & 0.426 \\
\hline 2688 & 0.486 & & & & & & & & & 1705 & 0.486 & & & 2164 & 0.000 & 1737 & 0.365 \\
\hline 2705 & 0.426 & & & & & & & & & 1723 & 0.426 & & & & & 1750 & 0.304 \\
\hline 2722 & 0.365 & & & & & & & & & 1737 & 0.365 & & & & & 1763 & 0.243 \\
\hline 2741 & 0.304 & & & & & & & & & 1750 & 0.304 & & & & & 1783 & 0.182 \\
\hline 2762 & 0.243 & & & & & & & & & 1763 & 0.243 & & & & & 1810 & 0.122 \\
\hline 2785 & 0.182 & & & & & & & & & 1783 & 0.182 & & & & & 1841 & 0.079 \\
\hline 2816 & 0.122 & & & & & & & & & 1809 & 0.122 & & & & & 1906 & 0.036 \\
\hline 2869 & 0.061 & & & & & & & & & 1856 & 0.061 & & & & & 1970 & 0.018 \\
\hline 2922 & 0.030 & & & & & & & & & 1906 & 0.027 & & & & & 2035 & 0.009 \\
\hline 2999 & 0.013 & & & & & & & & & 1970 & 0.009 & & & & & 2164 & 0.000 \\
\hline & & & & & & & & & & 2035 & 0.000 & & & & & & \\
\hline
\end{tabular}

Fig. 139. continued 


\section{JHKL'M ESO - Bouchet et al. - 1991}

Infrared photometry at ESO.

\section{GENERAL INFORMATION}
AUTHORS
P. Bouchet, J. Manfroid and F.X. Schmider
TELESCOPE $1 \mathrm{~m}, 2.2 \mathrm{~m}$ and $3.6 \mathrm{~m}$ (reflectors), ESO (La Silla)
DETECTOR $\mathrm{InSb}$
MAIN ARTICLE $\quad$ Bouchet, P., Manfroid, J., Schmider, F.X. 1991, A\&AS 91, 409

\section{SYSTEM DESCRIPTION}

\begin{tabular}{|c|ccc|cc|}
\hline \multicolumn{3}{|c|}{ BANDS DESCRIPTION [38] } & \multicolumn{2}{c|}{ ABSOLUTE CALIBRATION (\#) [38] } \\
\hline band & $\lambda_{0}(\mu \mathrm{m})$ & $\lambda_{\text {eff }}(\mu \mathrm{m})(\# \#)$ & FWHM $(\mu \mathrm{m})$ & $\mathrm{F}_{\lambda, 0}\left(\mathrm{~W} \mathrm{~m}^{-2} \mathrm{~nm}^{-1}\right)$ & $\mathrm{F}_{\mathrm{v}, 0}(\mathrm{Jy})$ \\
\hline$J$ & 1.228 & 1.210 & 0.190 & $3.4410^{-12}$ & 1680 \\
\hline$H$ & 1.651 & 1.635 & 0.269 & $1.2110^{-12}$ & 1070 \\
\hline$K$ & 2.216 & 2.197 & 0.360 & $4.1210^{-13}$ & 664 \\
\hline$L$ & 3.771 & 3.740 & 0.580 & $5.5810^{-14}$ & 260 \\
\hline$M$ & 4.772 & 4.759 & 0.381 & $2.2110^{-14}$ & 167 \\
\hline
\end{tabular}

(\#) Fluxes for a 0.00 mag star.

(\#\#) For a 11400 K blackbody.

ZERO POINT: HR 3314 (A0 V) is taken to have:

$V=3.89,(V-K)=-0.05,(J-K)=-0.01,(H-K)=-0.01,\left(H-L^{\prime}\right)=0.00,(K-M)=0.00 \quad[38]$

\section{SYSTEM ANALYSIS}

RELATIONS WITH OTHER SYSTEMS [48]

MSSO - Thomas et al - 1973

for 15 stars in common:

\begin{tabular}{|rrr|}
\hline$J_{E S O}-J_{M S S O}$ & $=0.007( \pm 0.006)+0.003( \pm 0.027)(J-K)_{M S S O}$ \\
\hline$H_{E S O}-H_{M S S O}$ & $=0.008( \pm 0.005)-0.013( \pm 0.024)(J-K)_{M S S O}$ \\
\hline$K_{E S O}-K_{M S S O}$ & $=-0.002( \pm 0.005)-0.021( \pm 0.024)(J-K)_{M S S O}$ \\
\hline
\end{tabular}

JHKL CTIO - Elias et al- 1982

for 15 stars in common:

\begin{tabular}{|r|}
\hline$J_{E S O}-J_{C T I O}=0.006( \pm 0.008)+0.134( \pm 0.033)(J-K)_{C T I O}$ \\
\hline$H_{E S O}-H_{C T I O}=0.028( \pm 0.007)+0.025( \pm 0.027)(J-K)_{C T I O}$ \\
\hline$K_{E S O}-K_{C T I O}=0.019( \pm 0.005)-0.010( \pm 0.018)(J-K)_{C T I O}$ \\
\hline$L_{E S O}-L_{C T I O}=0.015( \pm 0.007)+0.011( \pm 0.025)(J-K)_{C T I O}$ \\
\hline
\end{tabular}

Fig. 140. The photometric system JHKL'M ESO - Bouchet et al. - 1991 
JHKL' AAO - Allen and Cragg - 1983

for 22 stars in common:

\begin{tabular}{|rcr|}
\hline$J_{E S O}-J_{A A O}$ & $=-0.003( \pm 0.006)-0.017( \pm 0.018)(J-K)_{A A O}$ \\
\hline$H_{E S O}-H_{A A O}$ & $=0.009( \pm 0.005)+0.006( \pm 0.018)(J-K)_{A A O}$ \\
\hline$K_{E S O}-K_{A A O}$ & $=0.001( \pm 0.005)-0.002( \pm 0.017)(J-K)_{A A O}$ \\
\hline$L_{E S O}-L_{A A O}$ & $=$ & $-0.014( \pm 0.007)-0.042( \pm 0.023)(J-K)_{A A O}$ \\
\hline
\end{tabular}

JHKL SAAO - Carter - 1990

for 119 stars in common:

\begin{tabular}{|rcc|}
\hline$J_{E S O}-J_{S A A O}$ & $=0.014( \pm 0.006)-0.024( \pm 0.011)(J-K)_{S A A O}$ \\
\hline$H_{E S O}-H_{S A A O}$ & $=0.034( \pm 0.005)+0.013( \pm 0.009)(J-K)_{S A A O}$ \\
\hline$K_{E S O}-K_{S A A O}$ & $=0.019( \pm 0.005)-0.015( \pm 0.009)(J-K)_{S A A O}$ \\
\hline$L_{E S O}-L_{S A A O}$ & $=-0.002( \pm 0.004)-0.002( \pm 0.008)(J-K)_{S A A O}$ \\
\hline
\end{tabular}

Fig. 140. continued 


\section{POSS II - Reid et al. - 1991}

Second photographic Palomar Observatory Sky Survey.

\section{GENERAL INFORMATION}

AUTHORS I. N. Reid, C. Brewer, R. J. Brucato,

W. R. McKinley, A. Maury, D. Mendenhall, J. R. Mould, J. Mueller,

G. Neugebauer, J. Phinney, W. L. W. Sargent, J. Schombert and R. Thicksten

TELESCOPE

1.2m Oschin Schmidt, Palomar Obs.; 1.2m UK Schmidt; 1.0m Schmidt, ESO

DETECTOR

Kodak IIIa-J, IIIa-F, and IV-N photographic plates (hypersensitized)

MAIN ARTICLE Reid, I. N., et al. 1991, PASP 103, 661

\section{SYSTEM DESCRIPTION}

\begin{tabular}{|l|lcc|cc|}
\hline \multicolumn{7}{|c|}{ BANDS DESCRIPTION [250] } & & \\
\hline \multicolumn{1}{|c|}{ plate } & emulsion & lter & $\lambda_{\text {eff }}(\mathrm{A})$ & WHM (A) & $\lambda_{c}(\mathrm{~A})$ \\
\hline blue & IIIaJ & GG 395 & 4800 & 1430 & 4970 \\
\hline red & IIIaF & RG 610 & 6500 & 770 & 6720 \\
\hline infrared & IV-N & RG 9 & 8500 & 1760 & 8660 \\
\hline
\end{tabular}

TRANSMISSION CURVES

As derived from Fig 1 of [250].

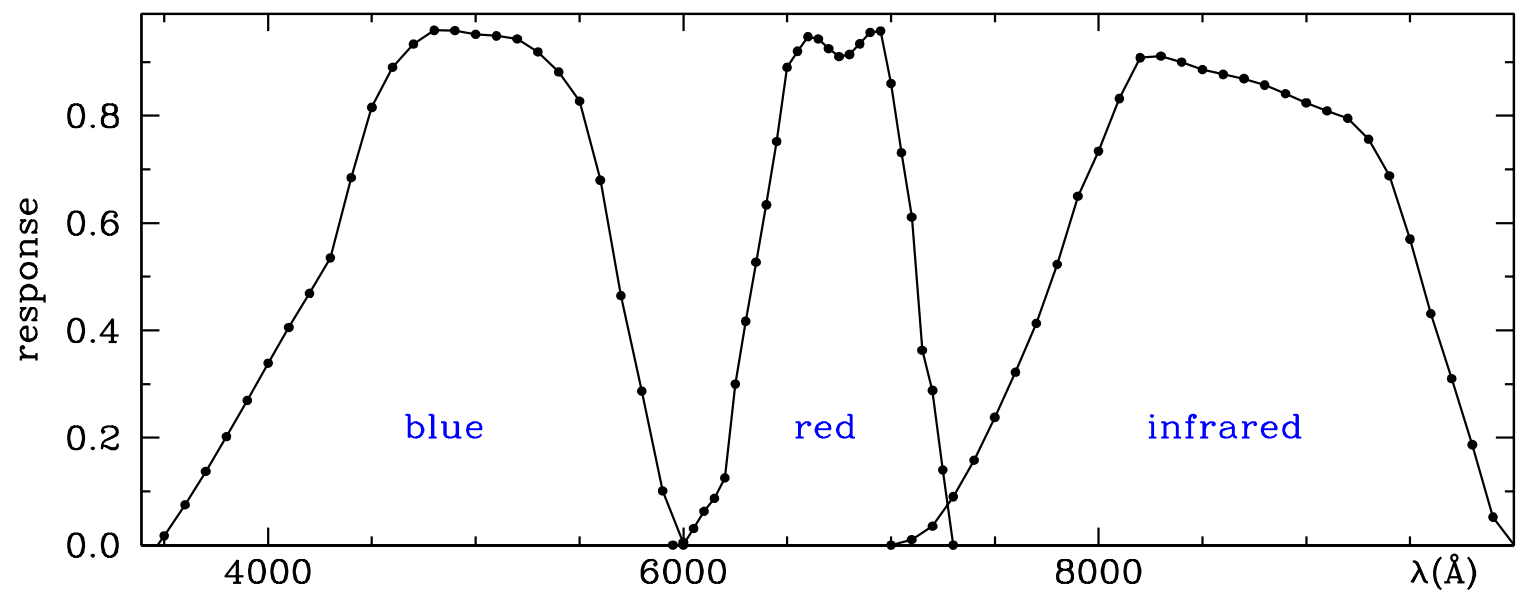

\begin{tabular}{|cc|cc|cc|cc|cc|cc|rc|}
\hline \multicolumn{4}{|c|}{ blue } & \multicolumn{6}{c|}{ red } & \multicolumn{6}{c|}{ infrared } \\
\hline$\lambda(\mathrm{A})$ & $\Upsilon$ & $\lambda(\mathrm{A})$ & $\Upsilon$ & $\lambda(\mathrm{A})$ & $\Upsilon$ & $\lambda(\mathrm{A})$ & $\Upsilon$ & $\lambda(\mathrm{A})$ & $\Upsilon$ & $\lambda(\mathrm{A})$ & $\Upsilon$ & $\lambda(\mathrm{A})$ & $\Upsilon$ \\
\hline 3400 & 0.000 & 4800 & 0.959 & 5950 & 0.000 & 6650 & 0.943 & 7000 & 0.000 & 8400 & 0.900 & 9800 & 0.187 \\
3500 & 0.017 & 4900 & 0.959 & 6000 & 0.000 & 6700 & 0.925 & 7100 & 0.010 & 8500 & 0.886 & 9900 & 0.052 \\
3600 & 0.075 & 5000 & 0.952 & 6050 & 0.031 & 6750 & 0.910 & 7200 & 0.035 & 8600 & 0.877 & 10000 & 0.000 \\
3700 & 0.137 & 5100 & 0.949 & 6100 & 0.063 & 6800 & 0.914 & 7300 & 0.090 & 8700 & 0.869 & & \\
3800 & 0.202 & 5200 & 0.943 & 6150 & 0.087 & 6850 & 0.934 & 7400 & 0.158 & 8800 & 0.857 & & \\
3900 & 0.269 & 5300 & 0.919 & 6200 & 0.125 & 6900 & 0.955 & 7500 & 0.238 & 8900 & 0.841 & & \\
4000 & 0.339 & 5400 & 0.881 & 6250 & 0.300 & 6950 & 0.958 & 7600 & 0.322 & 9000 & 0.824 & & \\
4100 & 0.405 & 5500 & 0.827 & 6300 & 0.417 & 7000 & 0.860 & 7700 & 0.413 & 9100 & 0.809 & & \\
4200 & 0.469 & 5600 & 0.680 & 6350 & 0.527 & 7050 & 0.731 & 7800 & 0.523 & 9200 & 0.795 & & \\
4300 & 0.535 & 5700 & 0.465 & 6400 & 0.634 & 7100 & 0.611 & 7900 & 0.650 & 9300 & 0.756 & & \\
4400 & 0.684 & 5800 & 0.287 & 6450 & 0.752 & 7150 & 0.363 & 8000 & 0.734 & 9400 & 0.688 & & \\
4500 & 0.815 & 5900 & 0.101 & 6500 & 0.890 & 7200 & 0.288 & 8100 & 0.832 & 9500 & 0.570 & & \\
4600 & 0.890 & 6000 & 0.005 & 6550 & 0.920 & 7250 & 0.140 & 8200 & 0.908 & 9600 & 0.431 & & \\
4700 & 0.933 & & & 6600 & 0.947 & 7300 & 0.000 & 8300 & 0.911 & 9700 & 0.310 & & \\
\hline
\end{tabular}

Fig. 141. The photometric system POSS II - Reid et al. - 1991 


\section{CaII - Twarog et al. - 1991}

A CaII-based metallicity index is added to the $u v b y H \beta$ - Strömgren and Crawford - 1956 system.

\section{GENERAL INFORMATION}

AUTHORS

TELESCOPE

DETECTOR

B. J. Anthony-Twarog, J. Laird, D. Payne and B. A. Twarog

$0.9 \mathrm{~m}$ (reector), KPNO; $0.6 \mathrm{~m}$ and $1.0 \mathrm{~m}$ (reectors), CTIO

1P21 (S-20 cathode, refrigerated)

MAIN ARTICLE Anthony-Twarog, B.J., Laird, J.B., Payne, D., Twarog, B.A. 1991, AJ 101, 1902

\section{SYSTEM DESCRIPTION}

\begin{tabular}{|c|c|c|c|c|}
\hline \multicolumn{3}{|c|}{ BANDS DESCRIPTION [10] } & \multirow[b]{2}{*}{$W H M(\mathrm{~A})$} & \multirow[b]{2}{*}{$\lambda_{c}(\mathrm{~A}$} \\
\hline band & $\lambda_{e f f}(\mathrm{~A})$ & FWHM (A) & & \\
\hline Ca old & 3959 & $\approx 90$ & 79 & 3953 \\
\hline Ca new & 3945 & $\approx 90$ & 90 & 3949 \\
\hline
\end{tabular}

Filters are appropriately blocked against red leaks.

\section{SYSTEM ANALYSIS}

\section{COLOR INDICES [10]}

If $\mathrm{CaII}$ is the magnitude in the present system and b, y are from uvbyH - Str̈mgren and Crawford - 1956:

$$
h k=(C a I I-b)-(b-y)
$$

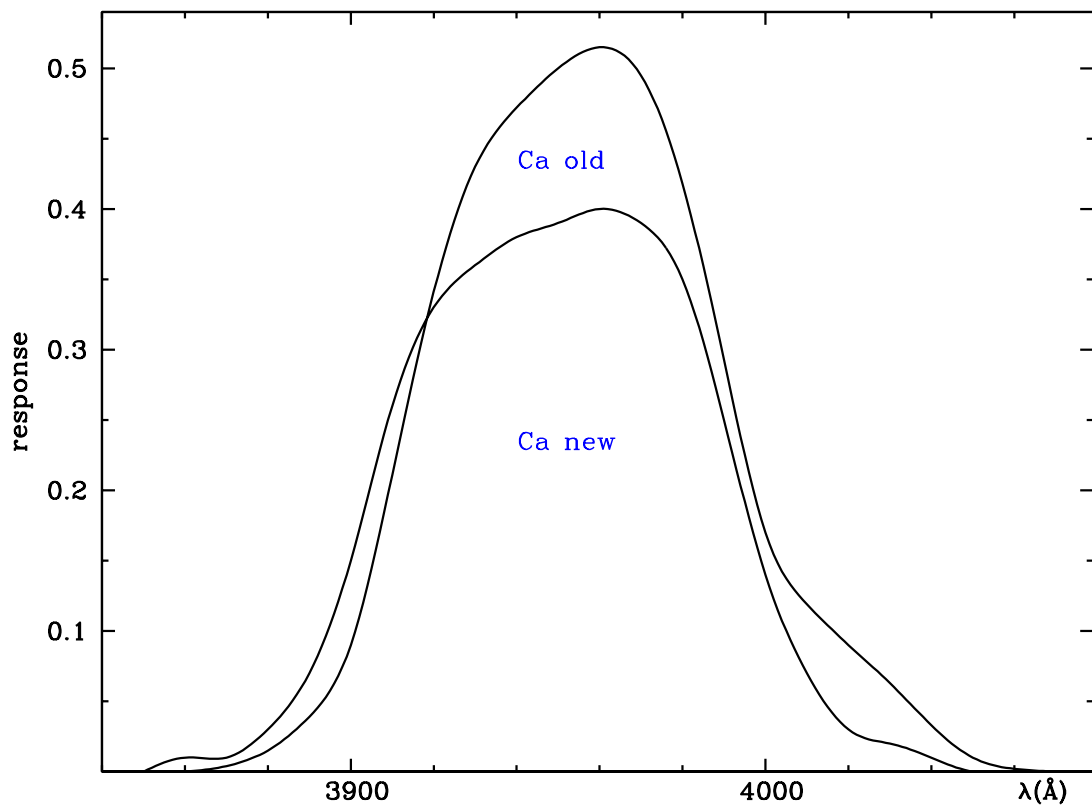

\begin{tabular}{|cc|cc|}
\hline Ca & old & Ca & new \\
\hline$\lambda(\mathrm{A})$ & $\Upsilon$ & $\lambda$ (A) & $\Upsilon$ \\
\hline 3850 & 0.000 & 3850 & 0.000 \\
3860 & 0.000 & 3860 & 0.010 \\
3870 & 0.004 & 3870 & 0.010 \\
3880 & 0.015 & 3880 & 0.030 \\
3890 & 0.039 & 3890 & 0.070 \\
3900 & 0.090 & 3900 & 0.150 \\
3910 & 0.211 & 3910 & 0.260 \\
3920 & 0.341 & 3920 & 0.330 \\
3930 & 0.430 & 3930 & 0.360 \\
3940 & 0.472 & 3940 & 0.380 \\
3950 & 0.500 & 3950 & 0.390 \\
3960 & 0.515 & 3960 & 0.400 \\
3970 & 0.495 & 3970 & 0.390 \\
3980 & 0.418 & 3980 & 0.350 \\
3990 & 0.293 & 3990 & 0.250 \\
4000 & 0.170 & 4000 & 0.140 \\
4010 & 0.119 & 4010 & 0.070 \\
4020 & 0.090 & 4020 & 0.030 \\
4030 & 0.063 & 4030 & 0.020 \\
4040 & 0.033 & 4040 & 0.010 \\
4050 & 0.010 & 4050 & 0.000 \\
4060 & 0.002 & 4060 & 0.000 \\
4070 & 0.000 & 4070 & 0.000 \\
\hline & & & \\
\hline
\end{tabular}

Fig. 142. The photometric system CaII - Twarog et al. - 1991 


\section{0 colors - Bastiaansen - 1992}

Narrow band photometric system. Used to derive the interstellar extinction toward hot stars.

\section{GENERAL INFORMATION}

AUTHORS

TELESCOPE

DETECTOR

P. A. Bastiaansen

$0.50 \mathrm{~m}$ (reflector), ESO (La Silla)

340-520 nm range: EMI 6256B, (S-11 cathode, thermo-electrically cooled)

541-787 nm range: EMI 9558B, (S-20 cathode, refrigerated)

MAIN ARTICLE Bastiaansen, P. A. 1992, A\&AS 93, 449

\section{SYSTEM DESCRIPTION}

\begin{tabular}{|c|c|c|c|c|c|c|c|}
\hline \multicolumn{8}{|c|}{ BANDS DESCRIPTION [23] } \\
\hline band & $\lambda_{0}(\AA)$ & band-width $(\AA)$ & feature & band & $\lambda_{0}(\AA)$ & band-width $(\AA)$ & feature \\
\hline 340 & 3402 & 42 & & 523 & 5205 & 94 & \\
\hline 350 & 3493 & 40 & & 543 & 5407 & 100 & HeII 5415 \\
\hline 360 & 3600 & 52 & & 560 & 5601 & 104 & \\
\hline 378 & 3785 & 48 & & 582 & 5821 & 98 & HeI 5876 \\
\hline 405 & 4038 & 46 & $\mathrm{H} \delta, \mathrm{H} \varepsilon, \mathrm{HeI} 4026$ & 609 & 6107 & 84 & \\
\hline 418 & 4192 & 104 & Hס, HeII 4200 & 637 & 6400 & 114 & $\mathrm{H} \alpha$ \\
\hline 438 & 4395 & 44 & $\mathrm{H} \gamma$ & 666 & 6681 & 86 & H $\alpha$, HeI 6687 \\
\hline 451 & 4496 & 84 & HeI 4471, HeII 4541 & 710 & 7102 & 92 & HeI 7065 \\
\hline 468 & 4708 & 96 & H $\beta$, HeII 4686 & 749 & 7505 & 78 & \\
\hline 502 & 4999 & 82 & H $\beta$, HeI 4922, HeI 5015 & 790 & 7873 & 80 & \\
\hline
\end{tabular}

TRANSMISSION CURVES

As derived from Fig 1 of [23].

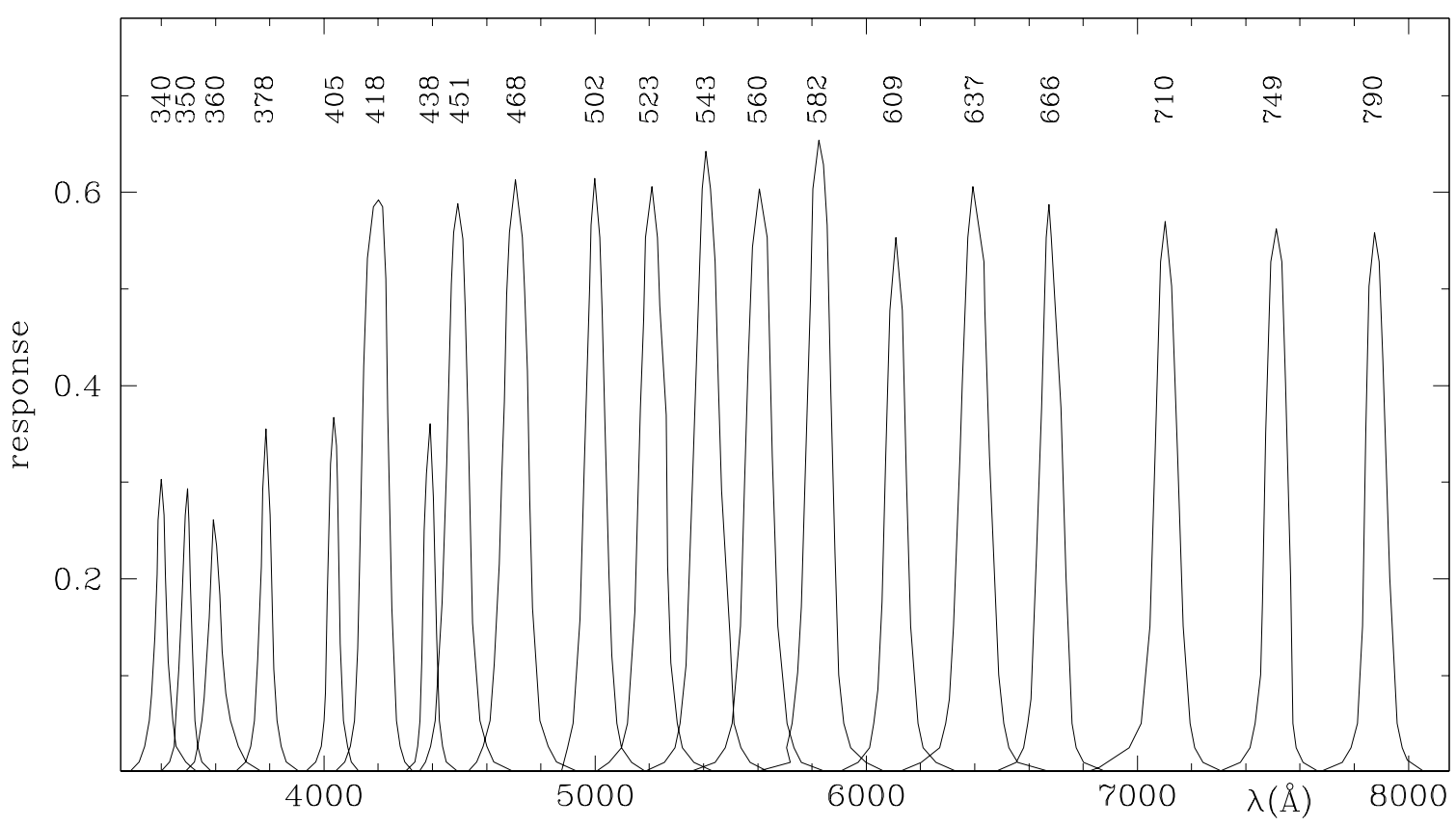

Fig. 143. The photometric system 20 colors - Bastiaansen - 1992 


\begin{tabular}{|c|c|c|c|c|c|c|c|c|c|c|c|c|c|}
\hline \multicolumn{2}{|c|}{340} & \multicolumn{2}{|c|}{350} & \multicolumn{2}{|c|}{360} & \multicolumn{2}{|c|}{378} & \multicolumn{2}{|c|}{405} & \multicolumn{2}{|c|}{418} & \multicolumn{2}{|c|}{438} \\
\hline$\lambda(\AA)$ & $\Upsilon$ & $\lambda(\AA)$ & $\Upsilon$ & $\lambda(\AA)$ & $\Upsilon$ & $\lambda(\AA)$ & $\Upsilon$ & $\lambda(\AA)$ & $\Upsilon$ & $\lambda(\AA)$ & $\Upsilon$ & $\lambda(\AA)$ & $\Upsilon$ \\
\hline 3282 & 0.000 & 3396 & 0.000 & 3487 & 0.000 & 3672 & 0.000 & 3931 & 0.000 & 4039 & 0.000 & 4298 & 0.000 \\
\hline 3318 & 0.005 & 3430 & 0.011 & 3522 & 0.011 & 3711 & 0.011 & 3969 & 0.011 & 4077 & 0.011 & 4336 & 0.011 \\
\hline 3339 & 0.011 & 3447 & 0.027 & 3535 & 0.027 & 3730 & 0.027 & 3989 & 0.027 & 4097 & 0.027 & 4345 & 0.027 \\
\hline 3356 & 0.027 & 3453 & 0.053 & 3550 & 0.053 & 3744 & 0.053 & 4000 & 0.053 & 4112 & 0.053 & 4354 & 0.053 \\
\hline 3364 & 0.053 & 3465 & 0.136 & 3558 & 0.081 & 3755 & 0.106 & 4004 & 0.133 & 4124 & 0.170 & 4362 & 0.144 \\
\hline 3376 & 0.114 & 3477 & 0.197 & 3577 & 0.125 & 3770 & 0.165 & 4011 & 0.245 & 4131 & 0.372 & 4370 & 0.285 \\
\hline 3384 & 0.202 & 3488 & 0.258 & 3585 & 0.184 & 3774 & 0.266 & 4024 & 0.301 & 4146 & 0.511 & 4378 & 0.361 \\
\hline 3389 & 0.266 & 3496 & 0.294 & 3593 & 0.234 & 3787 & 0.355 & 4037 & 0.338 & 4161 & 0.585 & 4392 & 0.309 \\
\hline 3399 & 0.303 & 3502 & 0.266 & 3605 & 0.261 & 3801 & 0.293 & 4046 & 0.367 & 4183 & 0.592 & 4403 & 0.250 \\
\hline 3410 & 0.261 & 3508 & 0.180 & 3616 & 0.219 & 3809 & 0.213 & 4049 & 0.319 & 4201 & 0.585 & 4415 & 0.117 \\
\hline 3416 & 0.205 & 3513 & 0.106 & 3624 & 0.160 & 3816 & 0.116 & 4053 & 0.184 & 4217 & 0.532 & 4426 & 0.053 \\
\hline 3426 & 0.139 & 3523 & 0.053 & 3638 & 0.077 & 3827 & 0.053 & 4060 & 0.082 & 4228 & 0.420 & 4438 & 0.027 \\
\hline 3442 & 0.081 & 3535 & 0.027 & 3655 & 0.053 & 3841 & 0.027 & 4073 & 0.053 & 4236 & 0.213 & 4451 & 0.011 \\
\hline 3454 & 0.053 & 3549 & 0.011 & 3683 & 0.027 & 3861 & 0.011 & 4087 & 0.027 & 4251 & 0.128 & 4495 & 0.000 \\
\hline 3489 & 0.027 & 3588 & 0.000 & 3713 & 0.011 & 3911 & 0.000 & 4101 & 0.011 & 4266 & 0.053 & & \\
\hline 3510 & 0.011 & & & 3773 & 0.000 & & & 4131 & 0.000 & 4281 & 0.027 & & \\
\hline 3534 & 0.000 & & & & & & & & & 4298 & 0.011 & & \\
\hline \multicolumn{2}{|c|}{451} & \multicolumn{2}{|c|}{468} & \multicolumn{2}{|c|}{502} & \multicolumn{2}{|c|}{523} & \multicolumn{2}{|c|}{543} & \multicolumn{2}{|c|}{560} & & \\
\hline$\lambda(\AA)$ & $\Upsilon$ & $\lambda(\AA)$ & $\Upsilon$ & $\lambda(\AA)$ & $\Upsilon$ & $\lambda(\AA)$ & $\Upsilon$ & $\lambda(\AA)$ & $\Upsilon$ & $\lambda(\AA)$ & $\Upsilon$ & $\lambda(\AA)$ & $\Upsilon$ \\
\hline 4350 & 0.000 & 4530 & 0.000 & 4823 & 0.000 & 5000 & 0.000 & 5179 & 0.000 & 5347 & 0.000 & 5590 & 0.000 \\
\hline 4374 & 0.011 & 4563 & 0.011 & 4876 & 0.010 & 5051 & 0.010 & 5256 & 0.010 & 5442 & 0.010 & 5702 & 0.010 \\
\hline 4393 & 0.027 & 4588 & 0.027 & 4898 & 0.025 & 5098 & 0.025 & 5294 & 0.025 & 5481 & 0.025 & 5707 & 0.025 \\
\hline 4411 & 0.053 & 4612 & 0.053 & 4918 & 0.050 & 5119 & 0.050 & 5313 & 0.050 & 5505 & 0.050 & 5727 & 0.050 \\
\hline 4436 & 0.154 & 4628 & 0.170 & 4945 & 0.121 & 5146 & 0.113 & 5336 & 0.143 & 5536 & 0.151 & 5747 & 0.101 \\
\hline 4453 & 0.261 & 4647 & 0.282 & 4959 & 0.201 & 5168 & 0.214 & 5359 & 0.292 & 5552 & 0.327 & 5760 & 0.226 \\
\hline 4470 & 0.370 & 4656 & 0.415 & 4980 & 0.362 & 5179 & 0.370 & 5382 & 0.402 & 5565 & 0.468 & 5774 & 0.392 \\
\hline 4478 & 0.481 & 4665 & 0.497 & 4985 & 0.478 & 5186 & 0.483 & 5394 & 0.528 & 5580 & 0.553 & 5794 & 0.566 \\
\hline 4493 & 0.552 & 4674 & 0.553 & 4999 & 0.553 & 5209 & 0.553 & 5408 & 0.604 & 5605 & 0.604 & 5803 & 0.629 \\
\hline 4513 & 0.588 & 4684 & 0.613 & 5018 & 0.615 & 5229 & 0.606 & 5426 & 0.642 & 5635 & 0.543 & 5825 & 0.654 \\
\hline 4521 & 0.559 & 4706 & 0.559 & 5025 & 0.566 & 5239 & 0.553 & 5442 & 0.604 & 5641 & 0.427 & 5842 & 0.604 \\
\hline 4530 & 0.505 & 4731 & 0.495 & 5036 & 0.503 & 5262 & 0.468 & 5454 & 0.503 & 5654 & 0.302 & 5856 & 0.493 \\
\hline 4539 & 0.319 & 4740 & 0.383 & 5051 & 0.312 & 5267 & 0.377 & 5466 & 0.302 & 5674 & 0.151 & 5870 & 0.302 \\
\hline 4548 & 0.176 & 4750 & 0.301 & 5061 & 0.158 & 5278 & 0.166 & 5496 & 0.111 & 5708 & 0.050 & 5884 & 0.171 \\
\hline 4576 & 0.053 & 4760 & 0.215 & 5080 & 0.050 & 5303 & 0.050 & 5512 & 0.050 & 5733 & 0.025 & 5898 & 0.103 \\
\hline 4600 & 0.027 & 4769 & 0.112 & 5098 & 0.025 & 5322 & 0.025 & 5537 & 0.025 & 5760 & 0.010 & 5918 & 0.050 \\
\hline 4626 & 0.011 & 4797 & 0.053 & 5135 & 0.001 & 5365 & 0.010 & 5571 & 0.010 & 5856 & 0.000 & 5942 & 0.025 \\
\hline 4702 & 0.000 & 4826 & 0.027 & 5189 & 0.000 & 5442 & 0.000 & 5641 & 0.000 & & & 5999 & 0.010 \\
\hline & & 4858 & 0.011 & & & & & & & & & 6073 & 0.000 \\
\hline & & & & & & & & & & & & & \\
\hline$\lambda(\AA)$ & $\Upsilon$ & $\lambda(\AA)$ & $\Upsilon$ & $\lambda(\AA)$ & $\Upsilon$ & $\lambda(\AA)$ & $\Upsilon$ & $\lambda(\AA)$ & $\Upsilon$ & $\lambda(\AA)$ & $\Upsilon$ & & \\
\hline 5898 & 0.000 & 6118 & 0.000 & 6472 & 0.000 & 6812 & 0.000 & 7296 & 0.000 & 7671 & 0.000 & & \\
\hline 5970 & 0.010 & 6203 & 0.010 & 6553 & 0.010 & 6859 & 0.010 & 7379 & 0.010 & 7756 & 0.013 & & \\
\hline 6013 & 0.025 & 6271 & 0.025 & 6581 & 0.025 & 6970 & 0.025 & 7415 & 0.025 & 7788 & 0.025 & & \\
\hline 6028 & 0.050 & 6293 & 0.050 & 6596 & 0.050 & 7014 & 0.050 & 7435 & 0.050 & 7812 & 0.050 & & \\
\hline 6043 & 0.151 & 6306 & 0.101 & 6607 & 0.201 & 7046 & 0.151 & 7455 & 0.201 & 7830 & 0.201 & & \\
\hline 6058 & 0.302 & 6322 & 0.327 & 6628 & 0.377 & 7066 & 0.327 & 7462 & 0.402 & 7842 & 0.427 & & \\
\hline 6073 & 0.478 & 6346 & 0.478 & 6646 & 0.553 & 7086 & 0.503 & 7473 & 0.528 & 7855 & 0.528 & & \\
\hline 6088 & 0.553 & 6355 & 0.528 & 6664 & 0.587 & 7103 & 0.570 & 7492 & 0.562 & 7880 & 0.558 & & \\
\hline 6109 & 0.478 & 6374 & 0.606 & 6673 & 0.553 & 7127 & 0.528 & 7512 & 0.528 & 7893 & 0.503 & & \\
\hline 6133 & 0.327 & 6393 & 0.553 & 6682 & 0.377 & 7148 & 0.327 & 7533 & 0.352 & 7905 & 0.352 & & \\
\hline 6148 & 0.176 & 6434 & 0.402 & 6719 & 0.226 & 7169 & 0.151 & 7547 & 0.176 & 7931 & 0.151 & & \\
\hline 6164 & 0.085 & 6438 & 0.327 & 6737 & 0.075 & 7194 & 0.050 & 7565 & 0.101 & 7959 & 0.050 & & \\
\hline 6192 & 0.050 & 6455 & 0.151 & 6759 & 0.050 & 7211 & 0.025 & 7575 & 0.050 & 7977 & 0.025 & & \\
\hline 6210 & 0.025 & 6489 & 0.075 & 6774 & 0.025 & 7243 & 0.005 & 7586 & 0.025 & 7995 & 0.010 & & \\
\hline 6258 & 0.010 & 6508 & 0.050 & 6802 & 0.010 & 7318 & 0.000 & 7612 & 0.010 & 8061 & 0.000 & & \\
\hline 6338 & 0.000 & 6526 & 0.025 & 6888 & 0.000 & & & 7671 & 0.000 & & & & \\
\hline & & 6558 & 0.010 & & & & & & & & & & \\
\hline
\end{tabular}

Fig. 143. continued 


\section{MACHO - 1992}

MACHO Project photometric system.

\section{GENERAL INFORMATION}

TELESCOPE $\quad 1.27 \mathrm{~m}$ (reector), Mt. Stromlo Obs.

DETECTOR 2048 X 2048 CCDs

MAIN ARTICLE Bessell, M. S., Germany, L. M. 1999, PASP 111, 1421

\section{SYSTEM DESCRIPTION}

\begin{tabular}{|c|cc|}
\hline \multicolumn{3}{|c|}{ BANDS DESCRIPTION } \\
\hline band & $W H M(A)$ & $\lambda_{c}(\mathrm{~A})$ \\
\hline$B_{m a}$ & 1350 & 5225 \\
\hline$R_{m a}$ & 1600 & 6950 \\
\hline
\end{tabular}

\section{SYSTEM ANALYSIS}

\section{REDDENING RATIOS [35]}

$E\left(B_{m a}-R_{m a}\right) / E(B-R)_{\text {Cousins }}=1.01$ derived from Landolt's F stars.

\section{RELATIONS WITH OTHER SYSTEMS}

$$
\text { RI - Cousins - } 1976 \text { [35] }
$$

\begin{tabular}{|lll|}
\hline$V=B_{m a}-0.18\left(B_{m a}-R_{m a}\right)$ & for $\left(B_{m a}-R_{m a}\right)<1.0$ \\
\hline$V=B_{m a}-0.07-0.10\left(B_{m a}-R_{m a}\right)$ & for $\left(B_{m a}-R_{m a}\right)>1.0$ \\
\hline$R=R_{m a}+0.19\left(B_{m a}-R_{m a}\right)$ & for $\left(B_{m a}-R_{m a}\right)<1.0$ \\
\hline$R=R_{m a}-0.07+0.26\left(B_{m a}-R_{m a}\right)$ & for $\left(B_{m a}-R_{m a}\right)>1.0$ \\
\hline
\end{tabular}

\section{TRANSMISSION CURVES [35]}

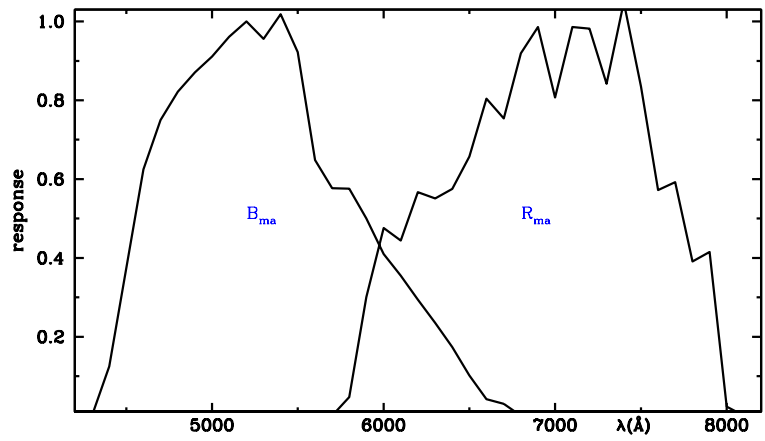

\begin{tabular}{|cc|cc|cc|cc|}
\hline \multicolumn{4}{|c|}{$B_{m a}$} & \multicolumn{4}{c|}{$R_{m a}$} \\
\hline$\lambda(\mathrm{A})$ & $\Upsilon$ & $\lambda(\mathrm{A})$ & $\Upsilon$ & $\lambda(\mathrm{A})$ & $\Upsilon$ & $\lambda(\mathrm{A})$ & $\Upsilon$ \\
\hline 4300 & 0.000 & 5600 & 0.648 & 5700 & 0.000 & 7000 & 0.807 \\
4400 & 0.125 & 5700 & 0.577 & 5800 & 0.047 & 7100 & 0.986 \\
4500 & 0.375 & 5800 & 0.576 & 5900 & 0.301 & 7200 & 0.982 \\
4600 & 0.625 & 5900 & 0.500 & 6000 & 0.476 & 7300 & 0.842 \\
4700 & 0.750 & 6000 & 0.410 & 6100 & 0.444 & 7400 & 1.049 \\
4800 & 0.822 & 6100 & 0.355 & 6200 & 0.567 & 7500 & 0.835 \\
4900 & 0.871 & 6200 & 0.294 & 6300 & 0.551 & 7600 & 0.572 \\
5000 & 0.911 & 6300 & 0.235 & 6400 & 0.575 & 7700 & 0.592 \\
5100 & 0.961 & 6400 & 0.174 & 6500 & 0.657 & 7800 & 0.391 \\
5200 & 1.000 & 6500 & 0.102 & 6600 & 0.804 & 7900 & 0.415 \\
5300 & 0.956 & 6600 & 0.042 & 6700 & 0.754 & 8000 & 0.023 \\
5400 & 1.018 & 6700 & 0.030 & 6800 & 0.919 & 8100 & 0.000 \\
5500 & 0.922 & 6800 & 0.000 & 6900 & 0.986 & & \\
\hline
\end{tabular}

Fig. 144. The photometric system MACHO - 1992 


\section{SCAS - Clark et al. - 1993}

Taxonomy of asteroids.

\section{GENERAL INFORMATION}

AUTHORS $\quad$ B. E. Clark, J. F. Bell, P. Fanale, and P. G. Lucey

TELESCOPE 2.3m IRTF, Mauna Kea Obs.

MAIN ARTICLE Clark, B. E., Bell, J. F., Fanale, F. P., Lucey, P. G. Lun. Plan. Inst. 24, 299

\section{SYSTEM DESCRIPTION}

\begin{tabular}{|c|cc|}
\hline \multicolumn{3}{|c|}{ BANDS DESCRIPTION } \\
\hline band & $W H M(\mu \mathrm{m})$ & $\lambda_{c}(\mu \mathrm{m})$ \\
\hline$A$ & 0.085 & 0.913 \\
\hline$B$ & 0.086 & 1.052 \\
\hline$C$ & 0.105 & 1.300 \\
\hline$D$ & 0.137 & 1.545 \\
\hline$E$ & 0.141 & 1.648 \\
\hline$F$ & 0.172 & 2.157 \\
\hline$G$ & 0.204 & 2.281 \\
\hline
\end{tabular}

TRANSMISSION CURVES

As derived from Fig 1 of [68].

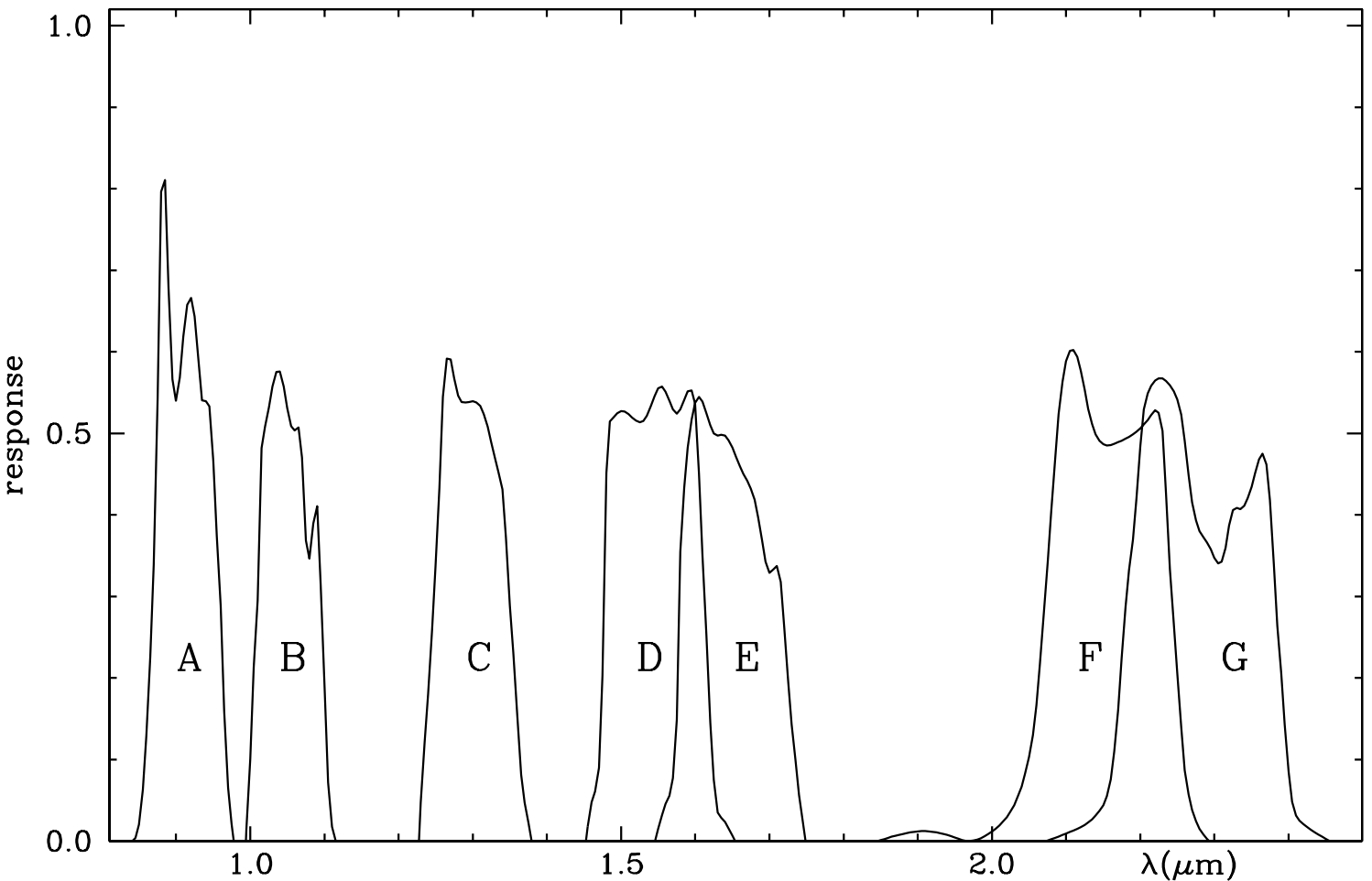

Fig. 145. The photometric system SCAS - Clark et al. - 1993 


\begin{tabular}{|c|c|c|c|c|c|c|c|c|c|c|c|c|c|}
\hline \multicolumn{2}{|c|}{$A$} & \multicolumn{2}{|c|}{$B$} & \multicolumn{2}{|c|}{$C$} & \multicolumn{2}{|c|}{$D$} & \multicolumn{2}{|c|}{$E$} & \multicolumn{2}{|c|}{$F$} & \multicolumn{2}{|c|}{$G$} \\
\hline$\lambda(\mu \mathrm{m})$ & $\Upsilon$ & $\lambda(\mu \mathrm{m})$ & $\Upsilon$ & $\lambda(\mu \mathrm{m})$ & $\Upsilon$ & $\lambda(\mu \mathrm{m})$ & $\Upsilon$ & $\lambda(\mu \mathrm{m})$ & $\Upsilon$ & $\lambda(\mu \mathrm{m})$ & $\Upsilon$ & $\lambda(\mu \mathrm{m})$ & $\Upsilon$ \\
\hline 0.791 & 0.000 & 0.994 & 0.000 & 1.227 & 0.000 & 1.453 & 0.000 & 1.546 & 0.000 & 1.846 & 0.000 & 2.074 & 0.000 \\
\hline 0.842 & 0.000 & 1.000 & 0.101 & 1.233 & 0.101 & 1.461 & 0.050 & 1.562 & 0.050 & 1.887 & 0.010 & 2.110 & 0.013 \\
\hline 0.854 & 0.050 & 1.004 & 0.201 & 1.242 & 0.201 & 1.471 & 0.101 & 1.572 & 0.101 & 1.907 & 0.012 & 2.147 & 0.039 \\
\hline 0.858 & 0.101 & 1.010 & 0.302 & 1.248 & 0.302 & 1.475 & 0.201 & 1.576 & 0.201 & 1.928 & 0.010 & 2.165 & 0.111 \\
\hline 0.864 & 0.201 & 1.012 & 0.402 & 1.254 & 0.402 & 1.477 & 0.302 & 1.578 & 0.302 & 1.948 & 0.005 & 2.173 & 0.201 \\
\hline 0.868 & 0.302 & 1.018 & 0.503 & 1.258 & 0.503 & 1.479 & 0.402 & 1.583 & 0.402 & 1.968 & 0.000 & 2.181 & 0.302 \\
\hline 0.872 & 0.402 & 1.039 & 0.578 & 1.278 & 0.553 & 1.481 & 0.492 & 1.593 & 0.503 & 1.988 & 0.005 & 2.194 & 0.402 \\
\hline 0.874 & 0.503 & 1.059 & 0.503 & 1.288 & 0.538 & 1.483 & 0.513 & 1.605 & 0.545 & 2.009 & 0.018 & 2.202 & 0.503 \\
\hline 0.876 & 0.603 & 1.071 & 0.452 & 1.315 & 0.525 & 1.501 & 0.528 & 1.623 & 0.503 & 2.033 & 0.050 & 2.212 & 0.553 \\
\hline 0.878 & 0.724 & 1.075 & 0.367 & 1.335 & 0.452 & 1.534 & 0.520 & 1.639 & 0.497 & 2.049 & 0.101 & 2.228 & 0.568 \\
\hline 0.892 & 0.613 & 1.085 & 0.392 & 1.343 & 0.402 & 1.554 & 0.558 & 1.664 & 0.452 & 2.064 & 0.201 & 2.244 & 0.553 \\
\hline 0.913 & 0.643 & 1.093 & 0.362 & 1.349 & 0.302 & 1.576 & 0.525 & 1.684 & 0.402 & 2.072 & 0.302 & 2.258 & 0.503 \\
\hline 0.929 & 0.603 & 1.095 & 0.302 & 1.357 & 0.201 & 1.599 & 0.543 & 1.700 & 0.329 & 2.080 & 0.402 & 2.265 & 0.452 \\
\hline 0.933 & 0.553 & 1.099 & 0.201 & 1.363 & 0.101 & 1.603 & 0.503 & 1.714 & 0.322 & 2.088 & 0.503 & 2.273 & 0.402 \\
\hline 0.947 & 0.513 & 1.104 & 0.101 & 1.369 & 0.050 & 1.607 & 0.402 & 1.725 & 0.201 & 2.120 & 0.575 & 2.293 & 0.362 \\
\hline 0.953 & 0.402 & 1.116 & 0.000 & 1.380 & 0.000 & 1.613 & 0.302 & 1.735 & 0.101 & 2.131 & 0.528 & 2.313 & 0.352 \\
\hline 0.959 & 0.302 & & & & & 1.617 & 0.201 & 1.741 & 0.050 & 2.165 & 0.487 & 2.323 & 0.402 \\
\hline 0.963 & 0.201 & & & & & 1.623 & 0.101 & 1.749 & 0.000 & 2.202 & 0.508 & 2.334 & 0.407 \\
\hline 0.968 & 0.101 & & & & & 1.627 & 0.050 & & & 2.216 & 0.524 & 2.354 & 0.447 \\
\hline 0.972 & 0.050 & & & & & 1.633 & 0.030 & & & 2.230 & 0.503 & 2.376 & 0.402 \\
\hline 0.978 & 0.000 & & & & & 1.654 & 0.000 & & & 2.232 & 0.482 & 2.382 & 0.302 \\
\hline & & & & & & & & & & 2.236 & 0.402 & 2.390 & 0.201 \\
\hline & & & & & & & & & & 2.242 & 0.302 & 2.399 & 0.101 \\
\hline & & & & & & & & & & 2.250 & 0.201 & 2.405 & 0.050 \\
\hline & & & & & & & & & & 2.258 & 0.101 & 2.415 & 0.025 \\
\hline & & & & & & & & & & 2.267 & 0.050 & 2.455 & 0.000 \\
\hline & & & & & & & & & & 2.293 & 0.000 & & \\
\hline
\end{tabular}

Fig. 145. continued 


\section{WFPC2 HST - 1993}

Photometric bands of the Wide Field and Planetary Camera 2 on board HST.

\section{GENERAL INFORMATION}

TELESCOPE $\quad 2.4 \mathrm{~m}$ Hubble Space Telescope

MAIN ARTICLE Biretta, J. A. et al. 1996, WFPC2 Instrument Handbook, Version 4.0 (Baltimore, STScI)

\section{SYSTEM DESCRIPTION}

\begin{tabular}{|c|c|c|c|c|c|c|}
\hline \multicolumn{7}{|c|}{ BANDS DESCRIPTION [36], pg 37} \\
\hline band & $\lambda_{0}(\AA)$ & $\lambda_{\text {peak }}(\AA)$ & FWHM $(\AA)$ & $\Upsilon_{\text {peak }}(\%)$ & Notes & In WF/PC-1 (\#) \\
\hline F122M & 1292 & 1240 & 263.5 & 18.9 & Ly $\alpha$ & $\mathrm{Y}$ \\
\hline F160AW & 1471 & 1403 & 457.2 & 10.1 & Woods A & \\
\hline$F 160 B W$ & 1471 & 1403 & 457.2 & 10.1 & Woods B & \\
\hline F170W & 1689 & 1667 & 434.9 & 30.3 & & \\
\hline F185W & 1907 & 1849 & 302.9 & 23.7 & & \\
\hline F218W & 2136 & 2091 & 355.9 & 21.3 & Interstellar Feature & \\
\hline$F 255 W$ & 2557 & 2483 & 408.2 & 15.5 & & \\
\hline F300W & 2924 & 2760 & 727.9 & 51.8 & Wide U & \\
\hline F336W & 3327 & 3447 & 370.7 & 80.3 & WFPC2 U, Strömgren $u$ & $\mathrm{Y}$ \\
\hline F343N & 3430 & 3433 & 24.3 & 19.7 & $\mathrm{NeV}$ & \\
\hline F375N & 3736 & 3736 & 26.2 & 17.2 & [OII] $3727 \mathrm{RS}$ & $\mathrm{Y}$ \\
\hline F380W & 3934 & 3981 & 694.7 & 65.6 & & \\
\hline F390N & 3889 & 3886 & 45.3 & 37.8 & $\mathrm{CN}$ & \\
\hline F410M & 4088 & 4098 & 146.9 & 70.1 & Strömgren $v$ & \\
\hline$F 437 N$ & 4369 & 4368 & 25.2 & 52.0 & [OIII] & $\mathrm{Y}$ \\
\hline F439W & 4292 & 4176 & 464.4 & 67.3 & WFPC2 B & $\mathrm{Y}$ \\
\hline F450W & 4445 & 5061 & 925.0 & 92.4 & Wide B & \\
\hline F467M & 4682 & 4728 & 171.5 & 75.3 & Strömgren $b$ & \\
\hline F469N & 4695 & 4699 & 24.9 & 52.4 & He II & $\mathrm{Y}$ \\
\hline F487N & 4865 & 4863 & 25.8 & 58.6 & $\mathrm{H} \beta$ & $\mathrm{Y}$ \\
\hline F502N & 5012 & 5009 & 26.8 & 63.8 & [OIII] & $\mathrm{Y}$ \\
\hline F547M & 5454 & 5361 & 486.6 & 91.3 & Strömgren $y$, but wider & $\mathrm{Y}$ \\
\hline F555W & 5252 & 5151 & 1222.5 & 94.8 & WFPC2 V & $\mathrm{Y}$ \\
\hline F569W & 5554 & 5309 & 965.8 & 94.2 & & $\mathrm{Y}$ \\
\hline F588N & 5892 & 5895 & 49.1 & 91.5 & $\mathrm{He}$ I \& Na I (NaD) & $\mathrm{Y}$ \\
\hline
\end{tabular}

Fig. 146. The photometric system WFPC2 HST - 1993 


\begin{tabular}{|c|c|c|c|c|c|c|}
\hline \multicolumn{7}{|c|}{ BANDS DESCRIPTION [36], pg 37} \\
\hline band & $\lambda_{0}(\mathrm{~A})$ & $\lambda_{\text {peak }}(\mathrm{A})$ & FWHM (A) & $\Upsilon_{\text {peak }}(\%)$ & Notes & In WF/PC-1 (\#) \\
\hline F606W & 5843 & 6183 & 1578.7 & 98.3 & Wide V & $\mathrm{Y}$ \\
\hline F622W & 6157 & 6034 & 935.4 & 95.6 & & $\mathrm{Y}$ \\
\hline F631N & 6306 & 6302 & 30.8 & 85.7 & [OI] & $\mathrm{Y}$ \\
\hline F656N & 6562 & 6561 & 22.0 & 77.9 & $\mathrm{H} \alpha$ & $\mathrm{Y}$ \\
\hline F658N & 6590 & 6592 & 28.5 & 79.7 & {$[\mathrm{NII}]$} & $\mathrm{Y}$ \\
\hline F673N & 6733 & 6733 & 47.2 & 87.0 & {$[\mathrm{SII}]$} & $\mathrm{Y}$ \\
\hline F675W & 6735 & 6796 & 889.4 & 97.9 & WFPC2 R & $\mathrm{Y}$ \\
\hline F702W & 6997 & 6539 & 1480.7 & 98.5 & Wide R & $\mathrm{Y}$ \\
\hline F791W & 8006 & 8081 & 1304.2 & 99.7 & & $\mathrm{Y}$ \\
\hline F814W & 8269 & 8386 & 1758.0 & 98.4 & WFPC2 I & $\mathrm{Y}$ \\
\hline F953N & 9546 & 9528 & 52.5 & 95.6 & [SiIII] & \\
\hline F1042M & 10443 & 10139 & 610.9 & 95.2 & & $\mathrm{Y}$ \\
\hline
\end{tabular}

(\#) It is indicated if an equivalent lter was available in the Wide Field and Planetary Camera 1.

The long-pass lters (F130LP, F165LP, F785LP, F850LP), the Quad and the Ramp lters are not listed.

\section{TRANSMISSION CURVES [36]}

To save space the transmission proles of the bands are plotted but not tabulated. The proles can be obtained in tabular form via STSDAS (inside Iraf) or from the ADPS $w w w$ site.

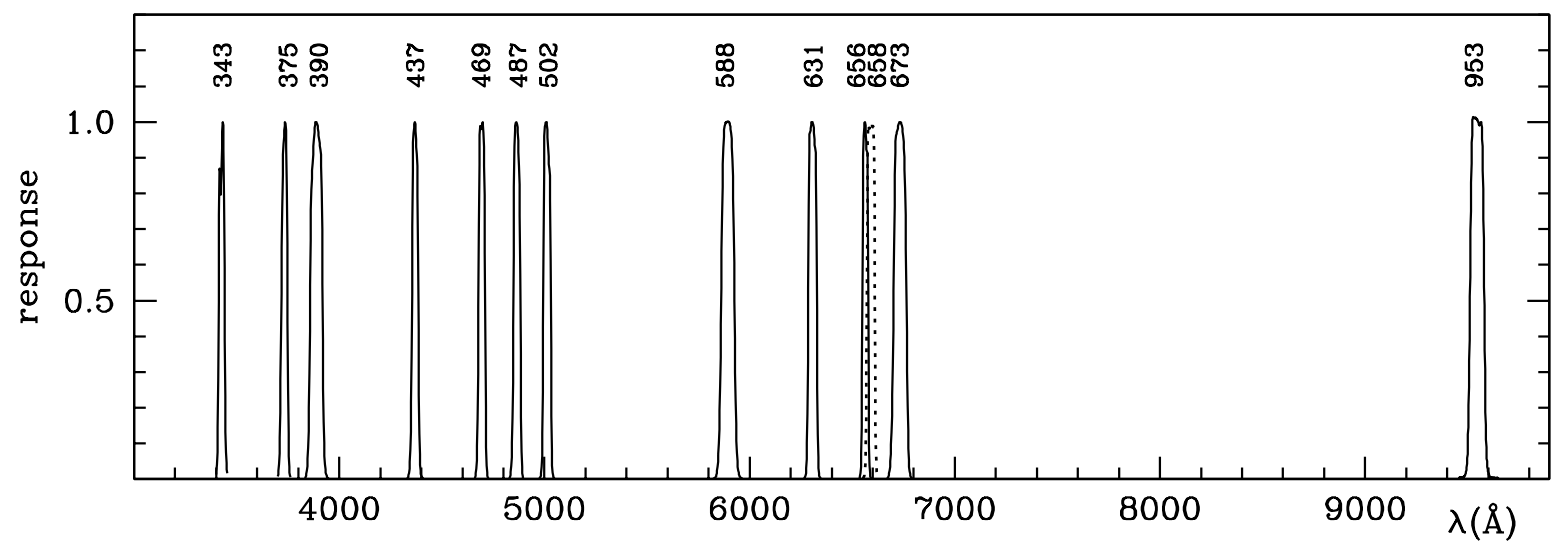

Fig. 146. continued 

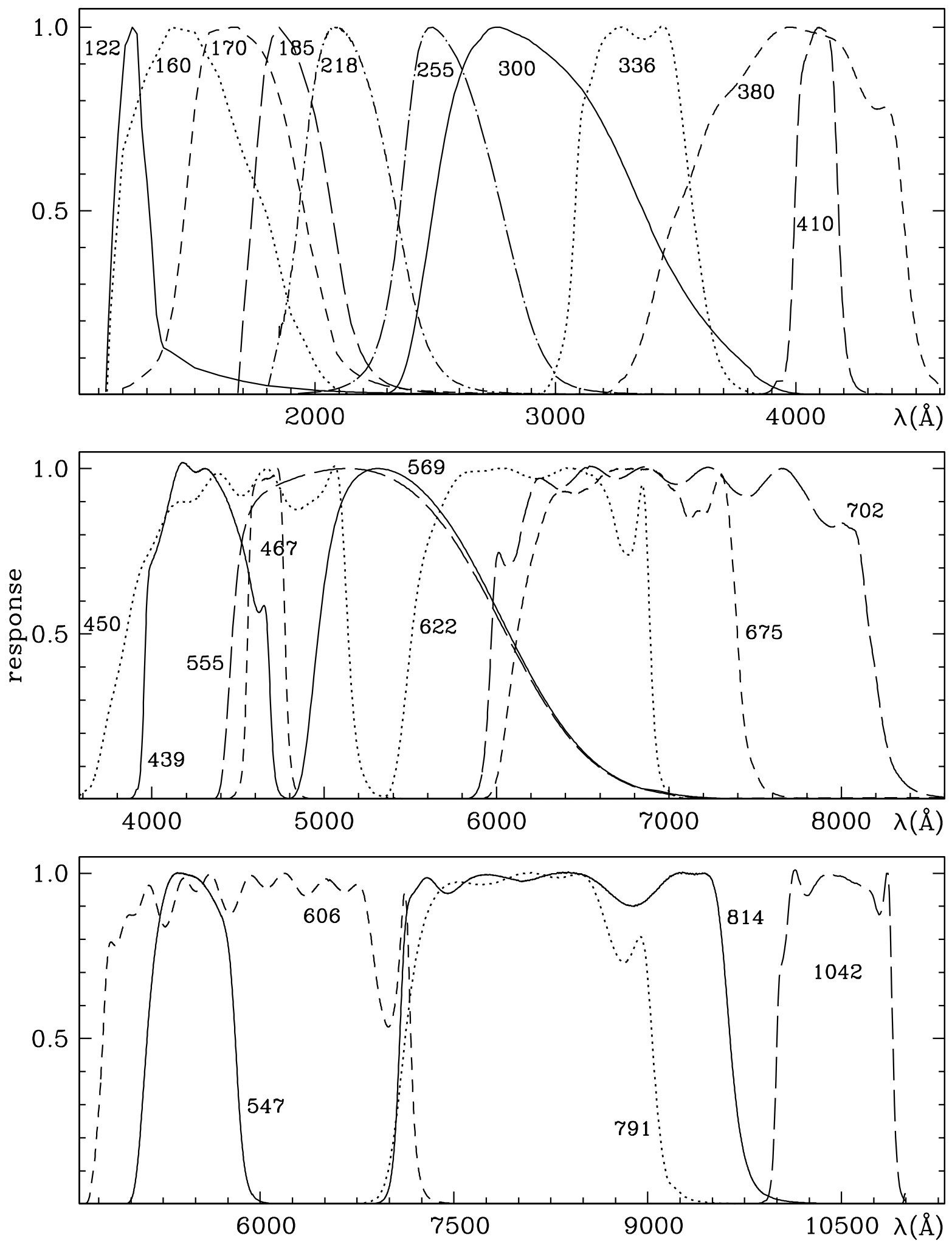

Fig. 146. continued 


\section{JHKL' CST - Alonso et al. - 1994}

Infrared photometry at Teide Observatory.

\section{GENERAL INFORMATION}

AUTHORS

TELESCOPE

DETECTOR

MAIN ARTICLE
A. Alonso, S. Arribas, C. Martinez-Roger

$1.54 \mathrm{~m}$ (reflector) Carlos Sánchez Telescope, Teide Obs. $\mathrm{InSb}$

Alonso, A., Arribas, S., Martinez-Roger, C. 1994, A\&AS 107, 365

\section{SYSTEM DESCRIPTION}

\begin{tabular}{|c|c|cc|c|}
\hline \multicolumn{2}{|c|}{ BANDS DESCRIPTION [7] } & & & FLUX CALIBRATION [7] \\
\hline band & $\lambda_{\text {eff }}(\mu \mathrm{m})(\#)$ & $W H M(\mu \mathrm{m})$ & $\lambda_{c}(\mu \mathrm{m})$ & $\mathrm{F}(\lambda)\left(\mathrm{erg} \mathrm{cm}^{-2} \mathrm{~s}^{-1} \mathrm{~nm}^{-1}\right)(\#)$ \\
\hline$J$ & 1.2790 & 0.128 & 1.310 & $29.1210^{-10}$ \\
\hline$H$ & 1.6483 & 0.274 & 1.661 & $10.9210^{-10}$ \\
\hline$K$ & 2.1869 & 0.399 & 2.166 & $4.2610^{-10}$ \\
\hline$L^{\prime}$ & & 0.614 & 3.678 & \\
\hline
\end{tabular}

(\#) Calculated for $\alpha$ Lyr.

\section{SYSTEM ANALYSIS}

REDDENING RATIOS [8]
$E_{J}=1.172 E_{H}+0.045$
$E_{J}=1.350 E_{K}-0.003$
$E_{L^{\prime}}=0.55 E_{J}+0.04$

RELATIONS WITH OTHER SYSTEMS [8]

\section{UBVRI(JHKLMN) - Johnson - 1965}

\begin{tabular}{|rcr|}
\hline$J_{C S T}$ & $=J_{J}-0.040-0.064(J-K)_{J}$ & \\
\hline$H_{C S T}$ & $=H_{J}-0.020-0.034(J-K)_{J}$ & \\
\hline$K_{C S T}$ & $=K_{J}-0.042+0.019(J-K)_{J}$ & \\
\hline$L_{C S T}^{\prime}$ & $=L_{J}+0.04-0.016(J-K)_{J}$ & \\
\hline$(J-K)_{C S T}$ & $=0.008+0.910(J-K)_{J}$ & for $-0.20<(J-K)_{C S T}<1.20$ \\
\hline$(J-H)_{C S T}$ & $=-0.010+0.942(J-H)_{J}$ & for $-0.10<(J-H)_{C S T}<0.90$ \\
\hline$\left(J-L^{\prime}\right)_{C S T}$ & $=-0.080+0.955(J-L)_{J}$ & for $-0.25<(J-L)_{C S T}<1.30$ \\
\hline$(V-K)_{C S T}$ & $=0.050+0.993(V-K)_{J}$ & for $-0.60<(V-K)_{C S T}<5.50$ \\
\hline
\end{tabular}

JHKL CTIO - Elias et al. - 1982

\begin{tabular}{|rll|}
\hline$J_{C S T}$ & $=J_{C T I O}-0.035+0.019(J-K)_{C T I O}$ & \\
\hline$H_{C S T}$ & $=H_{C T I O}-0.025+0.030(J-K)_{C T I O}$ & \\
\hline$K_{C S T}$ & $=K_{C T I O}-0.022+0.006(J-K)_{C T I O}$ & \\
\hline$(J-K)_{C S T}$ & $=-0.015+1.014(J-K)_{C T I O}$ & for $-0.15<(J-K)_{C S T}<1.10$ \\
\hline$(J-H)_{C S T}$ & $=-0.008+0.980(J-H)_{C T I O}$ & for $-0.08<(J-H)_{C S T}<0.85$ \\
\hline$(V-K)_{C S T}$ & $=0.022+0.998(V-K)_{C T I O}$ & for $-1.50<(V-K)_{C S T}<6.00$ \\
\hline
\end{tabular}

Fig. 147. The photometric system JHKL' CST - Alonso et al. - 1994 
$J_{n} K_{n} L_{n} M_{n}$ - Leggett et al. 1986

\begin{tabular}{|rlll|}
\hline$J_{C S T}$ & $=J_{n}-0.035+0.009(J-K)_{n}$ & for $-0.2<(J-K)_{C S T}<1.1$ \\
\hline$K_{C S T}$ & $=K_{n}-0.035+0.075(J-K)_{n}$ & & for $-0.2<(J-K)_{C S T}<1.3$ \\
\hline$L_{C S T}^{\prime}$ & $=L_{n}^{\prime}+0.04+0.015(J-K)_{n}$ & & \\
\hline$(V-K)_{C S T}$ & $=0.043+0.978(V-K)_{n}$ & for $-0.5<(V-K)_{C S T}<4.8$ \\
\hline
\end{tabular}

JHKL'M ESO - Bouchet et al - 1991

\begin{tabular}{|c|c|c|c|}
\hline$J_{C S T}$ & $=$ & $J_{E S O}-0.047-0.102(J-K)_{E S O}$ & \\
\hline$H_{C S T}$ & $=$ & $H_{E S O}-0.068+0.016(J-K)_{E S O}$ & \\
\hline$K_{C S T}$ & $=$ & $K_{E S O}-0.042+0.006(J-K)_{E S O}$ & \\
\hline$L_{C S T}^{\prime}$ & $=$ & $L_{E S O}^{\prime}+0.037-0.057(J-K)_{E S O}$ & \\
\hline$(J-K)_{C S T}$ & $=$ & $-0.012+0.910(J-K)_{E S O}$ & for $-0.15<(J-K)_{C S T}<0.90$ \\
\hline$(J-H)_{C S T}$ & $=$ & $0.010+0.883(J-H)_{E S O}$ & for $-0.10<(J-H)_{C S T}<0.70$ \\
\hline$\left(J-L^{\prime}\right)_{C S T}$ & $=$ & $-0.073+0.946\left(J-L^{\prime}\right)_{E S O}$ & for $-0.2<(J-H)_{C S T}<1.0$ \\
\hline$(V-K)_{C S T}$ & $=$ & $0.039+(V-K)_{E S O}$ & for $-0.9<(V-K)_{C S T}<3.5$ \\
\hline
\end{tabular}

TRANSMISSION CURVES

$J, H$ and $K$ bands are from [7]. $L$ ' band is from [8].

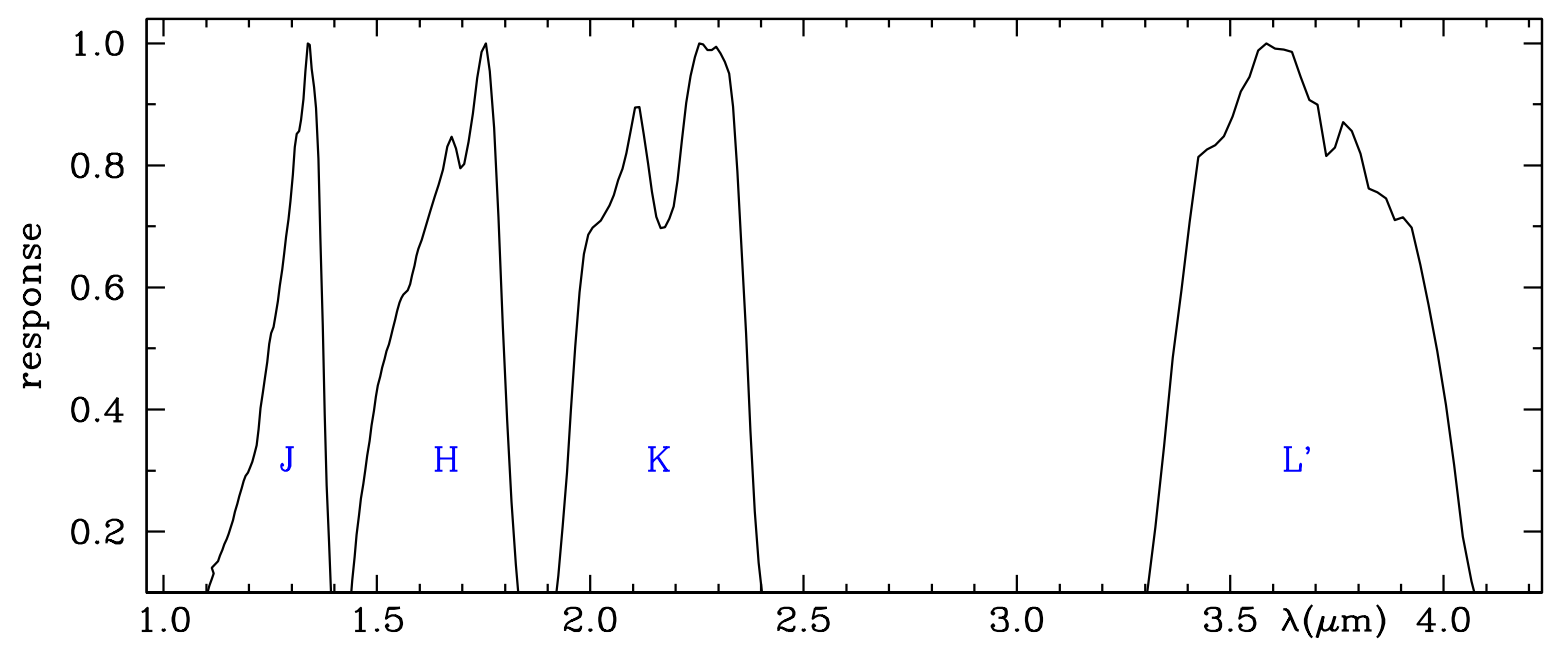

\begin{tabular}{|cc|cc|cc|cc|cc|cc|}
\hline \multicolumn{10}{|c|}{$J$} \\
\hline$\lambda(\mu \mathrm{m})$ & $\Upsilon$ & $\lambda(\mu \mathrm{m})$ & $\Upsilon$ & $\lambda(\mu \mathrm{m})$ & $\Upsilon$ & $\lambda(\mu \mathrm{m})$ & $\Upsilon$ & $\lambda(\mu \mathrm{m})$ & $\Upsilon$ & $\lambda(\mu \mathrm{m})$ & $\Upsilon$ \\
\hline 0.98500 & 0.0000 & 1.04750 & 0.0186 & 1.13250 & 0.1611 & 1.21750 & 0.3411 & 1.30250 & 0.7840 & 1.38750 & 0.1827 \\
0.98700 & 0.0001 & 1.05250 & 0.0252 & 1.13750 & 0.1703 & 1.22250 & 0.3679 & 1.30750 & 0.8299 & 1.39250 & 0.1010 \\
0.98900 & 0.0002 & 1.05750 & 0.0315 & 1.14250 & 0.1793 & 1.22750 & 0.4021 & 1.31250 & 0.8513 & 1.39750 & 0.0375 \\
0.99100 & 0.0003 & 1.06250 & 0.0375 & 1.14750 & 0.1880 & 1.23250 & 0.4287 & 1.31750 & 0.8559 & 1.40250 & 0.0054 \\
0.99300 & 0.0004 & 1.06750 & 0.0434 & 1.15250 & 0.1966 & 1.23750 & 0.4507 & 1.32250 & 0.8750 & 1.40750 & 0.0033 \\
0.99500 & 0.0005 & 1.07250 & 0.0511 & 1.15750 & 0.2060 & 1.24250 & 0.4782 & 1.32750 & 0.9084 & 1.41250 & 0.0018 \\
0.99700 & 0.0007 & 1.07750 & 0.0598 & 1.16250 & 0.2185 & 1.24750 & 0.5074 & 1.33250 & 0.9542 & 1.41750 & 0.0010 \\
0.99900 & 0.0009 & 1.08250 & 0.0674 & 1.16750 & 0.2328 & 1.25250 & 0.5249 & 1.33750 & 1.0000 & 1.42250 & 0.0007 \\
1.00250 & 0.0010 & 1.08750 & 0.0741 & 1.17250 & 0.2460 & 1.25750 & 0.5353 & 1.34250 & 0.9970 & 1.42750 & 0.0003 \\
1.00750 & 0.0013 & 1.09250 & 0.0804 & 1.17750 & 0.2583 & 1.26250 & 0.5534 & 1.34750 & 0.9580 & 1.42350 & 0.0000 \\
1.01250 & 0.0014 & 1.09750 & 0.0875 & 1.18250 & 0.2712 & 1.26750 & 0.5778 & 1.35250 & 0.9282 & \\
1.01750 & 0.0015 & 1.10250 & 0.0984 & 1.18750 & 0.2831 & 1.27250 & 0.6030 & 1.35750 & 0.8940 & \\
1.02250 & 0.0021 & 1.10750 & 0.1114 & 1.19250 & 0.2909 & 1.27750 & 0.6289 & 1.36250 & 0.8104 & \\
1.02750 & 0.0031 & 1.11250 & 0.1221 & 1.19750 & 0.2963 & 1.28250 & 0.6558 & 1.36750 & 0.6811 & \\
1.03250 & 0.0045 & 1.11750 & 0.1311 & 1.20250 & 0.3044 & 1.28750 & 0.6836 & 1.37250 & 0.5375 & \\
1.03750 & 0.0068 & 1.11250 & 0.1410 & 1.20750 & 0.3146 & 1.29250 & 0.7115 & 1.37750 & 0.3920 & \\
1.04250 & 0.0118 & 1.12750 & 0.1514 & 1.21250 & 0.3259 & 1.29750 & 0.7412 & 1.38250 & 0.2754 &
\end{tabular}

Fig. 147. continued 


\begin{tabular}{|c|c|c|c|c|c|c|c|c|c|c|c|}
\hline \multicolumn{4}{|c|}{$H$} & \multicolumn{4}{|c|}{$K$} & \multicolumn{4}{|c|}{$L^{\prime}$} \\
\hline$\lambda(\mu \mathrm{m})$ & $\Upsilon$ & $\lambda(\mu \mathrm{m})$ & $\Upsilon$ & $\lambda(\mu \mathrm{m})$ & $\Upsilon$ & $\lambda(\mu \mathrm{m})$ & $\Upsilon$ & $\lambda(\mu \mathrm{m})$ & $\Upsilon$ & $\lambda(\mu \mathrm{m})$ & $\Upsilon$ \\
\hline 1.3425 & 0.0000 & 1.5625 & 0.5879 & 1.8550 & 0.0000 & 2.2950 & 0.9939 & 3.160 & 0.000 & 3.925 & 0.698 \\
\hline 1.3475 & 0.0004 & 1.5675 & 0.5918 & 1.8650 & 0.0020 & 2.3050 & 0.9834 & 3.170 & 0.001 & 3.945 & 0.637 \\
\hline 1.3525 & 0.0005 & 1.5725 & 0.5952 & 1.8750 & 0.0054 & 2.3150 & 0.9692 & 3.180 & 0.002 & 3.965 & 0.571 \\
\hline 1.3575 & 0.0007 & 1.5775 & 0.6053 & 1.8850 & 0.0105 & 2.3250 & 0.9505 & 3.190 & 0.002 & 3.985 & 0.496 \\
\hline 1.3625 & 0.0008 & 1.5825 & 0.6198 & 1.8950 & 0.0150 & 2.3350 & 0.8961 & 3.200 & 0.003 & 4.005 & 0.408 \\
\hline 1.3675 & 0.0011 & 1.5875 & 0.6353 & 1.9050 & 0.0306 & 2.3450 & 0.7898 & 3.210 & 0.002 & 4.025 & 0.309 \\
\hline 1.3725 & 0.0014 & 1.5925 & 0.6514 & 1.9150 & 0.0678 & 2.3550 & 0.6575 & 3.220 & 0.003 & 4.045 & 0.192 \\
\hline 1.3775 & 0.0018 & 1.5975 & 0.6635 & 1.9250 & 0.1278 & 2.3650 & 0.5251 & 3.230 & 0.002 & 4.065 & 0.118 \\
\hline 1.3825 & 0.0026 & 1.6050 & 0.6776 & 1.9350 & 0.2086 & 2.3750 & 0.3653 & 3.240 & 0.004 & 4.085 & 0.064 \\
\hline 1.3875 & 0.0036 & 1.6150 & 0.7015 & 1.9450 & 0.2969 & 2.3850 & 0.2336 & 3.250 & 0.004 & 4.105 & 0.033 \\
\hline 1.3925 & 0.0046 & 1.6250 & 0.7259 & 1.9550 & 0.3987 & 2.3950 & 0.1495 & 3.260 & 0.009 & 4.125 & 0.014 \\
\hline 1.3975 & 0.0057 & 1.6350 & 0.7489 & 1.9650 & 0.5027 & 2.4050 & 0.0834 & 3.270 & 0.016 & 4.145 & 0.006 \\
\hline 1.4025 & 0.0075 & 1.6450 & 0.7680 & 1.9750 & 0.5921 & 2.4150 & 0.0464 & 3.285 & 0.034 & 4.165 & 0.001 \\
\hline 1.4075 & 0.0100 & 1.6550 & 0.7925 & 1.9850 & 0.6548 & 2.4250 & 0.0256 & 3.305 & 0.097 & 4.185 & 0.000 \\
\hline 1.4125 & 0.0130 & 1.6650 & 0.8305 & 1.9950 & 0.6863 & 2.4350 & 0.0120 & 3.325 & 0.207 & 4.205 & 0.000 \\
\hline 1.4175 & 0.0175 & 1.6750 & 0.8466 & 2.0050 & 0.6981 & & & 3.345 & 0.340 & & \\
\hline 1.4225 & 0.0275 & 1.6850 & 0.8275 & 2.0150 & 0.7029 & & & 3.365 & 0.485 & & \\
\hline 1.4275 & 0.0429 & 1.6950 & 0.7951 & 2.0250 & 0.7093 & & & 3.385 & 0.595 & & \\
\hline 1.4325 & 0.0627 & 1.7050 & 0.8020 & 2.0350 & 0.7218 & & & 3.405 & 0.707 & & \\
\hline 1.4375 & 0.0875 & 1.7150 & 0.8387 & 2.0450 & 0.7341 & & & 3.425 & 0.814 & & \\
\hline 1.4425 & 0.1199 & 1.7250 & 0.8846 & 2.0550 & 0.7512 & & & 3.445 & 0.826 & & \\
\hline 1.4475 & 0.1583 & 1.7350 & 0.9433 & 2.0650 & 0.7755 & & & 3.465 & 0.833 & & \\
\hline 1.4525 & 0.1951 & 1.7450 & 0.9854 & 2.0750 & 0.7946 & & & 3.485 & 0.848 & & \\
\hline 1.4575 & 0.2261 & 1.7550 & 1.0000 & 2.0850 & 0.8199 & & & 3.505 & 0.880 & & \\
\hline 1.4625 & 0.2539 & 1.7650 & 0.9543 & 2.0950 & 0.8571 & & & 3.525 & 0.921 & & \\
\hline 1.4675 & 0.2777 & 1.7750 & 0.8618 & 2.1050 & 0.8947 & & & 3.545 & 0.945 & & \\
\hline 1.4725 & 0.3003 & 1.7850 & 0.7145 & 2.1150 & 0.8955 & & & 3.565 & 0.988 & & \\
\hline 1.4775 & 0.3241 & 1.7950 & 0.5363 & 2.1250 & 0.8507 & & & 3.585 & 1.000 & & \\
\hline 1.4825 & 0.3486 & 1.8050 & 0.3825 & 2.1350 & 0.8030 & & & 3.605 & 0.991 & & \\
\hline 1.4875 & 0.3737 & 1.8150 & 0.2487 & 2.1450 & 0.7562 & & & 3.625 & 0.990 & & \\
\hline 1.4925 & 0.3980 & 1.8250 & 0.1458 & 2.1550 & 0.7160 & & & 3.645 & 0.986 & & \\
\hline 1.4975 & 0.4208 & 1.8350 & 0.0771 & 2.1650 & 0.6972 & & & 3.665 & 0.945 & & \\
\hline 1.5025 & 0.4393 & 1.8450 & 0.0324 & 2.1750 & 0.6990 & & & 3.685 & 0.907 & & \\
\hline 1.5075 & 0.4537 & 1.8550 & 0.0151 & 2.1850 & 0.7126 & & & 3.705 & 0.899 & & \\
\hline 1.5125 & 0.4684 & 1.8650 & 0.0061 & 2.1950 & 0.7327 & & & 3.725 & 0.815 & & \\
\hline 1.5175 & 0.4828 & 1.8750 & 0.0043 & 2.2050 & 0.7753 & & & 3.745 & 0.829 & & \\
\hline 1.5225 & 0.4955 & 1.8850 & 0.0033 & 2.2150 & 0.8404 & & & 3.765 & 0.871 & & \\
\hline 1.5275 & 0.5067 & 1.8950 & 0.0030 & 2.2250 & 0.9025 & & & 3.785 & 0.856 & & \\
\hline 1.5325 & 0.5187 & 1.9050 & 0.0024 & 2.2350 & 0.9460 & & & 3.805 & 0.819 & & \\
\hline 1.5375 & 0.5317 & 1.9150 & 0.0014 & 2.2450 & 0.9770 & & & 3.825 & 0.762 & & \\
\hline 1.5425 & 0.5473 & 1.9250 & 0.0012 & 2.2550 & 1.0000 & & & 3.845 & 0.756 & & \\
\hline 1.5475 & 0.5616 & 1.9350 & 0.0005 & 2.2650 & 0.9980 & & & 3.865 & 0.746 & & \\
\hline 1.5525 & 0.5750 & 1.9450 & 0.0000 & 2.2750 & 0.9890 & & & 3.885 & 0.710 & & \\
\hline 1.5575 & 0.5831 & & & 2.2850 & 0.9891 & & & 3.905 & 0.715 & & \\
\hline
\end{tabular}

Fig. 147. continued 


\section{DENIS - Epchtein et al. - 1994}

Photometric system for the Deep Infrared Southern Sky Survey.

\section{GENERAL INFORMATION}

AUTHORS N. Epchtein, B. de Batz, E. Copet, et al.

TELESCOPE $\quad 1.0 \mathrm{~m}$ (reflector), ESO (La Silla)

DETECTOR Tektronix $1 \mathrm{~K} \mathrm{CCD} \mathrm{(i} \mathrm{band),} \mathrm{(cooled} \mathrm{at} 180 \mathrm{~K}$ );

NICMOS-3 CCD (cooled at 80K)

MAIN ARTICLE Epchtein, N., de Batz, B., Copet, E., et al. 1994, Ap\&SS 217, 3

\section{SYSTEM DESCRIPTION}

\begin{tabular}{|c|cc|cc|cc|}
\hline \multicolumn{3}{|c|}{ BANDS DESCRIPTION [107] } & & \multicolumn{2}{c|}{ FLUX CALIBRATION [107](\#\#) } \\
\hline band & $\lambda_{0}(\mu \mathrm{m})$ & $\lambda_{\text {eff }}(\mu \mathrm{m})(\#)$ & $W H M(\mu \mathrm{m})$ & $\lambda_{c}(\mu \mathrm{m})$ & $\mathrm{F}_{\lambda}\left(\mathrm{W} \mathrm{m}^{-2} \mu \mathrm{m}^{-1}\right)$ & $\mathrm{F}_{v}(\mathrm{Jy})$ \\
\hline$I$ & 0.795 & 0.788 & 0.1312 & 0.925 & $1.20 \cdot 10^{-8}$ & 2499 \\
\hline$J$ & 1.235 & 1.221 & 0.199 & 1.249 & $3.17 \cdot 10^{-9}$ & 1595 \\
\hline$K_{S}$ & 2.160 & 2.144 & 0.330 & 2.151 & $4.34 \cdot 10^{-10}$ & 665 \\
\hline
\end{tabular}

(\#) For $\alpha$ Lyr.

(\#\#) For a 0.0mag star.

Fig. 148. The photometric system DENIS - Epchtein et al. - 1994 
As derived from Fig 3 of [107]

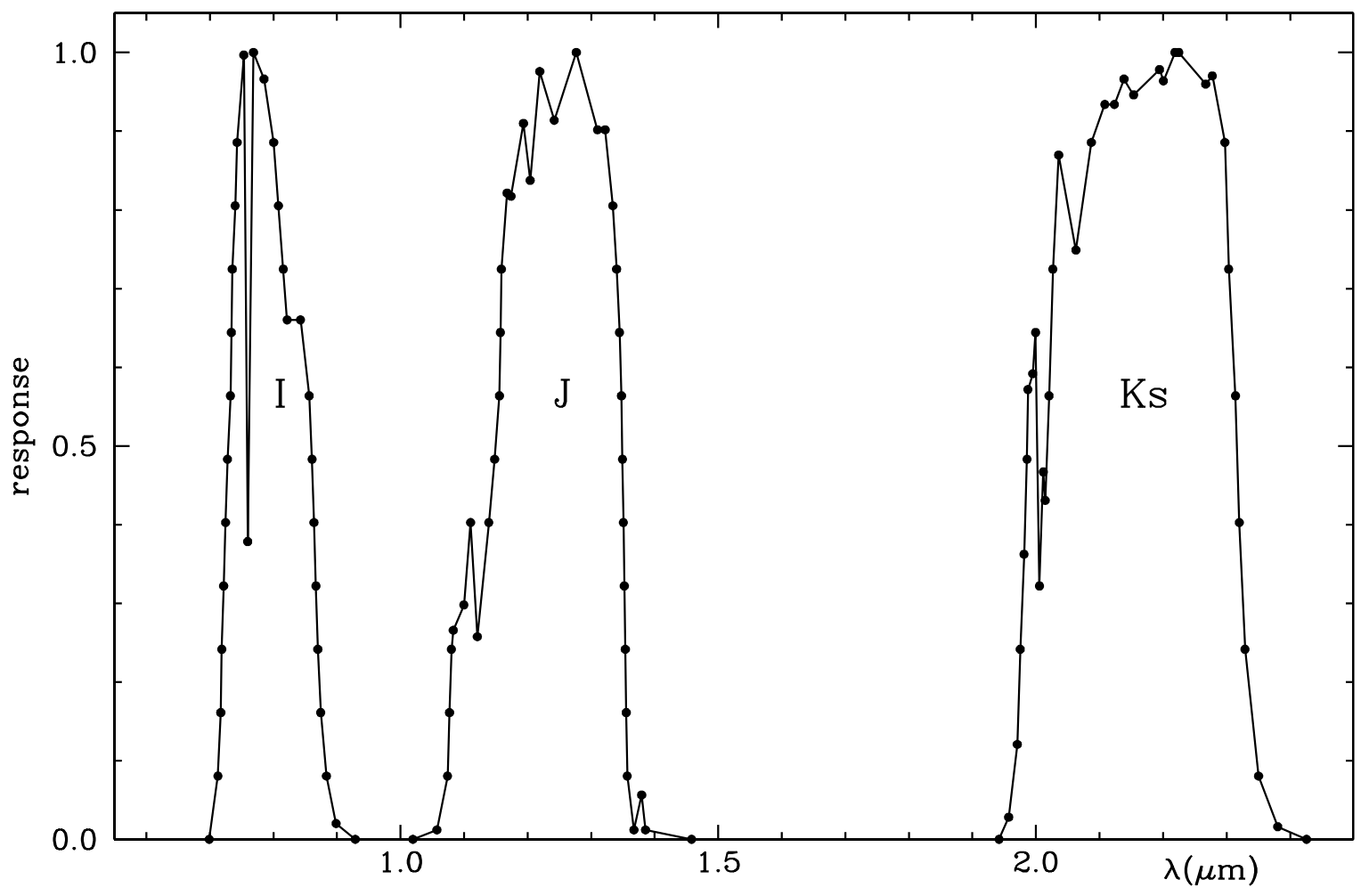

\begin{tabular}{|cc|cc|cc|ccc|cc|cc|}
\hline \multicolumn{7}{|c}{$I$} & \multicolumn{9}{c|}{$J$} & \multicolumn{4}{c|}{$K S$} \\
\hline$\lambda(\mu \mathrm{m})$ & $\Upsilon$ & $\lambda(\mu \mathrm{m})$ & $\Upsilon$ & $\lambda(\mu \mathrm{m})$ & $\Upsilon$ & $\lambda(\mu \mathrm{m})$ & $\Upsilon$ & $\lambda(\mu \mathrm{m})$ & $\Upsilon$ & $\lambda(\mu \mathrm{m})$ & $\Upsilon$ \\
\hline 0.699 & 0.000 & 0.822 & 0.660 & 1.020 & 0.000 & 1.242 & 0.914 & 1.942 & 0.000 & 2.139 & 0.966 \\
0.713 & 0.081 & 0.843 & 0.660 & 1.057 & 0.012 & 1.277 & 1.000 & 1.957 & 0.028 & 2.154 & 0.946 \\
0.717 & 0.161 & 0.856 & 0.564 & 1.074 & 0.081 & 1.310 & 0.902 & 1.971 & 0.121 & 2.195 & 0.978 \\
0.719 & 0.242 & 0.861 & 0.483 & 1.077 & 0.161 & 1.322 & 0.902 & 1.975 & 0.242 & 2.201 & 0.964 \\
0.722 & 0.322 & 0.864 & 0.403 & 1.080 & 0.242 & 1.334 & 0.805 & 1.981 & 0.362 & 2.219 & 1.000 \\
0.725 & 0.403 & 0.867 & 0.322 & 1.083 & 0.266 & 1.340 & 0.725 & 1.986 & 0.483 & 2.225 & 1.000 \\
0.728 & 0.483 & 0.870 & 0.242 & 1.100 & 0.298 & 1.345 & 0.644 & 1.987 & 0.572 & 2.267 & 0.960 \\
0.732 & 0.564 & 0.875 & 0.161 & 1.110 & 0.403 & 1.348 & 0.564 & 1.995 & 0.592 & 2.278 & 0.970 \\
0.734 & 0.644 & 0.884 & 0.081 & 1.121 & 0.258 & 1.349 & 0.483 & 1.999 & 0.644 & 2.297 & 0.886 \\
0.735 & 0.725 & 0.899 & 0.020 & 1.139 & 0.403 & 1.351 & 0.403 & 2.006 & 0.322 & 2.303 & 0.725 \\
0.740 & 0.805 & 0.929 & 0.000 & 1.148 & 0.483 & 1.352 & 0.322 & 2.012 & 0.467 & 2.314 & 0.564 \\
0.743 & 0.886 & & & 1.156 & 0.564 & 1.354 & 0.242 & 2.015 & 0.431 & 2.320 & 0.403 \\
0.754 & 0.997 & & & 1.157 & 0.644 & 1.355 & 0.161 & 2.021 & 0.564 & 2.329 & 0.242 \\
0.760 & 0.378 & & & 1.159 & 0.725 & 1.357 & 0.081 & 2.027 & 0.725 & 2.350 & 0.081 \\
0.769 & 1.000 & & & 1.168 & 0.821 & 1.367 & 0.012 & 2.036 & 0.870 & 2.381 & 0.016 \\
0.785 & 0.966 & & & 1.174 & 0.817 & 1.380 & 0.056 & 2.063 & 0.749 & 2.426 & 0.000 \\
0.800 & 0.886 & & & 1.194 & 0.910 & 1.386 & 0.012 & 2.087 & 0.886 & \\
0.808 & 0.805 & & & 1.204 & 0.837 & 1.458 & 0.000 & 2.108 & 0.934 & & \\
0.816 & 0.725 & & & 1.219 & 0.976 & & & 2.123 & 0.934 & \\
\hline
\end{tabular}

Fig. 148. continued 


\section{JHKL MSSSO - McGregor - 1994}

Infrared photometry at Mount Stromlo and Siding Spring Observatories.

\section{GENERAL INFORMATION}

$\begin{array}{ll}\text { AUTHORS } & \text { P. J. McGregor } \\ \text { TELESCOPE } & \text { ANU 2.3m (reflector), Siding Spring Obs. } \\ \text { DETECTOR } & \text { InSb (cooled) } \\ \text { MAIN ARTICLE } & \text { Mc Gregor, P. J. 1994, PASP 106, 508 }\end{array}$

\section{SYSTEM ANALYSIS}

\section{RELATIONS WITH OTHER SYSTEMS [204]}

JHKL CTIO - Elias et al - 1982

\begin{tabular}{|c|c|c|c|}
\hline$K_{\text {CTIO }}$ & $=$ & $K_{M S S S O}+0.004( \pm 0.006)(J-K)_{M S S S O}-0.015( \pm 0.007)$ & for $(J-K)<3.0$ \\
\hline$(J-K)_{C T I O}$ & $=$ & $0.947( \pm 0.004)(J-K)_{M S S S O}+0.006( \pm 0.004)$ & for $(J-K)<3.0$ \\
\hline$(H-K)_{C T I O}$ & $=$ & $0.966( \pm 0.009)(H-K)_{M S S S O}+0.010( \pm 0.003)$ & for $(H-K)<0.9$ \\
\hline$(J-H)_{C T I O}$ & $=$ & $0.937( \pm 0.005)(J-H)_{M S S S O}-0.003( \pm 0.004)$ & for $(J-H)<2.1$ \\
\hline$(K-L)_{C T I O}$ & $=$ & $0.751( \pm 0.10)(K-L)_{M S S S O}+0.02( \pm 0.01)$ & for $(K-L)<0.2$ \\
\hline
\end{tabular}

JHKL MSO - Jones and Hyland - 1982

\begin{tabular}{|rll|}
\hline$K_{M S O}$ & $=K_{M S S O}-0.010( \pm 0.008)(J-K)_{M S S S O}+0.000( \pm 0.004)$ & for $(J-K)<0.9$ \\
\hline$(J-K)_{M S O}$ & $=0.997( \pm 0.006)(J-K)_{M S S O}+0.009( \pm 0.003)$ & for $(J-K)<0.9$ \\
\hline$(H-K)_{M S O}=0.971( \pm 0.023)(H-K)_{M S S S O}+0.014( \pm 0.003)$ & for $(H-K)<0.3$ \\
\hline$(J-H)_{M S O}=1.002( \pm 0.007)(J-H)_{M S S S O}-0.004( \pm 0.003)$ & for $(J-H)<0.6$ \\
\hline
\end{tabular}

JHKL' AAO - Allen and Cragg - 1983

\begin{tabular}{|c|c|c|c|}
\hline$K_{A A O}$ & $=$ & $K_{M S S S O}+0.003( \pm 0.004)(J-K)_{M S S S O}+0.005( \pm 0.005)$ & for $(J-K)<4.0$ \\
\hline$(J-K)_{A A O}$ & - & $1.051( \pm 0.004)(J-K)_{M S S S O}+0.006( \pm 0.004)$ & for $(J-K)<4.0$ \\
\hline$(H-K)_{A A O}$ & $=$ & $1.018( \pm 0.007)(H-K)_{M S S S O}+0.009( \pm 0.003)$ & for $(H-K)<1.2$ \\
\hline$(J-H)_{A A O}$ & $=$ & $1.065( \pm 0.004)(J-H)_{M S S S O}-0.005( \pm 0.004)$ & for $(J-H)<2.8$ \\
\hline$\left(K-L^{\prime}\right)_{A A O}$ & $=$ & $1.496( \pm 0.037)(K-L)_{M S S S O}+0.010( \pm 0.004)$ & for $(K-L)<0.27$ \\
\hline
\end{tabular}

JHKLM - Bessell and Brett - 1988

\begin{tabular}{rl|}
\hline$(J-K)$ & $=1.026(J-K)_{M S S O}+0.005$ \\
\hline$(H-K)$ & $=0.997(H-K)_{M S S O}+0.007$ \\
\hline$(J-H)$ & $=1.028(J-H)_{M S S O}$ \\
\hline
\end{tabular}

Fig. 149. The photometric system JHKL MSSSO - McGregor - 1994 
JHKL SAAO - Carter - 1990

\begin{tabular}{|rrrr|}
\hline$K_{S A A O}$ & $=K_{M S S S O}+0.012( \pm 0.004)(J-K)_{M S S S O}-0.015( \pm 0.003)$ & & for $(J-K)<3.0$ \\
\hline$(J-K)_{\text {SAAO }}$ & $=1.063( \pm 0.003)(J-K)_{M S S S O}+0.011( \pm 0.002)$ & & for $(J-K)<3.0$ \\
\hline$(H-K)_{\text {SAAO }}$ & $=0.979( \pm 0.011)(H-K)_{M S S O}-0.001( \pm 0.002)$ & for $(H-K)<0.9$ \\
\hline$(J-H)_{\text {SAAO }}$ & $=1.096( \pm 0.005)(J-K)_{M S S O}+0.009( \pm 0.003)$ & & for $(J-H)<2.1$ \\
\hline$(K-L)_{S A A O}$ & $=0.863( \pm 0.098)(K-L)_{M S S S O}+0.020( \pm 0.005)$ & & for $(K-L)<0.2$ \\
\hline
\end{tabular}

TRANSMISSION CURVES

As derived from Fig 2 of [204]

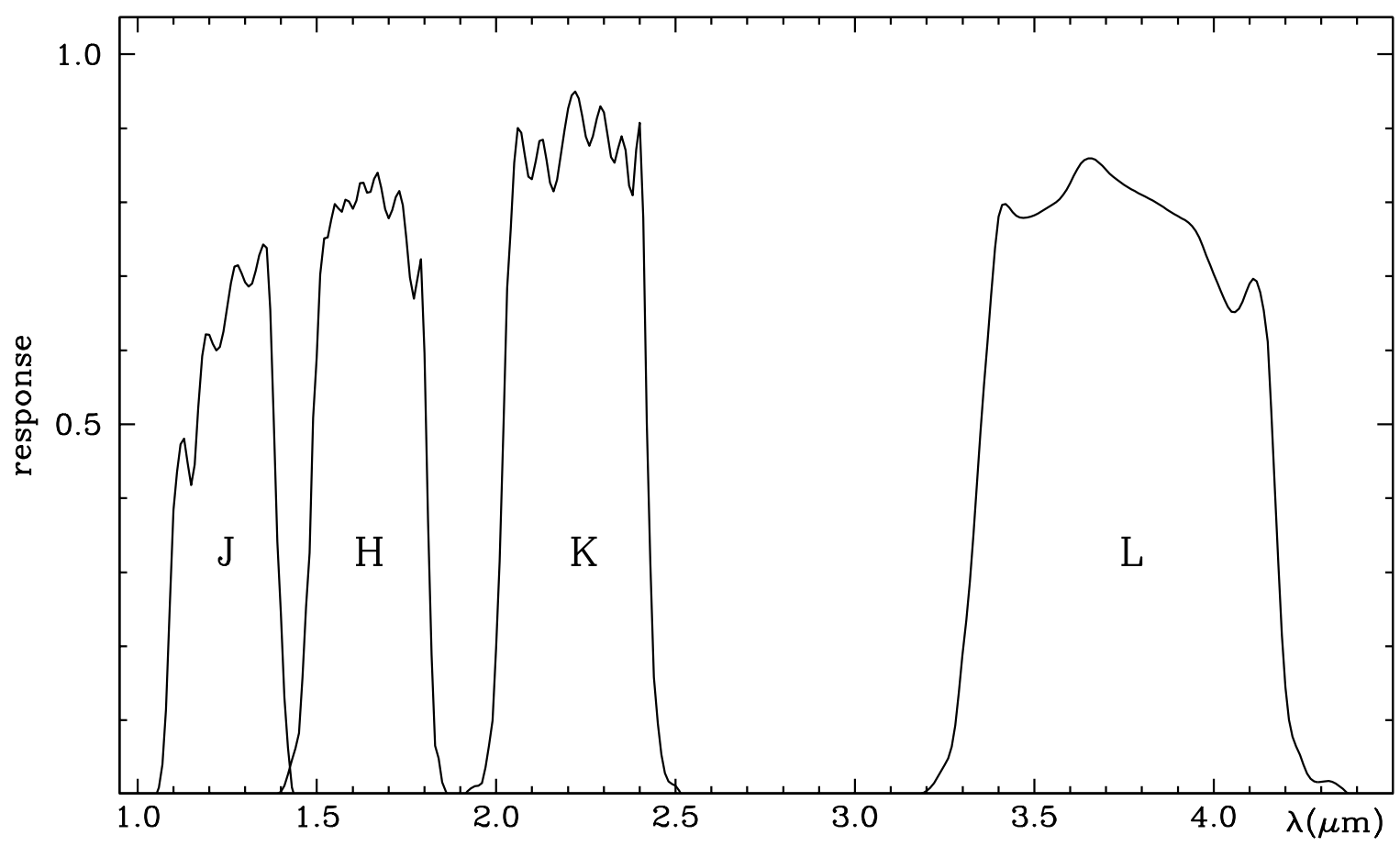

\begin{tabular}{|cc|c|cc|c|c|c|cc|ccc|ccc|c|}
\hline \multicolumn{7}{|c|}{$J$} & \multicolumn{9}{c|}{$H$} & \multicolumn{5}{c|}{$K$} & \multicolumn{4}{c|}{$L$} \\
\hline$\lambda(\mu \mathrm{m})$ & $\Upsilon$ & $\lambda(\mu \mathrm{m})$ & $\Upsilon$ & $\lambda(\mu \mathrm{m})$ & $\Upsilon$ & $\lambda(\mu \mathrm{m})$ & $\Upsilon$ & $\lambda(\mu \mathrm{m})$ & $\Upsilon$ & $\lambda(\mu \mathrm{m})$ & $\Upsilon$ & $\lambda(\mu \mathrm{m})$ & $\Upsilon$ & $\lambda(\mu \mathrm{m})$ & $\Upsilon$ \\
\hline 1.053 & 0.000 & 1.373 & 0.600 & 1.379 & 0.000 & 1.752 & 0.738 & 1.912 & 0.000 & 2.294 & 0.930 & 3.180 & 0.000 & 3.844 & 0.798 \\
1.073 & 0.060 & 1.382 & 0.480 & 1.415 & 0.018 & 1.776 & 0.678 & 1.948 & 0.012 & 2.309 & 0.894 & 3.216 & 0.012 & 3.903 & 0.780 \\
1.081 & 0.120 & 1.389 & 0.360 & 1.438 & 0.060 & 1.794 & 0.696 & 1.966 & 0.024 & 2.327 & 0.852 & 3.245 & 0.036 & 3.962 & 0.750 \\
1.085 & 0.180 & 1.400 & 0.240 & 1.456 & 0.120 & 1.800 & 0.600 & 1.977 & 0.060 & 2.357 & 0.882 & 3.281 & 0.096 & 3.986 & 0.720 \\
1.089 & 0.240 & 1.411 & 0.120 & 1.468 & 0.240 & 1.806 & 0.480 & 1.993 & 0.120 & 2.380 & 0.810 & 3.299 & 0.180 & 4.021 & 0.678 \\
1.092 & 0.300 & 1.421 & 0.060 & 1.482 & 0.360 & 1.810 & 0.360 & 2.004 & 0.240 & 2.403 & 0.894 & 3.322 & 0.300 & 4.051 & 0.652 \\
1.097 & 0.360 & 1.427 & 0.024 & 1.488 & 0.480 & 1.818 & 0.240 & 2.013 & 0.360 & 2.410 & 0.780 & 3.340 & 0.420 & 4.081 & 0.666 \\
1.107 & 0.420 & 1.450 & 0.000 & 1.501 & 0.600 & 1.823 & 0.120 & 2.019 & 0.480 & 2.416 & 0.600 & 3.358 & 0.540 & 4.116 & 0.696 \\
1.130 & 0.480 & & & 1.512 & 0.720 & 1.835 & 0.060 & 2.025 & 0.600 & 2.428 & 0.360 & 3.378 & 0.660 & 4.140 & 0.654 \\
1.154 & 0.420 & & & 1.527 & 0.750 & 1.847 & 0.024 & 2.033 & 0.720 & 2.434 & 0.240 & 3.393 & 0.756 & 4.152 & 0.600 \\
1.178 & 0.580 & & & 1.551 & 0.798 & 1.865 & 0.000 & 2.043 & 0.780 & 2.445 & 0.120 & 3.417 & 0.798 & 4.163 & 0.480 \\
1.207 & 0.612 & & & 1.569 & 0.786 & & & 2.049 & 0.840 & 2.469 & 0.030 & 3.441 & 0.786 & 4.175 & 0.360 \\
1.225 & 0.600 & & & 1.581 & 0.804 & & & 2.066 & 0.900 & 2.499 & 0.012 & 3.488 & 0.780 & 4.187 & 0.240 \\
1.251 & 0.660 & & & 1.604 & 0.792 & & & 2.096 & 0.828 & 2.517 & 0.000 & 3.536 & 0.792 & 4.205 & 0.120 \\
1.267 & 0.708 & & & 1.622 & 0.828 & & & 2.126 & 0.888 & & & 3.589 & 0.816 & 4.235 & 0.060 \\
1.296 & 0.696 & & & 1.646 & 0.810 & & & 2.155 & 0.816 & & & 3.630 & 0.852 & 4.258 & 0.030 \\
1.320 & 0.690 & & & 1.669 & 0.840 & & & 2.191 & 0.900 & & & 3.666 & 0.858 & 4.318 & 0.018 \\
1.355 & 0.744 & & & 1.695 & 0.780 & & & 2.232 & 0.936 & & & 3.707 & 0.840 & 4.377 & 0.000 \\
1.370 & 0.660 & & & 1.735 & 0.810 & & & 2.259 & 0.876 & & & 3.784 & 0.814 & \\
\hline
\end{tabular}

Fig. 149. continued 


\section{IRTF NSFCAM - Shure et al. - 1994}

Photometry with the NSFCAM infrared array camera at the NASA Infrared Telescope Facility

\section{GENERAL INFORMATION}

AUTHORS

TELESCOPE

DETECTOR

MAIN ARTICLE
M. A. Shure, D. W. Toomey, J. T. Rayner, P. M. Onaka, A. J. Denault

3.0m (reflector) NASA Infrared Telescope Facility, Mauna Kea

$256 \times 256 \mathrm{InSb}$ infrared array

Shure, M. A., Toomey, D. W., Rayner, J. T., Onaka, P. M.,

Denault, A. J., 1994, Proc. SPIE Vol. 2198, p. 614

NSFCAM User's Guide 1999

\section{SYSTEM DESCRIPTION}

\begin{tabular}{|c|cc|}
\hline \multicolumn{3}{|c|}{ BANDS DESCRIPTION [269][144] } \\
\hline band & $\lambda_{c}(\mu \mathrm{m}) \quad$ band width $(\mu \mathrm{m})$ \\
\hline \multicolumn{3}{|c|}{ broad bands } \\
\hline$J$ & 1.26 & 0.31 \\
\hline$H$ & 1.62 & 0.28 \\
\hline$K^{\prime}$ & 2.12 & 0.34 \\
\hline$K_{s}$ & 2.15 & 0.32 \\
\hline$K$ & 2.21 & 0.39 \\
\hline$L$ & 3.50 & 0.61 \\
\hline$L^{\prime}$ & 3.78 & 0.59 \\
\hline$M^{\prime}$ & 4.78 & 0.22 \\
\hline$M$ & 4.85 & 0.62 \\
\hline \multicolumn{3}{|c|}{ narrow bands } \\
\hline HeI & 1.08 & 0.01 \\
\hline Paschen $\gamma$ & 1.09 & 0.01 \\
\hline Paschen $\beta$ & 1.28 & 0.01 \\
\hline FeII & 1.64 & 0.02 \\
\hline$H_{2}$ & 2.12 & 0.02 \\
\hline Brackett $\gamma$ & 2.16 & 0.02 \\
\hline$H_{2}$ & 2.25 & 0.02 \\
\hline CO $O_{\text {cont }}$ & 2.26 & 0.05 \\
\hline CO & 2.30 & 0.03 \\
\hline
\end{tabular}

\section{SYSTEM ANALYSIS}

RELATIONS WITH OTHER SYSTEMS [144]

JHKL CTIO - Elias - 1982

\begin{tabular}{|c|l|}
\hline$J_{U K I R T}$ & $=J_{I R T F}$ \\
\hline$K_{U K I R T}$ & $=K_{I R T F}$ \\
\hline$L_{U K I R T}^{\prime}$ & $=L_{I R T F}^{\prime}$ \\
\hline$(H-K)_{U K I R T}$ & $=0.82( \pm 0.02) \times(H-K)_{I R T F}$ \\
\end{tabular}

\begin{tabular}{|rl|}
\hline$K_{C T I O}$ & $=K_{\text {IRTF }}-0.018 \times(J-K)_{\text {IRTF }}$ \\
\hline$(J-K)_{C T I O}$ & $=0.936 \times(J-K)_{\text {IRTF }}$ \\
\hline$(H-K)_{C T I O}$ & $=0.790 \times(H-K)_{\text {IRTF }}$ \\
\hline$(K-L)_{C T I O}$ & $=0.820 \times(K-L)_{\text {IRTF }}$
\end{tabular}

Fig. 150. The photometric system IRTF NSFCAM - Shure et al. - 1994 


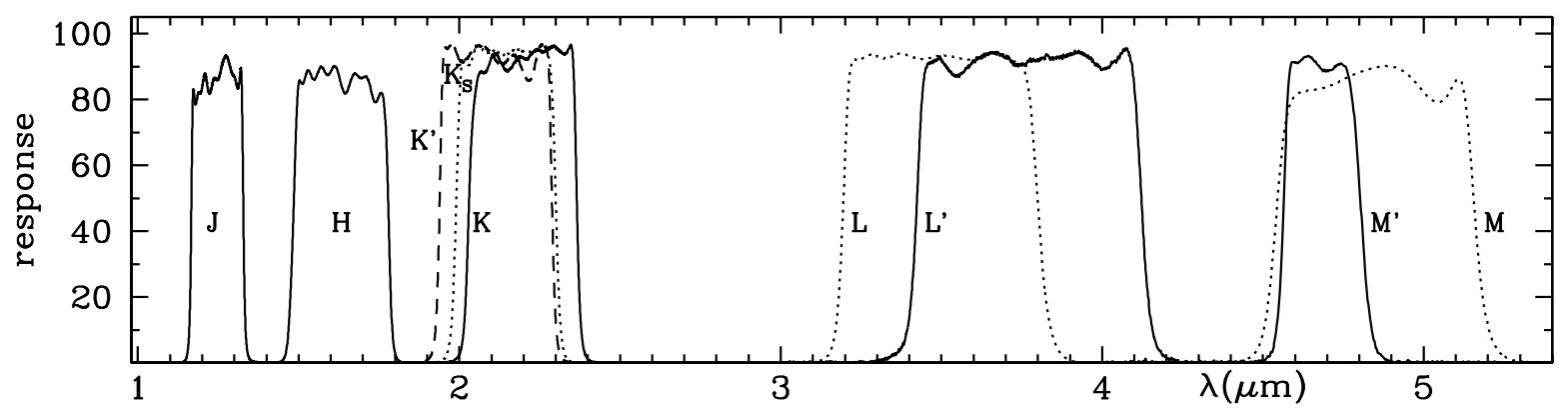

\begin{tabular}{|c|c|c|c|c|c|c|c|c|c|c|c|c|c|c|c|c|c|}
\hline \multicolumn{2}{|c|}{$J$} & \multicolumn{2}{|c|}{$H$} & \multicolumn{2}{|c|}{$K$} & \multicolumn{2}{|c|}{$K^{\prime}$} & \multicolumn{2}{|c|}{$K_{S}$} & \multicolumn{2}{|c|}{$L$} & \multicolumn{2}{|c|}{$L^{\prime}$} & \multicolumn{2}{|c|}{$M$} & \multicolumn{2}{|c|}{$M^{\prime}$} \\
\hline$\lambda(\mu \mathrm{m})$ & $\Upsilon$ & $\lambda(\mu \mathrm{m})$ & $\Upsilon$ & $\lambda(\mu \mathrm{m})$ & $\Upsilon$ & $\lambda(\mu \mathrm{m})$ & $\Upsilon$ & $\lambda(\mu \mathrm{m})$ & $\Upsilon$ & $\lambda(\mu \mathrm{m})$ & $\Upsilon$ & $\lambda(\mu \mathrm{m})$ & $\Upsilon$ & $\lambda(\mu \mathrm{m})$ & $\Upsilon$ & $\lambda(\mu \mathrm{m})$ & $\Upsilon$ \\
\hline 1.140 & 0.3 & 1.479 & 0.6 & 1.900 & 1.3 & 1.950 & 0.9 & 2.040 & 0.5 & 3.121 & 0.7 & 3.300 & 1.4 & 4.444 & 0.9 & 4.430 & 0.9 \\
\hline 1.150 & 1.0 & 1.488 & 1.7 & 1.910 & 3.8 & 1.960 & 2.5 & 2.050 & 1.2 & 3.142 & 1.7 & 3.322 & 5.2 & 4.466 & 2.1 & 4.440 & 1.7 \\
\hline 1.155 & 2.0 & 1.497 & 6.1 & 1.920 & 12.2 & 1.970 & 8.0 & 2.060 & 3.3 & 3.163 & 6.0 & 3.344 & 28.0 & 4.488 & 5.3 & 4.450 & 2.9 \\
\hline 1.160 & 4.5 & 1.506 & 21.8 & 1.930 & 39.4 & 1.980 & 27.4 & 2.070 & 10.3 & 3.184 & 25.4 & 3.366 & 80.9 & 4.510 & 13.9 & 4.460 & 4.5 \\
\hline 1.165 & 12.3 & 1.515 & 54.6 & 1.940 & 82.2 & 1.990 & 70.7 & 2.080 & 30.5 & 3.205 & 73.4 & 3.388 & 94.9 & 4.532 & 33.1 & 4.470 & 10.0 \\
\hline 1.170 & 36.9 & 1.524 & 75.6 & 1.950 & 96.4 & 2.000 & 94.2 & 2.090 & 64.5 & 3.226 & 91.9 & 3.410 & 91.7 & 4.554 & 59.1 & 4.480 & 17.9 \\
\hline 1.175 & 78.7 & 1.533 & 82.0 & 1.960 & 95.1 & 2.010 & 96.3 & 2.100 & 86.8 & 3.247 & 91.9 & 3.432 & 89.5 & 4.576 & 75.6 & 4.490 & 31.5 \\
\hline 1.180 & 88.8 & 1.542 & 80.9 & 1.970 & 93.5 & 2.020 & 96.4 & 2.110 & 93.7 & 3.268 & 93.4 & 3.454 & 90.2 & 4.598 & 80.9 & 4.500 & 48.0 \\
\hline 1.185 & 84.4 & 1.551 & 79.1 & 1.980 & 94.3 & 2.030 & 94.4 & 2.120 & 94.8 & 3.289 & 93.5 & 3.476 & 93.5 & 4.620 & 82.3 & 4.510 & 64.0 \\
\hline 1.190 & 83.8 & 1.560 & 80.3 & 1.990 & 95.2 & 2.040 & 89.7 & 2.130 & 94.6 & 3.310 & 92.8 & 3.498 & 94.1 & 4.642 & 82.7 & 4.520 & 77.4 \\
\hline 1.195 & 85.9 & 1.569 & 83.7 & 2.000 & 96.1 & 2.050 & 86.3 & 2.140 & 94.4 & 3.331 & 92.8 & 3.520 & 93.2 & 4.664 & 83.0 & 4.530 & 85.1 \\
\hline 1.200 & 87.0 & 1.578 & 86.3 & 2.010 & 96.0 & 2.060 & 85.9 & 2.150 & 94.5 & 3.352 & 93.4 & 3.542 & 92.3 & 4.686 & 83.4 & 4.540 & 89.4 \\
\hline 1.205 & 87.7 & 1.587 & 86.9 & 2.020 & 95.1 & 2.070 & 86.7 & 2.160 & 94.6 & 3.373 & 94.0 & 3.564 & 92.6 & 4.708 & 84.1 & 4.550 & 90.5 \\
\hline 1.210 & 88.8 & 1.596 & 86.3 & 2.030 & 95.1 & 2.080 & 89.5 & 2.170 & 94.4 & 3.394 & 93.5 & 3.586 & 92.7 & 4.730 & 85.1 & 4.560 & 90.8 \\
\hline 1.215 & 91.1 & 1.605 & 86.9 & 2.040 & 94.7 & 2.090 & 92.1 & 2.180 & 94.4 & 3.415 & 92.8 & 3.608 & 91.9 & 4.752 & 86.1 & 4.570 & 90.0 \\
\hline 1.220 & 92.6 & 1.614 & 87.7 & 2.050 & 94.9 & 2.100 & 93.3 & 2.190 & 94.6 & 3.436 & 92.2 & 3.630 & 91.8 & 4.774 & 87.2 & 4.580 & 89.1 \\
\hline 1.225 & 93.2 & 1.623 & 87.2 & 2.060 & 94.8 & 2.110 & 93.5 & 2.200 & 95.2 & 3.457 & 92.4 & 3.652 & 90.6 & 4.796 & 88.1 & 4.590 & 88.8 \\
\hline 1.230 & 92.8 & 1.632 & 85.0 & 2.070 & 94.2 & 2.120 & 92.0 & 2.210 & 95.0 & 3.478 & 92.8 & 3.674 & 90.8 & 4.818 & 88.9 & 4.600 & 88.6 \\
\hline 1.235 & 91.3 & 1.641 & 82.4 & 2.080 & 93.2 & 2.130 & 91.2 & 2.220 & 94.9 & 3.499 & 93.3 & 3.696 & 92.6 & 4.840 & 89.4 & 4.610 & 88.9 \\
\hline 1.240 & 89.3 & 1.650 & 82.0 & 2.090 & 92.2 & 2.140 & 90.6 & 2.230 & 94.1 & 3.520 & 93.3 & 3.718 & 93.9 & 4.862 & 89.9 & 4.620 & 90.1 \\
\hline 1.245 & 87.3 & 1.659 & 84.3 & 2.100 & 92.8 & 2.150 & 90.8 & 2.240 & 93.6 & 3.541 & 92.9 & 3.740 & 94.1 & 4.884 & 90.2 & 4.630 & 91.5 \\
\hline 1.250 & 86.1 & 1.668 & 87.8 & 2.110 & 92.5 & 2.160 & 91.8 & 2.250 & 93.5 & 3.562 & 92.4 & 3.762 & 93.0 & 4.906 & 89.9 & 4.640 & 92.2 \\
\hline 1.255 & 86.1 & 1.677 & 90.0 & 2.120 & 92.0 & 2.170 & 93.5 & 2.260 & 94.1 & 3.583 & 92.1 & 3.784 & 92.6 & 4.928 & 89.1 & 4.650 & 93.0 \\
\hline 1.260 & 86.2 & 1.686 & 89.6 & 2.130 & 90.7 & 2.180 & 94.5 & 2.270 & 94.7 & 3.604 & 92.4 & 3.806 & 91.2 & 4.950 & 87.6 & 4.660 & 93.0 \\
\hline 1.265 & 85.7 & 1.695 & 87.9 & 2.140 & 89.5 & 2.190 & 95.3 & 2.280 & 94.8 & 3.625 & 93.3 & 3.828 & 89.3 & 4.972 & 85.5 & 4.670 & 92.4 \\
\hline 1.270 & 83.6 & 1.704 & 87.7 & 2.150 & 88.8 & 2.200 & 95.9 & 2.290 & 94.3 & 3.646 & 93.9 & 3.850 & 87.8 & 4.994 & 83.0 & 4.680 & 91.5 \\
\hline 1.275 & 82.0 & 1.713 & 88.9 & 2.160 & 89.7 & 2.210 & 96.4 & 2.300 & 94.1 & 3.667 & 93.9 & 3.872 & 87.4 & 5.016 & 80.7 & 4.690 & 91.2 \\
\hline 1.280 & 82.3 & 1.722 & 90.1 & 2.170 & 91.3 & 2.220 & 96.0 & 2.310 & 94.7 & 3.688 & 93.3 & 3.894 & 89.6 & 5.038 & 79.2 & 4.700 & 91.4 \\
\hline 1.285 & 85.4 & 1.731 & 88.4 & 2.180 & 93.6 & 2.230 & 95.2 & 2.320 & 96.1 & 3.709 & 92.2 & 3.916 & 91.3 & 5.060 & 79.7 & 4.710 & 89.9 \\
\hline 1.290 & 87.9 & 1.740 & 86.4 & 2.190 & 93.8 & 2.240 & 94.2 & 2.330 & 96.3 & 3.730 & 91.4 & 3.938 & 92.1 & 5.082 & 82.5 & 4.720 & 79.0 \\
\hline 1.295 & 86.4 & 1.749 & 86.4 & 2.200 & 92.4 & 2.250 & 92.6 & 2.340 & 94.2 & 3.751 & 90.3 & 3.960 & 91.3 & 5.104 & 86.0 & 4.730 & 56.9 \\
\hline 1.300 & 83.3 & 1.758 & 88.4 & 2.210 & 89.6 & 2.260 & 91.5 & 2.350 & 91.1 & 3.772 & 82.2 & 3.982 & 89.7 & 5.126 & 82.0 & 4.740 & 32.2 \\
\hline 1.305 & 81.8 & 1.767 & 88.1 & 2.220 & 88.0 & 2.270 & 91.4 & 2.360 & 90.7 & 3.793 & 58.3 & 4.004 & 73.3 & 5.148 & 60.2 & 4.750 & 16.3 \\
\hline 1.310 & 82.0 & 1.776 & 85.3 & 2.230 & 88.3 & 2.280 & 93.5 & 2.370 & 92.9 & 3.814 & 30.0 & 4.026 & 39.9 & 5.170 & 32.4 & 4.760 & 8.2 \\
\hline 1.315 & 80.2 & 1.785 & 85.2 & 2.240 & 86.8 & 2.290 & 95.8 & 2.380 & 89.1 & 3.835 & 12.9 & 4.048 & 17.2 & 5.192 & 15.0 & 4.770 & 3.8 \\
\hline 1.320 & 78.5 & 1.794 & 83.1 & 2.250 & 80.5 & 2.300 & 96.6 & 2.390 & 66.2 & 3.856 & 5.4 & 4.070 & 7.7 & 5.214 & 6.9 & 4.780 & 2.1 \\
\hline 1.325 & 82.5 & 1.803 & 61.4 & 2.260 & 68.2 & 2.310 & 95.5 & 2.400 & 33.0 & 3.877 & 2.3 & 4.092 & 4.2 & 5.236 & 3.3 & 4.790 & 1.5 \\
\hline 1.330 & 71.9 & 1.812 & 33.5 & 2.270 & 41.5 & 2.320 & 94.8 & 2.410 & 12.1 & 3.898 & 0.9 & 4.114 & 2.4 & 5.258 & 1.7 & 4.800 & 0.7 \\
\hline 1.335 & 29.4 & 1.821 & 13.6 & 2.280 & 15.6 & 2.330 & 74.2 & 2.420 & 4.2 & & & 4.136 & 2.0 & 5.280 & 0.8 & & \\
\hline 1.340 & 8.9 & 1.830 & 4.0 & 2.290 & 5.3 & 2.340 & 28.2 & 2.430 & 1.6 & & & 4.158 & 1.1 & & & & \\
\hline 1.345 & 3.1 & 1.839 & 1.2 & 2.300 & 2.1 & 2.350 & 8.2 & 2.440 & 0.7 & & & 4.180 & 0.6 & & & & \\
\hline 1.350 & 1.1 & 1.848 & 0.4 & 2.310 & 1.2 & 2.360 & 2.8 & & & & & & & & & & \\
\hline 1.355 & 0.3 & & & 2.320 & 0.6 & & & & & & & & & & & & \\
\hline
\end{tabular}

Fig. 150. continued 


\title{
FPBS - Brewer et al. - 1995
}

Absorption bands in late type stars.

\section{GENERAL INFORMATION}

\author{
AUTHORS J. P. Brewer, H. B. Richer and D. R. Crabtree \\ TELESCOPE $\quad 3.6 \mathrm{~m}$ Canada French Hawaii Telescope (reflector), Mauna Kea \\ DETECTOR Loral3 2048X2048 CCD \\ MAIN ARTICLE $\quad$ Brewer, J., Richer, H. B., Crabtree, D.R. 1995, AJ 109, 2480
}

\section{SYSTEM DESCRIPTION}

\begin{tabular}{|c|cc|}
\hline \multicolumn{3}{|c|}{ BANDS DESCRIPTION [53] } \\
\hline band & $\lambda_{c}(\AA)$ & bandpass $(\AA)$ \\
\hline $\mathrm{TiO}$ & 7800 & 140 \\
\hline $\mathrm{CN}$ & 8100 & 140 \\
\hline
\end{tabular}

In addition to the $\mathrm{TiO}$ and the $\mathrm{CN}$ bands the system includes the $\mathrm{V}$ and the $I$ bands from $R I$ - Cousins - 1976.

Fig. 151. The photometric system FPBS - Brewer et al. - 1995 


\section{ISOCAM ISO - 1995}

Infrared photometric system of the ISO satellite.

\section{GENERAL INFORMATION}

TELESCOPE $\quad 0.60 \mathrm{~cm}$ (reflector)

DETECTOR InSb $32 X 32$ pixels Charge Injection Device (Short-Wavelength range);

32X32 Gallium doped Silicon photo-conductor array hybridized by Indium bumps

(Long-Wavelength range)

MAIN ARTICLE ISOCAM Observer's Manual V1.0

\section{SYSTEM DESCRIPTION}

\begin{tabular}{|l|cc|}
\hline \multicolumn{4}{|c|}{ BANDS DESCRIPTION [145] } \\
\hline band & $\lambda_{c}(\mu \mathrm{m})$ & bandpass $(\mu \mathrm{m})$ \\
\hline \multicolumn{3}{|c|}{ Short-Wavelength channel } \\
\hline SW1 & 3.57 & $3.05-4.10$ \\
\hline SW2 & 3.30 & $3.20-3.40$ \\
\hline SW3 & 4.50 & $4.00-5.00$ \\
\hline SW4 & 2.77 & $2.50-3.05$ \\
\hline SW5 & \multicolumn{3}{|c|}{$3.00-5.5$} \\
\hline SW6 & 3.72 & $3.45-4.00$ \\
\hline SW7 & 3.05 & $2.90-3.20$ \\
\hline SW8 & 4.06 \\
\hline SW9 & 3.88 & $3.76-4.00$ \\
\hline SW10 & 4.70 & $4.53-4.88$ \\
\hline SW11 & 4.26 & $4.16-4.37$ \\
\hline \multicolumn{2}{|c|}{ Long-Wavelength channel } \\
\hline LW1 & 4.50 & $4.00-5.00$ \\
\hline LW2 & 6.75 & $5.00-8.50$ \\
\hline LW3 & 15.00 & $12.0-18$ \\
\hline LW4 & 6.00 & $5.50-6.50$ \\
\hline LW5 & 6.75 & $6.50-7.00$ \\
\hline LW6 & 7.75 & $7.00-8.50$ \\
\hline LW7 & 9.62 & $8.50-10.7$ \\
\hline LW8 & 11.40 & $10.7-12.0$ \\
\hline LW9 & 15.00 & $14.0-16.0$ \\
\hline LW10 & 11.50 & $8.00-15.0$ \\
\hline
\end{tabular}

Fig. 152. The photometric system ISOCAM ISO - 1995 
TRANSMISSION CURVES [145]
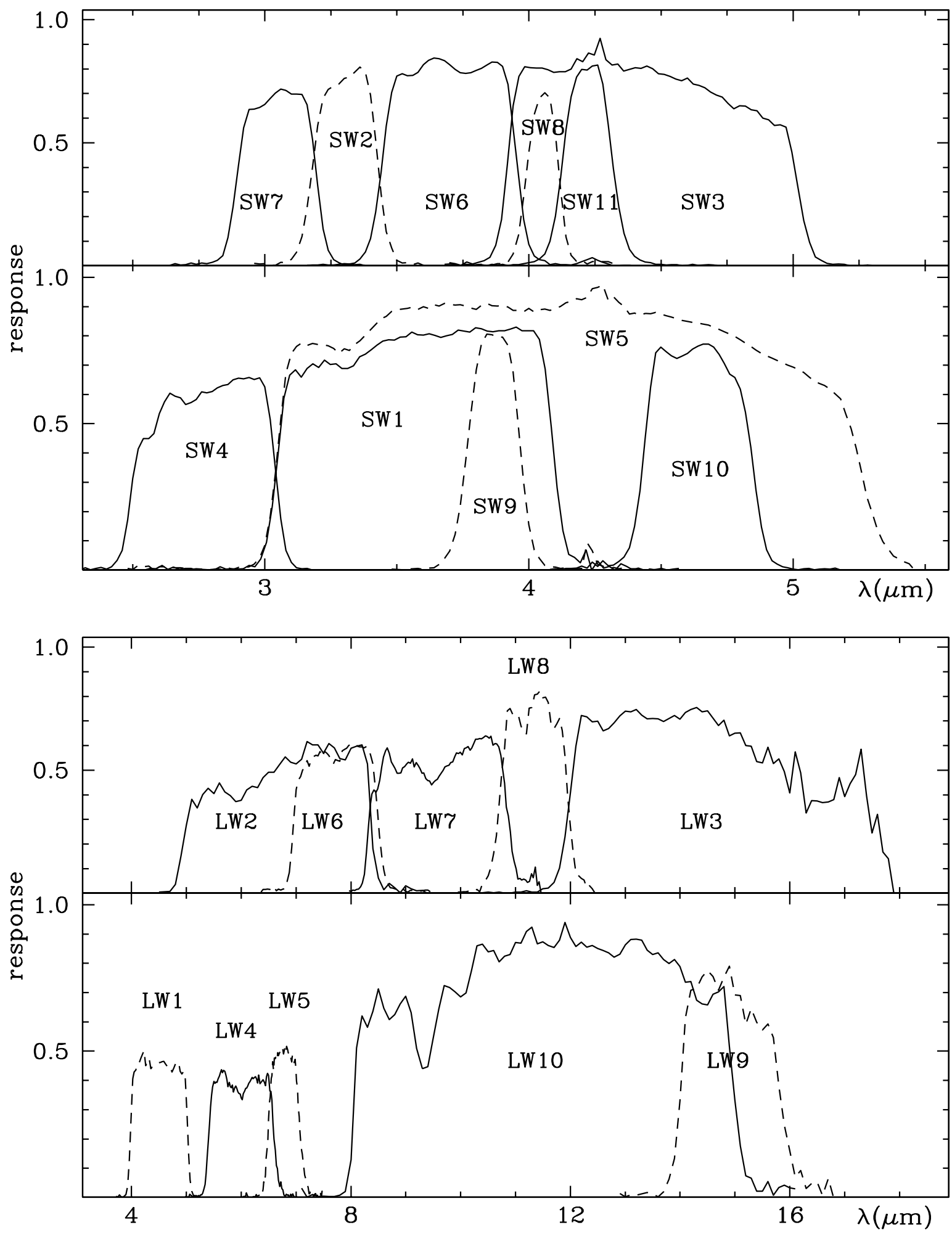

Fig. 152. continued 


\begin{tabular}{|c|c|c|c|c|c|c|c|c|c|c|c|c|c|c|c|c|c|c|c|}
\hline \multicolumn{2}{|c|}{ SW1 } & \multicolumn{2}{|c|}{ SW2 } & \multicolumn{2}{|c|}{ SW3 } & \multicolumn{2}{|c|}{ SW4 } & \multicolumn{2}{|c|}{ SW5 } & \multicolumn{2}{|c|}{ SW6 } & \multicolumn{2}{|c|}{ SW7 } & \multicolumn{2}{|c|}{ SW8 } & \multicolumn{2}{|c|}{ SW10 } & \multicolumn{2}{|c|}{ SW11 } \\
\hline$\lambda(\mu \mathrm{m})$ & $\Upsilon$ & $\lambda(\mu \mathrm{m})$ & $\Upsilon$ & $\lambda(\mu \mathrm{m})$ & $\Upsilon$ & $\lambda(\mu \mathrm{m})$ & $\Upsilon$ & $\lambda(\mu \mathrm{m})$ & r & $\lambda(\mu \mathrm{m})$ & r & $\lambda(\mu \mathrm{m})$ & $\Upsilon$ & $\lambda(\mu \mathrm{m})$ & $\Upsilon$ & $\lambda(\mu \mathrm{m})$ & r & $\lambda(\mu \mathrm{m})$ & r \\
\hline 2.90 & 0.00 & 3.06 & 0.01 & 3.80 & 0.01 & 2.40 & 0.00 & 2.90 & 0.01 & 3.30 & 0.00 & 2.70 & 0.00 & 3.85 & 0.00 & 4.25 & 0.02 & 3.95 & 0.01 \\
\hline 2.95 & 0.01 & 3.09 & 0.02 & 3.85 & 0.03 & 2.43 & 0.02 & 2.99 & 0.06 & 3.33 & 0.01 & 2.73 & 0.01 & 3.89 & 0.01 & 4.29 & 0.03 & 3.97 & 0.01 \\
\hline 3.00 & 0.07 & 3.12 & 0.05 & 3.90 & 0.22 & 2.46 & 0.07 & 3.08 & 0.67 & 3.36 & 0.03 & 2.76 & 0.01 & 3.93 & 0.03 & 4.33 & 0.02 & 3.99 & 0.01 \\
\hline 3.05 & 0.40 & 3.15 & 0.17 & 3.95 & 0.72 & 2.49 & 0.24 & 3.17 & 0.77 & 3.39 & 0.08 & 2.79 & 0.01 & 3.97 & 0.20 & 4.37 & 0.06 & 4.01 & 0.01 \\
\hline 3.10 & 0.68 & 3.18 & 0.40 & 4.00 & 0.81 & 2.52 & 0.41 & 3.26 & 0.76 & 3.42 & 0.22 & 2.82 & 0.02 & 4.01 & 0.55 & 4.41 & 0.20 & 4.03 & 0.02 \\
\hline 3.15 & 0.67 & 3.21 & 0.64 & 4.05 & 0.80 & 2.55 & 0.45 & 3.35 & 0.78 & 3.45 & 0.48 & 2.85 & 0.07 & 4.05 & 0.70 & 4.45 & 0.54 & 4.05 & 0.04 \\
\hline 3.20 & 0.69 & 3.24 & 0.72 & 4.10 & 0.79 & 2.58 & 0.47 & 3.44 & 0.86 & 3.48 & 0.71 & 2.88 & 0.24 & 4.09 & 0.62 & 4.49 & 0.76 & 4.07 & 0.07 \\
\hline 3.25 & 0.70 & 3.27 & 0.73 & 4.15 & 0.79 & 2.61 & 0.56 & 3.53 & 0.89 & 3.51 & 0.78 & 2.91 & 0.49 & 4.13 & 0.20 & 4.53 & 0.74 & 4.09 & 0.14 \\
\hline 3.30 & 0.69 & 3.30 & 0.76 & 4.20 & 0.83 & 2.64 & 0.60 & 3.62 & 0.89 & 3.54 & 0.77 & 2.94 & 0.64 & 4.17 & 0.03 & 4.57 & 0.73 & 4.11 & 0.27 \\
\hline 3.35 & 0.72 & 3.33 & 0.78 & 4.25 & 0.86 & 2.67 & 0.59 & 3.71 & 0.91 & 3.57 & 0.78 & 2.97 & 0.64 & 4.21 & 0.01 & 4.61 & 0.75 & 4.13 & 0.45 \\
\hline 3.40 & 0.75 & 3.36 & 0.81 & 4.30 & 0.82 & 2.70 & 0.57 & 3.80 & 0.89 & 3.60 & 0.82 & 3.00 & 0.66 & 4.25 & 0.02 & 4.65 & 0.77 & 4.15 & 0.63 \\
\hline 3.45 & 0.78 & 3.39 & 0.75 & 4.35 & 0.80 & 2.73 & 0.58 & 3.89 & 0.90 & 3.63 & 0.84 & 3.03 & 0.69 & & & 4.69 & 0.77 & 4.17 & 0.73 \\
\hline 3.50 & 0.79 & 3.42 & 0.51 & 4.40 & 0.81 & 2.76 & 0.61 & 3.98 & 0.88 & 3.66 & 0.84 & 3.06 & 0.72 & & & 4.73 & 0.72 & 4.19 & 0.79 \\
\hline 3.55 & 0.81 & 3.45 & 0.20 & 4.45 & 0.81 & 2.79 & 0.61 & 4.07 & 0.89 & 3.69 & 0.83 & 3.09 & 0.70 & & & 4.77 & 0.66 & 4.21 & 0.80 \\
\hline 3.60 & 0.80 & 3.48 & 0.07 & 4.50 & 0.78 & 2.82 & 0.62 & 4.16 & 0.92 & 3.72 & 0.80 & 3.12 & 0.70 & & & 4.81 & 0.58 & 4.23 & 0.80 \\
\hline 3.65 & 0.80 & 3.51 & 0.01 & 4.55 & 0.76 & 2.85 & 0.63 & 4.25 & 0.96 & 3.75 & 0.78 & 3.15 & 0.69 & & & 4.85 & 0.35 & 4.25 & 0.82 \\
\hline 3.70 & 0.80 & 3.54 & 0.00 & 4.60 & 0.76 & 2.88 & 0.65 & 4.34 & 0.91 & 3.78 & 0.78 & 3.18 & 0.51 & & & 4.89 & 0.10 & 4.27 & 0.79 \\
\hline 3.75 & 0.82 & & & 4.65 & 0.73 & 2.91 & 0.65 & 4.43 & 0.87 & 3.81 & 0.80 & 3.21 & 0.23 & & & 4.93 & 0.03 & 4.29 & 0.66 \\
\hline 3.80 & 0.83 & & & 4.70 & 0.70 & 2.94 & 0.66 & 4.52 & 0.87 & 3.84 & 0.82 & 3.24 & 0.06 & & & 4.97 & 0.01 & 4.31 & 0.48 \\
\hline 3.85 & 0.81 & & & 4.75 & 0.67 & 2.97 & 0.65 & 4.61 & 0.85 & 3.87 & 0.83 & 3.27 & 0.02 & $S V$ & & & & 4.33 & 0.31 \\
\hline 3.90 & 0.82 & & & 4.80 & 0.65 & 3.00 & 0.63 & 4.70 & 0.83 & 3.90 & 0.81 & 3.30 & 0.00 & $\lambda(\mu \mathrm{m})$ & r & & & 4.35 & 0.18 \\
\hline 3.95 & 0.83 & & & 4.85 & 0.63 & 3.03 & 0.44 & 4.79 & 0.79 & 3.93 & 0.66 & & & 3.65 & 0.01 & & & 4.37 & 0.09 \\
\hline 4.00 & 0.82 & & & 4.90 & 0.60 & 3.06 & 0.17 & 4.88 & 0.74 & 3.96 & 0.37 & & & 3.69 & 0.05 & & & 4.39 & 0.05 \\
\hline 4.05 & 0.75 & & & 4.95 & 0.57 & 3.09 & 0.04 & 4.97 & 0.70 & 3.99 & 0.13 & & & 3.73 & 0.16 & & & 4.41 & 0.03 \\
\hline 4.10 & 0.33 & & & 5.00 & 0.44 & 3.12 & 0.01 & 5.06 & 0.66 & 4.02 & 0.04 & & & 3.77 & 0.45 & & & 4.43 & 0.02 \\
\hline 4.15 & 0.05 & & & 5.05 & 0.14 & 3.15 & 0.00 & 5.15 & 0.61 & 4.05 & 0.02 & & & 3.81 & 0.74 & & & 4.45 & 0.01 \\
\hline 4.20 & 0.04 & & & 5.10 & 0.02 & & & 5.24 & 0.42 & 4.08 & 0.01 & & & 3.85 & 0.81 & & & 4.47 & 0.01 \\
\hline 4.25 & 0.01 & & & 5.15 & 0.01 & & & 5.33 & 0.11 & 4.11 & 0.00 & & & 3.89 & 0.80 & & & 4.49 & 0.01 \\
\hline 4.30 & 0.00 & & & 5.20 & 0.00 & & & 5.42 & 0.03 & & & & & 3.93 & 0.73 & & & & \\
\hline & & & & & & & & & & & & & & 3.97 & 0.39 & & & & \\
\hline & & & & & & & & & & & & & & 4.01 & 0.10 & & & & \\
\hline & & & & & & & & & & & & & & 4.05 & 0.03 & & & & \\
\hline & & & & & & & & & & & & & & 4.09 & 0.00 & & & & \\
\hline
\end{tabular}

\begin{tabular}{|c|c|c|c|c|c|c|c|c|c|c|c|c|c|c|c|c|c|c|c|}
\hline \multicolumn{2}{|c|}{ LW1 } & \multicolumn{2}{|c|}{ LW2 } & \multicolumn{2}{|c|}{ LW3 } & \multicolumn{2}{|c|}{ LW4 } & \multicolumn{2}{|c|}{ LW5 } & \multicolumn{2}{|c|}{ LW6 } & \multicolumn{2}{|c|}{ LW7 } & \multicolumn{2}{|c|}{ LW8 } & \multicolumn{2}{|c|}{ LW9 } & \multicolumn{2}{|c|}{ LW10 } \\
\hline$\lambda(\mu \mathrm{m})$ & $\Upsilon$ & $\lambda(\mu \mathrm{m})$ & r & $\lambda(\mu \mathrm{m})$ & $\Upsilon$ & $\lambda(\mu \mathrm{m})$ & $\Upsilon$ & $\lambda(\mu \mathrm{m})$ & r & $\lambda(\mu \mathrm{m})$ & r & $\lambda(\mu \mathrm{m})$ & $\Upsilon$ & $\lambda(\mu \mathrm{m})$ & r & $\lambda(\mu \mathrm{m})$ & r & $\lambda(\mu \mathrm{m})$ & r \\
\hline 3.85 & 0.00 & 4.70 & 0.01 & 11.50 & 0.02 & 5.20 & 0.00 & 6.30 & 0.00 & 6.70 & 0.00 & 8.20 & 0.04 & 10.30 & 0.00 & 13.65 & 0.01 & 7.70 & 0.00 \\
\hline 3.90 & 0.01 & 4.85 & 0.09 & 11.70 & 0.04 & 5.25 & 0.01 & 6.35 & 0.02 & 6.77 & 0.02 & 8.30 & 0.22 & 10.37 & 0.02 & 13.75 & 0.04 & 8.00 & 0.13 \\
\hline 3.95 & 0.09 & 5.00 & 0.28 & 11.90 & 0.23 & 5.30 & 0.02 & 6.40 & 0.04 & 6.84 & 0.05 & 8.40 & 0.41 & 10.44 & 0.05 & 13.85 & 0.09 & 8.30 & 0.58 \\
\hline 4.00 & 0.32 & 5.15 & 0.37 & 12.10 & 0.60 & 5.35 & 0.06 & 6.45 & 0.12 & 6.91 & 0.16 & 8.50 & 0.43 & 10.51 & 0.08 & 13.95 & 0.21 & 8.60 & 0.65 \\
\hline 4.05 & 0.43 & 5.30 & 0.40 & 12.30 & 0.72 & 5.40 & 0.19 & 6.50 & 0.27 & 6.98 & 0.37 & 8.60 & 0.56 & 10.58 & 0.14 & 14.05 & 0.48 & 8.90 & 0.66 \\
\hline 4.10 & 0.45 & 5.45 & 0.41 & 12.50 & 0.70 & 5.45 & 0.33 & 6.55 & 0.41 & 7.05 & 0.46 & 8.70 & 0.57 & 10.65 & 0.23 & 14.15 & 0.68 & 9.20 & 0.51 \\
\hline 4.15 & 0.46 & 5.60 & 0.45 & 12.70 & 0.67 & 5.50 & 0.40 & 6.60 & 0.46 & 7.12 & 0.50 & 8.80 & 0.51 & 10.72 & 0.42 & 14.25 & 0.71 & 9.50 & 0.55 \\
\hline 4.20 & 0.49 & 5.75 & 0.40 & 12.90 & 0.72 & 5.55 & 0.39 & 6.65 & 0.47 & 7.19 & 0.54 & 8.90 & 0.49 & 10.79 & 0.57 & 14.35 & 0.73 & 9.80 & 0.72 \\
\hline 4.25 & 0.47 & 5.90 & 0.37 & 13.10 & 0.74 & 5.60 & 0.41 & 6.70 & 0.50 & 7.26 & 0.53 & 9.00 & 0.52 & 10.86 & 0.76 & 14.45 & 0.77 & 10.10 & 0.70 \\
\hline 4.30 & 0.47 & 6.05 & 0.40 & 13.30 & 0.72 & 5.65 & 0.43 & 6.75 & 0.52 & 7.33 & 0.56 & 9.10 & 0.54 & 10.93 & 0.73 & 14.55 & 0.77 & 10.40 & 0.87 \\
\hline 4.35 & 0.44 & 6.20 & 0.44 & 13.50 & 0.71 & 5.70 & 0.43 & 6.80 & 0.52 & 7.40 & 0.56 & 9.20 & 0.53 & 11.00 & 0.73 & 14.65 & 0.73 & 10.70 & 0.81 \\
\hline 4.40 & 0.45 & 6.35 & 0.45 & 13.70 & 0.70 & 5.75 & 0.39 & 6.85 & 0.50 & 7.47 & 0.56 & 9.30 & 0.49 & 11.07 & 0.71 & 14.75 & 0.72 & 11.00 & 0.87 \\
\hline 4.45 & 0.46 & 6.50 & 0.49 & 13.90 & 0.72 & 5.80 & 0.38 & 6.90 & 0.48 & 7.54 & 0.58 & 9.40 & 0.46 & 11.14 & 0.66 & 14.85 & 0.79 & 11.30 & 0.92 \\
\hline 4.50 & 0.46 & 6.65 & 0.50 & 14.10 & 0.73 & 5.85 & 0.37 & 6.95 & 0.48 & 7.61 & 0.56 & 9.50 & 0.45 & 11.21 & 0.67 & 14.95 & 0.74 & 11.60 & 0.86 \\
\hline 4.55 & 0.46 & 6.80 & 0.55 & 14.30 & 0.76 & 5.90 & 0.38 & 7.00 & 0.43 & 7.68 & 0.53 & 9.60 & 0.47 & 11.28 & 0.76 & 15.05 & 0.69 & 11.90 & 0.94 \\
\hline 4.60 & 0.46 & 6.95 & 0.53 & 14.50 & 0.74 & 5.95 & 0.36 & 7.05 & 0.29 & 7.75 & 0.54 & 9.70 & 0.51 & 11.35 & 0.81 & 15.15 & 0.64 & 12.20 & 0.87 \\
\hline 4.65 & 0.45 & 7.10 & 0.54 & 14.70 & 0.68 & 6.00 & 0.34 & 7.10 & 0.16 & 7.82 & 0.56 & 9.80 & 0.53 & 11.42 & 0.82 & 15.25 & 0.61 & 12.50 & 0.85 \\
\hline 4.70 & 0.44 & 7.25 & 0.62 & 14.90 & 0.64 & 6.05 & 0.36 & 7.15 & 0.09 & 7.89 & 0.58 & 9.90 & 0.56 & 11.49 & 0.80 & 15.35 & 0.63 & 12.80 & 0.82 \\
\hline 4.75 & 0.43 & 7.40 & 0.60 & 15.10 & 0.65 & 6.10 & 0.37 & 7.20 & 0.05 & 7.96 & 0.60 & 10.00 & 0.57 & 11.56 & 0.80 & 15.45 & 0.58 & 13.10 & 0.88 \\
\hline 4.80 & 0.46 & 7.55 & 0.59 & 15.30 & 0.60 & 6.15 & 0.39 & 7.25 & 0.01 & 8.03 & 0.61 & 10.10 & 0.59 & 11.63 & 0.72 & 15.55 & 0.58 & 13.40 & 0.84 \\
\hline 4.85 & 0.46 & 7.70 & 0.59 & 15.50 & 0.53 & 6.20 & 0.41 & 7.30 & 0.02 & 8.10 & 0.60 & 10.20 & 0.59 & 11.70 & 0.67 & 15.65 & 0.58 & 13.70 & 0.81 \\
\hline 4.90 & 0.43 & 7.85 & 0.54 & 15.70 & 0.53 & 6.25 & 0.41 & & & 8.17 & 0.60 & 10.30 & 0.63 & 11.77 & 0.70 & 15.75 & 0.47 & 14.00 & 0.79 \\
\hline 4.95 & 0.43 & 8.00 & 0.59 & 15.90 & 0.49 & 6.30 & 0.39 & & & 8.24 & 0.59 & 10.40 & 0.63 & 11.84 & 0.68 & 15.85 & 0.31 & 14.30 & 0.67 \\
\hline 5.00 & 0.33 & 8.15 & 0.60 & 16.10 & 0.57 & 6.35 & 0.39 & & & 8.31 & 0.59 & 10.50 & 0.63 & 11.91 & 0.53 & 15.95 & 0.20 & 14.60 & 0.69 \\
\hline 5.05 & 0.11 & 8.30 & 0.53 & 16.30 & 0.33 & 6.40 & 0.40 & & & 8.38 & 0.55 & 10.60 & 0.60 & 11.98 & 0.31 & 16.05 & 0.10 & 14.90 & 0.51 \\
\hline 5.10 & 0.02 & 8.45 & 0.09 & 16.50 & 0.37 & 6.45 & 0.40 & & & 8.45 & 0.42 & 10.70 & 0.59 & 12.05 & 0.17 & 16.15 & 0.08 & 15.20 & 0.07 \\
\hline 5.15 & 0.01 & 8.60 & 0.01 & 16.70 & 0.37 & 6.50 & 0.42 & & & 8.52 & 0.24 & 10.80 & 0.45 & 12.12 & 0.07 & 16.25 & 0.07 & 15.50 & 0.02 \\
\hline 5.20 & 0.00 & & & 16.90 & 0.47 & 6.55 & 0.36 & & & 8.59 & 0.11 & 10.90 & 0.26 & 12.19 & 0.06 & 16.35 & 0.03 & 15.80 & 0.03 \\
\hline & & & & 17.10 & 0.45 & 6.60 & 0.20 & & & 8.66 & 0.05 & 11.00 & 0.11 & 12.26 & 0.05 & 16.45 & 0.06 & 16.10 & 0.02 \\
\hline & & & & 17.30 & 0.58 & 6.65 & 0.10 & & & 8.73 & 0.02 & 11.10 & 0.06 & 12.33 & 0.02 & 16.55 & 0.02 & 16.40 & 0.01 \\
\hline & & & & 17.50 & 0.24 & 6.70 & 0.04 & & & 8.80 & 0.00 & 11.20 & 0.05 & 12.40 & 0.00 & & & 16.70 & 0.00 \\
\hline & & & & 17.70 & 0.17 & 6.75 & 0.03 & & & & & 11.30 & 0.07 & & & & & & \\
\hline & & & & 17.90 & 0.00 & 6.80 & 0.00 & & & & & 11.40 & 0.03 & & & & & & \\
\hline
\end{tabular}

Fig. 152. continued 


\section{BATC - Fan et al. - 1996}

General purpose system.

\section{GENERAL INFORMATION}

$\begin{array}{ll}\text { AUTHORS } & \text { X. Fan } \text { et al. } \\ \text { TELESCOPE } & \text { 0.6/0.9m Schmidt, Beijing Astron. Obs. } \\ \text { DETECTOR } & \text { 2048X2048, UV-coated CCD } \\ \text { MAIN ARTICLE } & \text { Fan, X. et al. 1996, AJ 112, 628 }\end{array}$

\section{SYSTEM DESCRIPTION}

\begin{tabular}{|c|cc|}
\hline \multicolumn{3}{|c|}{ BANDS DESCRIPTION [102] } \\
\hline band & $\lambda_{c}(\AA)$ & FWHM $(\AA)$ \\
\hline 1 & 3360 & 360 \\
\hline 2 & 3890 & 340 \\
\hline 3 & 4210 & 320 \\
\hline 4 & 4550 & 340 \\
\hline 5 & 4925 & 390 \\
\hline 6 & 5270 & 340 \\
\hline 7 & 5795 & 310 \\
\hline 8 & 6075 & 310 \\
\hline 9 & 6660 & 480 \\
\hline $10 a$ & 7050 & 300 \\
\hline 10 & 7215 & 550 \\
\hline $10 b$ & 7490 & 330 \\
\hline 11 & 8020 & 260 \\
\hline 12 & 8480 & 180 \\
\hline 13 & 9190 & 260 \\
\hline 14 & 9745 & 270 \\
\hline
\end{tabular}

\section{SYSTEM ANALYSIS}

RELATIONS WITH OTHER SYSTEMS [102]

UBV - Johnson and Morgan - 1953

$(B-V)_{J}=0.127+0.489\left(m_{3890}-m_{5795}\right)-0.033\left(m_{3890}-m_{5795}\right)^{2}$

$R I$ - Cousins - 1976

\begin{tabular}{|ccc|}
\hline$(V-I)_{C}$ & $=+0.343( \pm 0.015)+1.090( \pm 0.072)\left(m_{5795}-m_{8020}\right)+0.196( \pm 0.083)\left(m_{5795}-m_{8020}\right)^{2}$ \\
\hline$(V-R)_{C}$ & $=+0.002( \pm 0.012)+1.299( \pm 0.04)\left(m_{5795}-m_{6660}\right)$
\end{tabular}

Fig. 153. The photometric system BATC - Fan et al. - 1996 


\section{TRANSMISSION CURVES [102]}

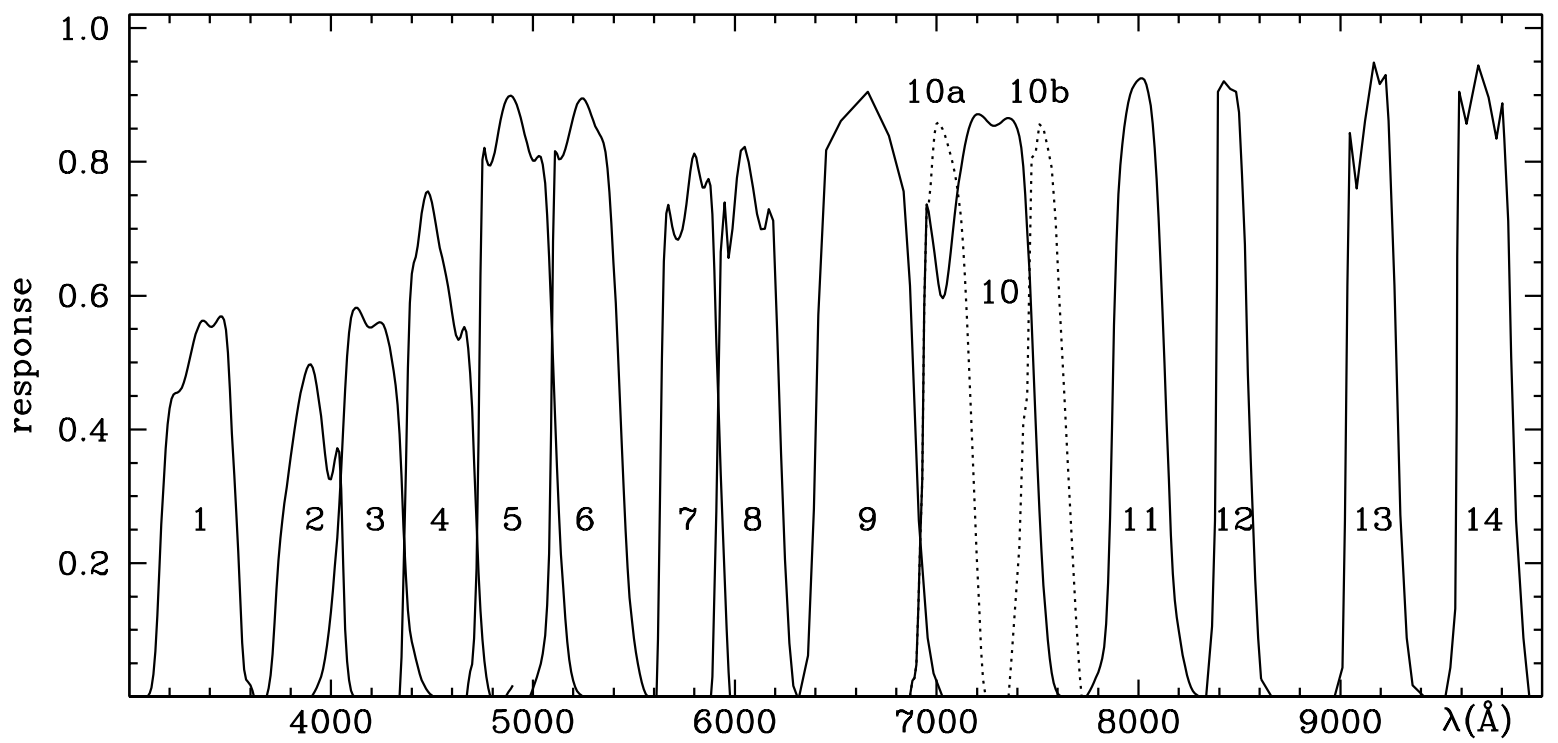

\begin{tabular}{|c|c|c|c|c|c|c|c|c|c|c|c|c|c|c|c|}
\hline \multicolumn{2}{|c|}{1} & \multicolumn{2}{|c|}{2} & \multicolumn{2}{|c|}{3} & \multicolumn{2}{|c|}{4} & \multicolumn{2}{|c|}{5} & \multicolumn{2}{|c|}{6} & \multicolumn{2}{|c|}{7} & \multicolumn{2}{|c|}{8} \\
\hline$\lambda(\mathrm{A})$ & $\Upsilon$ & $\lambda(\mathrm{A})$ & $\Upsilon$ & $\lambda(\mathrm{A})$ & $\Upsilon$ & $\lambda(\mathrm{A})$ & $r$ & $\lambda(\mathrm{A})$ & $r$ & $\lambda(\mathrm{A})$ & $\Upsilon$ & $\lambda(\mathrm{A})$ & $\Upsilon$ & $\lambda(\mathrm{A})$ & $\Upsilon$ \\
\hline 3087 & 0.000 & 3680 & 0.000 & 3902 & 0.000 & 4317 & 0.000 & 4673 & 0.000 & 4984 & 0.000 & 5577 & 0.000 & 5844 & 0.000 \\
\hline 3134 & 0.088 & 3709 & 0.062 & 3961 & 0.044 & 4353 & 0.088 & 4688 & 0.035 & 5035 & 0.044 & 5622 & 0.119 & 5892 & 0.044 \\
\hline 3161 & 0.264 & 3733 & 0.176 & 4006 & 0.145 & 4385 & 0.554 & 4718 & 0.158 & 5073 & 0.158 & 5637 & 0.439 & 5904 & 0.176 \\
\hline 3182 & 0.378 & 3760 & 0.264 & 4050 & 0.343 & 4391 & 0.598 & 4732 & 0.439 & 5088 & 0.351 & 5652 & 0.668 & 5918 & 0.439 \\
\hline 3250 & 0.457 & 3813 & 0.387 & 4080 & 0.510 & 4421 & 0.659 & 4747 & 0.773 & 5103 & 0.747 & 5681 & 0.719 & 5933 & 0.710 \\
\hline 3360 & 0.562 & 3858 & 0.466 & 4119 & 0.582 & 4460 & 0.743 & 4762 & 0.817 & 5118 & 0.815 & 5732 & 0.690 & 5963 & 0.672 \\
\hline 3413 & 0.554 & 3902 & 0.496 & 4184 & 0.554 & 4540 & 0.672 & 4792 & 0.795 & 5147 & 0.808 & 5764 & 0.747 & 6001 & 0.747 \\
\hline 3466 & 0.567 & 3953 & 0.413 & 4279 & 0.540 & 4584 & 0.606 & 4836 & 0.857 & 5192 & 0.857 & 5800 & 0.813 & 6052 & 0.822 \\
\hline 3487 & 0.527 & 3991 & 0.325 & 4302 & 0.501 & 4635 & 0.534 & 4881 & 0.898 & 5236 & 0.895 & 5844 & 0.760 & 6111 & 0.721 \\
\hline 3517 & 0.351 & 4021 & 0.356 & 4347 & 0.351 & 4667 & 0.550 & 4955 & 0.848 & 5325 & 0.844 & 5874 & 0.773 & 6165 & 0.719 \\
\hline 3546 & 0.176 & 4050 & 0.308 & 4371 & 0.176 & 4688 & 0.492 & 5005 & 0.801 & 5370 & 0.791 & 5889 & 0.729 & 6185 & 0.731 \\
\hline 3561 & 0.075 & 4065 & 0.141 & 4406 & 0.075 & 4703 & 0.413 & 5038 & 0.808 & 5429 & 0.457 & 5918 & 0.439 & 6215 & 0.501 \\
\hline 3591 & 0.022 & 4080 & 0.053 & 4451 & 0.025 & 4718 & 0.281 & 5059 & 0.773 & 5474 & 0.176 & 5948 & 0.193 & 6245 & 0.246 \\
\hline \multirow[t]{4}{*}{3620} & 0.000 & 4125 & 0.000 & 4510 & 0.000 & 4747 & 0.097 & 5118 & 0.360 & 5518 & 0.053 & 5969 & 0.044 & 6274 & 0.062 \\
\hline & & & & & & 4777 & 0.018 & 5168 & 0.088 & 5548 & 0.013 & 6046 & 0.000 & 6319 & 0.000 \\
\hline & & & & & & 4866 & 0.000 & 5207 & 0.018 & 5607 & 0.000 & & & & \\
\hline & & & & & & & & 5251 & 0.000 & & & & & & \\
\hline \multicolumn{2}{|c|}{9} & \multicolumn{2}{|c|}{$10 \mathrm{a}$} & \multicolumn{2}{|c|}{10} & \multicolumn{2}{|c|}{$10 b$} & \multicolumn{2}{|c|}{11} & \multicolumn{2}{|c|}{12} & \multicolumn{2}{|c|}{13} & \multicolumn{2}{|c|}{14} \\
\hline$\lambda(\mathrm{A})$ & $\Upsilon$ & $\lambda(\mathrm{A})$ & $\Upsilon$ & $\lambda(\mathrm{A})$ & $\Upsilon$ & $\lambda(\mathrm{A})$ & $\Upsilon$ & $\lambda(\mathrm{A})$ & $\Upsilon$ & $\lambda(\mathrm{A})$ & $\Upsilon$ & $\lambda(\mathrm{A})$ & $\Upsilon$ & $\lambda(\mathrm{A})$ & $\Upsilon$ \\
\hline 6319 & 0.000 & 6867 & 0.000 & 6867 & 0.000 & 7357 & 0.000 & 7742 & 0.000 & 8335 & 0.000 & 8973 & 0.000 & 9521 & 0.000 \\
\hline 6363 & 0.062 & 6882 & 0.026 & 6882 & 0.026 & 7401 & 0.185 & 7816 & 0.053 & 8365 & 0.105 & 9011 & 0.044 & 9545 & 0.044 \\
\hline 6393 & 0.281 & 6906 & 0.088 & 6906 & 0.088 & 7416 & 0.264 & 7846 & 0.141 & 8380 & 0.264 & 9023 & 0.264 & 9569 & 0.132 \\
\hline 6414 & 0.571 & 6918 & 0.220 & 6918 & 0.220 & 7445 & 0.475 & 7867 & 0.351 & 8388 & 0.615 & 9035 & 0.615 & 9577 & 0.422 \\
\hline 6452 & 0.817 & 6935 & 0.439 & 6935 & 0.439 & 7460 & 0.606 & 7884 & 0.615 & 8394 & 0.905 & 9047 & 0.844 & 9580 & 0.659 \\
\hline 6526 & 0.861 & 6941 & 0.624 & 6941 & 0.624 & 7463 & 0.747 & 7914 & 0.808 & 8424 & 0.921 & 9082 & 0.760 & 9583 & 0.791 \\
\hline 6660 & 0.905 & 6956 & 0.734 & 6956 & 0.734 & 7469 & 0.808 & 7944 & 0.879 & 8454 & 0.909 & 9124 & 0.861 & 9589 & 0.905 \\
\hline 6764 & 0.839 & 6986 & 0.830 & 6992 & 0.659 & 7511 & 0.857 & 8000 & 0.923 & 8483 & 0.905 & 9165 & 0.949 & 9625 & 0.857 \\
\hline 6838 & 0.756 & 7016 & 0.857 & 7024 & 0.598 & 7546 & 0.826 & 8062 & 0.879 & 8498 & 0.874 & 9195 & 0.917 & 9684 & 0.945 \\
\hline 6867 & 0.615 & 7075 & 0.804 & 7084 & 0.703 & 7579 & 0.764 & 8098 & 0.729 & 8513 & 0.782 & 9225 & 0.930 & 9735 & 0.896 \\
\hline 6912 & 0.264 & 7134 & 0.659 & 7134 & 0.815 & 7609 & 0.598 & 8113 & 0.628 & 8528 & 0.677 & 9239 & 0.861 & 9773 & 0.835 \\
\hline 6956 & 0.088 & 7164 & 0.475 & 7193 & 0.870 & 7638 & 0.413 & 8127 & 0.518 & 8543 & 0.483 & 9269 & 0.615 & 9803 & 0.888 \\
\hline 6986 & 0.035 & 7208 & 0.149 & 7282 & 0.854 & 7668 & 0.246 & 8142 & 0.395 & 8557 & 0.351 & 9299 & 0.272 & 9812 & 0.835 \\
\hline 7030 & 0.000 & 7223 & 0.053 & 7357 & 0.866 & 7698 & 0.088 & 8157 & 0.264 & 8578 & 0.176 & 9328 & 0.088 & 9832 & 0.712 \\
\hline & & 7253 & 0.000 & 7401 & 0.848 & 7718 & 0.000 & 8202 & 0.097 & 8593 & 0.088 & 9358 & 0.018 & 9847 & 0.510 \\
\hline & & & & 7431 & 0.791 & & & 8231 & 0.044 & 8608 & 0.026 & 9417 & 0.000 & 9871 & 0.264 \\
\hline & & & & 7499 & 0.351 & & & 8305 & 0.000 & 8661 & 0.000 & & & 9907 & 0.088 \\
\hline & & & & 7549 & 0.088 & & & & & & & & & 9921 & 0.044 \\
\hline & & & & 7623 & 0.000 & & & & & & & & & 9936 & 0.000 \\
\hline
\end{tabular}

Fig. 153. continued 


\section{Sloan DSS - Fukugita et al. - 1996}

Photometric system of the SLOAN Digital Sky Survey.

\section{GENERAL INFORMATION}

AUTHORS M. Fukugita, T. Ichikawa, J. E. Gunn,

M. Doi, K. Shimasaku and D. P. Schneider

TELESCOPE 2.5m (reflector), Apache Point Obs.

DETECTOR thinnned, antireflection UV-coated CCD

MAIN ARTICLE Fukugita, M., Ichikawa, T., Gunn, J. E., Doi, M., Shimasaku, K., Schneider, D. P. 1996, AJ 111, 1748

\section{SYSTEM DESCRIPTION}

\begin{tabular}{|c|c|c|c|c|c|c|}
\hline \multicolumn{7}{|c|}{ BANDS DESCRIPTION [109] } \\
\hline band & filter & coating & $\lambda_{e f f}$ & $\AA$ ̊) (\#) & FWH & $(\AA)(\#)$ \\
\hline$u^{\prime}$ & $1 \mathrm{~mm}$ UG11 + 1mm BG38 + 3mm quarz & suppresses $6600-8200 \AA$ & 3557 & 3522 & 599 & 634 \\
\hline$g^{\prime}$ & $2 \mathrm{~mm}$ GG400 + 3mm BG38 & short-pass coating cut off $\lambda 5500 \AA$ & 4825 & 4803 & 1379 & 1409 \\
\hline$r^{\prime}$ & $4 \mathrm{~mm}$ OG550 + 1mm BK7 & short-pass coating cut off $\lambda 7000 \AA$ & 6261 & 6254 & 1382 & 1388 \\
\hline$i^{\prime}$ & 4mm RG695 + 1mm BK7 & short-pass coating cut off $\lambda 8500 \AA$ & 7672 & 7668 & 1535 & 1535 \\
\hline$z^{\prime}$ & 4mm RG830 + 1mm BK7 & & 9097 & 9114 & 1370 & 1409 \\
\hline
\end{tabular}

(\#) left-side values comprise atmospheric extinction, those at right not.

\section{SYSTEM ANALYSIS}

RELATIONS WITH OTHER SYSTEMS [109]

UBV - Johnson and Morgan - 1953, RI - Cousins - 1976

For stars with $(B-V) \leq+1.5$ :

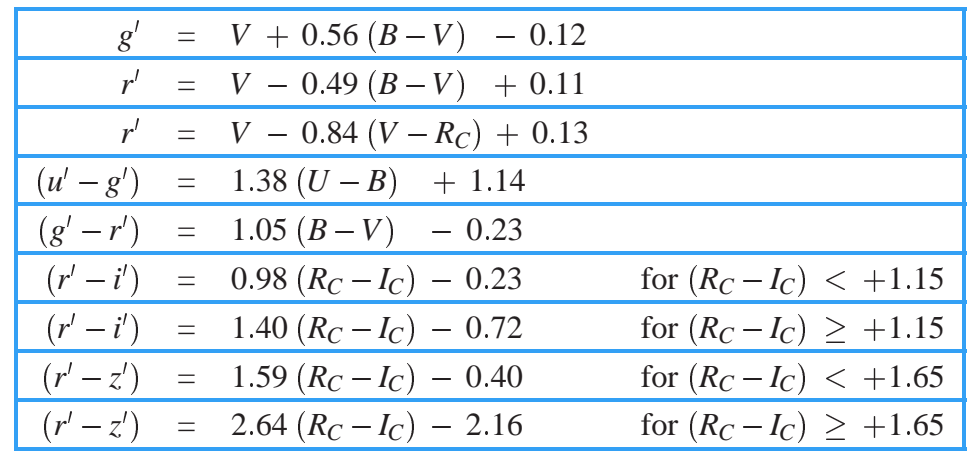

Fig. 154. The photometric system Sloan DSS - Fukugita el al. - 1996 


\section{TRASMISSION CURVES [109]}

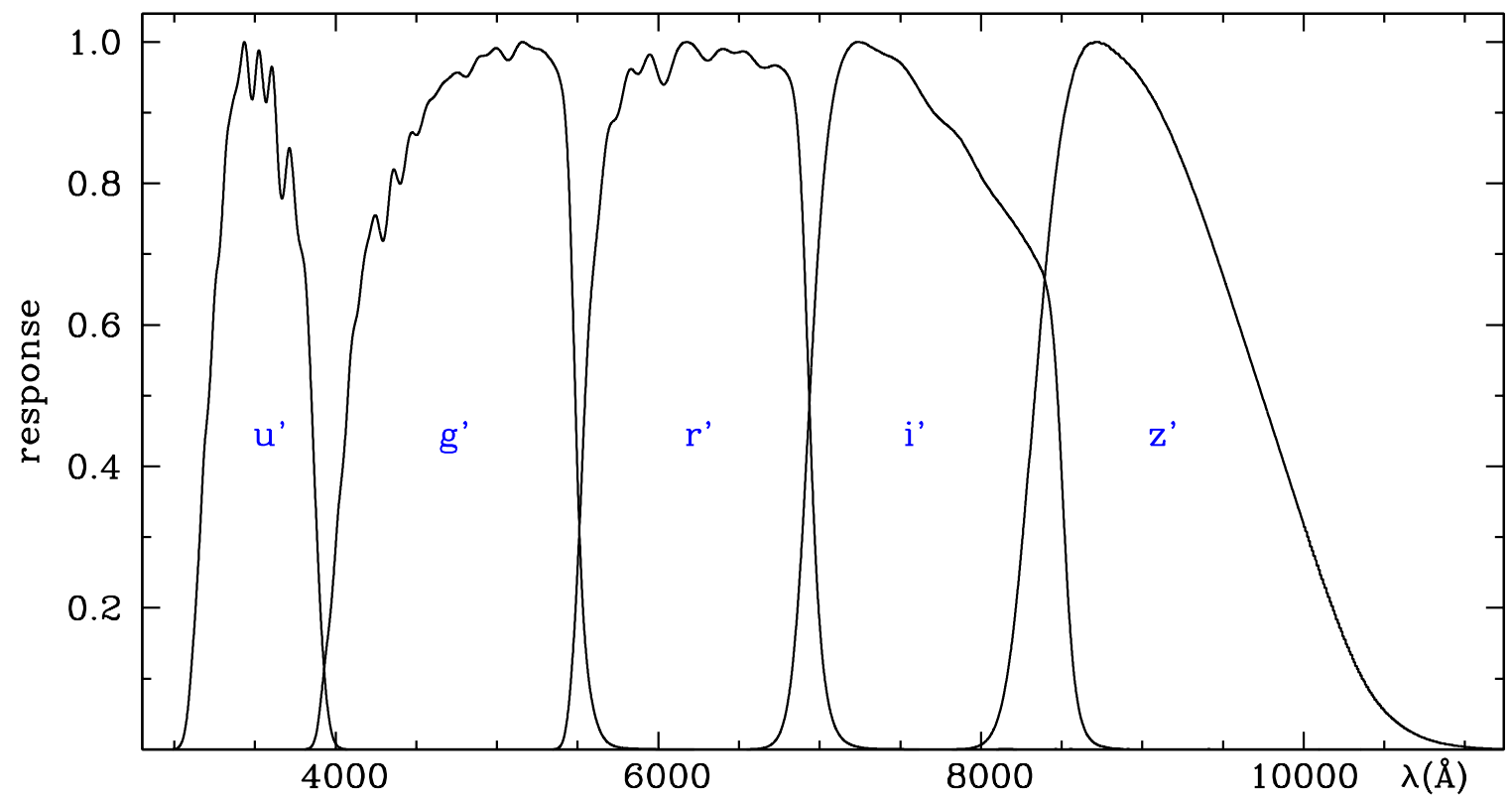

\begin{tabular}{|c|c|c|c|c|c|c|c|c|c|c|c|}
\hline \multicolumn{2}{|c|}{$u^{\prime}$} & \multicolumn{2}{|c|}{$g^{\prime}$} & \multicolumn{2}{|c|}{$r^{\prime}$} & \multicolumn{2}{|c|}{$i^{\prime}$} & \multicolumn{4}{|c|}{$z^{\prime}$} \\
\hline$\lambda(\mathrm{A})$ & $\Upsilon$ & $\lambda(\mathrm{A})$ & $\Upsilon$ & $\lambda(\mathrm{A})$ & $\Upsilon$ & $\lambda(\mathrm{A})$ & $\Upsilon$ & $\lambda(\mathrm{A})$ & $\Upsilon$ & $\lambda(\mathrm{A})$ & $\Upsilon$ \\
\hline 2935 & 0.000 & 3760 & 0.000 & 5280 & 0.000 & 6540 & 0.000 & 7830 & 0.000 & 10430 & 0.077 \\
\hline 2985 & 0.000 & 3860 & 0.008 & 5380 & 0.002 & 6640 & 0.002 & 7930 & 0.001 & 10530 & 0.049 \\
\hline 3035 & 0.005 & 3960 & 0.176 & 5480 & 0.171 & 6740 & 0.017 & 8030 & 0.012 & 10630 & 0.030 \\
\hline 3085 & 0.060 & 4060 & 0.442 & 5580 & 0.633 & 6840 & 0.136 & 8130 & 0.065 & 10730 & 0.018 \\
\hline 3135 & 0.211 & 4160 & 0.663 & 5680 & 0.861 & 6940 & 0.505 & 8230 & 0.232 & 10830 & 0.010 \\
\hline 3185 & 0.420 & 4260 & 0.748 & 5780 & 0.926 & 7040 & 0.843 & 8330 & 0.491 & 10930 & 0.006 \\
\hline 3235 & 0.581 & 4360 & 0.820 & 5880 & 0.955 & 7140 & 0.973 & 8430 & 0.748 & 11030 & 0.003 \\
\hline 3285 & 0.718 & 4460 & 0.868 & 5980 & 0.968 & 7240 & 1.000 & 8530 & 0.912 & 11130 & 0.001 \\
\hline 3335 & 0.881 & 4560 & 0.907 & 6080 & 0.962 & 7340 & 0.990 & 8630 & 0.987 & 11230 & 0.000 \\
\hline 3385 & 0.934 & 4660 & 0.940 & 6180 & 1.000 & 7440 & 0.979 & 8730 & 1.000 & & \\
\hline 3435 & 1.000 & 4760 & 0.957 & 6280 & 0.977 & 7540 & 0.960 & 8830 & 0.986 & & \\
\hline 3485 & 0.919 & 4860 & 0.968 & 6380 & 0.989 & 7640 & 0.923 & 8930 & 0.964 & & \\
\hline 3535 & 0.977 & 4960 & 0.985 & 6480 & 0.985 & 7740 & 0.892 & 9030 & 0.934 & & \\
\hline 3585 & 0.937 & 5060 & 0.975 & 6580 & 0.978 & 7840 & 0.872 & 9130 & 0.891 & & \\
\hline 3635 & 0.868 & 5160 & 1.000 & 6680 & 0.964 & 7940 & 0.837 & 9230 & 0.840 & & \\
\hline 3685 & 0.801 & 5260 & 0.990 & 6780 & 0.960 & 8040 & 0.796 & 9330 & 0.782 & & \\
\hline 3735 & 0.813 & 5360 & 0.965 & 6880 & 0.819 & 8140 & 0.764 & 9430 & 0.717 & & \\
\hline 3785 & 0.707 & 5460 & 0.705 & 6980 & 0.250 & 8240 & 0.731 & 9630 & 0.579 & & \\
\hline 3835 & 0.579 & 5560 & 0.117 & 7080 & 0.042 & 8340 & 0.693 & 9730 & 0.508 & & \\
\hline 3885 & 0.297 & 5660 & 0.017 & 7180 & 0.008 & 8440 & 0.602 & 9830 & 0.438 & & \\
\hline 3935 & 0.094 & 5760 & 0.003 & 7280 & 0.002 & 8540 & 0.213 & 9930 & 0.367 & & \\
\hline 3985 & 0.016 & 5860 & 0.001 & 7380 & 0.001 & 8640 & 0.030 & 10030 & 0.300 & & \\
\hline 4035 & 0.001 & 5960 & 0.000 & 7480 & 0.000 & 8740 & 0.005 & 10130 & 0.233 & & \\
\hline 4085 & 0.000 & & & & & 8840 & 0.001 & 10230 & 0.171 & & \\
\hline 4135 & 0.000 & & & & & 8940 & 0.000 & 10330 & 0.118 & & \\
\hline
\end{tabular}

Fig. 154. continued 


\section{StrömVil - Straizys et al. - 1996}

Combines the uvby Hß - Strömgren and Crawford -1956 system with three bands from Vilnius - Straizys et al. - 1965.

\section{GENERAL INFORMATION}

AUTHORS $\quad$ V. Straizys, D. L. Crawford and A. G. Davis Philip

MAIN ARTICLE Straizys V., Crawford, D. L., Davis Philip A. G. 1996, Baltic Astron. 5, 83

\section{SYSTEM DESCRIPTION}

\begin{tabular}{|l|lcc|}
\hline \multicolumn{4}{|c|}{ BANDS DESCRIPTION [290] } \\
\hline band & band (\#) & $\lambda_{0}(\mathrm{~A})$ & FWHM (A) \\
\hline$u$ & 35 & 3500 & 300 \\
\hline$P$ & 37 & 3740 & 260 \\
\hline$V$ & 41 & 4110 & 190 \\
\hline$b$ & 47 & 4670 & 180 \\
\hline$Z$ & 51 & 5160 & 210 \\
\hline$y$ & 55 & 5470 & 230 \\
\hline$S$ & 66 & 6560 & 200 \\
\hline
\end{tabular}

(\#) Alternative name.

It has been proposed for implementation on the GAIA mission by ESA. [290]

\section{TRANSMISSION CURVES [211]}

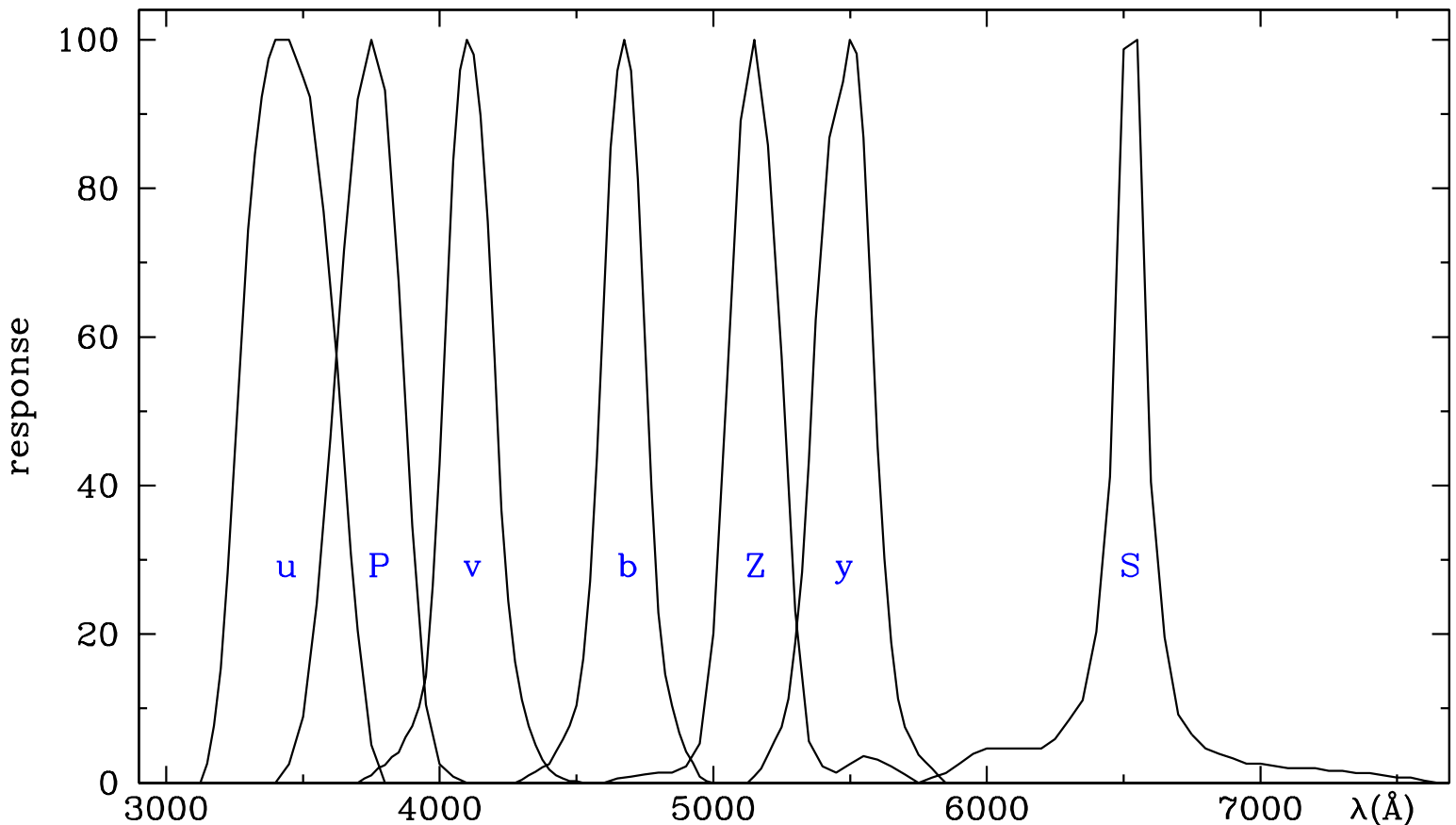

Fig. 155. The photometric system StrömVil - Straižys et al. - 1996 


\begin{tabular}{|c|c|c|c|c|c|c|c|c|c|c|c|c|c|c|c|}
\hline \multicolumn{2}{|c|}{$u$} & \multicolumn{2}{|c|}{$P$} & \multicolumn{2}{|c|}{ V } & \multicolumn{2}{|c|}{$b$} & \multicolumn{2}{|c|}{$Z$} & \multicolumn{2}{|c|}{$y$} & \multicolumn{4}{|c|}{$S$} \\
\hline$\lambda(\AA)$ & $\Upsilon$ & $\lambda(\AA)$ & $\Upsilon$ & $\lambda(\AA)$ & $\Upsilon$ & $\lambda(\AA)$ & $\Upsilon$ & $\lambda(\AA)$ & $\Upsilon$ & $\lambda(\AA)$ & $\Upsilon$ & $\lambda(\AA)$ & $\Upsilon$ & $\lambda(\AA)$ & $\Upsilon$ \\
\hline 3125 & 0.0 & 3400 & 0.0 & 3700 & 0.0 & 4275 & 0.0 & 4600 & 0.0 & 5150 & 0.0 & 5750 & 0.0 & 6950 & 2.6 \\
\hline 3150 & 2.6 & 3450 & 2.5 & 3750 & 1.0 & 4300 & 0.4 & 4650 & 0.6 & 5200 & 3.8 & 5800 & 0.7 & 7000 & 2.6 \\
\hline 3200 & 15.4 & 3500 & 8.9 & 3800 & 2.4 & 4350 & 1.5 & 4700 & 0.8 & 5250 & 7.5 & 5850 & 1.3 & 7050 & 2.3 \\
\hline 3250 & 43.6 & 3550 & 24.1 & 3850 & 4.1 & 4400 & 2.5 & 4750 & 1.1 & 5300 & 18.9 & 5900 & 2.6 & 7100 & 2.0 \\
\hline 3300 & 74.4 & 3600 & 46.4 & 3900 & 7.6 & 4450 & 5.8 & 4800 & 1.4 & 5350 & 43.4 & 5950 & 3.9 & 7150 & 2.0 \\
\hline 3350 & 92.3 & 3650 & 71.7 & 3950 & 14.3 & 4500 & 10.4 & 4850 & 1.4 & 5400 & 77.4 & 6000 & 4.6 & 7200 & 2.0 \\
\hline 3400 & 100.0 & 3700 & 92.0 & 4000 & 42.9 & 4550 & 27.1 & 4900 & 2.2 & 5450 & 90.6 & 6050 & 4.6 & 7250 & 1.6 \\
\hline 3450 & 100.0 & 3750 & 100.0 & 4050 & 83.7 & 4600 & 64.6 & 4950 & 5.3 & 5500 & 100.0 & 6100 & 4.6 & 7300 & 1.6 \\
\hline 3500 & 94.9 & 3800 & 93.2 & 4100 & 100.0 & 4650 & 95.8 & 5000 & 20.1 & 5550 & 86.8 & 6150 & 4.6 & 7350 & 1.3 \\
\hline 3550 & 84.6 & 3850 & 67.5 & 4150 & 89.8 & 4675 & 100.0 & 5050 & 53.8 & 5600 & 45.3 & 6200 & 4.6 & 7400 & 1.3 \\
\hline 3600 & 66.7 & 3900 & 34.6 & 4200 & 57.1 & 4700 & 95.8 & 5100 & 89.1 & 5650 & 18.9 & 6250 & 5.9 & 7450 & 1.0 \\
\hline 3650 & 43.6 & 3950 & 10.5 & 4250 & 24.5 & 4750 & 60.4 & 5150 & 100.0 & 5700 & 7.5 & 6300 & 8.5 & 7500 & 0.7 \\
\hline 3700 & 20.5 & 4000 & 2.5 & 4300 & 11.2 & 4800 & 22.9 & 5200 & 85.8 & 5750 & 3.8 & 6350 & 11.1 & 7550 & 0.7 \\
\hline 3750 & 5.1 & 4050 & 0.8 & 4350 & 5.1 & 4850 & 10.4 & 5250 & 57.4 & 5800 & 1.9 & 6400 & 20.3 & 7600 & 0.3 \\
\hline 3800 & 0.0 & 4100 & 0.0 & 4400 & 1.8 & 4900 & 4.2 & 5300 & 23.1 & 5850 & 0.0 & 6450 & 41.2 & 7650 & 0.0 \\
\hline & & & & 4450 & 0.6 & 4950 & 0.8 & 5350 & 5.6 & & & 6500 & 98.7 & & \\
\hline & & & & 4500 & 0.2 & 5000 & 0.0 & 5400 & 2.2 & & & 6550 & 100.0 & & \\
\hline & & & & 4525 & 0.0 & & & 5450 & 1.4 & & & 6600 & 40.5 & & \\
\hline & & & & & & & & 5500 & 2.5 & & & 6650 & 19.6 & & \\
\hline & & & & & & & & 5550 & 3.6 & & & 6700 & 9.2 & & \\
\hline & & & & & & & & 5600 & 3.1 & & & 6750 & 6.5 & & \\
\hline & & & & & & & & 5650 & 2.2 & & & 6800 & 4.6 & & \\
\hline & & & & & & & & 5700 & 1.1 & & & 6850 & 3.9 & & \\
\hline & & & & & & & & 5750 & 0.0 & & & 6900 & 3.3 & & \\
\hline
\end{tabular}

Fig. 155. continued 


\section{ESO MIR - Van der Bliek et al - 1996}

Mid-infrared photometry at ESO.

\section{GENERAL INFORMATION}

AUTHORS

TELESCOPE

DETECTOR

MAIN ARTICLE Van der Bliek, N. S., Manfroid, J., Bouchet, P. 1996, A\&AS 109, 547

N. S. Van der Bliek, J. Manfroid and P. Bouchet

$3.6 \mathrm{~m}, 2.2 \mathrm{~m}, 1.0 \mathrm{~m}$ (reflectors), ESO (La Silla)

bolometer

\section{SYSTEM DESCRIPTION}

\begin{tabular}{|l|ccc|cc|}
\hline \multicolumn{4}{|c|}{ BANDS DESCRIPTION [38] } & \multicolumn{2}{c|}{ FLUX CALIBRATION (\#) [38] } \\
\hline band & $\lambda_{0}(\mu \mathrm{m})$ & $\lambda_{\text {eff } f}(\mu \mathrm{m})(\# \#)$ & FWHM $(\mu \mathrm{m})$ & $\mathrm{F}_{\lambda, 0}\left(\mathrm{~W} \mathrm{~m}^{-2} \mathrm{~nm}^{-1}\right)$ & $\mathrm{F}_{v, 0}(\mathrm{Jy})$ \\
\hline$N$ & 11.055 & 9.682 & 5.47 & $1.2910^{-15}$ & $4.1710^{1}$ \\
\hline$N_{1}$ & 8.361 & 8.328 & 0.72 & $2.5010^{-15}$ & $5.7810^{1}$ \\
\hline$N_{2}$ & 9.787 & 9.650 & 1.57 & $1.4010^{-15}$ & $4.3510^{1}$ \\
\hline$N_{3}$ & 12.819 & 12.774 & 1.15 & $4.6410^{-16}$ & $2.5310^{1}$ \\
\hline$Q_{0}$ & 18.666 & 18.425 & 3.23 & $1.0910^{-16}$ & $1.2310^{1}$ \\
\hline
\end{tabular}

(\#) Fluxes for a 0.0 mag star.

(\#\#) For a $11400 \mathrm{~K}$ blackbody.

ZERO POINT: The colors of $\beta$ Hyi (G2 IV) and $\alpha$ Cen (G2 V) are taken equal to the colors of the Sun. [38]

Fig. 156. The photometric system ESO MIR - Van der Bliek et al. - 1996 


\section{ESO NIR - Van der Bliek et al. - 1996}

Infrared line photometry via CWF at ESO.

\section{GENERAL INFORMATION}

AUTHORS

TELESCOPE

DETECTOR

MAIN ARTICLE Van der Bliek, N. S., Manfroid, J., Bouchet, P. 1996, A\&AS 109, 547

N. S. Van der Bliek, J. Manfroid and P. Bouchet ESO 3.6m, ESO/MPI 2.2m, ESO 1m $\mathrm{InSb}$

\section{SYSTEM DESCRIPTION}

\begin{tabular}{|l|ccc|cc|}
\hline \multicolumn{3}{|c|}{ BANDS DESCRIPTION [38] } & \multicolumn{2}{|c|}{ FLUX CALIBRATION (\#) [38] } \\
\hline band & $\lambda_{0}(\mu \mathrm{m})$ & $\lambda_{\text {eff }}(\mu \mathrm{m})(\# \#)$ & FWHM $(\mu \mathrm{m})$ & $\mathrm{F}_{\lambda, 0}\left(\mathrm{~W} \mathrm{~m}^{-2} \mathrm{~nm}^{-1}\right)$ & $\mathrm{F}_{\mathrm{v}, 0}(\mathrm{Jy})$ \\
\hline $\mathrm{H}_{0}$ & 1.577 & 1.577 & 0.031 & $1.3710^{-12}$ & $1.1410^{3}$ \\
\hline$B r_{\gamma}$ & 2.161 & 2.161 & 0.037 & $4.3810^{-13}$ & $6.8310^{2}$ \\
\hline$K_{0}$ & 2.221 & 2.220 & 0.038 & $3.9710^{-13}$ & $6.5310^{2}$ \\
\hline$C O$ & 2.291 & 2.290 & 0.039 & $3.5410^{-13}$ & $6.1910^{2}$ \\
\hline$L_{0}$ & 3.706 & 3.706 & 0.050 & $5.7810^{-14}$ & $2.6510^{2}$ \\
\hline
\end{tabular}

(\#) Fluxes for a 0.0 mag. star.

(\#\#) Best fit values with a $11400 \mathrm{~K}$ black body.

The bands are obtained via a Continous Wheel Filter (CWF), at fixed positions.

ZERO POINT: For the star HR 3314 (A0 V) it is $\mathrm{H}_{0}=H, B r_{\gamma}=K_{0}=C O=K$ and $L_{0}=L^{\prime}$ where $H, K$, and $L^{\prime}$, are from the JHKL'M ESO - Bouchet et al. - 1991 system. [38]

Fig. 157. The photometric system ESO NIR - Van der Bliek et al. - 1996 


\section{MANIAC - Böker et al. - 1997}

Photometric system for the Mid And Near Infrared Array Camera (MANIAC).

\section{GENERAL INFORMATION}

AUTHORS

TELESCOPE

DETECTOR

T. Böker, J. W. V. Storey, A. Krabbe and T. Lehmann

$2.2 \mathrm{~m}$ (reflector), ESO (La Silla)

Si:As CCD, 128 X 128 pixels

MAIN ARTICLE Böker, T., Storey, J. W. V., Krabbe, A., Lehmann, T. 1997, PASP 109, 827

\section{SYSTEM DESCRIPTION}

\begin{tabular}{|c|cccl|}
\hline \multicolumn{5}{|c|}{ BANDS DESCRIPTION [40] } \\
\hline band & $\lambda_{0}(\mu \mathrm{m})$ & width $(\mu \mathrm{m})$ & $\Upsilon_{\text {peak }}(\%)$ & feature \\
\hline $803(\#)$ & 8.03 & 0.15 & 45 & \\
\hline 1036 & 10.36 & 0.12 & 60 & continuum \\
\hline$N$ & 10.50 & 5.0 & 80 & N-band \\
\hline 1053 & 10.53 & 0.12 & 60 & SIV \\
\hline 1068 & 10.68 & 0.12 & 60 & continuum \\
\hline 1256 & 12.56 & 0.15 & 54 & continuum \\
\hline 1282 & 12.82 & 0.15 & 54 & NeII \\
\hline 1295 & 12.95 & 0.12 & 62 & continuum \\
\hline$Q^{\prime}$ & 17.60 & 1.80 & 60 & Q'-band \\
\hline 1843 & 18.43 & 0.19 & 40 & continuum \\
\hline 1873 & 18.73 & 0.19 & 40 & SIII \\
\hline 1900 & 19.00 & 0.21 & 40 & continuum \\
\hline$Q$ & 20.00 & 5.40 & 45 & Q-band \\
\hline
\end{tabular}

(\#) The 803 band is also called "the Sweet Spot".

Fig. 158. The photometric system MANIAC - Böker et al. - 1997 


\section{Damineli et al. - 1997}

Near-infrared photometry of Wolf-Rayet stars.

\section{GENERAL INFORMATION}

AUTHORS A. Damineli, F. Jablonski, L. C. de Freitas and J. A. de Freitas-Pacheco

TELESCOPE $\quad 1.6 \mathrm{~m}$ (reector), Laboratorio Nacional de Astrosica, Brazil

DETECTOR GEC 385 x 578 pixel, front illuminated CCD

MAIN ARTICLE Damineli, A., Jablonski, F., de Freitas, L. C., de Freitas-Pacheco, J. A. 1997, PASP 109, 633

\section{SYSTEM DESCRIPTION}

\begin{tabular}{|c|crrrc|}
\hline \multicolumn{5}{|c|}{ BANDS DESCRIPTION [82] } \\
\hline band & \multicolumn{2}{|c|}{$\lambda_{c}(\mathrm{~A})(\#)$} & FWHM $(\mathrm{A})(\#)$ & feature \\
\hline 970 & 9716 & 9709 & 143 & 150 & CIII \\
\hline 990 & 9901 & 9894 & 192 & 198 & continuum \\
\hline 1013 & 10133 & 10126 & 90 & 96 & HeII \\
\hline
\end{tabular}

(\#) Left side values are for an unobstructed parallel incident beam, right side quantities are for an f/5.6 and $20 \%$ central obstruction incident beam.

Filters by Boston Electronics.

ZERO POINT: Colors are zero for $\theta$ Vir (A1 IV) [82].

\section{TRANSMISSION CURVES}

As derived from Fig 3 of [82] for the case of a parallel beam.

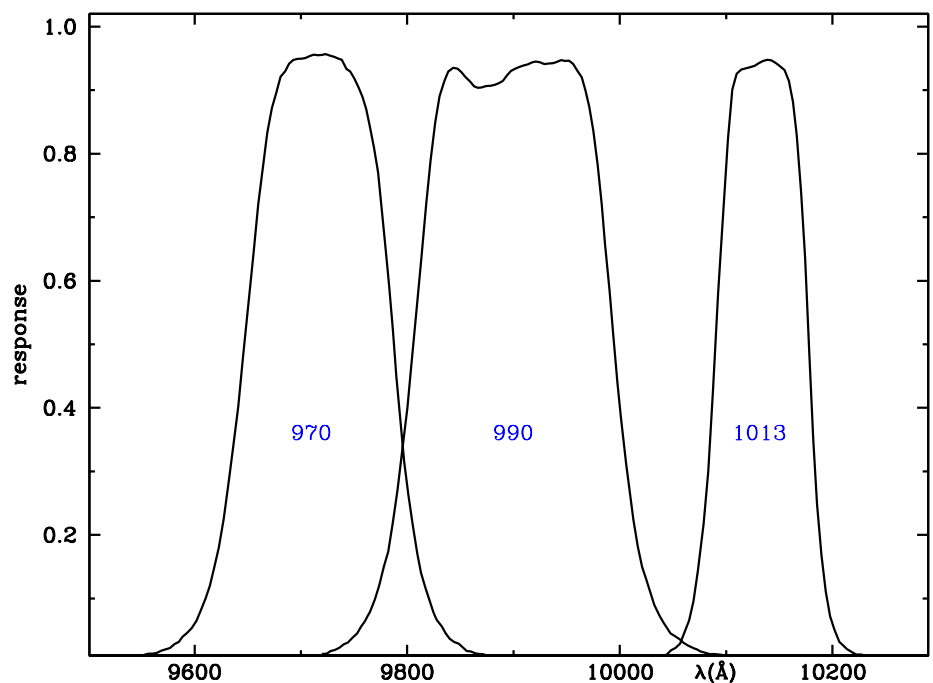

\begin{tabular}{|cc|cc|cc|}
\hline \multicolumn{2}{|c|}{970} & \multicolumn{2}{|c|}{990} & \multicolumn{2}{c|}{1013} \\
\hline$\lambda(\mathrm{A})$ & $\Upsilon$ & $\lambda(\mathrm{A})$ & $\Upsilon$ & $\lambda(\mathrm{A})$ & $\Upsilon$ \\
\hline 9500 & 0.000 & 9690 & 0.000 & 10010 & 0.000 \\
9515 & 0.004 & 9700 & 0.005 & 10020 & 0.006 \\
9530 & 0.007 & 9715 & 0.013 & 10030 & 0.008 \\
9545 & 0.009 & 9730 & 0.018 & 10040 & 0.010 \\
9560 & 0.013 & 9745 & 0.032 & 10050 & 0.020 \\
9575 & 0.021 & 9760 & 0.063 & 10060 & 0.042 \\
9590 & 0.042 & 9775 & 0.128 & 10070 & 0.103 \\
9605 & 0.077 & 9790 & 0.261 & 10080 & 0.235 \\
9620 & 0.159 & 9805 & 0.487 & 10090 & 0.499 \\
9635 & 0.321 & 9820 & 0.757 & 10100 & 0.781 \\
9650 & 0.554 & 9835 & 0.915 & 10110 & 0.926 \\
9665 & 0.787 & 9850 & 0.930 & 10120 & 0.935 \\
9680 & 0.917 & 9865 & 0.904 & 10130 & 0.942 \\
9695 & 0.949 & 9880 & 0.907 & 10140 & 0.948 \\
9710 & 0.955 & 9895 & 0.926 & 10150 & 0.938 \\
9725 & 0.956 & 9910 & 0.939 & 10160 & 0.908 \\
9740 & 0.943 & 9925 & 0.944 & 10170 & 0.752 \\
9755 & 0.902 & 9940 & 0.948 & 10180 & 0.418 \\
9770 & 0.798 & 9955 & 0.944 & 10190 & 0.162 \\
9785 & 0.553 & 9970 & 0.884 & 10200 & 0.060 \\
9800 & 0.277 & 9985 & 0.681 & 10210 & 0.026 \\
9815 & 0.128 & 10000 & 0.401 & 10220 & 0.014 \\
9830 & 0.061 & 10015 & 0.200 & 10230 & 0.010 \\
9845 & 0.031 & 10030 & 0.102 & 10240 & 0.007 \\
9860 & 0.016 & 10045 & 0.051 & 10250 & 0.003 \\
9875 & 0.010 & 10060 & 0.030 & 10260 & 0.000 \\
9890 & 0.005 & 10075 & 0.017 & & \\
9890 & 0.000 & 10090 & 0.008 & & \\
& & 10105 & 0.000 & & \\
\hline & & & & & \\
\hline
\end{tabular}

Fig. 159. The photometric system Damineli et al. - 1997 


\section{TNG - Marchetti et al. - 1997}

Discrimination between M, S and Carbon stars in observations obtained with the Speckle Camera at TNG.

\section{GENERAL INFORMATION}

AUTHORS

TELESCOPE

DETECTOR

MAIN ARTICLE
E. Marchetti, S. Mallucci, A. Ghedina, J. Farinato, A. Baruffolo, U. Munari, R. Ragazzoni 3.6m (reflector) Telescopio Nazionale Galileo, La Palma

CCD

Marchetti, E., Mallucci, S., Ghedina, A., Farinato, J.,

Baruffolo, A., Munari, U., Ragazzoni, R. 1997, proc. "The Three Galileos:

the Man, the Spacecraft, the Telescope" (Padova 1997),

Dordrecht Kluwer Academic Publishers, Ap\&SpSci Lib. vol 220, 383

(C. Barbieri, J. H. Rahe, T. V. Johnson, A. M. Sohus ed.s)

\section{SYSTEM DESCRIPTION}

\begin{tabular}{|l|cccl|}
\hline \multicolumn{5}{|c|}{ BANDS DESCRIPTION [196] } \\
\hline \multicolumn{1}{|c|}{ band } & filter & $\lambda_{0}(\AA)$ & half-width $(\AA)$ & feature \\
\hline$b(\#)$ & Oriel 51690 & 4750 & 300 & \\
\hline$y(\#)$ & Oriel 51300 + Oriel 51970 & 5470 & 300 & \\
\hline$C_{2}$ & interference & 5500 & 100 & $\mathrm{C}_{2}$ band \\
\hline$C_{2}$ cont & interference & 5700 & 100 & continuum \\
\hline broad blue & Oriel 57377 (short-pass) & 5800 & 1000 & \\
\hline $\mathrm{ZrO}$ & interference & 6500 & 100 & ZrO band \\
\hline 660 & interference & 6600 & 100 & continuum, $\mathrm{H} \alpha$ and $[\mathrm{NII}]$ \\
\hline $\mathrm{TiO}$ & interference & 6700 & 100 & TiO band \\
\hline
\end{tabular}

(\#) b, y intended to match the corresponding bands of the uvbyH $\beta$ - Strömgren and Crawford - 1956 system.

\section{SYSTEM ANALYSIS}

COLOR INDICES AND PARAMETERS [196]

$$
\begin{aligned}
& \left(\mathrm{C}_{2}-\mathrm{C}_{2} \text { cont }\right): \mathrm{C}_{2} \text { band index } \\
& (\mathrm{TiO}-660): \mathrm{TiO} \text { band index } \\
& (\mathrm{ZrO}-660): \mathrm{ZrO} \text { band index }
\end{aligned}
$$

Fig. 160. The photometric system TNG - Marchetti et al. - 1997 


\section{$\mathrm{UBV}(\mathrm{RI})_{M W}-$ Sandage - 1997}

Standard UBVRI photometry at Mount Wilson.

\section{GENERAL INFORMATION}

$\begin{array}{ll}\text { AUTHORS } & \text { A. Sandage } \\ \text { TELESCOPE } & 1.52 \mathrm{~m} \text { and 2.54m (reflectors), Mount Wilson Obs. } \\ \text { DETECTOR } & \text { extended S-20 } \\ \text { MAIN ARTICLE } & \text { Sandage, A. 1997, PASP 109, 1193 }\end{array}$

\section{SYSTEM ANALYSIS}

RELATIONS WITH OTHER SYSTEMS [255]

UBVRI(JHKLMN) - Johnson - 1965

\begin{tabular}{|c|c|c|c|}
\hline$(V-R)_{M W}$ & $=$ & $0.70(V-R)_{J}$ & for $(V-R)_{M W}<0.80$ \\
\hline$(V-R)_{M W}$ & $=$ & $0.43(V-R)_{J}+0.312$ & for $(V-R)_{M W}>0.80$ \\
\hline
\end{tabular}

RI - Eggen - 1965

$(R-I)_{M W}=1.20(R-I)_{E}+0.08 \quad 0<(R-I)_{M W}<2.3$

Fig. 161. The photometric system UBV $(\mathrm{RI})_{M W}-$ Sandage - 1997 


\section{UWTAT - Strassmeier et al. - 1997}

Photometry with the Twin Automatic Photoelectric Telescope of the University of Wien.

\section{GENERAL INFORMATION}

AUTHORS

TELESCOPE

DETECTOR

K. Strassmeier, L. J. Boyd, D. H. Epand and Th. Granzer

$0.75 \mathrm{~m}$ (reflectors), Fairborn Obs.

EMI-9124QB (telescope Wolfgang)

EMI-9828 (telescope Amadeus)

MAIN ARTICLE Strassmeier, K. G., Boyd, L. J., Epand, D. H., Granzer, Th. 1997, PASP 109, 697

\section{SYSTEM DESCRIPTION}

\begin{tabular}{|c|rrr|}
\hline \multicolumn{4}{|c|}{ BANDS DESCRIPTION [291] } \\
\hline band & $\lambda_{c}(\AA)$ & FWHM $(\AA)$ & $\Upsilon_{\text {peak }}$ \\
\hline$u$ & 3500 & 340 & \\
\hline$U$ & 3540 & 675 & 79 \\
\hline$V$ & 4102 & 165 & 52 \\
\hline$B$ & 4340 & 985 & 57 \\
\hline$H \beta$ wide & 4862 & 180 & 72 \\
\hline$H \beta$ narrow & 4864 & 30 & 66 \\
\hline$b$ & 4870 & 240 & 78 \\
\hline$V$ & 5500 & 1150 & 78 \\
\hline b & 5491 & 248 & 77 \\
\hline$R_{C}$ & 6255 & 1700 & 75 \\
\hline$H \alpha \quad$ wide & 6562 & 285 & 82 \\
\hline$H \alpha$ narrow & 6564 & 31 & 77 \\
\hline$I_{C}$ & 7875 & 1350 & 82 \\
\hline
\end{tabular}

Fig. 162. The photometric system UWTAT - Strassmeier et al. - 1997 


\section{NICMOS HST - 1997}

Filter system for the Near-Infrared Camera and Multi-Object Spectrograph on board HST.

\section{GENERAL INFORMATION}

TELESCOPE

DETECTOR

MAIN ARTICLE 2.4m Hubble Space Telescope

HgCdTe arrays (NIC1, NIC2, NIC3)

Calzetti et al 1999, NICMOS Instrument Handbook, Version 3.0 (Baltimore: STScI)

\section{SYSTEM DESCRIPTION}

\begin{tabular}{|c|c|c|c|c|}
\hline \multicolumn{5}{|c|}{ BANDS DESCRIPTION [56], pg. 41} \\
\hline band & $\lambda_{c}(\mu \mathrm{m})$ & bandwidth $(\mu \mathrm{m})$ & NIC & feature \\
\hline F090M & 0.9 & $0.8-1.0$ & 1 & \\
\hline F095N & 0.953 & $1 \%$ & 1 & {$[\mathrm{~S}$ III] } \\
\hline F097N & 0.97 & $1 \%$ & 1 & [S III] continuum \\
\hline F108N & 1.083 & $1 \%$ & 1,3 & He I \\
\hline F110M & 1.1 & $1.0-1.2$ & 1 & \\
\hline F110W & 1.025 & $0.8-1.35$ & $1,2,3$ & \\
\hline$F 113 N$ & 1.13 & $1 \%$ & 1,3 & He I continuum \\
\hline F140W & 1.3 & $0.8-1.8$ & 1 & \\
\hline F145M & 1.45 & $1.35-1.55$ & 1 & $\mathrm{H}_{2} \mathrm{O}$ \\
\hline F150W & 1.5 & $1.1-1.9$ & 3 & \\
\hline F160W & 1.55 & $1.35-1.75$ & $1,2,3$ & \\
\hline F164N & 1.644 & $1 \%$ & 1,3 & {$[\mathrm{Fe} \mathrm{II}]$} \\
\hline F165M & 1.6 & $1.55-1.75$ & 1,2 & \\
\hline$F 166 N$ & 1.66 & $1 \%$ & 1,3 & [Fe II] continuum \\
\hline F170M & 1.7 & $1.6-1.8$ & 1 & \\
\hline F171M & 1.715 & $1.68-1.75$ & 2 & $\mathrm{HCO}_{2}$ and $\mathrm{C}_{2}$ continuum \\
\hline F175W & 1.75 & $1.2-2.3$ & 3 & \\
\hline F180M & 1.80 & $1.765-1.835$ & 2 & $\mathrm{HCO}_{2}$ and $\mathrm{C}_{2}$ band \\
\hline F187N & 1.87 & $1 \%$ & $1,2,3$ & Pashen $\alpha$ \\
\hline F187W & 1.875 & $1.75-2.0$ & 2 & \\
\hline F190N & 1.90 & $1 \%$ & $1,2,3$ & Pashen $\alpha$ continuum \\
\hline F196N & 1.962 & $1 \%$ & 3 & {$[\mathrm{Si} \mathrm{VI}]$} \\
\hline F200N & 2.0 & $1 \%$ & 3 & [Si VI] continuum \\
\hline F204M & 2.04 & $1.9-2.09$ & 2 & $\mathrm{CH}_{4}$ \\
\hline F205M & 2.05 & $1.8-2.3$ & 3 & \\
\hline F205W & 1.9 & $1.75-2.35$ & 2 & \\
\hline
\end{tabular}

continues

Fig. 163. The photometric system NICMOS HST - 1997 


\begin{tabular}{|c|cclll|}
\hline \multicolumn{5}{|c|}{ BANDS DESCRIPTION [56], pg. 41 } \\
\hline band & $\lambda_{c}(\mu \mathrm{m})$ & bandwidth $(\mu \mathrm{m})$ & NIC & feature \\
\hline F207M & 2.1 & $2.0-2.15$ & 2 & & \\
\hline F212N & 2.121 & $1 \%$ & 2,3 & $\mathrm{H}_{2}$ \\
\hline F215N & 2.15 & $1 \%$ & 2,3 & $\mathrm{H}_{2}$ and Br $\gamma$ continuum \\
\hline F216N & 2.165 & $1 \%$ & 2 & $\mathrm{Br} \gamma$ \\
\hline F222M & 2.3 & $2.15-2.30$ & 2,3 & $\mathrm{CO}$ continuum \\
\hline F237M & 2.375 & $2.3-2.45$ & 2 & $\mathrm{CO}$ \\
\hline F240M & 2.4 & $2.3-2.5$ & 3 & $\mathrm{CO}$ \\
\hline
\end{tabular}

The "NIC" column lists the cameras for which the given lter is available

\section{SYSTEM ANALYSIS}

RELATIONS WITH OTHER SYSTEMS [282]

JHKL' CTIO - Elias et al. - 1982

from observations obtained with the NIC2 camera:

for $-1.3<(F 110 W-F 222 M)<0.0$
\begin{tabular}{|c|c|}
\hline$J_{C I T}$ & $=F 110 W-0.198( \pm 0.036)(F 110 W-F 222 M)+21.754( \pm 0.030)$ \\
\hline$H_{C I T}=$ & $F 160 W-0.177( \pm 0.037)(F 110 W-F 222 M)+21.450( \pm 0.028)$ \\
\hline$K_{C I T}=F 222 W-0.074( \pm 0.037)(F 110 W-F 222 M)+20.115( \pm 0.031)$ \\
\hline
\end{tabular}

\begin{tabular}{|c|c|}
\hline$J_{C I T}$ & $=F 110 W-0.344( \pm 0.063)(F 110 W-F 160 W)+22.054( \pm 0.034)$ \\
\hline$H_{C I T}$ & $=F 160 W-0.305( \pm 0.065)(F 110 W-F 160 W)+21.715( \pm 0.037)$ \\
\hline
\end{tabular}

\section{TRANSMISSION CURVES [56]}

To save space the transmission proles of the bands are plotted but not tabulated. The proles can be obtained in tabular form via STSDAS (inside Iraf) or from the ADPS $w w w$ site.

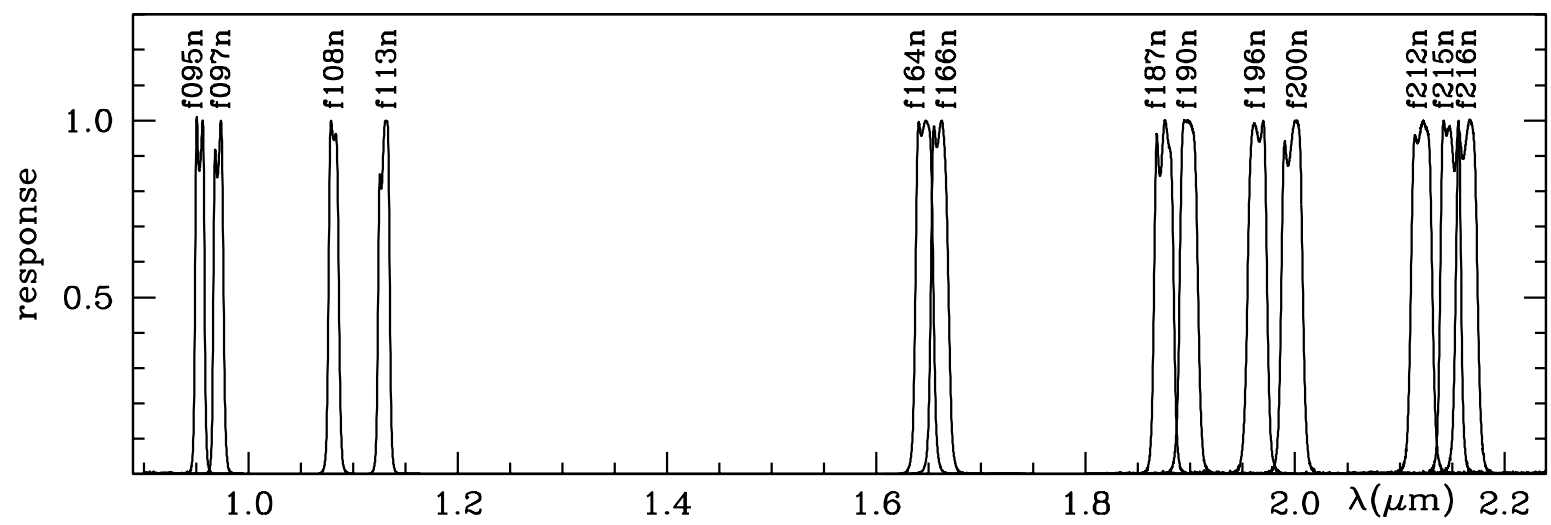

Fig. 163. continued 

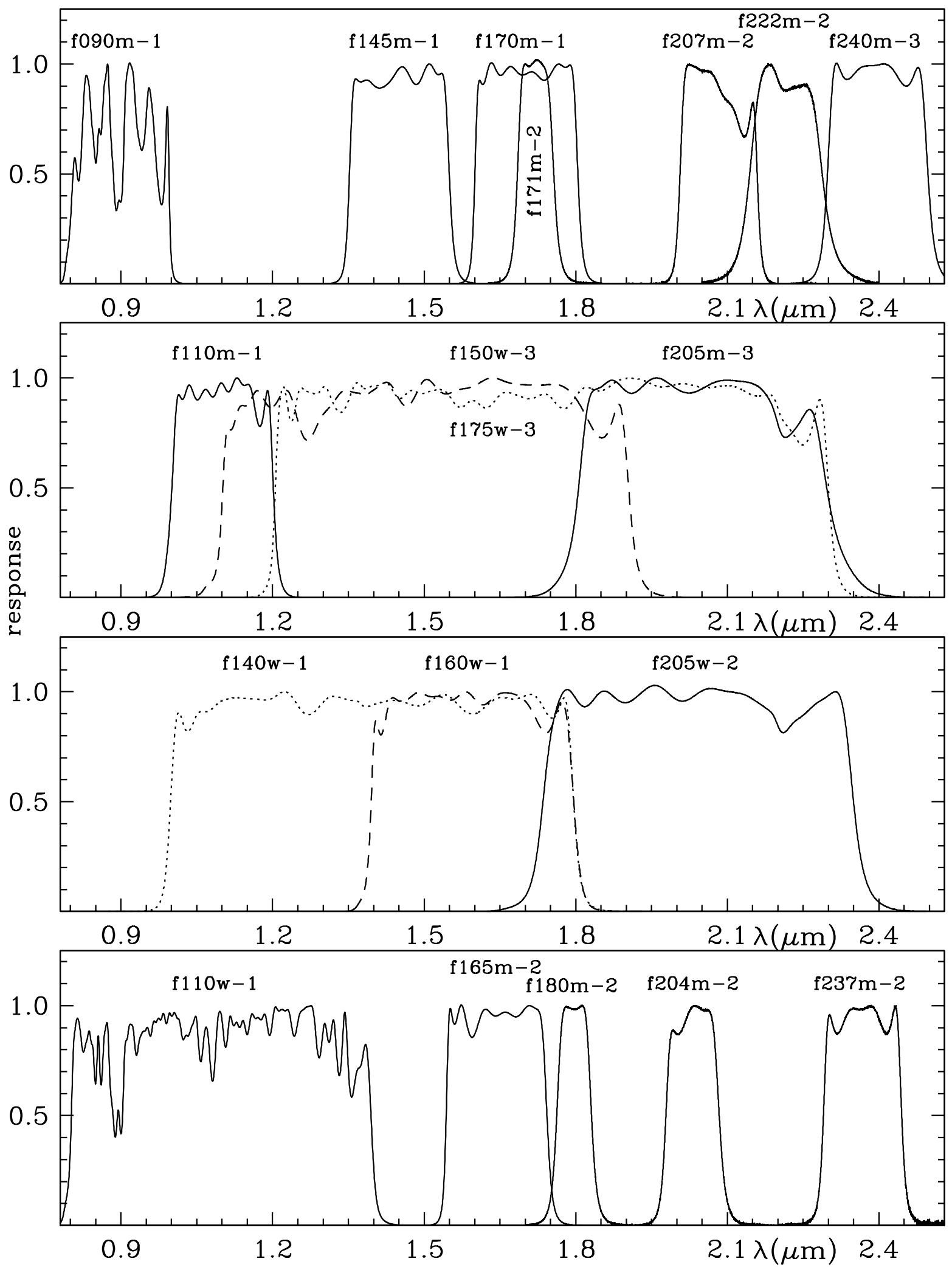

Fig. 163. continued 


\section{STIS HST - 1997}

Filter system of the Space Telescope Imaging Spectrograph (STIS).

\section{GENERAL INFORMATION}

TELESCOPE

DETECTOR

2.4m Hubble Space Telescope

SITe $1024 \times 1024$ thinned CCD

MAIN ARTICLE Baum, S., et al 1996 STIS intrument Handbook, Version 1.0 (Baltimore, STScI)

\section{SYSTEM DESCRIPTION}

\begin{tabular}{|c|c|c|c|c|}
\hline \multicolumn{5}{|c|}{ BANDS DESCRIPTION [24], pg. 48} \\
\hline \multicolumn{2}{|l|}{ band } & $\lambda_{c}(\AA)$ & FWHM $(\AA)$ & feature \\
\hline \multicolumn{5}{|c|}{ Visible } \\
\hline $50 C C D$ & Oclear & \multicolumn{2}{|c|}{$\approx 3750-8300 \AA$} & Clear \\
\hline F28X50LP & Oclear-lp & \multicolumn{2}{|c|}{$\lambda>5550 \AA$} & longpass \\
\hline F28X500II & [OII] & 3740 & 80 & [OII] \\
\hline F28X50OIII & {$[\mathrm{OIII}]$} & & 5 & [OIII] \\
\hline \multicolumn{5}{|c|}{ Ultraviolet } \\
\hline 25MAMA-F & Fclear & \multicolumn{2}{|c|}{$\approx 1150-1700 \AA$} & Clear \\
\hline F25SRF2 & Fsrf2 & \multicolumn{2}{|c|}{$\lambda>1280 \AA$} & longpass \\
\hline F25QTZ & Fqtz & \multicolumn{2}{|c|}{$\lambda>1450 \AA$} & longpass \\
\hline 25MAMA-N & Nclear & \multicolumn{2}{|c|}{$\approx 1750-3100 \AA$} & Clear \\
\hline N25SRF2 & Nsrf2 & \multicolumn{2}{|c|}{$\lambda>1280 \AA$} & longpass \\
\hline N25QTZ & Nqtz & \multicolumn{2}{|c|}{$\lambda>1450 \AA$} & longpass \\
\hline F25LYA & Lya & 1216 & 85 & Lyman $\alpha$ \\
\hline F25CN182 & $\mathrm{cn} 182$ & 1820 & 350 & continuum at $1800 \AA$ \\
\hline F25CIII & CIII & 1909 & 70 & [CIII] \\
\hline F25CN270 & cn270 & 2700 & 350 & continuum at $2700 \AA$ \\
\hline F25MGII & MgII & 2800 & 70 & $\mathrm{Mg}$ II \\
\hline
\end{tabular}

Fig. 164. The photometric system STIS HST - 1997 


\section{TRANSMISSION CURVES [24]}

To save space the transmission proles of the bands are plotted but not tabulated. The proles can be obtained in tabular form via STSDAS (inside Iraf) or from the ADPS $w w w$ site.
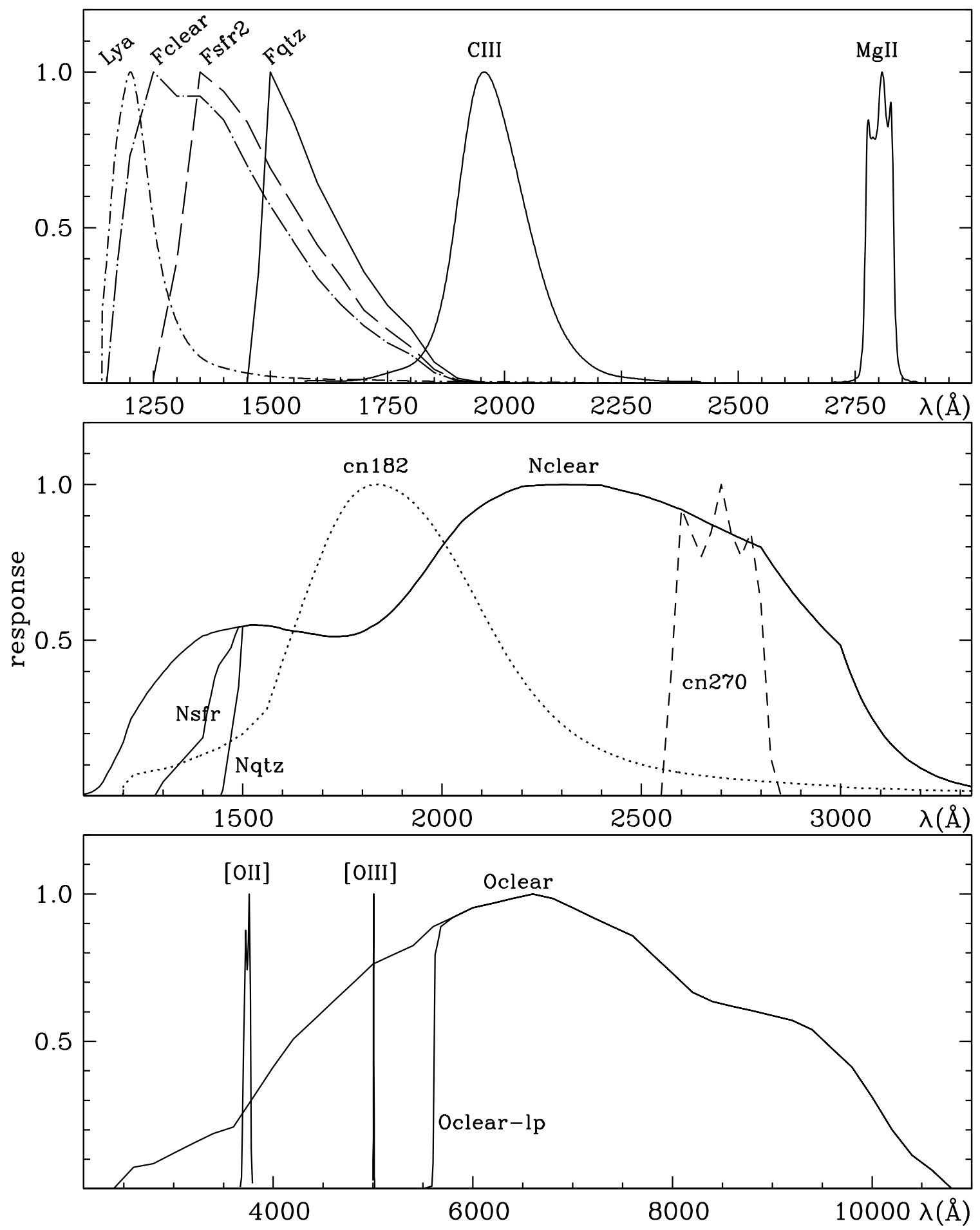

Fig. 164. continued 


\section{Royer et al. - 1998}

Classification of Wolf-Rayet stars.

\section{GENERAL INFORMATION}

AUTHORS P. Royer, J. -M. Vreux and J. Manfroid

TELESCOPE

1m (reflector), ESO (La Silla)

DETECTOR RCA 31034

MAIN ARTICLE Royer, P., Vreux, J.-M., Manfroid, F. 1998, A\&AS 130, 407

SYSTEM DESCRIPTION

\begin{tabular}{|c|ccl|}
\hline \multicolumn{4}{|c|}{ BANDS DESCRIPTION [253] } \\
\hline filter & $\lambda_{c}(\AA)$ & FWHM $(\AA)$ & \multicolumn{1}{c|}{ feature } \\
\hline$r_{H e I I}$ & 4648 & 30 & HeII 4686 \\
\hline$c_{1}$ & 5057 & 53 & continuum \\
\hline$r_{C I V}$ & 5806 & 27 & CIV 5801- 12 \\
\hline$r_{H e I}$ & 5881 & 26 & HeI 5876 \\
\hline$c_{2}$ & 6051 & 28 & continuum \\
\hline
\end{tabular}

Interference filters.

\section{SYSTEM ANALYSIS}

COLOR INDICES AND PARAMETERS [253]

$l(\mathrm{HeII})=\left[c_{1}+0.373\left(c_{1}-c_{2}\right)\right]-r_{H e I I}$ : normalized intensity of the HeII line at $4686 \AA$.

$l(C I V)=\left[c_{2}+0.2475\left(c_{1}-c_{2}\right)\right]-r_{C I V}:$ normalized intensity of the CIV line at $5807 \AA$.

$l(\mathrm{HeI})=\left[c_{2}+0.177\left(c_{1}-c_{2}\right)\right]-r_{H e I}$ : normalized intensity of the HeI line at $5876 \AA$.

Fig. 165. The photometric system Royer et al. - 1998 


\section{Asiago GAIA - Munari - 1998}

Conceptual study of a 5 broad +5 intermediate bands photometric system for the GAIA satellite by ESA.

The study by [221] is a revision of a preliminary design by [216].

GENERAL INFORMATION

\section{AUTHORS U. Munari}

MAIN ARTICLE Munari, U. 1998, Proc. ESA Leiden Workshop on GAIA, Baltic Astron. 8, 123

\section{SYSTEM DESCRIPTION}

\begin{tabular}{|c|cc|c|cc|}
\hline \multicolumn{7}{|c|}{ BANDS DESCRIPTION $[221]$} \\
\hline band & $\lambda_{\text {peak }}(\AA)$ & FWHM $(\AA)$ & band & $\lambda_{\text {peak }}(\AA)$ & FWHM $(\AA)$ \\
\hline \multicolumn{5}{|c|}{ broad bands } & \multicolumn{3}{c|}{ intermediate bands } \\
\hline b300 & 3000 & 1415 & $i 387$ & 3865 & 190 \\
\hline b480 & 4800 & 1500 & 1410 & 4095 & 178 \\
\hline b630 & 6300 & 1500 & 1430 & 4302 & 118 \\
\hline b792 & 7920 & 1720 & 1517 & 5173 & 84 \\
\hline b964 & 9640 & 1700 & 1531 & 5307 & 168 \\
\hline
\end{tabular}

\begin{tabular}{|r|rr|r|rr|}
\hline \multicolumn{5}{|c|}{ substitute and supplementary bands } \\
\hline b340 & 3400 & 615 & 1656 & 6560 & 75 \\
\hline & & 1839 & 8390 & 180 \\
\hline & & 1853 & 8535 & 118 \\
\hline
\end{tabular}

ZERO POINT: $\alpha$ Lyr has all colors equal to 0.00 .

b340 is a substitute for b300 if the latter could not be accommodated in the final Focal Plane Assembly design. i839, i853 are substitute bands for i517, i531. $i 656$ is a supplementary band aimed to detect $\mathrm{H} \alpha$ in emission and in combination with $b 480, b 630, b 792$ bands to measure the strength of 6200 and $6700 \AA$ TiO absorption bands.

\section{SYSTEM ANALYSIS}

\section{COLOR INDICES AND PARAMETERS [221]}

Metallicity indices from intermediate bands:

$m_{a}=i 378-i 410-0.10:$ Fe discontinuity index.

$m_{b}=(i 430-i 410)-(i 410-i 387)-0.11=i 430-2 i 410+i 387-0.11:$ reddening-free index based on

$m_{c}=i 517-i 531-0.10: \mathrm{Mg}$ index. $\mathrm{Fe}$ and $\mathrm{CH}$ abundances.

$m_{d}=i 853-i 839-0.02:$ Ca II index. $\quad m_{e}=(i 656-b 630)-0.12(b 480-b 792):$ TiO index.

metallicity of $\mathrm{G}$ dwarfs: $\quad\left[\mathrm{Z} / \mathrm{Z}_{\odot}\right]=-2.53+4.13 m_{a} \quad\left[\mathrm{Z} / \mathrm{Z}_{\odot}\right]=-2.80+3.98 m_{b}$

metallicity of $\mathrm{G}$ supergiants: $\quad\left[\mathrm{Z} / \mathrm{Z}_{\odot}\right]=-2.32+3.60 m_{a} \quad\left[\mathrm{Z} / \mathrm{Z}_{\odot}\right]=-2.33+3.86 m_{b}$

REDDENING RATIOS [221]

$$
\begin{aligned}
& E_{b 300-b 480}=1.82 E_{B-V} \quad E_{b 480-b 630}=1.04 E_{B-V} \quad E_{b 630-b 792}=0.76 E_{B-V} \quad E_{b 792-b 964}=0.58 E_{B-V} \\
& A_{b 300}=5.55 E_{B-V} \quad A_{b 480}=3.73 E_{B-V} \quad A_{b 630}=2.69 E_{B-V} \quad A_{b 792}=1.93 E_{B-V} \quad A_{b 964}=1.35 E_{B-V}
\end{aligned}
$$

REDDENING-FREE PARAMETERS [221]

$$
Q_{a}=(b 300-b 480)-1.75(b 480-b 630) \quad Q_{b}=(b 480-b 630)-1.79(b 792-b 964)
$$

Fig. 166. The photometric system Asiago GAIA - Munari - 1998 


\section{RELATIONS WITH OTHER SYSTEMS [221]}

UBV - Johnson and Morgan - 1953

\begin{tabular}{|lll|}
\hline$b 300-b 480$ & $=-0.043+1.961(U-B)$ & for $(U-B)<-0.05$ \\
& $=+0.701+1.181(U-B)+0.644(U-B)^{2}$ & for $(U-B)>+0.02$ \\
\hline$b 480-b 630$ & $=+0.004+1.061(B-V)+0.092(B-V)^{2}$ & \\
\hline
\end{tabular}

RI - Cousins - 1976

\begin{tabular}{|c|c|c|c|c|}
\hline$b 630-b 792$ & $=$ & $-0.011+1.550(V-R)-1.138(V-R)^{2}$ & $-0.625(V-R)^{3}$ & for $(V-R)<0.70$ \\
\hline$b 792-$ & $=$ & $+0.021+2.064(R-I)+2.712(R-I)^{2}$ & & for $(R-I)<0.00$ \\
\hline & $=$ & $-0.028+0.113(R-I)+0.806(R-I)^{2}$ & $-0.127(R-I)^{3}$ & for $(R-I)>0.00$ \\
\hline
\end{tabular}

Sloan DSS - Fukugita et al. - 1996

\begin{tabular}{|c|c|c|c|}
\hline$b 300-b 480$ & $\begin{array}{l}= \\
=\end{array}$ & $\begin{array}{l}+0.005+1.527(u-g) \\
-0.405+1.374(u-g)\end{array}$ & $\begin{array}{l}\text { for }(u-g)<-0.10 \\
\text { for }(u-g)>+0.10\end{array}$ \\
\hline$b 480-b 630$ & $=$ & $+0.002+1.021(g-r)$ & \\
\hline$b 630-b 792$ & $=$ & $-0.002+1.108(r-i)$ & \\
\hline$b 792-b 964$ & $\begin{array}{l}= \\
=\end{array}$ & $\begin{array}{l}+0.007+1.433(i-z) \\
-0.033+1.243(i-z)\end{array}$ & $\begin{array}{l}\text { for }(i-z)<-0.02 \\
\text { for }(i-z)>+0.05\end{array}$ \\
\hline
\end{tabular}

TRANSMISSION CURVES [221]

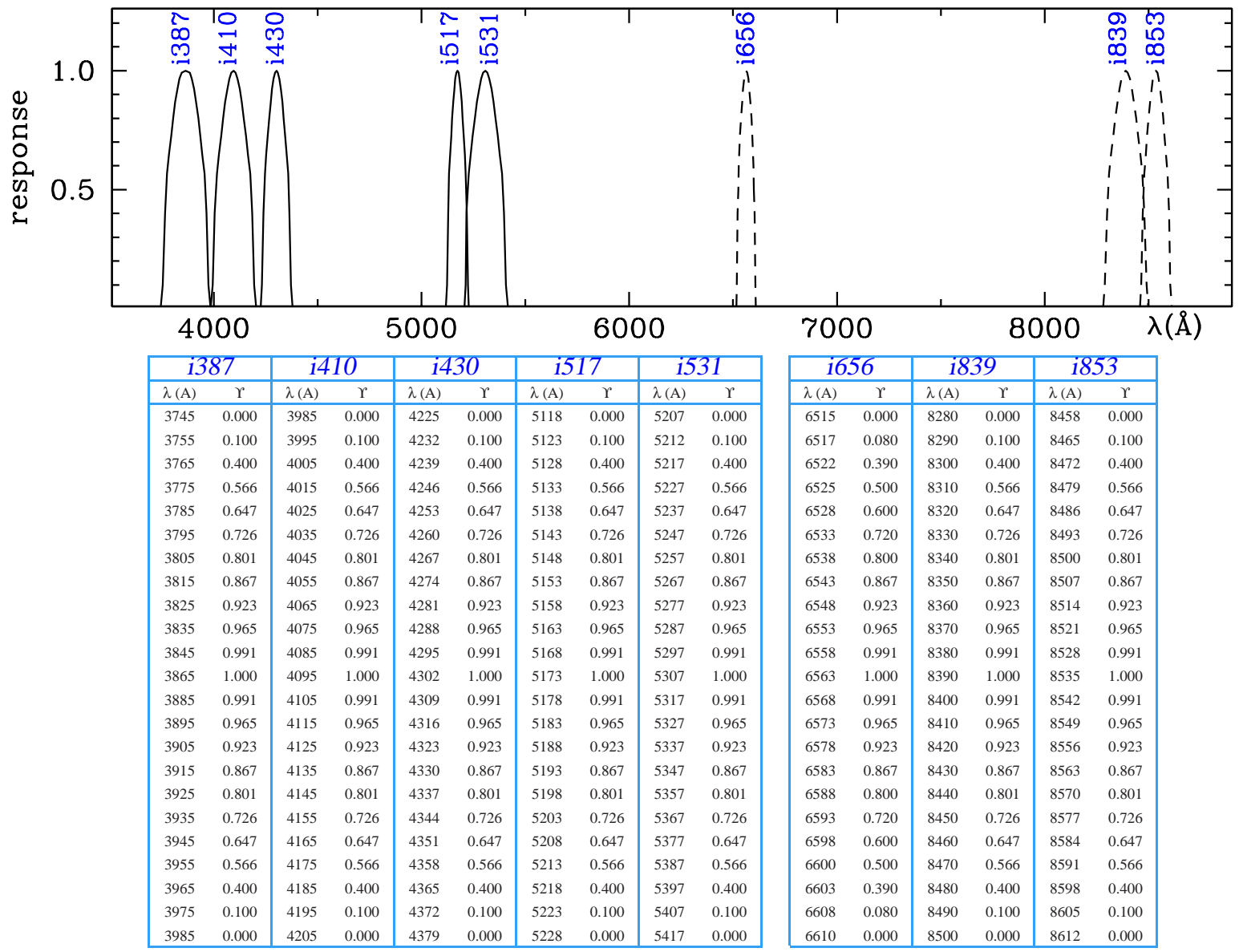

Fig. 166. continued 


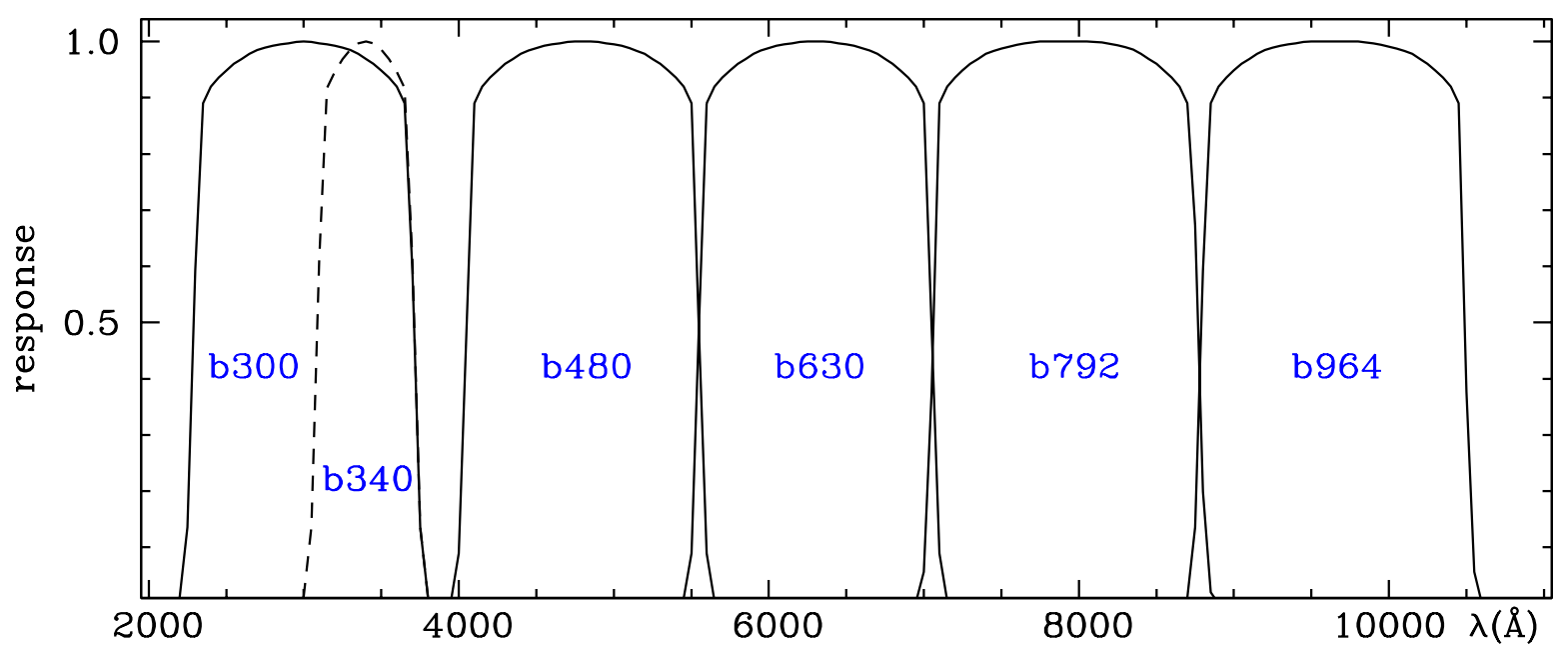

\begin{tabular}{|c|c|c|c|c|c|c|c|c|c|}
\hline \multicolumn{2}{|c|}{ b300 } & \multicolumn{2}{|c|}{ b480 } & \multicolumn{2}{|c|}{ b630 } & \multicolumn{2}{|c|}{ b792 } & \multicolumn{2}{|c|}{ b964 } \\
\hline$\lambda(\mathrm{A})$ & $\Upsilon$ & $\lambda(\mathrm{A})$ & $\Upsilon$ & $\lambda(\mathrm{A})$ & $\Upsilon$ & $\lambda(\mathrm{A})$ & $\Upsilon$ & $\lambda(\mathrm{A})$ & $\Upsilon$ \\
\hline 2150 & 0.000 & 3900 & 0.000 & 5400 & 0.000 & 6900 & 0.000 & 8650 & 0.000 \\
\hline 2200 & 0.011 & 3950 & 0.006 & 5450 & 0.006 & 6950 & 0.003 & 8700 & 0.011 \\
\hline 2250 & 0.136 & 4000 & 0.089 & 5500 & 0.089 & 7000 & 0.056 & 8750 & 0.136 \\
\hline 2300 & 0.592 & 4050 & 0.489 & 5550 & 0.489 & 7050 & 0.377 & 8800 & 0.592 \\
\hline 2350 & 0.890 & 4100 & 0.890 & 5600 & 0.890 & 7100 & 0.890 & 8850 & 0.890 \\
\hline 2400 & 0.920 & 4150 & 0.920 & 5650 & 0.920 & 7150 & 0.920 & 8900 & 0.920 \\
\hline 2450 & 0.936 & 4200 & 0.936 & 5700 & 0.936 & 7200 & 0.936 & 8950 & 0.936 \\
\hline 2500 & 0.948 & 4250 & 0.948 & 5750 & 0.948 & 7250 & 0.948 & 9000 & 0.948 \\
\hline 2550 & 0.960 & 4300 & 0.960 & 5800 & 0.960 & 7300 & 0.960 & 9050 & 0.960 \\
\hline 2600 & 0.969 & 4350 & 0.969 & 5850 & 0.969 & 7350 & 0.969 & 9100 & 0.969 \\
\hline 2650 & 0.978 & 4400 & 0.978 & 5900 & 0.978 & 7400 & 0.978 & 9150 & 0.978 \\
\hline 2700 & 0.985 & 4450 & 0.984 & 5950 & 0.984 & 7450 & 0.983 & 9200 & 0.985 \\
\hline 2750 & 0.989 & 4500 & 0.988 & 6000 & 0.988 & 7500 & 0.987 & 9250 & 0.989 \\
\hline 2800 & 0.992 & 4550 & 0.992 & 6050 & 0.992 & 7550 & 0.991 & 9300 & 0.992 \\
\hline 2850 & 0.995 & 4600 & 0.995 & 6100 & 0.995 & 7600 & 0.994 & 9350 & 0.995 \\
\hline 2900 & 0.997 & 4650 & 0.997 & 6150 & 0.997 & 7650 & 0.997 & 9400 & 0.997 \\
\hline 2950 & 0.999 & 4700 & 0.999 & 6200 & 0.999 & 7700 & 0.998 & 9450 & 0.999 \\
\hline 3000 & 1.000 & 4750 & 1.000 & 6250 & 1.000 & 7750 & 1.000 & 9500 & 1.000 \\
\hline 3050 & 0.999 & 4800 & 1.000 & 6300 & 1.000 & 7900 & 1.000 & 9650 & 1.000 \\
\hline 3100 & 0.997 & 4850 & 1.000 & 6350 & 1.000 & 8050 & 1.000 & 9800 & 1.000 \\
\hline 3150 & 0.995 & 4900 & 0.999 & 6400 & 0.999 & 8100 & 0.999 & 9850 & 0.998 \\
\hline 3200 & 0.992 & 4950 & 0.997 & 6450 & 0.997 & 8150 & 0.998 & 9900 & 0.997 \\
\hline 3250 & 0.989 & 5000 & 0.995 & 6500 & 0.995 & 8200 & 0.996 & 9950 & 0.994 \\
\hline 3300 & 0.985 & 5050 & 0.992 & 6550 & 0.992 & 8250 & 0.993 & 10000 & 0.991 \\
\hline 3350 & 0.978 & 5100 & 0.988 & 6600 & 0.988 & 8300 & 0.990 & 10050 & 0.987 \\
\hline 3400 & 0.969 & 5150 & 0.983 & 6650 & 0.984 & 8350 & 0.986 & 10100 & 0.983 \\
\hline 3450 & 0.960 & 5200 & 0.978 & 6700 & 0.978 & 8400 & 0.978 & 10150 & 0.978 \\
\hline 3500 & 0.948 & 5250 & 0.969 & 6750 & 0.969 & 8450 & 0.969 & 10200 & 0.969 \\
\hline 3550 & 0.936 & 5300 & 0.960 & 6800 & 0.960 & 8500 & 0.960 & 10250 & 0.960 \\
\hline 3600 & 0.920 & 5350 & 0.948 & 6850 & 0.948 & 8550 & 0.948 & 10300 & 0.948 \\
\hline 3650 & 0.890 & 5400 & 0.936 & 6900 & 0.936 & 8600 & 0.936 & 10350 & 0.936 \\
\hline 3700 & 0.592 & 5450 & 0.920 & 6950 & 0.920 & 8650 & 0.920 & 10400 & 0.920 \\
\hline 3750 & 0.136 & 5500 & 0.890 & 7000 & 0.890 & 8700 & 0.890 & 10450 & 0.890 \\
\hline 3800 & 0.011 & 5550 & 0.489 & 7050 & 0.489 & 8750 & 0.673 & 10500 & 0.377 \\
\hline 3850 & 0.000 & 5600 & 0.089 & 7100 & 0.089 & 8800 & 0.199 & 10550 & 0.056 \\
\hline & & 5650 & 0.006 & 7150 & 0.006 & 8850 & 0.020 & 10600 & 0.003 \\
\hline & & 5700 & 0.000 & 7200 & 0.000 & 8900 & 0.000 & 10650 & 0.000 \\
\hline
\end{tabular}

\begin{tabular}{|c|c|}
\hline \multicolumn{2}{|c|}{ b340 } \\
\hline$\lambda(\mathrm{A})$ & $\Upsilon$ \\
\hline 2950 & 0.000 \\
\hline 3000 & 0.011 \\
\hline 3050 & 0.136 \\
\hline 3100 & 0.610 \\
\hline 3150 & 0.917 \\
\hline 3200 & 0.946 \\
\hline 3250 & 0.969 \\
\hline 3300 & 0.986 \\
\hline 3350 & 0.997 \\
\hline 3400 & 1.000 \\
\hline 3450 & 0.997 \\
\hline 3500 & 0.986 \\
\hline 3550 & 0.969 \\
\hline 3600 & 0.946 \\
\hline 3650 & 0.917 \\
\hline 3700 & 0.610 \\
\hline 3750 & 0.136 \\
\hline 3800 & 0.011 \\
\hline 3850 & 0.000 \\
\hline & \\
\hline & \\
\hline & \\
\hline & \\
\hline & \\
\hline & \\
\hline & \\
\hline & \\
\hline & \\
\hline & \\
\hline
\end{tabular}

Fig. 166. continued 


\section{Geneva GAIA - Grenon et al. - 1999}

Conceptual study of a photometric system for the GAIA satellite by ESA.

\section{GENERAL INFORMATION}

AUTHORS M. Grenon, C. Jordi, F. Figueras and J. Torra

MAIN ARTICLE Grenon, M., Jordi, C., Figueras, F., Torra, J. 1999

MG-PWG-002 Tech. Rep. to the GAIA Photom. Working Group

\section{SYSTEM DESCRIPTION}

\begin{tabular}{|l|ccc|l|lrl|}
\hline \multicolumn{7}{|c|}{ BANDS DESCRIPTION $[18],[122]$} \\
\hline band & $\lambda_{c}(\mathrm{~A})$ & FWHM (A) & $\Upsilon_{\text {peak }}$ & band & $\lambda_{c}(\mathrm{~A})$ & FWHM (A) & $\Upsilon_{\text {peak }}$ \\
\hline 33 & 3260 & 820 & 0.92 & 66 & 6560 & 240 & 0.72 \\
\hline 37 & 3750 & 1460 & 0.96 & 67 & 6740 & 1160 & 0.94 \\
\hline 41 & 4050 & 600 & 0.90 & 73 & 7330 & 1850 & 0.97 \\
\hline 47 & 4645 & 450 & 0.86 & 75 & 7470 & 280 & 0.79 \\
\hline 51 & 5075 & 270 & 0.78 & 78 & 7775 & 310 & 0.81 \\
\hline 53 & 5250 & 2070 & 0.97 & 82 & 8160 & 480 & 0.87 \\
\hline 57 & 5700 & 900 & 0.93 & 89 & 8940 & 480 & 0.87 \\
\hline
\end{tabular}

The bands 37, 53, 66 and 73 are not included in the original proposal by [122].

They are reported by [18] to have been added later.

\section{TRANSMISSION CURVES}

To plot and tabulate the bands a gaussian prole is adopted following [18].

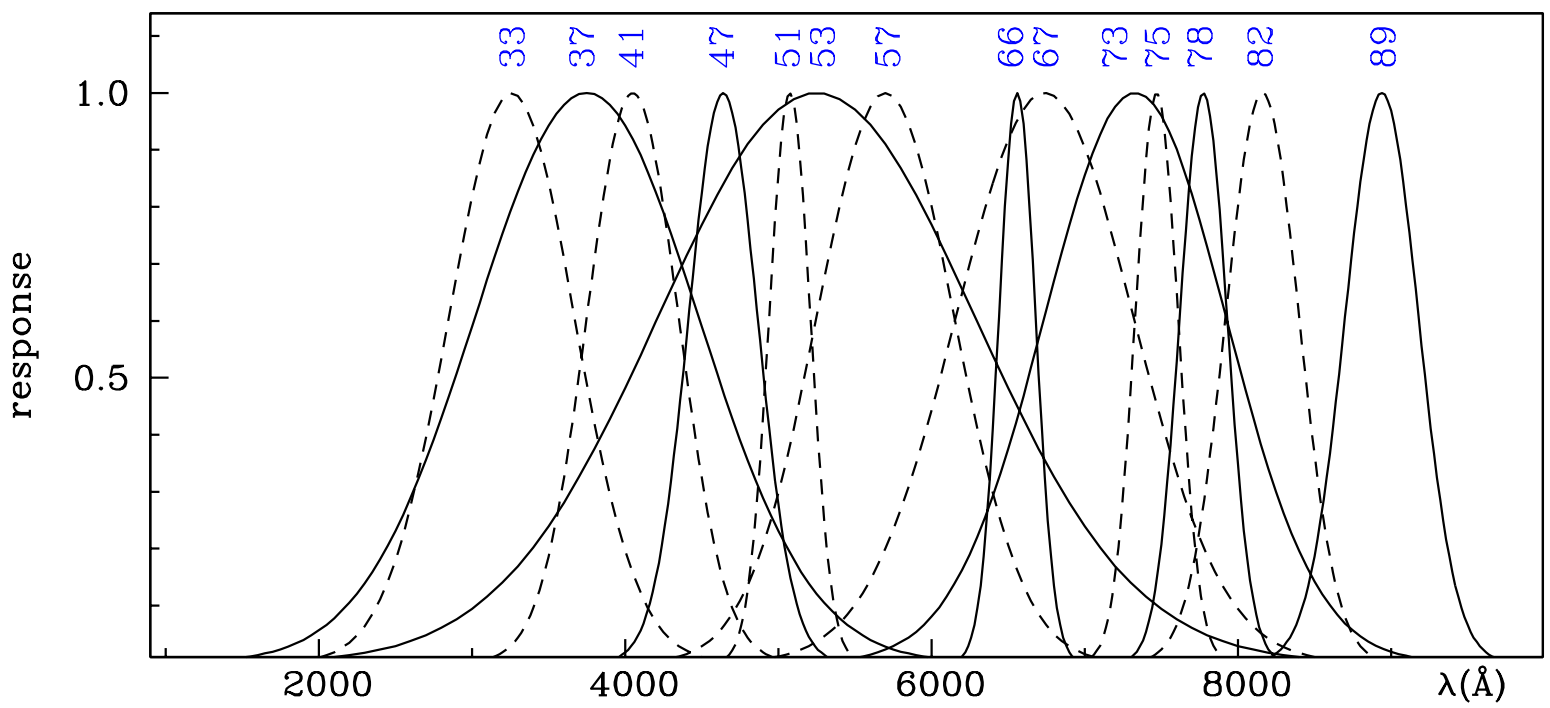

Fig. 167. The photometric system Geneva GAIA - Grenon et al. - 1999 


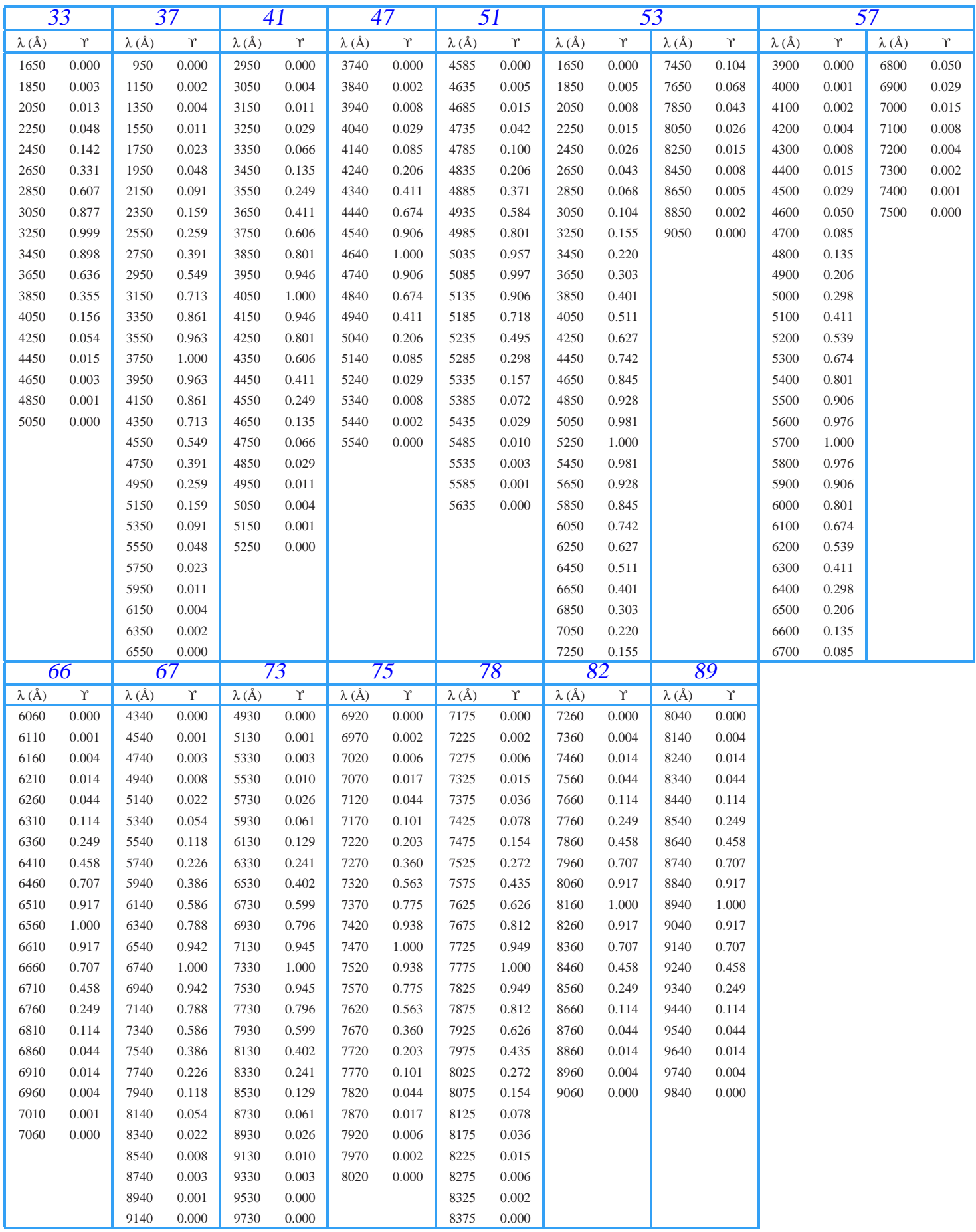

Fig. 167. continued 
Table 2. List of references used in the figures describing the various photometric systems

[1] Aaronson, M. Mould, J. 1980, ApJ 240, 804

[2] Abt, H.A., Golson J. C. 1965, ApJ 143, 106

[3] Alexander, J. B., Branch, D. 1974, MNRAS 167, 539

[4] Alexander, J. B., Jones, D. H. P., Sinclair, J. E. 1983, RGO Bull. 191

[5] Allen, C. W. 1973, Astrophysical Quantities, 3rd ed., Univ. of London, Athlone Press

[6] Allen, D. A., Cragg, T. A. 1983, MNRAS 203, 777

[7] Alonso, A., Arribas, S., Martinez-Roger, C. 1994, A\&AS 107, 365

[8] Alonso, A., Arribas, S., Martinez-Roger, C. 1998, A\&AS 131, 209

[9] Andrews, P. J. 1968, MemRAS 72, 35

[10] Anthony-Twarog, B. J., Laird, J. B., Payne, D., Twarog, B. A. 1991, AJ 101, 1902

[11] Argue A. N. 1967, MNRAS 135, 23

[12] Arp, H. C. 1958, AJ 63, 118

[13] Avertisian, A. Kh., Zalinyan, V. P., Melik-Alaverdyan, Y. K., Oganesyan, R. Kh., Tovmasya, G. M. 1981, Astrofizika 17, 118

[14] Avertisian, A. Kh., Melik-Alaverdyan, Y. K. 1982, Astrofizika 18, 235

[15] Ažusienis, A., Straižys, V. 1969, Soviet Astron. 13, 316

[16] Bahng, J. D. R. 1958, MNRAS 14, 572

[17] Bahng, J. 1969, MNRAS 143, 73

[18] Bailer-Jones, C. A. L. 2000, A\&A 357, 197

[19] Baldwin, J. R., Frogel, J. A., Persson, S. E. 1973, ApJ 184, 427

[20] Baliunas, S. L., Ciccone, M. A., Guinan, E. F. 1975, PASP 87, 969

[21] Bappu, M. K. V., Chandra, S., Sanwal, N. B., Sinvhal, S. D. 1962, MNRAS 123, 521

[22] Barbier, D., Morguleff, N. 1964, Compt. Rend. Acad. Sci. Paris 258, 4925

[23] Bastiaansen, P. A. 1992, A\&AS 93, 449

[24] Baum, S., et al 1996, STIS Intrument Handbook, Version 1.0 (Baltimore, STScI)

[25] Becker, W. 1946, Veröff. Univ. Strernwarte Göttingen 79

[26] Becker, W. 1963, in Basic Astronomical Data, K. A. Strand ed., Univ. Chicago Press, pag. 241

[27] Beer, A. 1964, MNRAS 128, 261

[28] Beichman, C. A., Neugebauer, G., Habing, H. J., Clegg, P. E., Chester, T. J. 1988 Infrared Astronomical Satellite (IRAS) Catalogs and Atlases, NASA RP-1190, Vol. 1

[29] Bell, R. A. 1972, MNRAS 159, 357

[30] Bersanelli, M., Bouchet, P., Falomo, R. 1991, A\&A 252 
Table 2. continued

[31] Bessell, M. S. 1979, PASP 91, 589

[32] Bessell, M. S. 1990, PASP 102, 1181

[33] Bessell M. S. 1993, in Stellar photometry-Current tecniques and future developments, IAU Colloquium 136, C.J. Butler, I. Elliott ed.s, Cambridge Univ. Press, pag.22

[34] Bessell, M. S., Brett, J. M. 1988, PASP 100, 1134

[35] Bessell, M. S., Germany, L. M. 1999, PASP 111, 1421

[36] Biretta, J. A. et al. 1996, WFPC2 Instrument Handbook, Version 4.0 (Baltimore, STScI)

[37] Bless, R. C., Code, A. D., Houck, T. E., McNall, J. F., Taylor, D. J. 1965, AJ 70, 666

[38] Van der Bliek, N. S., Manfroid, J., Bouchet, P. 1996, A\&AS 119, 547

[39] Boggess, A., Dunkelmann, L. 1958, AJ 63, 303

[40] Böker, T., Storey, J. W. V., Krabbe, A., Lehmann, T. 1997, PASP 109, 827

[41] Boksenberg, A., Evans, R. G., Fowler, R. G., Gardner, I. S. K., Honziaux, L., Humphries, C. M., Jamar, C., Macau, D., Malaise, D., Monfils, A., Nandy, K., Thompson, G. I., Wilson, R., Wroe, H. 1973, MNRAS 163, 291

[42] Borgman, J. 1959, ApJ 129, 362

[43] Borgman, J. 1960, BAN 15, 255

[44] Borgman, J. 1961, BAN 16, 99

[45] Borgman, J. 1963, BAN 17, 58

[46] Borgman, J. 1978 A\&A 69, 245

[47] Borgman, J., Blaauw, A. 1963, BAN. 17, 358

[48] Bouchet, P., Manfroid, J., Schmider, F. X. 1991, A\&AS 91, 409

[49] Boyce, P. B., Olsen, E. H., Helt, B. E. 1967, PASP 79, 473

[50] Brand, J., Wouterloot, J. G. A. 1988, A\&AS 75, 117

[51] Breger, M. 1975, in Multicolor photometry and theoretical HR diagram, A. G. Davis Philips, D. S. Hayes ed.s, Dudley Observatory report No. 9, pag. 31

[52] Brewer, J., Richer, H. B., Crabtree, D. R. 1995, AJ 109, 2480

[53] Brewer, J., Richer, H. B., Crabtree, D. R. 1996, AJ 112, 491

[54] Buser, R. 1978, A\&A 62, 411

[55] Buser, R. 1978, A\&A 62, 425

[56] Calzetti, D. et al. 1999, NICMOS Instrument Handbook, Version 3.0 (Baltimore, STScI)

[57] Canterna, R. 1976, AJ 81, 228

[58] Canterna, R., Harris, H. 1979, in Problems of calibration of multicolor photometric systems, ed. A. G. D. Philips, Dudley Obs. Reports No. 14, pag. 199

[59] Caplan, J. G. 1973, A\&A 28, 213

[60] Carnochan, D. J. 1982, MNRAS 201, 1139 
Table 2. continued

[61] Carrasco, L., Recillas-Cruz, E., Garcia-Barreto, A., Cruz-Gonzales, I., Serrano P. G., A. 1991, PASP 103, 987

[62] Carter, B. S. 1990, MNRAS 242, 1

[63] Cathey, L. 1974, AJ 79, 1370

[64] Cester, B., Giuricin, G., Mardirossian, F., Pucillo, M., Castelli, F., Flora, U. 1977, A\&AS 30, 1

[65] Chalonge D., Divan L. 1952, Ann. Astron. 15, 201

[66] Chapman, C. R., McCord, B., Johnson, T. V. 1973, AJ 78, 126

[67] Cherepashcuk, A. M., Khaliullin, Kh., F. 1973, Soviet Astron. 17, 330

[68] Clark, B. E., Bell, J. F., Fanale, F. P., Lucey, P. G. Lun. Plan. Inst. 24, 299

[69] Clark, J. P. A, and McClure, R. D. 1979, PASP 91, 507

[70] Code, A. D., Houck, T. E., McNall, J. F., Bless, R. C., Lillie, C. F. 1970, ApJ 161, 377

[71] Code, A. D., Holm, A. V., Bottemiller, R. L. 1980, ApJS 43, 501

[72] Cohen, M. 1979, MNRAS 186, 837

[73] Cook, K. H., Aaroson, M. 1989, AJ 97, 923

[74] Couch, W. J., Newell, E. B. 1980, PASP 92, 746

[75] Cousins A. W. J. 1976, MemRAS 81, 25

[76] Cousins A. W. J. 1987, Obs. 107, 80

[77] Cousins A. W. J. 1993, SAAO Circulars, No 15, 30

[78] Cramer, N., Mander, J. 1979 A\&A 78305

[79] Crawford, D. L., Mander, J. 1966, AJ 71, 114

[80] Crawford, D. L., Barnes, J. V. 1970, AJ 75, 978

[81] Dachs, J., Schmidt-Kaler, Th. 1975, A\&AS 21, 81

[82] Damineli, A., Jablonski, F., de Freitas, L. C., de Freitas-Pacheco, J. A. 1997, PASP 109, 633

[83] Dean, J. F., Warren, P. R., Cousins, A. W. J. 1978, MNRAS 183, 569

[84] Deeming, T. J. 1960, MNRAS 121, 52

[85] De Martino, D., Buson, L. 1999, MemS.A.It 70, 315

[86] Dickow, P., Gyldenkerne, K., Hansen, L., Jacobsen ,P.-U., Johansen, K. T., Kjaergaard, P., Olsen, E. H. 1970, A\&AS 2, 1

[87] van Dijk, W., Keirssies, A., Hammerschlag-Hensberge, G., Wesselius, P. R. 1978, A\&A 66, 187

[88] van Duinen, R. J, Aalders, J. W. G., Wesselius, P. R., Windeman, K. J., Wu, C. C., Luinge, W., Snel, D. 1975, A\&A 39, 159

[89] Dzervitis, U. 1977, Investig. of the Sun and Red Stars, Riga, 6, 43

[90] Edvardsson, B., Bell, R. A. 1989, MNRAS 238, 1121

[91] Eggen, O. J. 1955, AJ 60, 65 
Table 2. continued

[92] Eggen, O. J. 1965, AJ 70, 19

[93] Eggen, O. J. 1967, ApJS 14, 307

[94] Eggen, O. J. 1976, PASP 88, 732

[95] Eggen, O., Sandage, A. 1960, MNRAS 120, 79

[96] Elias, J. H, Frogel, J. A., Matthews K., Neugebauer, G. 1982, AJ 87, 1029

[97] Elias, J. H, Frogel, J. A., Hyland, A. R., Jones, T. J. 1983, AJ 88, 1027

[98] Elsner, B., Bastian, U., Liubertas, R., Scholz, R. 1999, Baltic Astron. 8, 385

[99] Engels, D., Sherwood, W. A., Wamsteker, W., Schultz, G. V. 1981, A\&AS 45, 5

[100] Epchtein, N., de Batz, B., Copet, E., et al. 1994, Ap\&SS 217, 3

[101] Faber, S. M. 1973, A\&AS 10, 201

[102] Fan, X. et al. 1996, AJ 112, 628

[103] Faulkner, D. R., Honeycutt, R. K., Johnson, H. R. 1988, ApJ 324, 490

[104] Faÿ, T. D., Warren, W. H., Johnson, H. R., Honneycutt 1974, AJ 79, 634

[105] Feinstein, A. 1974, MNRAS 169, 171

[106] Fernie, J. D. 1974, PASP 86, 837

[107] Fouqué, P., Chevallier, L., Cohen, M., Galliano, E., Loup, C., Alard, C., de Bartz, B., Bertin, E., Borsenberger, J., Cioni, M. R., Copet, E., Dennefeld, M., Derrier, S., Deul, E., Duc, P.-A., Egret, D., Epchtein, N., Forveille, T., Garzón, F., Habing, H. J., Hron, J., Kimeswenger, S., Lacombe, F., Le Bertre, T., Mamon, G. A., Omont, A., Paturel, G., Pau, S., Persi, P., Robin, A. C., Rouan, D., Schultheis, M., Simon, G., Tiphène, D., Vuglin, I., Wagner, S. J. 2000, A\&AS 141, 313

[108] Frogel, J. A., Persson, S. E., Aaronson, M., and Matthews, K. 1978, ApJ 220, 75

[109] Fukugita, M., Ichikawa, T., Gunn, J. E., Doi, M., Shimasaku, K., and Schneider, D. P. 1996, AJ 111, 1748

[110] Geisler, D. 1996, AJ 111, 480

[111] Geisler, D., Kapradinis, S. 1983, AJ 88, 461

[112] de Geus, E. J., Lub, J., van de Grift, E. 1990, A\&AS 85, 915

[113] Glass, I. S. 1973, MNRAS 164, 155

[114] Glass, I. S. 1974, MNSSA 33, 53

[115] Golay, M. 1962, Pub. Obs. Genève No 15 (série A), 29

[116] Golay, M. 1972, Vistas in Astronomy 14, 13

[117] Golay, M. 1974, Introduction to astronomical photometry, Ap\&SS Lib. 41, Reidel

[118] Golay, M. 1980, Vistas in Astronomy 24, 141

[119] Gonzalez, G., Pichè, F. 1992, AJ 103, 2048

[120] Gray, R. O. 1998, AJ 116, 482

[121] Greenstein, J. L. 1976, AJ 81, 323 
Table 2. continued

[122] Grenon, M., Jordi, C., Figueras, F., Torra, J. 1999, MG-PWG-002 Technical Report to the ESA GAIA Photometric Working Group

[123] Griffin, R. F. 1961, MNRAS 122, 181

[124] Griffin, R. F., Redman, R. O. 1960, MNRAS 120,287

[125] Guinan, E. F., McCook, G. P. 1974, PASP 86, 947

[126] Gunther, S. 1933, Zeits. f. Astrophys. 7, 106

[127] Gustafsson, B., Nissen, P. E. 1972, A\&A 19, 261

[128] Gustafsson, B. Kjaergaard, P., Andersen, S. 1974, A\&A 34, 99

[129] Gutierrez-Moreno, A., Gutierrez-Moreno, H., Stock, J., 1967, Pub. Dep. Astron. Univ. Chile, No 2, 4

[130] Gyldenkerne, K., Helt, B. E. 1966, in Spectral Classification and Multicolour Photometry, IAU Symp 24, K. Lodén, L. O. Lodén, U. Sinnerstad ed.s, Reidel, pag. 162

[131] Häggkvist, L. 1971, A\&A 12, 5

[132] Häggkvist, L., Oja, T. 1970, A\&AS 1, 199

[133] Häggkvist, L., Oja, T. 1987, A\&AS 68, 259

[134] Hartwick, F. D. A., Cowley, A. P., Mould, J. R. 1984, ApJ 286, 269

[135] Hayes, D. S. 1970, ApJ 159, 165

[136] Helt, B. E., Gyldenkerne, K. 1975, A\&AS 22, 171

[137] Henry, R. C. 1969, ApJS 18, 47

[138] Herbst, W., Layden, A. C. 1987, AJ 94, 150

[139] Herbst, W., Miller, J. R. 1989, AJ 97, 981

[140] Hill, G., Morris, S. C., Walker, G. A. H. 1971, AJ 76, 246

[141] Hipparcos and Tycho Catalogues 1997, ESA Pub SP-1200, Volume 1

[142] Hortzmann, J. A., Burrows, C. J., Casertano, S., Hester, J. J., Trauger, J. T., Watson, A. M., Worthey, G. 1995, PASP 107, 1065

[143] Houck, J. R., Soifer, B. T., Pipher, J. L., Harwit, M. 1971, ApJ 169, L31

[144] IRTF-NSFCAM User's Guide 1999

[145] ISOCAM Observer's Manual V1.0 (http://www.iso.vilspa.esa.es/manuals/iso_cam/)

[146] Jacobsen, P. U. 1969, A\&A 4, 302

[147] Jennens P. A. 1975, MNRAS 172, 695

[148] Jennens P. A., Helfer H. L. 1975, MNRAS 172, 667

[149] Johnson, H. L. 1962, ApJ 135, 975

[150] Johnson, H. L. 1963, in Basic Astronomical Data, K. A. Strand ed., Univ. Chicago Press, pag. 204

[151] Johnson, H. L. 1965, ApJ 141, 923

[152] Johnson, H. L. 1965, Comm. Lunar and Planetary Lab. 53 
Table 2. continued

[153] Johnson, H. L. 1967, ARA\&A 4, 193

[154] Johnson, H. L., Mitchell, R. I., Latham, A. S. 1967, Comm. Lunar and Planetary Lab. No 92, 85

[155] Johnson, H. L., Mitchell, R.I. 1976, RMxAA 1, 299

[156] Johnson H. L., Morgan W. W. 1953, ApJ 117, 313

[157] Joncas J., Borra E. F. 1981, A\&A 94, 134

[158] Jones, D. H. P., Dixon, M. E. 1972, ApJ 177, 665

[159] Jones, D. H. P., Carrick D.W. 1973, in Problems of Calibration of Absolute Magnitudes and Temperature of Stars, IAU Symp. 54, B. Hauck and B.E. Westerlund ed.s, Reidel, pag. 36

[160] Jones, D. H. P., Sinclair, J. E., Alexander J. B. 1981, MNRAS 194, 403

[161] Jones, T. J., Hyland, A. R. 1982, MNRAS 200, 508

[162] Kent, S. M. 1985, PASP 97, 165

[163] Kenyon, S.J., Fernandez-Castro, T. 1987, AJ 93, 938

[164] Khaliullin, KH., Mironov, A. V., Moshkalyov, V. G. 1984, Ap\&SS 111, 291

[165] Khozov, G. V., Shalberova, V. V., Danilova, L. V. 1973, Trudy Leningrad Astron. Obs. 29, 80

[166] Kjaergaard, P. 1984, A\&AS 56, 313

[167] KODAK 1960, Photographic Plates for Scientific and Technical use, tech. pub.

[168] Koester, D., Schultz, H., Weidmann, V. 1979, A\&A 76, 262

[169] Kornilov, V., Mironov, A., Zakharov, A. 1996, Baltic Astron. 5, 379

[170] Kraft, R. P. 1960, ApJ 131, 330

[171] Kron, G. E., Gascoigne, S. C. B., White, H. S. 1951, AJ 62, 205

[172] Kron, G. E., Smith, J. L. 1951, ApJ 113, 324

[173] Kron, G. E., White, H. S., Gascoigne, S. C. B. 1953, ApJ 118, 502

[174] Kron, G. E., Mayall, N. U. 1960, AJ 65, 581

[175] Kruszewski, M. 1966, Acta Astronomica 16, 285

[176] Kunkel W. E., Rydgren A. E. 1979, AJ 84, 633

[177] Kupperian, J. E. Boggess, A., Milligan, J. E. 1958, ApJ 128, 453

[178] Lamla, E. 1982, in Landolt-Bornstein Series, vol 2b Stars and Star Clusters K.Schaifers and H.H.Voight ed.s, Springer-Verlag, Berlin

[179] Lampton, M., Margon, B., Paresce, F., Stern, R., Bowyer, S. 1976, ApJ 203, L71

[180] Landolt, A. U. 1970, AJ 75, 337

[181] Landolt, A. U. 1973, AJ 78, 959

[182] Landolt, A. U. 1983, AJ 88, 439

[183] Landolt, A. U. 1992, AJ 104, 372

[184] Lasker, B. M., Sturch, C. R., McClean, B. J., Russell, J. L., Jenkner, H., Shara, M. M. 1990, AJ 99, 2019 
Table 2. continued

[185] Leggett, S. K. 1985, A\&A 153, 273

[186] Leggett, S. K., Bartholomew, M., Mountain, C. M., Selby, M. J. 1986, MNRAS 223, 443

[187] Lindemann, E., and Hauck, B. 1973, A\&AS 11, 201

[188] Lockwood, G. W. 1972, ApJS 24, 375

[189] Lockwood. G. W., Wing. R. F. 1971, ApJ 169, 63

[190] Low, F. J., Rieke, G. H. 1974, in Methods of Experimental Physics Vol. 12, Part A, N. Carleton ed., Academic Press, New York, pag. 456

[191] Lub, J., Pel, J. W. 1977, A\&A 54, 137

[192] Lund, J. M., Dixon, R. S. 1973, PASP 85, 230

[193] Lundström, I., Stenholm, B. 1979 A\&AS 35, 303

[194] Lutz, J. H., Lutz, T. E., 1972, AJ 77, 376

[195] Maitzen, H. M. 1976, A\&A 51, 223

[196] Marchetti, E., Mallucci, S., Ghedina, A., Farinato, J., Baruffolo, A., Munari, U., Ragazzoni, R. 1997, in The Three Galileos: the Man, the Spacecraft, the Telescope, C. Barbieri, J. H. Rahe,

T. V. Johnson, A. M. Sohus ed.s, Kluwer Academic Publishers, Ap\&SS Lib. vol 220, pag. 383

[197] Massey, P. 1984, ApJ 281, 789

[198] Matthews, T. A., Sandage, A. R. 1963, ApJ 138, 30

[199] Maucherat-Joubert, M., Cruvellier, P., Deharveng, J. M. 1978, A\&A 70, 467

[200] Maucherat-Joubert, M., Deharveng, J. M., Cruvellier, P. 1979, A\&A 74, 218

[201] McClure R. D. 1976, AJ 81, 182

[202] McClure R. D. 1979, in Problems of Calibration of Multicolor Photometric Systems, A. G. D. Philip ed., Dudley Obs. Report 14, pag. 83

[203] McClure R. D., Van den Bergh, S. 1968, AJ 73, 313

[204] McGregor, P. 1994, PASP 106, 508

[205] McNamara, D. H., Helm, T. M., Wilcken, S. K. 1970, PASP 82, 293

[206] McWilliam, A., Lambert, D. L. 1984, PASP 96, 882

[207] Meistas, E., Zdanavicius, K., Straižys, V., Gurklyte, A. 1975, Bull. Vilnius Astr. Obs. 42, 3

[208] Mendoza, E. E. V. 1968, ApJ 151, 977

[209] Mendoza, E. E. V. 1971, Boletin de los observatorios de Tonantzintla y Tacubaya No 37, 6

[210] Mendoza, E. E. V. 1987, RMxAA 14, 385

[211] Mermillod, C. 1997, General Catalogue Photometric Data (http://obswww.unige.ch/gcpd/gcpd.html)

[212] Miner, E. D. 1965, ApJ 144, 1101

[213] Moffett T. J., Barnes T. G. 1978, PASP 91, 180

[214] Morguleff, N., Véron, M. P. 1970, A\&A 4, 391

[215] Morguleff, N., Gerbaldi, M. 1975, A\&AS 19, 189 
Table 2. continued

[216] Munari, U., Moro, D. 1997, UM-PWG-007 Technical Report to the ESA GAIA Photometric Working Group

[217] Mould, J. R. 1976, ApJ 207, 535

[218] Mould, J. R., Wallis, R. E. 1977, MNRAS 81, 625

[219] Mould, J. R., McElroy, D. B. 1978, ApJ 220, 935

[220] Mould, J. R., Aaronson, M. 1980, ApJ 240, 464

[221] Munari, U. 1998, in ESA Leiden Workshop on GAIA, V. Straižys ed., Baltic Astron. 8, 123

[222] Neckel, Th., and Chini, R. 1980, A\&AS 39, 411

[223] Neckel, Th., and Chini, R. 1981, A\&AS 45, 451

[224] Neff, J. S. 1966, AJ 71, 202

[225] Neff, J. S., Travis, L. D. 1967, AJ 72, 48

[226] Nersisian, S. E. 1984, Nauchnye Informatsii, Moscow, 56, 109

[227] Neugebauer, G., Habing, H. J., van Duinen, R., Aumann, H. H., Baud, B., Beichman, C. A., Beintema, D. A., Boggess, N., Clegg, P. E., de Long, T., Emerson, J. P., Gautier, T. N., Gillett, F. C., Harris, S., Hauser, M. G., Houck, J. R., Jennings, R. E., Low, F. J., Mardsen, P. L., Miley, G., Olnon, F. M., Pottasch, S. R., Raimond, E., Rowan-Robinson, M., Soifer, B. T., Walker, R. G., Wesselius, P. R., Young, E. 1984, ApJ 278, L1

[228] Newell, E. B. 1973, ApJS 26, 37

[229] Newell, E. B., Rodgers, A. W., Searle, L. 1969, ApJ 156, 597

[230] Nicollier, CL., Hauck, B. 1978, A\&AS 31, 437

[231] Nissen, P. E. 1970, A\&A 8, 476

[232] Nissen, P. E. 1974, A\&A 36, 57

[233] Nissen, P. E. 1976, A\&A 50, 343

[234] North, P., Hauck, B., Straižys, V. 1982, A\&A 108, 373

[235] Nota, A. et al. 1996, FOC Instrument Handbook Version 7.0 (Baltimore, STScI)

[236] Oke, J. B., Schild, R. E. 1970, ApJ 161, 1015

[237] Oke, J. B., Gunn, J. E. 1983, ApJ 266, 713

[238] Paltoglu, G., Bell, R. A. 1994, MNRAS 268, 793

[239] Park, N.-K., Lee, S.-W. 1986, J. Korean A.S. 19, 1

[240] Peat, D. W. 1964, MNRAS 128, 435

[241] Pedersen, H., Rudkjøbing, M. 1978, A\&A 34, 441

[242] Persson, S. E., Aaronson, M., Frogel, J.A. 1977, AJ 82, 729

[243] Peton, A., Bigay, J. H., Garnier, R., Paturel, G. 1972, A\&A 17, 47

[244] Petrie, R. M. 1953, Publ. Dominion Astrophys. Obs. Victoria, 9, 251

[245] Piccirillo, J. 1976, PASP 88, 680 
Table 2. continued

[246] Pilachowski, C. A. 1978, ApJ 224, 412

[247] Price, M. J. 1966, MNRAS 134, 135

[248] Redman, R. O. 1966, in Spectral Classification and Multicolour Photometry, IAU Symposium 24, K. Lodén, L. O. Lodén, U. Sinnerstad ed.s, Reidel, pag. 155

[249] Rego, M. E., Williams, P. M., Peat, D. W. 1972, MNRAS 160, 129

[250] Reid, I. N., Brewer, C., Brucato, R. J., McKinley, W. R., Maury, A., Mendenhall, D., Mould J. R., Mueller, J., Neugebauer, G., Phinney, J., Sargent, W. L. W., Schombert, J., Thicksten, R. 1991, PASP 103, 661

[251] Rieke, G. H., Lebofsky, M. J., Low, F. J. 1985, AJ 90, 900

[252] Roth, M., Iriarte, M., Resendiz, G. 1983, RMxAA 9, 25

[253] Royer, P., Vreux, J.-M., Manfroid, F. 1998, A\&AS 130, 407

[254] Rufener, F. 1989, A\&AS 78, 469

[255] Sandage, A. 1997, PASP 109, 1193

[256] Sandage, A., Smith, L. L. 1963, ApJ 137, 1057

[257] Sandage, A., Visvanathan, N. 1978, ApJ 223, 707

[258] Savage, B.D., Mathis, J. S. 1979, ARA\&A 17, 73

[259] Scarfe, C. D. 1966, MNRAS 133, 99

[260] Schild, R. E., Peterson, D. M., Oke, J. B. 1971, ApJ 166, 95

[261] Schneider, P., J. Gunn, J. E., Hoessel, J. G. 1983, ApJ 264, 337

[262] Schneider, D. P., Schmidt, M., Gunn, J. E. 1989, AJ 98, 1507

[263] SCHOTT 1957, Farb-und filterglas für wissenschaft und technik, tech. pub.

[264] Schuster, W. J. 1984, RMxAA 9, 53

[265] Seeds, M. A. 1972, in Conference on Red Stars,

H. R. Johnson, J. P. Mutschlecner, and B. F. Peery ed.s, Bloomington, pag. 192

[266] Selby, M. J., Hepburn, I., Blackwell, D. E., Booth, A. J., Haddock, D. J., Arribas, S., Leggett, S. K., Mountain, C. M. 1988, A\&AS 74, 127

[267] Sharpless, S. 1963, in Basic Astronomical Data, K. A. Strand ed., Univ. Chicago Press, pag. 225

[268] Sharpless, S., Wawrukiewicz, A. S. 1973, AJ 78, 477

[269] Shure, M. A., Toomey, D. W., Rayner, J. T., Onaka, P. M., Denault, A. J., 1994, Proc. SPIE Vol. 2198, pag. 614

[270] Sinnerstad U., Arkling J., Alm S.H., Brattlund P. 1968, Ark. Astr. 5, 105

[271] Smak, J. 1966, Acta Astron. 16, 109

[272] Smith, L. F. 1968, MNRAS 140, 409

[273] Solheim, J. -E., de Vaucouleurs, G., de Vaucouleurs, A. 1982, A\&AS 49, 109

[274] Sorvari, J. M. 1974, AJ 79, 1416

[275] Spinrad, H., Taylor, B. J. 1969, ApJ 157, 1279 
Table 2. continued

[276] Stebbins, J., Huffer, C. M., Whitford, A. E. 1940, ApJ 91, 20

[277] Stebbins, J., Whitford, A. E. 1943, ApJ 98, 20

[278] Stebbins, J., Whitford, A. E. 1945, ApJ 102, 318

[279] Stebbins, J., Kron, G. E. 1956, ApJ 123, 440

[280] Stecher et al. 1992, ApJ 395, L1

[281] Stecher et al. 1997, PASP 109, 584

[282] Stephens, A. W., Frogel, J. A., Ortolani, S., Davies, R., Jablonka, P., Renzini, A., Rich, M. R. 2000, AJ 119, 314 (erratum in AJ 119, 419)

[283] Stock, J. 1956, ApJ 123, 253

[284] Straižys, V. 1973, A\&A 28, 349

[285] Straižys, V. 1995, Multicolor Stellar Photometry Pachart Publishing House, Tucson

[286] Straižys, V., Zdanavičius, K. 1965 Bull. Vilnius Obs. 14, 1

[287] Straižys, V., Zdanavičius, K. 1970, Bull. Vilnius Obs. 29, 15

[288] Straižys, V., Sŭdžius, J., and Kuriliene, G. 1976, A\&A 50, 413

[289] Straižys, V., Crawford, D. L., Davis Philip, A. G. 1996, Baltic Astron. 5, 83

[290] Straižys, V., Høg, E., Davis Philip, A. G. 1997, in Hipparcos-Venice '97, ESA SP-402, 761

[291] Strassmeier, K. G., Boyd, L. J., Epand, D. H., Granzer, Th. 1997, PASP 109, 697

[292] Strauss, F. M., Ducati, J.R. 1981, A\&AS 44, 337

[293] Strömgren, B. 1956, Vistas in Astronomy 2, 1337

[294] Strömgren, B. 1963, in Basic Astronomical Data, K. A. Strand ed., Univ. Chicago Press, pag. 123

[295] Strömgren, B. 1966, ARA\&A 4, 433

[296] Taylor, B. 1986, ApJS 60, 577

[297] Taylor, B. J., Joner, M. D., Johnson, S. B. 1989, AJ 97, 1798

[298] Tebbe, P. L. 1969, AJ 74, 920

[299] Tedesco, F. E., Tholen, D. J., Zellner, B. 1982, AJ 87, 1585

[300] Thomas, J. A., Hyland, A. R., Robinson, G. 1973, MNRAS 165, 201

[301] Thuan, X. T., Gunn, J. E. 1976, PASP 88, 543

[302] Tifft, W. G. 1958, AJ 63, 127

[303] Tifft, W. G. 1961, AJ 66, 390

[304] Tobin, W., Viton, M., Silvan, J.-P. 1994, A\&AS 107, 385

[305] Trefzger, C. F., Cameron, L. M., Spaenhauer, A., Steinlin, U.W. 1983, A\&A 117, 347

[306] Trefzger, C. F., Pel, J. W., Gabi, S. 1995, A\&A 304, 381

[307] Turner, D. 1990, PASP 102, 1331

[308] Vansevičius, V., Vazdekis, A., Prada, F. 1999, Balt. Astron., in press (astro-ph/9906353) 
Table 2. continued

[309] Vidal, J.-L. 1974, A\&A 34, 401

[310] Visvanathan, N., Sandage, A. 1977, ApJ 216, 214

[311] Viton, M., Burgarella, D., Cassatella, A., Prévot, L. 1988, A\&A 205, 147

[312] Wade, R. A., Hoessel, J. G., Elias, J. H., Huchra, J. P. 1979, PASP 91, 35

[313] Walraven, Th., Walraven J. H. 1960, BAN 15, 67

[314] Wamsteker, W. 1981, A\&A 97, 329

[315] Wawrukiewicz, A. S. 1971, PASP 83, 57

[316] Wegner, W. 1993, Acta Astron. 43, 209

[317] Weistrop, D. 1975 PASP 87, 367

[318] Wesselius, P. R., van Duinen, R. J., Aalders, J. W., Kester, D. 1980, A\&A 85, 221

[319] Wesselius, P. R., van Duinen, R. J., de Jonge, Aalders, J. W. G., Luinge, W., Wildeman, K. J. 1982, A\&AS 49, 427

[320] Westerlund B. E. 1966, ApJ 145, 725

[321] Wevers, B. M. H. R., van der Kruit, P. C., Allen, R. J. 1986, A\&AS 66, 505

[322] White, N. M., Wing, R. F. 1978, ApJ 222, 209

[323] Wickramasinghe D. T., and Strittmatter, P. A. 1972, MNRAS 160, 42

[324] Williams, E. G. 1936, ApJ 83, 279

[325] Williams, P. M. 1971, MNRAS 153, 171

[326] Williams, P. M. 1975, MNRAS 170, 343

[327] Wing, R. F. 1967, in Colloquium on Late-Type Stars, M. Hack ed., Trieste, pag. 205

[328] Wing, R. F. 1971, in Conference on Late-Type Stars,

G. W. Lockwood and H. M. Dyck ed.s, KPNO Contr. 554, pag. 145

[329] Wing, R. F., Spinrad, H., Kuhi, L. V. 1967, ApJ 147, 117

[330] Wing, R. F., Rinsland, C. P. 1981, RMxAA 6, 145

[331] Wood, D. B. 1966, ApJ 145, 36

[332] Wood, D. B. 1969, AJ 74, 177

[333] Yamashita, Y., Nishimura, S., Shimizu, M., Noguchi, T., Watanabe, E., Okida, K. 1977, PASJ 29, 731

[334] Yorka, S. 1983, AJ 88, 1816

[335] Zinn, R. 1980, ApJS 42, 19

[336] Menzies, J. W., Cousins, A. W. J., Banfield, R. M., Laing, J. D. 1989, Circulars of the South. Afr. Astron. Obs. 13, 1

[337] Menzies, J. W., Marang F., Laing, J. D., Coulson I. M., Engelbrecht, C. A. 1991, MNARS 248, 642 